\title{
A MODEL FOLLOWING INVERSE CONTROLLER WITH ADAPTIVE COMPENSATION FOR GENERAL AVIATION AIRCRAFT
}

\author{
A Dissertation by \\ Hugh S. Bruner \\ Master of Science, Wichita State University, 1986 \\ Bachelor of Science, University of Kansas, 1979
}

Submitted to the Department of Aerospace Engineering and the faculty of the Graduate School of

Wichita State University in partial fulfillment of the requirements for the degree of Doctor of Philosophy 
(C) Copyright 2007 by Hugh S. Bruner

All Rights Reserved 


\section{A MODEL FOLLOWING INVERSE CONTROLLER WITH ADAPTIVE COMPENSATION FOR GENERAL AVIATION AIRCRAFT}

I have examined the final copy of this dissertation for form and content, and recommend that it be accepted in partial fulfillment of the requirement for the degree of Doctor of Philosophy with a major in Aerospace Engineering.

James Steck, Committee Chair

We have read this dissertation and recommend its acceptance:

Benham Bahr, Committee Member

Roy Myose, Committee Member

Gawad Nagati, Committee Member

Kamran Rokhsaz, Committee Member

Accepted for the College of Engineering

Zulma Toro-Ramos, Dean

Accepted for the Graduate School

Susan Kovar, Dean 


\section{ACKNOWLEGEMENTS}

Earning my Ph.D. has been a long process. It has been fun, but I never could have done it on my own. I have received help from so many people. Some are teachers, some are wellwishers, and some are family. Space doesn't allow me to name them all, so I must summarize the list. My apologies to those I don't name.

First, let me thank my advisor, Dr. James Steck. Dr. Steck sparked my first curiosities with neural networks and has been fanning the flames ever since with his encouragement and good advice. I appreciate his patience as I searched and searched, looking for a topic for which I could have a real passion. At last, I found it.

Next, I need to thank the Hawker Beechcraft Corporation for its support. I appreciate the financial support I received for my education through Hawker Beechcraft's tuition reimbursement program. It is a pleasure to work for an employer that recognizes the need to invest in the continuing education of its employees. But beyond that, I truly want to thank the people there that provided moral support and kept me going when my motivation was waning: Mr. Gary Bickel, Dr. Leland Johnson Jr., Mr. David Riemer, and Mr. Randy Nelson.

Most of all, I want to thank my loved ones that both cheered me on and, at the same time, endured my obsession with school: Diane Bruner, Heather and Justin Ryan, Stephanie and Tim Bruner, and my mother Maxine Bruner. I am grateful for their forbearance, and now I look forward to making amends for the time I didn't spend with them. 


\begin{abstract}
The theory for an adaptive inverse flight controller, suitable for use on General Aviation aircraft, is developed in this research. The objectives of this controller are to separate the normally coupled modes of the basic aircraft and thereby permit direct control of airspeed and flight-path angle, meet prescribed performance characteristics as defined by damping ratio and natural frequency, adapt to uncertainties in the physical plant, and be computationally efficient.

The three basic elements of the controller are a linear prefilter, an inverse transfer function, and an adaptive neural network compensator. The linear prefilter shapes accelerations required of the overall system in order to achieve the desired system performance characteristics. The inverse transfer function is used to compute the aircraft control inputs required to achieve the necessary accelerations. The adaptive neural network compensator is used to compensate for modeling errors during design or real-time changes in the physical plant. This architecture is patterned after the work of Calise, but differs by not requiring dynamic feedback of the state variables.

The controller is coded in ANSI C and integrated with a simulation of a typical General Aviation aircraft. Twenty-three cases are simulated to prove that the objectives for the controller are met. Among these cases are simulated stability and controllability failures in the physical plant, as well as several simulated failures of the neural network. With the exception of some bounded speed-tracking error, the controller is capable of continued flight with any foreseeable failure of the neural network.
\end{abstract}

Recommendations are provided for follow-on investigations by other researchers. 


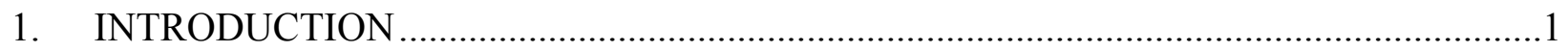

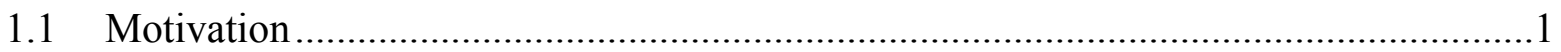

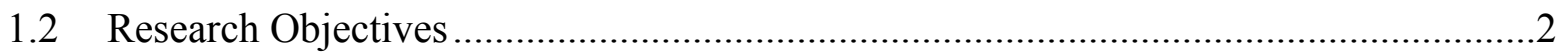

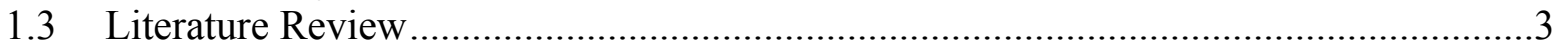

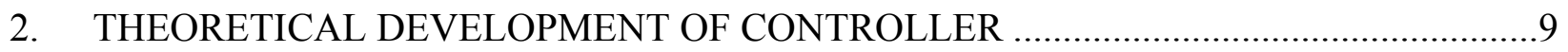

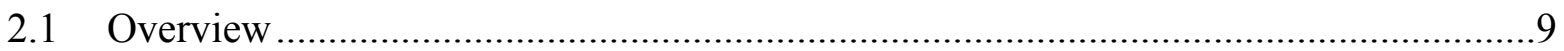

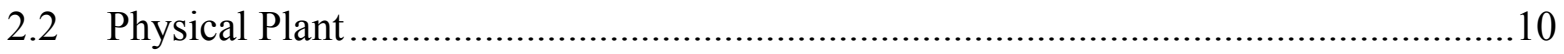

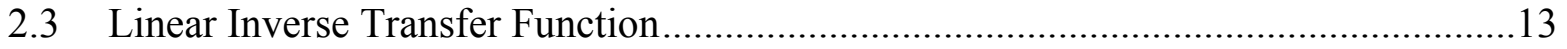

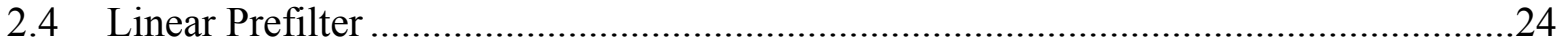

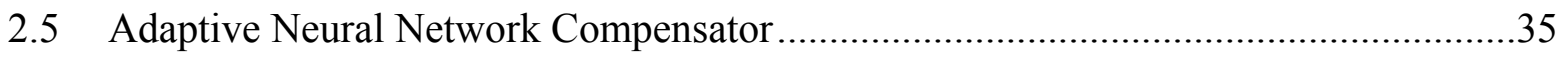

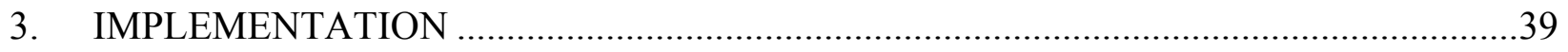

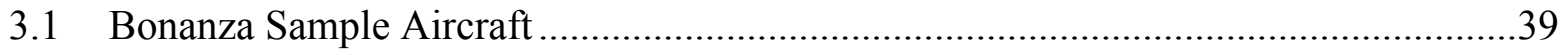

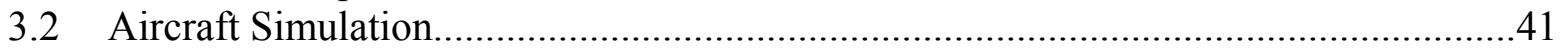

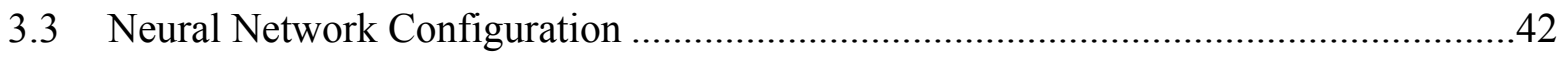

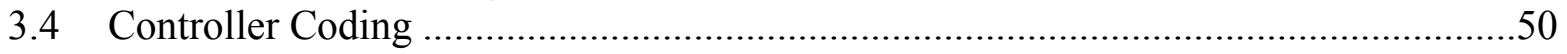

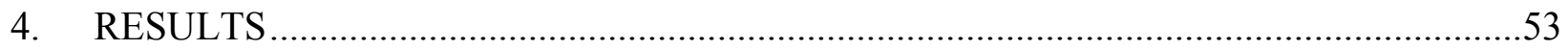

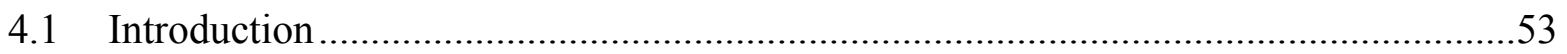

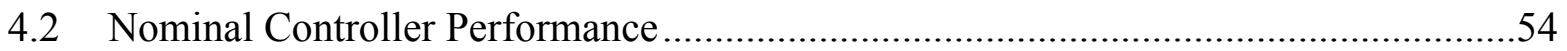

4.3 Failures Involving Stability and Controllability of the Physical Plant .........................75

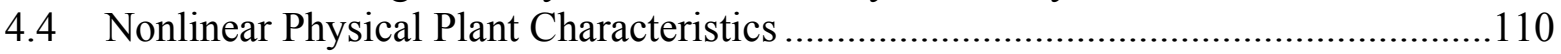

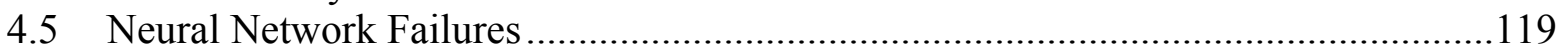

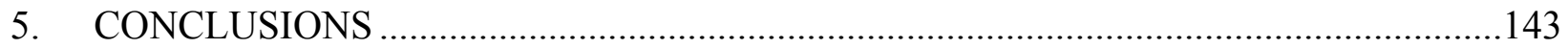

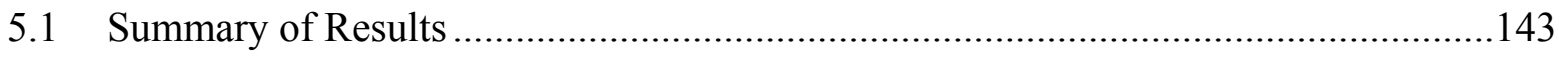

5.2 Recommendations for Future Study ....................................................................144

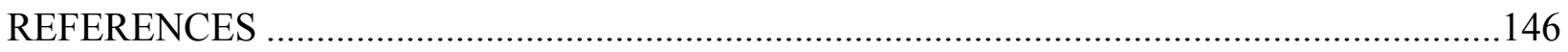

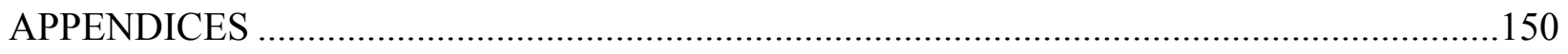

A. State Space Representation for the Linearized Aircraft Model .................................151

B. Equations of Motion Used in Modeling Aircraft Flight Dynamics ...........................155

C. Program Listing................................................................................................. 


\section{LIST OF TABLES}

Table

Page

1. Beechcraft Model F33C Summary Characteristics .........................................................11

2. Summary of Simulation Cases Examined ...................................................................56 


\section{LIST OF FIGURES}

Figure

Page

1. The decline of General Aviation deliveries since 1978 1

2. Typical configuration of dynamic inversion controller................................................5

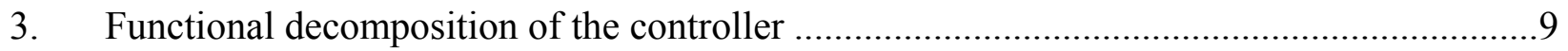

4. Summary architecture of adaptive inverse controller................................................10

5. Three-view drawing of the Beechcraft F33C Bonanza...................................................12

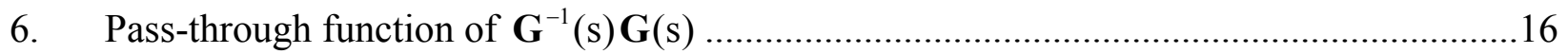

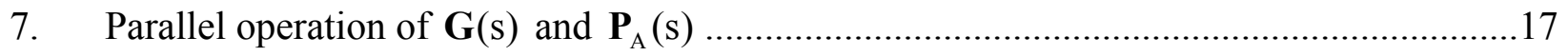

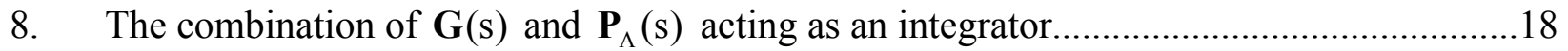

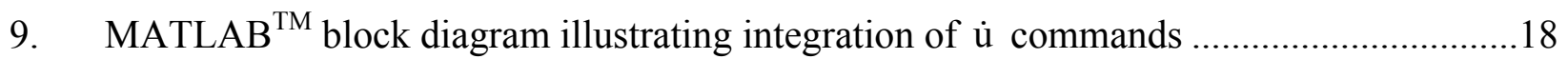

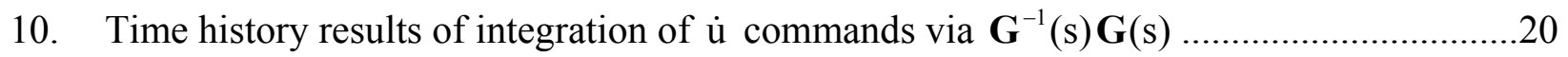

11. Time histories of state variables resulting from $\dot{\mathrm{u}}$ commands ......................................21

12. MATLAB ${ }^{\mathrm{TM}}$ block diagram illustrating integration of $\dot{\alpha}$ commands ..............................22

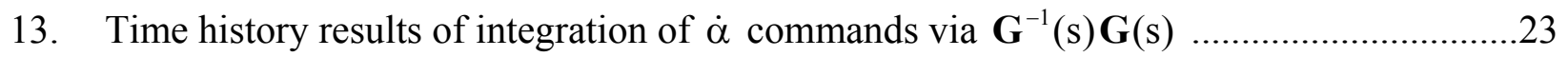

14. Time histories of state variables resulting from $\dot{\alpha}$ commands ....................................24

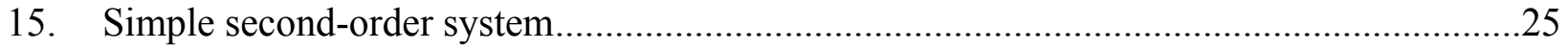

16. Rearrangement of second-order system, with no change in connectivity........................26

17. Customizing second-order system for speed control …........................................27

18. Functional grouping of elements within speed control …........................................27

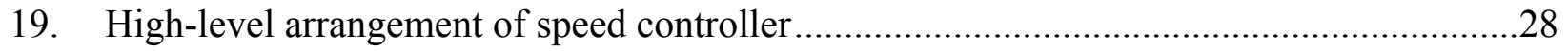

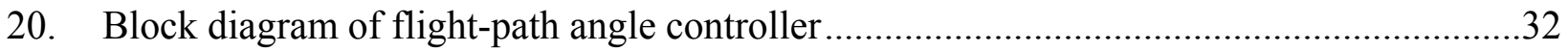




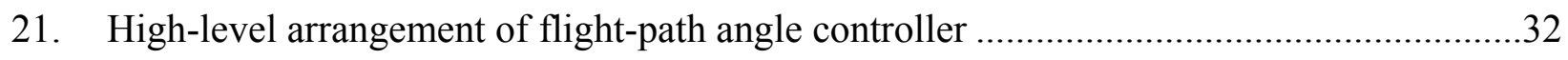

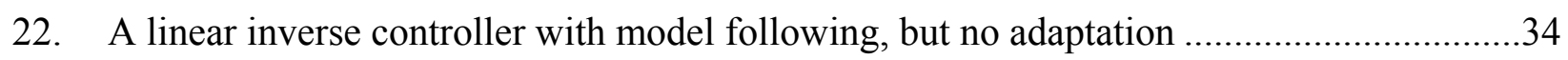

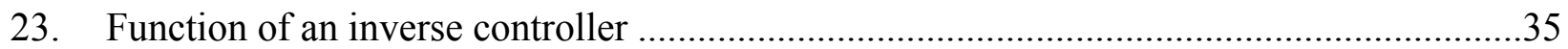

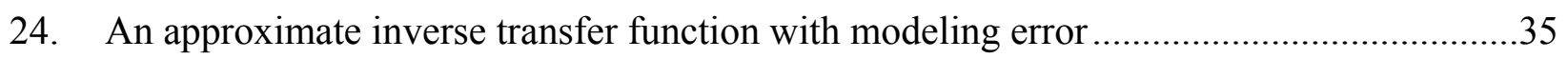

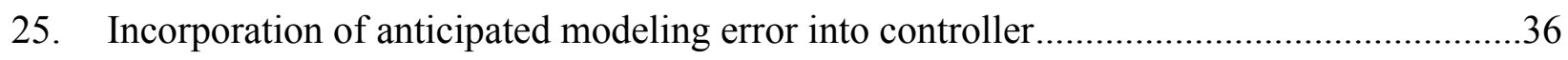

26. Incorporation of adaptive neural network to approximate modeling error ........................37

27. Addition of neural network preserves integration function of controller..........................38

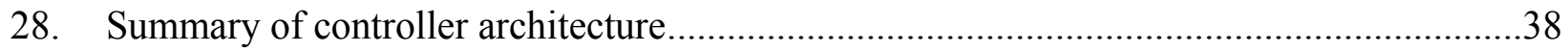

29. In-flight photo of Hawker Beechcraft Corporation's fly-by-wire Bonanza, CJ-144 ..........40

30. Cockpit of Hawker Beechcraft Corporation's fly-by-wire Bonanza, CJ-144 ...................40

31. Equivalency of neural network and state space matrix ...........................................43

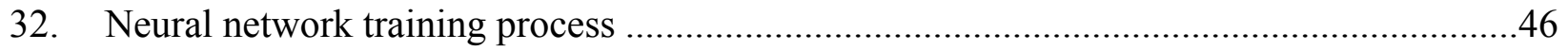

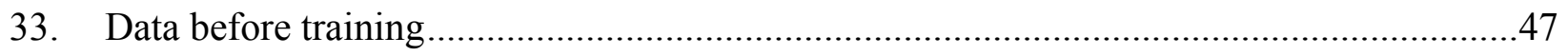

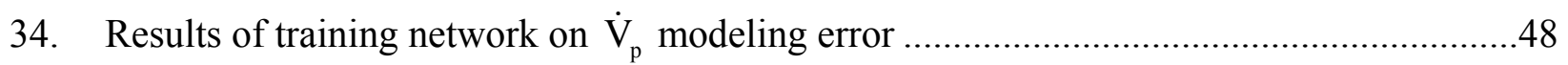

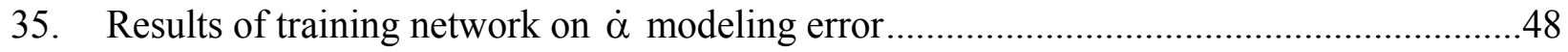

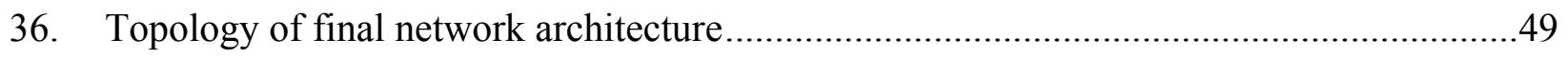

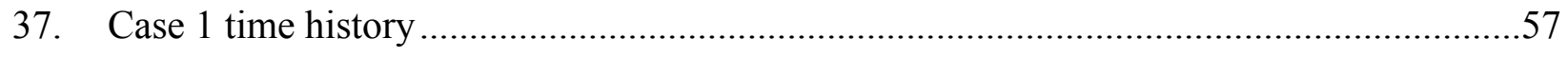

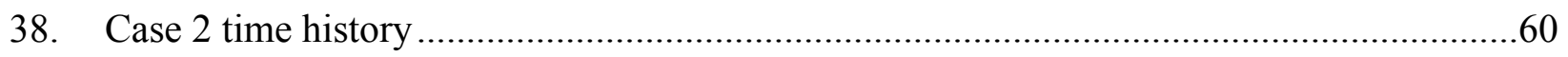

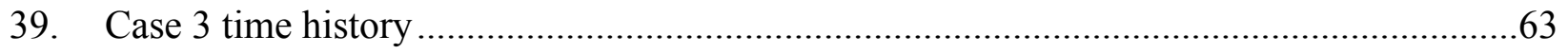

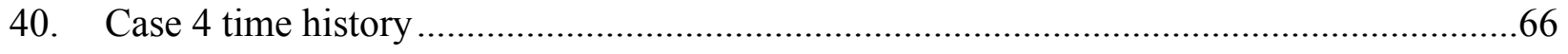

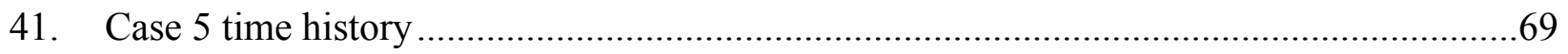

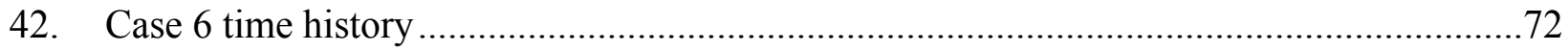




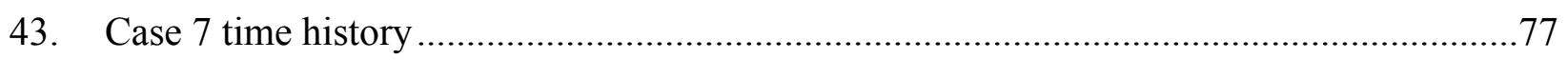

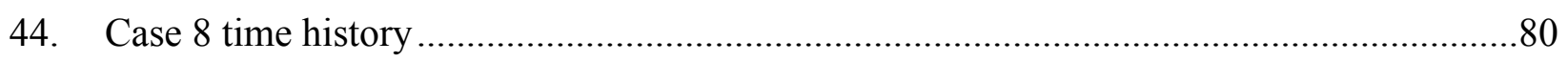

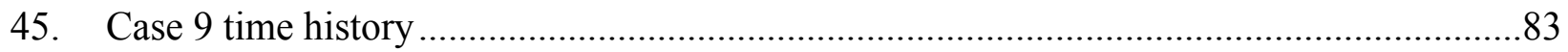

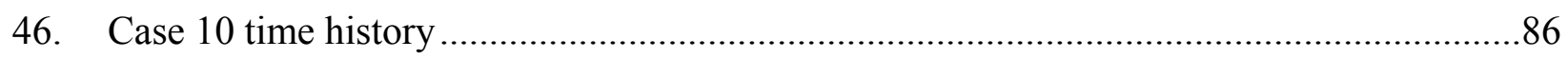

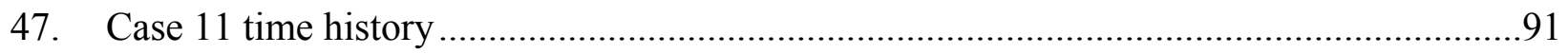

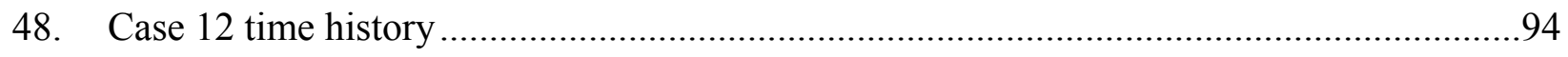

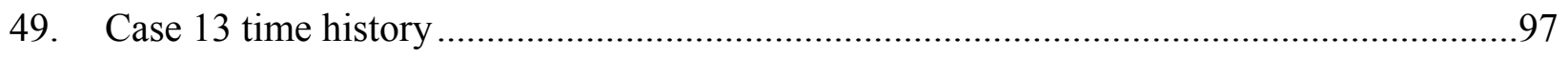

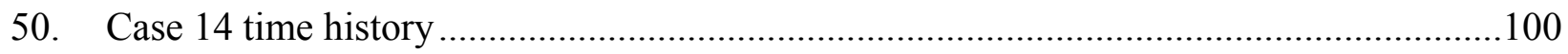

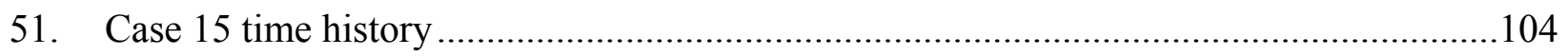

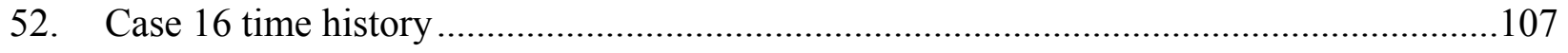

53. Simulated nonlinear lift coefficient characteristics .............................................110

54. Simulated nonlinear pitching moment coefficient characteristics ...............................111

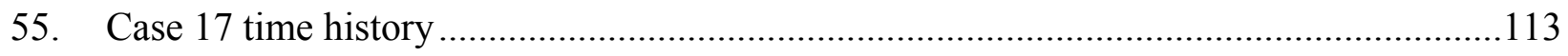

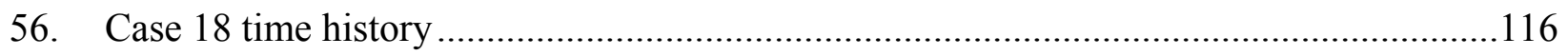

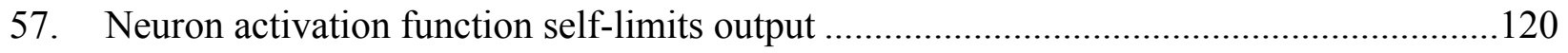

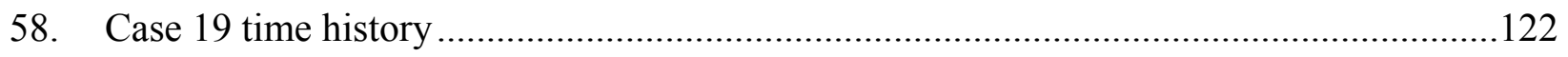

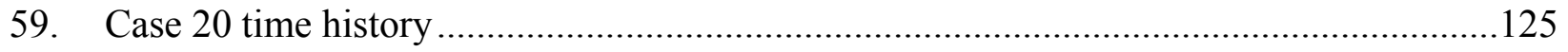

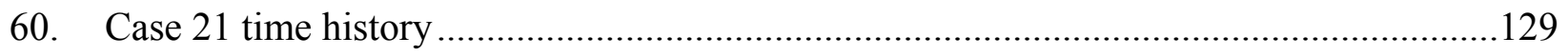

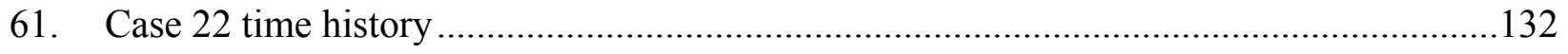

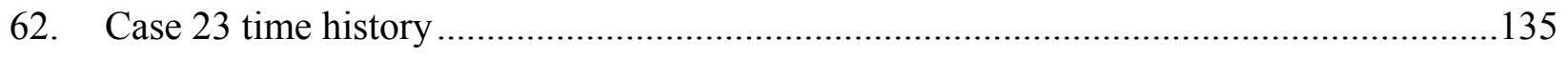

63. Speed controller block diagram with erroneous neural network signal .........................138

64. Speed sensitivity to oscillatory $\Delta \dot{\mathrm{u}}$ errors from the neural network.............................140 
65. Flight-path sensitivity to oscillatory $\Delta \dot{\mathrm{u}}$ errors from the neural network.......................142

66. Flight-path sensitivity to oscillatory $\Delta \dot{\alpha}$ errors from the neural network .......................142 


\section{LIST OF SYMBOLS}

b Wing span

$\mathrm{C}_{\mathrm{D}} \quad$ Drag coefficient

$\mathrm{C}_{\mathrm{D}_{\mathrm{K}}} \quad$ Partial derivative of drag coefficient with respect to lift coefficient squared

$\mathrm{C}_{\mathrm{D}_{0}} \quad$ Drag coefficient at zero lift

$\mathrm{C}_{\mathrm{L}} \quad$ Lift coefficient

$\mathrm{C}_{\mathrm{L}_{0}} \quad$ Lift coefficient at zero angle of attack

$\mathrm{C}_{\mathrm{L}_{\alpha}} \quad$ Partial derivative of lift coefficient with respect to angle of attack

$\mathrm{C}_{\mathrm{L}_{\dot{\alpha}}} \quad$ Partial derivative of lift coefficient with respect to non-dimensional rate of change of angle of attack

$\mathrm{C}_{\mathrm{L}_{\delta \mathrm{e}}} \quad$ Partial derivative of lift coefficient with respect to elevator deflection

$\mathrm{C}_{\mathrm{L}_{\delta \mathrm{tab}}} \quad$ Partial derivative of lift coefficient with respect to elevator tab deflection

$\mathrm{C}_{\mathrm{L}_{\delta_{\mathrm{t}}}} \quad$ Partial derivative of lift coefficient with respect to thrust

$\mathrm{C}_{\mathrm{L}_{\mathrm{q}}} \quad$ Partial derivative of lift coefficient with respect to non-dimensional pitch rate

$\mathrm{C}_{1} \quad$ Rolling moment coefficient, in stability axis

$\mathrm{C}_{1_{\beta}} \quad$ Partial derivative of rolling moment coefficient with respect to sideslip angle

$\mathrm{C}_{1_{\delta_{\mathrm{a}}}} \quad$ Partial derivative of rolling moment coefficient with respect to aileron deflection

$\mathrm{C}_{1_{\delta_{\mathrm{r}}}} \quad$ Partial derivative of rolling moment coefficient with respect to rudder deflection

$\mathrm{C}_{\mathrm{l}_{\mathrm{p}}} \quad$ Partial derivative of rolling moment coefficient with respect to non-dimensional roll rate 


\section{LIST OF SYMBOLS (continued)}

$\mathrm{C}_{\mathrm{lr}} \quad$ Partial derivative of rolling moment coefficient with respect to non-dimensional yaw rate

$\mathrm{C}_{\mathrm{M}} \quad$ Pitching moment coefficient

$\mathrm{C}_{\mathrm{M}_{0}} \quad$ Pitching moment coefficient at zero angle of attack

$\mathrm{C}_{\mathrm{M}_{\alpha}} \quad$ Partial derivative of pitching moment coefficient with respect to angle of attack

$\mathrm{C}_{\mathrm{M}_{\dot{\alpha}}} \quad$ Partial derivative of pitching moment coefficient with respect to non-dimensional rate of change of angle of attack

$\mathrm{C}_{\mathrm{M}_{\delta_{e}}} \quad$ Partial derivative of pitching moment coefficient with respect to elevator deflection

$\mathrm{C}_{\mathrm{M}_{\text {סेab }}} \quad$ Partial derivative of pitching moment coefficient with respect to elevator tab deflection

$\mathrm{C}_{\mathrm{M}_{\delta_{t}}} \quad$ Partial derivative of pitching moment coefficient with respect to thrust

$\mathrm{C}_{\mathrm{M}_{\mathrm{q}}} \quad$ Partial derivative of pitching moment coefficient with respect to non-dimensional pitch rate

$\mathrm{C}_{\mathrm{n}} \quad$ Yawing moment coefficient, in stability axis

$\mathrm{C}_{\mathrm{n}_{\beta}} \quad$ Partial derivative of yawing moment coefficient with respect to sideslip angle

$\mathrm{C}_{\mathrm{n}_{\delta_{a}}} \quad$ Partial derivative of yawing moment coefficient with respect to aileron deflection

$\mathrm{C}_{\mathrm{n}_{\delta_{r}}} \quad$ Partial derivative of yawing moment coefficient with respect to rudder deflection

$\mathrm{C}_{\mathrm{n}_{\mathrm{p}}} \quad$ Partial derivative of yawing moment coefficient with respect to non-dimensional roll rate

$\mathrm{C}_{\mathrm{n}_{\mathrm{r}}} \quad$ Partial derivative of yawing moment coefficient with respect to non-dimensional yaw rate 


\section{LIST OF SYMBOLS (continued)}

$\mathrm{C}_{\mathrm{y}} \quad$ Side force coefficient, in stability axis

$\mathrm{C}_{\mathrm{y}_{\beta}} \quad$ Partial derivative of side force coefficient with respect to sideslip angle

$\mathrm{C}_{\mathrm{y}_{\delta_{a}}} \quad$ Partial derivative of side force coefficient with respect to aileron deflection

$\mathrm{C}_{\mathrm{y}_{\delta_{\mathrm{r}}}} \quad$ Partial derivative of side force coefficient with respect to rudder deflection

$\mathrm{C}_{\mathrm{y}_{\mathrm{p}}} \quad$ Partial derivative of side force coefficient with respect to non-dimensional roll rate

$\mathrm{C}_{\mathrm{y}_{\mathrm{r}}} \quad$ Partial derivative of side force coefficient with respect to non-dimensional yaw rate

$\overline{\mathrm{c}} \quad$ Mean aerodynamic chord length

$\mathrm{F}_{\mathrm{X}_{\mathrm{b}}} \quad \mathrm{X}$ component of force in body axis

$\mathrm{F}_{\mathrm{Y}_{\mathrm{b}}} \quad \mathrm{Y}$ component of force in body axis

$\mathrm{F}_{\mathrm{Z}_{\mathrm{b}}} \quad \mathrm{Z}$ component of force in body axis

g Gravitational constant

h $\quad$ Altitude

$\dot{\mathrm{h}} \quad$ Rate of change in altitude

I Identity matrix

$\mathrm{I}_{\mathrm{xx}_{\mathrm{b}}} \quad$ Rolling moment of inertia, in body axis

$\mathrm{I}_{\mathrm{yy}_{\mathrm{b}}} \quad$ Pitching moment of inertia, in body axis

$\mathrm{I}_{\mathrm{zz}_{\mathrm{b}}} \quad$ Yawing moment of inertia, in body axis

KEAS Equivalent airspeed, in knots

$\mathrm{k} \quad$ Computational step in discrete time domain 


\section{LIST OF SYMBOLS (continued)}

knots Nautical miles per hour

$\mathrm{L}_{\mathrm{b}} \quad$ Rolling moment, in body axis

$\mathrm{L}_{\mathrm{s}} \quad$ Rolling moment, in stability axis

LTI Linear time invariant

$\mathrm{M}_{\mathrm{b}} \quad$ Pitching moment, in body axis

$\mathrm{M}_{\mathrm{s}} \quad$ Pitching moment, in stability axis

m Airplane mass

$\mathrm{N}_{\mathrm{b}} \quad$ Yawing moment, in body axis

$\mathrm{N}_{\mathrm{s}} \quad$ Yawing moment, in stability axis

$\mathrm{n}_{\mathrm{z}} \quad$ Load factor in direction of lift vector

$\mathrm{P}_{\mathrm{b}} \quad$ Roll rate, in body axis

$\mathrm{P}_{\mathrm{S}} \quad$ Roll rate, in stability axis

PLA Power-lever angle

$\hat{\mathrm{p}} \quad$ Non-dimensional roll rate

psf pounds per square foot

$\mathrm{Q}_{\mathrm{b}} \quad$ Pitch rate, in body axis

$\mathrm{Q}_{\mathrm{s}} \quad$ Pitch rate, in stability axis

$\hat{\mathrm{q}} \quad$ Non-dimensional pitch rate

$\overline{\mathrm{q}} \quad$ Dynamic pressure

$\mathrm{R}_{\mathrm{b}} \quad$ Yaw rate, in body axis 


\section{LIST OF SYMBOLS (continued)}

\begin{tabular}{ll}
$\mathrm{R}_{\mathrm{s}}$ & Yaw rate, in stability axis \\
$\hat{\mathrm{r}}$ & Non-dimensional yaw rate \\
$\mathbf{r}$ & Command vector \\
$\mathrm{rad}$ & radians \\
$\mathrm{S}$ & Wing area \\
$\mathrm{s}$ & Laplace variable \\
$\mathrm{sec}$ & seconds \\
$\mathrm{T}$ & Period \\
$\mathrm{t}$ & time \\
$\mathrm{U}_{\mathrm{b}}$ & X component of velocity in body axis \\
$\mathbf{u}$ & Control vector \\
$\mathrm{u}_{\mathrm{b}}$ & Perturbation $\mathrm{X}$ velocity in stability axis \\
$\mathrm{V}_{\mathrm{b}}$ & Y component of velocity in body axis \\
$\mathrm{V}_{\mathrm{p}}$ & Airplane true airspeed \\
$\mathrm{W}$ & Airplane weight \\
$\mathrm{W}_{\mathrm{b}}$ & Z component of velocity in body axis \\
$\mathbf{x}$ & State vector \\
$\mathbf{y}$ & Output vector \\
\hline
\end{tabular}

\section{Greek Symbols}
$\alpha \quad$ Angle of attack
$\dot{\alpha} \quad$ Rate of change of angle of attack 


\section{LIST OF SYMBOLS (continued)}

$\hat{\dot{\alpha}} \quad$ Non-dimensional rate of change of angle of attack

$\beta \quad$ Sideslip angle

$\gamma \quad$ Flight-path angle

$\Delta \quad$ Physical plant rate error vector

$\Delta \dot{\alpha} \quad$ Neural network angle of attack rate error

$\Delta \mathrm{u}_{\mathrm{b}} \quad$ Neural network velocity rate error

$\delta_{\mathrm{a}} \quad$ Aileron deflection

$\delta_{\mathrm{e}} \quad$ Elevator deflection

$\delta_{\mathrm{t}} \quad$ Thrust command

$\varepsilon \quad$ Simulated neural network error signal

$\zeta \quad$ Damping ratio

$\Theta \quad$ Airplane pitch attitude angle in Earth axis system

$\rho \quad$ Density of air

$\Phi \quad$ Airplane bank angle attitude in Earth axis system

$\Psi \quad$ Airplane heading angle in Earth axis system

$\omega \quad$ Frequency

$\omega_{\mathrm{n}} \quad$ Natural frequency

\section{Subscripts}

A Approximation

b Body axis

G Relating to system G(s) 


\section{LIST OF SYMBOLS (continued)}

I Inverse

P Physical plant

S Stability axis

xviii 


\section{CHAPTER 1}

\section{INTRODUCTION}

\subsection{Motivation}

Data from General Aviation manufacturers show that deliveries of new aircraft have significantly declined since 1978 [1]. This decline has been attributed to a number of factors: the price of fuel, product liability, the declining number of military veteran pilots, competing modes of transportation, the high cost of learning to fly, the high cost of maintaining pilot proficiency, and perceptions of safety.

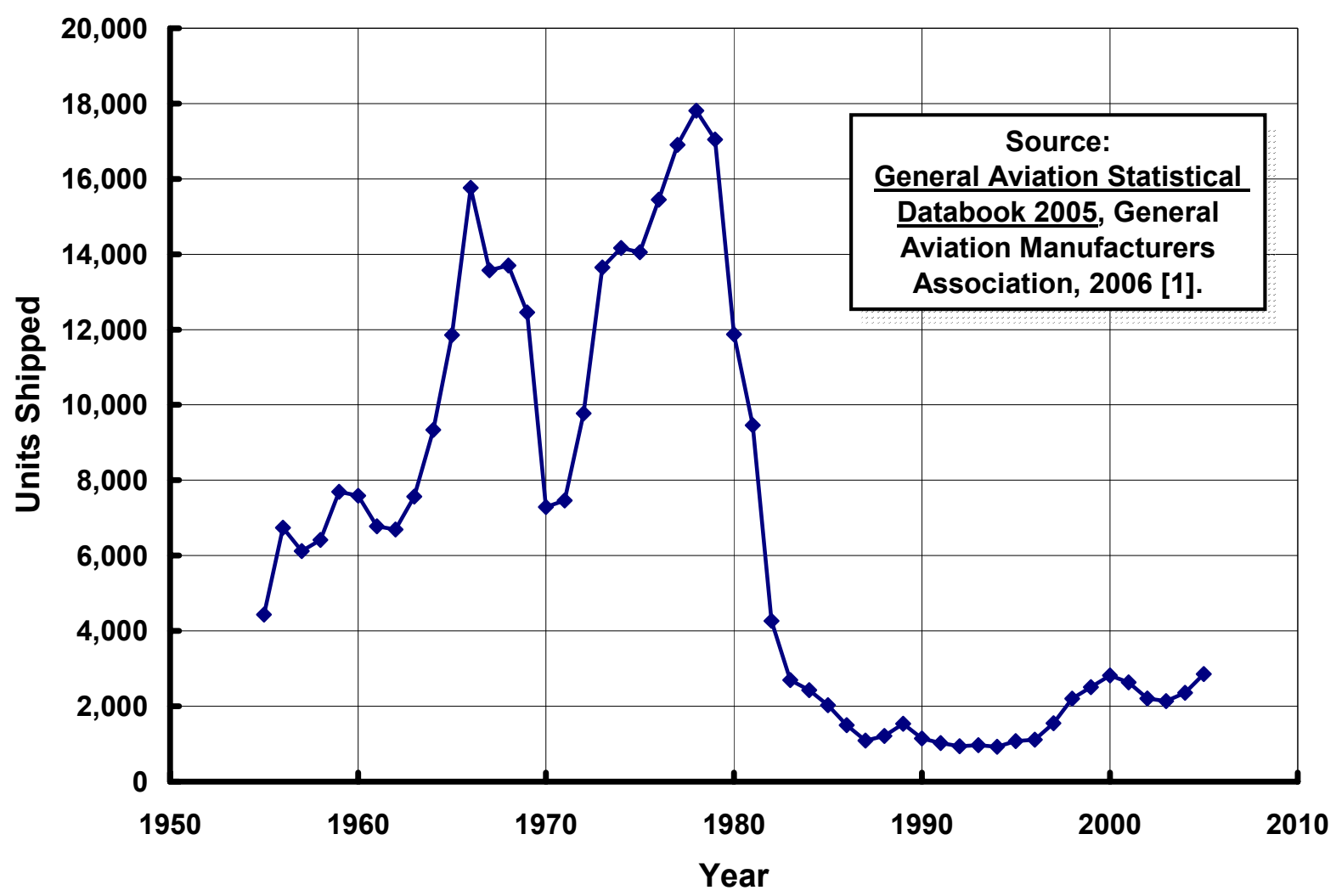

Figure 1: The decline of General Aviation deliveries since 1978 
NASA speculates that a renewed interest in personal aviation might be stimulated if these last three factors are addressed [2]. Aircraft that are easier to fly would reduce the associated cost of learning to fly and, additionally, eliminate the burdensome requirements of maintaining instrument proficiency. Furthermore, safety would be increased if consistent predictable flying qualities could be maintained, regardless of the aircraft failure state. These speculations by NASA suggest that a resurgence in personal flying may be possible if aircraft can be built with easy-to-use intuitive flight controls that adapt to changes in the aircraft operating state.

The research described herein is motivated by the desire to make aviation more accessible to the masses who have the desire for personal air transportation but do not have the free time available for learning the traditional piloting technique.

\subsection{Research Objectives}

Traditional aircraft controls have a one-to-one correspondence with the control surfaces and the throttle [3]. In this "stick and rudder" mode of flying, the pilot does not control the endgoal states of the aircraft: typically speed, altitude, and heading. The pilot serves as, at least, a second-order integrator as he "closes the loop" between aircraft accelerations and desired endstates. This task is further complicated by the coupled oscillatory modes typically found in General Aviation aircraft. Workload, accuracy, and safety are improved if the pilot is able to more directly command the desired end-state objectives.

The objectives of this research are intended to address these issues in order to enable more direct control of the aircraft. To achieve this, a controller architecture will be derived that does the following:

1. Separates the normally coupled modes of the aircraft.

2. Permits direct control of flight-path angle, $\gamma$, and speed, $\mathrm{V}_{\mathrm{p}}$. 
3. Achieves prescribed overall system dynamic characteristics.

4. Adapts to uncertainties in the system, including real-time changes to the physical plant, such as failures.

5. Is computationally efficient.

The overarching goal is to strive for a system that is simple, robust, and relevant to General Aviation aircraft.

This research will address only the longitudinal characteristics of the aircraft. It is expected that the same method can be applied to the lateral-directional modes.

\subsection{Literature Review}

Significant work has taken place over the past decade with respect to advanced flight controls. Beringer [3] looked at the application of "Performance Control Systems" (PCS) to General Aviation aircraft. Significant reductions in workload and piloting error were found if the pilot directly controlled "higher level performance variables," such as rate of climb or airspeed. Piloted simulations were conducted that permitted back-to-back evaluation of conventional controls versus decoupled fly-by-wire controls. Decoupled flight controls showed about one-third the control activity (reduced workload) and one-third the tracking error (reduced piloting error), when compared to conventional flight controls.

Duerksen examined the application of adaptive flight controls on General Aviation aircraft [4]. Duerksen accomplished his adaptation using fuzzy logic. Decoupled flight controls were implemented for speed and flight-path angle control. The primary objective for this effort was to develop a generic controller that could be independent of aircraft type. To illustrate the concept, the technique was applied to simulations of two disparate aircraft types: a Beechjet 400A and Beechcraft Bonanza. One aircraft is a jet, and the other a piston-powered single- 
engine aircraft. Using fuzzy logic, the two aircraft were made to handle similarly. The fuzzy logic algorithms developed by Duerksen are the algorithms later implemented by Beringer in his work on PCS controllers [3].

Calise, Rysdyk et al. have accomplished significant work in the control of nonlinear systems using a technique called "dynamic inversion" $[5,6,7,8,9]$. This control architecture is illustrated in Figure 2. For any physical system described by the transfer function $f$, the key element of this technique is to find an approximate inverse transfer function $\hat{f}^{-1}$. A "pseudo control variable," $v$, is calculated that closely represents the desired rates of change of the state variable $x$. This pseudo control variable is fed into the inverse transfer function to create control inputs, $\delta$, to the physical system. If $\widehat{f}^{-1}$ is a perfect inverse of $f$, then $\hat{f}^{-1} f=\mathbf{I}$, and the output from $f$ would be precisely $v$. The integration of $v$ would give $x$. However, it is stated that $\widehat{f}^{-1}$ is only an approximation of a perfect inverse, and therefore there is some inversion error. This inversion error might arise from modeling error or uncertainties in the physical system. To compensate for this inversion error, an adaptive neural network is used to "learn" what the error is and, in turn, provide a compensating signal back into the controller. The pseudo control variable, $v$, has the beneficial addition of this compensating signal. The intent is to null out the inversion error. The handling qualities and linear control blocks upstream convert the pilot control inputs into the desired rates, as required to achieve the desired handling characteristics. 


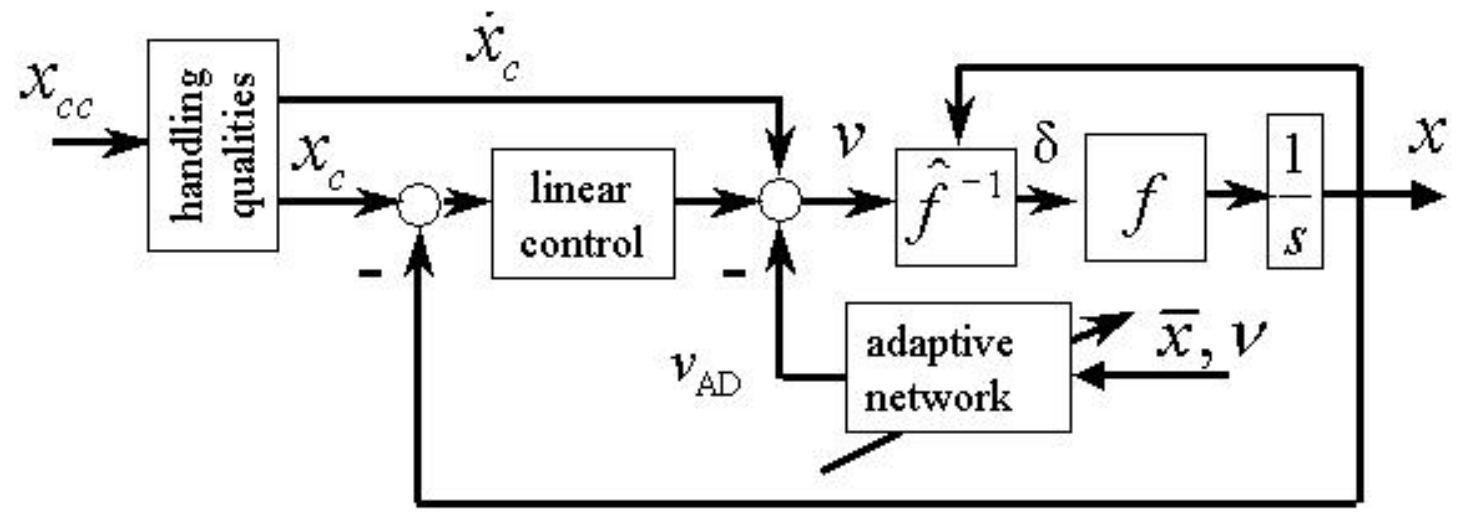

Figure 2: Typical configuration of dynamic inversion controller [9]

Various applications of this technique have been demonstrated in simulation. A rate and attitude command system was demonstrated by Calise and Rysdyk for the highly nonlinear XV15 tilt-rotor aircraft [5]. MacFarland and Calise demonstrated angle of attack and bank angle control for an agile anti-air missile in regions of significant aerodynamic nonlinearity [6]. Hovakimyan et al. used dynamic inversion techniques for the control of an R-50 helicopter [7]. Hovakimyan's application is interesting in that an observer was included as part of the controller to estimate plant states not directly measurable. In this instance, the neural network learned from the observer outputs, not from direct measurement of the states. Rysdyk expanded the XV-15 controller to include lateral directional control [8]. This work included an expansion of the work by Calise and Rysdyk [5] but, more importantly, included a proof that showed the outputs of a neural network are bounded. Rysdyk and Agarwal applied the technique to implementation of a "Total Energy Control System" [9], as originally proposed by Lambregts [10]. In this work, the concept of "pseudo control hedging" was proposed as a means for "anti-windup protection" in the neural network. 
Research at Wichita State University (WSU) by Steck, Rokhsaz et al., has involved integration of the Calise methods with the works of Lambregts and Duerksen to implement adaptive feedback control into General Aviation aircraft $[11,12,13,14]$. Investigations have been made into the FAA certification of neural networks in General Aviation aircraft [13]. Considerable effort has been invested in simulations, both desktop simulations with MATLAB ${ }^{\mathrm{TM}}$ and pilot-in-the loop simulations [14]. These simulations investigated the ability of the WSU algorithms to cope with aircraft failures involving both stability ("A matrix" failures) and/or controllability ("B matrix" failures). Work is ongoing to flight-test an adaptive controller in a fly-by-wire test-bed aircraft owned by Hawker Beechcraft Corporation.

While not directly related to aircraft, another work of interest is that conducted by Hess [15]. These investigations looked at the application of recursive neural networks (RNN) in the control of large warships. Recursion is of interest because it allows for dynamics to be included in the neural network. This suggests that a better representation of modeling error, particularly dynamic errors, may be obtained with a recursive neural network. Inputs to the neural network are taken from a number of prior time steps. Finite differences between time steps enable the network to approximate first-order derivatives, or higher, depending on the number of preceding time steps included. This work also provides good examples of substantial pre- and postprocessing of network parameters in generalizing the complexity of maneuvering a large ship in extreme sea-states. The final neural network for this application involved 88 inputs, two hidden layers, and 134 computational nodes.

Many observations were gleaned from these past works that are applicable to the current topic. 
First, the work of Calise et al. was applied to highly nonlinear systems, where the inverse transfer function depended on dynamic state feedback to adequately characterize the inverse. For General Aviation aircraft, the problem is generally linear. Assuming linearity suggests that a simple inverse transfer function can be formulated to enable strictly feed-forward operations. Where this assumption of linearity may not be valid, it is proposed that a greater emphasis can be placed on the neural network to address residual nonlinearities.

Second, once again taking credit for largely linear aerodynamics, it is proposed that the algebraic expressions for the inverse transfer function developed by Steck et al. can be replaced by a linear time invariant (LTI) state space representation of the inverse transfer function. Linear algebra should provide for faster execution within the controller. Furthermore, opportunities are identified to improve upon the flight-path control laws by controlling normal load factor, $\mathrm{n}_{\mathrm{z}}$, rather than pitch rate acceleration, $\ddot{\theta}$, as the means to achieve a desired flight-path. Steck addressed the entire six degree of freedom problem. This work only addresses the longitudinal case.

Finally, it is proposed that since the inverse transfer function is a dynamic system, it is safe to assume that the inversion error is likely to involve dynamics also. Consequently, the recursive neural networks discussed by Hess may provide benefit in accurately capturing any dynamics present in the inversion error.

With these observations in mind, the following items are addressed in this dissertation:

1. An adaptive inverse controller similar to those presented by Calise et al. will be used as a starting point.

2. A closed form of the inverse transfer function will be implemented, not dependent on dynamic feedback. 
3. A refined control law for flight-path angle will be derived using normal load factor, $\mathrm{n}_{\mathrm{z}}$, as the key variable.

4. Inversion errors will be cancelled using a recursive neural network.

This approach will be used to derive a control architecture that satisfies the objectives identified in subchapter 1.2. 


\section{CHAPTER 2}

\section{THEORETICAL DEVELOPMENT OF CONTROLLER}

\subsection{Overview}

The functional objectives of the controller are to enable the pilot to directly control the aircraft end-states and to have the overall system react with the desired dynamic response. These objectives are decomposed in Figure 3 into a number of subfunctions. The pilot commands his desired end-state. These commands are interpreted in the context of the desired overall system dynamics. Once the path from the current-state to the end-state is determined, aircraft control movements are computed and fed into the aircraft. The aircraft will react in response to these control inputs. Feedback from the airplane response is used to assure consistency with the desired dynamic characteristics. Furthermore, feedback from the airplane response may lead to modification of the control inputs to achieve better overall system performance.

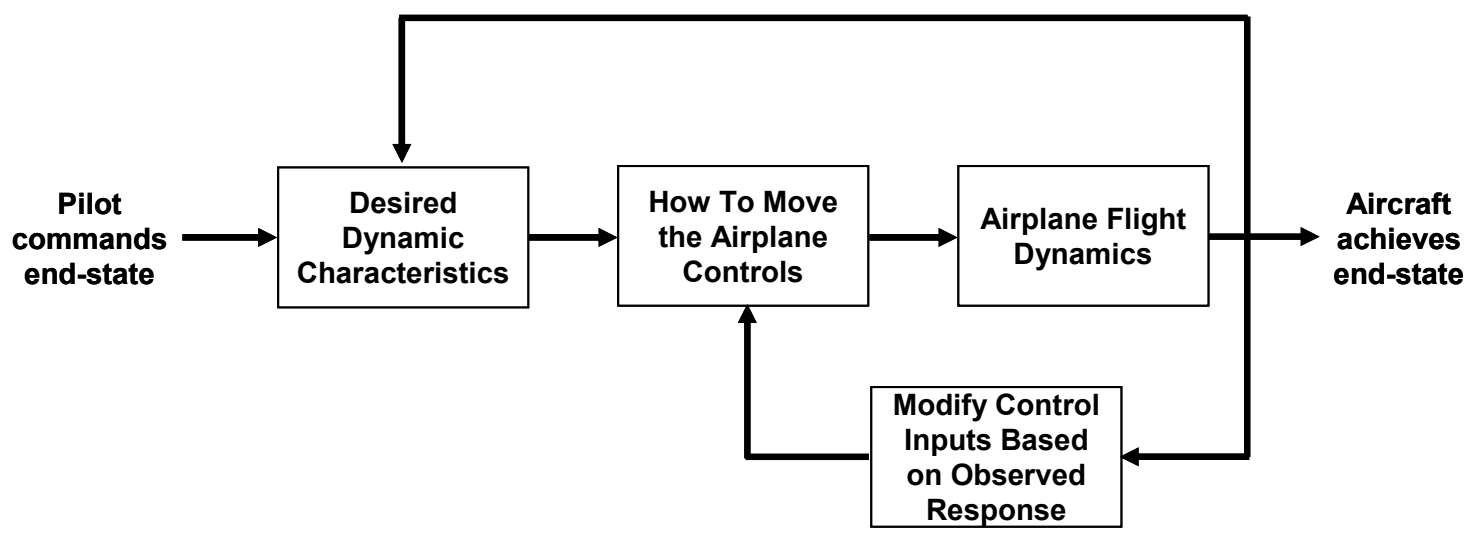

Figure 3: Functional decomposition of the controller

These subfunctions map one-for-one onto the architecture of the overall system. The controller consists of three major elements: a linear model prefilter, a linear inverse transfer function, and an adaptive neural network compensator. A block diagram showing the 
configuration of the controller, plus the physical plant, is shown in Figure 4. In summary, the linear prefilter computes the rates "desired" of the system to achieve the commanded values of speed and flight-path angle. These desired rates are combined with the compensating output of the neural network to compute the "required" rates. The adaptive neural network compensates for modeling error or changes in the physical plant that may occur during operation. Given these rates, the inverse transfer function then computes the control inputs to the physical plant necessary to achieve the desired rates and, as a consequence of integration, to achieve the commanded speed and flight-path angle. A detailed description of each element follows.

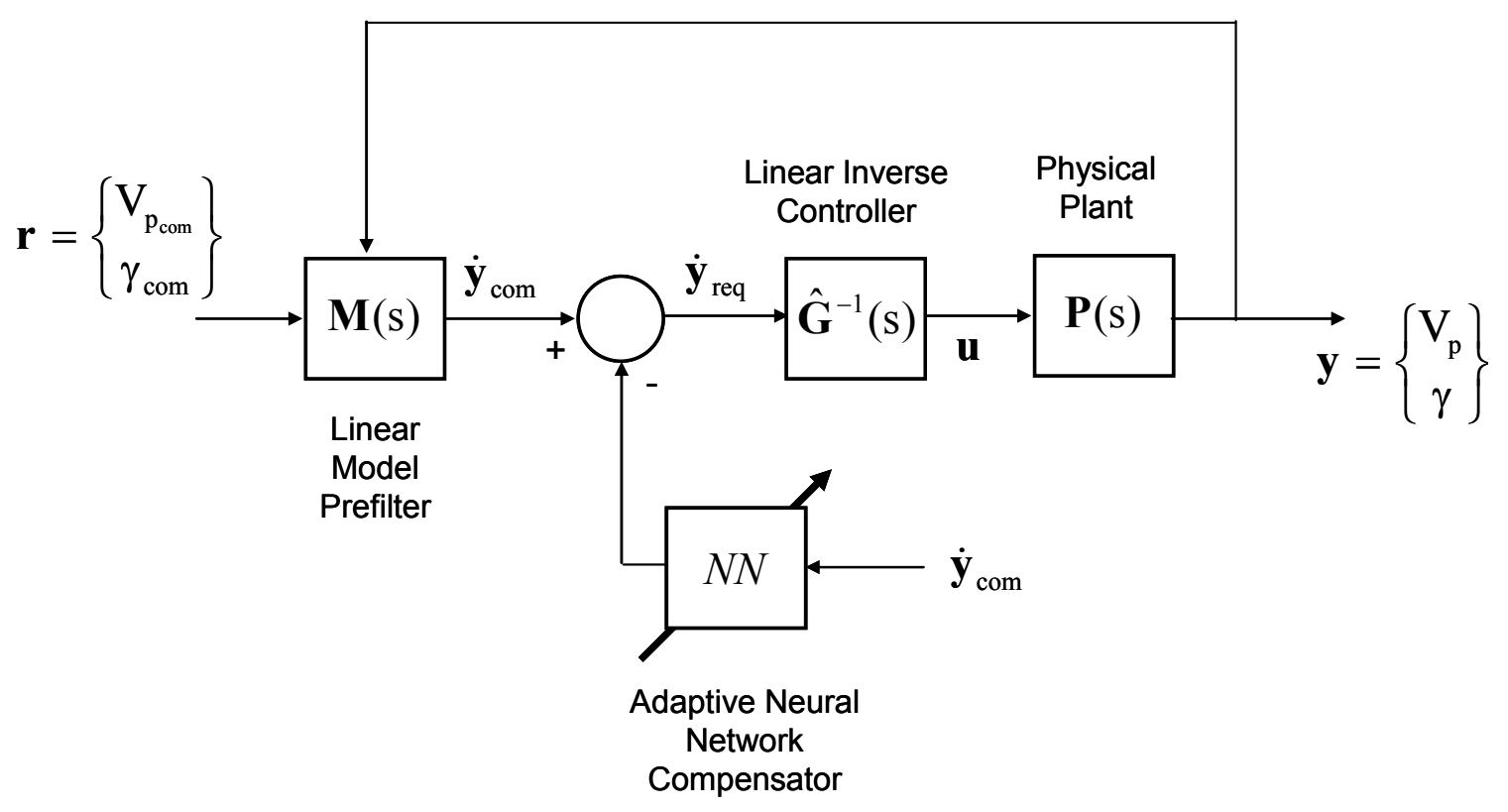

Figure 4: Summary architecture of adaptive inverse controller

\subsection{Physical Plant}

The physical plant, $\mathrm{P}(\mathrm{s})$, may be any physical system. For this research, the physical plant is assumed to be a Beechcraft F-33C Bonanza. The use of this subject aircraft is consistent with previous work accomplished at Wichita State University [11, 12, 13, 14] and complements 
ongoing flight research being conducted jointly by Wichita State University and Hawker Beechcraft Corporation. The F-33C Bonanza is a four-passenger, low-wing monoplane, typical of high-performance General Aviation airplanes [16]. The technical specifications of the aircraft are summarized in Table 1. A three-view illustration of the aircraft is shown in Figure 5.

\section{TABLE 1}

\section{BEECHCRAFT MODEL F33C SUMMARY CHARACTERISTICS}

\begin{tabular}{|l|c|}
\hline Wing Area & 181 square feet \\
\hline Wing Span & 33 feet 6 inches \\
\hline Engine & $\begin{array}{c}\text { One Teledyne Continental Motors } \\
\text { Corporation IO-520, rated at 285 } \\
\text { horsepower }\end{array}$ \\
\hline Maximum Takeoff Weight & 3400 pounds \\
\hline Maximum Structural Cruising Speed & 167 KIAS \\
\hline $\begin{array}{l}\text { Stall Speed (Max Takeoff Weight, Landing } \\
\text { Flaps) }\end{array}$ & 52 KIAS \\
\hline
\end{tabular}

The controls available for the aircraft are elevator deflection, $\delta_{\mathrm{e}}$, aileron deflection, $\delta_{\mathrm{a}}$, rudder deflection, $\delta_{\mathrm{r}}$, and thrust command, $\delta_{\mathrm{t}}$. Since this research is limited to the longitudinal mode, only elevator and thrust commands are utilized here.

The aerodynamics of this airplane are essentially linear up to the angle of attack for aerodynamic stall. However, the drag characteristics are nonlinear, exhibiting a typical parabolic drag polar. The validity of the proposed controller is not limited to physical plants that are linear in behavior. In fact, it will be shown that the controller shows satisfactory performance even when artificial nonlinearities are introduced into the Bonanza aerodynamic characteristics. 

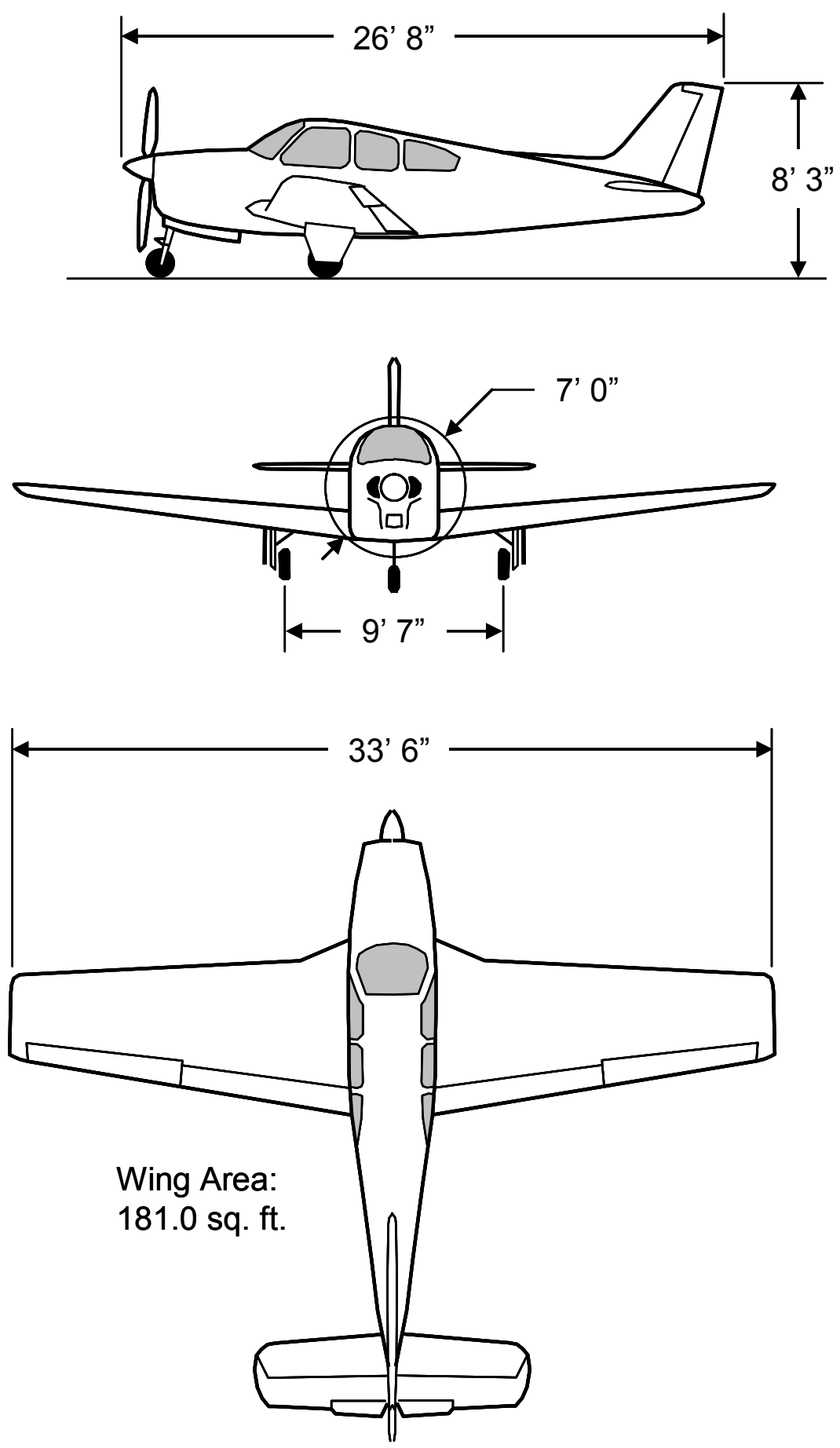

Figure 5: Three-view drawing of the Beechcraft F33C Bonanza [16] 
For some purposes, it is convenient to invoke a strictly linear approximation of the physical plant. This linear approximation is noted as $\mathbf{P}_{\mathrm{A}}(\mathrm{s})$. Furthermore, it is convenient to express $\mathbf{P}_{\mathrm{A}}(\mathrm{s})$ as an LTI state space model, such that

$$
\begin{aligned}
& \dot{\mathbf{x}}_{\mathrm{A}}=\mathbf{A}_{\mathrm{A}} \mathbf{x}_{\mathrm{A}}+\mathbf{B}_{\mathrm{A}} \mathbf{u} \\
& \mathbf{y}_{\mathrm{A}}=\mathbf{C}_{\mathrm{A}} \mathbf{x}_{\mathrm{A}}+\mathbf{D}_{\mathrm{A}} \mathbf{u}
\end{aligned}
$$

where

$$
\mathbf{u}=\left[\begin{array}{ll}
\delta_{\mathrm{e}} & \delta_{\mathrm{t}}
\end{array}\right]^{\mathrm{T}}
$$

This state space model can be represented by the notation

$$
\mathbf{P}_{\mathrm{A}}(\mathrm{s})=\left[\begin{array}{c|c}
\mathbf{A}_{\mathrm{A}} & \mathbf{B}_{\mathrm{A}} \\
\hline \mathbf{C}_{\mathrm{A}} & \mathbf{D}_{\mathrm{A}}
\end{array}\right]
$$

Derivation of the state space model for a typical General Aviation aircraft with conventional controls, such as the Bonanza, is shown in Appendix A. This state space model will be used in the derivation of the controller. It is shown that

$$
\mathbf{x}_{\mathrm{A}}=\left\{\begin{array}{c}
\mathrm{u}_{\mathrm{b}} \\
\alpha \\
\mathrm{q} \\
\theta
\end{array}\right\} \text {, and } \mathbf{y}_{\mathrm{A}}=\left\{\begin{array}{c}
\mathrm{u}_{\mathrm{b}} \\
\gamma
\end{array}\right\}
$$

It is important to note at this point that $\mathbf{D}_{\mathrm{A}}=0$ for such aircraft.

\subsection{Linear Inverse Transfer Function}

The transfer function of a physical system calculates system outputs, $\mathbf{y}$, based on given inputs, u. The inverse transfer function does the inverse, as the name implies. The inverse transfer function calculates the system inputs, $\mathbf{u}$, necessary to achieve a given system output, $\mathbf{y}$. In the Laplace domain, the transfer function is represented as

$$
\mathbf{Y}(\mathrm{s})=\mathbf{G}(\mathrm{s}) \mathbf{U}(\mathrm{s})
$$


The inverse transfer function is represented as

$$
\mathbf{U}(\mathrm{s})=\mathbf{G}^{-1}(\mathrm{~s}) \mathbf{Y}(\mathrm{s})
$$

Inserting equation (2.6) into equation (2.7), it is seen that

$$
\mathbf{G}^{-1}(\mathrm{~s}) \mathbf{G}(\mathrm{s})=\mathbf{I}
$$

where I is the identity matrix. It is important to note that $\mathbf{G}(\mathrm{s})$ is an operator in the Laplace domain. Consequently, its multiplicative inverse, $\mathbf{G}^{-1}(\mathrm{~s})$, exists only in the Laplace domain. If the choice is to work in the time domain, $\mathbf{G}(\mathrm{s})$ is represented as an LTI system $\mathbf{G}(\mathrm{t})$. Another LTI system may be found that is the inverse LTI operator $\mathbf{G}^{-1}(t)$ such that

$$
\mathbf{G}^{-1}(\mathbf{G}(\mathrm{t}))=\mathbf{I}
$$

If $\mathbf{G}(\mathrm{s})$ is realized as an LTI system, such as $\mathbf{P}_{\mathrm{A}}$ (s), described in state space format, the existence of $\mathbf{G}^{-1}(\mathrm{~s})$ is dependent on satisfying both of the following two conditions [17]:

1. D must be square.

2. D must be invertible.

A consequence of these two conditions is that $\mathbf{D}$ must be a square matrix of rank $\mathbf{N}$, where $\mathrm{N}$ is the number of controls. In the case of typical General Aviation aircraft, such as the subject aircraft, there are two longitudinal controls. Therefore, $\mathrm{N}$ equals two: one for the elevator and one for the throttle. Consequently, only $\mathrm{N}$ output variables can be controlled. In the case of the subject aircraft, two output variables can be controlled.

If $\mathbf{G}(\mathrm{s})$ is realized as a state space system

$$
\mathbf{G}(\mathrm{s})=\left[\begin{array}{l|l}
\mathbf{A} & \mathbf{B} \\
\hline \mathbf{C} & \mathbf{D}
\end{array}\right]
$$

it can be shown [17] that the inverse transfer function is 


$$
\mathbf{G}^{-1}(\mathbf{s}) \equiv\left[\begin{array}{c|c}
\mathbf{A}_{\mathrm{I}} & \mathbf{B}_{\mathrm{I}} \\
\hline \mathbf{C}_{\mathrm{I}} & \mathbf{D}_{\mathrm{I}}
\end{array}\right]=\left[\begin{array}{c|c}
\mathbf{A}-\mathbf{B D}^{-1} \mathbf{C} & -\mathbf{B D}^{-1} \\
\hline \mathbf{D}^{-1} \mathbf{C} & \mathbf{D}^{-1}
\end{array}\right]
$$

At this point, a judicious choice is required for $\mathbf{G}(\mathrm{s})$. A first reaction would be to choose $\mathbf{G}(\mathrm{s})=\mathbf{P}_{\mathrm{A}}(\mathrm{s})$. This choice would suggest direct control of the end-states via the inverse transfer function. However, this will not work here because $\mathbf{D}_{\mathrm{A}}=\mathbf{0}$ and therefore violates condition (2) above. A different transfer function $\mathbf{G}(\mathrm{s})$ must be found. A function that produces $\mathbf{y}=\left[\begin{array}{ll}\dot{\mathrm{u}}_{\mathrm{b}} & \dot{\alpha}\end{array}\right]^{\mathrm{T}}$ satisfies the conditions. It will be shown in subchapter 2.4 that controlling $\dot{\mathrm{u}}_{\mathrm{b}}$ and $\dot{\alpha}$ enables control of the end-states of $\mathrm{V}_{\mathrm{p}}$ and $\gamma$ as well. For this case the state space matrices are constructed like this:

1. $\mathbf{A}$ and $\mathbf{B}$ are the same as $\mathbf{A}_{\mathrm{A}}$ and $\mathbf{B}_{\mathrm{A}}$.

2. $\mathbf{C}$ is constructed of the first two rows of $\mathbf{A}_{\mathrm{A}}$ (the $\dot{\mathrm{u}}_{\mathrm{b}}$ and $\dot{\alpha}$ rows).

3. $\mathbf{D}$ is similarly constructed of the first two rows of $\mathbf{B}_{\mathrm{A}}$ (the $\dot{\mathrm{u}}_{\mathrm{b}}$ and $\dot{\alpha}$ rows).

D now satisfies conditions (1) and (2), and therefore an inverse of this transfer function exists. Specifically,

$$
\mathbf{G}(\mathbf{s})=\left[\begin{array}{c|c}
\mathbf{A}_{\mathrm{G}} & \mathbf{B}_{\mathrm{G}} \\
\hline \mathbf{C}_{\mathrm{G}} & \mathbf{D}_{\mathrm{G}}
\end{array}\right]
$$

After making the substitutions as noted above,

$$
\mathbf{G}(\mathrm{s})=\left[\begin{array}{llll|ll}
\multicolumn{5}{c}{\mathbf{A}_{\mathrm{A}}} & \multicolumn{2}{c}{\mathbf{B}_{\mathrm{A}}} \\
\hline \mathrm{A}_{\mathrm{A}}(1,1) & \mathrm{A}_{\mathrm{A}}(1,2) & \mathrm{A}_{\mathrm{A}}(1,3) & \mathrm{A}_{\mathrm{A}}(1,4) & \mathrm{B}_{\mathrm{A}}(1,1) & \mathrm{B}_{\mathrm{A}}(1,2) \\
\mathrm{A}_{\mathrm{A}}(2,1) & \mathrm{A}_{\mathrm{A}}(2,2) & \mathrm{A}_{\mathrm{A}}(2,3) & \mathrm{A}_{\mathrm{A}}(2,4) & \mathrm{B}_{\mathrm{A}}(2,1) & \mathrm{B}_{\mathrm{A}}(2,2)
\end{array}\right]
$$

From the definition of the inverse transfer function in equation (2.11),

$$
\mathbf{G}^{-1}(\mathbf{s})=\left[\begin{array}{c|c}
\mathbf{A}_{\mathrm{G}}-\mathbf{B}_{\mathrm{G}} \mathbf{D}_{\mathrm{G}}{ }^{-1} \mathbf{C}_{\mathrm{G}} & -\mathbf{B}_{\mathrm{G}} \mathbf{D}_{\mathrm{G}}{ }^{-1} \\
\hline \mathbf{D}_{\mathrm{G}}{ }^{-1} \mathbf{C}_{\mathrm{G}} & \mathbf{D}_{\mathrm{G}}{ }^{-1}
\end{array}\right]
$$




$$
\begin{aligned}
& \mathbf{G}^{-1}(\mathrm{~s})=\left[\begin{array}{c|c}
\mathbf{A}_{\mathrm{I}} & \mathbf{B}_{\mathrm{I}} \\
\hline \mathbf{C}_{\mathrm{I}} & \mathbf{D}_{\mathrm{I}}
\end{array}\right] \\
& \dot{\mathbf{x}}_{\mathrm{I}}=\mathbf{A}_{\mathrm{I}} \mathbf{x}_{\mathrm{I}}+\mathbf{B}_{\mathrm{I}} \mathbf{u} \\
& \mathbf{y}_{\mathrm{I}}=\mathbf{C}_{\mathrm{I}} \mathbf{x}_{\mathrm{I}}+\mathbf{D}_{\mathrm{I}} \mathbf{u} \\
& \text { and } \\
& \mathbf{u}=\left[\begin{array}{ll}
\dot{\mathrm{u}}_{\mathrm{b}} & \dot{\alpha}
\end{array}\right]_{\mathrm{req}}^{\mathrm{T}} \\
& \mathbf{y}_{\mathrm{I}}=\left[\begin{array}{ll}
\delta_{\mathrm{e}} & \delta_{\mathrm{t}}
\end{array}\right]^{\mathrm{T}}
\end{aligned}
$$

While not obvious, it should be pointed out that $\mathbf{x}_{\mathrm{I}}=-\mathbf{x}_{\mathrm{A}}$. At the same time the LTI system $\mathbf{G}^{-1}$ (s) is computing the necessary control inputs, it is calculating its own internal representation of the physical plant state variables. The dynamics of the physical plant enter into the calculations via this internal representation. This is in contrast with the dynamic inverse methods used by Calise or Steck where the airplane state is explicitly fed back for purposes of calculating the dynamic inverse.

$\mathbf{G}^{-1}(\mathrm{~s})$ acts upon $\left[\begin{array}{ll}\dot{\mathrm{u}}_{\mathrm{b}} & \dot{\alpha}\end{array}\right]_{\text {req }}^{\mathrm{T}}$ to produce a set of control inputs $\left[\begin{array}{ll}\delta_{\mathrm{e}} & \delta_{\mathrm{t}}\end{array}\right]^{\mathrm{T}}$. When fed into $\mathbf{G}(\mathrm{s})$, these control inputs will produce $\left[\begin{array}{ll}\dot{\mathrm{u}}_{\mathrm{b}} & \dot{\alpha}\end{array}\right]^{\mathrm{T}}$. A block diagram of this sequence of operations is illustrated in Figure 6.

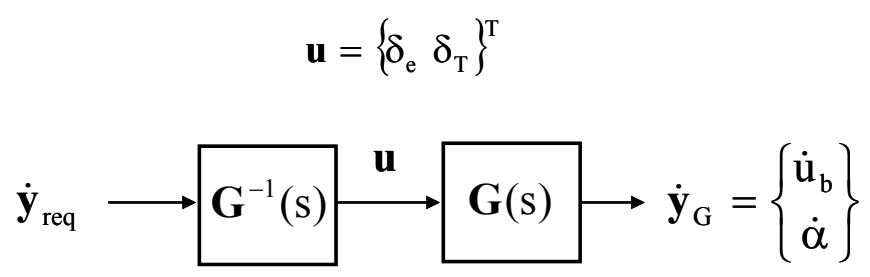

Figure 6: Pass-through function of $\mathbf{G}^{-1}(\mathrm{~s}) \mathbf{G}(\mathrm{s})$ 
By a change to the $\mathbf{C}_{\mathrm{A}}$ matrix (refer to Appendix A, equation (A23)), $\mathbf{P}_{\mathrm{A}}(\mathrm{s})$ can also produce $\mathbf{y}=\left[\begin{array}{ll}\dot{\mathbf{u}}_{\mathrm{b}} & \dot{\alpha}\end{array}\right]^{\mathrm{T}}$. With this context in mind, a parallel set of transfer functions may be envisioned, as shown in Figure 7.

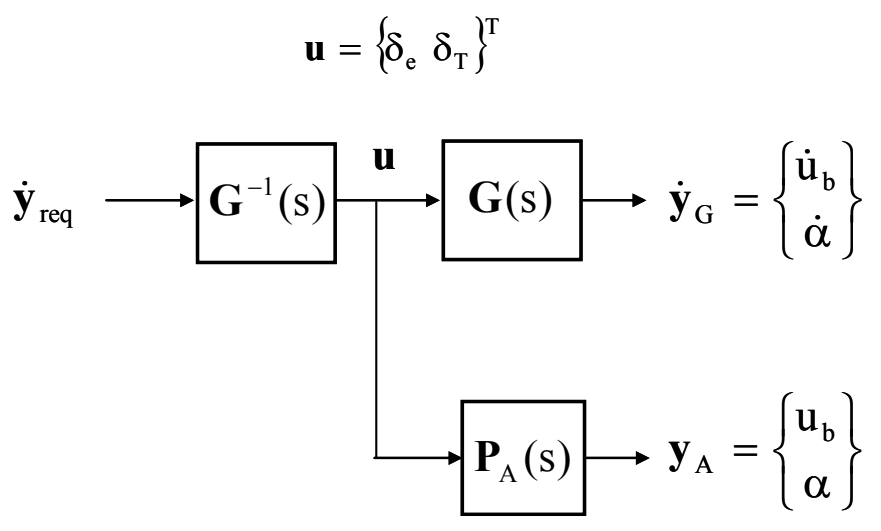

Figure 7: Parallel operation of $\mathbf{G}(\mathbf{s})$ and $\mathbf{P}_{\mathrm{A}}(\mathrm{s})$.

A vector of required rates, $\dot{\mathbf{y}}_{\text {req }}$, is input into $\mathbf{G}^{-1}(\mathrm{~s})$. The inverse transfer function produces control inputs which, in turn, go to both $\mathbf{G}(\mathrm{s})$ and $\mathbf{P}_{\mathrm{A}}(\mathrm{s})$. $\mathbf{G}(\mathrm{s})$ produces the rates, while $\mathbf{P}_{\mathrm{A}}(\mathrm{s})$ produces $\mathrm{u}_{\mathrm{b}}$ and $\alpha$. This parallel structure is effectively an integrator, as shown in Figure 8. It should be noted that $\mathbf{G}(\mathrm{s})$ is only an abstraction used in formulating $\mathbf{G}^{-1}(\mathrm{~s})$. No physical instance of $\mathbf{G}(\mathrm{s})$ is found in the system. 


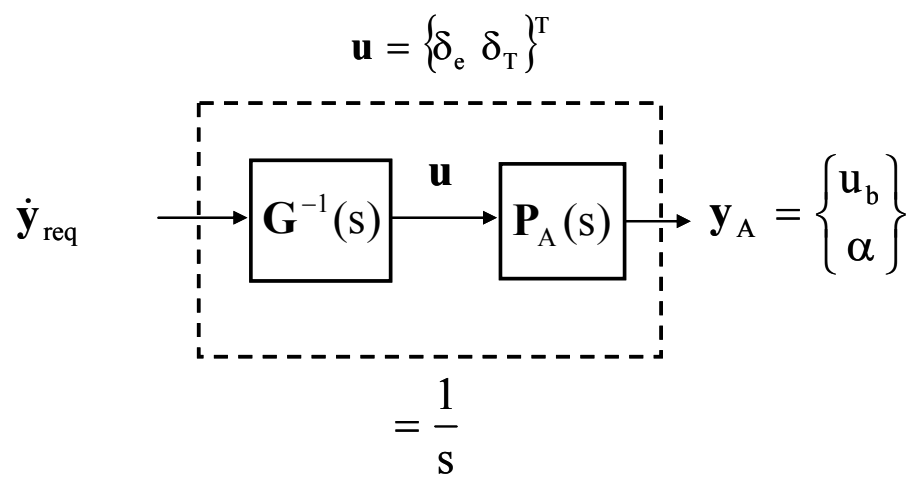

Figure 8: The combination of $\mathbf{G}(\mathrm{s})$ and $\mathbf{P}_{\mathrm{A}}(\mathrm{s})$ acting as an integrator

The equivalency of $\mathbf{G}^{-1}(\mathrm{~s}) \mathbf{P}_{\mathrm{A}}$ (s) to an integrator is a key concept in the development of this controller. This equivalency is illustrated in the examples below.

A MATLAB ${ }^{\mathrm{TM}}$ model was constructed for the transfer function $\mathbf{G}^{-1}(\mathrm{~s}) \mathbf{P}_{\mathrm{A}}(\mathrm{s})$, as shown in Figure 9. The inputs to the transfer function are a $\dot{\mathrm{u}}$ command and an $\dot{\alpha}$ command. The command for $\dot{\mathrm{u}}$ is a repeating pulse, $10 \mathrm{feet} / \mathrm{sec}^{2}$ in amplitude and two seconds wide. The command for $\dot{\alpha}$ is zero. These inputs are plotted in Figure 10, as are the outputs from the system. It is obvious to see that the $\dot{\mathrm{u}}$ pulses, $10 \mathrm{feet} / \mathrm{sec}^{2}$ wide and 2 seconds wide, integrate into changes in velocity of $20 \mathrm{feet} / \mathrm{sec}$ with each pulse. Since no $\dot{\alpha}$ is commanded, $\alpha$ remains constant.

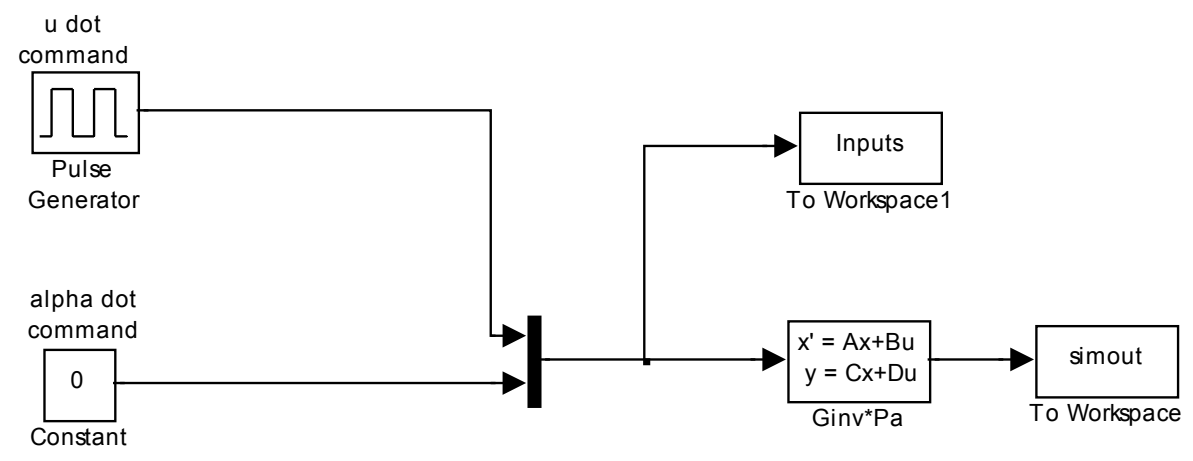

Figure 9: $M A T L A B{ }^{T M}$ block diagram illustrating integration of $\dot{u}$ commands 
Figure 11 shows all the state variables for the airplane system, $\mathbf{x}=\left[\begin{array}{llll}\mathrm{u} & \alpha & \mathrm{q} & \theta\end{array}\right]^{\mathrm{T}}$. It is important to note that only the "commanded" variables, $u$ and $\alpha$, are "under control." The other state variables will take on whatever values come as a consequence of achieving the commanded values of $\dot{\mathrm{u}}$ and $\dot{\alpha}$. For this example, where speed is increased at a fixed angle of attack, lift is no longer in equilibrium with weight. A vertical acceleration normal to the flight-path occurs, resulting in a curving flight-path as evident in the pitch rate and pitch angle traces. The mathematical relationships between these parameters will be examined in the following subchapter. As a reminder, flight-path angle, $\gamma$, is not a state variable but rather a linear combination of state variables $\alpha$ and $\theta$.

Figure 12 shows a similar model but with $\dot{\alpha}$ being stimulated with a pulse .05 radians/sec in amplitude and two seconds wide. The command for $\dot{\mathrm{u}}$ is zero. The inputs and corresponding outputs are shown in Figure 13. Once again, it can be seen that the integration of an $\dot{\alpha}$ pulse .05 radians/sec by two seconds results in a change in $\alpha$ of .10 radians with each pulse. Velocity stays constant at zero. Figure 14 shows how all of the state variables change in response.

Similar to the preceding example, a change in lift occurs, but as a result of changing the angle of attack in this case. An acceleration normal to the flight-path occurs, resulting in a similar but somewhat different curvilinear flight-path. 

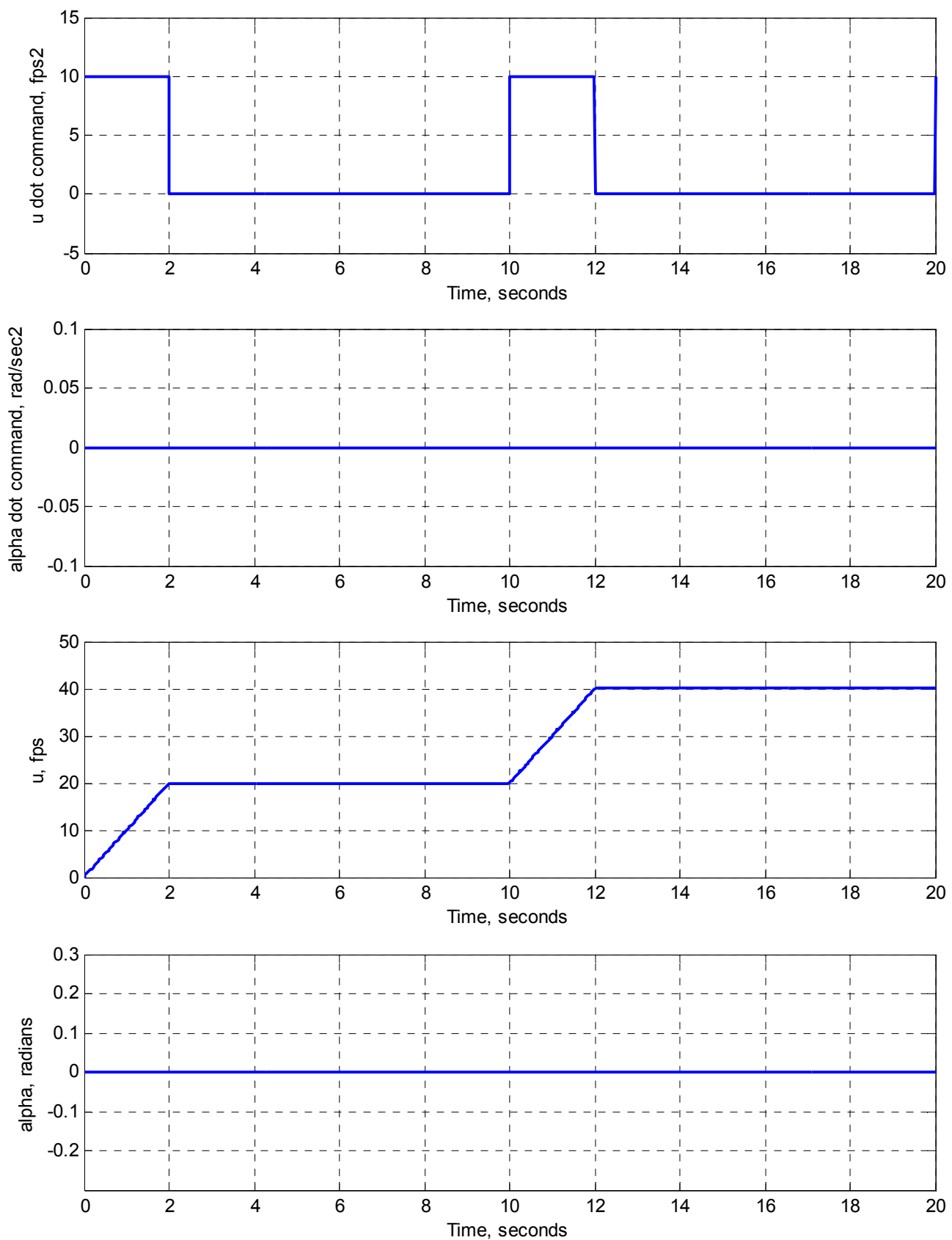

Figure 10: Time history results of integration of $\dot{\mathbf{u}}$ commands via $\mathbf{G}^{-1}(\mathrm{~s}) \mathbf{P}_{\mathrm{A}}(\mathrm{s})$ 

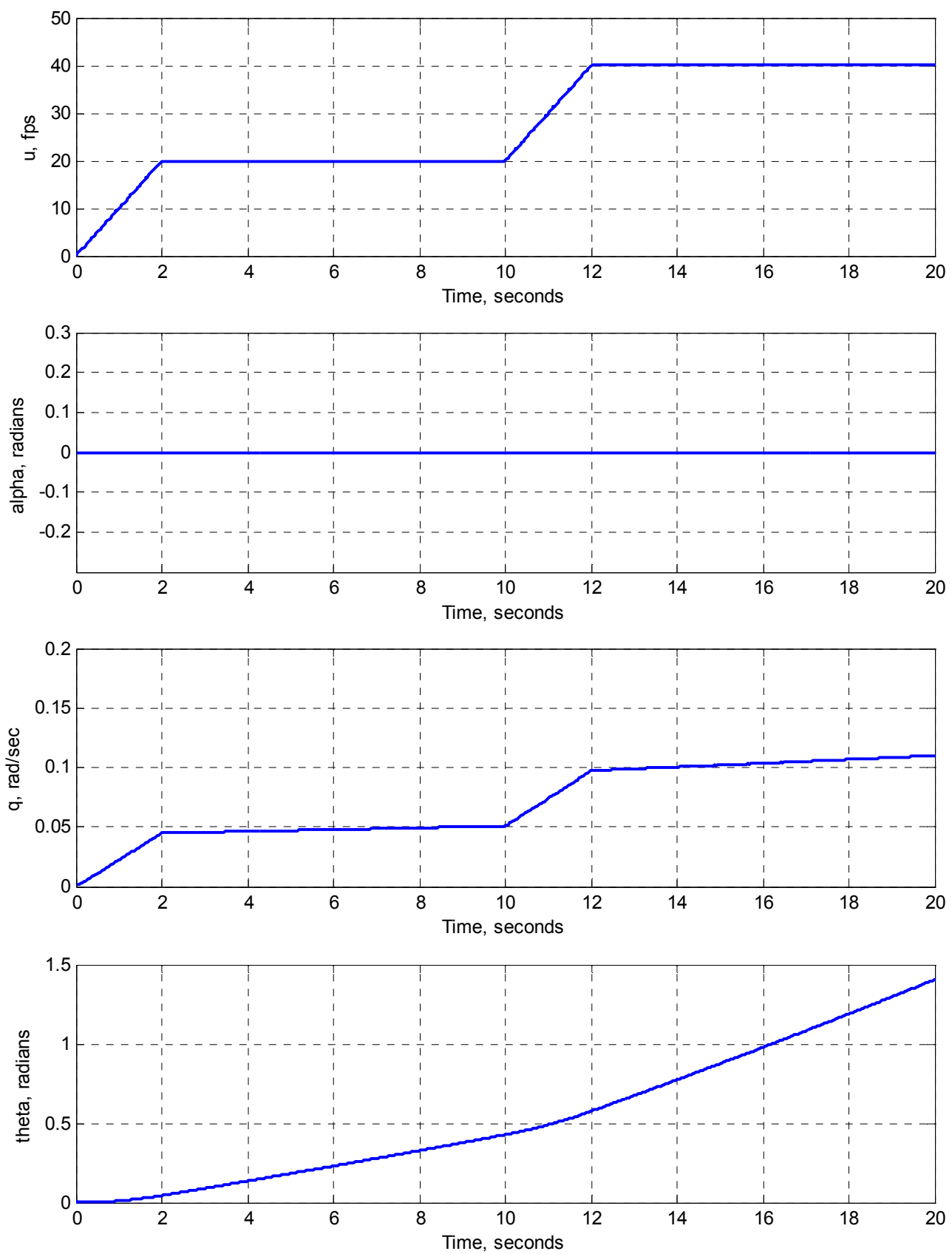

Figure 11: Time histories of state variables resulting from ù commands 


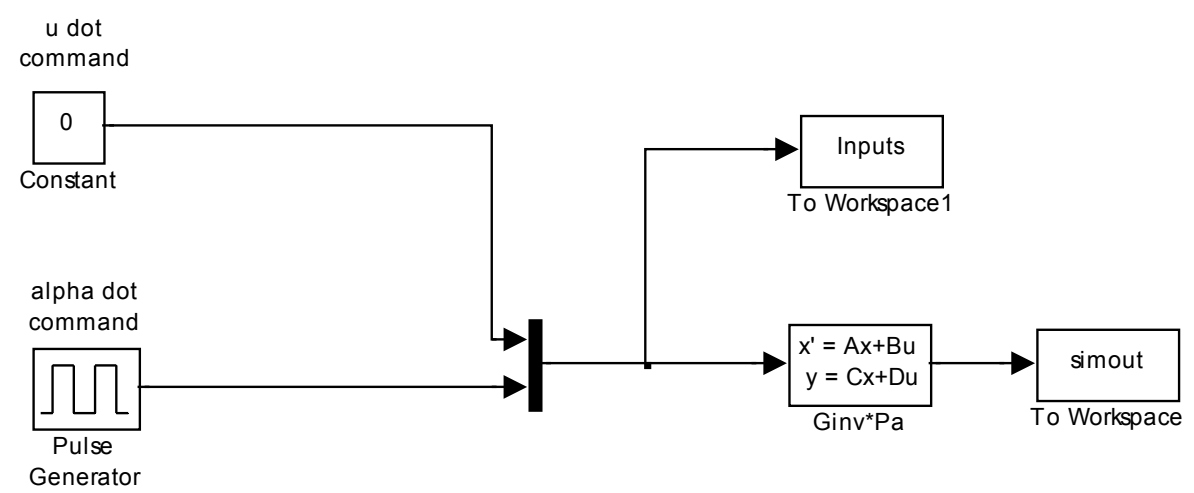

Figure 12: MATLAB $^{\mathrm{TM}}$ block diagram illustrating integration of $\dot{\alpha}$ commands

The important point to be learned from these examples is that the linear inverse transfer function, combined with the physical plant, achieves exactly the integral of the input parameters: no more, no less. No direct control of the other state variables is implied or expected. Some other control logic must be applied if the objective is to control the other state variables or, in the case of flight-path angle, a linear combination of the state variables. Hence, the role of the next element of the controller: the linear prefilter. 

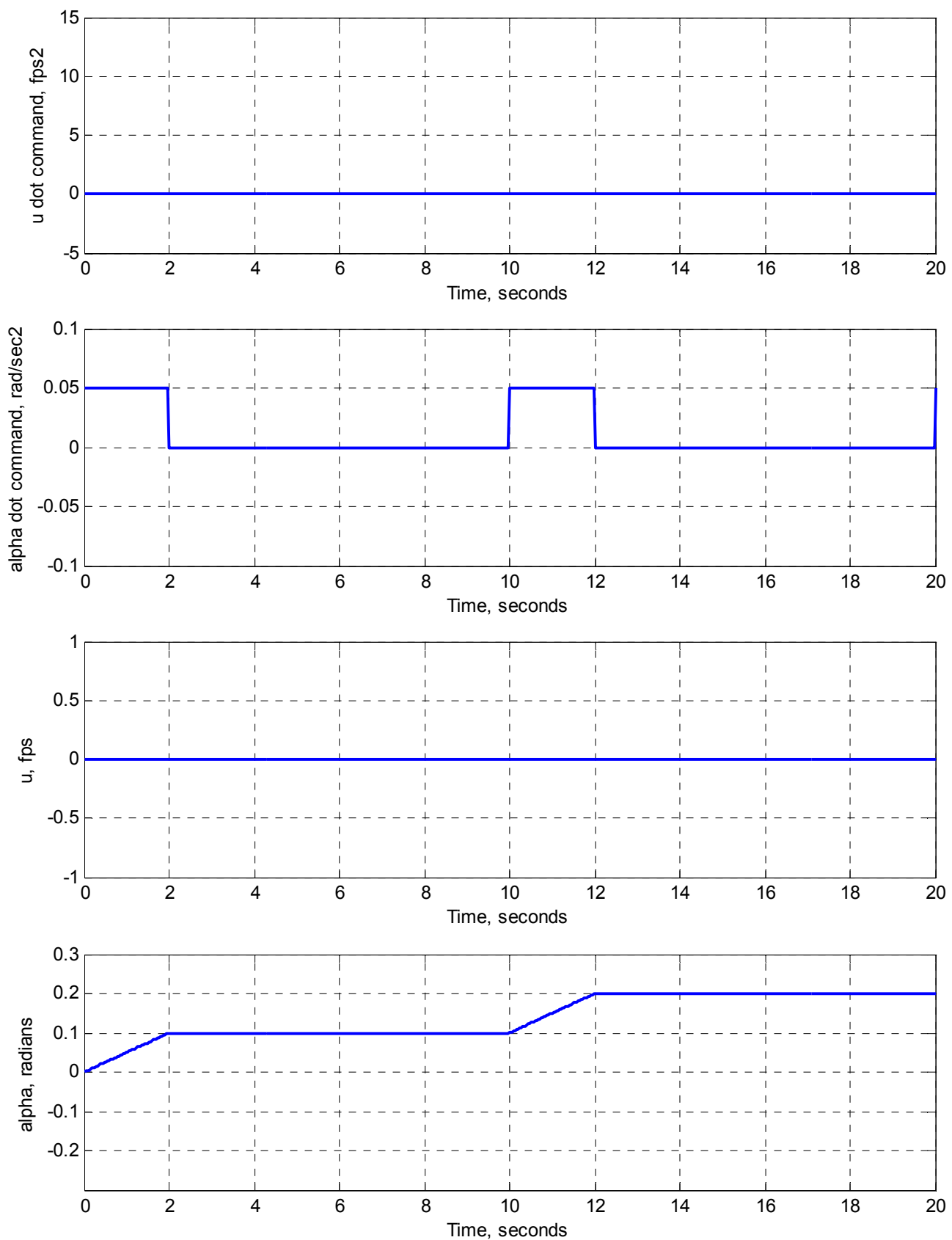

Figure 13: Time history results of integration of $\dot{\alpha}$ commands via $\mathbf{G}^{-1}(\mathrm{~s}) \mathbf{P}_{\mathrm{A}}(\mathrm{s})$ 

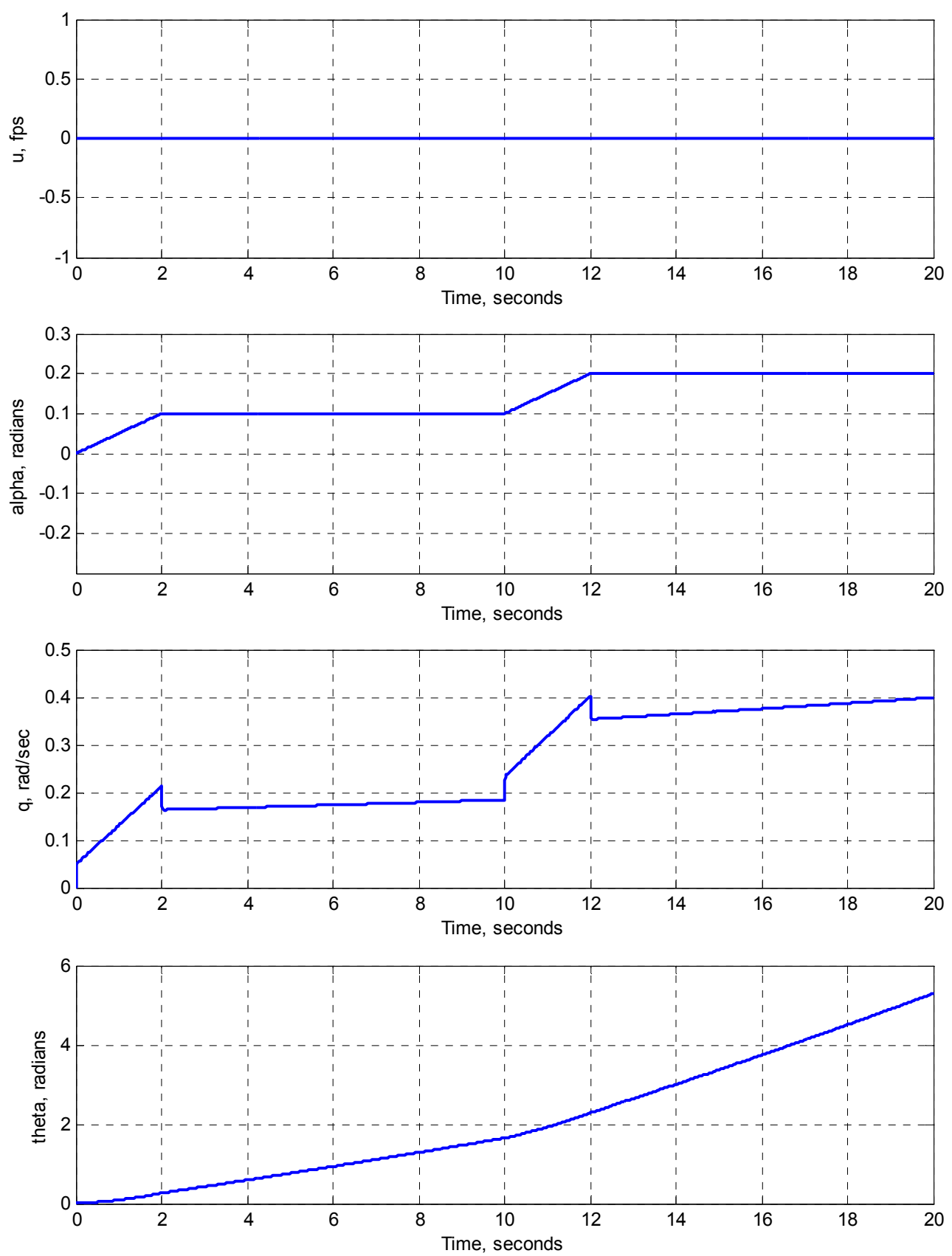

Figure 14: Time histories of state variables resulting from $\dot{\alpha}$ commands

\subsection{Linear Prefilter}

The linear prefilter computes the rates desired of the overall system to achieve the tracking characteristics of any arbitrary linear system. This prefilter performs a similar function to the command filter and handling qualities filter used by Calise and Rysdyk [6] and illustrated 
in Figure 2. These desired rates from this prefilter will be shown to be an input into the inverse linear transfer function. In essence, the overall controller becomes a model-following controller, following the characteristics embedded in the linear prefilter. For this work, it is assumed that the system is to have the characteristics of a second-order system with respect to tracking speed and flight-path angle, as described in equations (2.20) and (2.21).

$$
\begin{gathered}
\ddot{\mathrm{V}}_{\mathrm{p}}+2 \varsigma_{\mathrm{u}} \omega_{\mathrm{n}_{\mathrm{u}}} \dot{\mathrm{V}}_{\mathrm{p}}+\omega_{\mathrm{n}_{\mathrm{u}}}^{2} \mathrm{~V}_{\mathrm{p}}=\omega_{\mathrm{n}_{\mathrm{u}}}^{2} \mathrm{~V}_{\mathrm{p}_{\mathrm{com}}} \\
\ddot{\gamma}+2 \varsigma_{\gamma} \omega_{\mathrm{n}_{\gamma}} \dot{\gamma}+\omega_{\mathrm{n}_{\gamma}}^{2} \gamma=\omega_{\mathrm{n}_{\gamma}}^{2} \gamma_{\text {com }}
\end{gathered}
$$

These damping ratios and natural frequencies are totally sufficient to describe the dynamic characteristics of the overall system and can be arbitrarily chosen in order to have the desired overall system characteristics.

A block diagram for a simple second-order system is shown in Figure 15. This will be used as a starting point for the development of both the speed and flight-path prefilters.

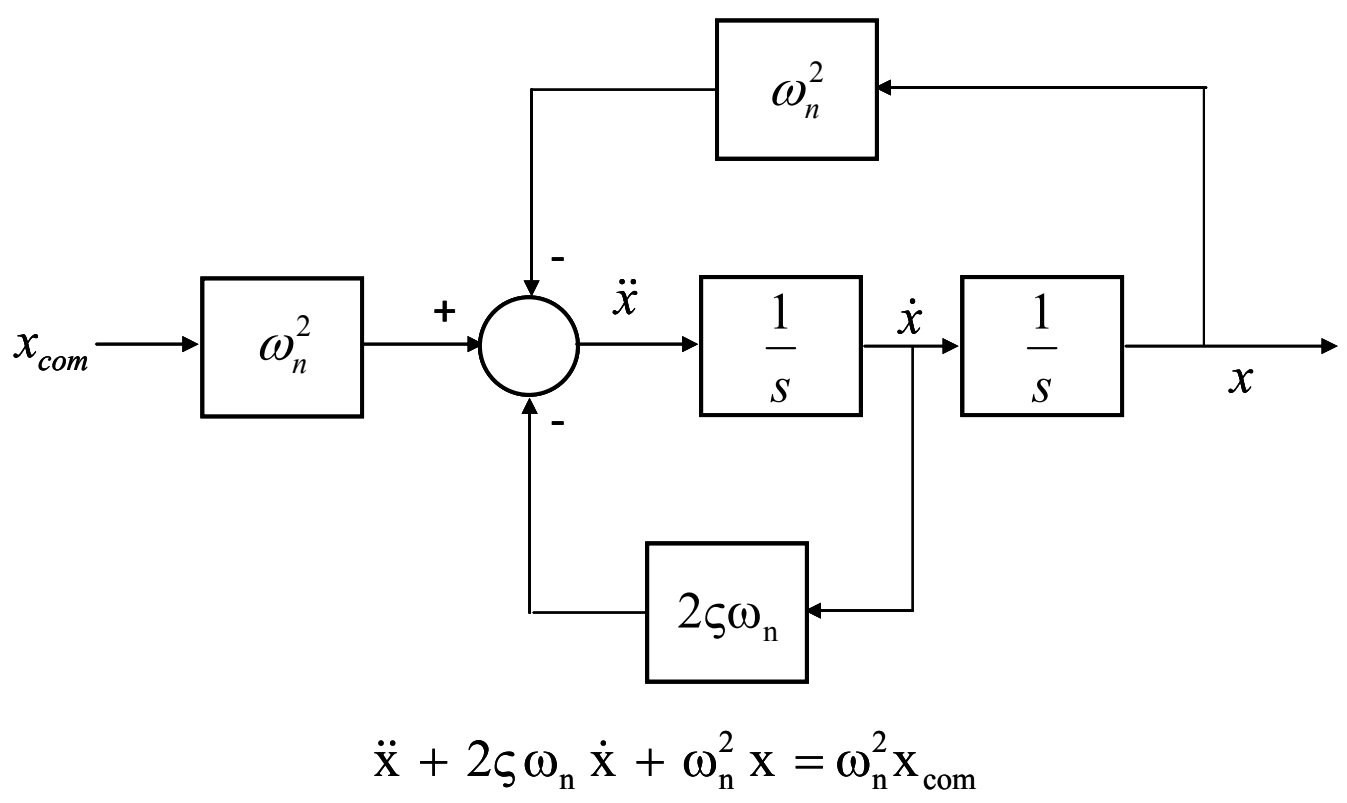

Figure 15: Simple second-order system 
The speed prefilter is considered first. The block diagram in Figure 15 may be rearranged, but with no change in connectivity, as shown in Figure 16.

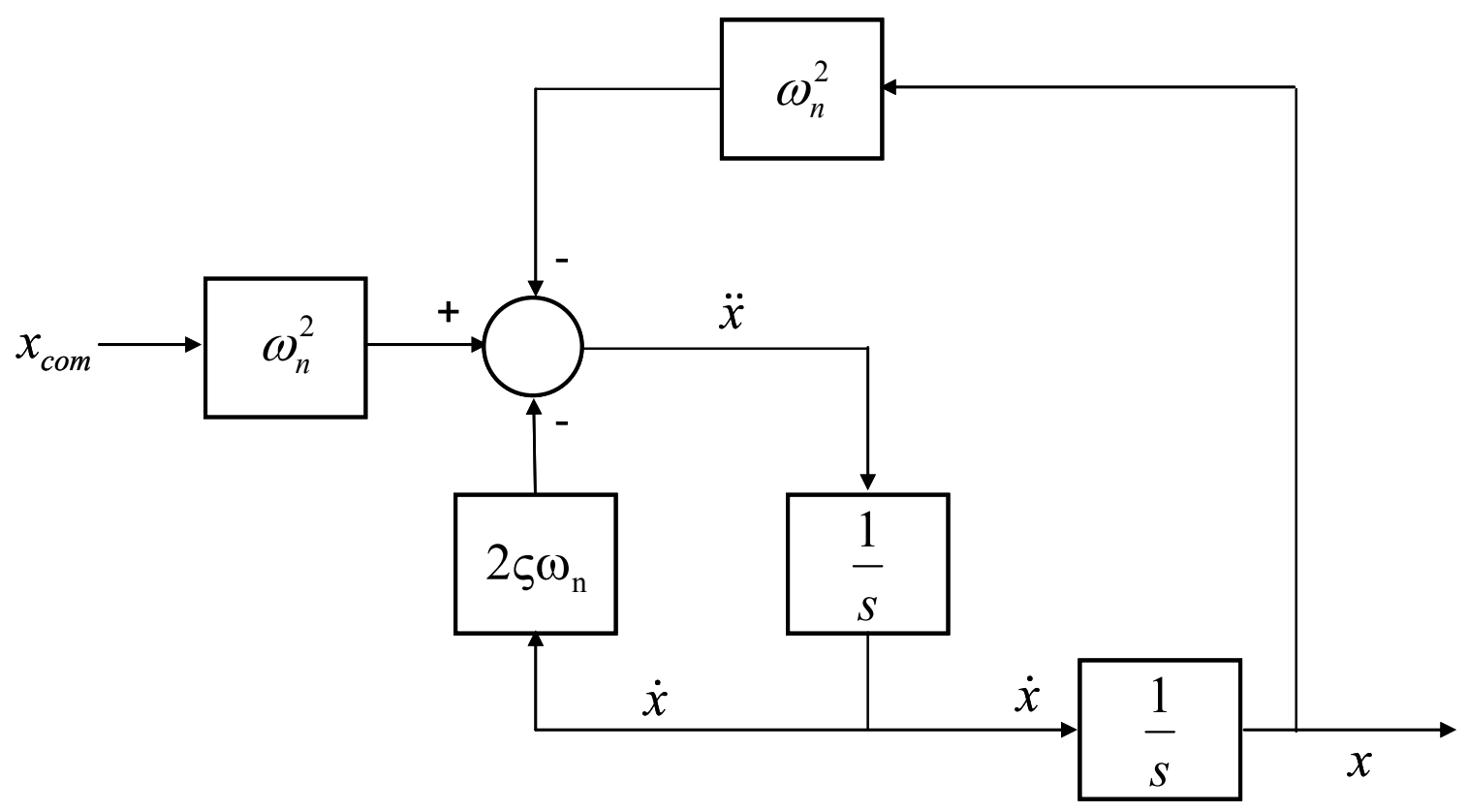

Figure 16: Rearrangement of second-order system, with no change in connectivity

Furthermore, this block diagram may be made specific to speed control by substituting $\mathrm{u}_{\mathrm{b}}$ for $\mathrm{x}$, introduce the variable, $\mathrm{u}_{\mathrm{b}_{\mathrm{m}}}$, and invoke the equivalency of $\mathbf{G}^{-1}(\mathrm{~s}) \mathbf{P}_{\mathrm{A}}(\mathrm{s})$ as an integrator. The results of this are shown in Figure 17.

Finally, the elements within this block diagram can be grouped by function, as shown in Figure 18. At a still higher level of abstraction, the block diagram for the speed prefilter can be viewed in Figure 19. 


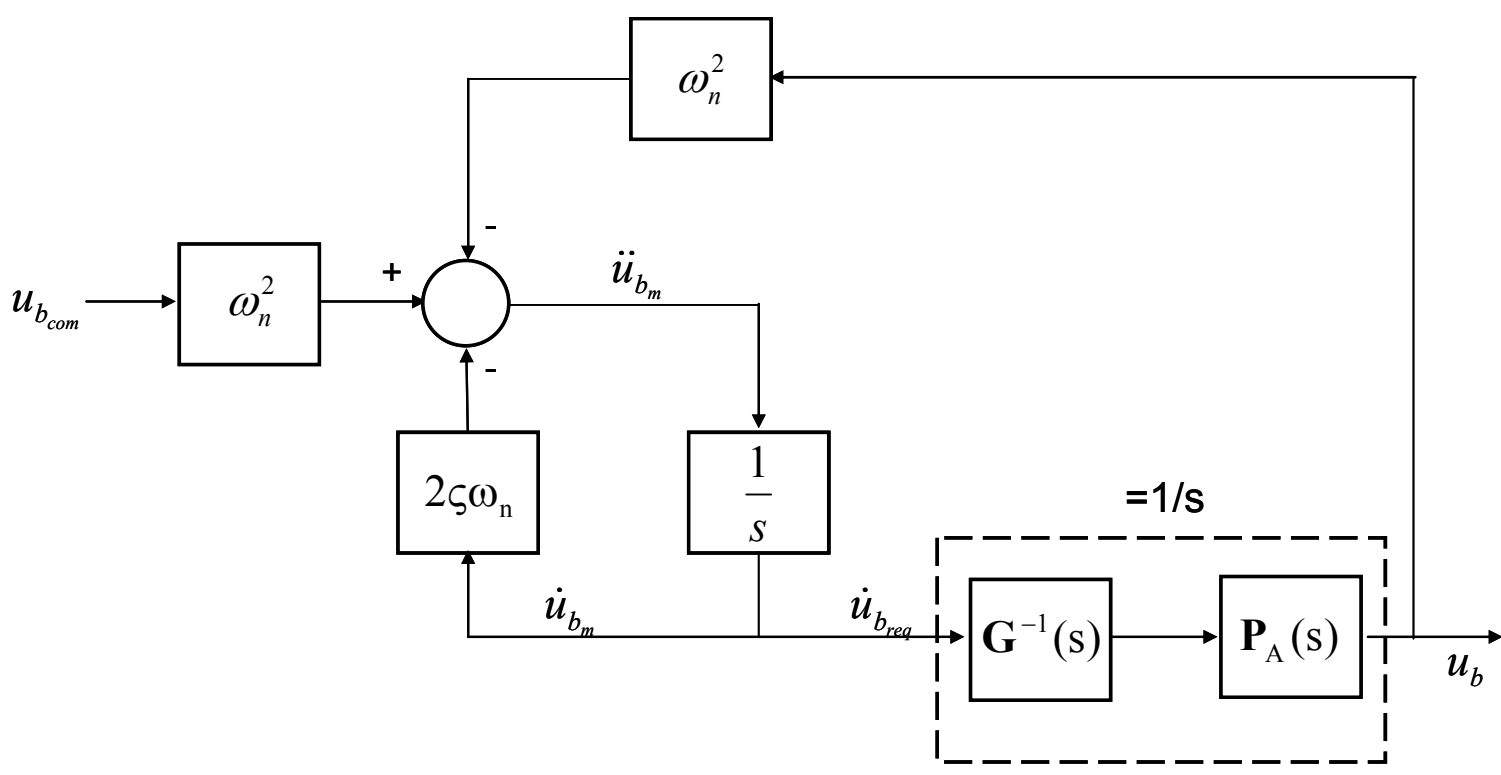

Figure 17: Customizing second-order system for speed control

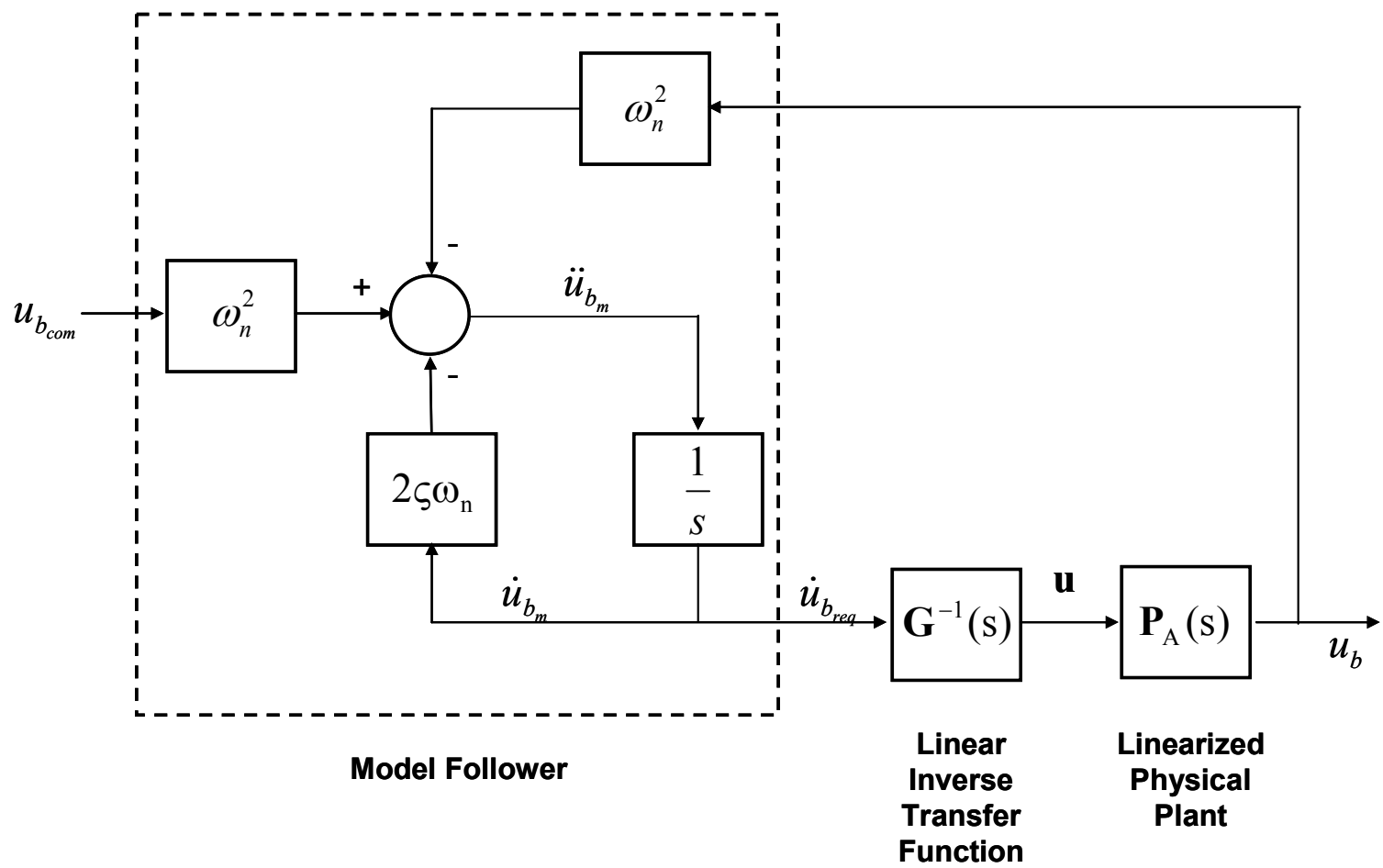

Figure 18: Functional grouping of elements within speed controller 


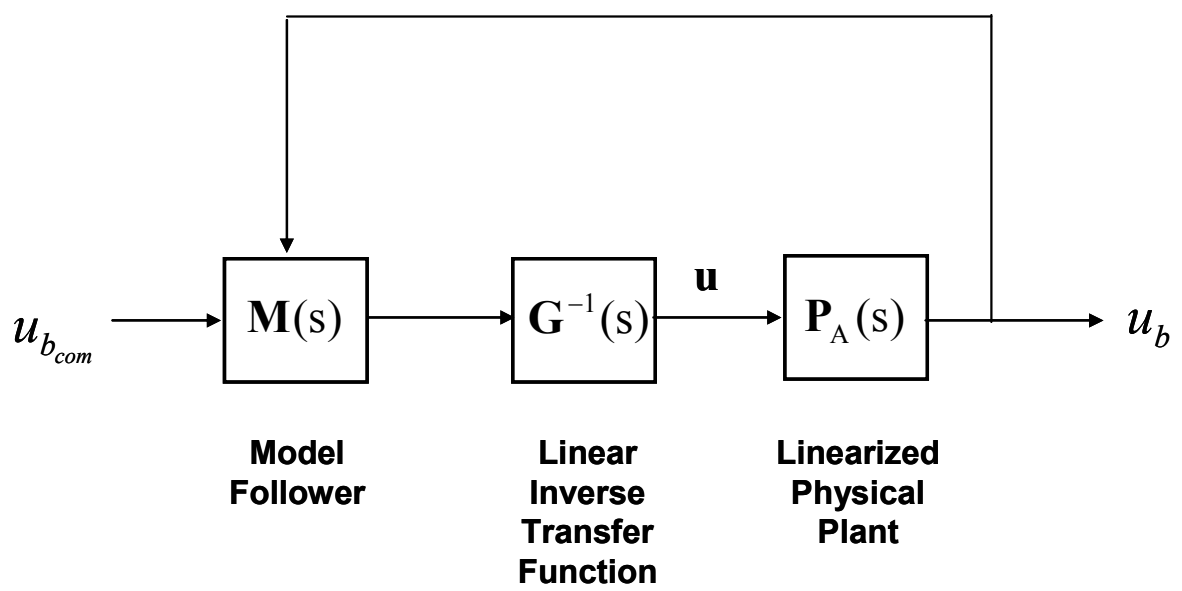

Figure 19: High-level arrangement of speed controller

The flight-path angle prefilter is more complex because flight-path angle is not controlled directly. Only $\dot{\mathrm{u}}_{\mathrm{b}}$ and $\dot{\alpha}$ are directly controlled by the inverse transfer function. To derive the control law for flight-path angle, it is necessary to first consider how flight-path angle is changed. If a pilot were flying the airplane, he would "pull g's." That is to say, the pilot would increase the angle of attack in order to increase the normal load factor, $\mathrm{n}_{\mathrm{z}}$, and thereby produce an imbalance in forces normal to the flight-path. This force imbalance causes an acceleration normal to the flight-path, thereby changing the flight-path angle. Mathematically,

$$
\begin{gathered}
\mathrm{mV}_{\mathrm{p}} \dot{\gamma}=\sum \sum_{\substack{\text { normal } \\
\text { to Vp }}} \\
\mathrm{mV}_{\mathrm{p}} \dot{\gamma}=\overline{\mathrm{q}} \mathrm{SC}_{\mathrm{L}}-\mathrm{W} \cos \gamma
\end{gathered}
$$

Taking the derivative of both sides with respect to time yields:

$$
\underline{\mathrm{mV}_{\mathrm{p}} \dot{\gamma}}+\mathrm{mV}_{\mathrm{p}} \ddot{\gamma}=\dot{\overline{\mathrm{q}}} \mathrm{SC}_{\mathrm{L}}+\overline{\mathrm{q}} \dot{\mathrm{C}}_{\mathrm{L}}+\underline{\mathrm{W} \sin \gamma \dot{\gamma}}
$$

If an order-of-magnitude analysis is performed on equation (2.24), with the flight conditions likely for the example aircraft, it can be shown that: $\mathrm{m} \dot{\mathrm{V}}_{\mathrm{p}} \dot{\gamma}$ is on the order of $10^{0} \mathrm{lbs} / \mathrm{sec}$. 
$\mathrm{mV}_{\mathrm{p}} \ddot{\gamma}$ is on the order of $10^{4} \mathrm{lbs} / \mathrm{sec}$.

$\dot{\overline{\mathrm{q}}} \mathrm{SC}_{\mathrm{L}}$ is on the order of $10^{2} \mathrm{lbs} / \mathrm{sec}$.

$\overline{\mathrm{q} S \dot{C}_{\mathrm{L}}}$ is on the order of $10^{4} \mathrm{lbs} / \mathrm{sec}$.

$\mathrm{W} \sin \gamma \dot{\gamma}$ is zero, if the equation is evaluated at $\gamma=0$.

The underlined terms in equation (2.24) are thus considered to be "small," and can be dismissed.

The time derivative of dynamic pressure can be decomposed into

$$
\begin{gathered}
\dot{\overline{\mathrm{q}}}=\frac{\mathrm{d}}{\mathrm{dt}}\left(\frac{1}{2} \rho \mathrm{V}_{\mathrm{p}}^{2}\right) \\
\dot{\overline{\mathrm{q}}}=\rho \mathrm{V}_{\mathrm{p}} \dot{\mathrm{V}}_{\mathrm{p}}
\end{gathered}
$$

Making the simplification that the lift coefficient is primarily a function of angle of attack, the time derivative of lift coefficient can be similarly decomposed as

$$
\begin{gathered}
\dot{\mathrm{C}}_{\mathrm{L}} \approx \frac{\mathrm{d}}{\mathrm{dt}}\left(\mathrm{C}_{\mathrm{L}_{0}}+\mathrm{C}_{\mathrm{L}_{\alpha}} \alpha\right) \\
\dot{\mathrm{C}}_{\mathrm{L}}=\mathrm{C}_{\mathrm{L}_{\alpha}} \dot{\alpha}
\end{gathered}
$$

Substituting equations (2.26) and (2.28) into equation (2.24) and dropping the underlined terms, results in

$$
\mathrm{mV}_{\mathrm{p}} \ddot{\gamma}=\rho \mathrm{V}_{\mathrm{p}} \dot{\mathrm{V}}_{\mathrm{p}} \mathrm{SC}_{\mathrm{L}}+\overline{\mathrm{q}} \mathrm{SC}_{\mathrm{L}_{\alpha}} \dot{\alpha}
$$

Rearranging, this becomes

$$
\begin{gathered}
\overline{\mathrm{q}} \mathrm{SC}_{\mathrm{L}_{\alpha}} \dot{\alpha}=\mathrm{mV}_{\mathrm{p}} \ddot{\gamma}-\rho \mathrm{V}_{\mathrm{p}} \dot{\mathrm{V}}_{\mathrm{p}} \mathrm{SC}_{\mathrm{L}} \\
\overline{\mathrm{q}}_{\mathrm{SC}_{\mathrm{L}_{\alpha}}} \dot{\alpha}=\mathrm{mV}_{\mathrm{p}} \ddot{\gamma}-2\left(\frac{\rho \mathrm{V}_{\mathrm{p}}^{2} \mathrm{SC}_{\mathrm{L}}}{2 \mathrm{~V}_{\mathrm{p}}}\right) \dot{\mathrm{V}}_{\mathrm{p}}
\end{gathered}
$$




$$
\begin{gathered}
\overline{\mathrm{qSC}}_{\mathrm{L}_{\alpha}} \dot{\alpha}=\mathrm{mV}_{\mathrm{p}} \ddot{\gamma}+\left(\frac{-2 \mathrm{n}_{\mathrm{z}} \mathrm{W}}{\mathrm{V}_{\mathrm{p}}}\right) \dot{\mathrm{V}}_{\mathrm{p}} \\
\dot{\alpha}=\left(\frac{\mathrm{mV}_{\mathrm{p}}}{\overline{\mathrm{q}} \mathrm{SC}_{\mathrm{L}_{\alpha}}}\right) \ddot{\gamma}+\left(\frac{-2 \mathrm{n}_{\mathrm{z}} \mathrm{W}}{\overline{\mathrm{q}} \mathrm{SC}_{\mathrm{L}_{\alpha}} \mathrm{V}_{\mathrm{p}}}\right) \dot{\mathrm{V}}_{\mathrm{p}} \\
\dot{\alpha}=\Lambda_{1} \ddot{\gamma}+\Lambda_{2} \dot{\mathrm{V}}_{\mathrm{p}}
\end{gathered}
$$

where

$$
\begin{gathered}
\Lambda_{1} \equiv \frac{\mathrm{mV}_{\mathrm{p}}}{\overline{\mathrm{q}} \mathrm{SC}_{\mathrm{L}_{\alpha}}} \\
\Lambda_{2} \equiv \frac{-2 \mathrm{n}_{\mathrm{z}} \mathrm{W}}{\overline{\mathrm{q} S C_{\mathrm{L}_{\alpha}} \mathrm{V}_{\mathrm{p}}}}
\end{gathered}
$$

Equation (2.34) provides the necessary link connecting angle of attack (which can be controlled) with flight-path angle (the desired variable to track). Recall that the objective is to have $\ddot{\gamma}=-2 \varsigma_{\gamma} \omega_{\mathrm{n}_{\gamma}} \dot{\gamma}-\omega_{\mathrm{n}_{\gamma}}^{2} \gamma+\omega_{\mathrm{n}_{\gamma}}^{2} \gamma_{\text {com }}$, as noted in equation (2.21). Making this substitution into equation (2.34)

$$
\begin{gathered}
\dot{\alpha}=\Lambda_{1}\left(-2 \varsigma_{\gamma} \omega_{\mathrm{n}_{\gamma}} \dot{\gamma}-\omega_{\mathrm{n}_{\gamma}}^{2} \gamma+\omega_{\mathrm{n}_{\gamma}}^{2} \gamma_{\text {com }}\right)+\Lambda_{2} \dot{\mathrm{V}}_{\mathrm{p}} \\
\dot{\alpha}=\Lambda_{2} \dot{\mathrm{V}}_{\mathrm{p}}-2 \Lambda_{1} \varsigma_{\gamma} \omega_{\mathrm{n}_{\gamma}} \dot{\gamma}-\Lambda_{1} \omega_{\mathrm{n}_{\gamma}}^{2} \gamma+\Lambda_{1} \omega_{\mathrm{n}_{\gamma}}^{2} \gamma_{\text {com }}
\end{gathered}
$$

In the event it is desired to match the system response to some other linear system response, such as a first-order system, a different but appropriate substitution could be made into equation (2.34).

Equation (2.38) now forms the basis of the flight-path angle control law. Observe that the last two terms are essentially an error term, suggesting that $\dot{\alpha}$ follows the error in flight-path angle, with all other parameters held constant. 
A problematic aspect of equation (2.38) is that it involves the derivative of the flight-path angle, $\dot{\gamma}$. This derivative is not available as an output from the physical plant. Taking the derivative of the flight-path angle directly is not good practice, since signal noise may make the derivative unreliable. To overcome this, a second-order filter is applied to the flight-path angle to produce a filtered flight-path angle, $\gamma_{\mathrm{f}}$, and its derivative, $\dot{\gamma}_{\mathrm{f}}$. This second-order filter is implemented using an LTI state space model of the form

$$
\mathbf{F}(\mathrm{s})=\left[\begin{array}{c|c}
\mathbf{A}_{\mathrm{F}} & \mathbf{B}_{\mathrm{F}} \\
\hline \mathbf{C}_{\mathrm{F}} & \mathbf{D}_{\mathrm{F}}
\end{array}\right]
$$

where

$$
\begin{gathered}
\mathbf{A}=\left[\begin{array}{cc}
-2 \varsigma_{\mathrm{f}} \omega_{\mathrm{n}_{\mathrm{f}}} & -\omega_{\mathrm{n}_{\mathrm{f}}}^{2} \\
1 & 0
\end{array}\right] \\
\mathbf{B}=\left[\begin{array}{c}
\omega_{\mathrm{n}_{\mathrm{f}}}^{2} \\
0
\end{array}\right] \\
\mathbf{C}=\left[\begin{array}{ll}
1 & 0 \\
0 & 1
\end{array}\right] \\
\mathbf{D}=\mathbf{0} \\
\mathbf{u}=\gamma \\
\mathbf{y}=\left[\begin{array}{l}
\dot{\gamma}_{\mathrm{f}} \\
\gamma_{\mathrm{f}}
\end{array}\right]
\end{gathered}
$$

Combining the gamma filter and the control law, as described in equations (2.29) through (2.45), completes the flight-path prefilter. A block diagram of the prefilter is illustrated in Figures 20 and 21. 


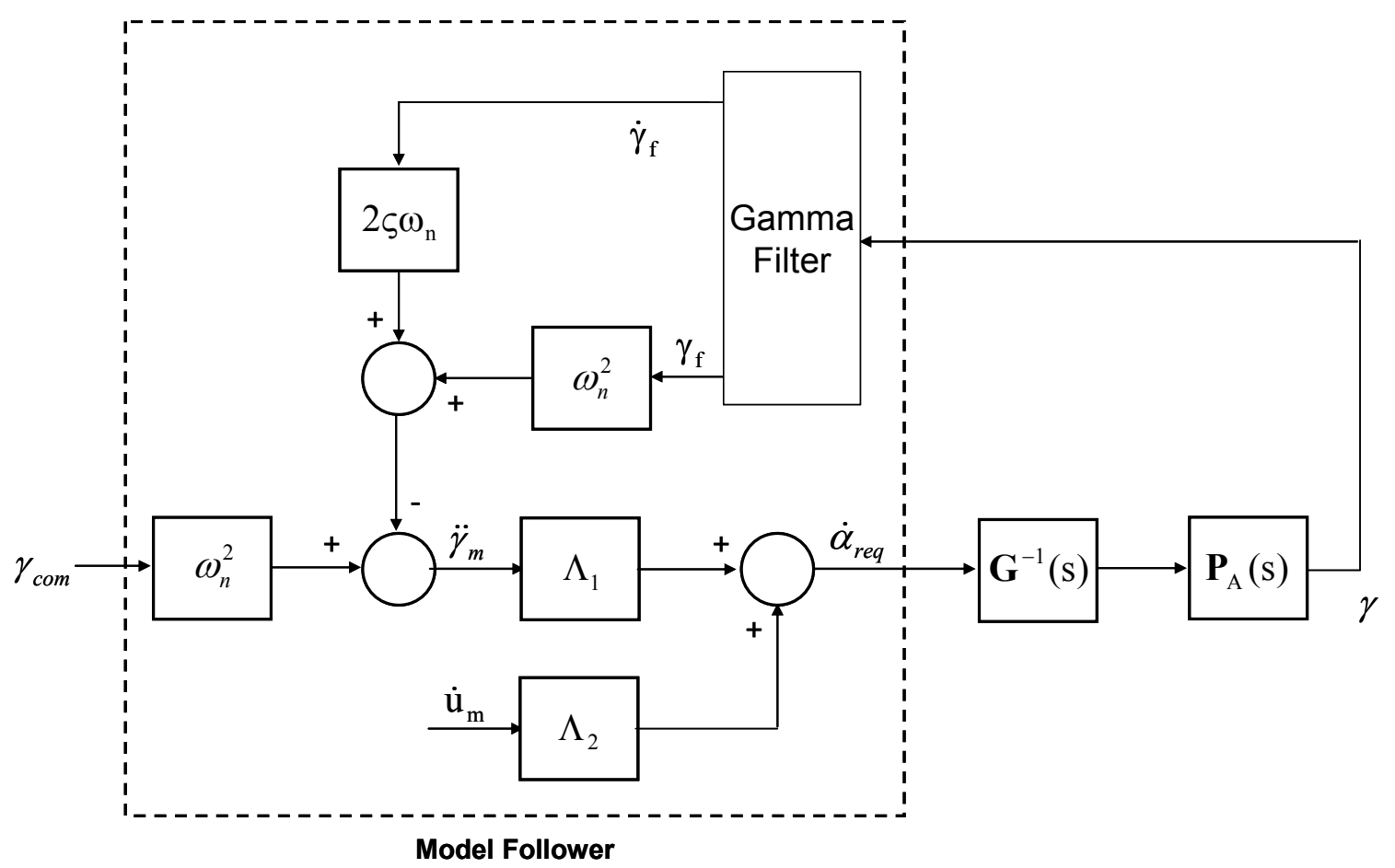

Figure 20: Block diagram of flight-path angle controller

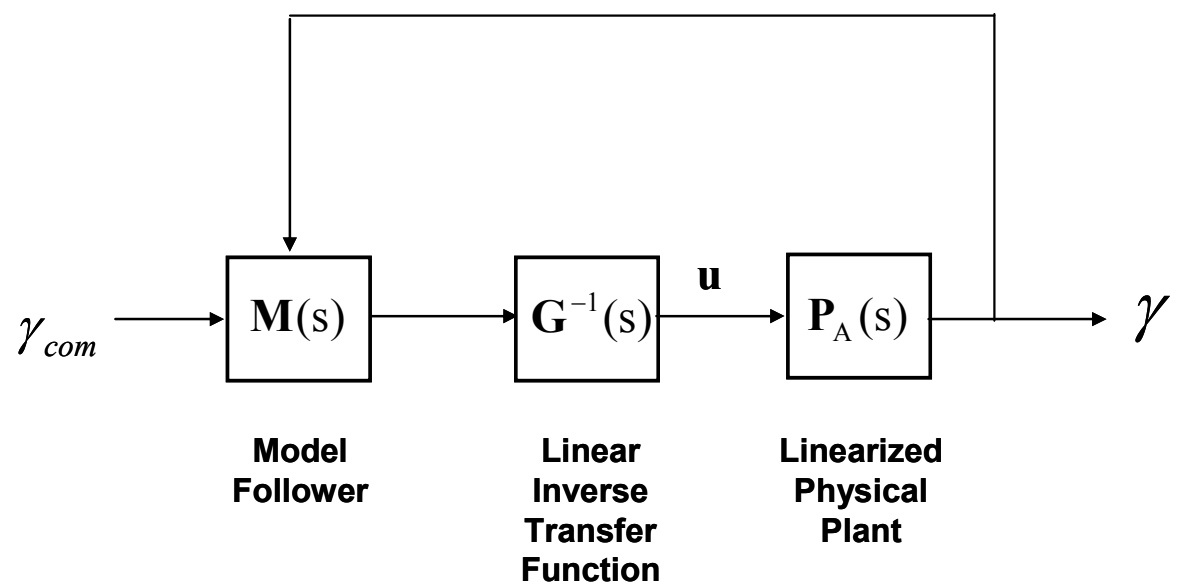

Figure 21: High-level arrangement of flight-path angle controller

The speed and flight-path channels of the linear prefilter can now be assembled into a single state space representation 


$$
\mathbf{M}(\mathrm{s})=\left[\begin{array}{c|c}
\mathbf{A}_{\mathrm{M}} & \mathbf{B}_{\mathrm{M}} \\
\hline \mathbf{C}_{\mathrm{M}} & \mathbf{D}_{\mathrm{M}}
\end{array}\right]
$$

where

$$
\begin{aligned}
& \mathbf{A}_{M}=\left[\begin{array}{cccc}
-2 \varsigma_{u} \omega_{n_{u}} & -\omega_{n_{u}}^{2} & 0 & 0 \\
1 & 0 & 0 & 0 \\
0 & 0 & -2 \varsigma_{f} \omega_{n_{f}} & -\omega_{n_{f}}^{2} \\
0 & 0 & 1 & 0
\end{array}\right] \\
& \mathbf{B}_{M}=\left[\begin{array}{cccc}
\omega_{n_{u}}^{2} & 0 & -\omega_{n_{u}}^{2} & 0 \\
0 & 0 & 0 & 0 \\
0 & 0 & 0 & \omega_{n_{f}}^{2} \\
0 & 0 & 0 & 0
\end{array}\right] \\
& \mathbf{C}_{\mathrm{M}}=\left[\begin{array}{cccc}
1 & 0 & 0 & 0 \\
\Lambda_{2} & 0 & -2 \varsigma_{\gamma} \omega_{\mathrm{n}_{\gamma}} \Lambda_{1} & -\omega_{\mathrm{n}_{\lambda}}^{2} \Lambda_{1}
\end{array}\right] \\
& \mathbf{D}_{\mathrm{M}}=\left[\begin{array}{cccc}
0 & 0 & 0 & 0 \\
0 & \Lambda_{1} \omega_{\mathrm{n}_{\gamma}}^{2} & 0 & 0
\end{array}\right] \\
& \mathbf{u}=\left[\begin{array}{c}
\mathrm{V}_{\mathrm{p}_{\mathrm{com}}} \\
\gamma_{\mathrm{com}} \\
\mathrm{u} \\
\gamma
\end{array}\right] \\
& \mathbf{y}_{\mathrm{M}}=\left[\begin{array}{l}
\dot{\mathrm{u}}_{\mathrm{b}_{\text {req }}} \\
\dot{\gamma}_{\text {req }}
\end{array}\right]
\end{aligned}
$$

Not withstanding any modeling errors or inversion errors, a controller could be assembled from the elements already described. The controller would take the form as shown in Figure 22. Commands from the pilot, plus feedback from the physical plant are received by the linear model prefilter. The prefilter calculates the rates required of the system in order to drive the tracking error to zero in a fashion consistent with the second-order system characteristics 
imbedded in the prefilter. The linear inverse transfer function computes control inputs to the physical plant necessary to achieve the desired rates. The physical plant responds accordingly.

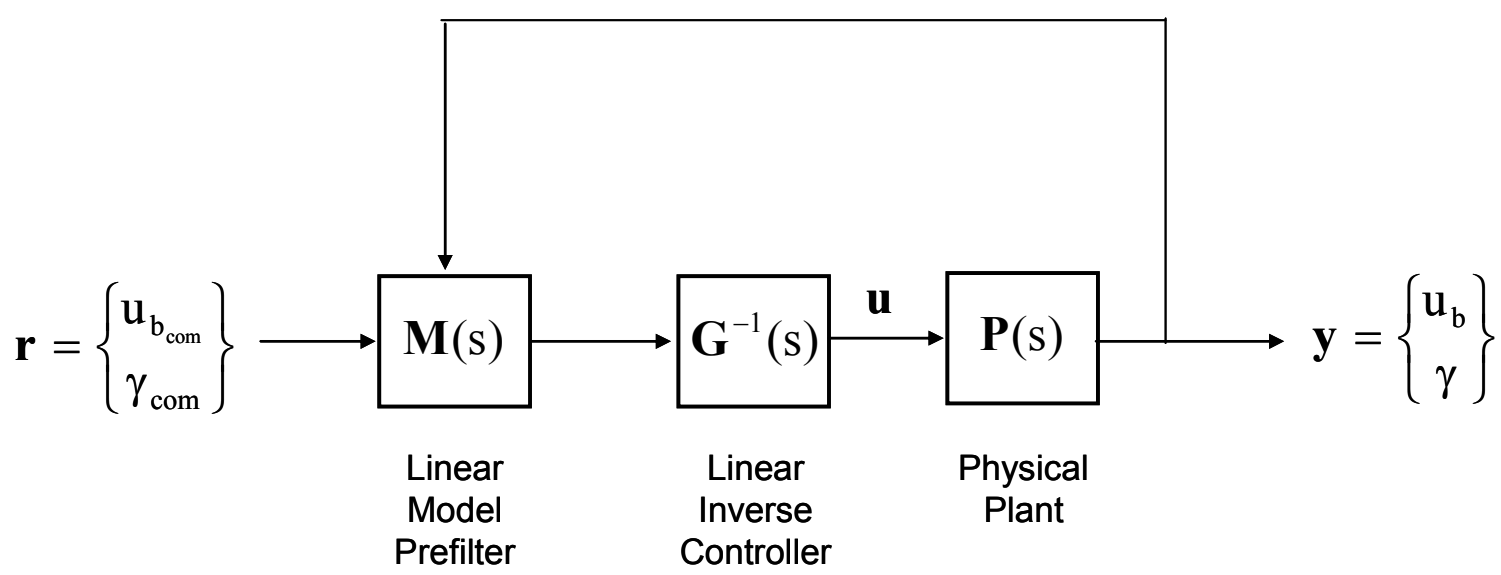

Figure 22: A linear inverse controller with model following, but no adaptation

There are two significant shortcomings in the system described in Figure 22. First, the inverse transfer function is based on a linear approximation of the physical plant. The degree of nonlinearity present in the real plant will influence the precision of tracking. Second, the controller is not adaptive to changes in the physical plant during operation. Even if the physical plant was linear, unforeseen changes may occur within the plant (wear, failures, or new operating modes) that cause the physical plant to operate differently than the characteristics assumed when creating the inverse transfer function. Both of these issues are satisfactorily addressed by the addition of the third element into the controller: the adaptive neural network compensator. 


\subsection{Adaptive Neural Network Compensator}

The existence of an inverse transfer function was discussed in subchapter 2.3. Under certain conditions, a linear inverse transfer function exists such that $\mathbf{G}^{-1}(\mathrm{~s}) \mathbf{G}(\mathrm{s})=\mathbf{I}$. A block diagram illustrating this is shown in Figure 23.

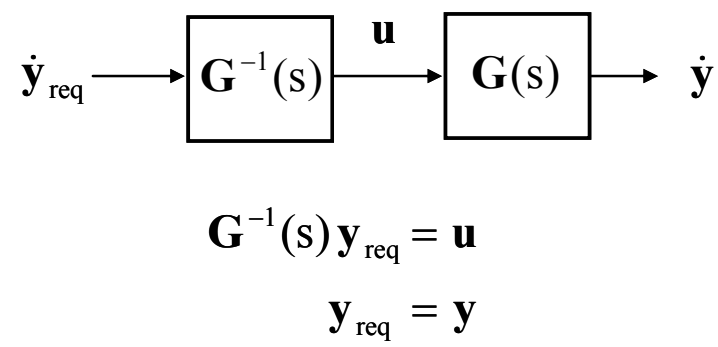

Figure 23: Function of an inverse transfer function

In practice, an exact inverse may not be realizable. This may be the result of approximating what is really a nonlinear function, or perhaps there has been some change in behavior of the physical plant. In cases such as this, the best that can be achieved is an approximate inverse function, $\widehat{\mathbf{G}}^{-1}(\mathrm{~s})$, illustrated in Figure 24 . Note that there is some modeling error, denoted as $\Delta$.

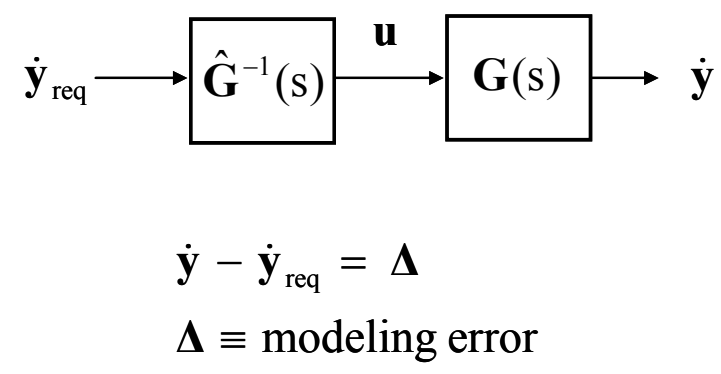

Figure 24: An approximate inverse transfer function with modeling error 
In a discretized computation environment, the definition for $\Delta$ is

$$
\begin{gathered}
\Delta(\mathrm{k})=\dot{\mathbf{y}}(\mathrm{k}+1)-\dot{\mathbf{y}}_{\text {req }}(\mathrm{k}), \text { or } \\
\dot{\mathbf{y}}(\mathrm{k}+1)-\Delta(\mathrm{k})=\dot{\mathbf{y}}_{\text {req }}(\mathrm{k})
\end{gathered}
$$

The intent of the controller is to produce a result $\dot{\mathbf{y}}(\mathrm{k}+1)$ that matches the command of $\dot{\mathbf{y}}_{\text {com }}(\mathrm{k})$, such that

$$
\dot{\mathbf{y}}_{\text {com }}(\mathrm{k})=\dot{\mathbf{y}}(\mathrm{k}+1)
$$

After substitution of equation (2.55) into equation (2.54), the result is

$$
\dot{\mathbf{y}}_{\text {com }}(\mathrm{k})-\Delta(\mathrm{k})=\dot{\mathbf{y}}_{\text {req }}(\mathrm{k})
$$

Figure 25 depicts this in block diagram form. It must be noted that $\Delta(\mathrm{k})$ anticipates the modeling error and adjusts the commanded rate appropriately.

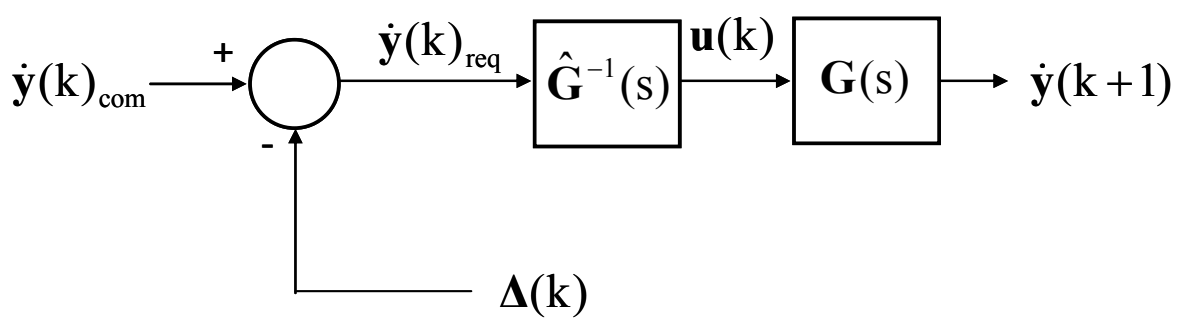

Figure 25: Incorporation of anticipated modeling error into controller

A neural network can be used to compute an estimate of $\Delta(\mathrm{k})$ based on past observations of the modeling error. This estimate is referred to as $\hat{\boldsymbol{\Delta}}(\mathrm{k})$. The difference between $\dot{\mathrm{y}}_{\mathrm{req}}(\mathrm{k})$ and $\dot{\mathrm{y}}(\mathrm{k}+1)$, the actual rate realized at one computation step ahead, is computed and fed into the network to be learned. This $\Delta(\mathrm{k})$ is learned as a function of $\dot{y}_{\text {req }}(\mathrm{k})$ and a variety of other training data. When used to estimate $\hat{\boldsymbol{\Delta}}(\mathrm{k})$, the input to the network is $\dot{\mathrm{y}}_{\text {com }}(\mathrm{k})$ plus these same 
other data, as shown in Figure 26. These other data are selected to best represent the dynamics of the modeling error. Selection of these other data is discussed in subchapter 3.3.

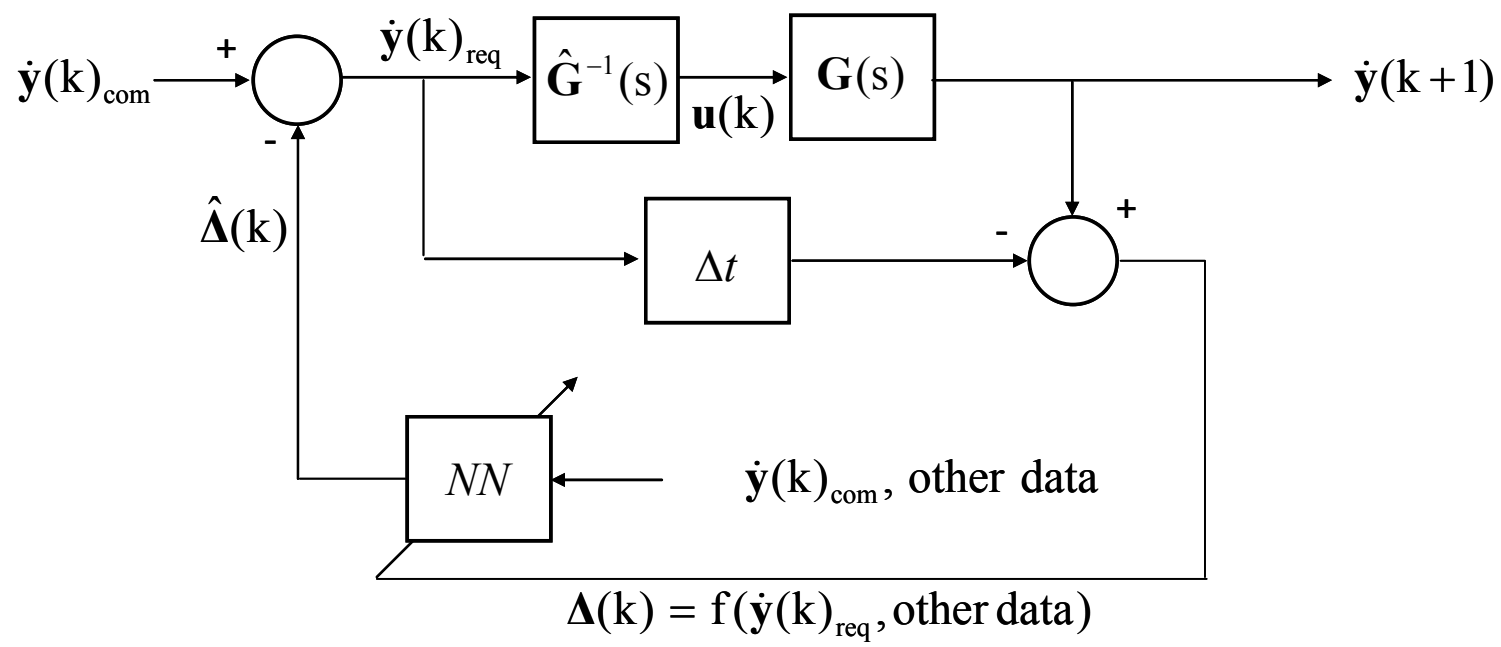

Figure 26: Incorporation of adaptive neural network to approximate modeling error

This entire structure can be viewed as an integrator, similar to the linear inverse transfer function in parallel with the physical plant. Commanded rates come into the system, and their integrals are produced as outputs. See Figure 27.

Online learning allows the neural network to adapt to new operating characteristics in the physical plant. Online learning can be accomplished by the network, whether or not it is actively engaged for compensation. The controller can operate without the benefit of the adaptive compensation, with some degradation in performance, for cases where the physical plant is closely matched to the inverse transfer function. Such operation is demonstrated in subchapter 4.3, example Case 7. While not typical, operation without compensation provides a mechanism for the network to be trained before being put into operation. A preferred method for initial training, however, is to pre-train the network offline to learn the nominal characteristics of the physical plant. 


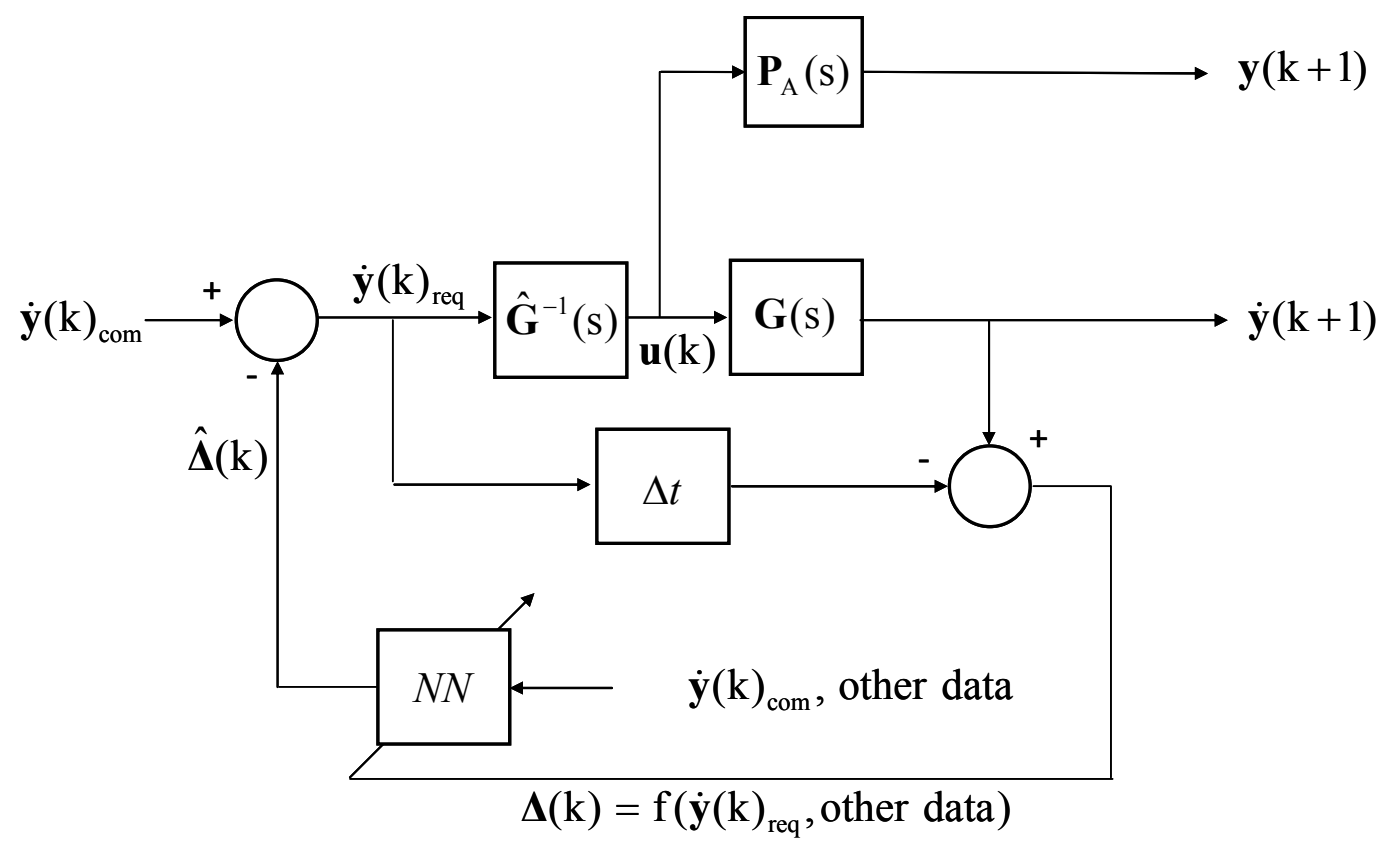

Figure 27: Addition of neural network preserves integration function of controller

At this point, the theory of all major elements of the controller has been discussed. The controller architecture is summarized once again in Figure 28. Now it is time to discuss the mechanics of implementing the controller.

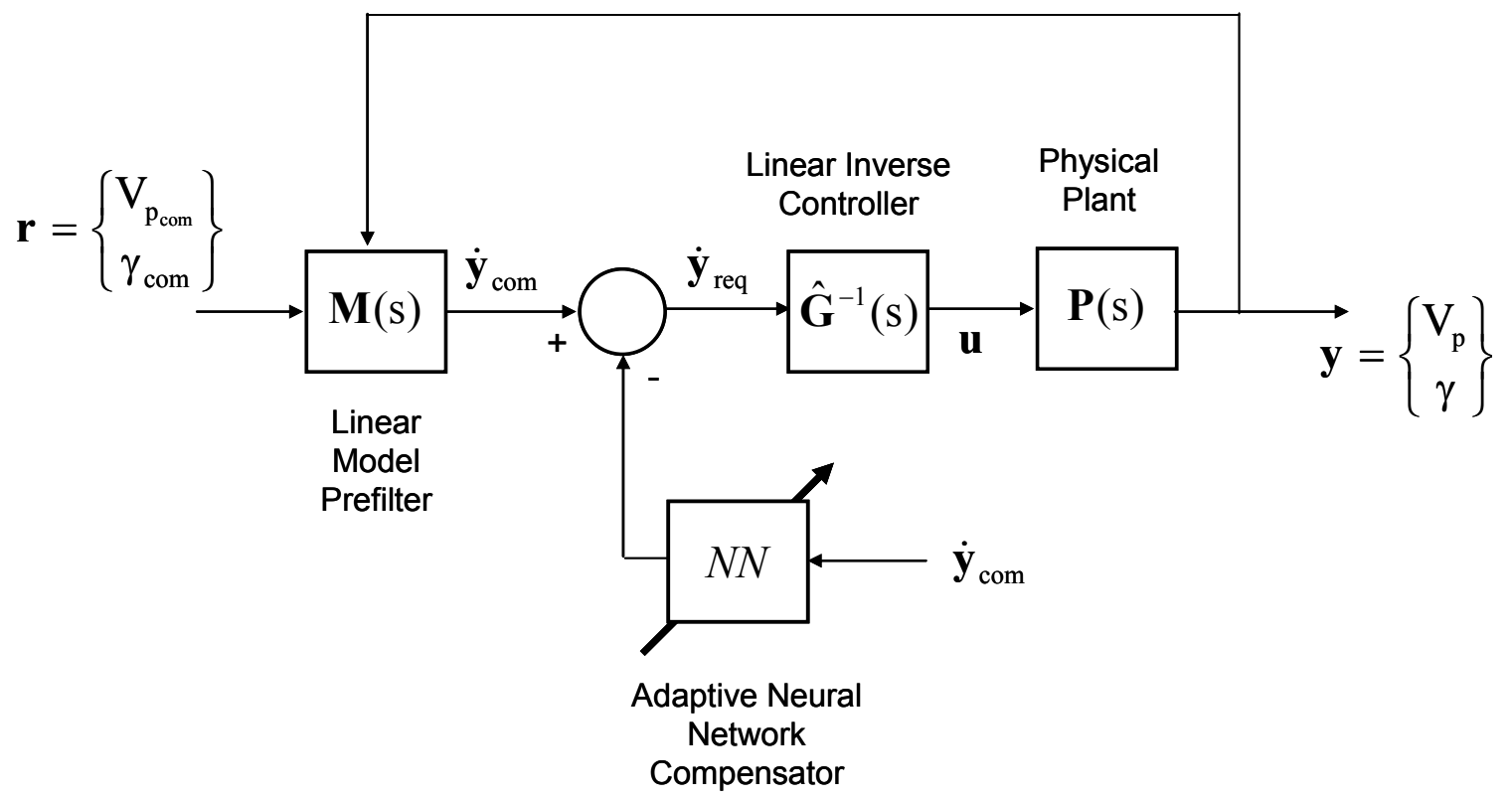

Figure 28: Summary of controller architecture 


\section{CHAPTER 3 \\ IMPLEMENTATION}

\subsection{Bonanza Sample Aircraft}

The Beechcraft Bonanza is a low-wing piston-powered monoplane typical of highperformance General Aviation aircraft. Wichita State University and Hawker Beechcraft Corporation have collaborated in flight research on advanced flight controls using a specially equipped Bonanza, serial number CJ-144. This same aircraft has been used successfully in NASA-sponsored research as part of the AGATE and SATS programs. CJ-144 has been modified to have a fly-by-wire control system on the pilot's side of the aircraft, while retaining traditional mechanical flight controls on the copilot's side. This architecture allows a subject pilot to evaluate flight control algorithms on the left side, while a safety pilot is standing by on the right side to take control in the event of a problem. Illustrations of CJ-144 and its cockpit arrangement are shown in Figures 29 and 30.

The pilot's controls in CJ-144 consist of a "sidestick" controller and a speed-command lever. In the CJ-144 configuration, the sidestick controller uses fore-and-aft motion to command the vertical flight-path angle, while side-to-side motion controls bank angle. The sidestick is spring-loaded to center, which in turn, commands straight and level flight. The speed command lever is simply an input device to command speed. Baseline CJ-144 control laws command elevator deflection and engine power as necessary to maintain the commanded flight-path angle (via the sidestick) and airspeed (via the speed-command lever). For this research, a similar control scheme is assumed. The pilot controls flight-path angle and airspeed as two independent inputs. Controlling the lateral-directional modes of the aircraft is outside the scope of this effort. 


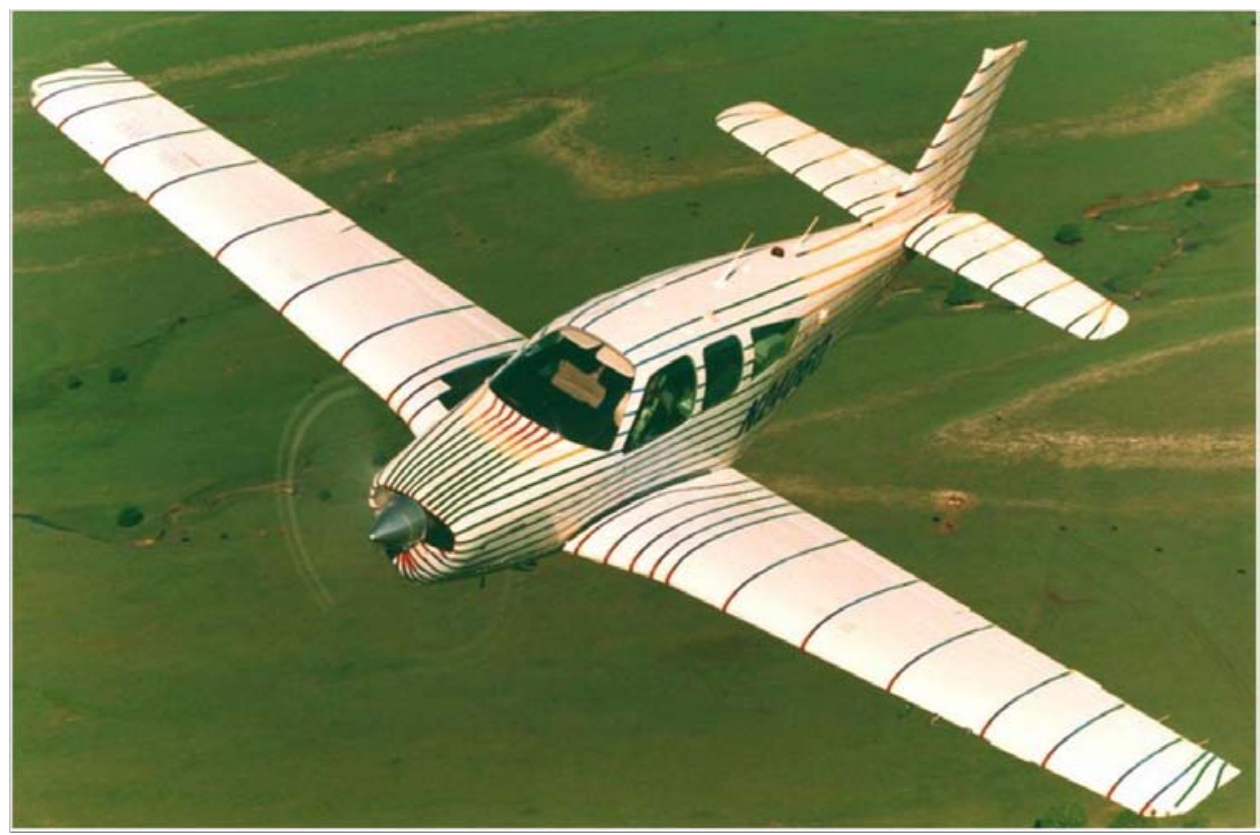

Figure 29: In-flight photo of Hawker Beechcraft Corporation's fly-by-wire Bonanza, CJ-144 (courtesy of Hawker Beechcraft Corporation)

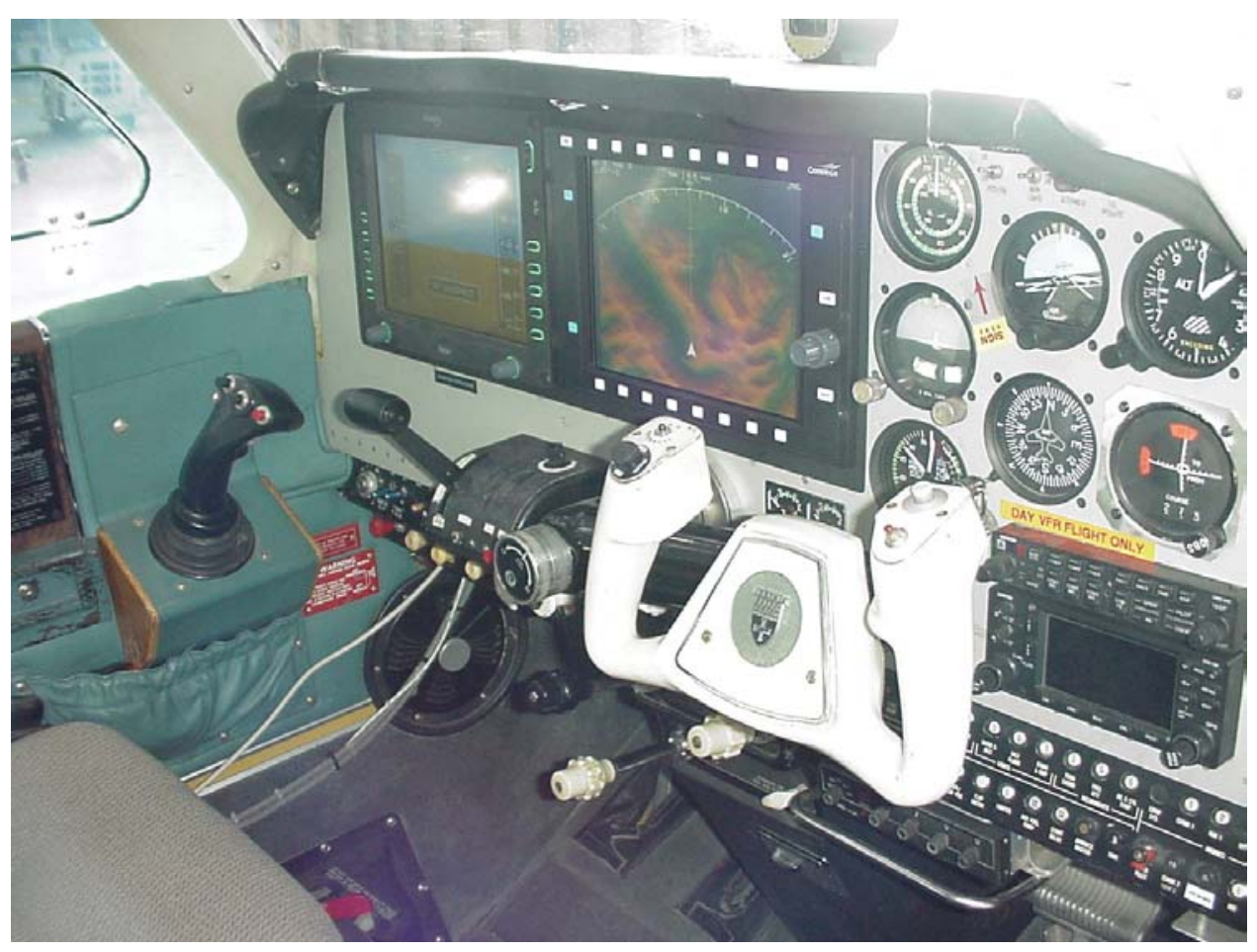

Figure 30: Cockpit of Hawker Beechcraft Corporation's fly-by-wire Bonanza, CJ-144 (courtesy of Hawker Beechcraft Corporation) 
The aerodynamic coefficients and mass properties of the aircraft are provided to Wichita State University for academic use only under license by Hawker Beechcraft Corporation. The data are still considered proprietary data by Hawker Beechcraft Corporation and will not be disclosed in this dissertation.

It should be noted that no flight test evaluation of this controller has taken place on the Bonanza. The purpose of the airplane is simply to serve as a "real-world" candidate platform for simulated evaluation of this controller.

\subsection{Aircraft Simulation}

The aircraft is simulated in a six degree of freedom simulation written in ANSI C. Even though the simulation is capable of running all six degrees of freedom, only the longitudinal modes are utilized for this effort. The equations of motion are largely taken from Roskam [18] and are described in detail in Appendix B. The simulation uses a fourth-order Adams predictorcorrector algorithm for solving the differential equations of motion. The dynamics of the simulation were validated by inserting the data for Roskam's sample "typical business jet." Program function "force_Roskam" inserts this sample airplane data into the simulation via hard code. Results from the simulation were compared with the results shown in Roskam's Table 5.4 and found to be in agreement. The static and trim solutions have been checked by hand. The simulation runs at 50 computation cycles per second of simulated time. On a PC with an Intel 3.2 GHz Pentium 4 processor, the simulation runs approximately 1000 times faster than real time.

Some key features of the code are as follows:

1. Batch mode operation for stacking of multiple cases.

2. Online training of the adaptive neural network. 
3. Creation of training data files for offline training, if desired.

4. Creation of output files for plotting and analysis of simulation results.

A complete source code listing, except for proprietary Hawker Beechcraft Corporation data, can be found in Appendix C.

\subsection{Neural Network Configuration}

The neural network used is a feed forward architecture using backpropagation for learning. The theory of neural networks is described in detail in Fausett's textbook [19]. The value of neural networks in this problem is their ability to approximate any mathematical function, their ability to model a phenomenon with no a priori knowledge of the physics, and their ability to learn in real time. All of these are essential attributes when the controller is tasked with responding quickly to an unspecified failure that may be unknown or not identified.

Defining the exact topology of the network involves some educated guesswork, along with some trial and error. The baseline inspiration for the topology is the state space model for the inverse transfer function. The reasoning is that if the intent of the neural network is to capture the modeling error, the modeling error should, at a minimum, be somewhat similar in functional dependence to the linear inverse transfer function.

The inverse transfer function is described as a state space model. While typically computed using matrices, this same state space model can be implemented exactly as a neural network, with one hidden layer and linear activation functions on each neuron. The weights between the fully connected input and hidden layers represent the coefficients of matrices $\mathbf{A}$ and B. Weights between the fully connected hidden and output layers represent the coefficients of matrices $\mathbf{C}$ and $\mathbf{D}$. This is illustrated in Figure 31. This reasoning immediately suggests that a three-layer network will be required for this application, with a matching number of neurons on 
the first and second layers. The output layer will, of course, have two output neurons: one for the modeling error on $\dot{V}_{p}$ and one for the modeling error on $\dot{\alpha}$.

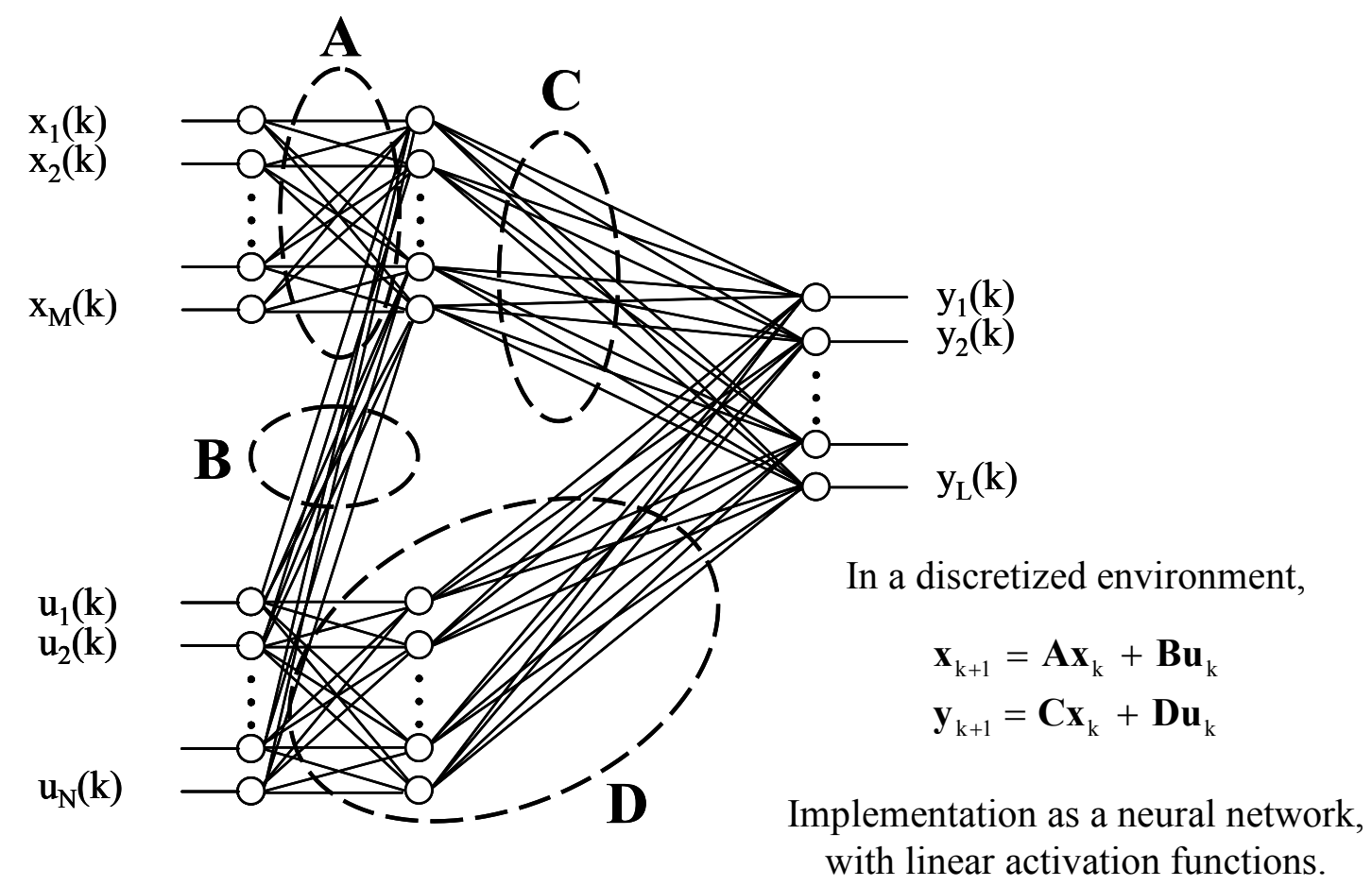

Figure 31: Equivalency of neural network and state space matrix

Anticipating nonlinearities in the modeling error, nonlinear activation functions are assumed for all neurons. A hyperbolic tangent (tanh) function is used.

Furthermore, it is anticipated that the modeling error will be a function of angle of attack (such as nonlinear stall characteristics) and airspeed (such as dynamic pressure, Reynolds number, or Mach effects). In fact, it can be hypothesized that, in general, modeling error could be a function of any of the aircraft state variables: $V_{p}, \alpha, q$, or $\theta$. Consequently, these state variables should be included as inputs to the network.

It is anticipated that the modeling error will involve dynamics of the aircraft states. A recursive neural network, as employed by Hess [15], is utilized to provide a mechanism to 
capture these dynamics. Recursion is accomplished externally to maintain a feed-forward network structure. It cannot be known a priori what the modeling error may be caused by, particularly in the case of system failures within the physical plant. These errors may involve first and second derivatives of the state variables or control inputs. Consequently, not only must the current values of the state variables and inputs be included, but the proceeding two time steps must be included. The network will have current values, plus first and second derivatives by means of finite differences, available for its computations. These could be important for modeling error resulting from hysteresis, computational delays, or backlash.

Trim speed and trim altitude are also likely candidates for inputs to the network, since the aerodynamic characteristics that are the building blocks for the inverse transfer function are dependent on these data.

With these concepts in mind, an iterative process begins to pre-train the neural network. Pre-training the network is analogous to teaching a human to fly. Pre-training instills initial intelligence into the controller. When humans fly an airplane, they generally take control prepared with previously learned flying skills. It would be unreasonable to expect a human to cope with complex failure scenarios in an airplane, and manage well, with no prior experience. So it is true with the adaptive inverse controller. It is unreasonable to expect good performance without pre-training. During the pre-training process, flying experiences are repetitively presented to the neural network, and over time, the network learns. This is not unlike the repetitive process humans go through in learning to fly.

A companion program was written to pre-train the network "offline." Offline implies post-processing the results of the airplane simulation. The flight dynamic modules are absent in the offline training program, and are replaced with file $\mathrm{I} / \mathrm{O}$ routines to handle large training data 
files. All neural network computational modules are the same in both the flight simulation and offline training programs. This offline process facilitates the iterative process of pre-training the network. During this pre-training process, the network architecture evolves. The ability to deal with unanticipated scenarios drives to a larger network configuration than would be required for a small bounded set of failure scenarios. As will be shown later, the final architecture proved to be robust and responsive to all nonlinearities and failures examined.

The pre-training process is shown graphically in Figure 32. The process follows this sequence:

1. The aircraft simulation is exercised over a range of speed and altitudes that cover the aircraft flight envelope. Training maneuvers, consisting of random commands for speed and flight-path, are flown by the simulation. The airplane is in its nominal no-failurestate condition with no neural network compensation. The necessary data for training are recorded in a data file. There are 11,550 training pairs in the final training data set.

2. Recorded data is divided into a training data set and test data set. The training data set is used for offline training of the network. The trained network is then exercised offline with the test data to see if the network can replicate the test outputs. The goal for training is to produce a network that works equally well on both the training data set and the test data set.

3. Candidate architectures are implemented into the simulation. The offline training program creates $\mathrm{C}$ code that can be "cut and paste" into the simulation code. This coding contains all the network parameters necessary to fully describe the network; such as the network topology, the value of all network weights, and all network input and output scaling factors. 
4. A series of validation maneuvers are flown with the simulation to evaluate the performance of the controller with the adaptive neural network compensator turned on. Unlike the random training maneuvers, these are a series of well-defined piloting tasks similar to the sample cases to be described in Chapter 4.

5. The training iteration is continued until the results of the validation maneuvers are satisfactory. If the results are not satisfactory, adjustments are made to either the network architecture or to the training experience, and then the process is repeated.

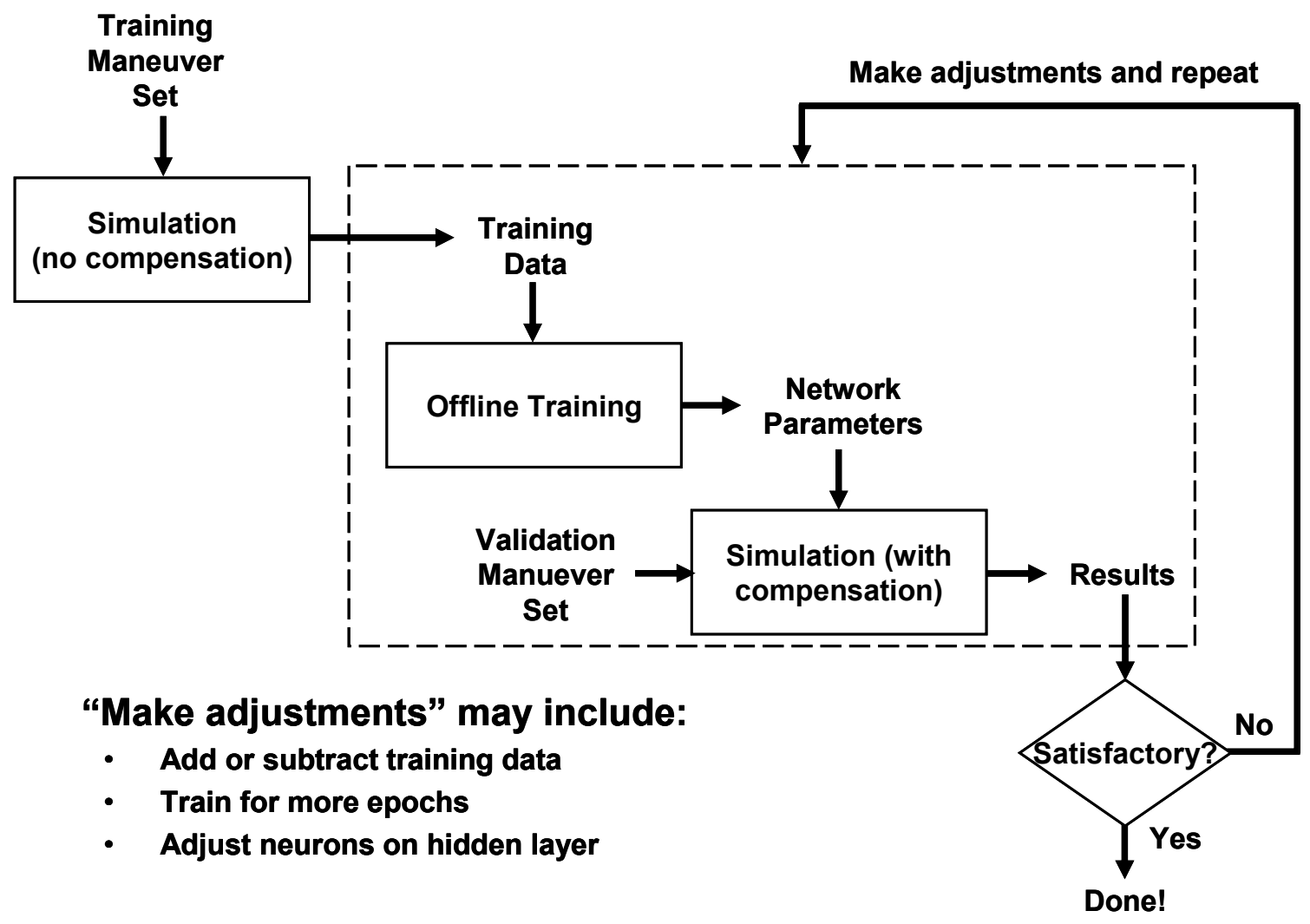

Figure 32: Neural network training process

Figure 33 characterizes the training data before the training process begins. The purpose of the training is to find some correlation between $\Delta \dot{\mathrm{u}}_{\mathrm{b}}$ and $\Delta \dot{\alpha}$ with the required values of 
$\dot{\mathrm{u}}_{\mathrm{b}}$ and $\dot{\alpha}$, respectively, plus whatever other data may be required to achieve a correlation. The magnitude of this task is evident. There is a weak correlation evident between $\Delta \dot{\mathrm{u}}_{\mathrm{b}}$ and the required $\dot{\mathrm{u}}_{\mathrm{b}}$, but considerable scatter remains. Other data must be involved to explain this scatter. On the other hand, there is no correlation evident between $\Delta \dot{\alpha}$ and the required $\dot{\alpha}$. An absolutely random scattering of $\Delta \dot{\alpha}$ points is apparent. The challenge for the offline training is to find a correlation such that a good estimate for $\Delta(\mathrm{k})$ may be obtained from some combination of available data.
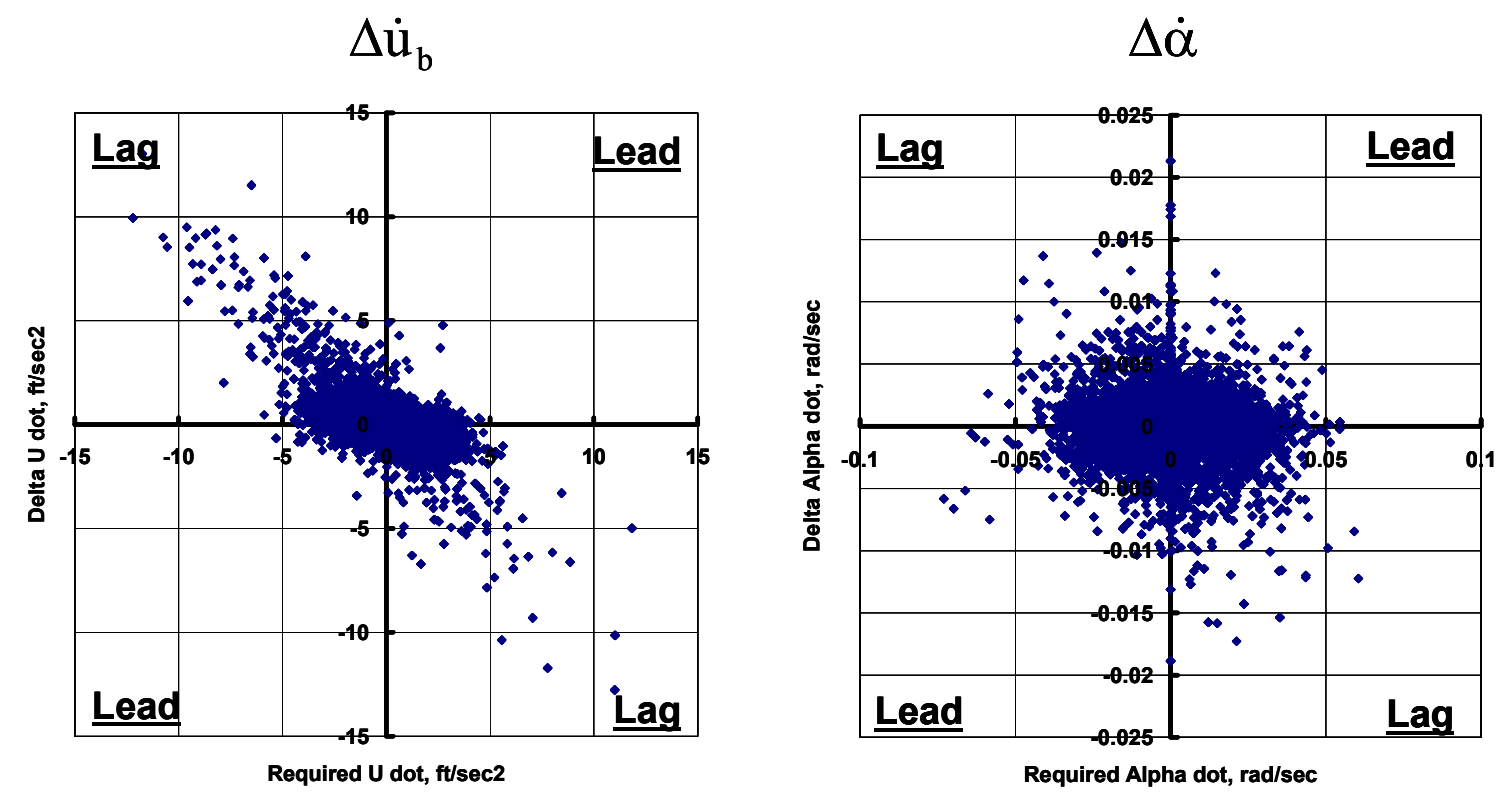

Figure 33: Data before training

Figures 34 and 35 show the final training results and the test results from the offline training. The known system outputs are plotted on the x-axis, and the estimated output by the network is plotted on the y-axis. Ideally, all points should lie along the " 45 degree line of correlation." While not perfect, this fit is quite good. The final RMS error for all data points is 
$.030 \%$. The Pearson product moment correlation coefficient $\left(\mathrm{R}^{2}\right)$ commonly used in Excel ${ }^{\mathrm{TM}}$ trend analysis is seen to be very close to one, also indicating a good correlation with the data.

The final architecture chosen is illustrated in Figure 36. There are 64 computational nodes in the network. The external recursion of the output can be seen.
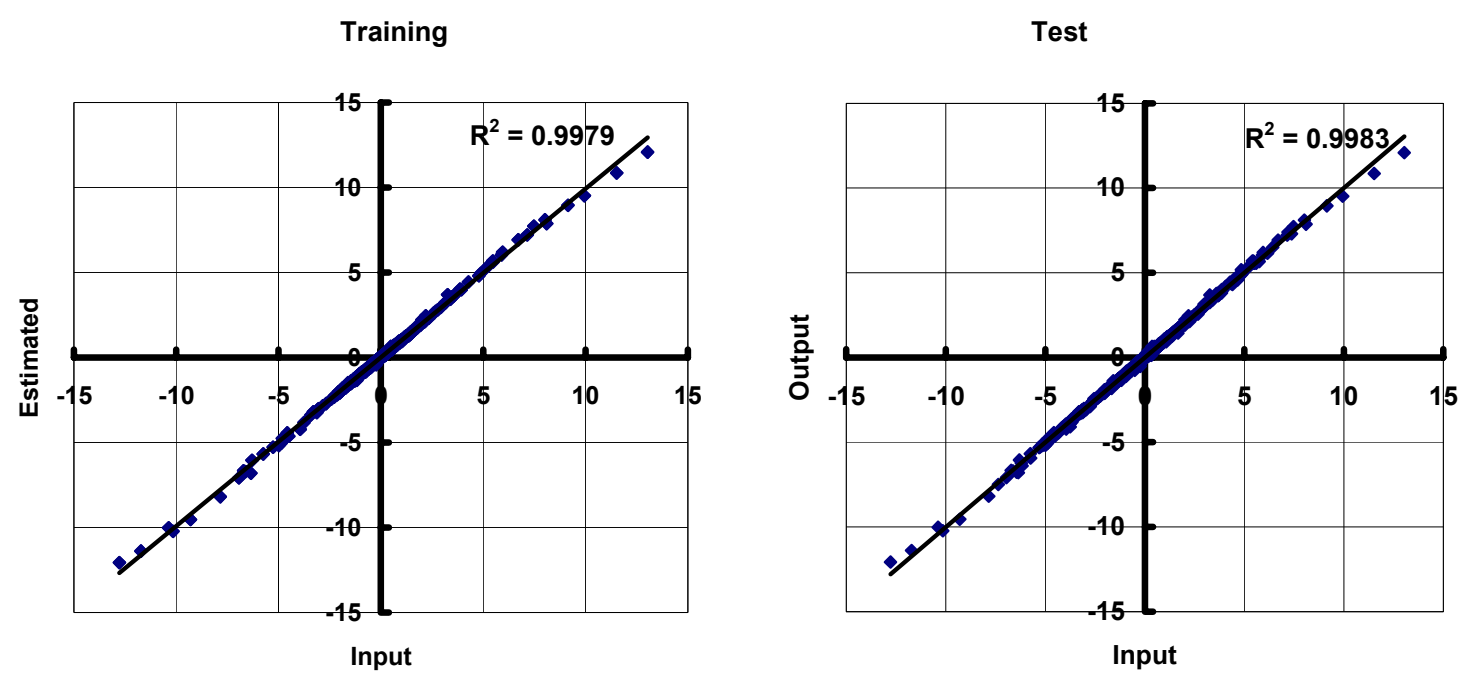

Figure 34: Results of training network on $\dot{V}_{p}$ modeling error
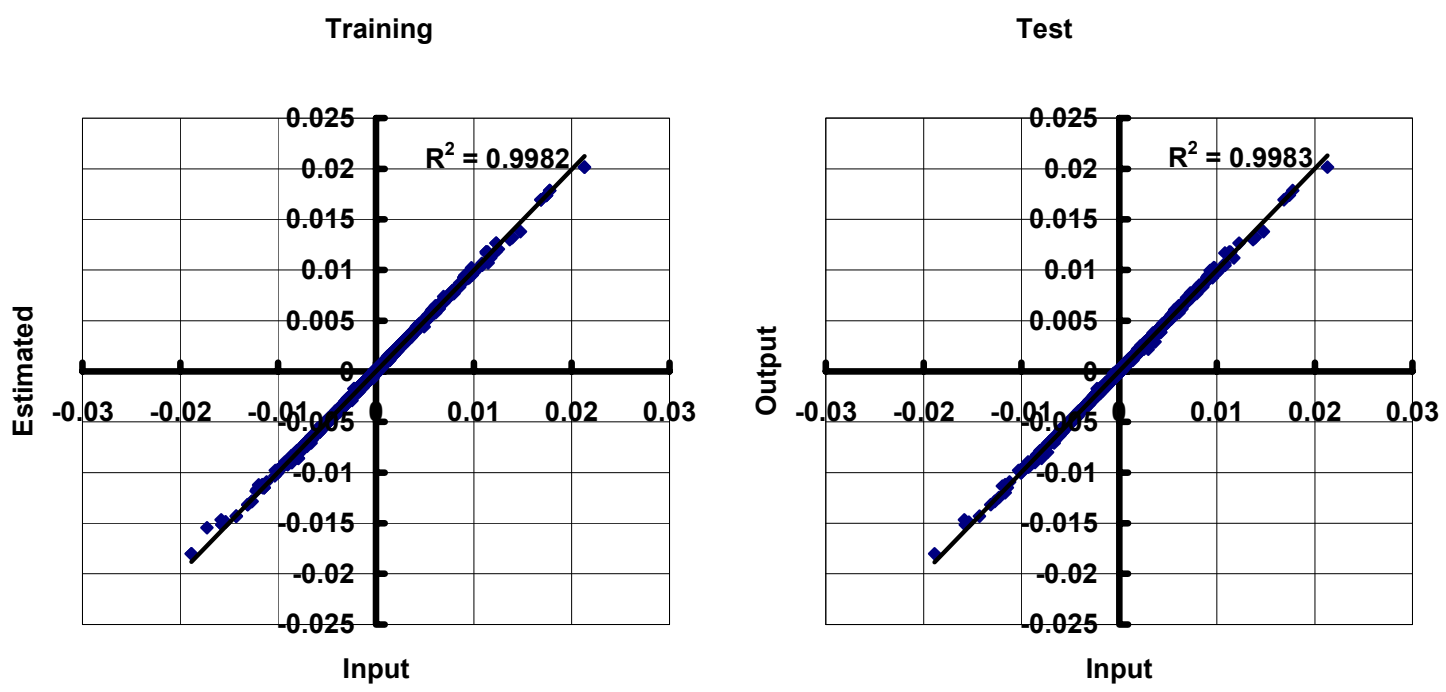

Figure 35: Results of training network on $\dot{\alpha}$ modeling error 

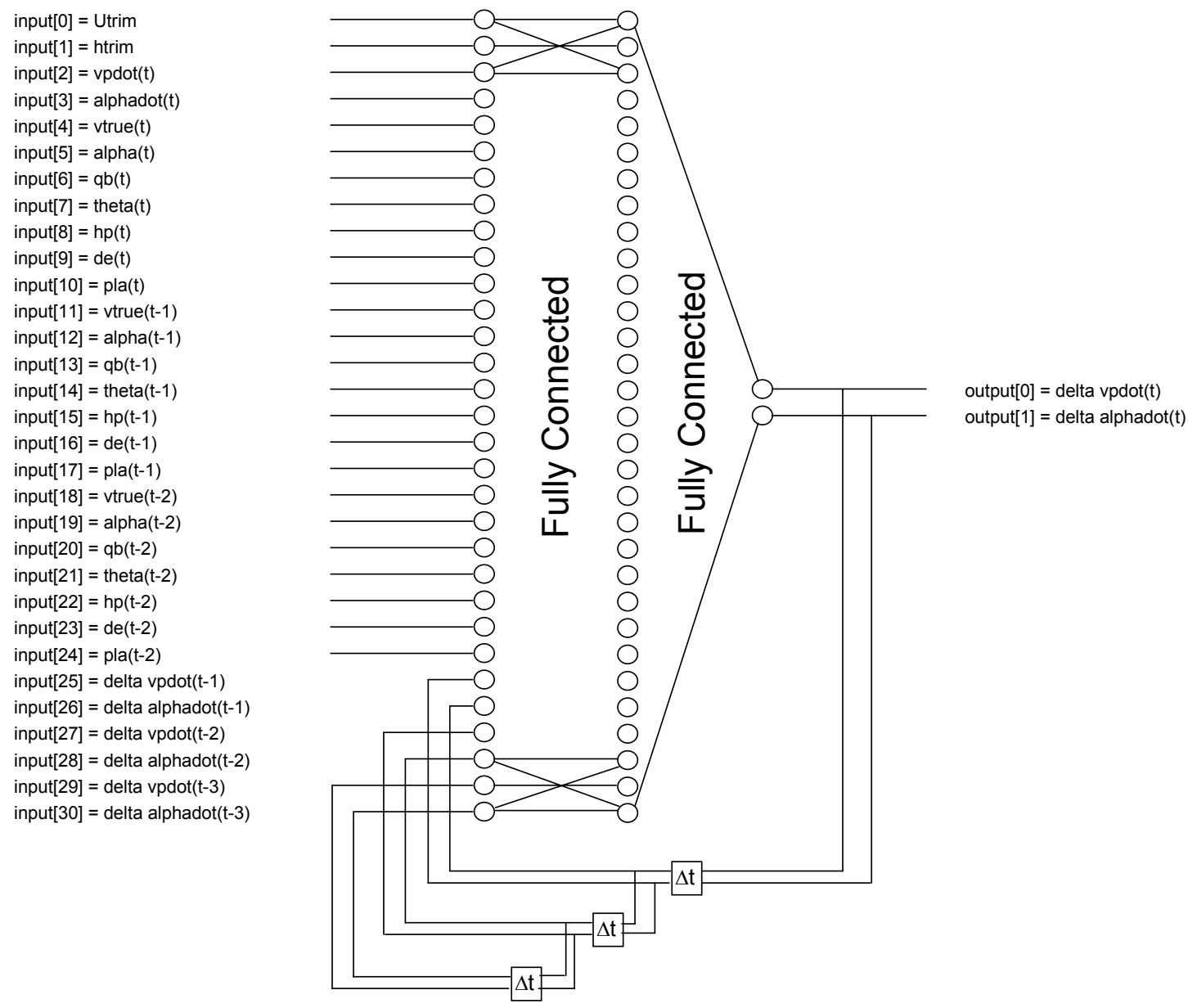

Figure 36: Topology of final network architecture 


\subsection{Controller Coding}

The controller is coded in ANSI C for future compatibility with the flight computer in Hawker Beechcraft Corporation's Bonanza CJ-144. The code is modularized to facilitate rapid integration into CJ-144 if the opportunity becomes available.

The linear prefilter is implemented in function "linear_prefilter_12." The code may be found in Appendix C. This function implements the state space model of the prefilter, as described in equations (2.46) through (2.52). All operations are implemented using linear algebra. The $\mathbf{A}$ and $\mathbf{B}$ matrices of the prefilter are static after initialization, since they are functions only of constant damping ratios and natural frequencies. The $\mathbf{C}$ and $\mathbf{D}$ matrices are sparse, and only the non-zero coefficients are explicitly coded. These non-zero coefficients are updated on each computation cycle to reflect the current values of true airspeed and atmospheric density. The inputs to this routine are commands for airspeed and flight-path angle, and the current values of airspeed and flight-path angle. The outputs are the commanded values of $\dot{\mathrm{V}}_{\mathrm{p}}$ and $\dot{\gamma}$. There are 25 lines of executable code in this function.

The inverse transfer function is implemented in function "inverse_xfunction." The code may be found in Appendix C. This function implements the state space model of the linear inverse transfer function, as described in equations (2.12) through (2.19). All operations are implemented using linear algebra. The $\mathbf{A}, \mathbf{B}, \mathbf{C}$, and $\mathbf{D}$ matrices are all static once initialized. They are a function of the initial trim true airspeed only, and consequently do not change

throughout a simulation run. The inputs to this routine are the required values of $\dot{V}_{p}$ and $\dot{\gamma}$. The outputs are elevator position and power-lever angle, PLA. "Power-lever angle" is an anachronism in this application, but refers to the historical use of "power levers" to control engine power. In this controller, power-lever angle is used to command a percent of maximum 
available thrust for the given altitude and airspeed. PLA of 100 equates to 100 percent of available thrust. There are 20 lines of executable code in this function.

The $\mathbf{A}, \mathbf{B}, \mathbf{C}$, and $\mathbf{D}$ matrices for the inverse transfer function are scheduled as functions of the trim airspeed. Scheduling is accomplished by explicitly calculating the coefficients for each matrix for ten different true airspeeds, from $100 \mathrm{feet} / \mathrm{sec}$ to $260 \mathrm{feet} / \mathrm{sec}$, at an altitude of 3000 feet. The assumption of 3000 feet highlights that this inverse transfer function is approximate at other altitudes. These matrices were calculated using a MATLAB ${ }^{\mathrm{TM}}$ script. Internal MATLAB ${ }^{\mathrm{TM}}$ routines were used to convert the state space model from the continuous time domain to the discrete time domain. Each coefficient of each matrix was curve fit against a polynomial in speed. These resulting polynomials are coded into the $\mathrm{C}$ program. Function "update_inv_state_space" expands these polynomials once at the beginning of a run. While the calculations sound daunting, this function requires only 25 lines of code.

The adaptive neural network compensator is implemented through three $\mathrm{C}$ functions. Function "initialize_by_code" initializes the network configuration only once at the beginning of each simulation. Functions "forward_pass" and "backprop" accomplish the forward pass and backpropagation computations for the neural network. Online learning occurs during backpropagation. Function forward_pass has seven lines of executable code. Function backprop has 21 lines of executable code.

Function "DEF_JRcontrol" assembles all components into the functional controller. The computational sequence is as follows:

1. Compute $\Delta$ based on results from the inputs and outputs to the last computation cycle.

2. If requested, write training data to a scratch file for later formatting.

3. If learning is enabled, back propagate to learn from $\Delta$ calculated above. 
4. Use the linear prefilter to compute the desired values of $\dot{V}_{p}$ and $\dot{\alpha}$.

5. Invoke angle of attack limiting, if requested.

6. Assemble the inputs to the network for this computation cycle.

7. If the adaptive neural network compensator is active, calculate values for $\hat{\Delta} \dot{\mathrm{V}}_{\mathrm{p}}$ and $\hat{\Delta} \dot{\alpha}$ and add to desired $\dot{V}_{\mathrm{p}}$ and $\dot{\alpha}$ to get required $\dot{\mathrm{V}}_{\mathrm{p}}$ and $\dot{\alpha}$. Otherwise, set the required $\dot{\mathrm{V}}_{\mathrm{p}}$ and $\dot{\alpha}$ values to be the same as the desired $\dot{V}_{\mathrm{p}}$ and $\dot{\alpha}$.

8. Use the linear inverse transfer function to calculate the control inputs for elevator and power-lever angle. The resulting control inputs are then returned to the simulation to fly the airplane. 


\section{CHAPTER 4}

\section{RESULTS}

\subsection{Introduction}

Twenty-three simulations are presented to demonstrate the controller's ability to meet the objectives outlined in subchapter 1.2. The scenarios examined in each of these cases are summarized in Table 2. Each case is addressed by a series of three pages of time histories. Page one illustrates the perturbations in speed, flight-path angle, and the controls (elevator and powerlever angle) from the trim values. The command inputs are over-plotted on speed and flight-path angle. Pages two and three show the full values of the flight parameters in common engineering units. It is important to note that the simulation works with the full values, and the perturbation quantities are simply shown for convenience when comparing back to an ideal second-order system. Page three also contains a running average of the network RMS error. This parameter is an indicator of the learning achieved by the network. Small values of this parameter indicate that the network is well trained and little additional learning is taking place. RMS errors on the order of one suggest that the network is experiencing significant new information and that significant learning is taking place. This organization applies to all example cases that follow.

As previously discussed, the current controller permits arbitrary values to be prescribed for the dynamic characteristics of the overall system. For the cases examined, the following characteristics are assigned: $\zeta_{\mathrm{u}}=.8, \omega_{\mathrm{nu}}=1 \mathrm{rad} / \mathrm{sec}, \quad \zeta_{\gamma}=.7$, and $\omega_{\mathrm{nu}}=2.5 \mathrm{rad} / \mathrm{sec}$. These values produce a rise time of 3.12 seconds for speed and .856 seconds for flight-path angle. These characteristics are chosen for illustrating the generality of the current controller but may not be the optimum for handling qualities. 
The controller as implemented in this work is intended to demonstrate that the academic objectives of the controller are satisfied: obtaining prescribed system performance and adaptation to changes in the physical plant. To make these objectives evident, some "real-world" considerations have been purposely neglected. No limits are imposed on elevator or thrust commands. No limits are placed on normal load factor or speed. Such limits would obscure the performance of the system toward achievement of the characteristics prescribed. A simple limiter is imposed on angle of attack at fourteen degrees. This angle of attack limiter is exercised in some of the simulations examined to demonstrate that the controller architecture is compatible with the types of limits that would need to be imposed in a real application.

\subsection{Nominal Controller Performance}

Six cases follow that illustrate the performance of the controller for the nominal physical plant. These cases span a range of altitude and speed and demonstrate the controller's performance across the aircraft flight envelope. The command inputs are a series of step inputs in speed or flight-path angle. In addition to the input commands, a reference response is overplotted on speed and flight-path. This reference response is the response that would be expected from an ideal second-order linear system with the specified characteristics. Cases 1 through 6 (Figures 37 through 42) demonstrate several important characteristics of the controller. First, the controller closely approximates the second-order performance desired. Examination of the perturbation velocity graph shows that the simulation almost perfectly overlays the reference. Examining the perturbation gamma graph shows a similar comparison. The flight-path angle is still good but does show some very slight tendency to overshoot the reference. This overshoot suggests that a somewhat lower damping ratio is achieved than is targeted. This small loss of damping can be traced to the gamma filter within the linear prefilter, and the phase shift it 
produces in flight-path angle feedback. If exact damping performance is required, the target value for damping ratio can be adjusted to compensate. Second, comparing the results of Cases 1 through 6 shows that controller performance is consistent across all speeds and altitudes. Third, little learning is occurring. The RMS error graph shows a low level of learning for all cases. This is to be expected since the adaptive neural network was pre-trained using the flight characteristics of the nominal aircraft. The step commands in flight-path angle show higher levels of learning taking place, since step inputs were not part of the training maneuvers and these are "new" to the neural network. Finally, it should be observed that there is no evidence of the phugoid or short period modes associated with typical General Aviation airplanes. These classically coupled modes have been totally suppressed. 
TABLE 2

SUMMARY OF SIMULATION CASES EXAMINED

\section{$\underline{\text { Case \# Description }}$}

$1 \quad$ Square Wave on Speed

2 Square Wave on Speed

3 Square Wave on Speed

4 Square Wave on Gamma

5 Square Wave on Gamma

6 Square Wave on Gamma

7 Square Wave on Speed, with Gamma Step at $\mathrm{t}=2$

8 Square Wave on Speed, with Gamma Step at $\mathrm{t}=3$

9 Square Wave on Speed, with Gamma Step at $\mathrm{t}=4$

10 Square Wave on Speed, with Gamma Step at $\mathrm{t}=5$

11 Steck Case 1

12 Steck Case 2

13 Steck Case 3

$14 \quad$ Steck Case 4

15 Long Duration Manuever

16 Long Duration Manuever

17 Non-Linear CLA and CMA

18 Non-Linear CLA and CMA

19 Sine Wave on Speed

20 Sine Wave on Speed

21 Sine Wave on Speed

22 Sine Wave on Speed

23 Sine Wave on Speed
NNet Failure State

Compensation

On None

On None

On None

On None

On None

Off None

On None

Off Halve CMA at $\mathrm{t}=6$

On Halve CMA at $\mathrm{t}=6$

120

6000

On As Noted

100

3000

On As Noted

100

3000

On

As Noted

100

3000

On

As Noted

100

3000

On

None

100

3000

On

$\mathrm{CMA}=0$ after $\mathrm{t}=400$

100

80

3000

On None

3000

On Halve CLDE and CMDE at $\mathrm{t}=400$

80

3000

On None

100

3000

On

Neural Network Udot

Hardover

On

Neural Network Udot

Oscillatory Failure

On

Neural Network Alphadot

Hardover

On

Neural Network Alphadot

000

\section{0} 000 000 000 000 000

Oscillatory Failure 


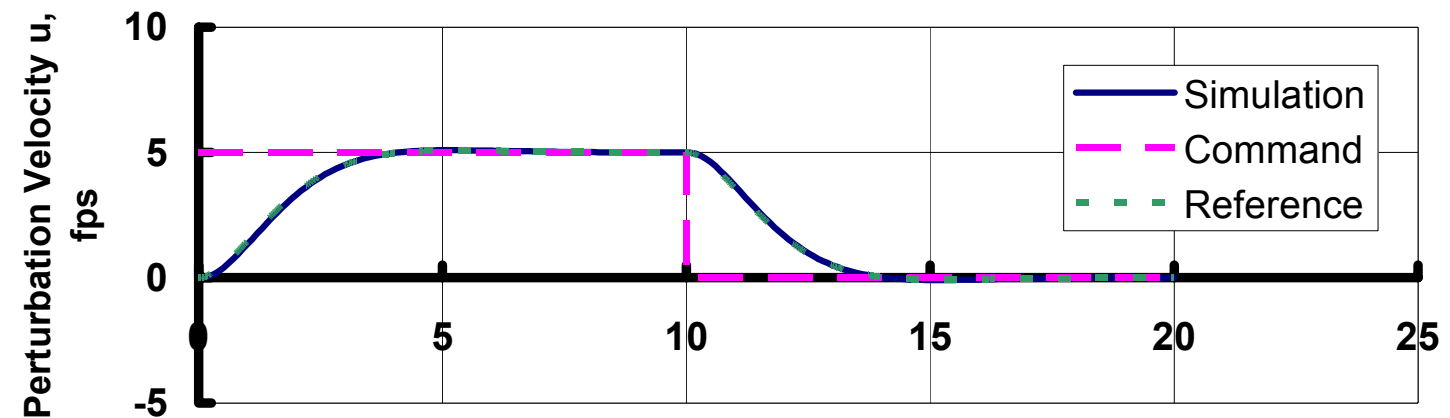

Time, Seconds

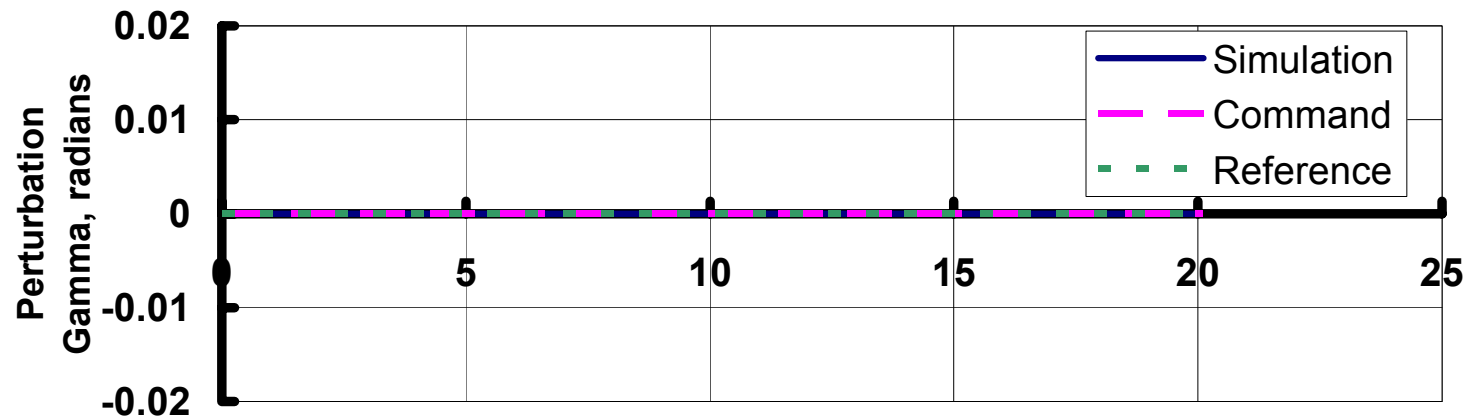

Time, Seconds

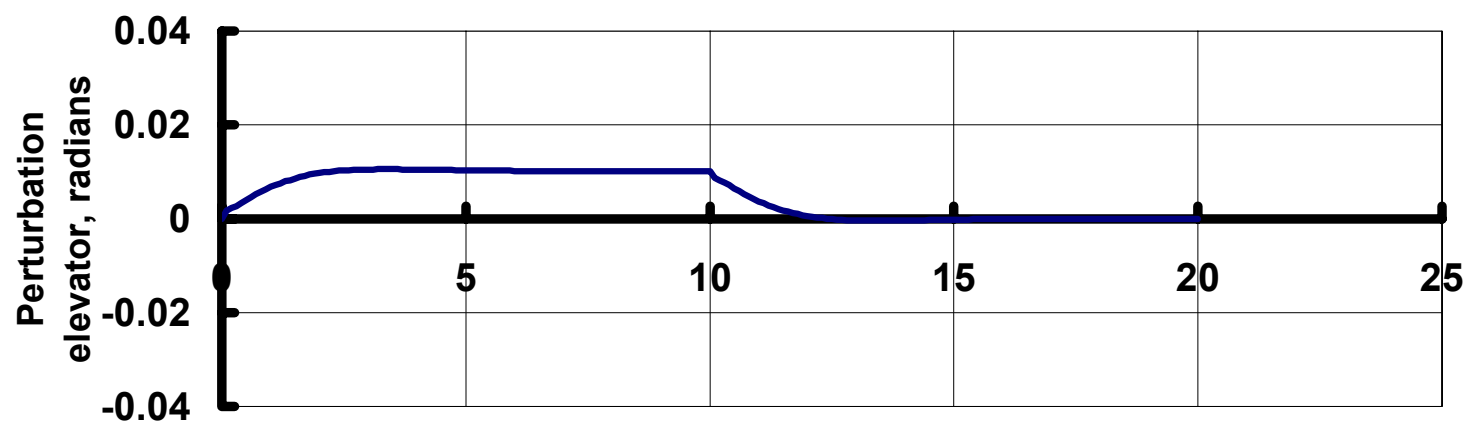

Time, Seconds

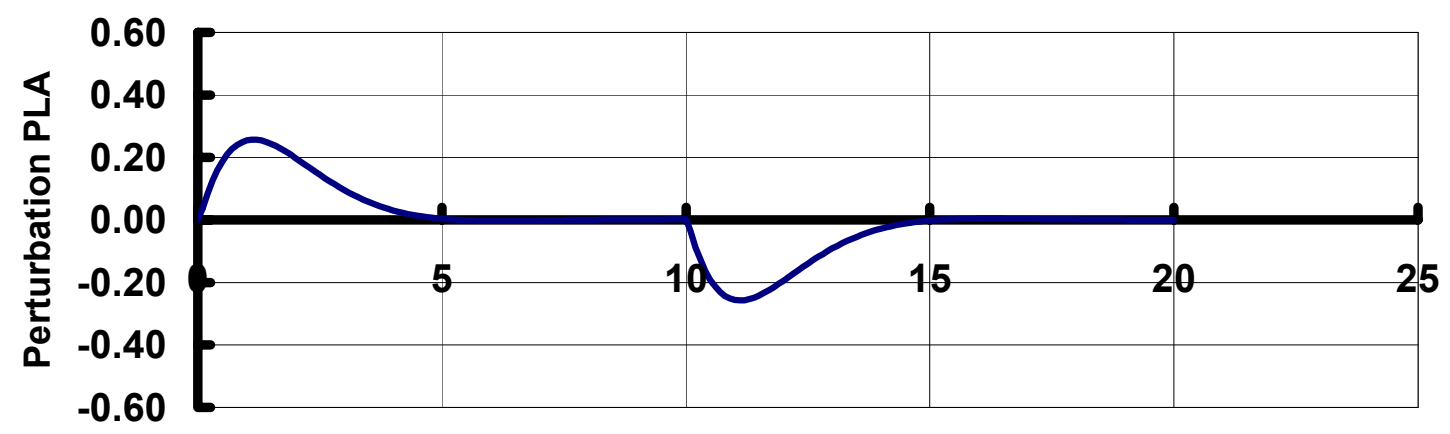

Time, Seconds

Figure 37 (a): Case 1 time history 

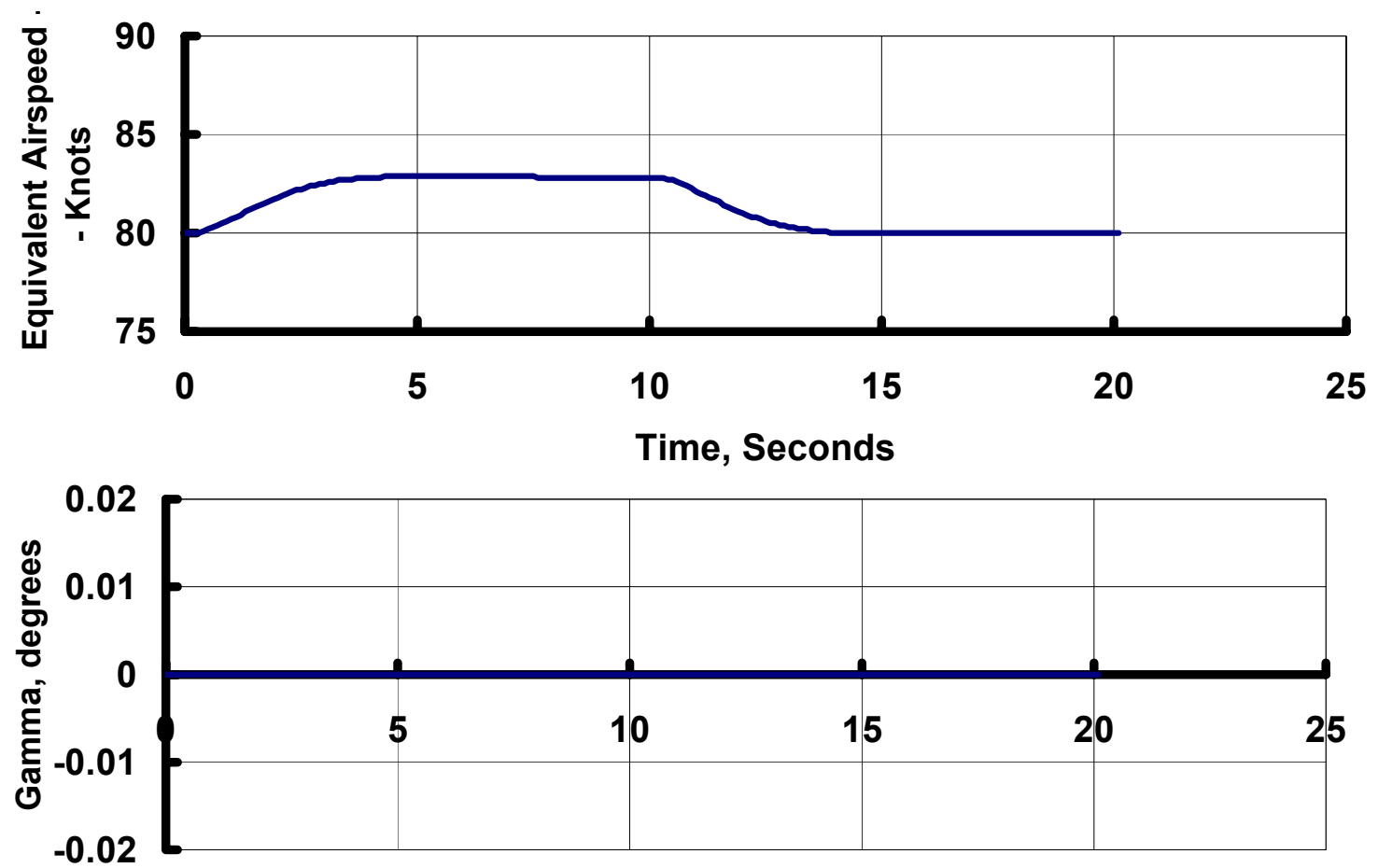

Time, Seconds

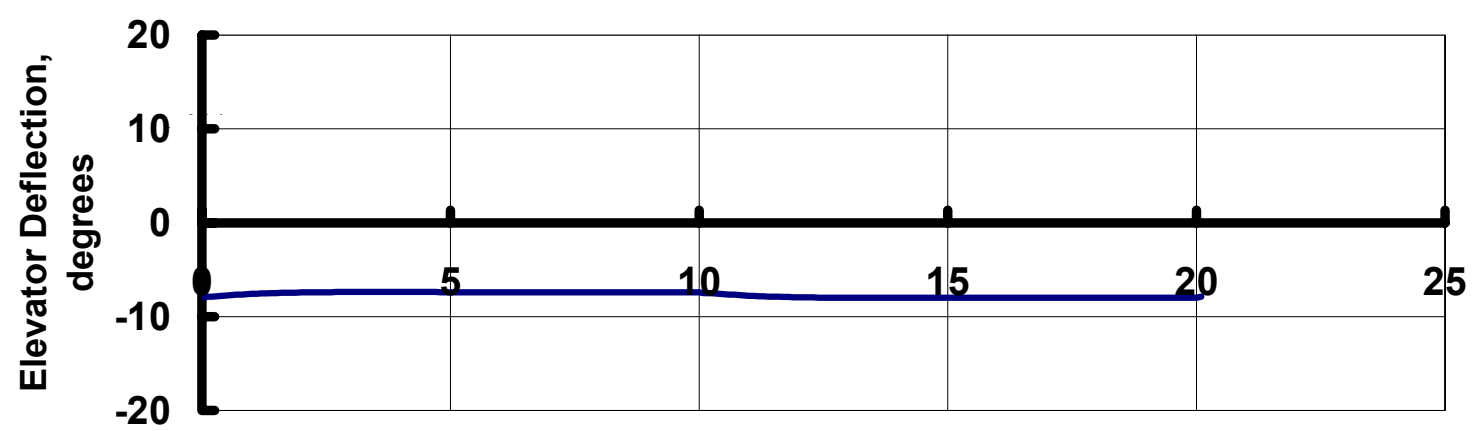

Time, Seconds

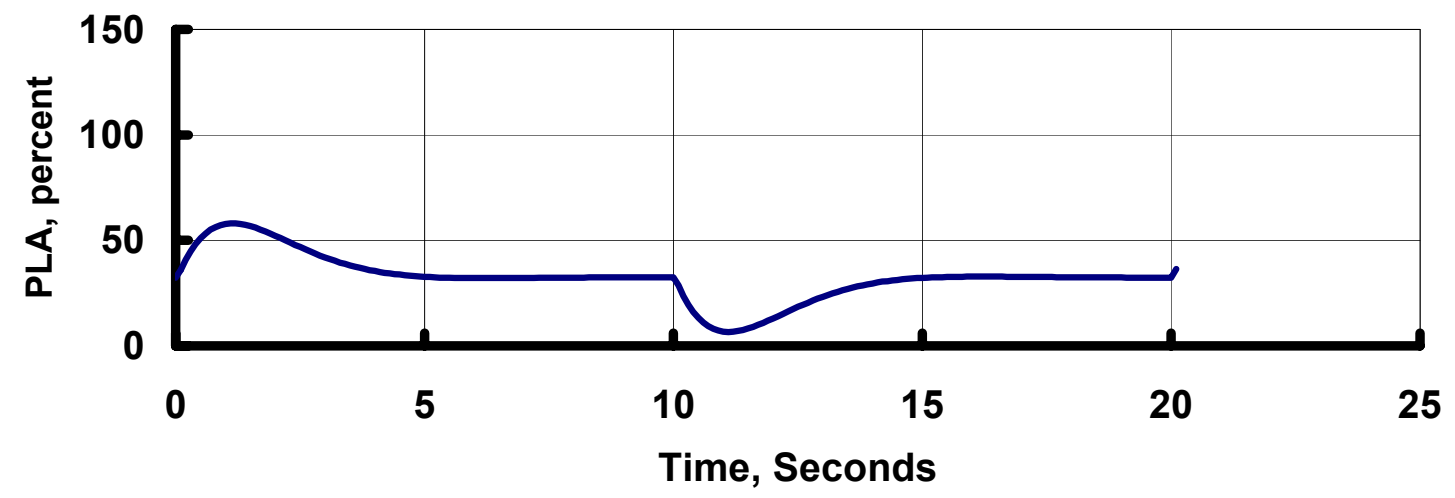

Figure 37 (b): Case 1 time history 

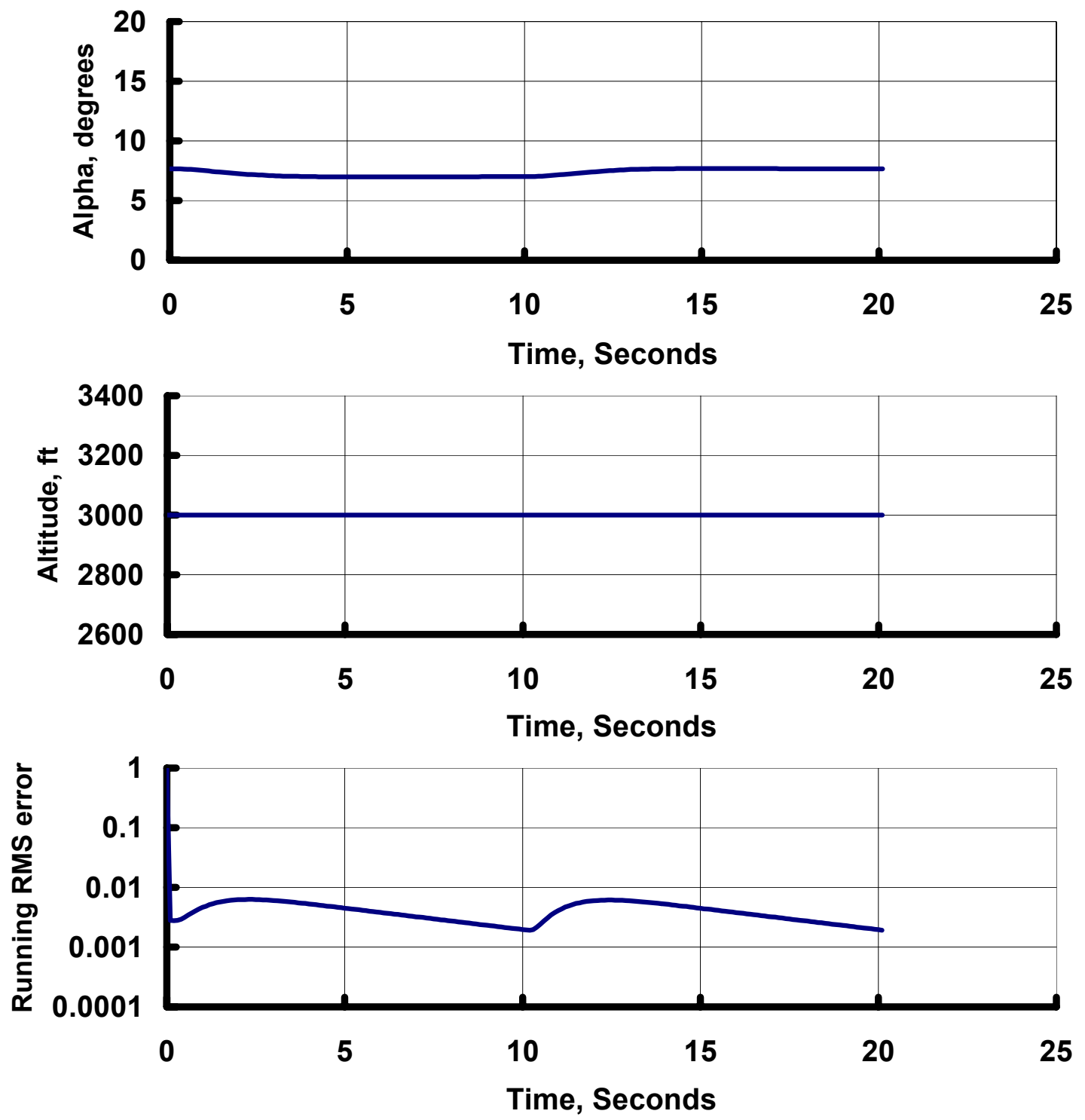

Figure 37 (c): Case 1 time history 


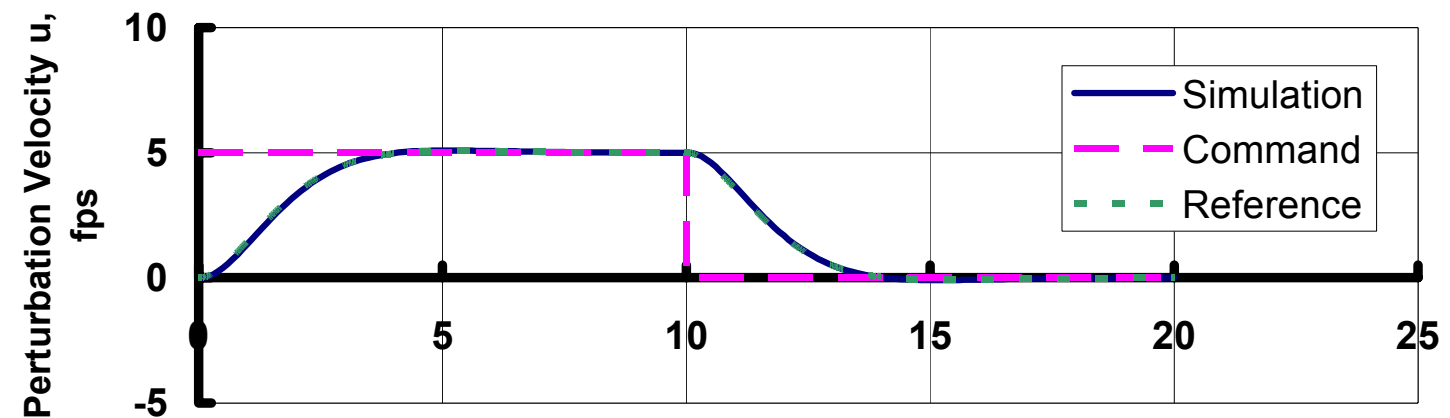

Time, Seconds

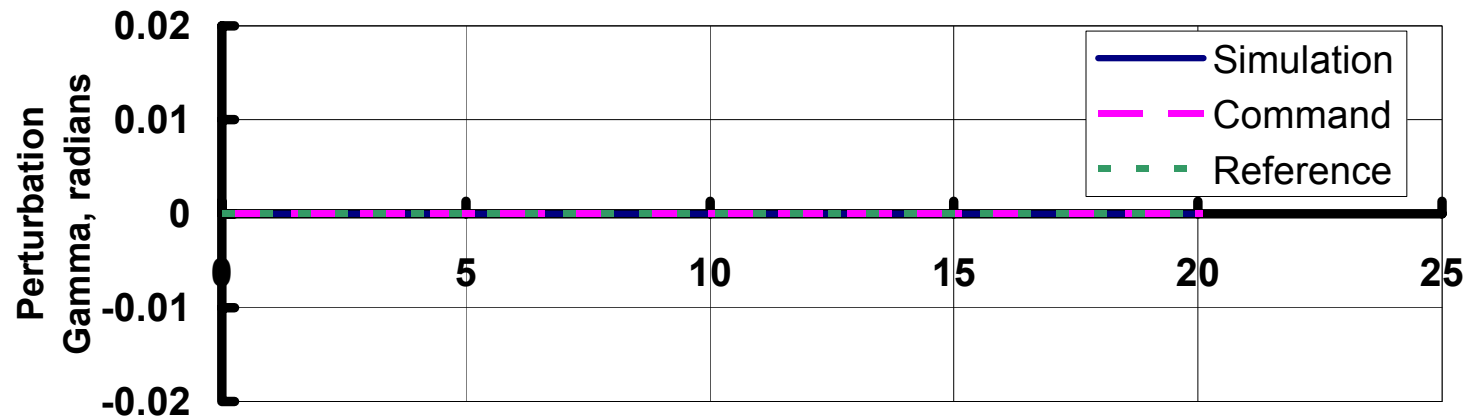

Time, Seconds

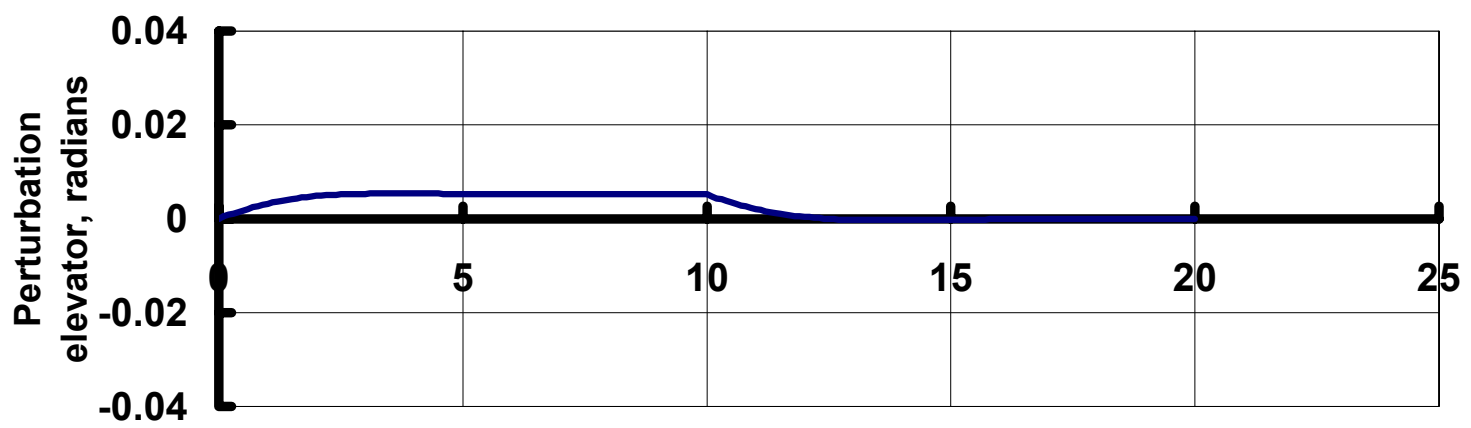

Time, Seconds

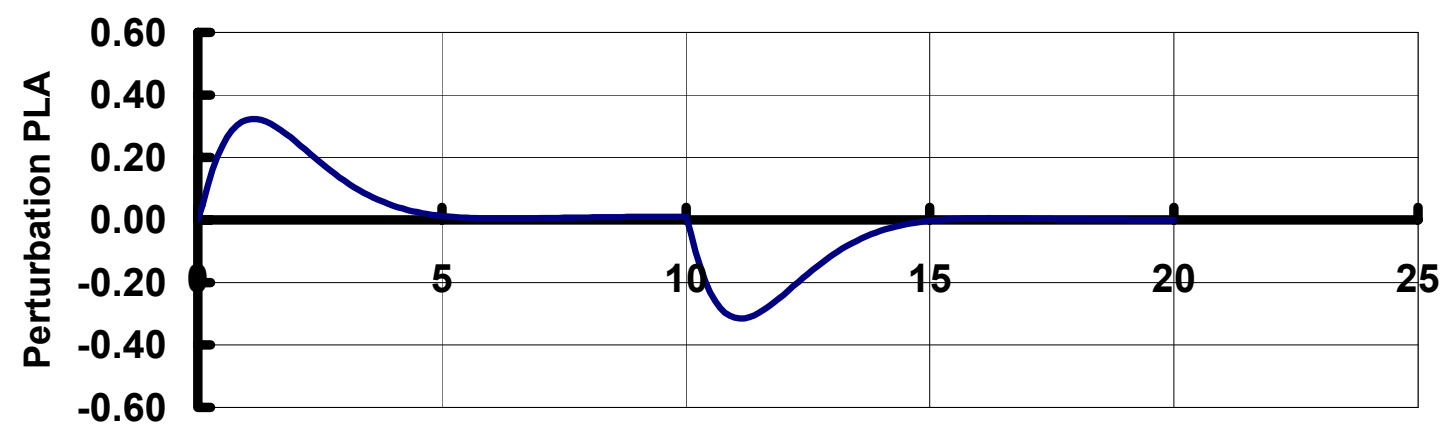

Time, Seconds

Figure 38 (a): Case 2 time history 

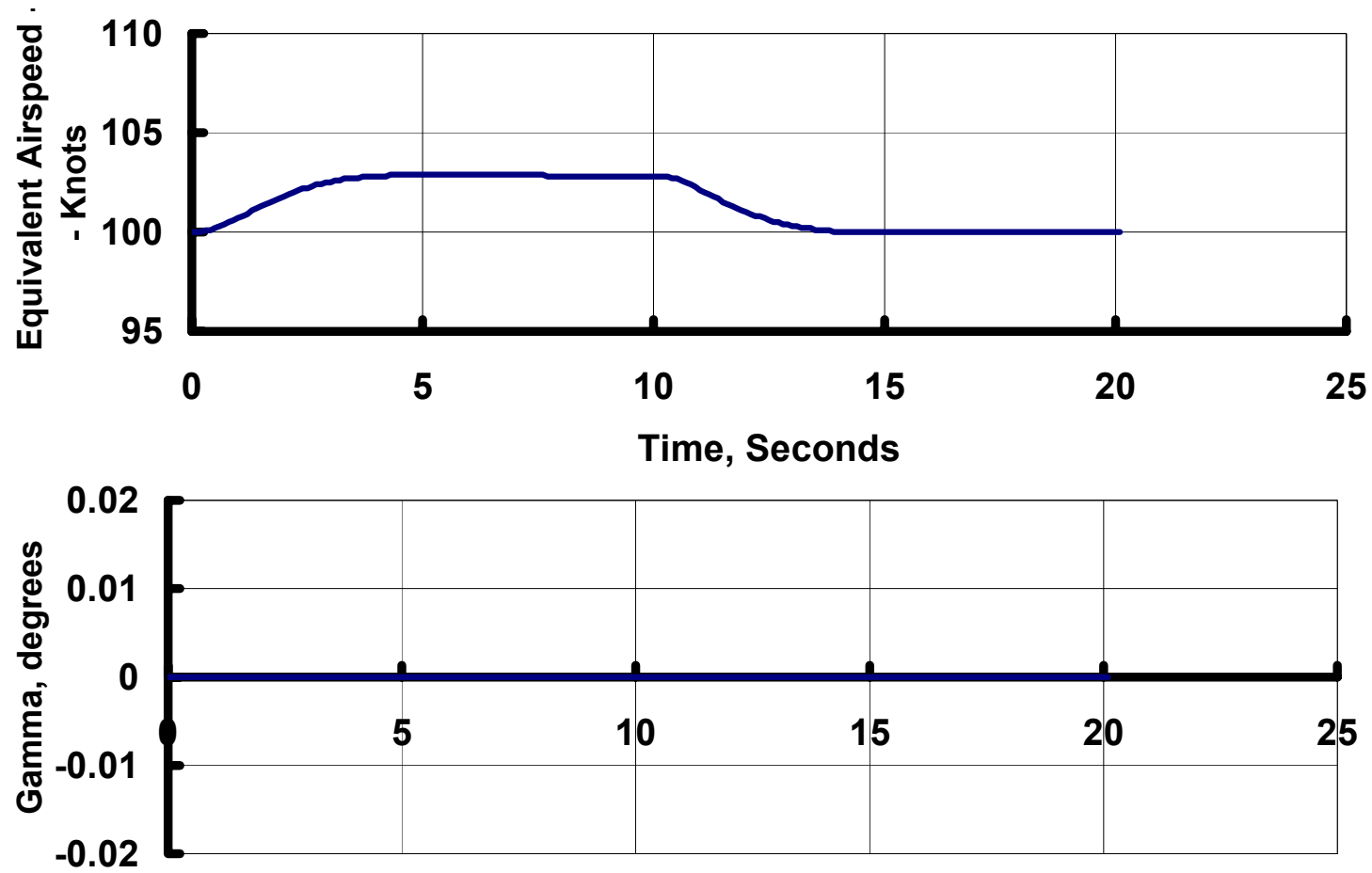

Time, Seconds

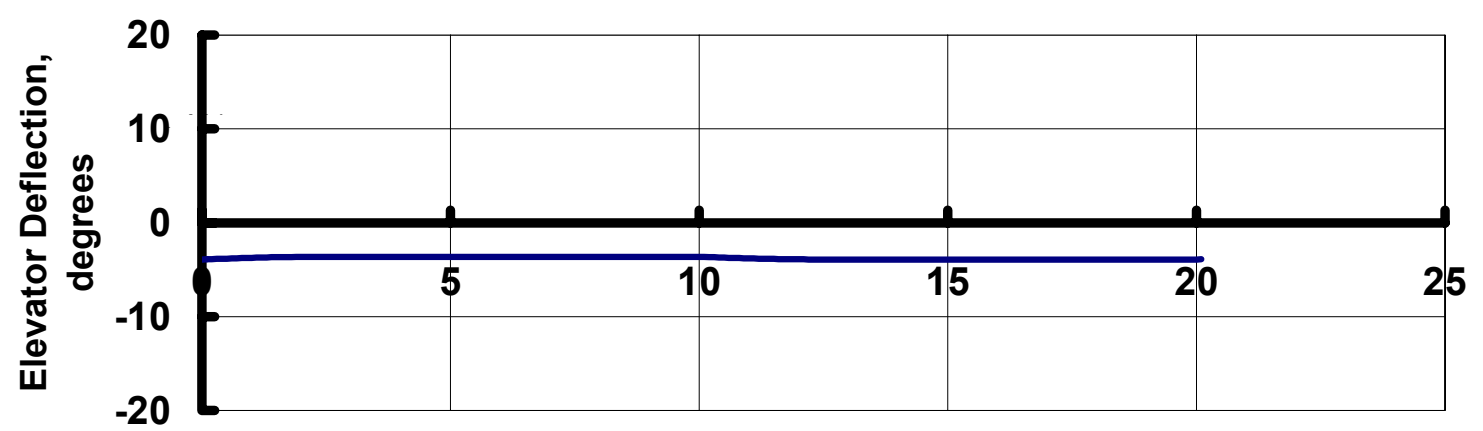

Time, Seconds

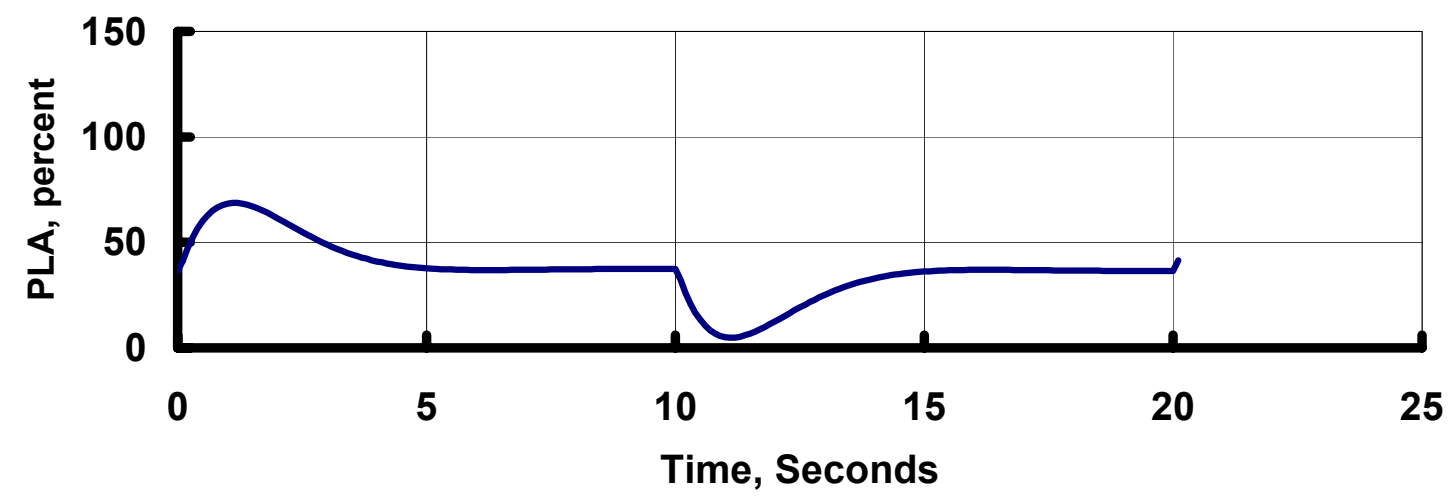

Figure 38 (b): Case 2 time history 

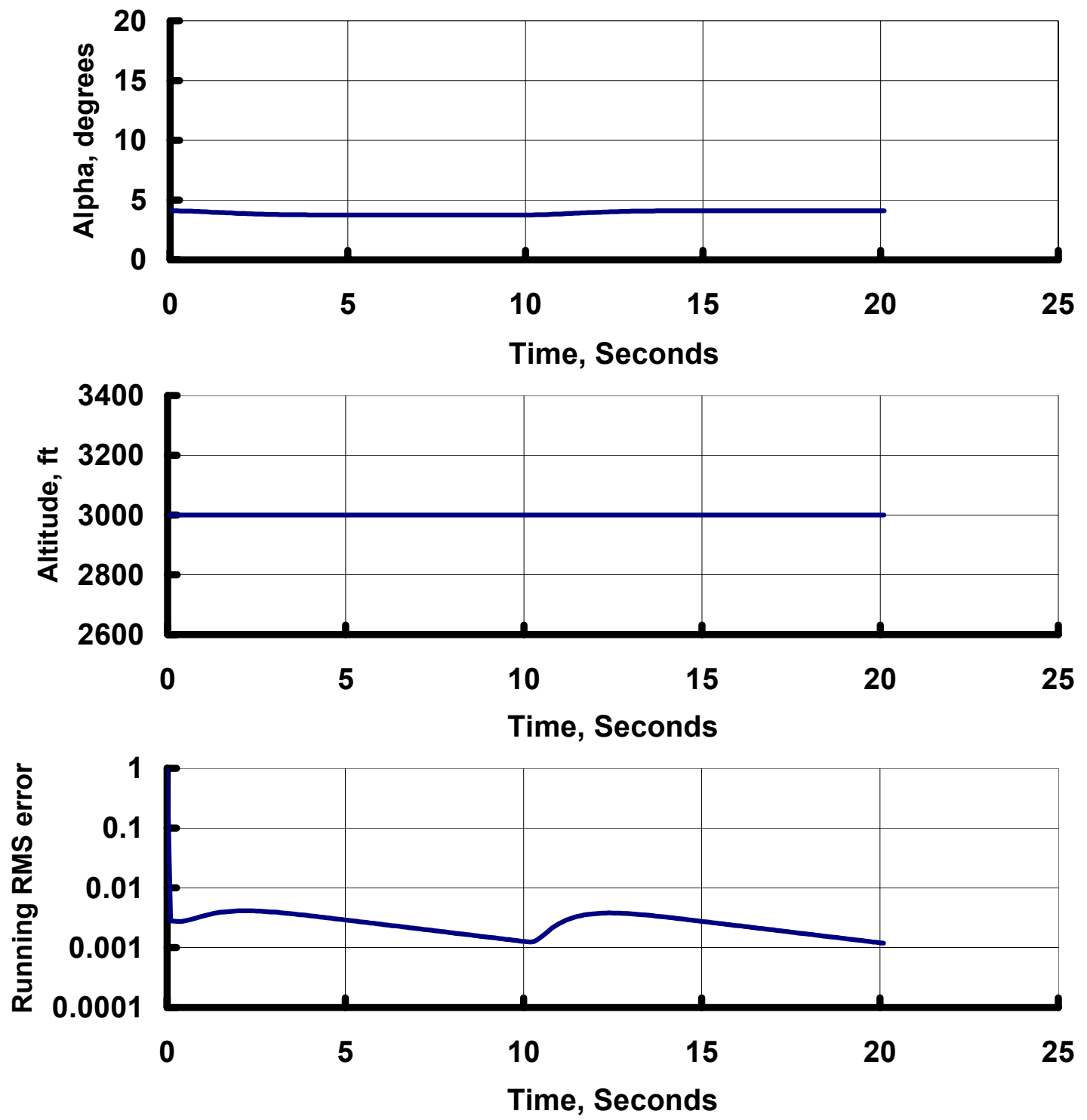

Figure 38 (c): Case 2 time history 


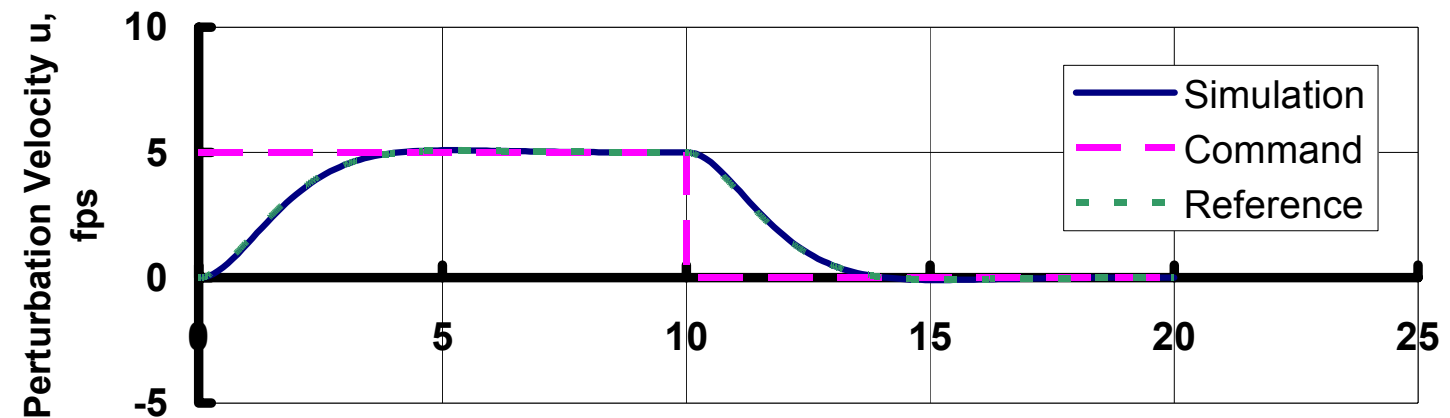

Time, Seconds

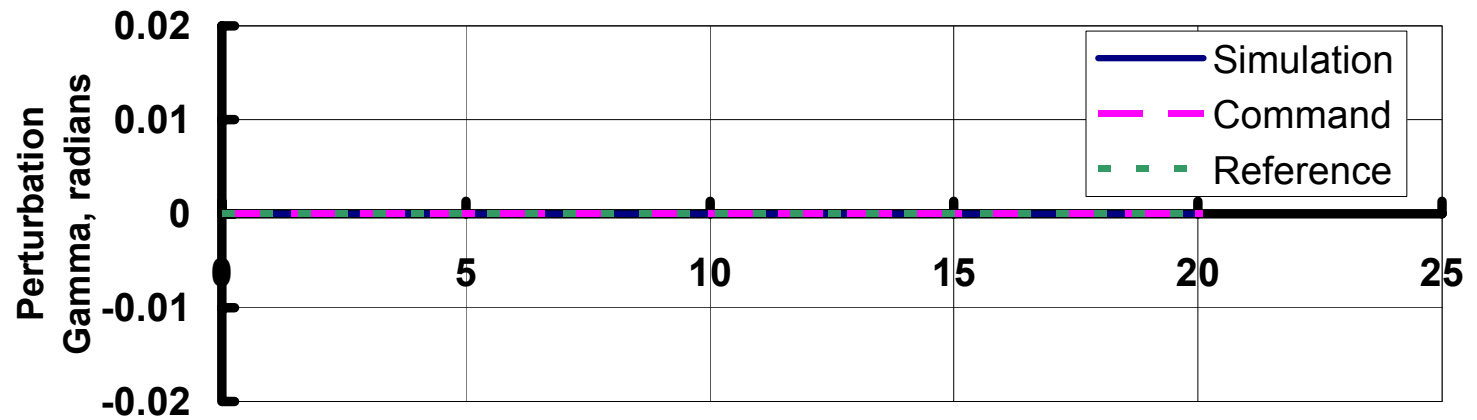

Time, Seconds

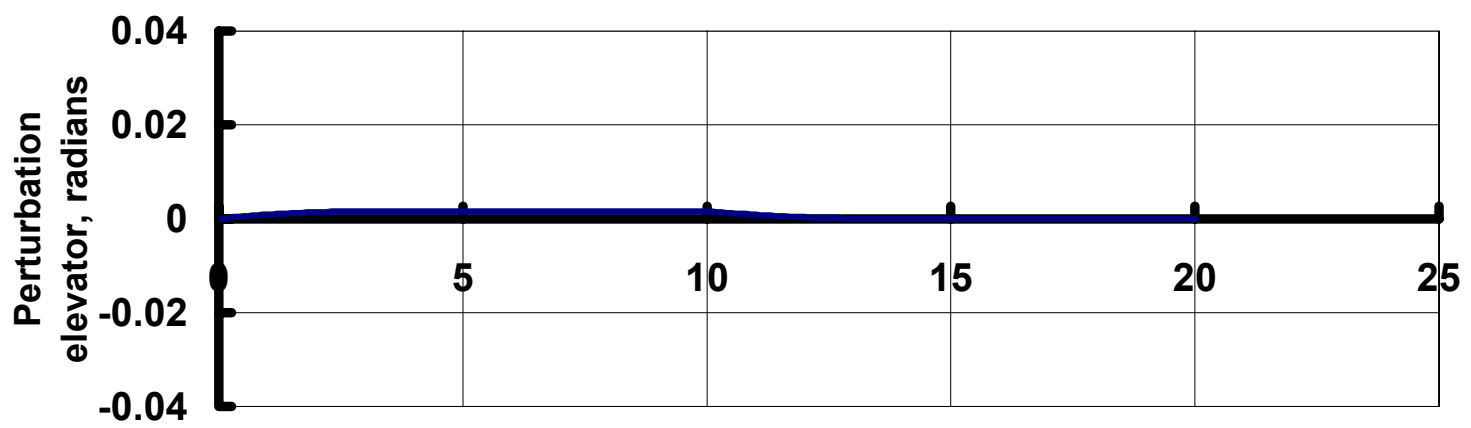

Time, Seconds

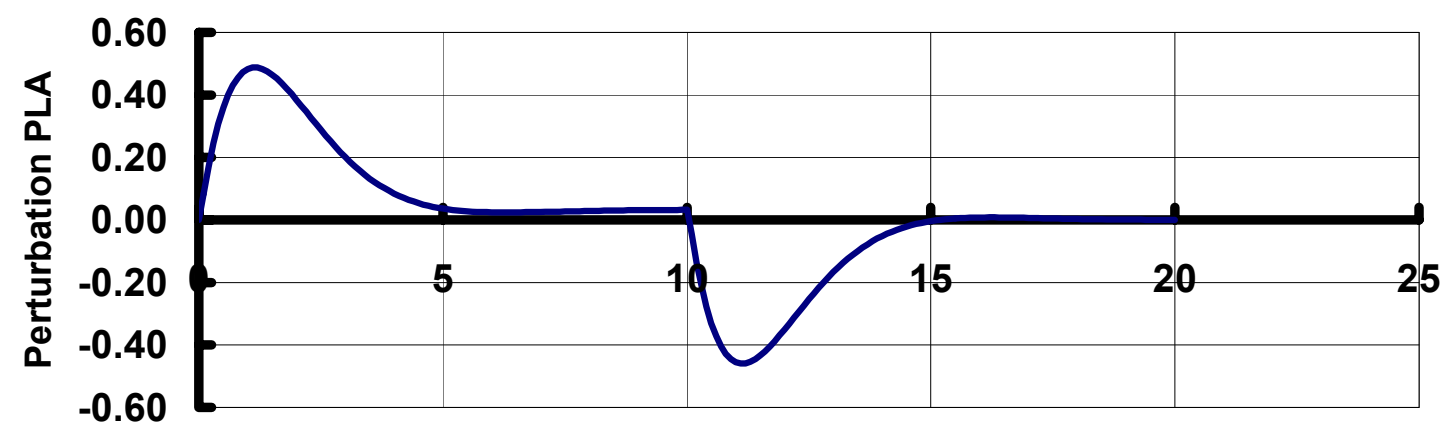

Time, Seconds

Figure 39 (a): Case 3 time history 

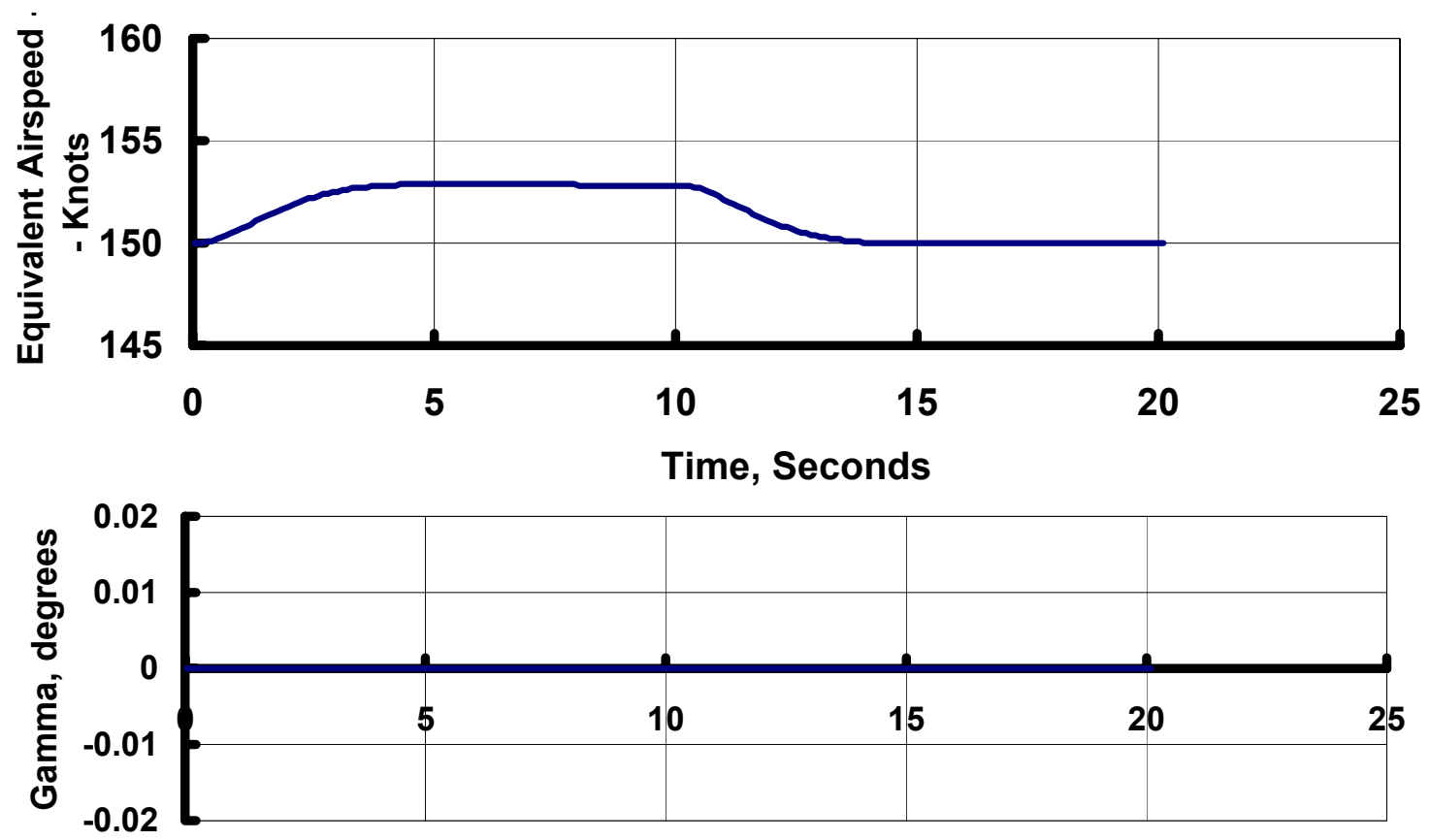

Time, Seconds

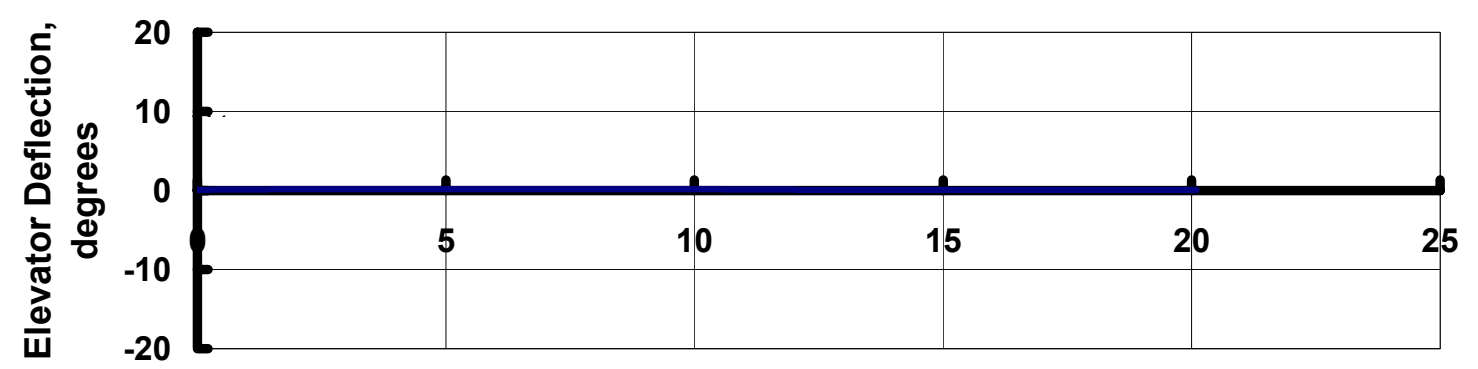

Time, Seconds

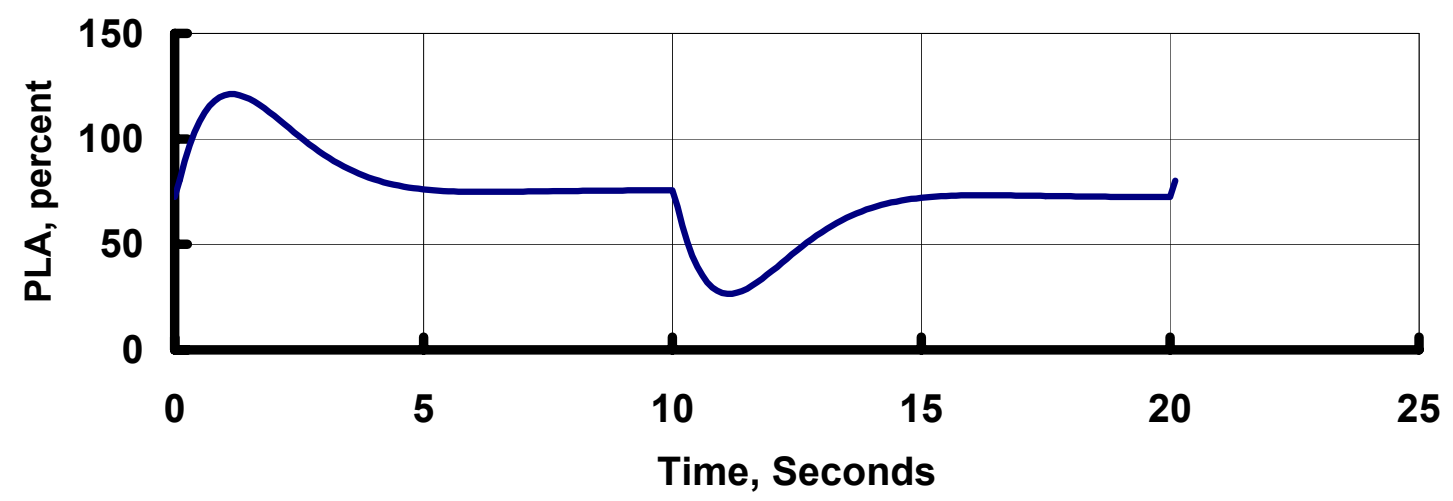

Figure 39 (b): Case 3 time history 

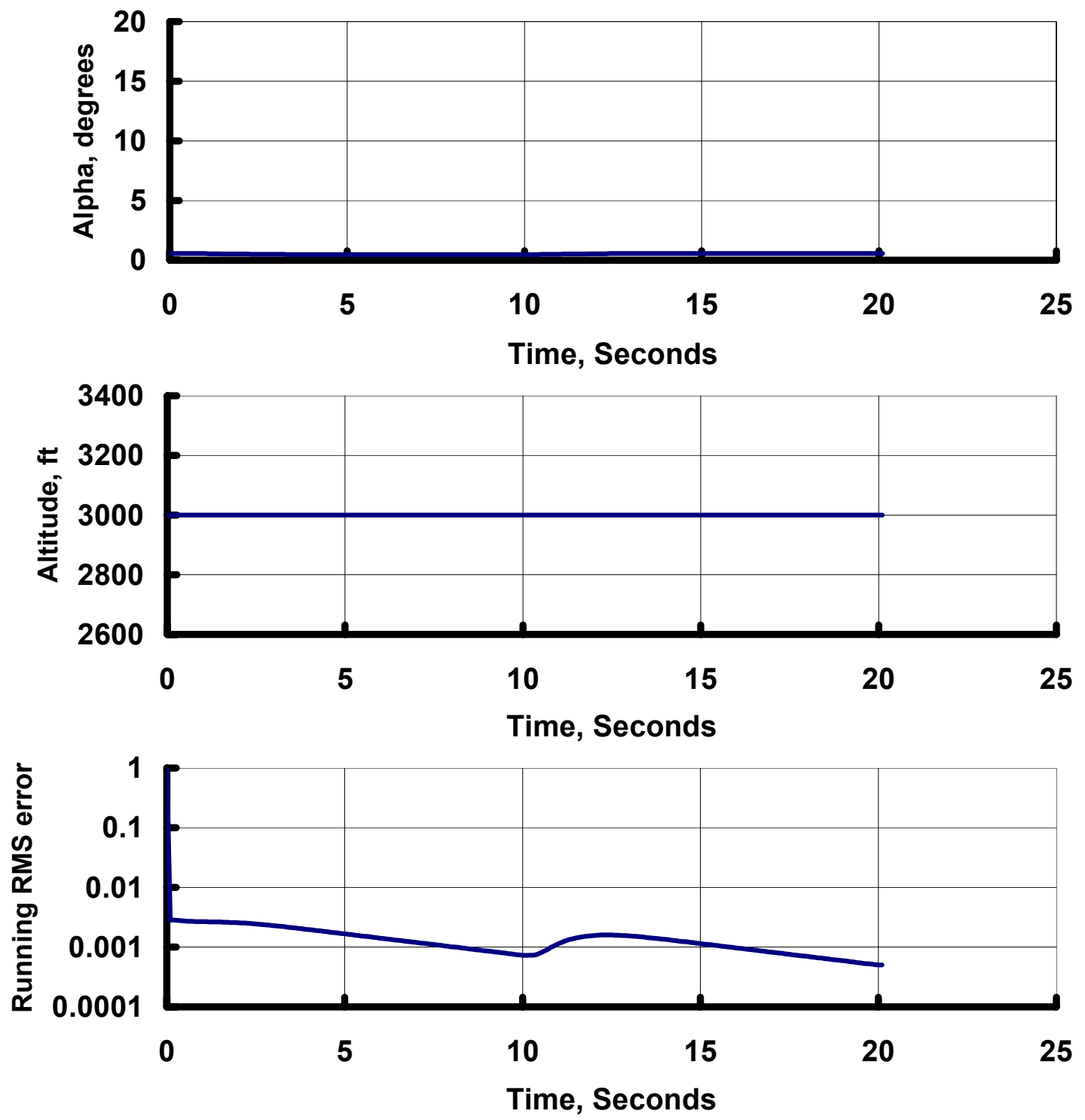

Figure 39 (c): Case 3 time history 


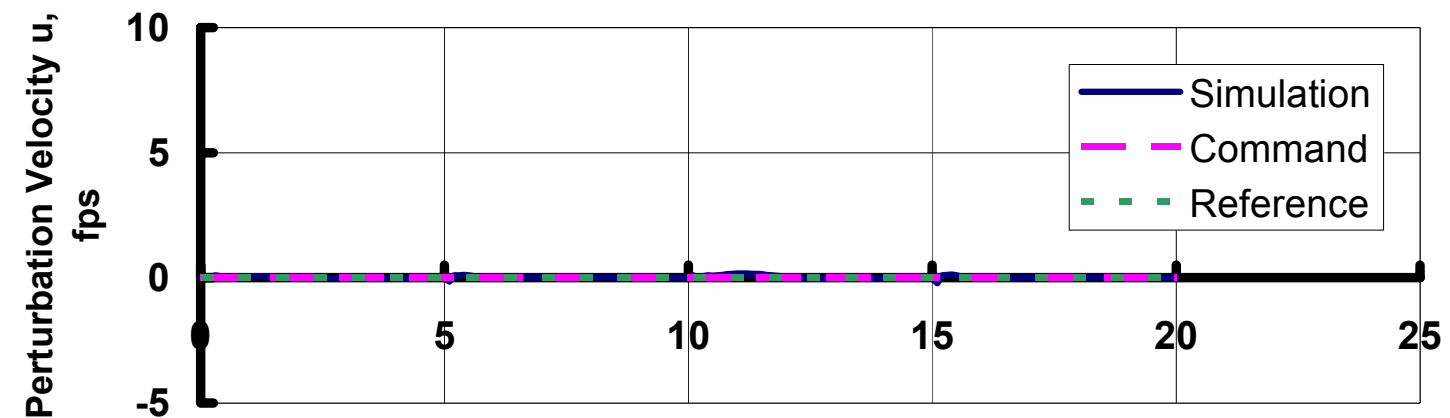

Time, Seconds

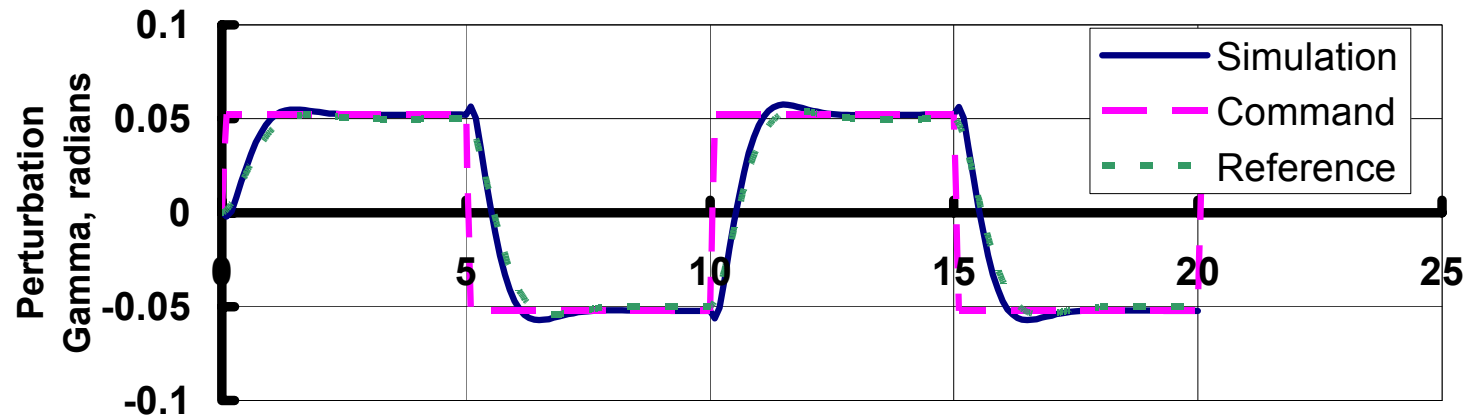

Time, Seconds

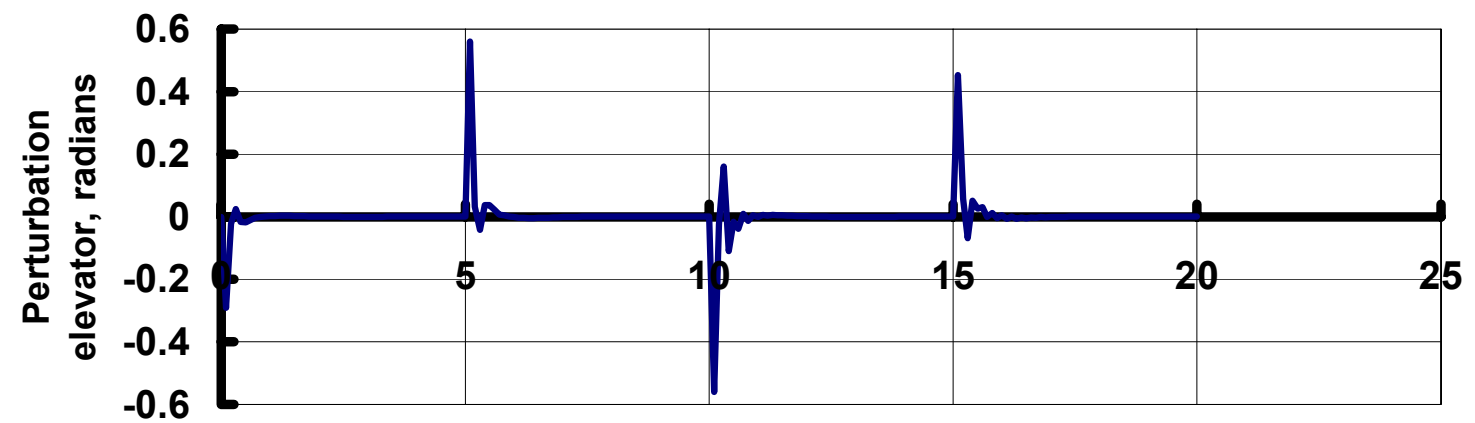

Time, Seconds

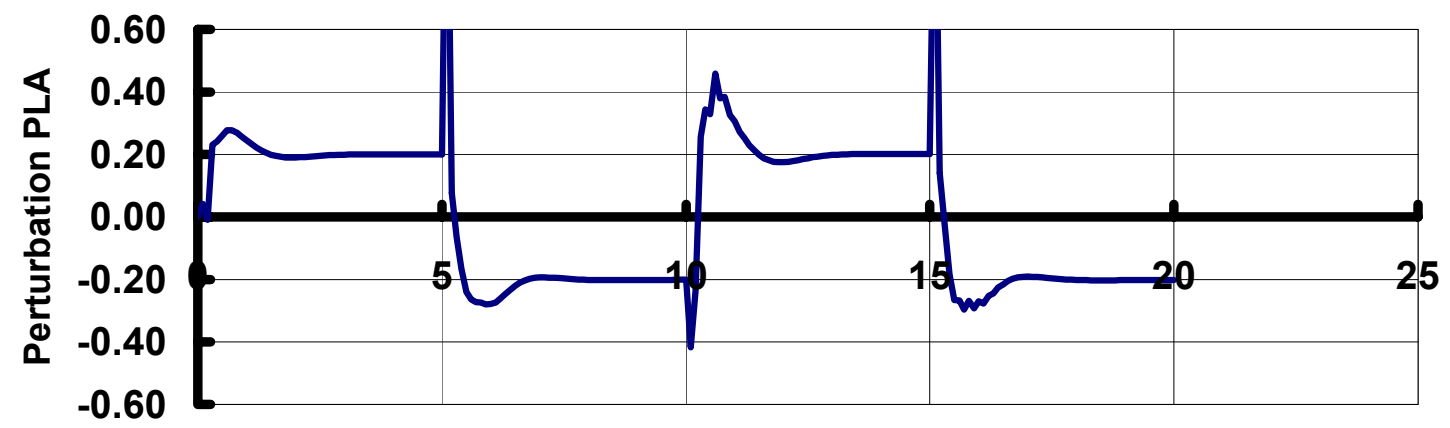

Time, Seconds

Figure 40 (a): Case 4 time history 

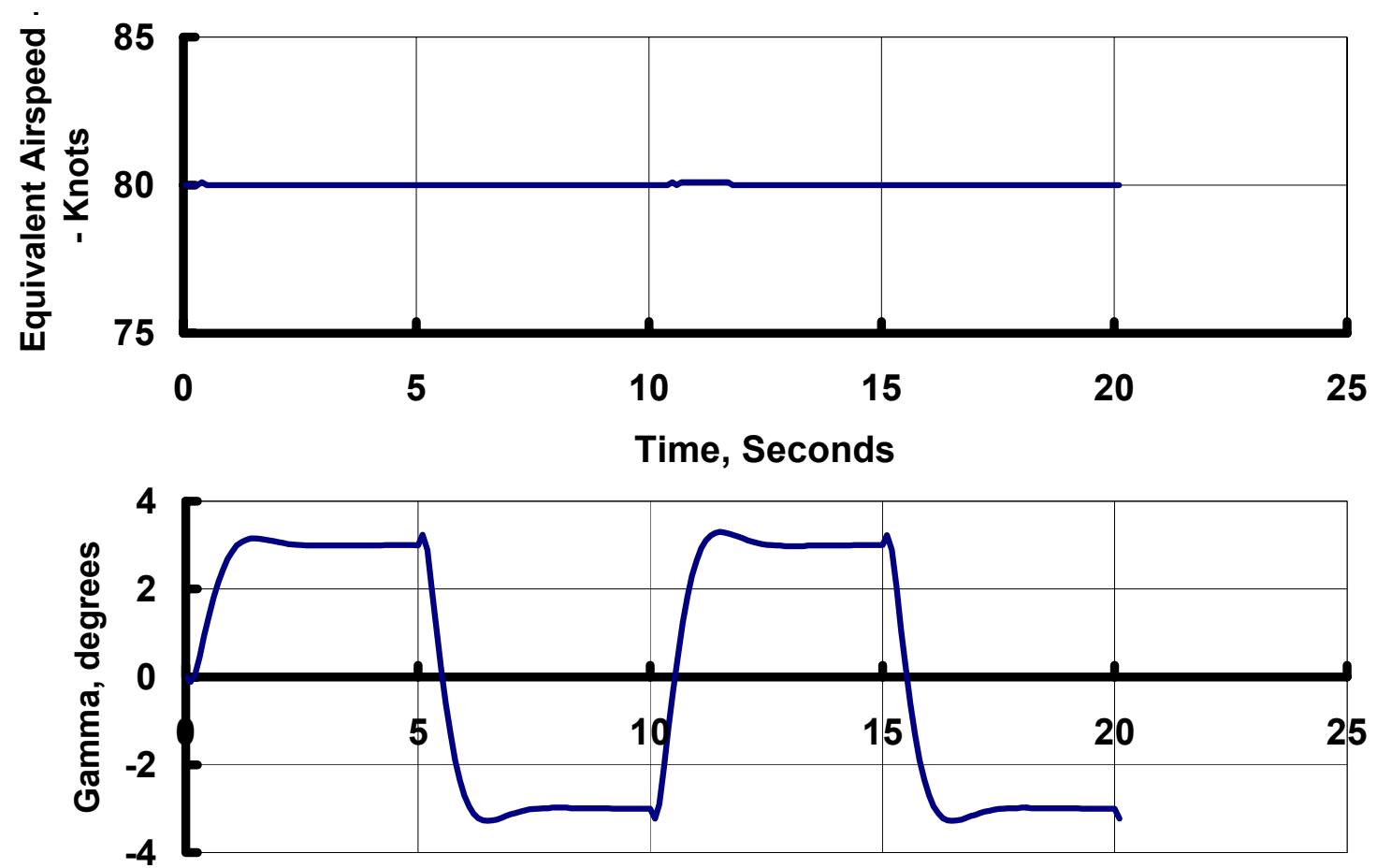

Time, Seconds

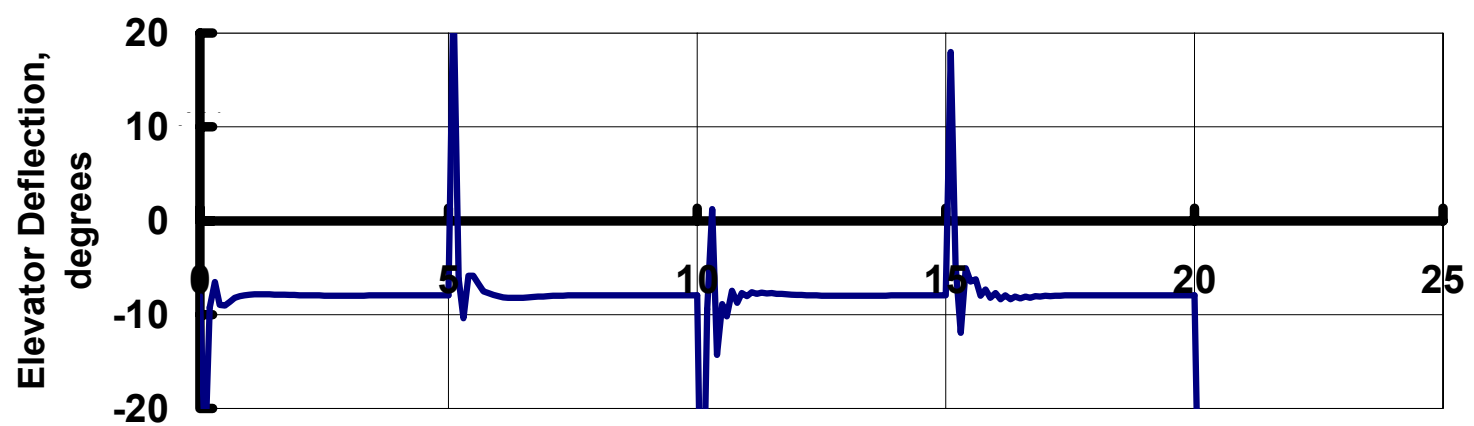

Time, Seconds

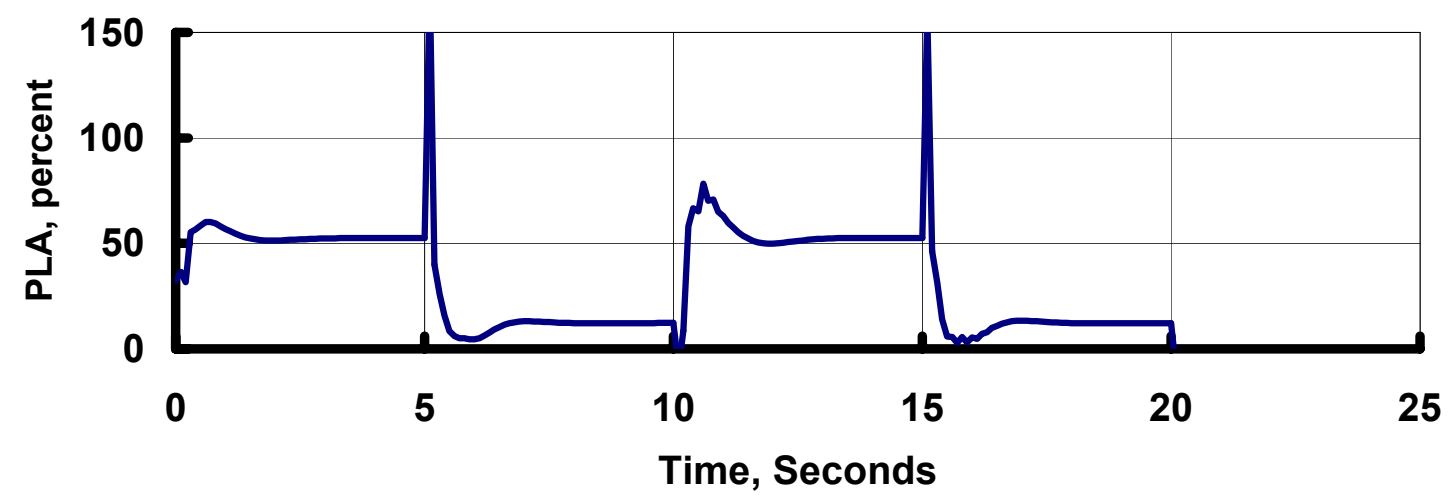

Figure 40 (b): Case 4 time history 


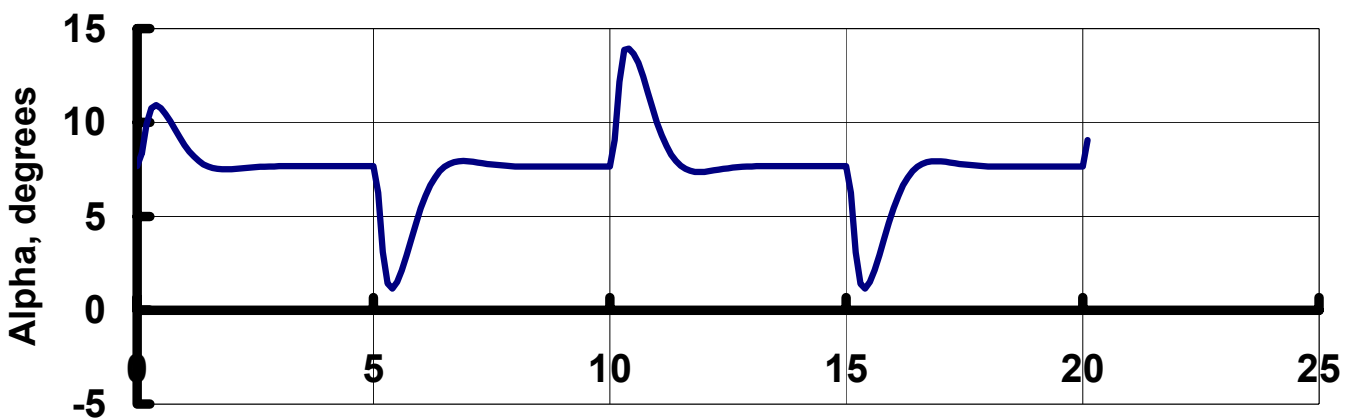

Time, Seconds
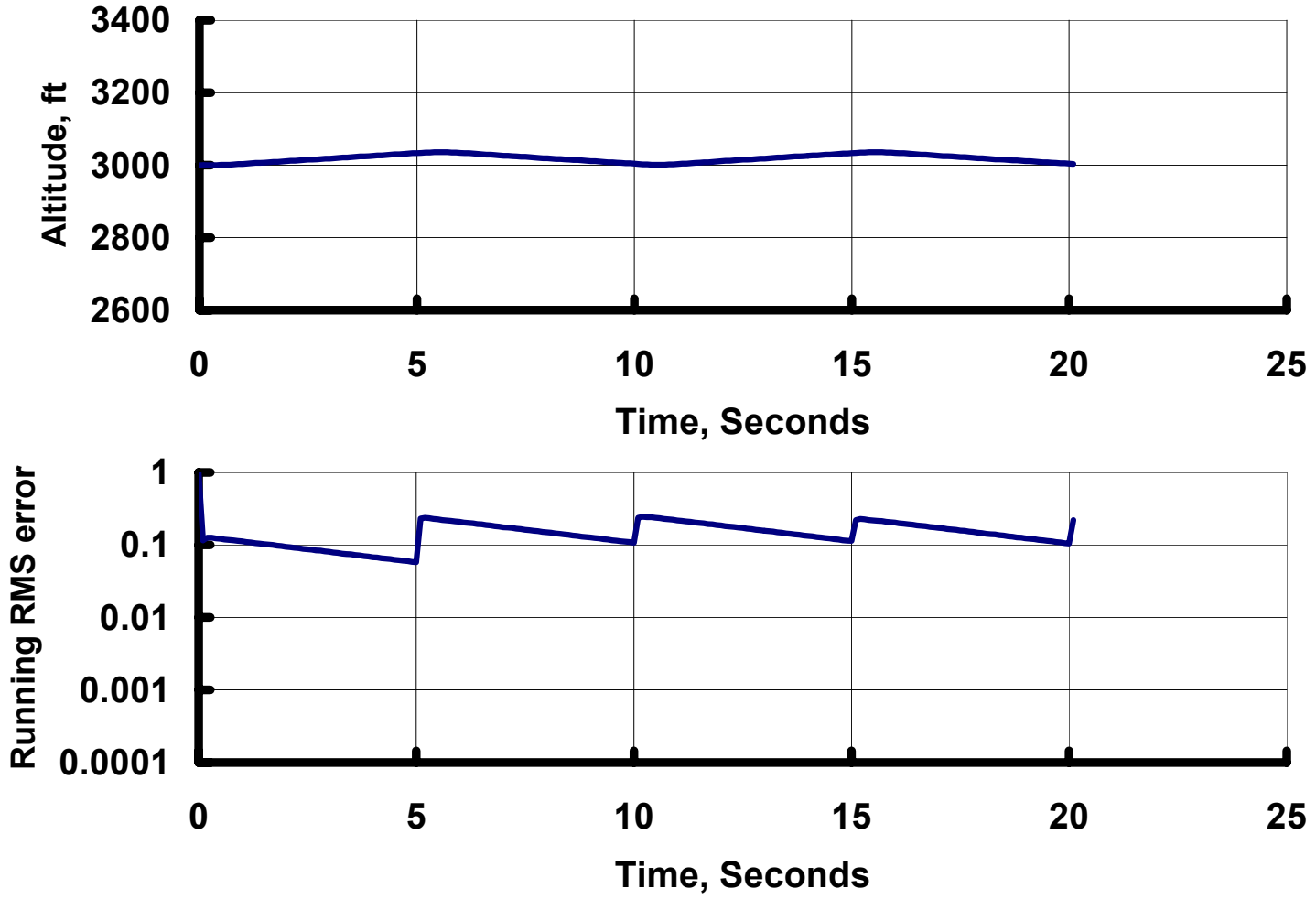

Figure 40 (c): Case 4 time history 


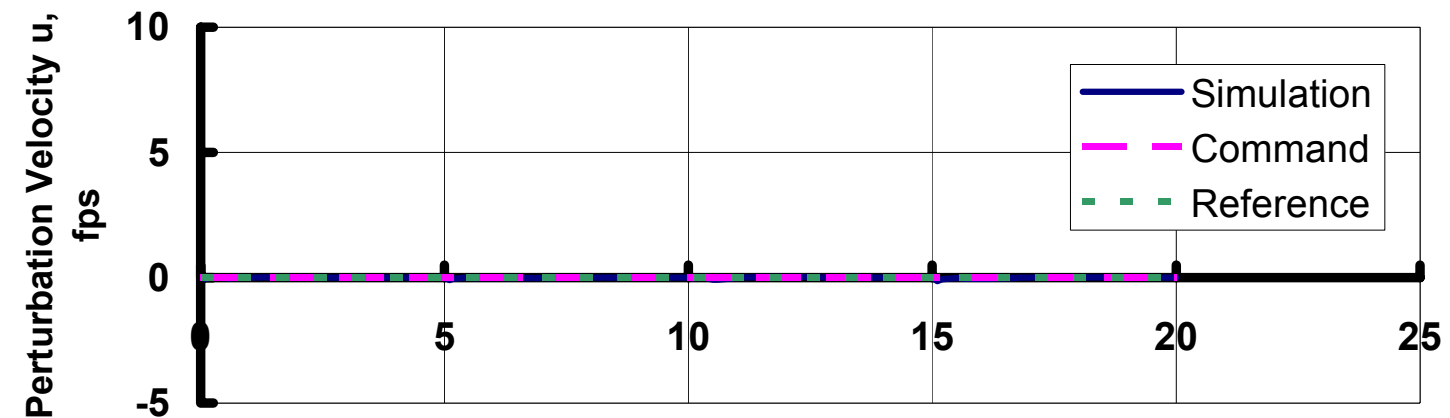

Time, Seconds

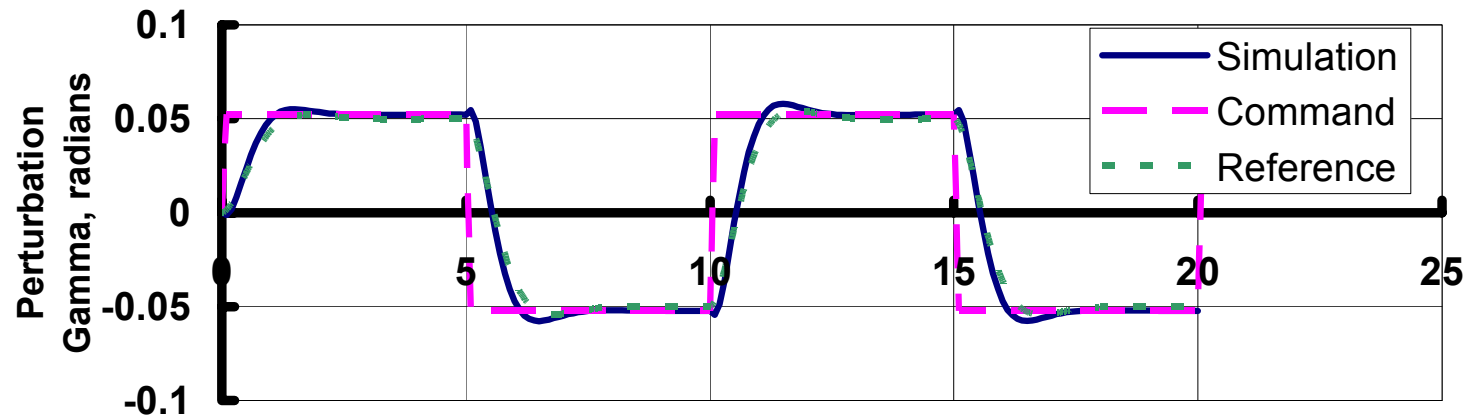

Time, Seconds

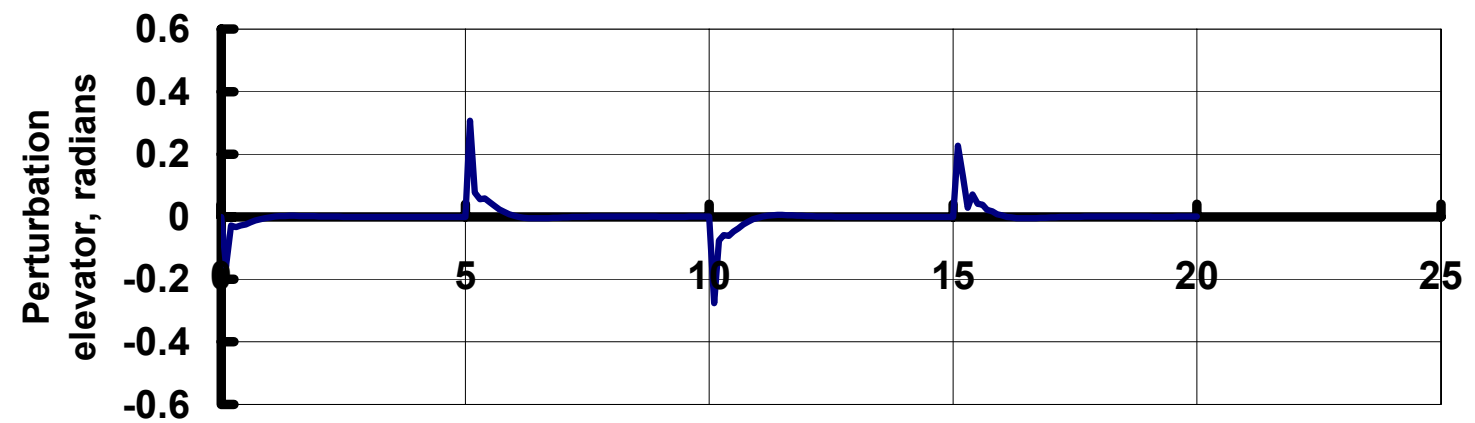

Time, Seconds

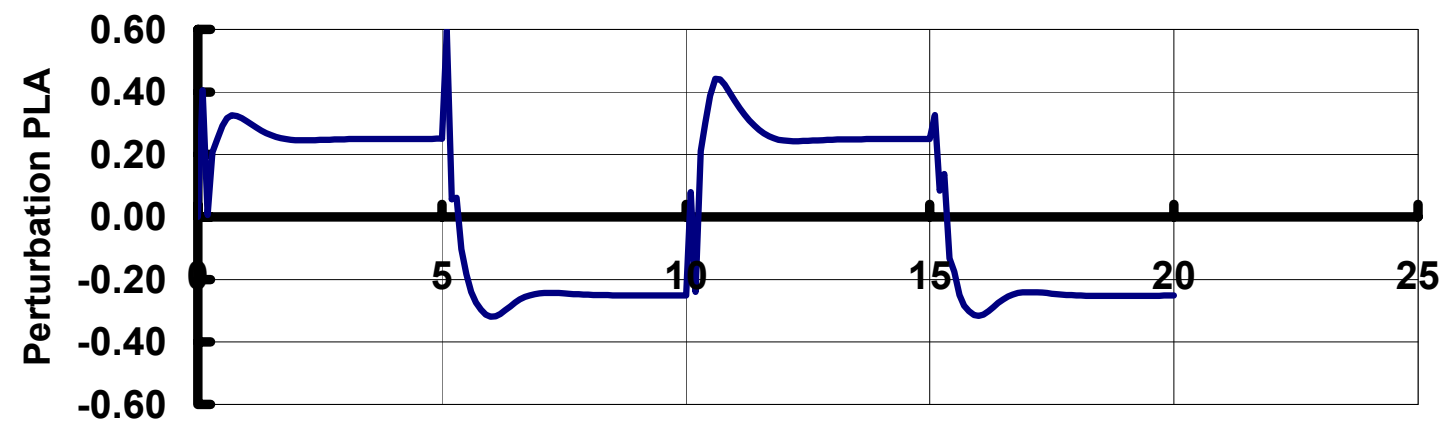

Time, Seconds

Figure 41 (a): Case 5 time history 

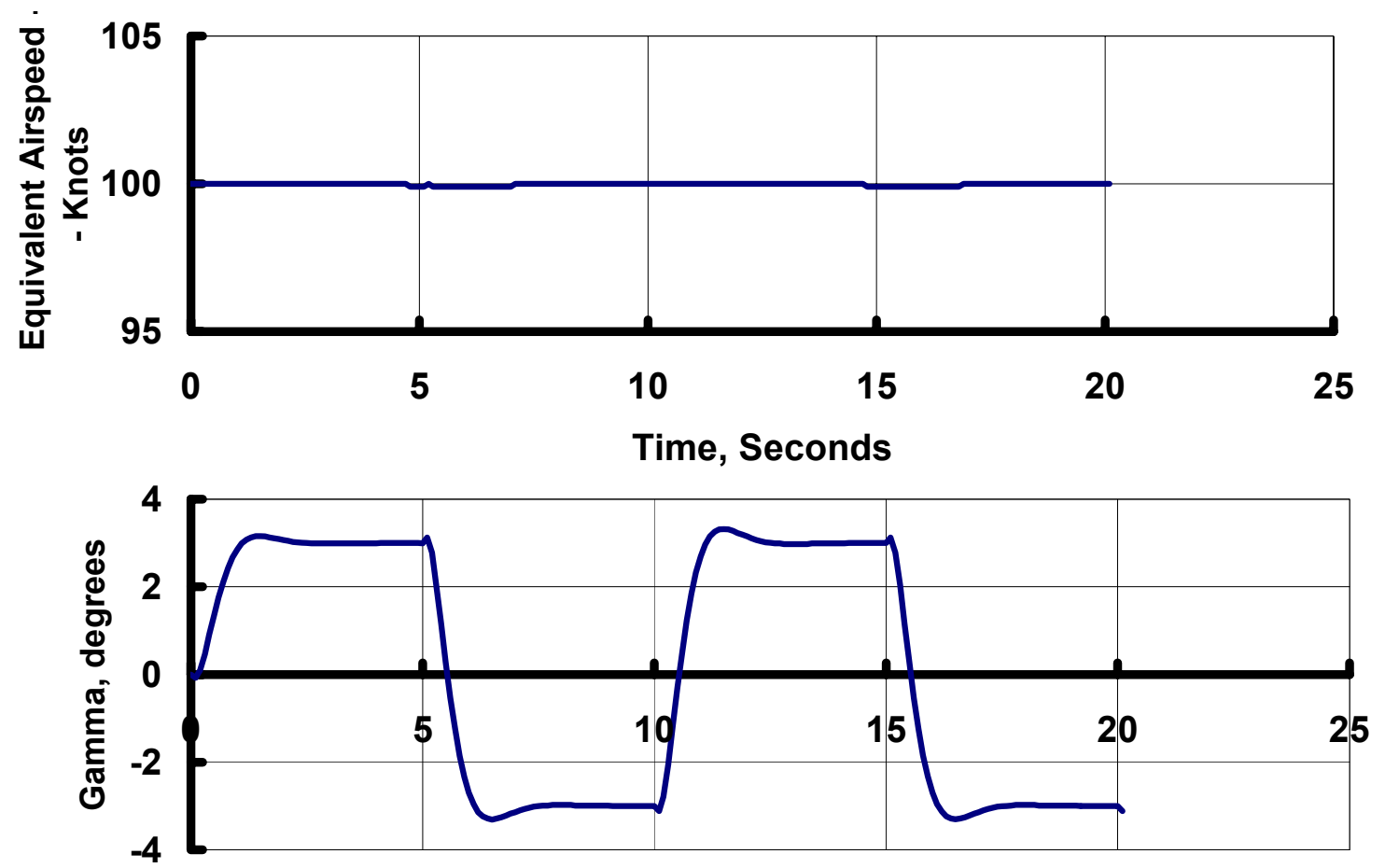

Time, Seconds

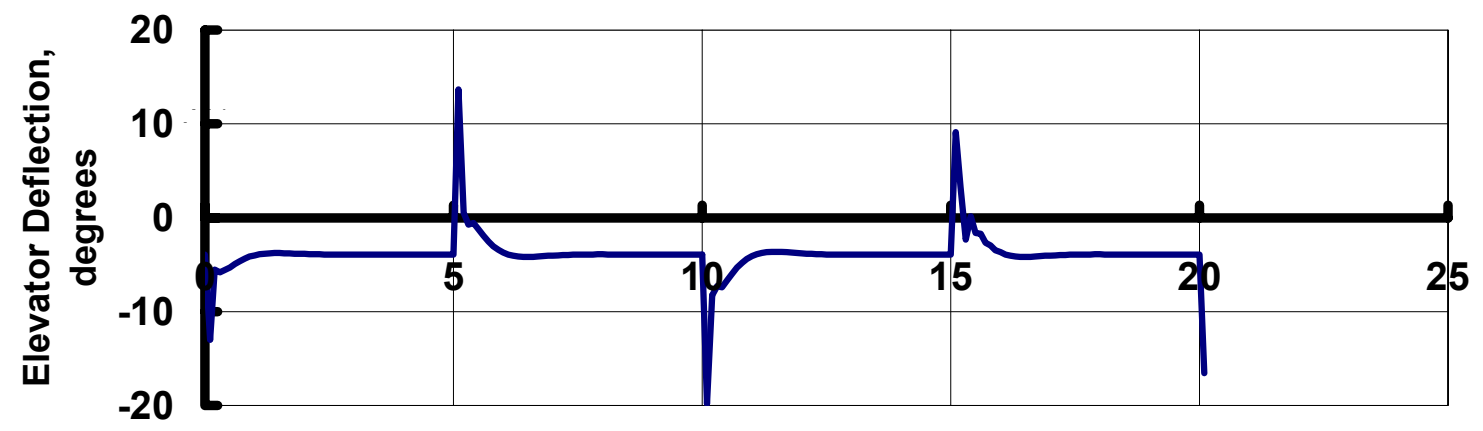

Time, Seconds

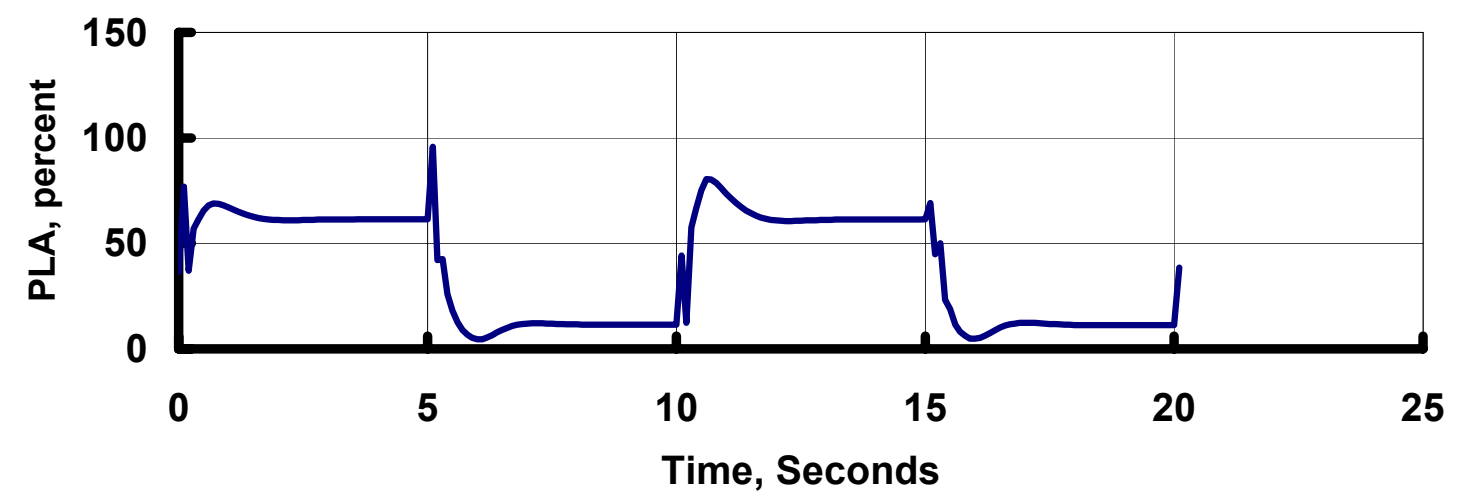

Figure 41 (b): Case 5 time history 


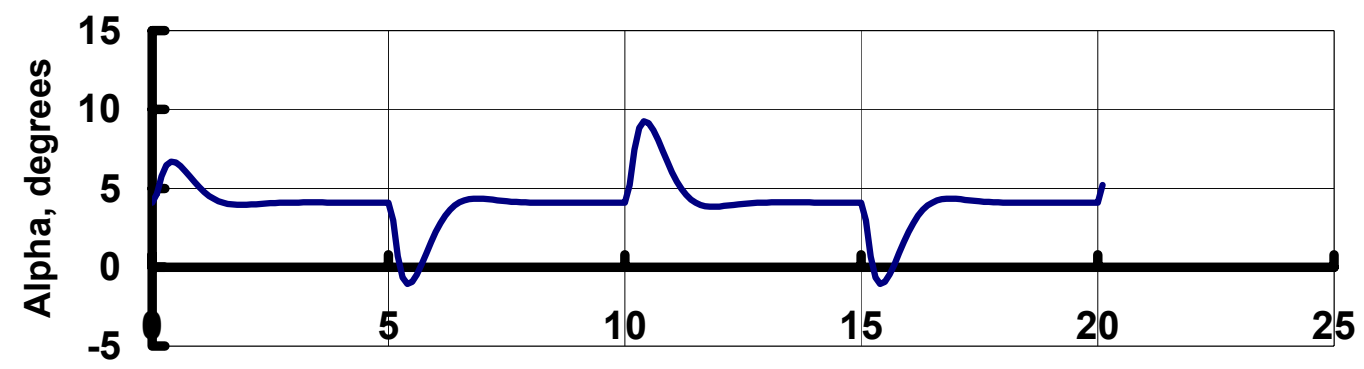

Time, Seconds
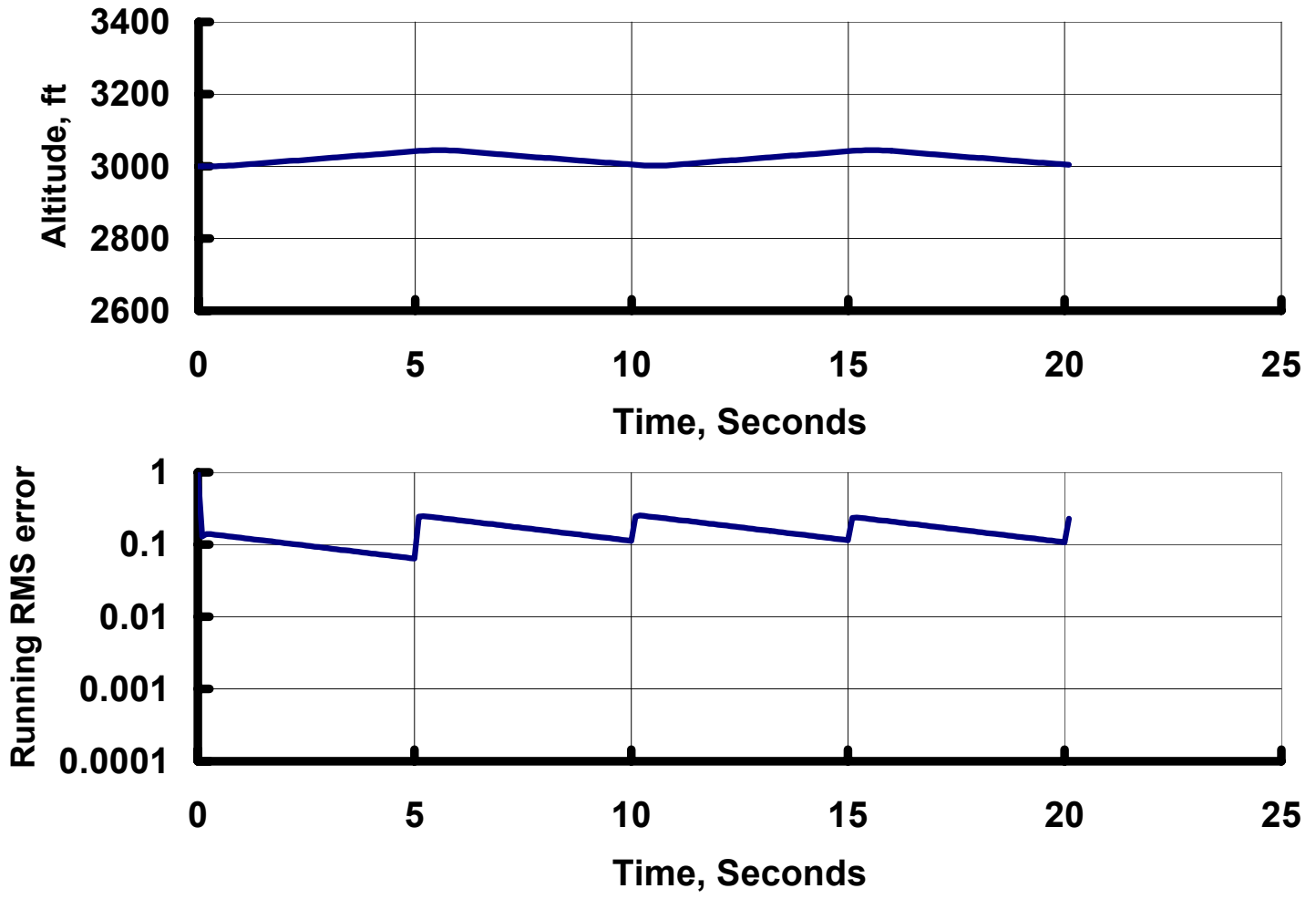

Figure $41(\mathrm{c})$ : Case 5 time history 


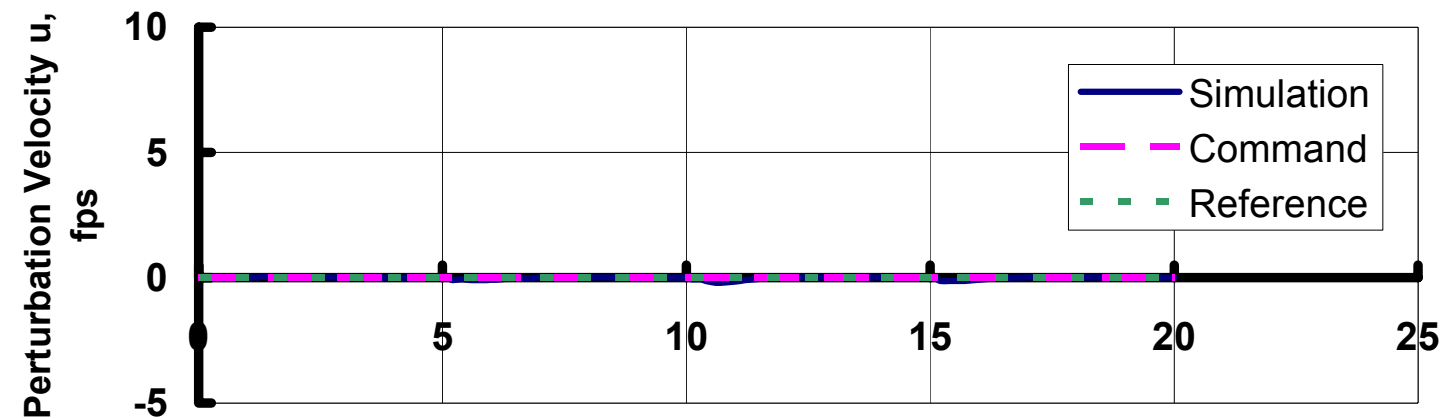

Time, Seconds

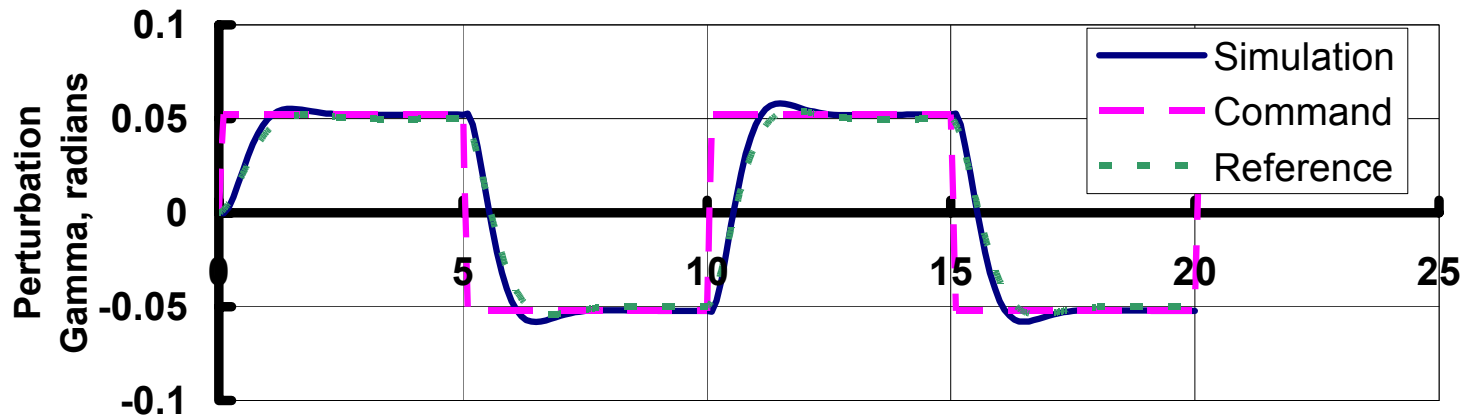

Time, Seconds

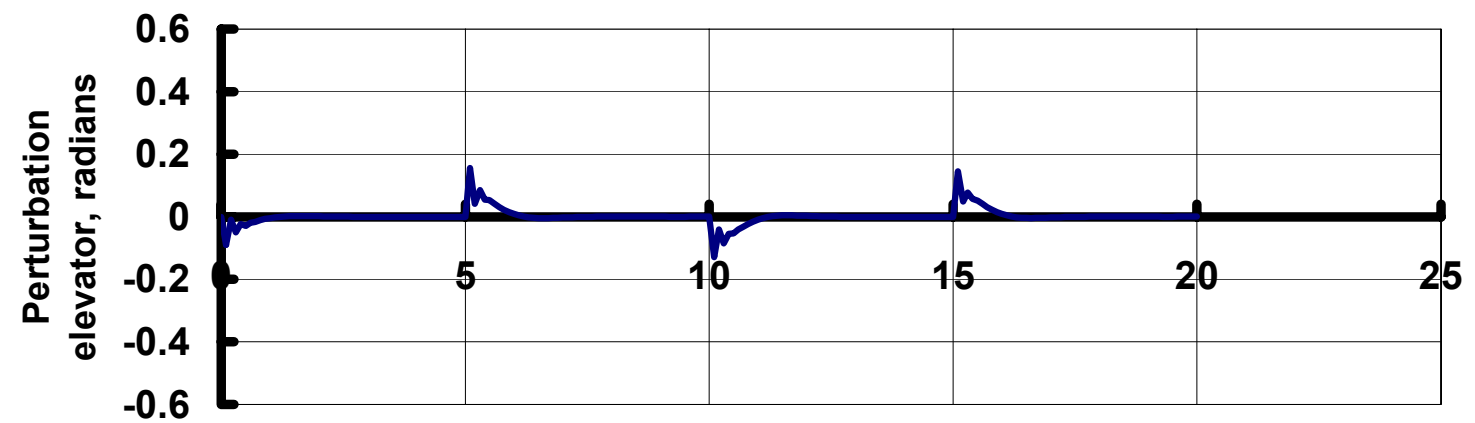

Time, Seconds

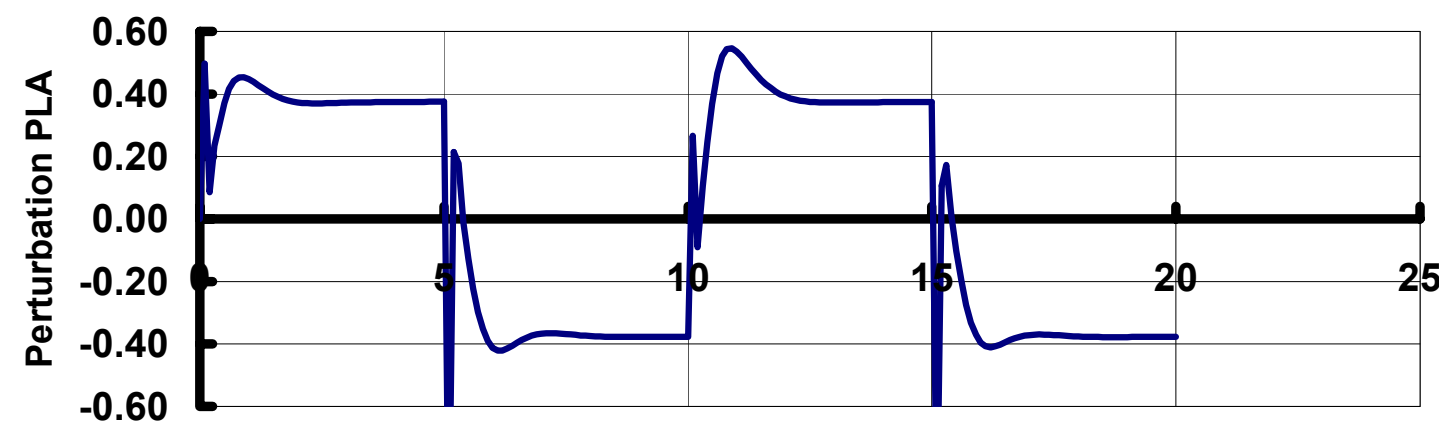

Time, Seconds

Figure 42 (a): Case 6 time history 

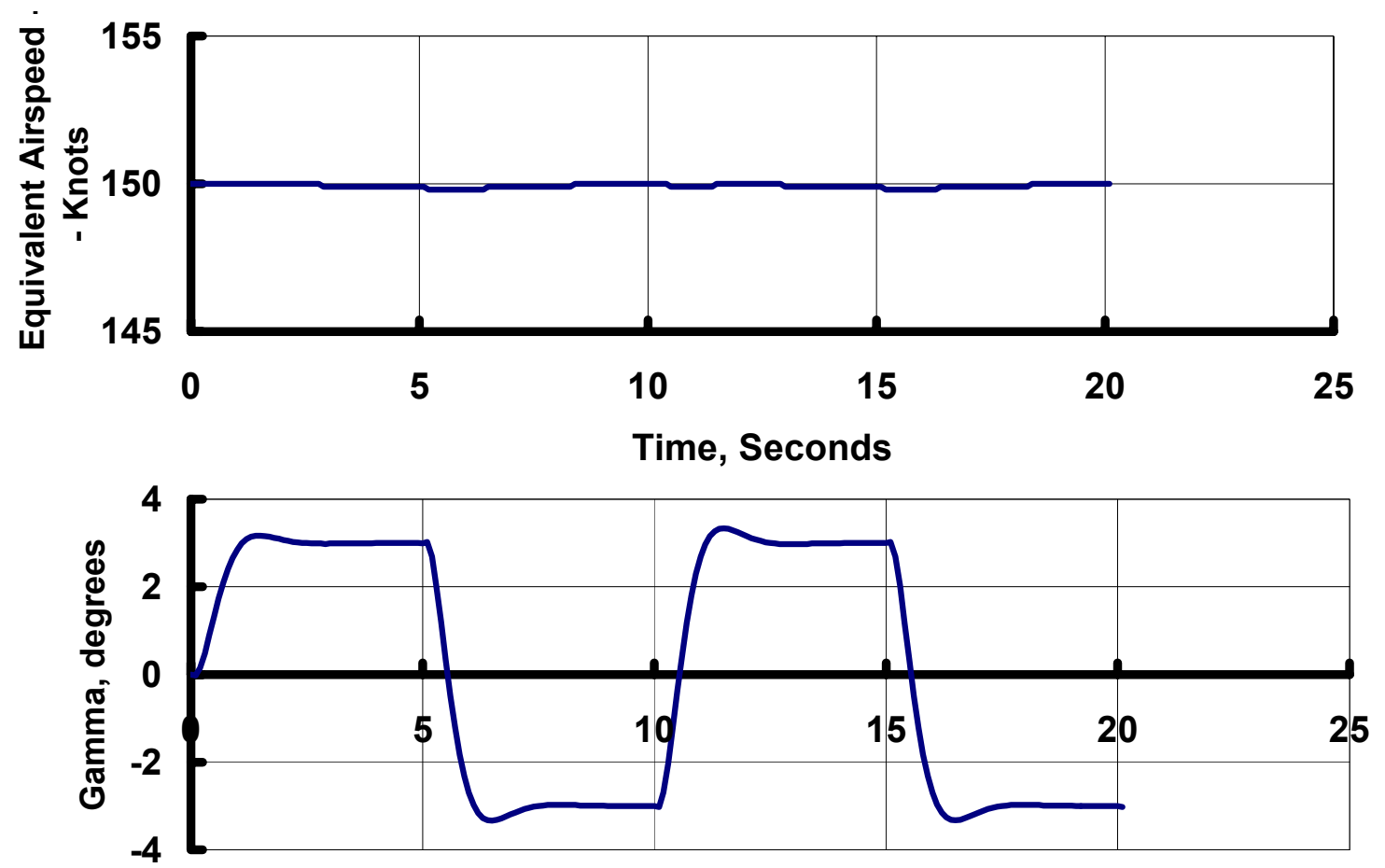

Time, Seconds

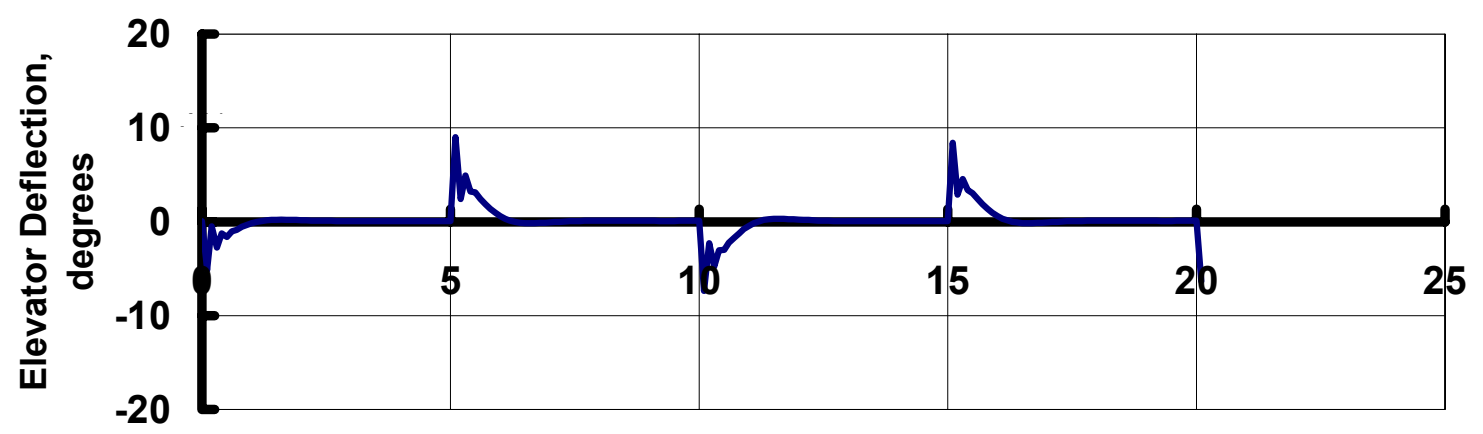

Time, Seconds

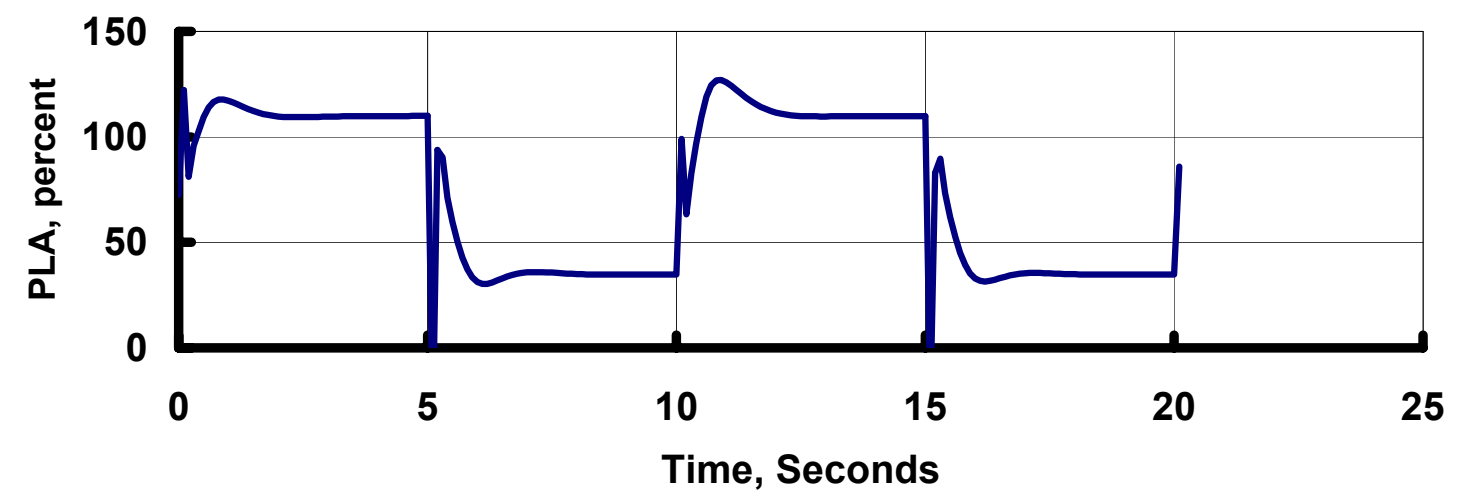

Figure 42 (b): Case 6 time history 


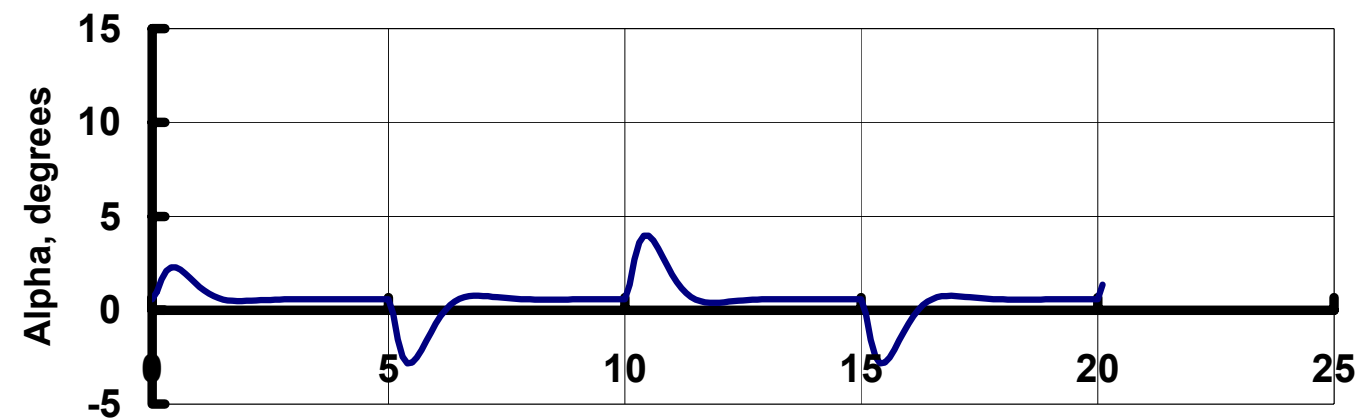

Time, Seconds
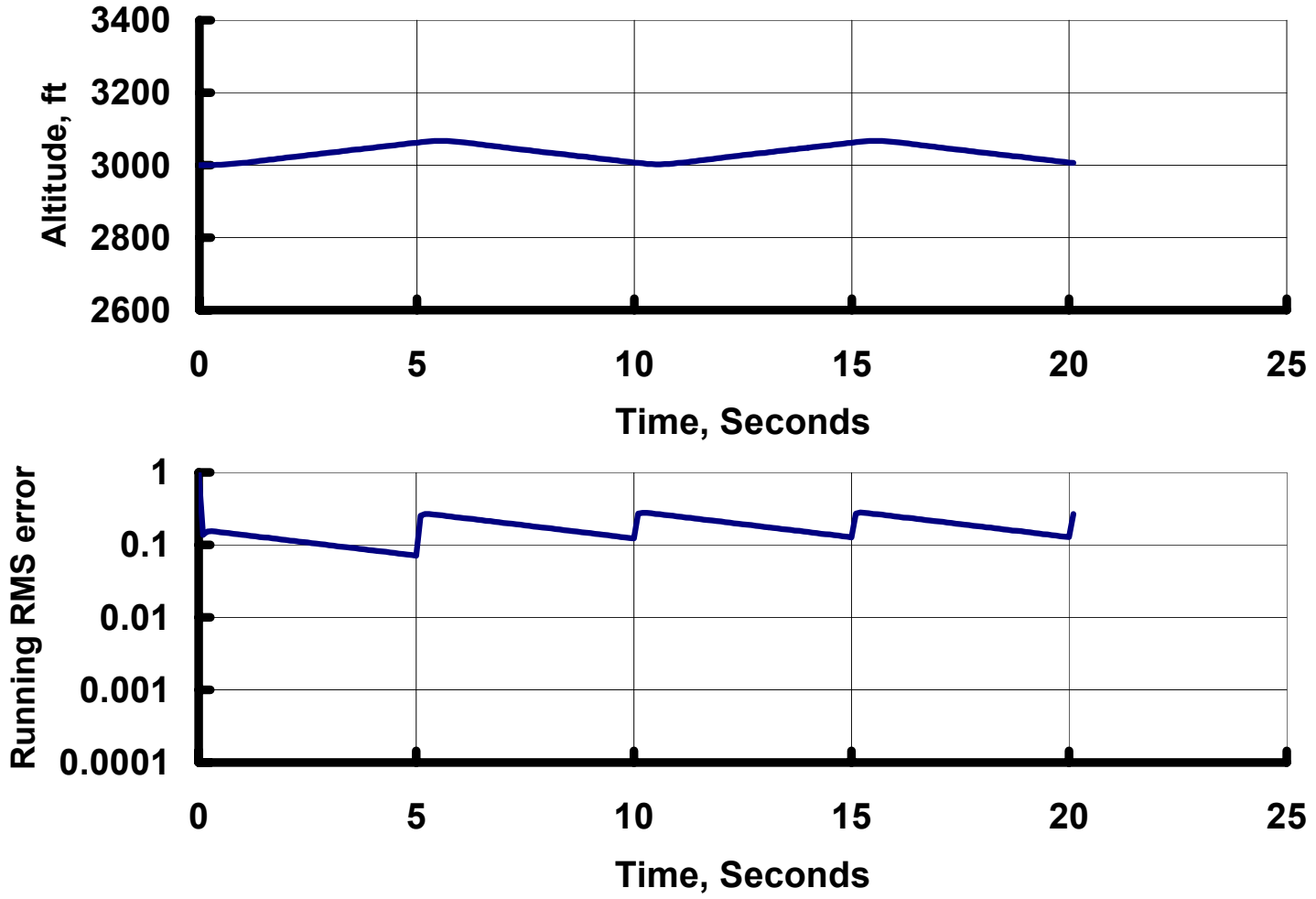

Figure 42 (c): Case 6 time history 


\subsection{Failures Involving Stability and Controllability of the Physical Plant}

Cases 7 through 14 examine the controller's ability to adapt to failures within the physical plant. Good performance will be evaluated by the controller's ability to maintain the desired second-order characteristics with a minimal transient between the nominal and the failure state.

Cases 7 through 10 (Figures 43 through 46) all address the same commanded maneuver. The airplane is commanded to fly a speed square wave, with speed steps of five feet/second. At two seconds into the simulation, a step change in flight-path is commanded equal to eight degrees.

Case 7 demonstrates the controller's performance for the nominal physical plant and no neural network compensation. This case can be compared with Case 8 where the neural network actively compensates for modeling errors. At first glance, it appears that whether the network is on or off makes little appreciable difference for these two cases. This is to be expected, since the linear inverse transfer function is built around the nominal aircraft state, therefore there should be little modeling or inversion error. However, on close inspection of the perturbation velocity trace it is possible to see that speed tracking accuracy is somewhat degraded in Case 7, without the neural network compensation. Furthermore, without the neural network compensation in Case 7, a defect in speed, relative to the reference, is apparent between about two seconds and five seconds. The neural network corrects this defect, and all "humps" in Case 8 are similar. Even with a nominal system, an improvement in tracking is available with the neural network on.

The neural network compensation is not involved in flying the aircraft in Case 7. However, the neural network is continuing to learn. This is apparent in the RMS error time history. This will be seen in all cases with the neural network compensation off. 
The time history for equivalent airspeed in Case 8 shows what might appear to be a drift in equivalent airspeed. This is not a drift, but rather reflects the general decrease in equivalent airspeed with increasing altitude as the controller tracks to true airspeed commands. The change in altitude is evident in the altitude trace.

Case 9 begins the same as Case 7. The airplane is initially in its nominal state with no neural network compensation. However, an anomaly of some kind is assumed to occur in Case 9 at $t=6$ seconds that reduces the stability of the airplane. The derivative $C_{M_{\alpha}}$ is cut in half. A vertical dashed line in Figure 45 graphically indicates the time of the failure. Since the linear transfer function incorrectly assumes nominal aircraft stability, and with the no neural network compensation, the airplane receives erroneous elevator commands and deviates from the commanded maneuver. Speed tracking is the most effected without the benefit of the neural network. Speed begins an apparent divergence from commanded speed, at least within the time window examined. Flight-path angle is also somewhat degraded. Comparison of the gamma perturbation plot with Case 8 shows a transient on the commanded flight-path angle after the failure.

Case 10 repeats the scenario of Case 9 , only now the adaptive neural network compensation is active. With the adaptive neural network compensation, there is no appreciable deviation from the commanded maneuver when the failure occurs at six seconds. Comparing speed and flight-path angle tracking for Case 10 with Case 8 shows them to be virtually the same, even with the failure present in Case 10. From the perturbation elevator trace, it is apparent that a change occurs at six seconds. A change in elevator deflection for Case 10 reflects what is essentially a new trim state based on the new value for $\mathrm{C}_{\mathrm{M}_{\alpha}}$. The neural network compensator quickly adapts to the failure and continues the maneuver with little interruption. 


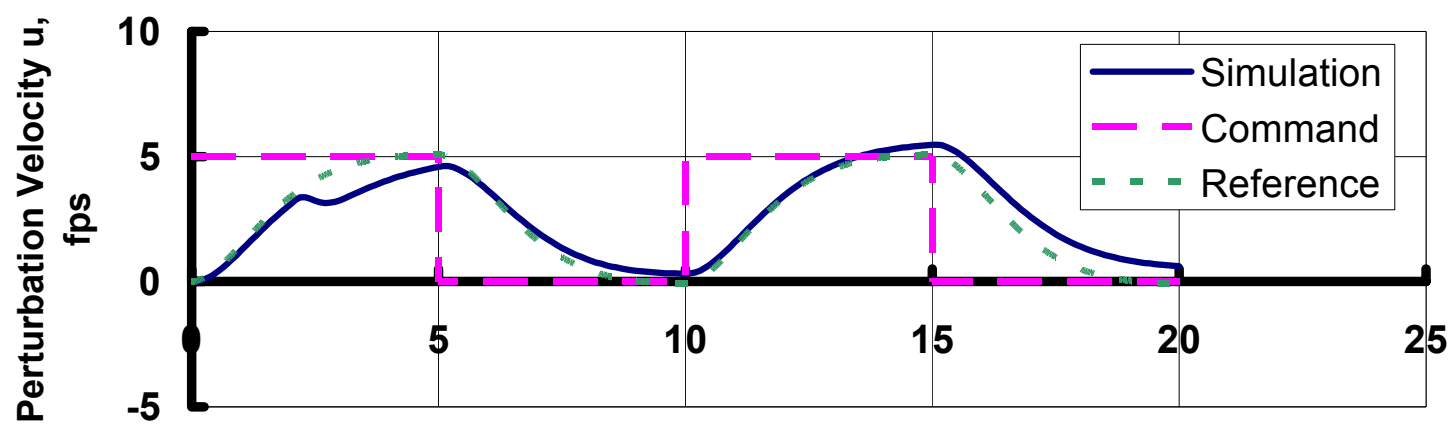

Time, Seconds
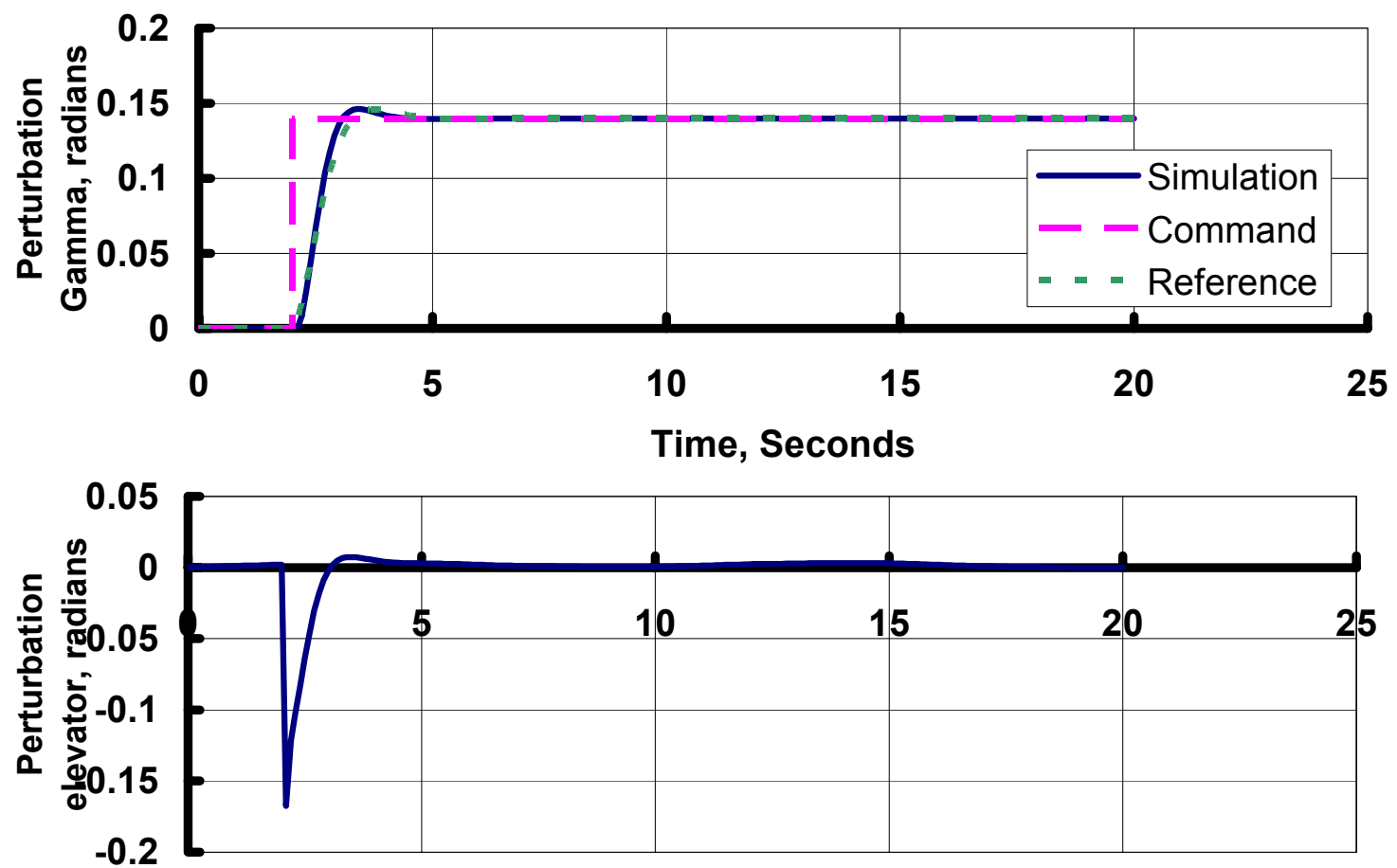

Time, Seconds

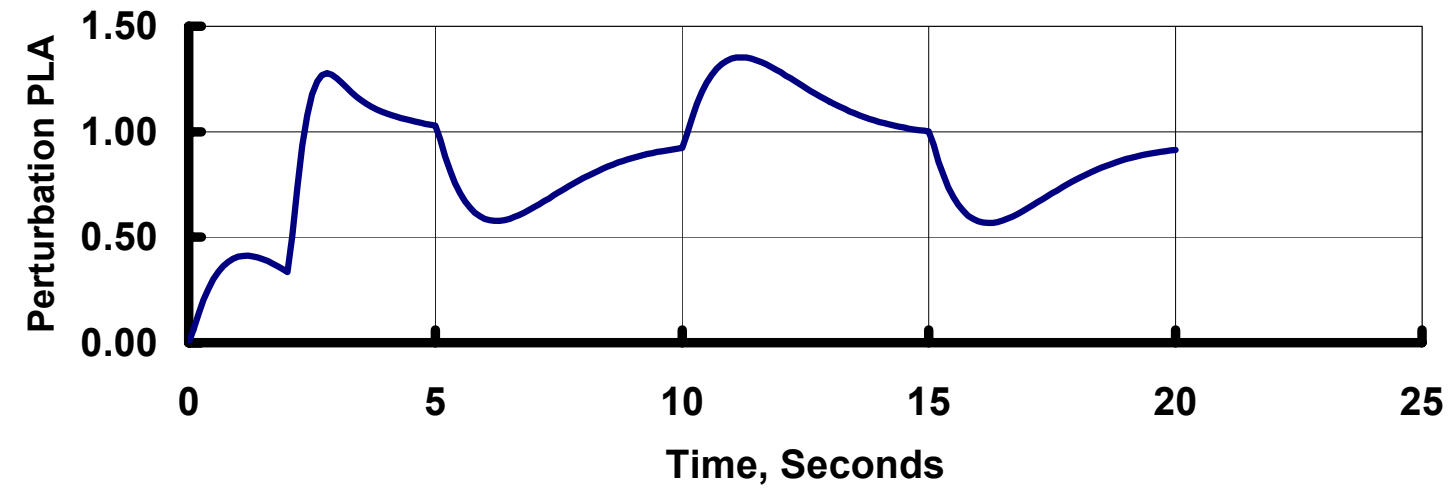

Figure 43 (a): Case 7 time history 

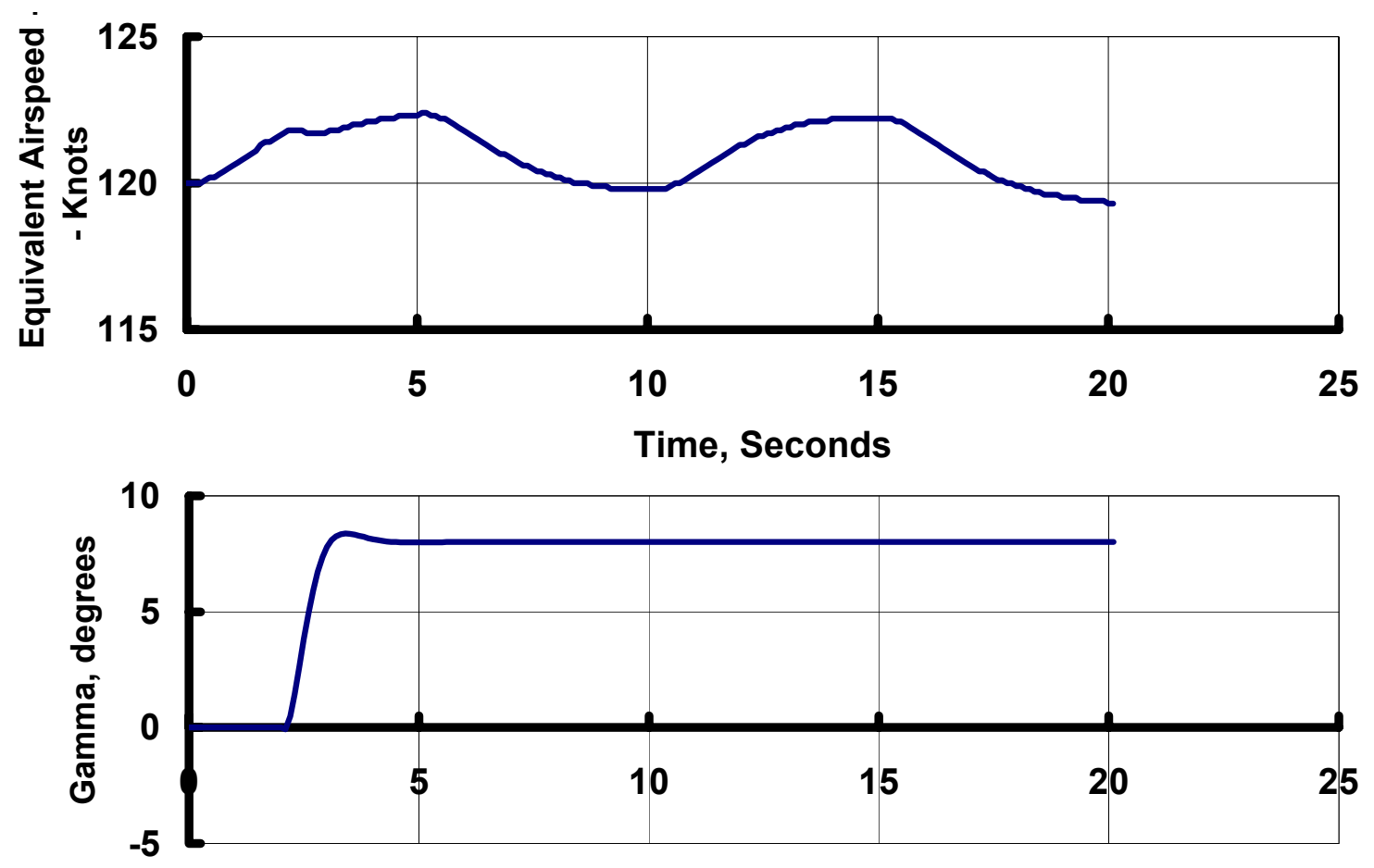

Time, Seconds

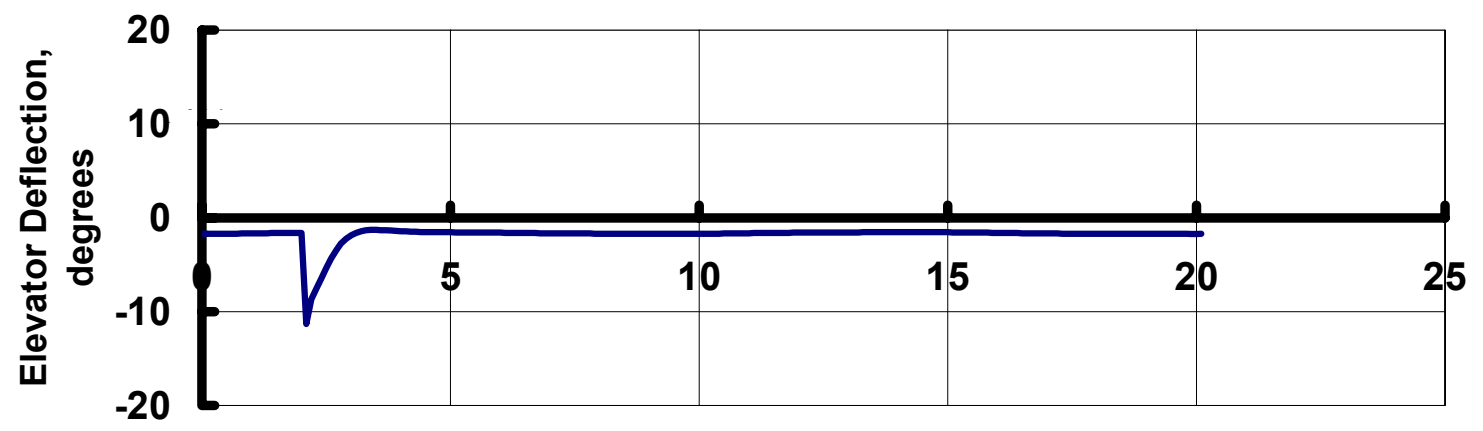

Time, Seconds

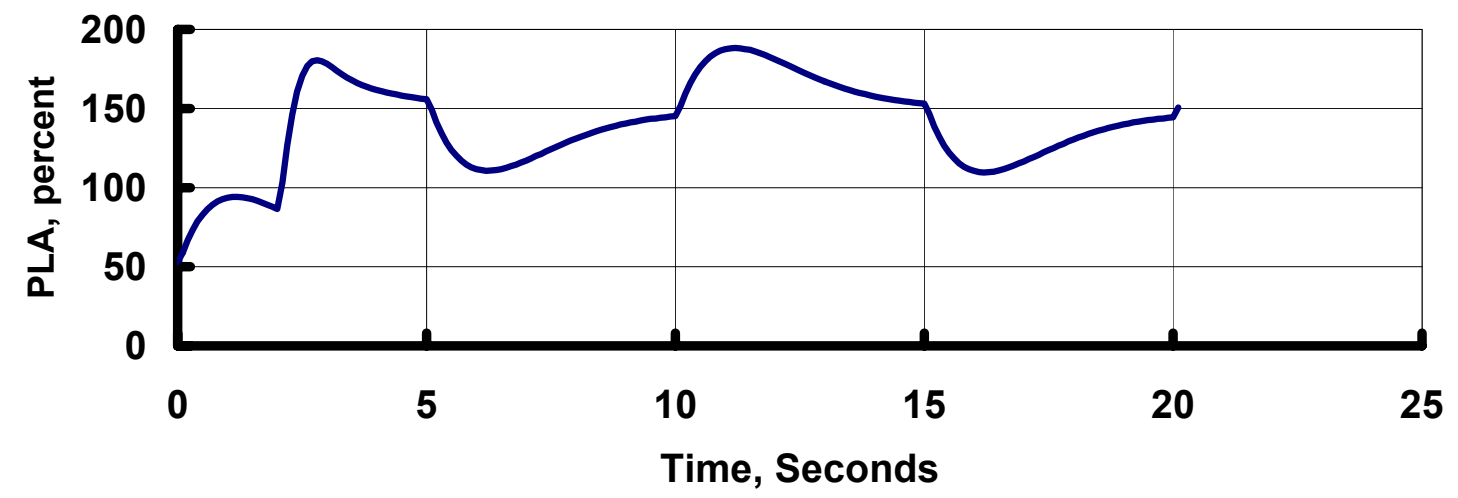

Figure 43 (b): Case 7 time history 


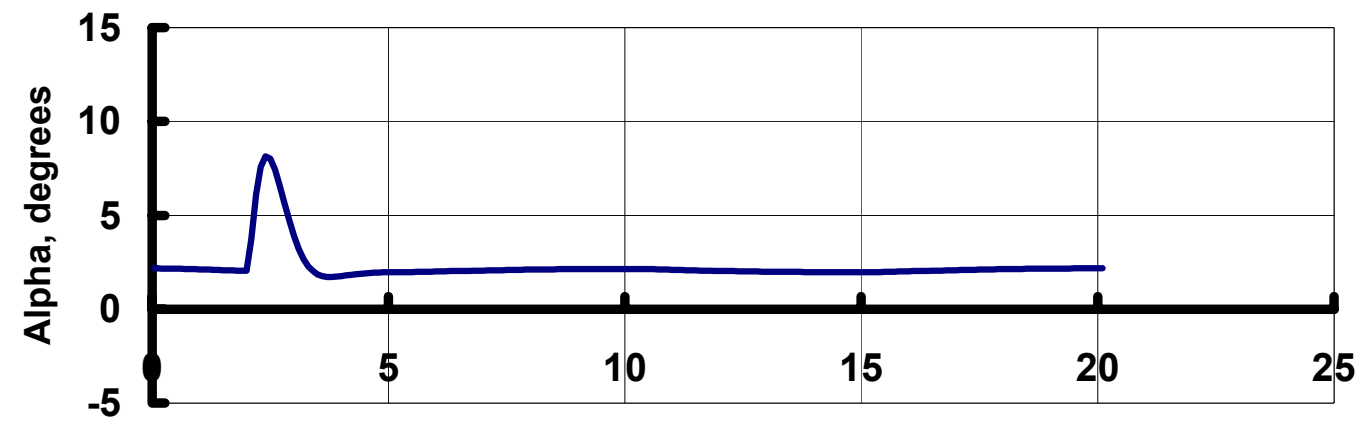

Time, Seconds
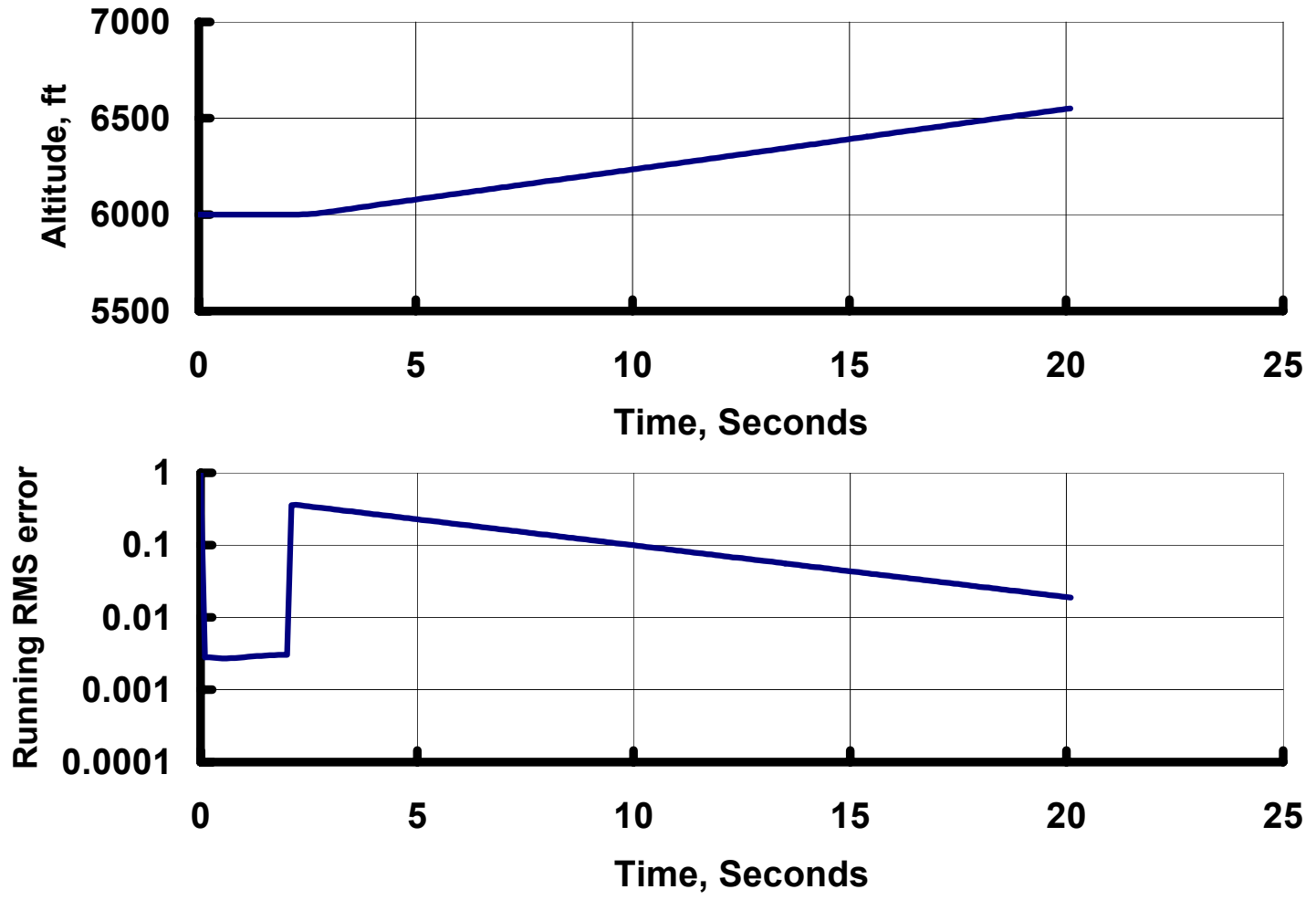

Figure 43 (c): Case 7 time history 


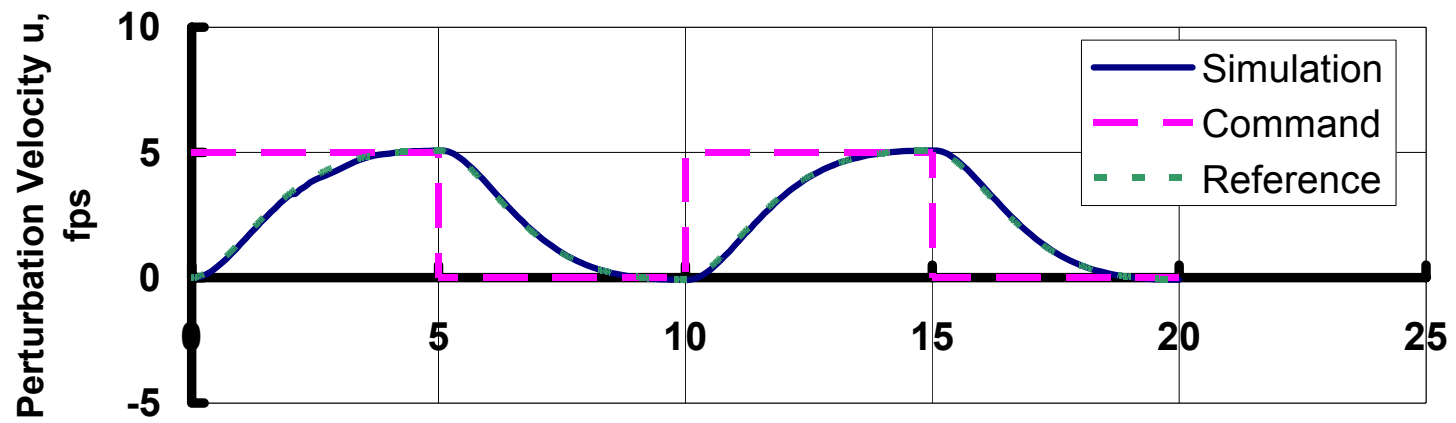

Time, Seconds
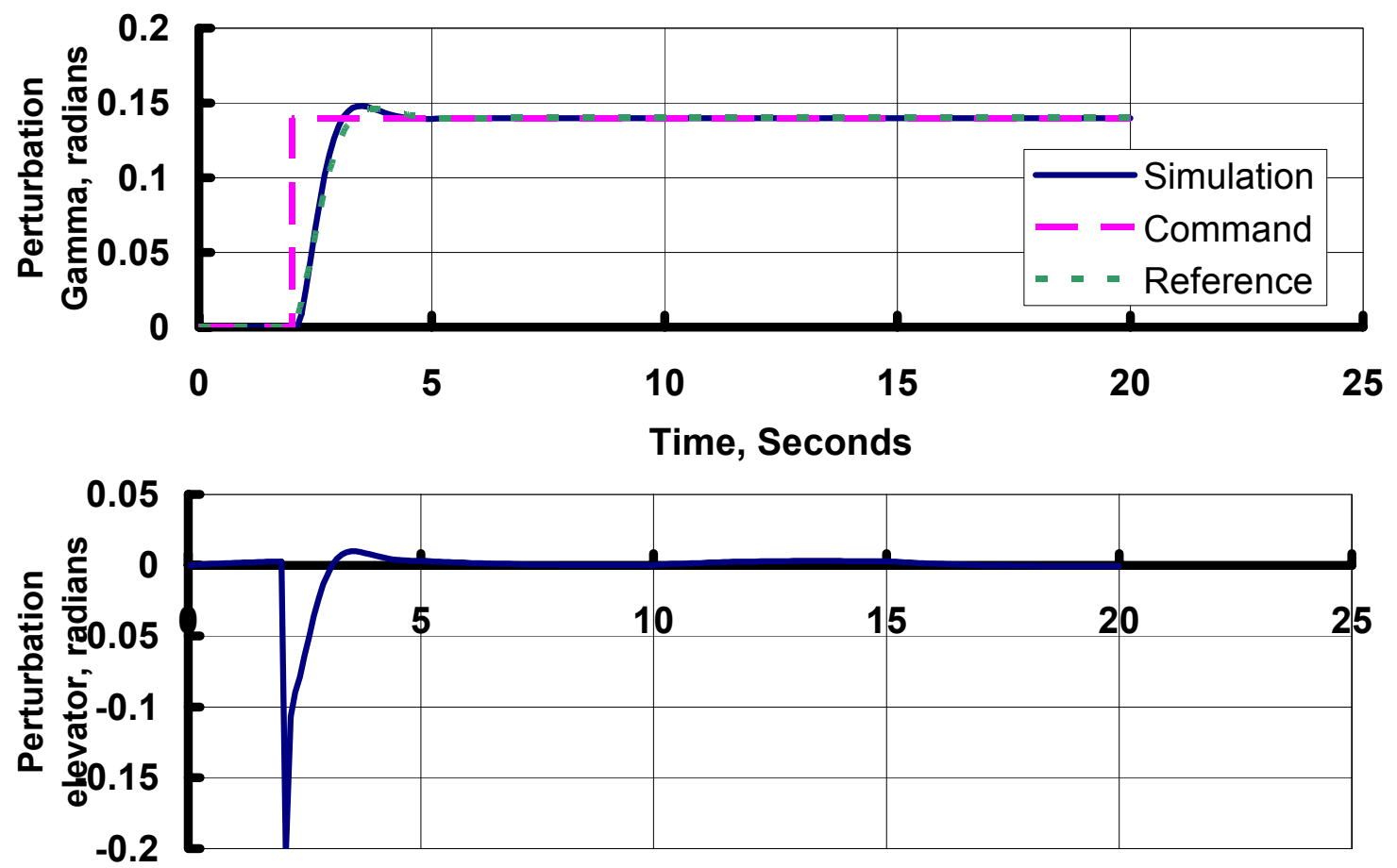

Time, Seconds

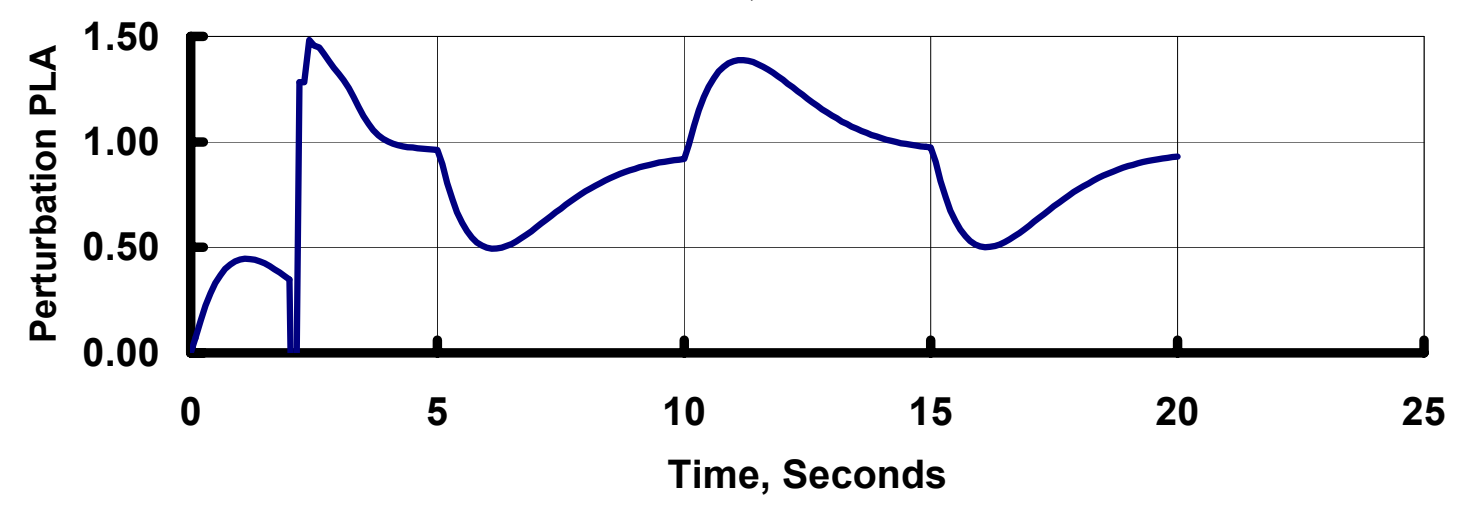

Figure 44 (a): Case 8 time history 

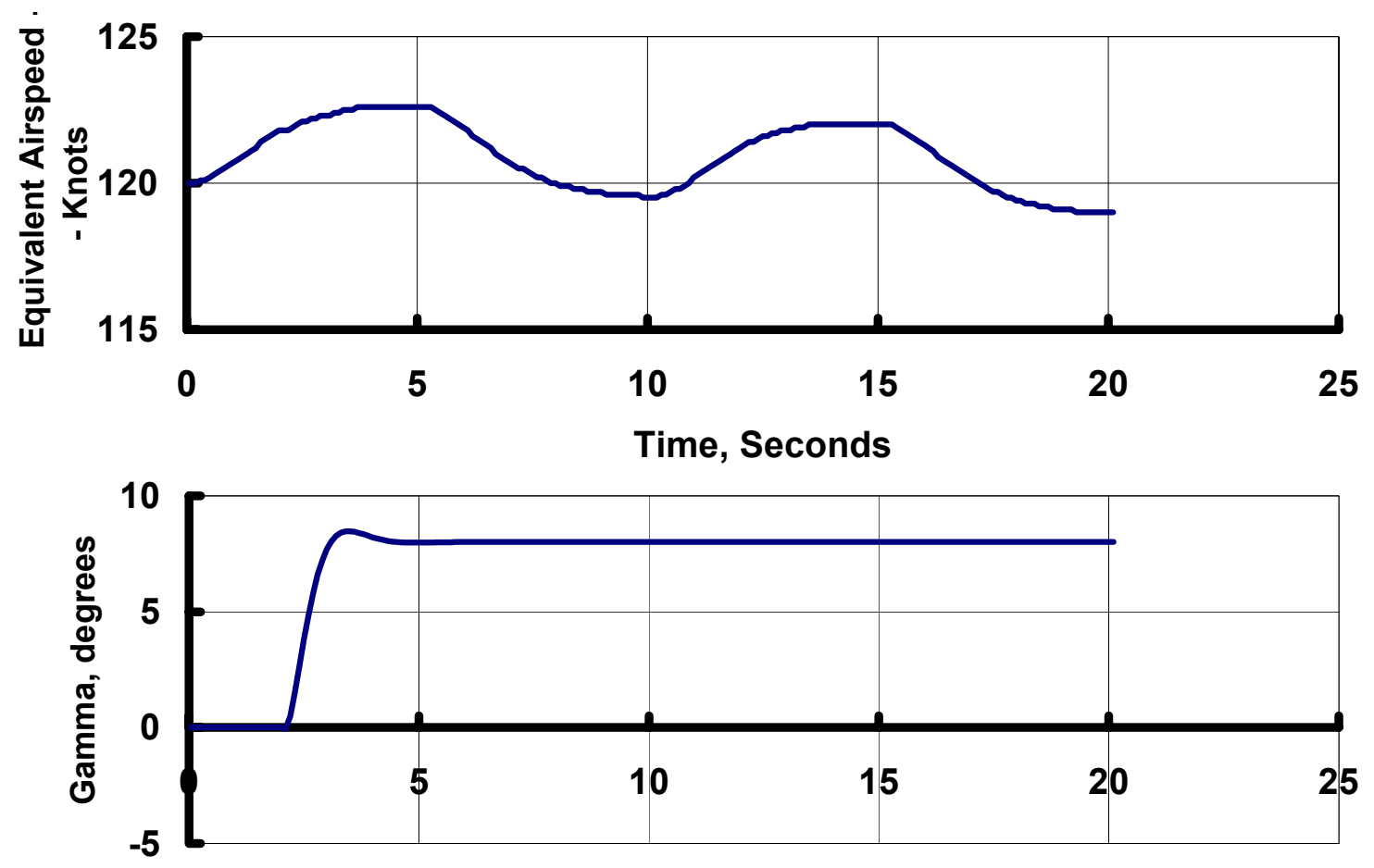

Time, Seconds

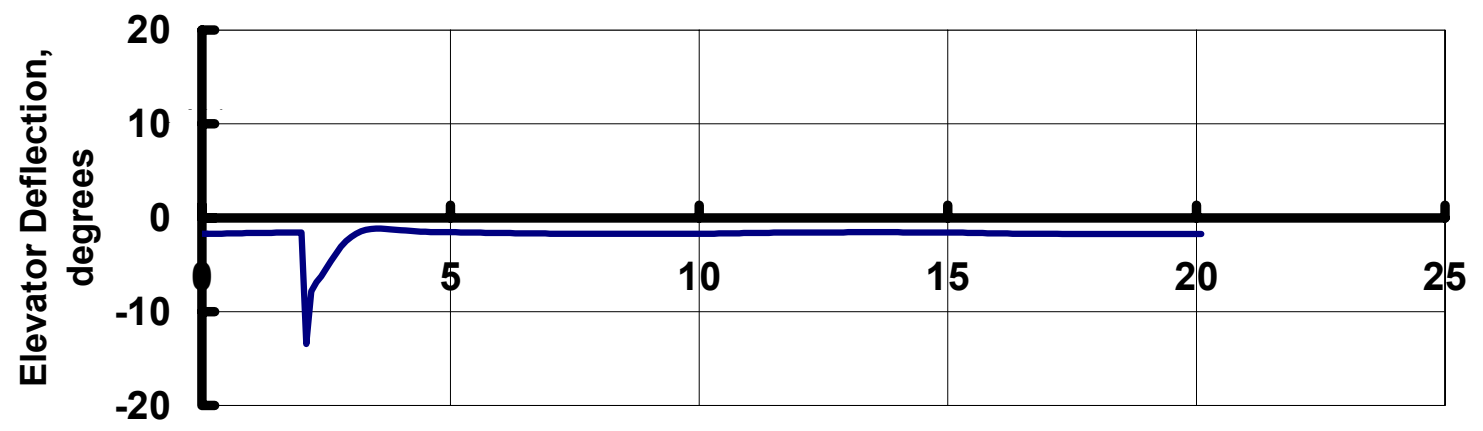

Time, Seconds

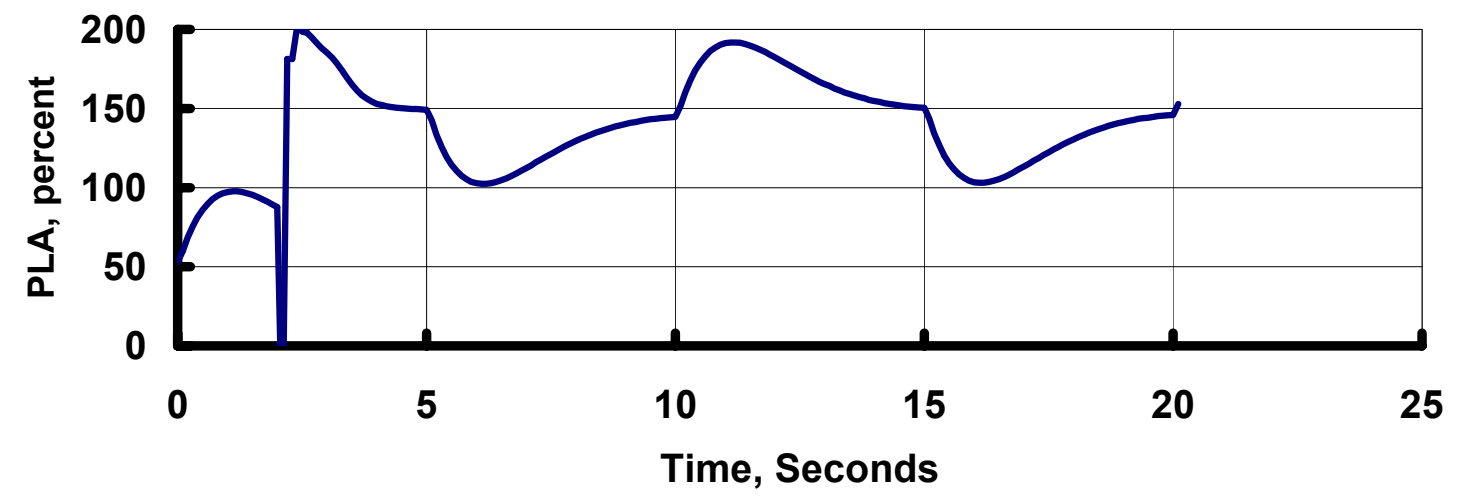

Figure 44 (b): Case 8 time history 


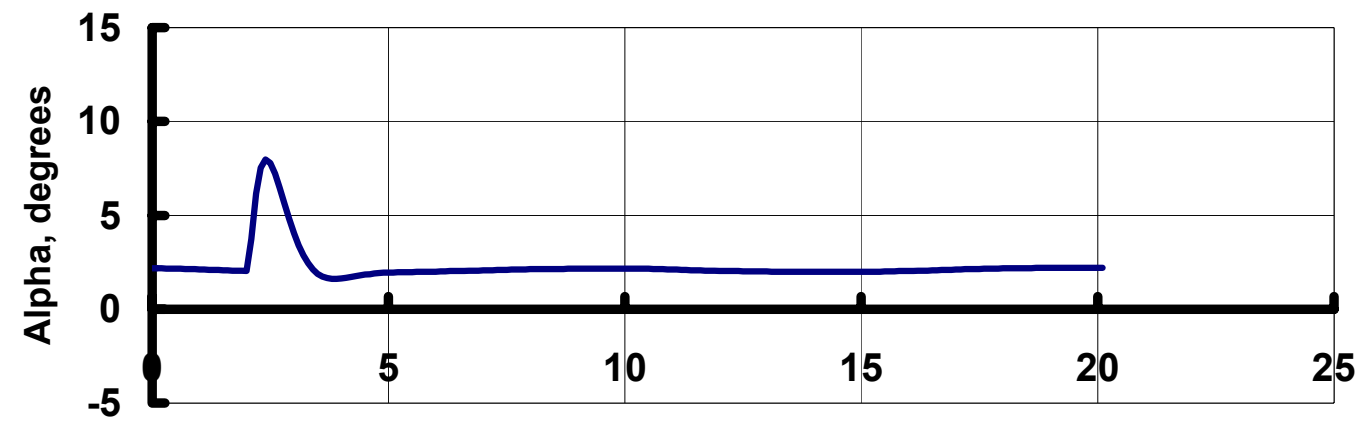

Time, Seconds
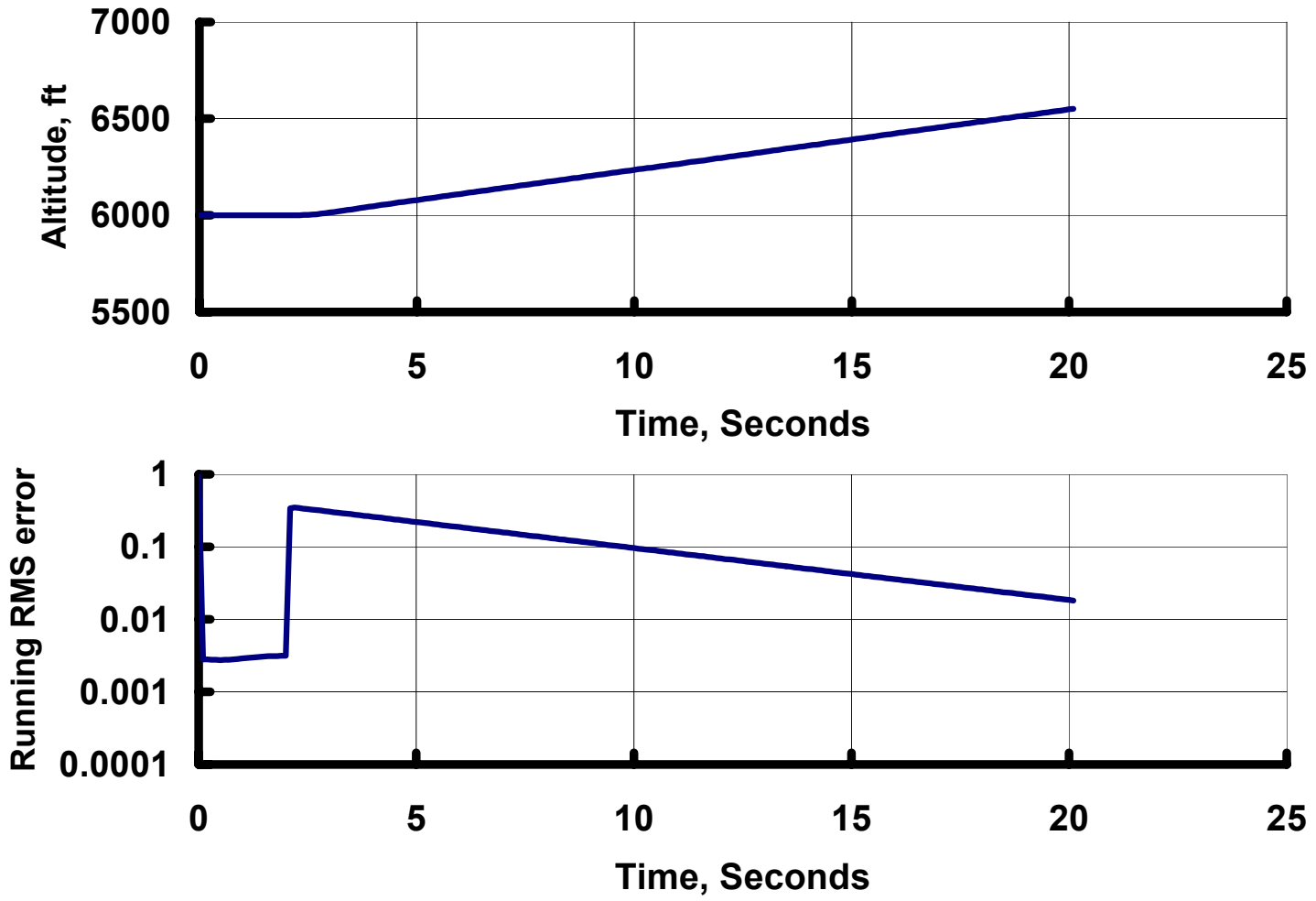

Figure 44 (c): Case 8 time history 


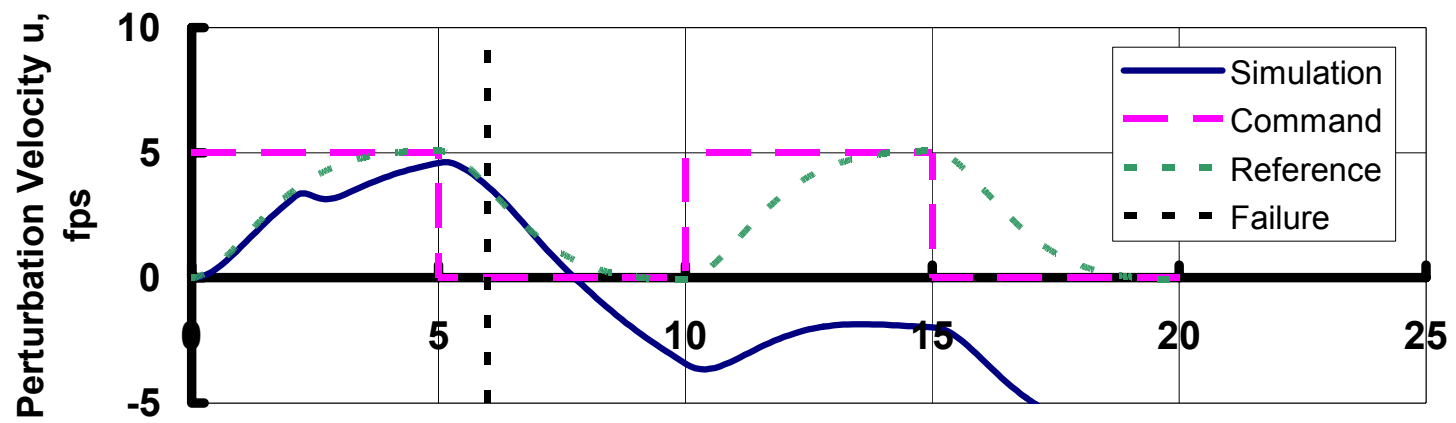

Time, Seconds

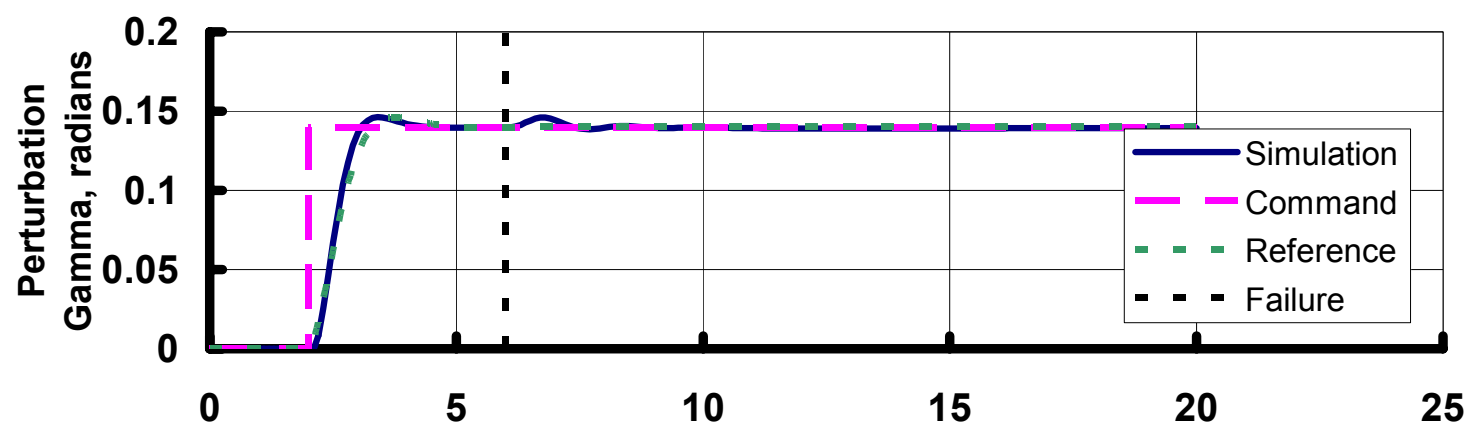

Time, Seconds

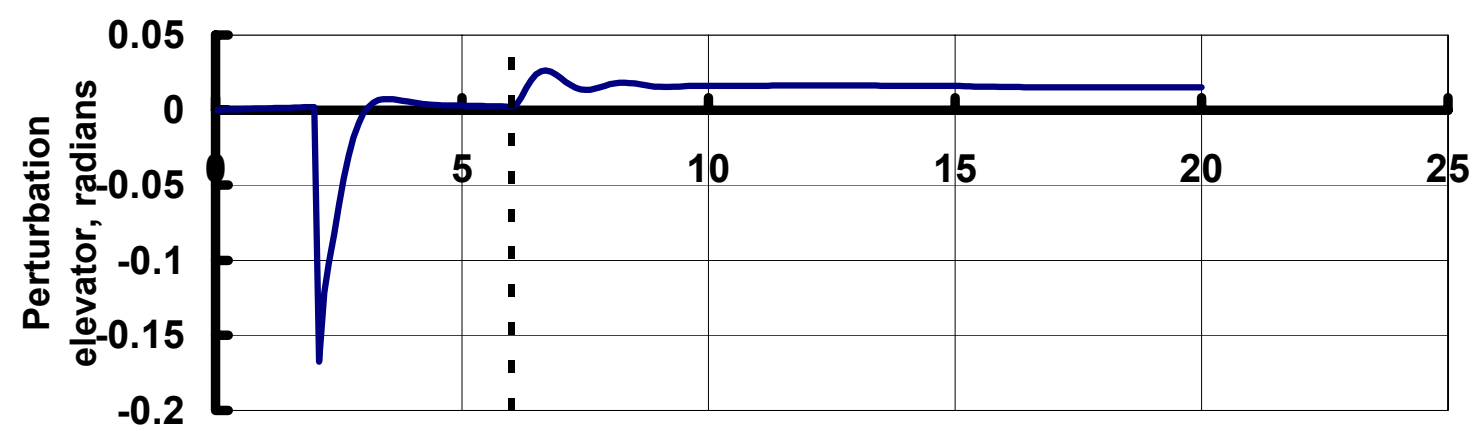

Time, Seconds

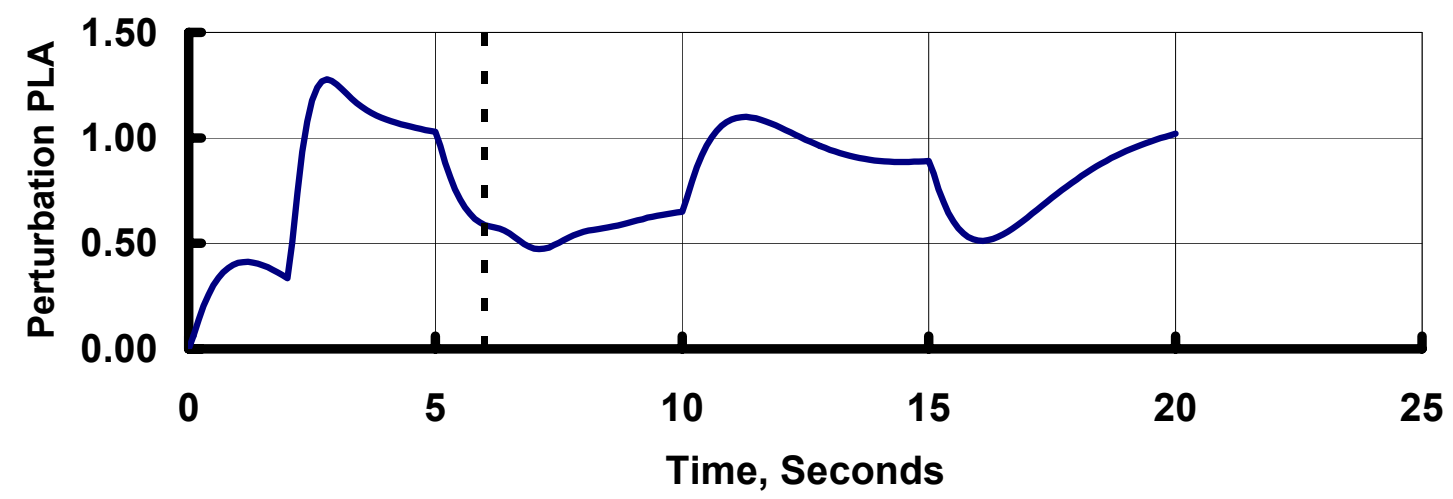

Figure 45 (a): Case 9 time history 

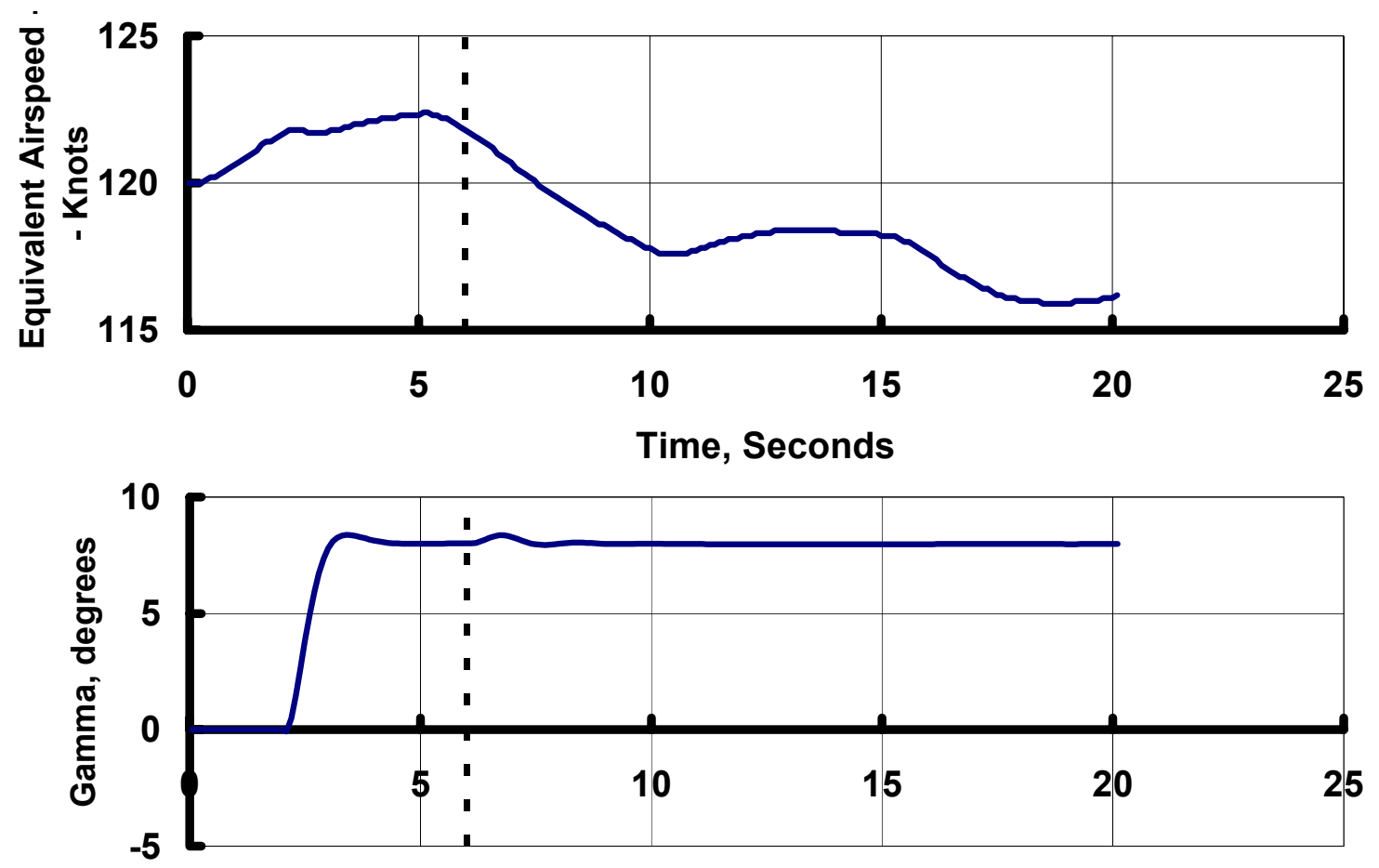

Time, Seconds

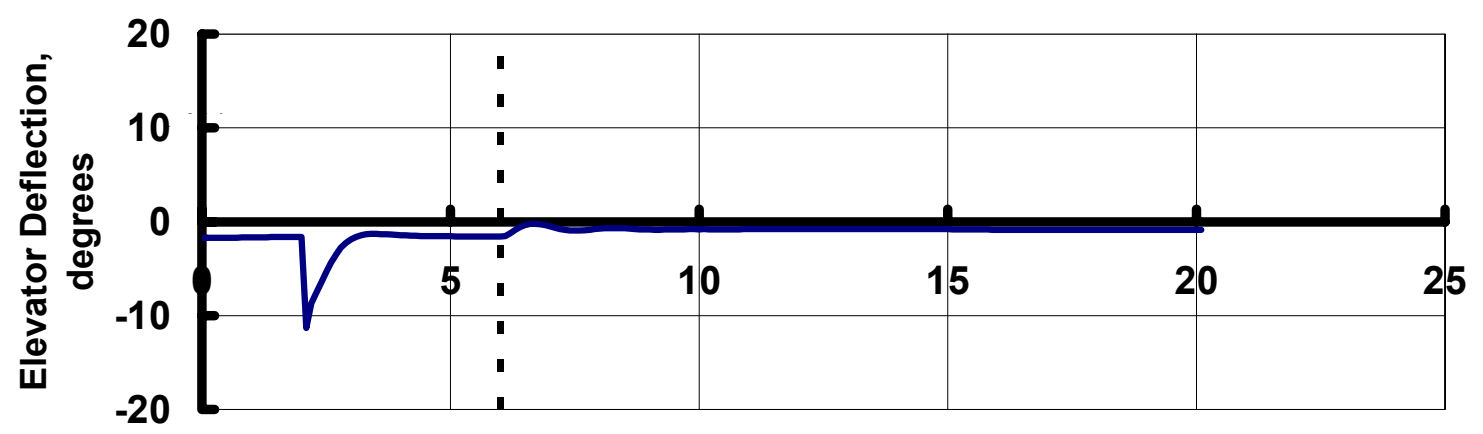

Time, Seconds

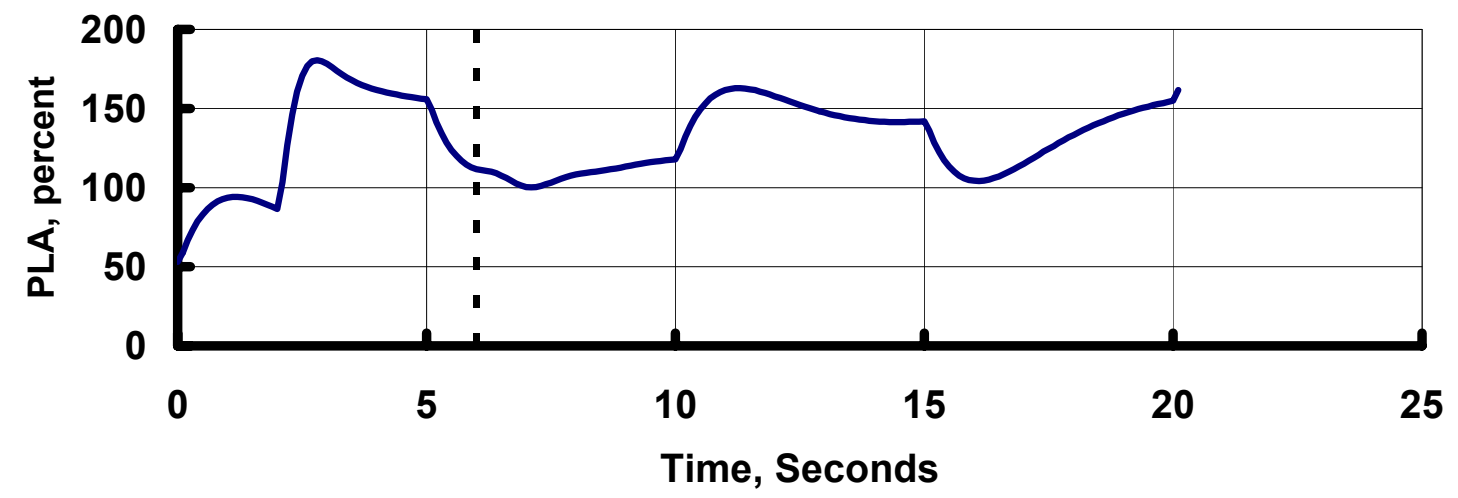

Figure 45 (b): Case 9 time history 


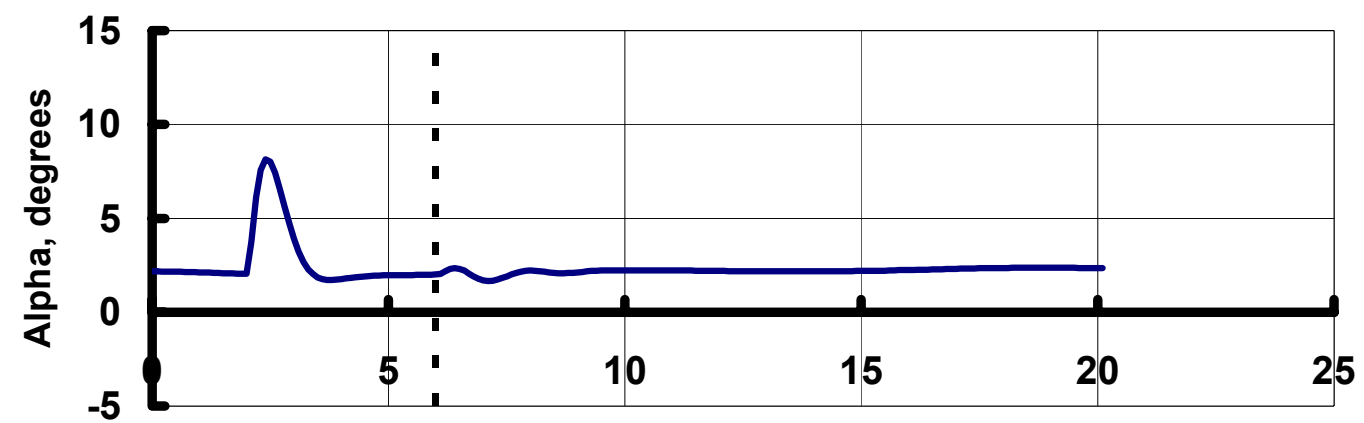

Time, Seconds
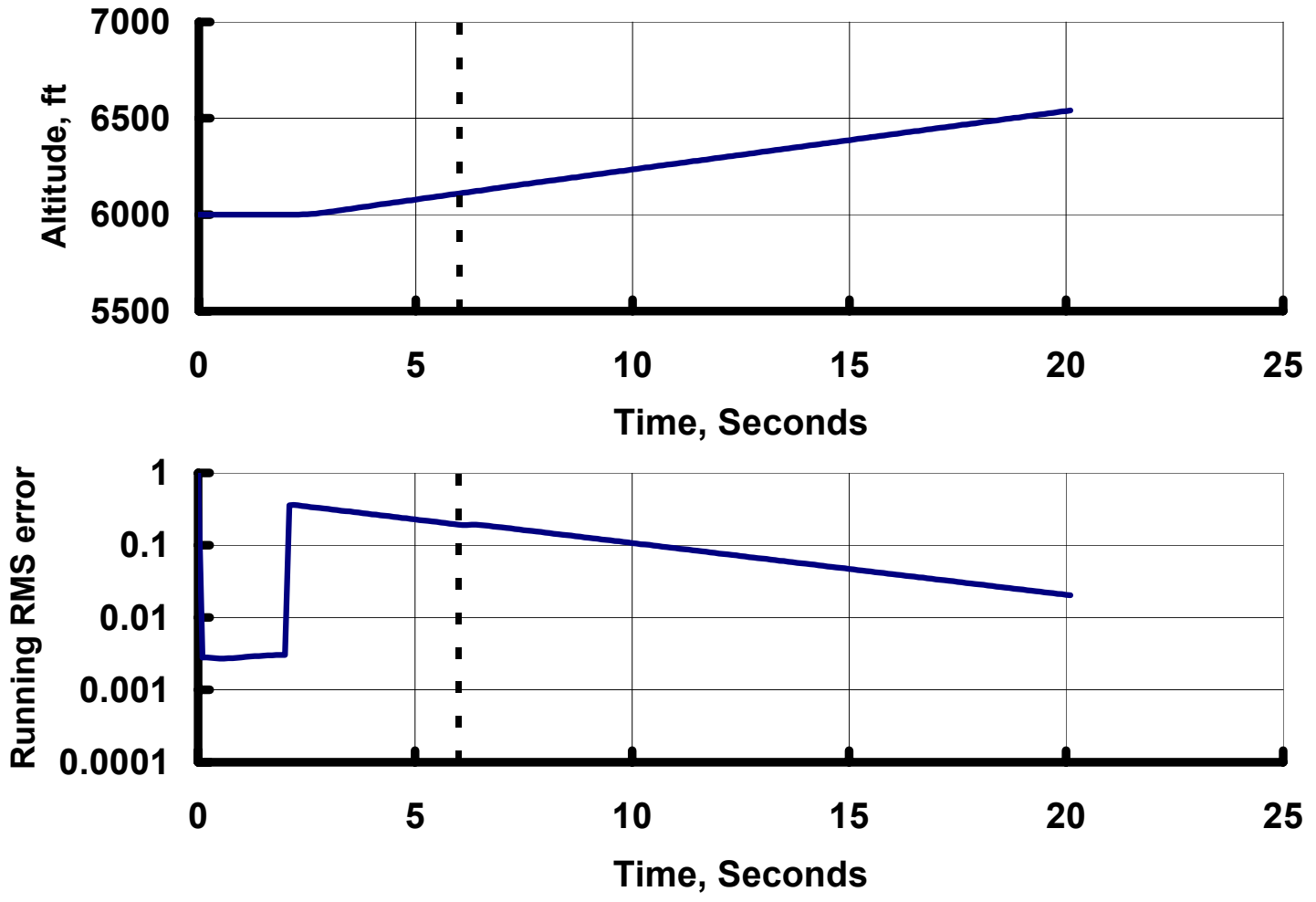

Figure 45 (c): Case 9 time history 


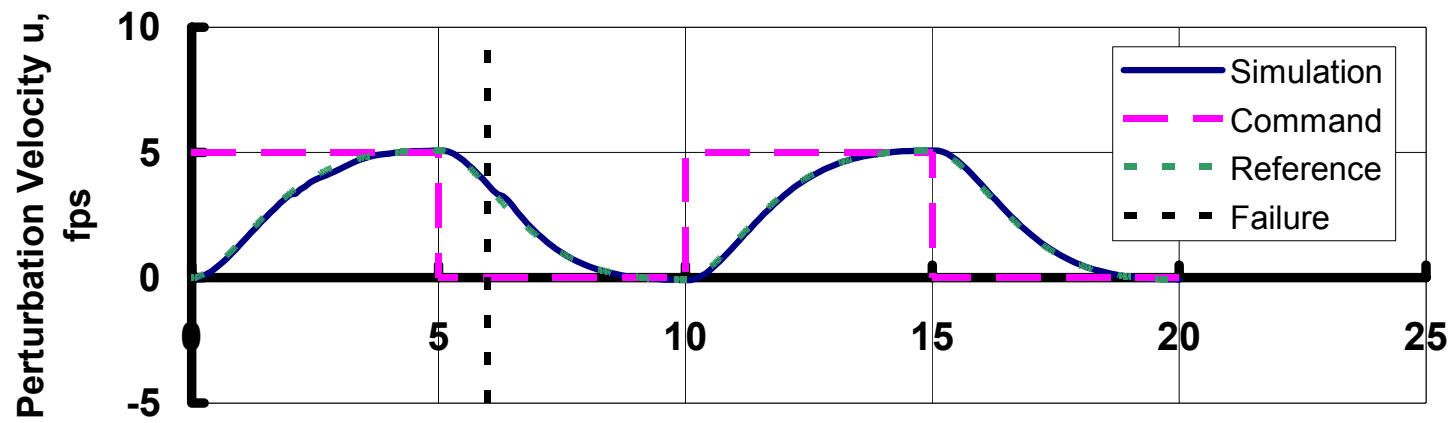

Time, Seconds

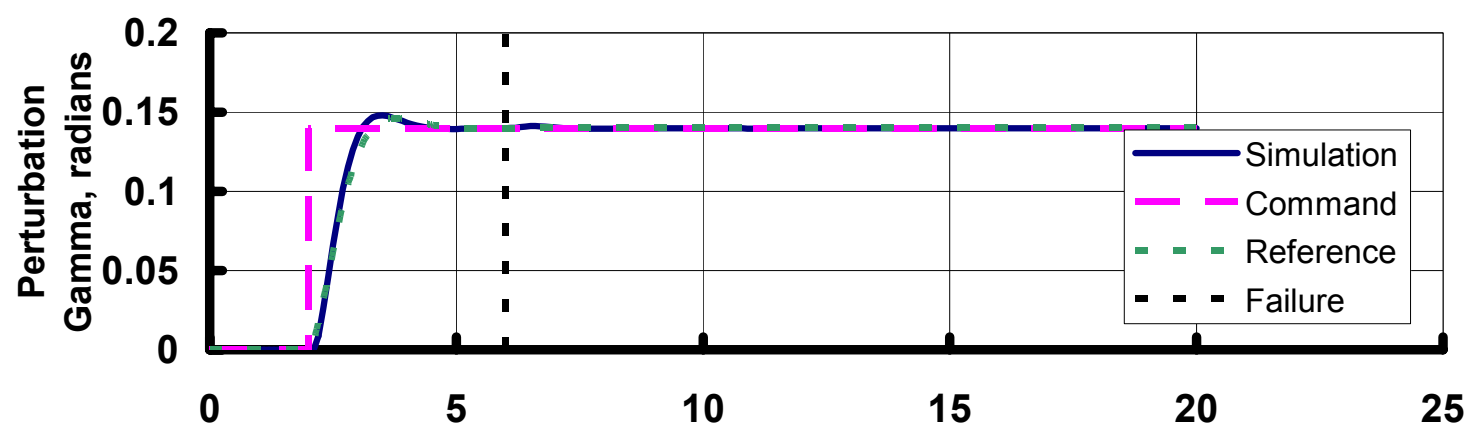

Time, Seconds

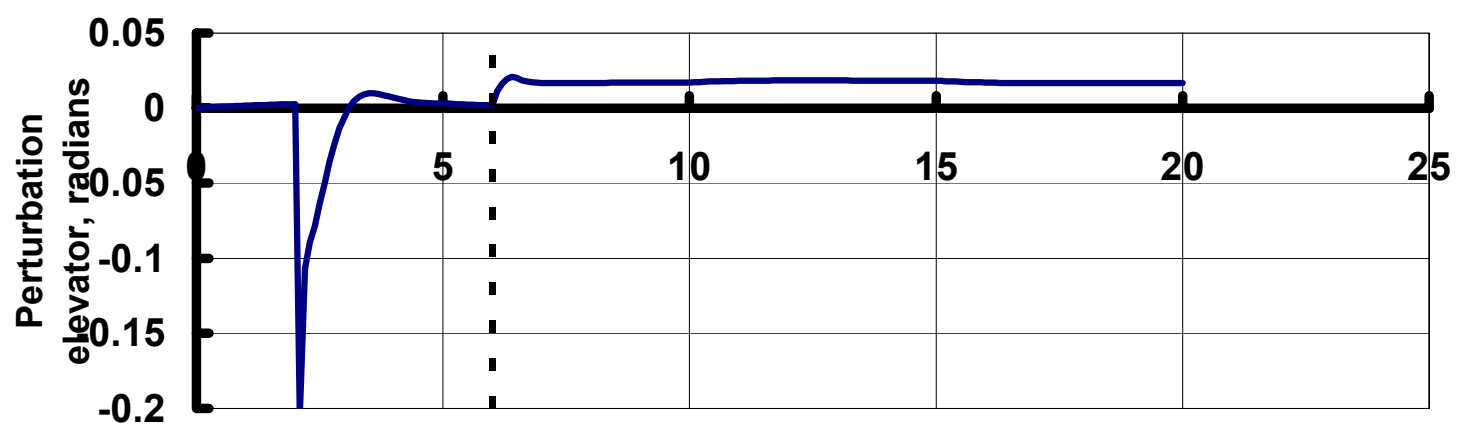

Time, Seconds

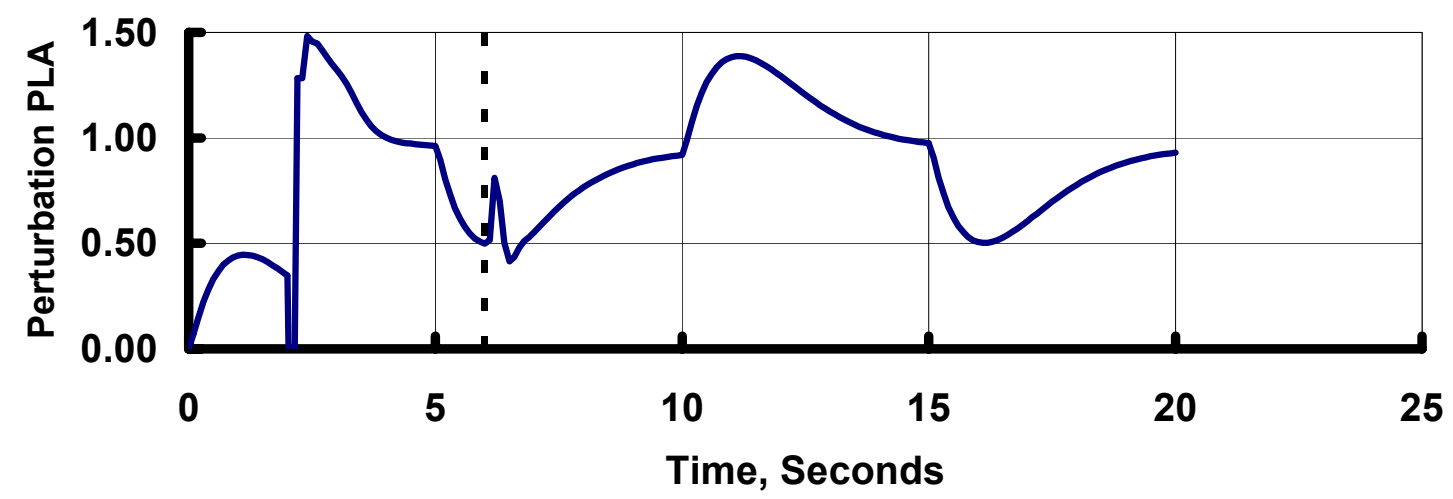

Figure 46 (a): Case 10 time history 

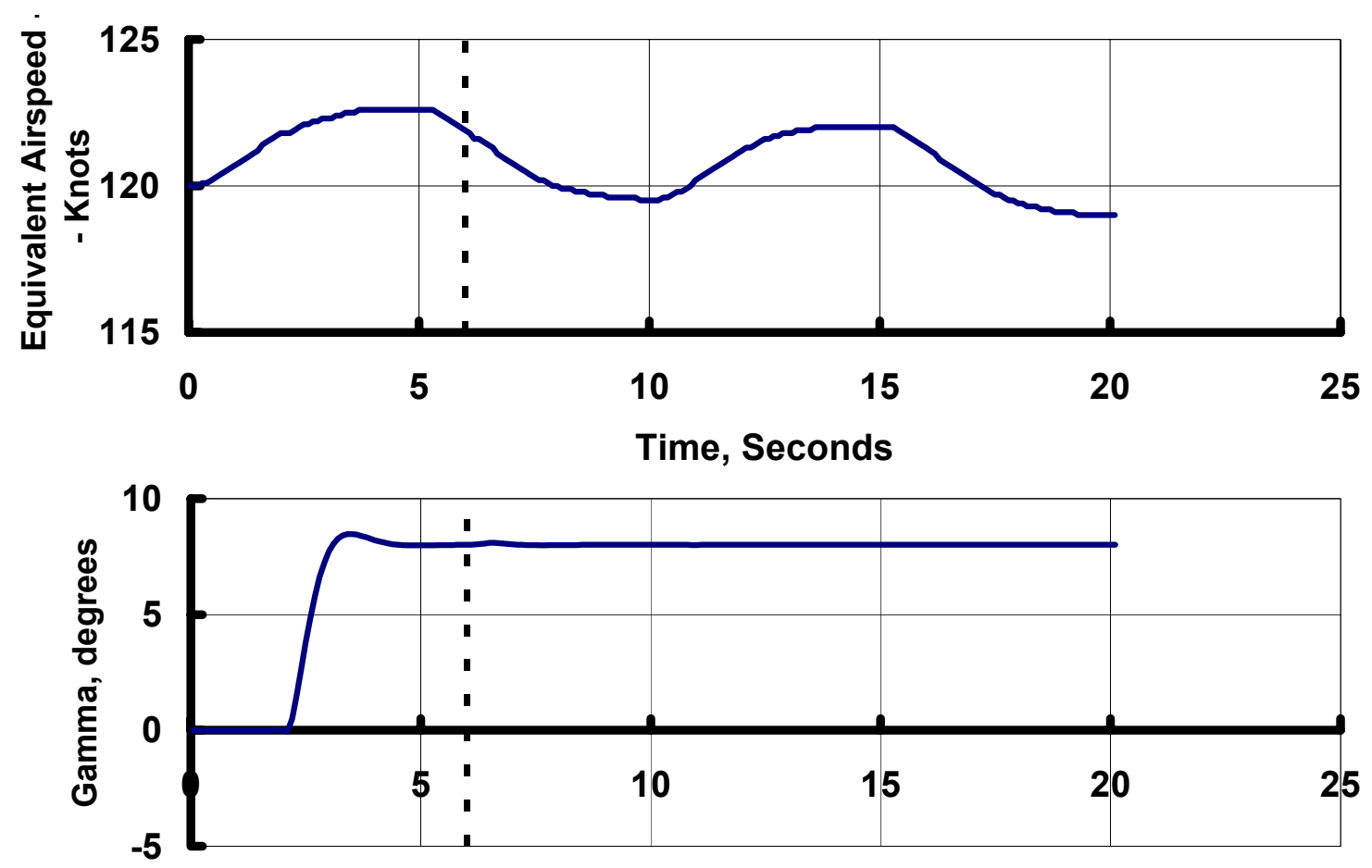

Time, Seconds

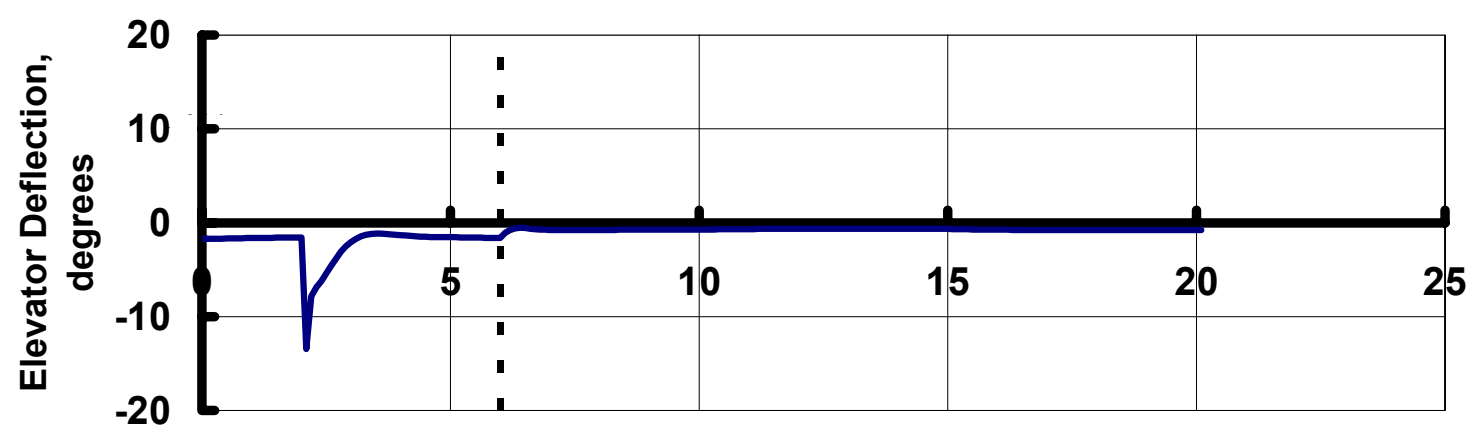

Time, Seconds

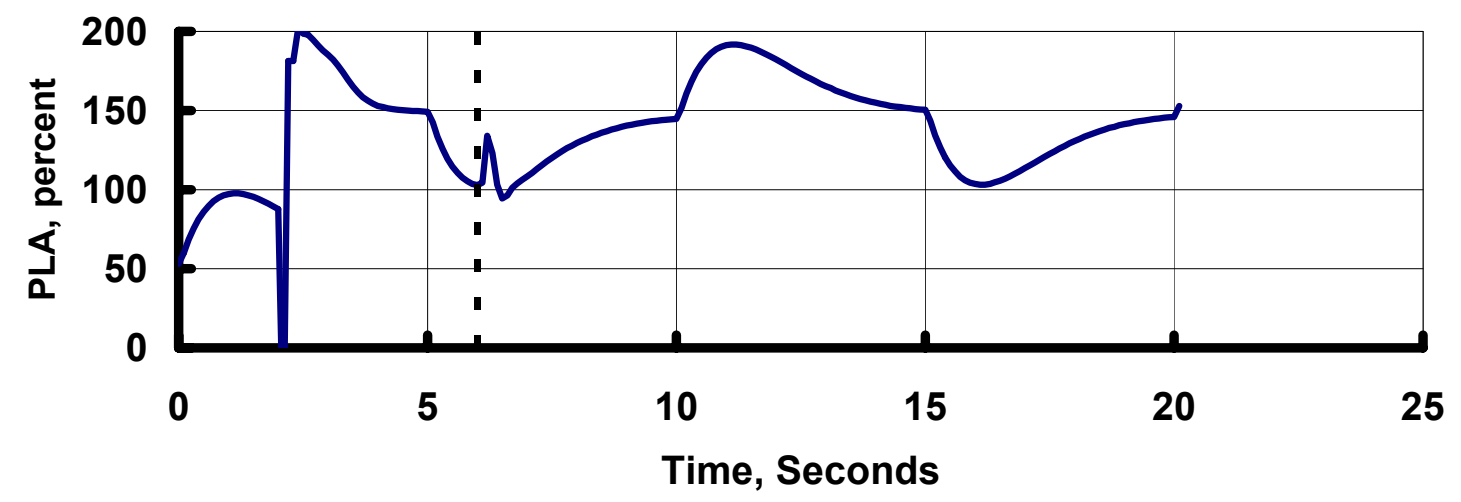

Figure 46 (b): Case 10 time history 


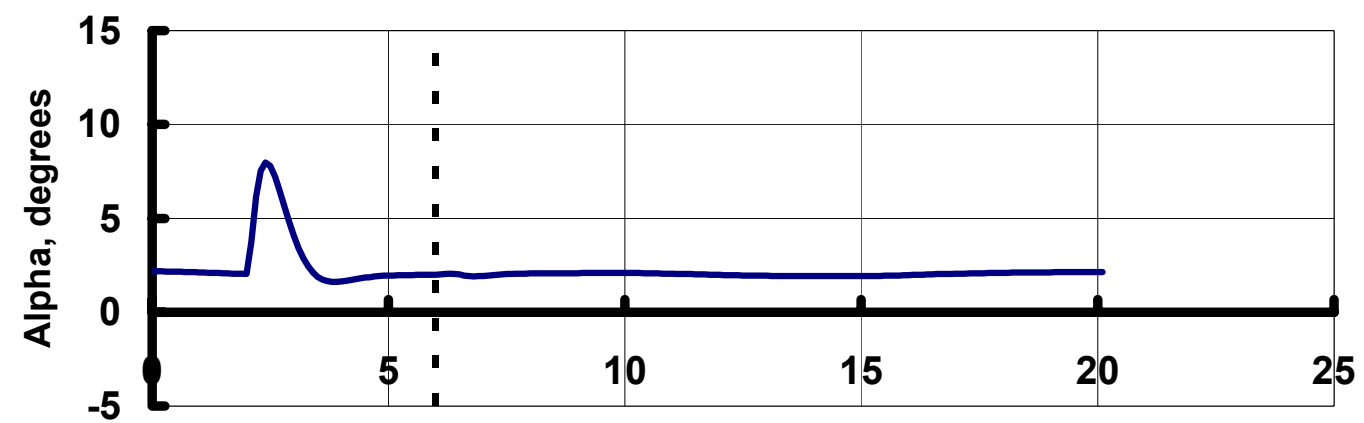

Time, Seconds
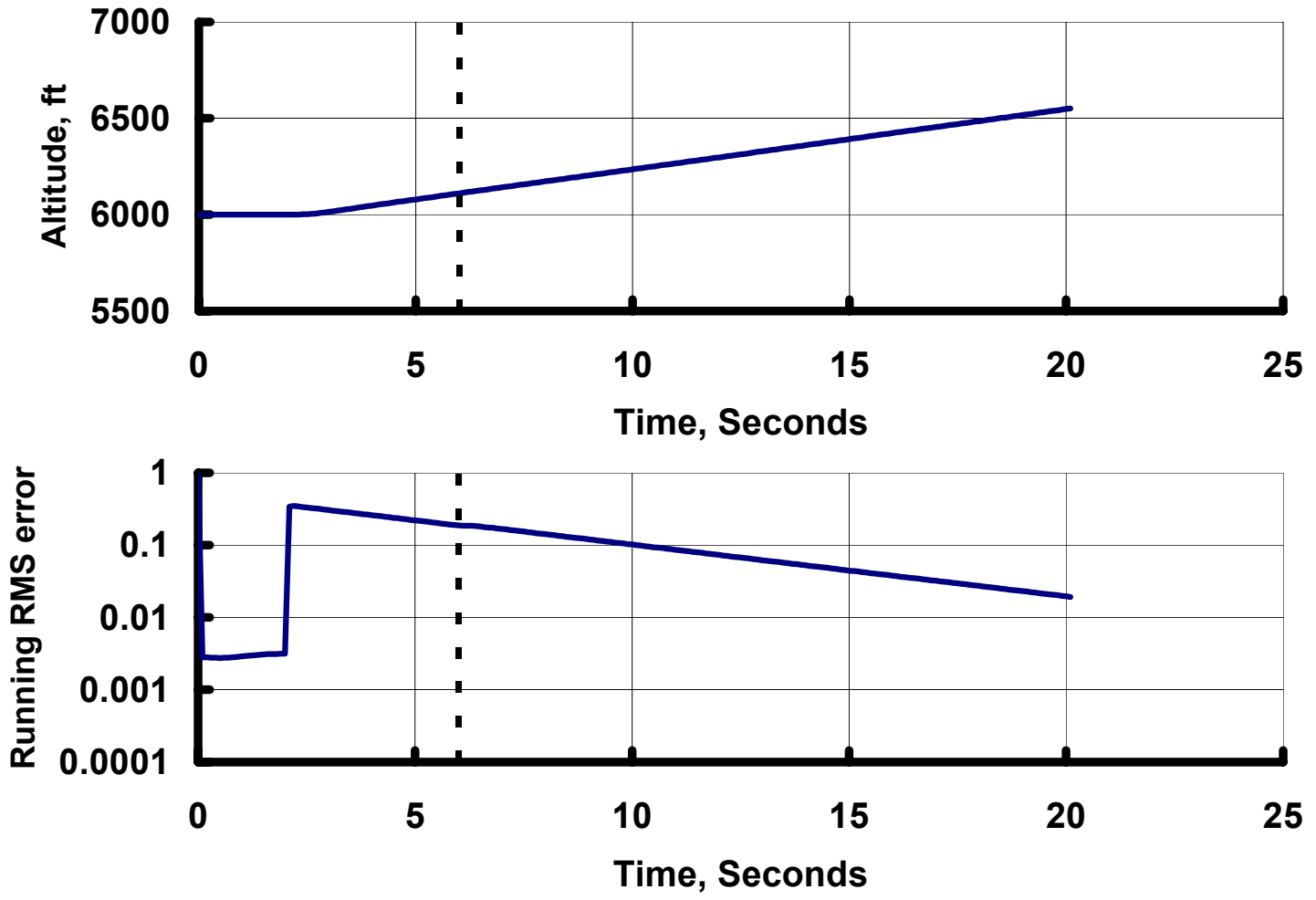

Figure 46 (c): Case 10 time history 
Cases 11 through 14 (Figures 47 through 50) reproduce failure scenarios examined by Steck [13]. The neural network is on at all times during these four cases to compensate for failures.

Case 11 represents maintaining level flight with the insertion of two failures. At five seconds into the simulation, $\mathrm{C}_{\mathrm{M}_{\alpha}}$ is reduced by ten percent. Ten seconds later, throttle gain is reduced by 25 percent. Vertical dashed lines in Figure 47 mark the time of the failures. The current controller adapts to these failures with very little transient behavior. Speed variations are less than one knot, and gamma variations are less than one degree. A small change in trim elevator setting is evident when the stability change occurs at six seconds. An increase in PLA is observed at fifteen seconds to offset the reduction in throttle gain. Learning is evident from the RMS error chart. Step changes in learning occur at the first and second failure.

Case 12 involves a pitch-up command at eight seconds to a five-degree flight-path angle, followed by a 20 feet/sec step command in speed at 20 seconds. The same failure sequence occurs as in Case 11. Once again, no significant deviations from the commanded speed and flight-path angle are observed. Similar behaviors for elevator and PLA are seen as in Case 11 as the system adapts to the failure. Learning is evident at the time of the failures based on the step changes in RMS error.

Case 13 starts with an immediate failure at time zero that reduces both $\mathrm{C}_{\mathrm{L}_{\alpha}}$ and $\mathrm{C}_{\mathrm{M}_{\alpha}}$ by 50 percent. The objective is to maintain steady level flight. Significant learning at time zero is evident in the RMS error trace. The transient errors after this immediate failure are somewhat larger with this scenario but still less than one knot in airspeed and less than one degree in flightpath angle. 
Case 14 (Figure 50) is a somewhat extreme scenario. A step decrease in speed of 70 feet/sec is commanded at three seconds. Failures involve a ten percent reduction in $\mathrm{C}_{\mathrm{M}_{\alpha}}$ at twenty seconds and throttle gain reduction of 25 percent at 25 seconds. This is an unrealistic scenario in that the final commanded speed is less than the stall speed for the real aircraft. None the less, the controller is able to achieve the desired speed reduction. This is an interesting case in that the maneuver causes the airplane to reach the angle of attack limiter implemented in the simulation. Almost simultaneously with reaching the target speed reduction at about eight seconds, the angle of attack reaches the 14 degree limit. Elevator and PLA traces are seen to have a change in character when the controller switches to compliance with the 14 degree angle of attack limit. Examination of the angle of attack trace shows good adherence to the 14 degree limit. The available lift coefficient at 14 degrees angle of attack is not quite sufficient to maintain equilibrium in lift, and the airplane is observed to begin a descent. Flight-path angle increases negatively, and the altitude begins to decrease. The failures seem almost anti-climactic after the speed reduction. However, the controller is observed to achieve a new steady-state elevator solution after the stability failure and a new PLA setting after the throttle gain failure. Even with the controller "distracted" by observing the angle of attack limit, it does a reasonable job of tracking speed in this very low-speed maneuver. 


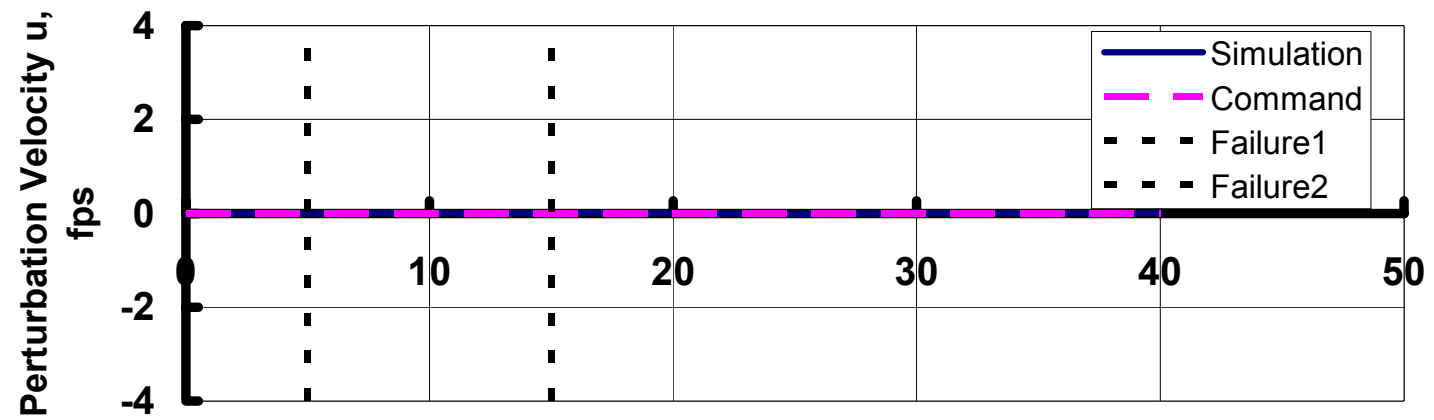

Time, Seconds

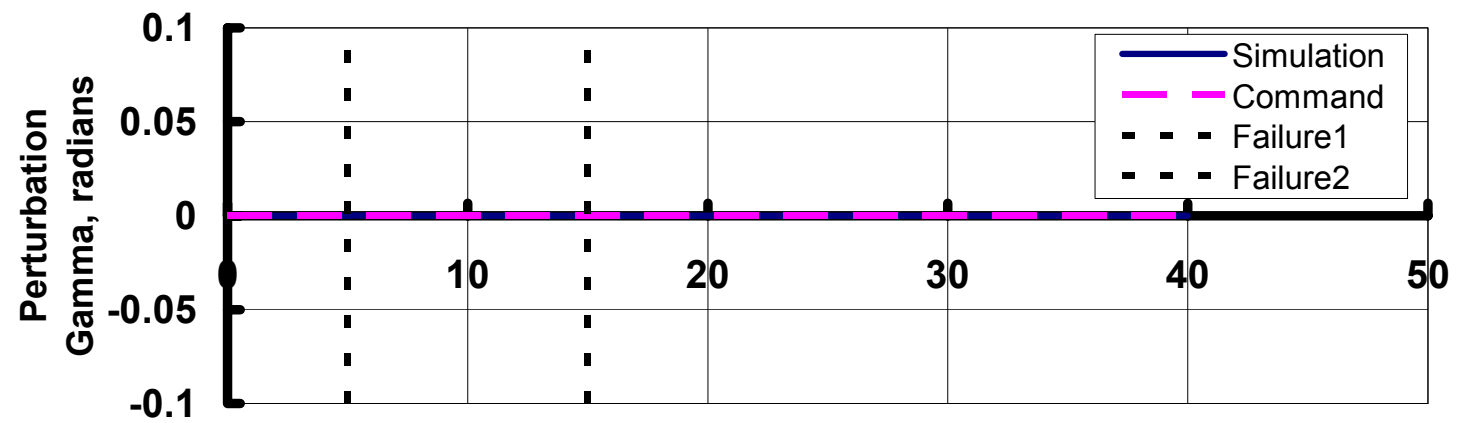

Time, Seconds

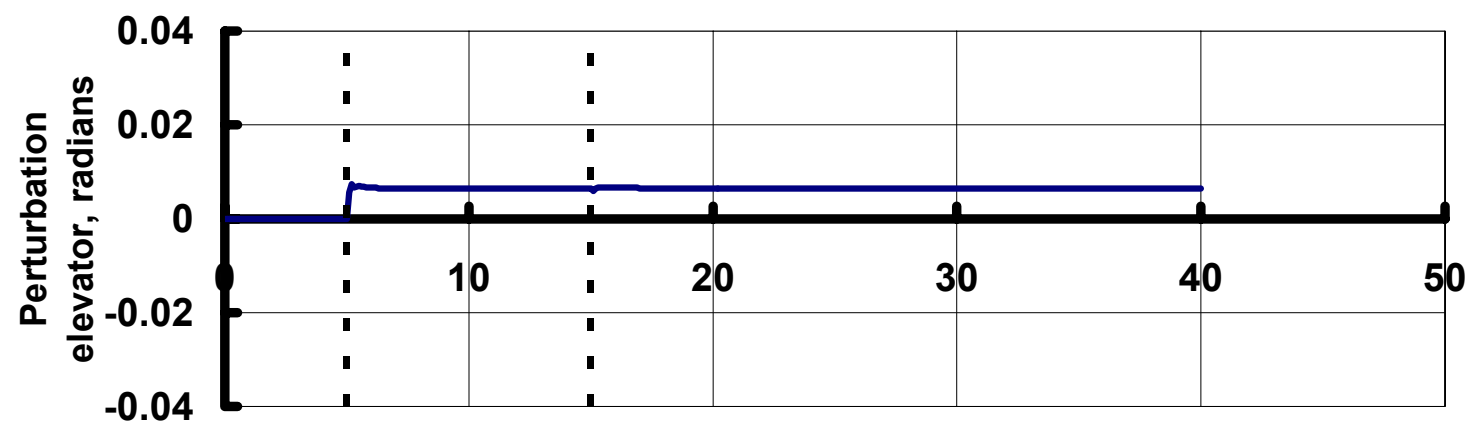

Time, Seconds

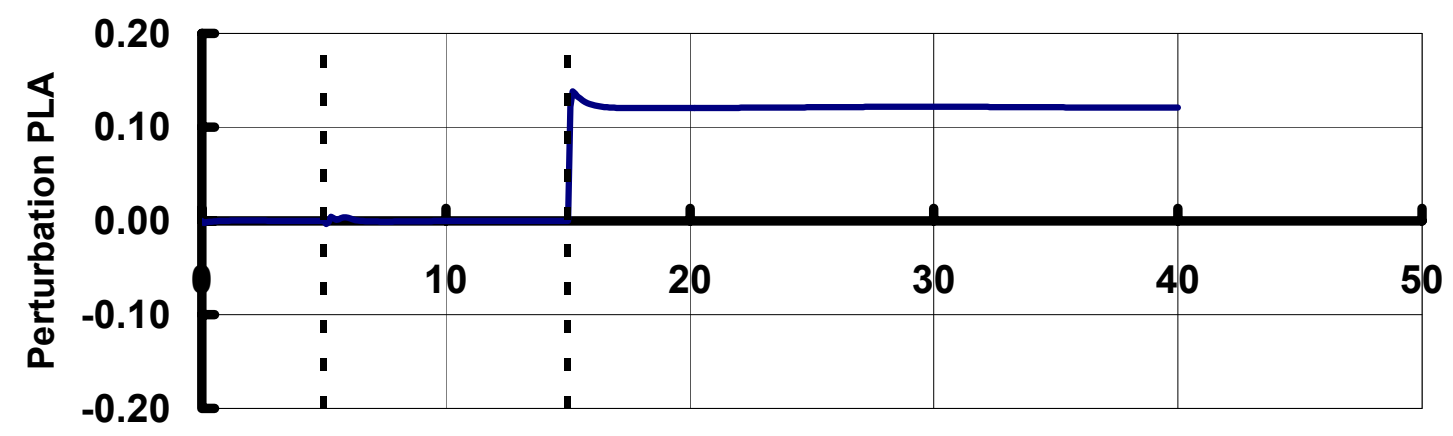

Time, Seconds

Figure 47 (a): Case 11 time history 

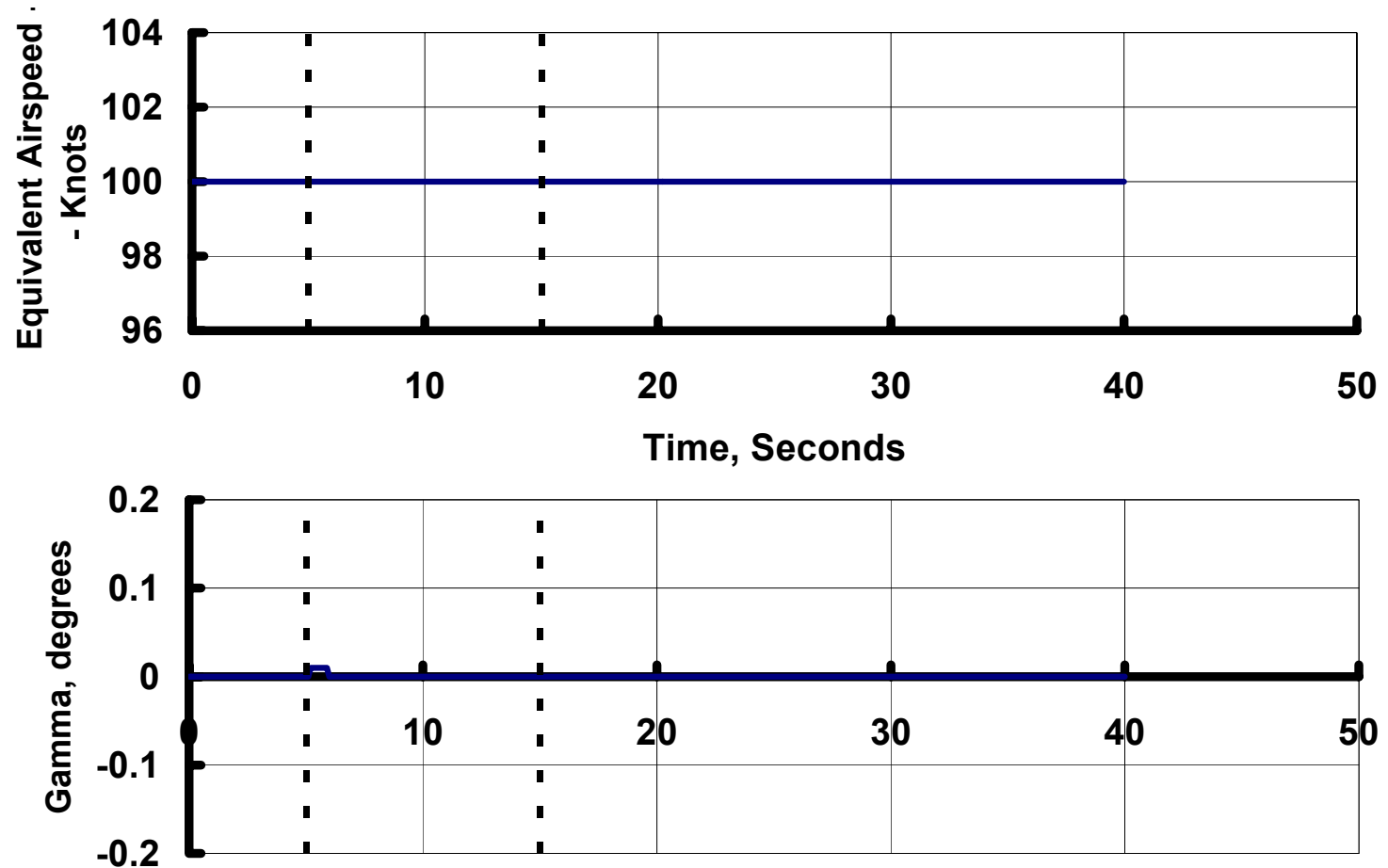

Time, Seconds

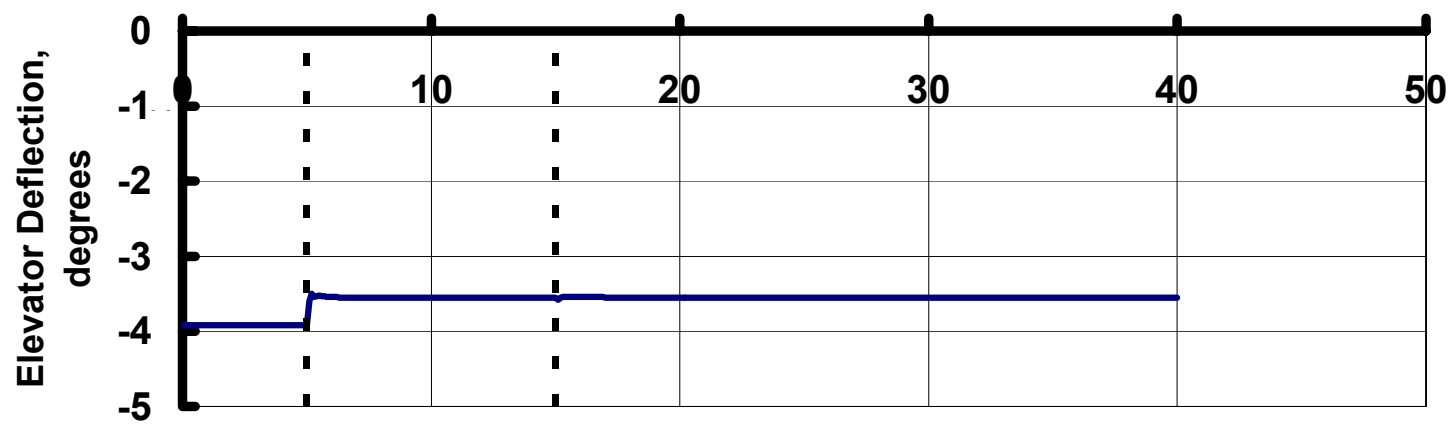

Time, Seconds

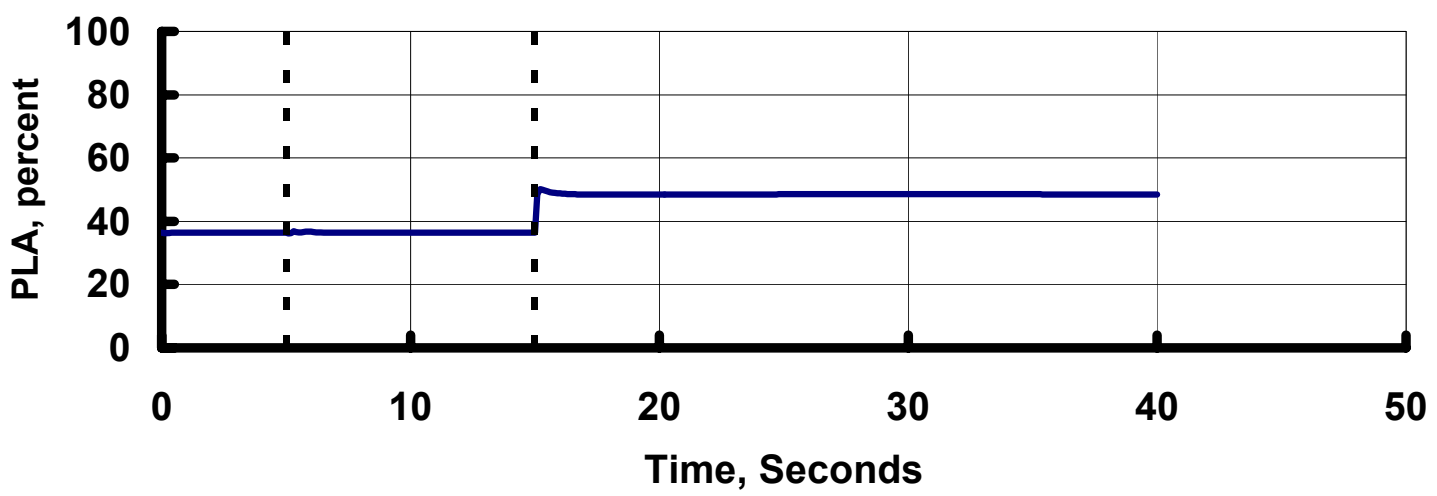

Figure 47 (b): Case 11 time history 


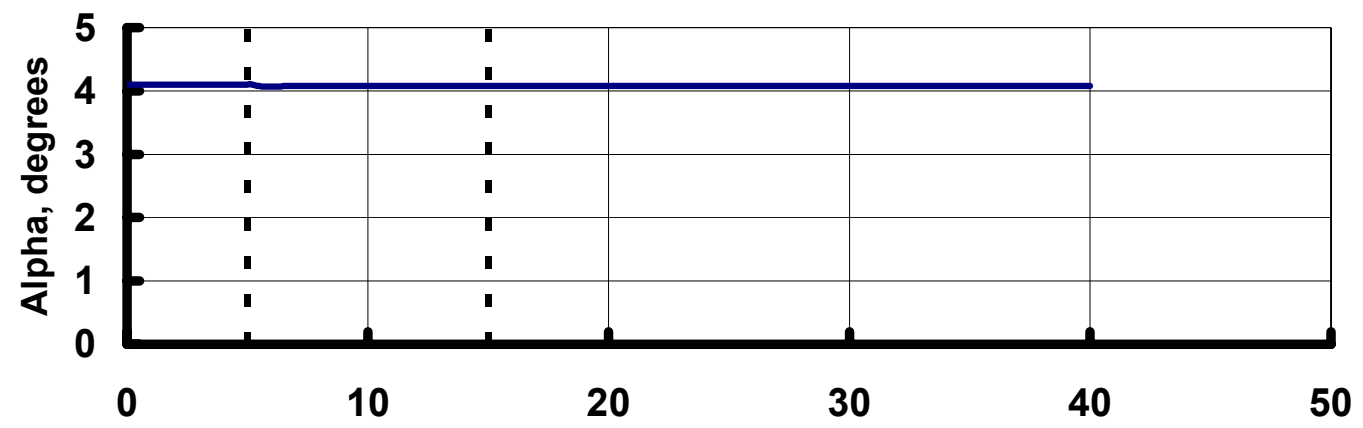

Time, Seconds

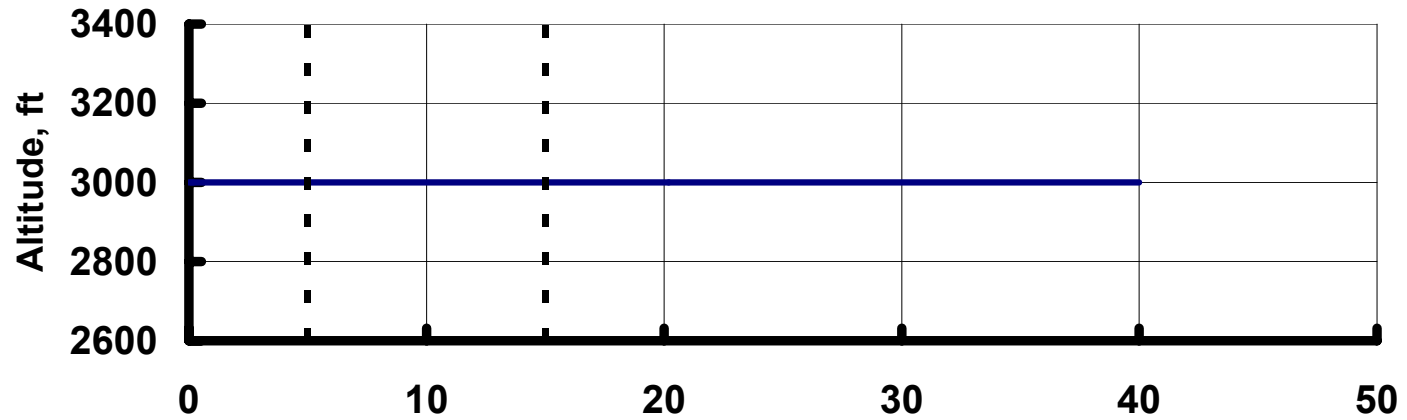

Time, Seconds

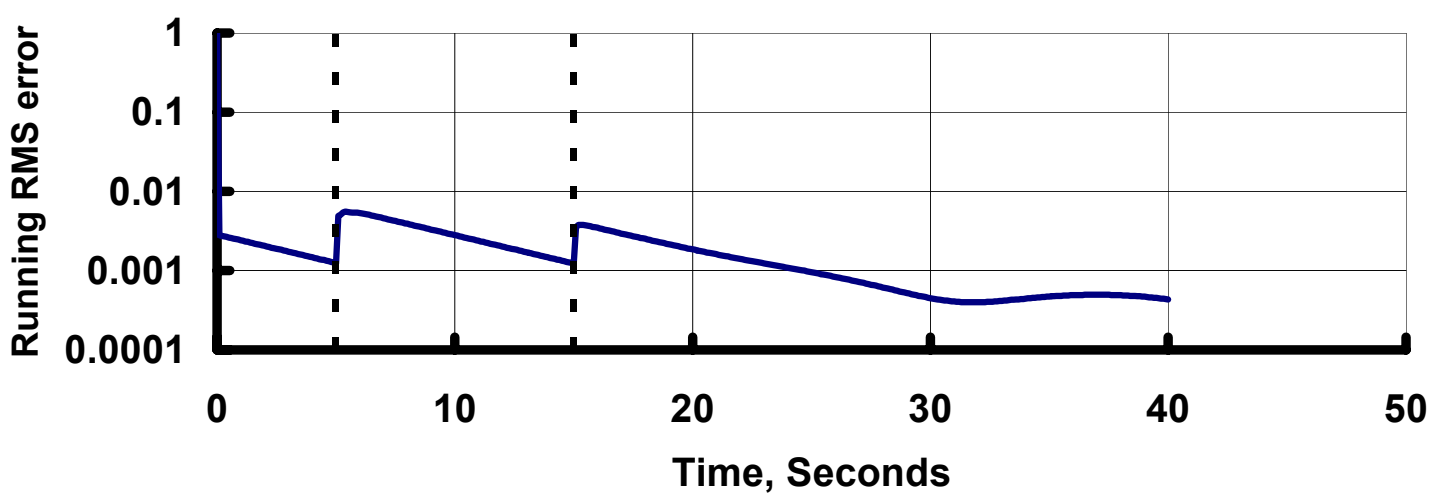

Figure 47 (c): Case 11 time history 


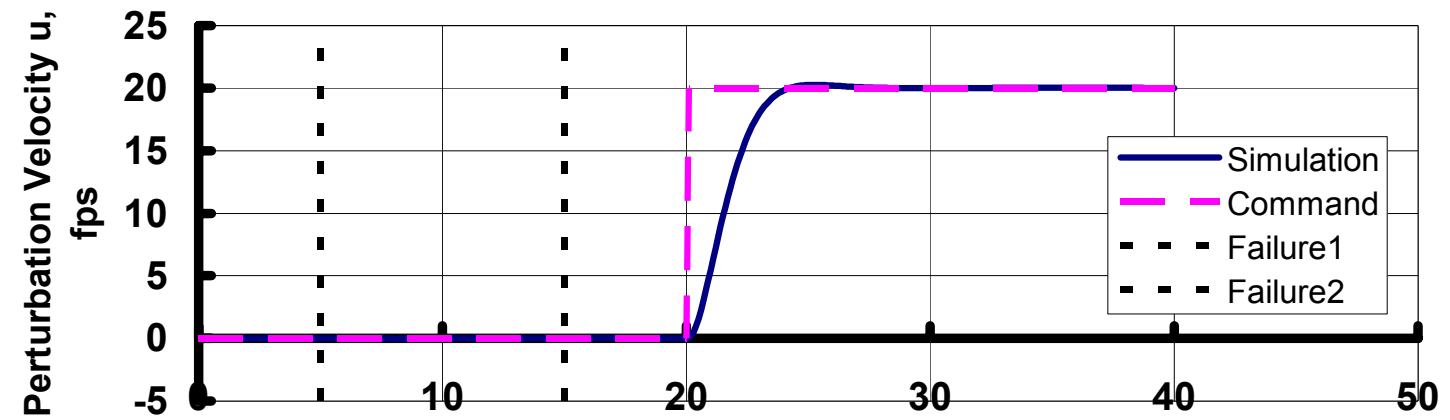

Time, Seconds

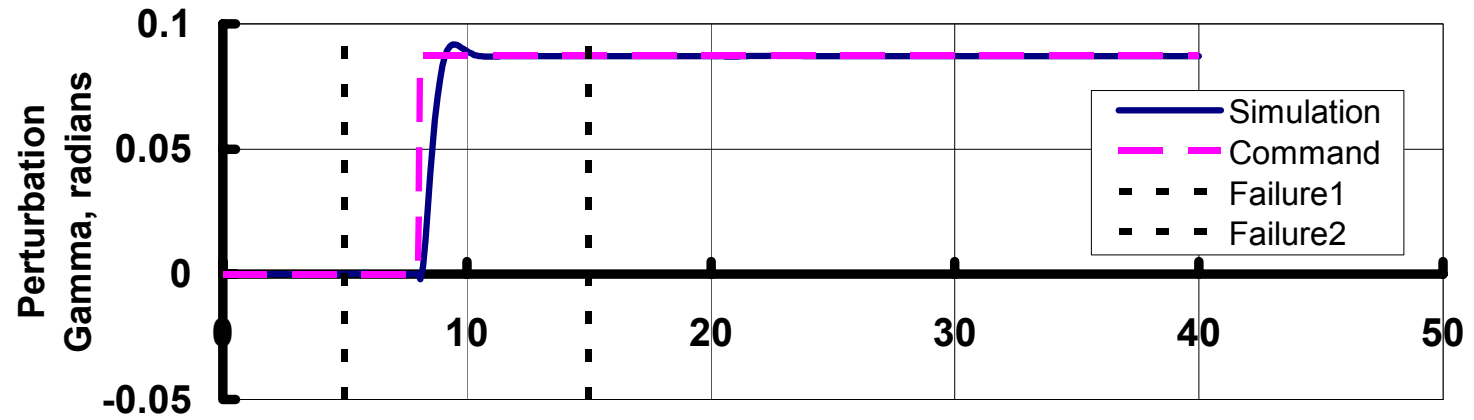

Time, Seconds

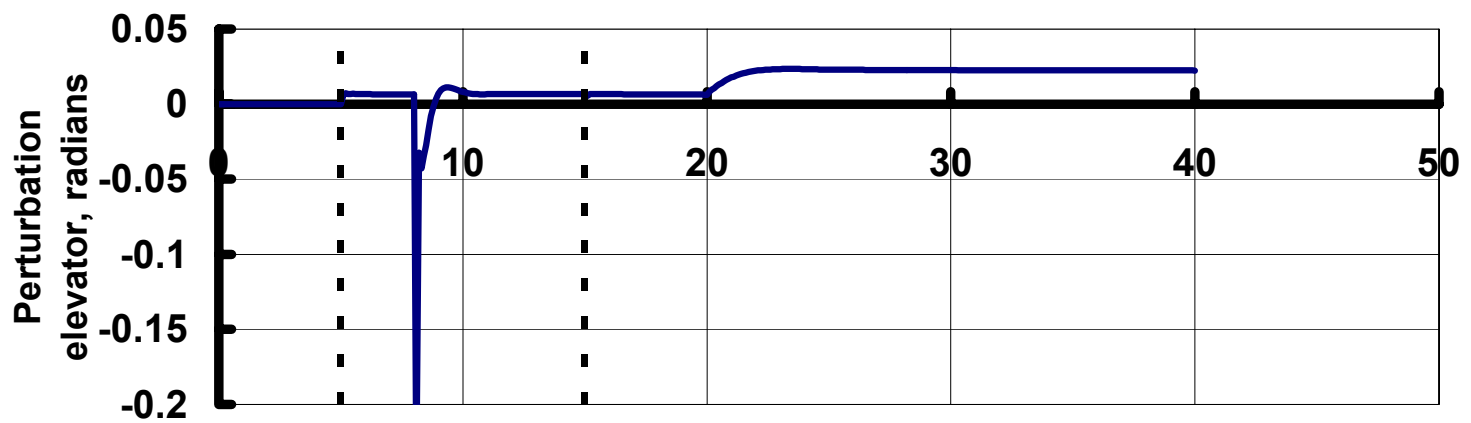

Time, Seconds

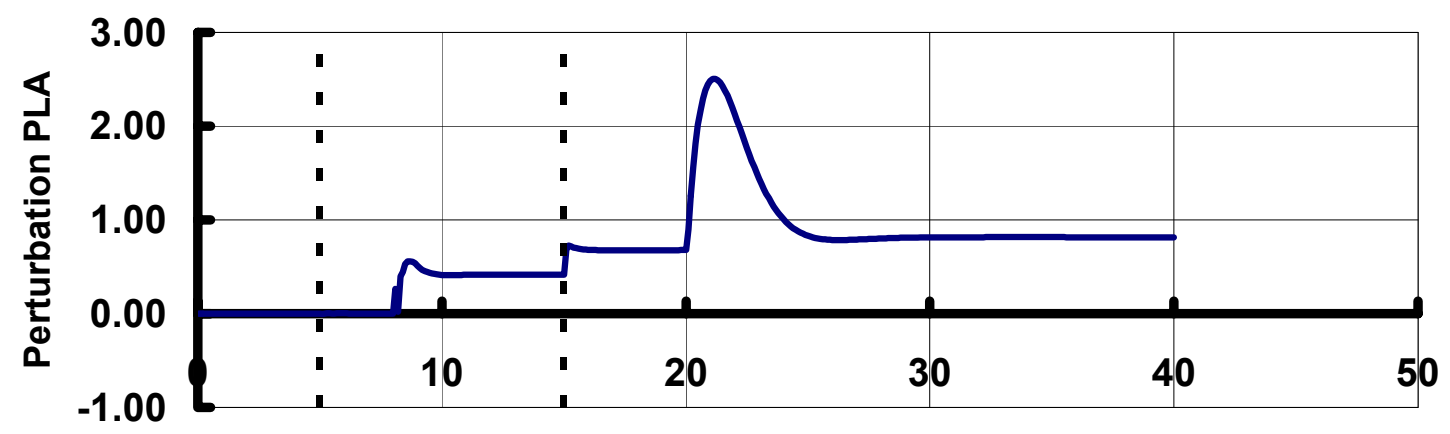

Time, Seconds

Figure 48 (a): Case 12 time history 

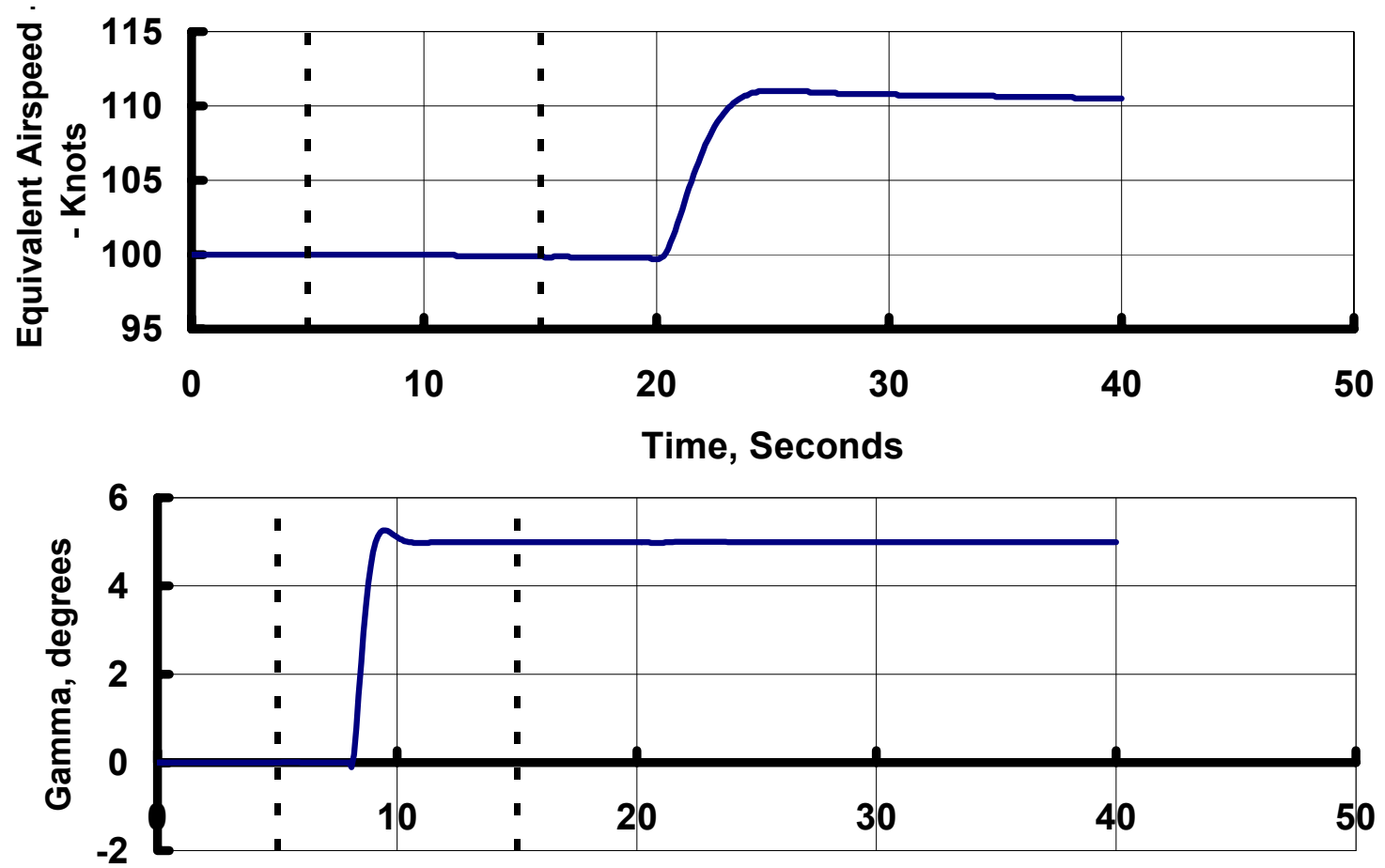

Time, Seconds

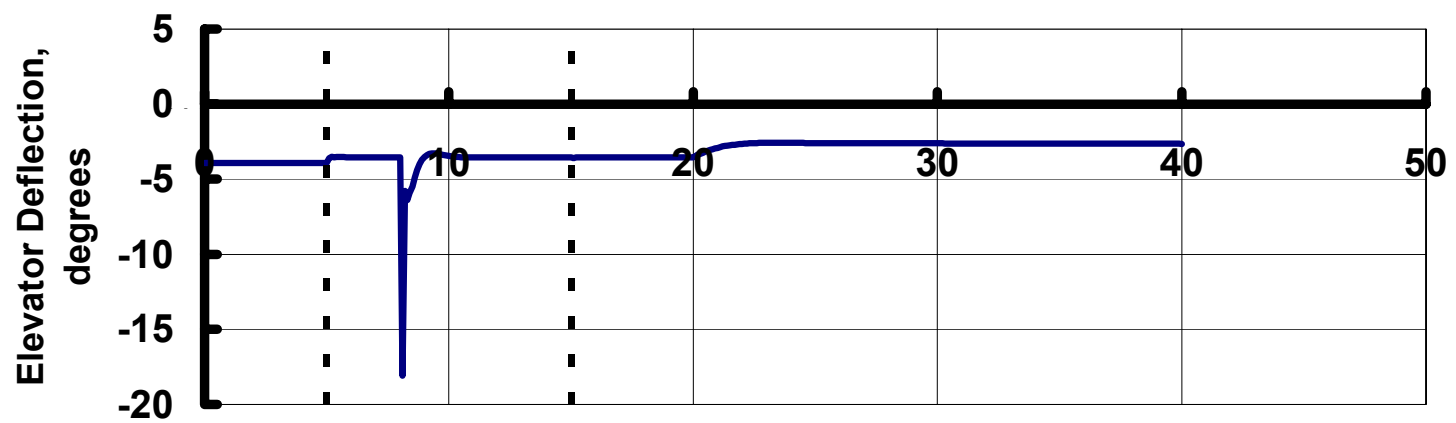

Time, Seconds

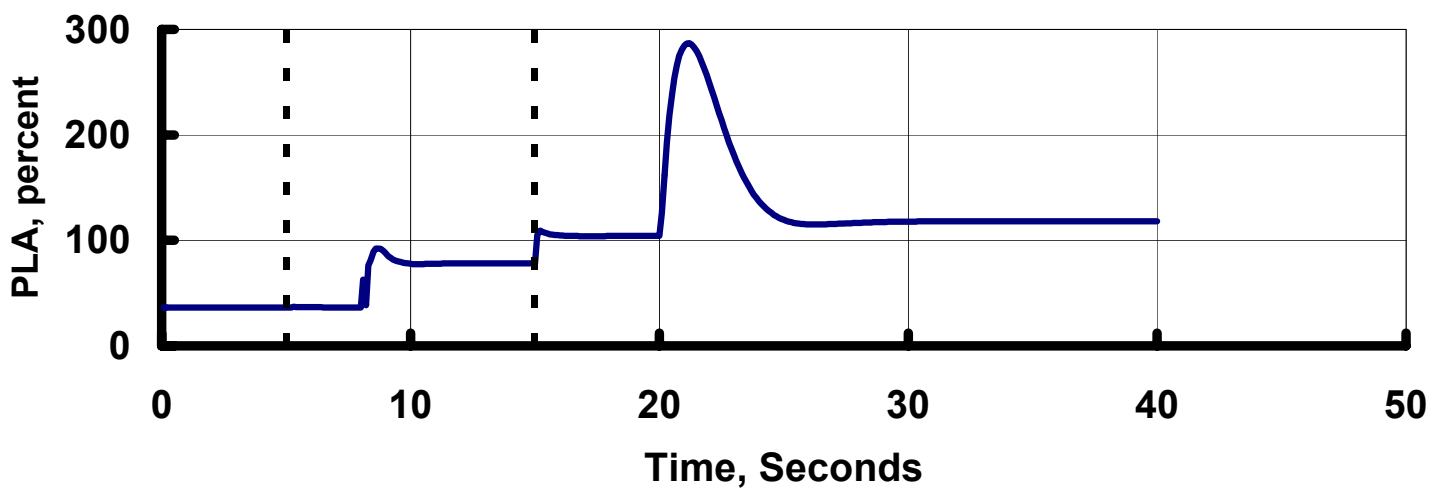

Figure 48 (b): Case 12 time history 


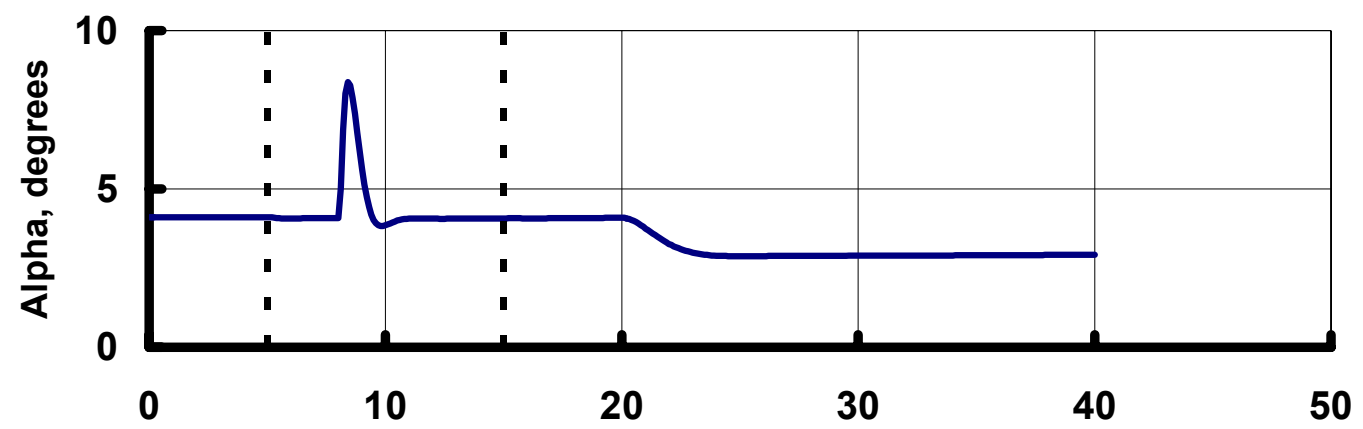

Time, Seconds

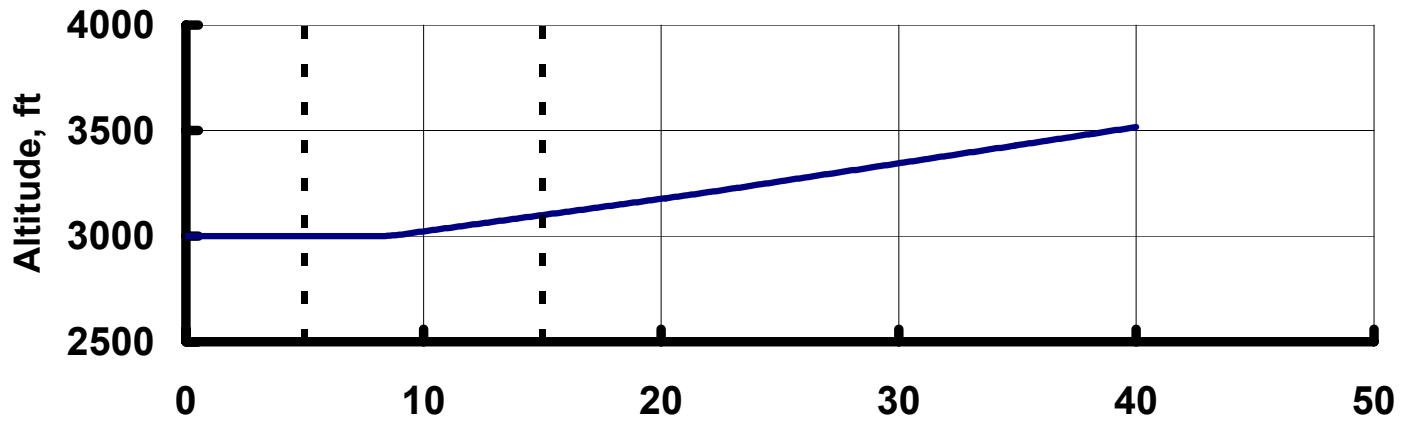

Time, Seconds

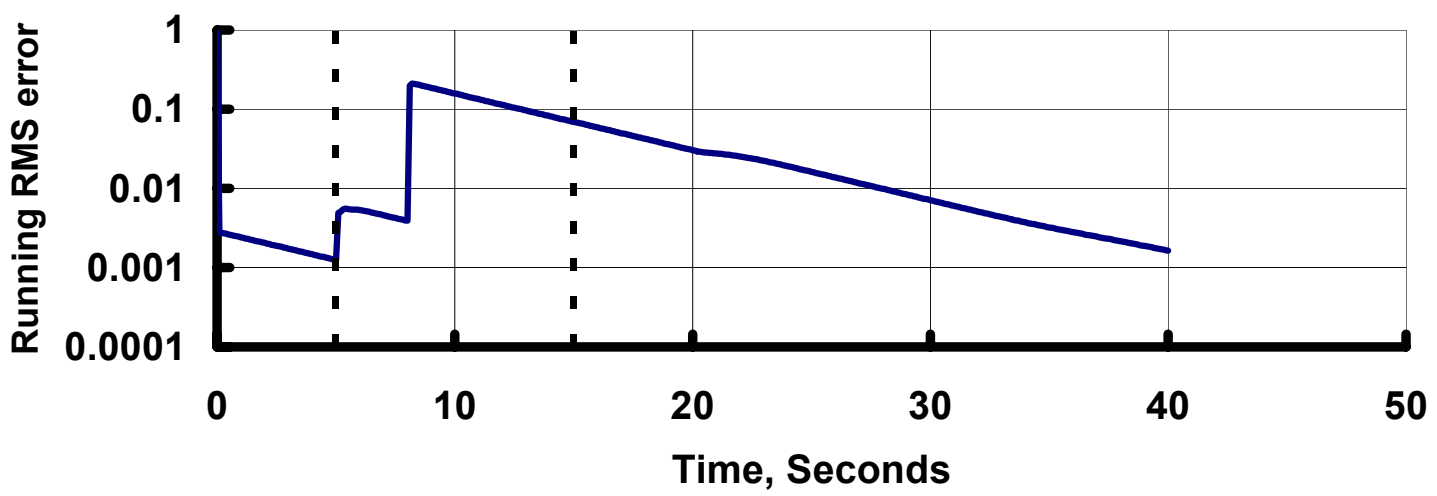

Figure 48 (c): Case 12 time history 


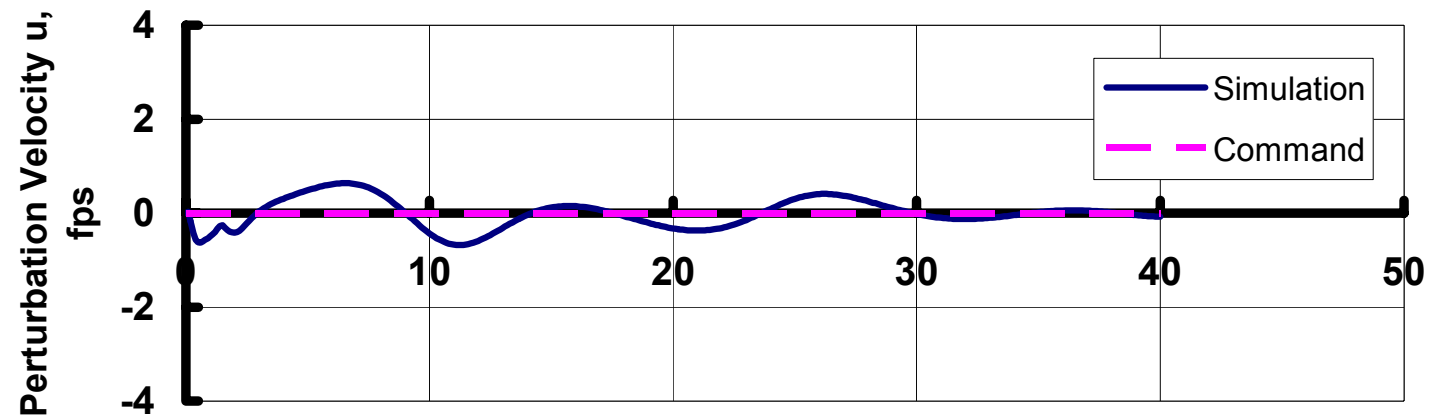

Time, Seconds

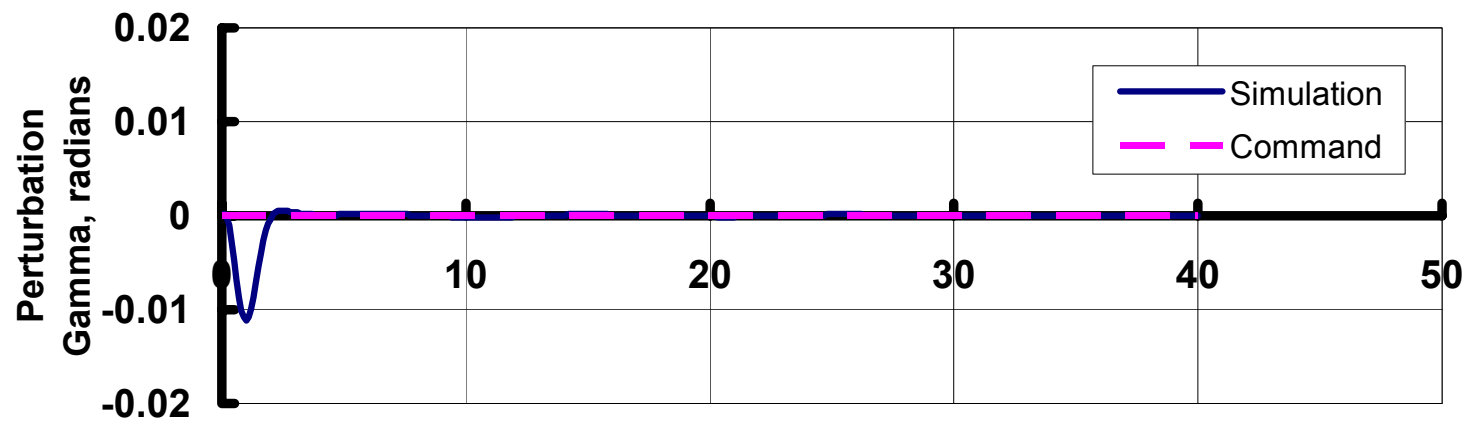

Time, Seconds

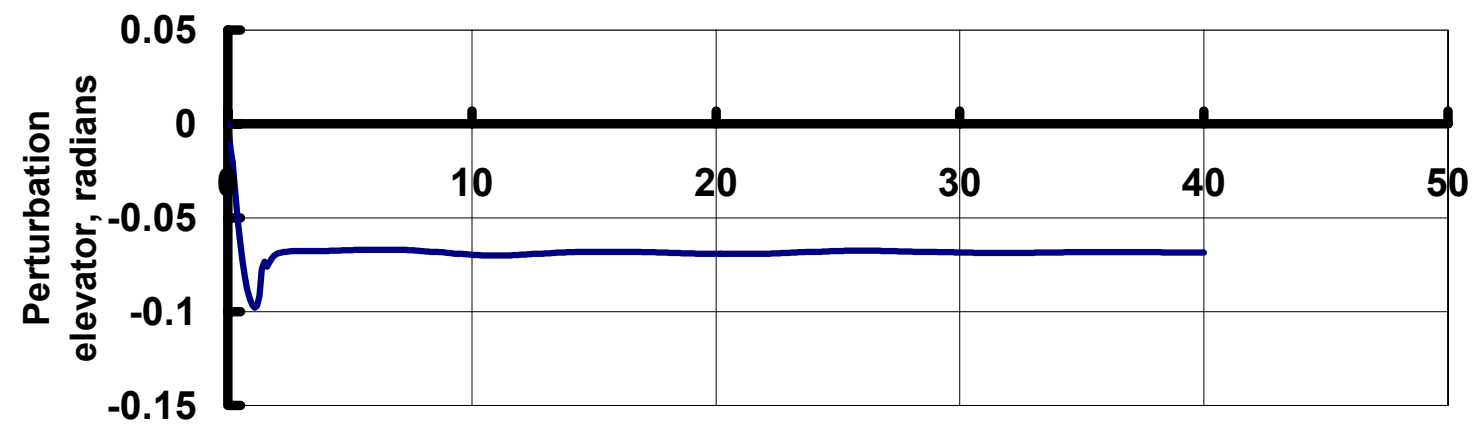

Time, Seconds

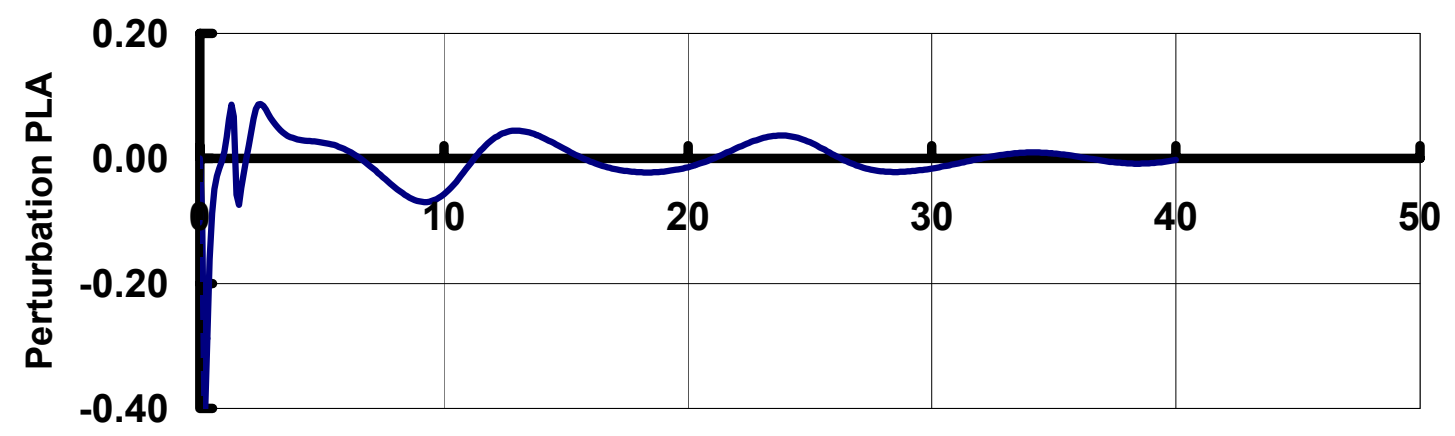

Time, Seconds

Figure 49 (a): Case 13 time history 

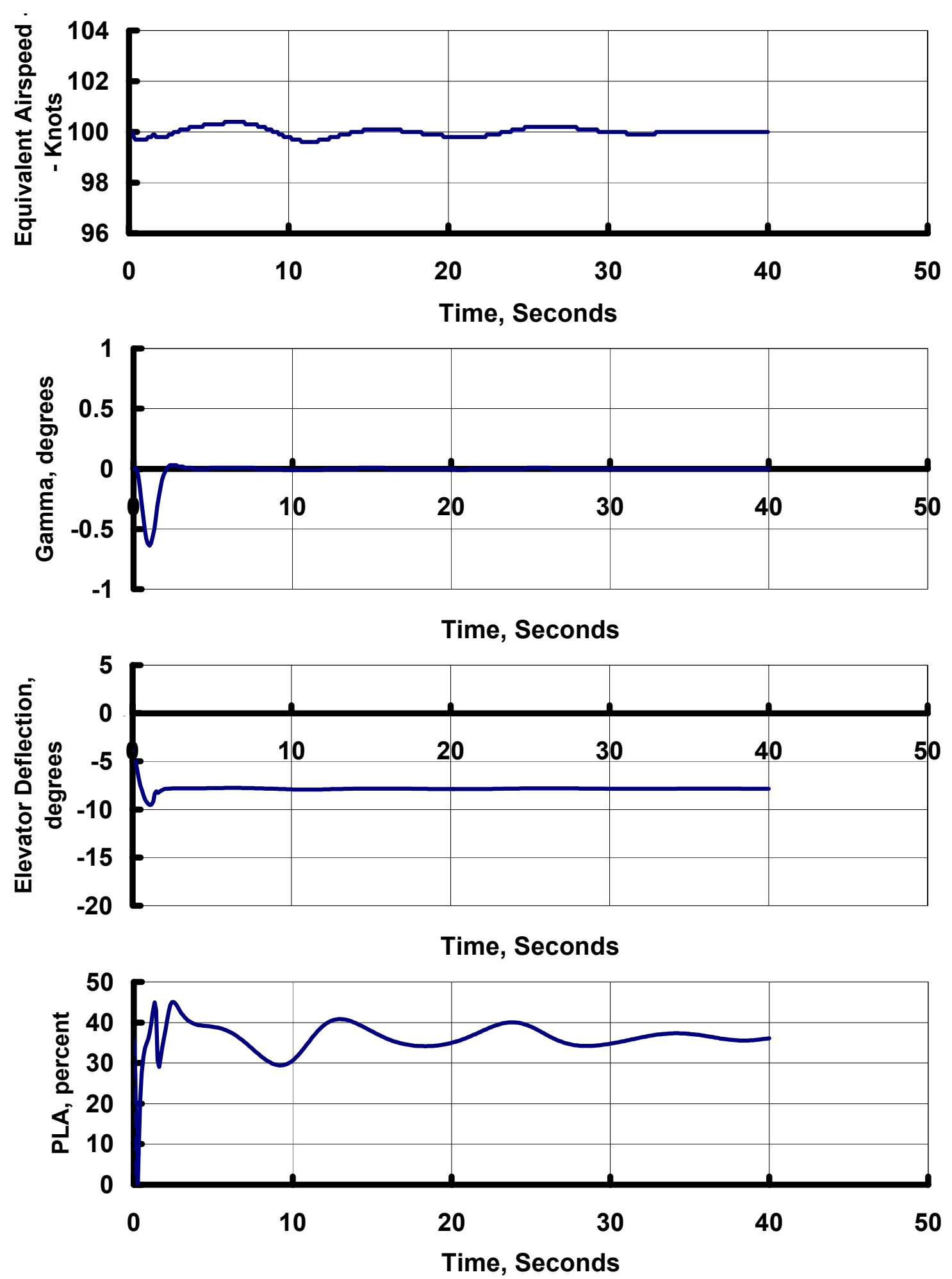

Figure 49 (b): Case 13 time history 


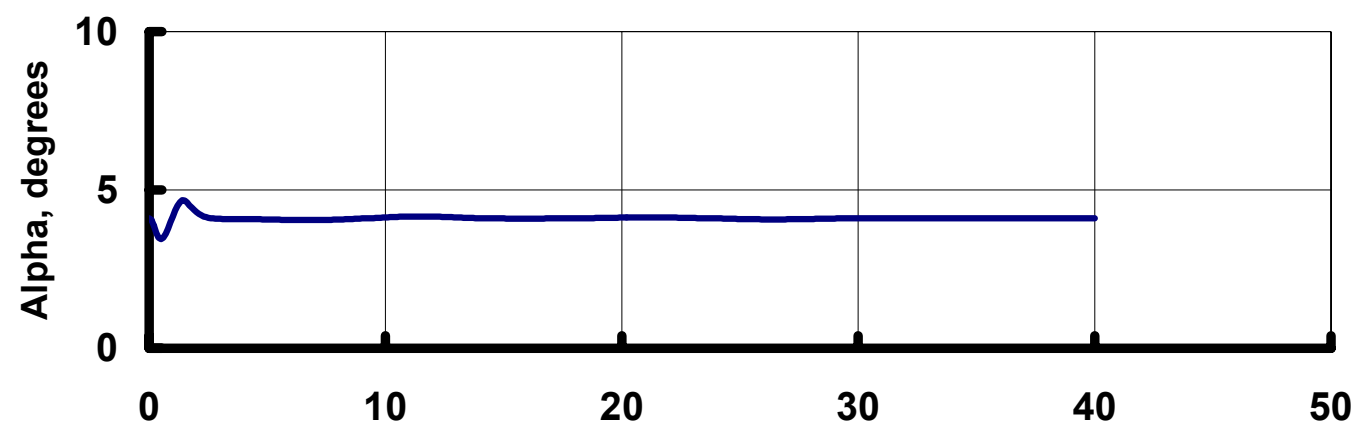

Time, Seconds

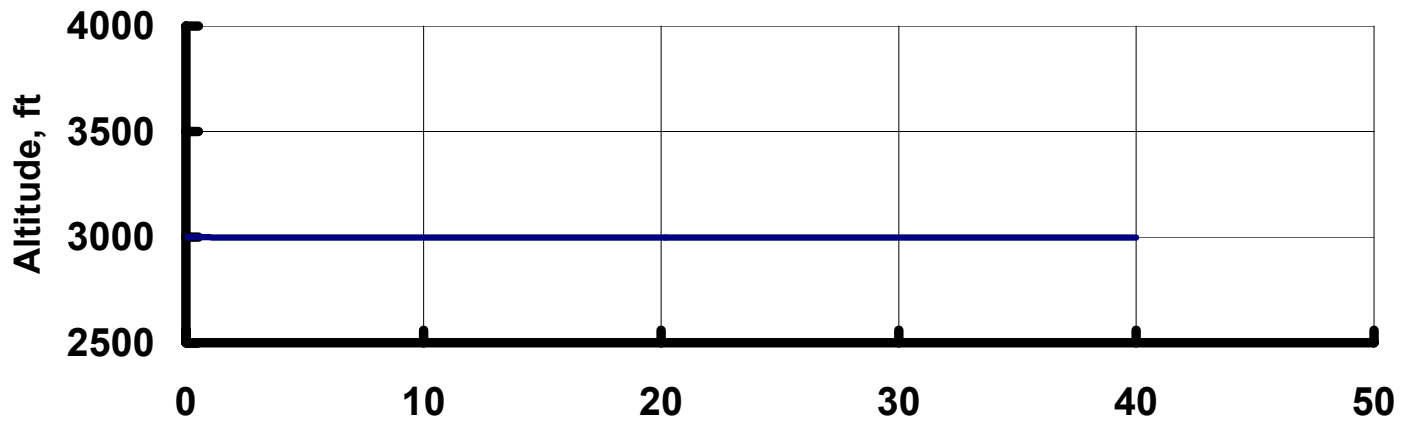

Time, Seconds

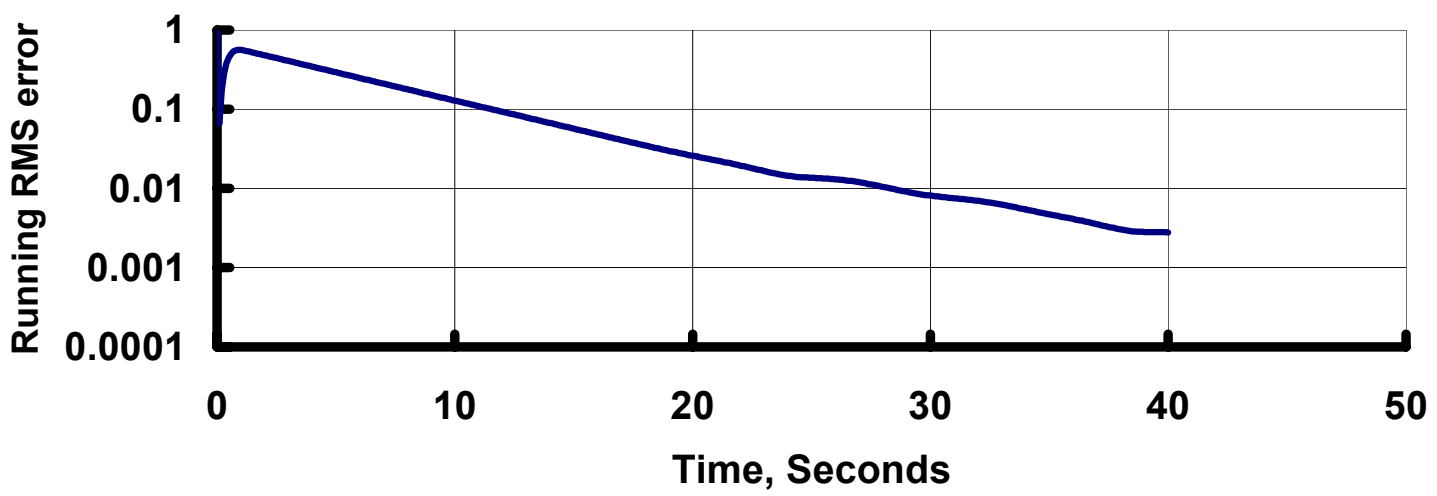

Figure 49 (c): Case 13 time history 


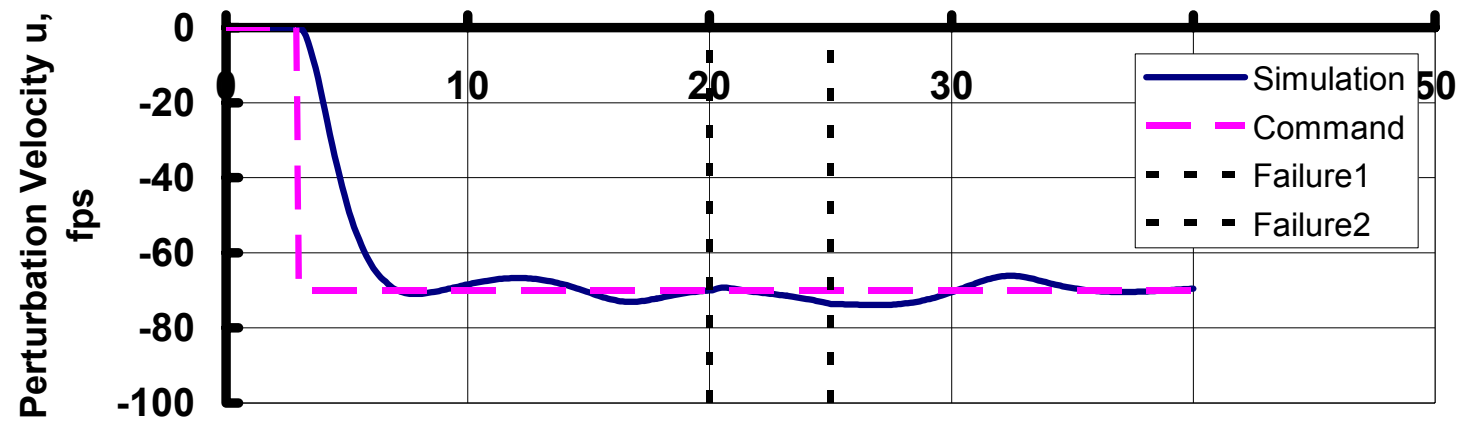

Time, Seconds

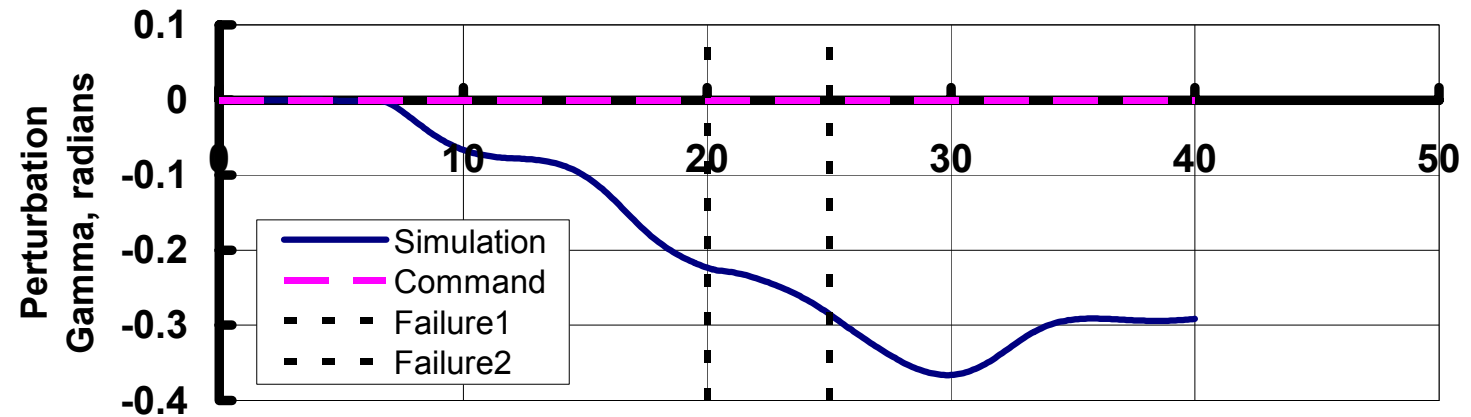

Time, Seconds

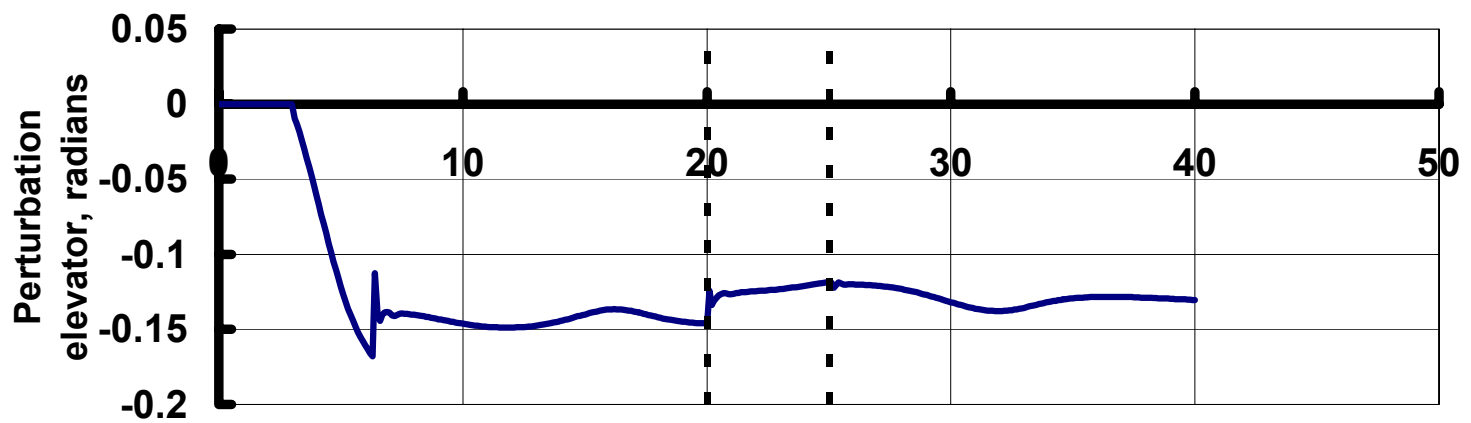

Time, Seconds

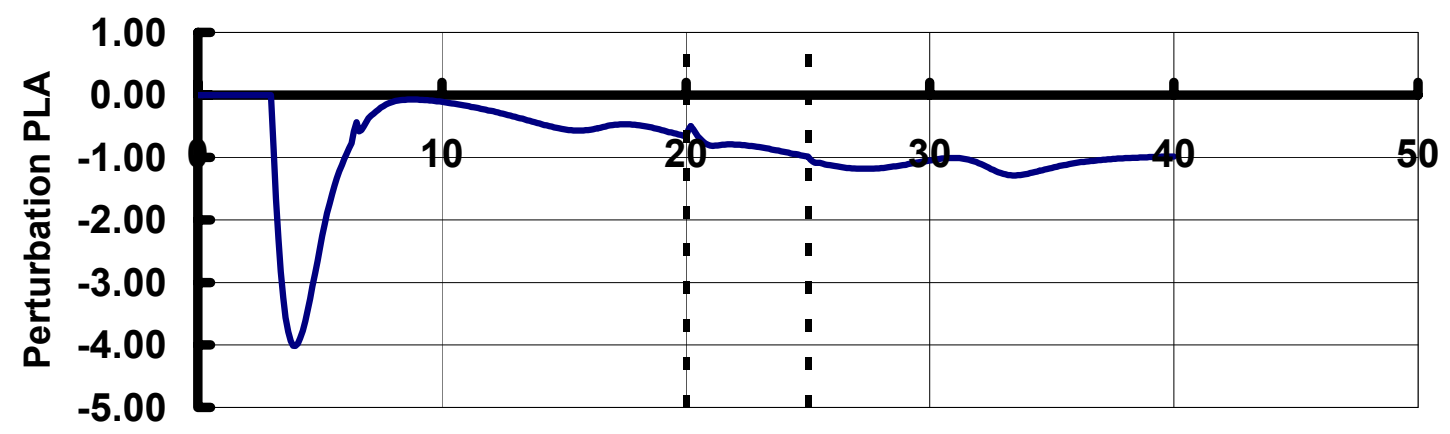

Time, Seconds

Figure 50 (a): Case 14 time history 

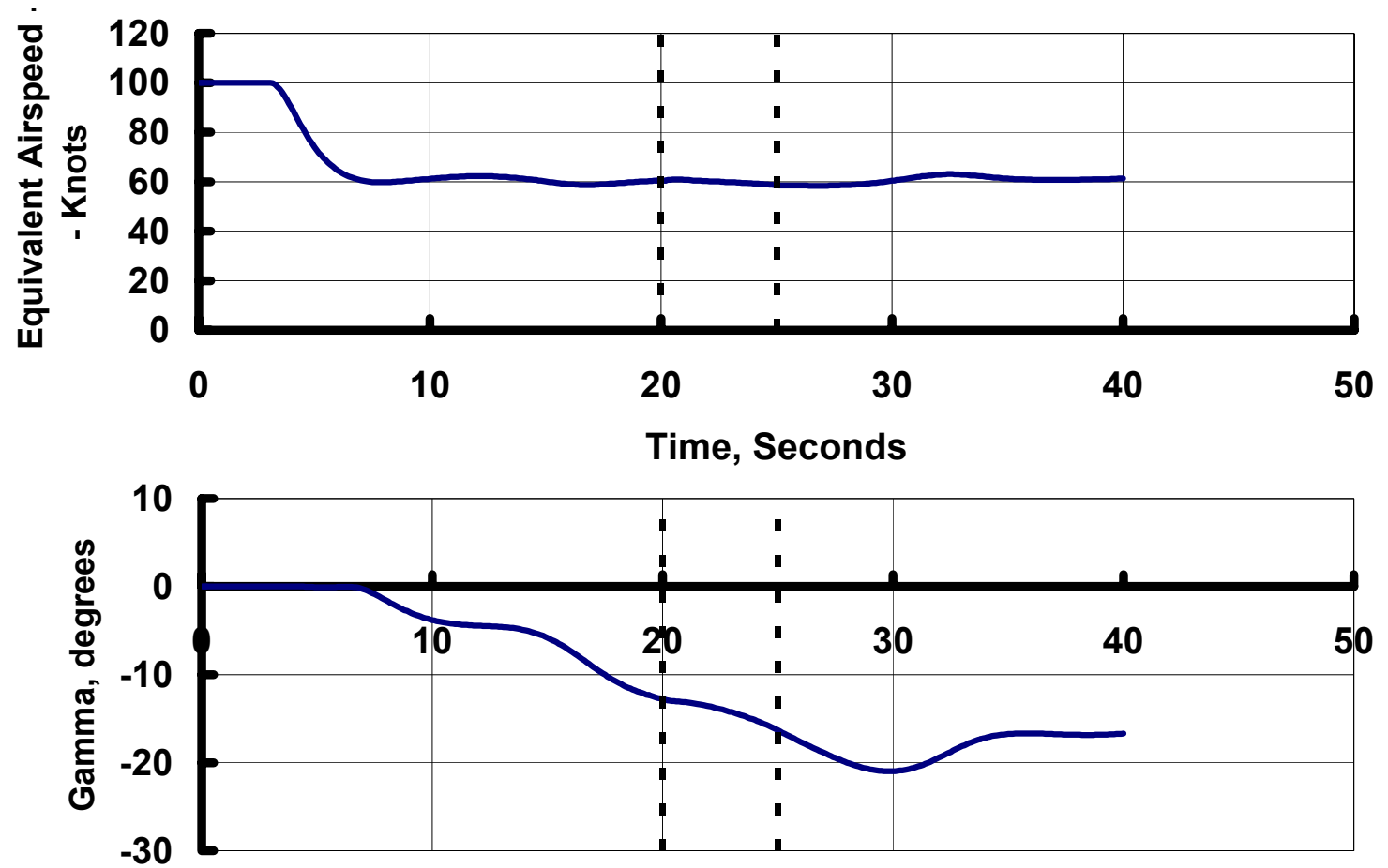

Time, Seconds

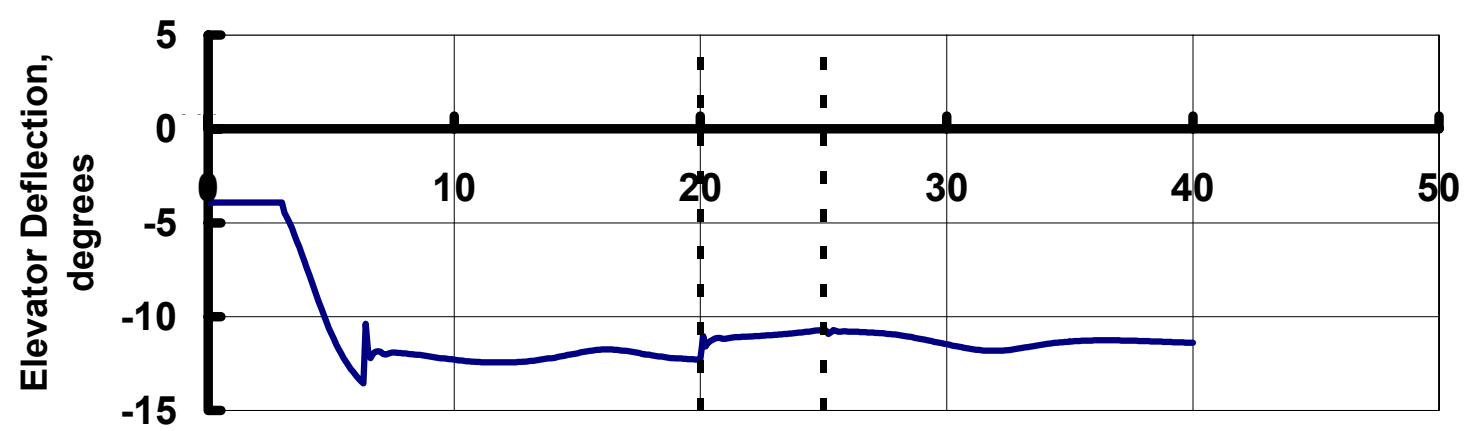

Time, Seconds

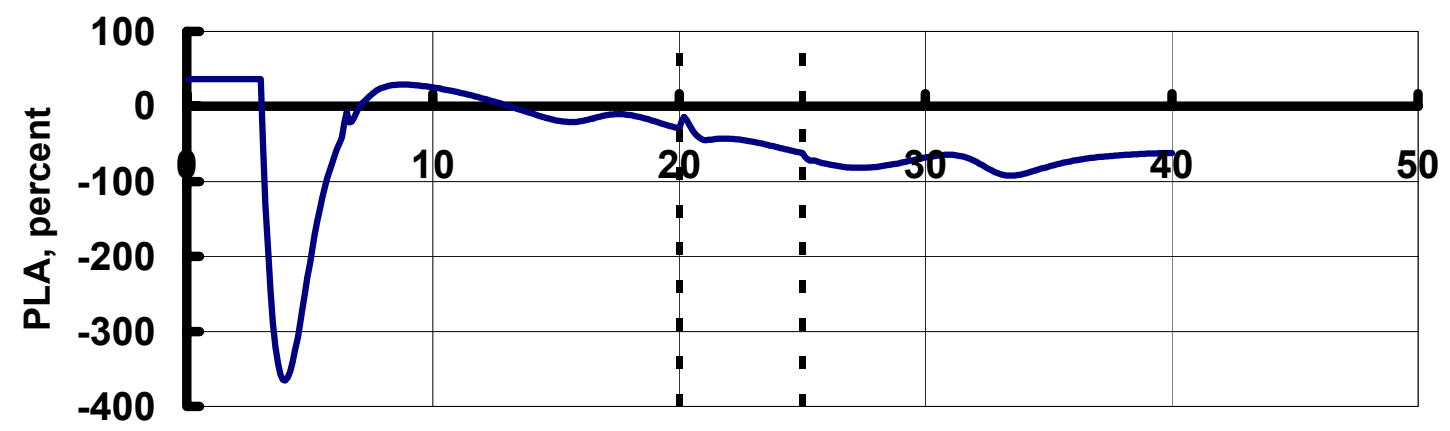

Time, Seconds

Figure 50 (b): Case 14 time history 


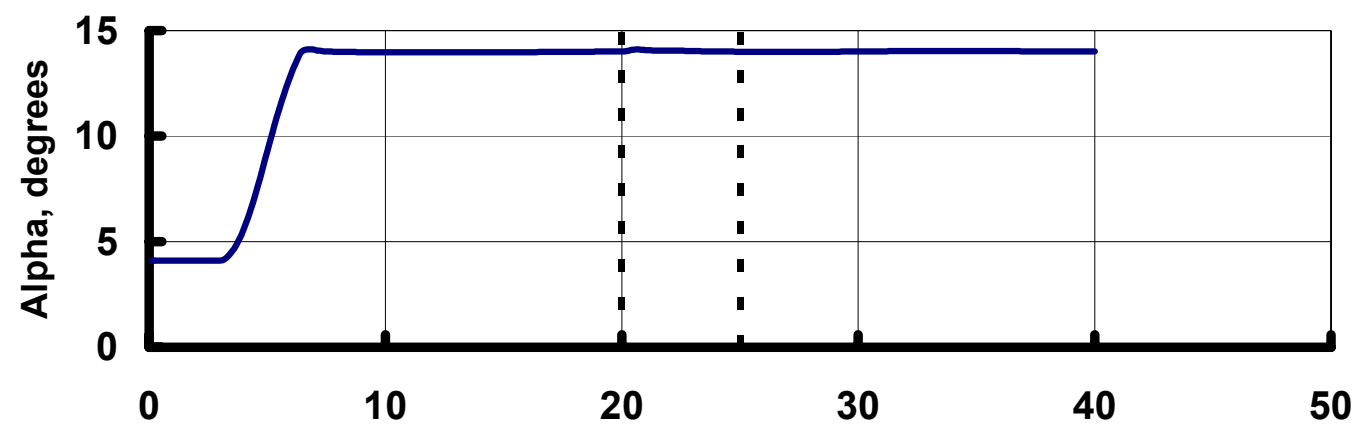

Time, Seconds

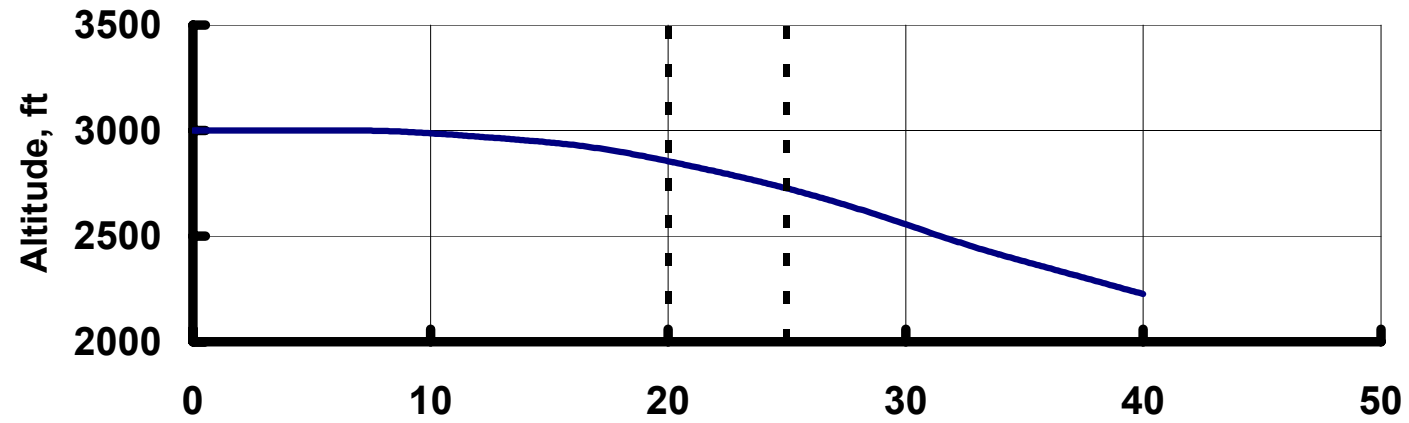

Time, Seconds

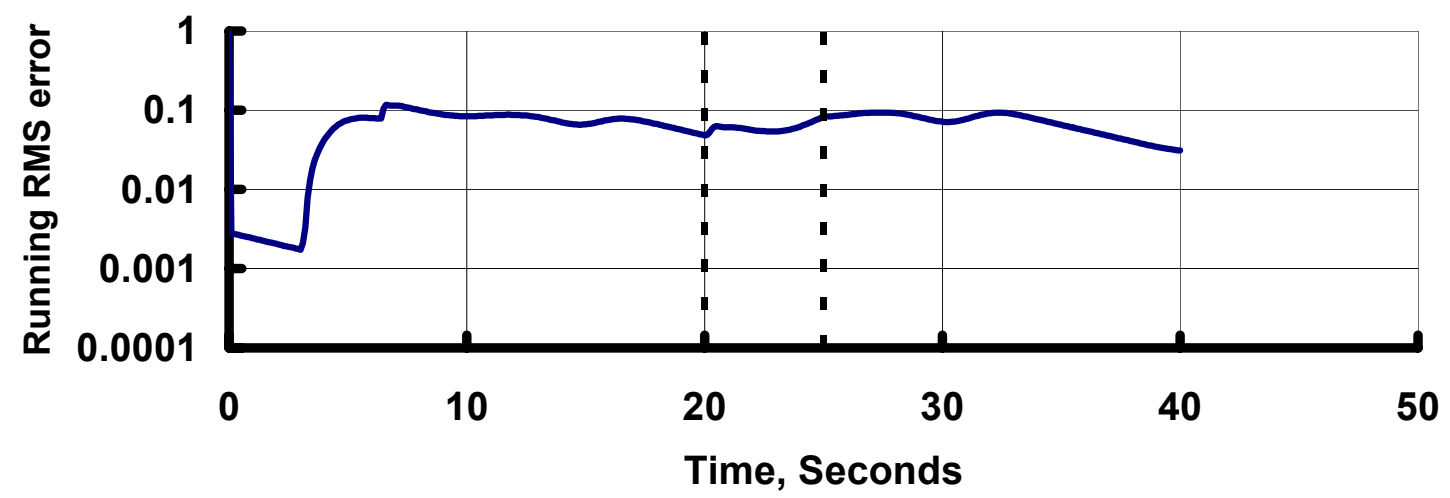

Figure 50 (c): Case 14 time history 
Case 15 (Figure 51) presents a long duration maneuver to illustrate the time stability of the controller. This case is also the baseline for the cases that follow. In Case 15, the aircraft is in its nominal state with the adaptive neural network compensation on. The commanded maneuver involves an 800 second sine wave command on airspeed, with an amplitude of ten feet/sec, followed by 300 seconds of a square wave command on flight-path angle, with an amplitude of .04 radians ( 2.3 degrees). The nominal airplane shows good tracking behavior and crisp response to commands. The simulation speed and flight-path angle can be seen to virtually overlay the commands. As noted in prior cases, the RMS error increases when step commands in flight-path angle are received, reflecting the fact that step changes were not part of the training data used to pre-train the neural network.

Case 16 (Figure 52) is the same command set as Case 15. However, in Case 16 the airplane loses all static stability $\left(\mathrm{C}_{\mathrm{M}_{\alpha}}=0\right)$ at 400 seconds. In spite of this significant change in stability, the controller quickly adapts, stabilizes the neutrally stable airplane, and maintains the commanded airspeed and flight-path angle. There are no visible transients in airspeed or flightpath angle. However, the failure is quite evident by examination of the elevator time history. Once again a significant change in the trim elevator setting is visible, as would be expected with a change in $\mathrm{C}_{\mathrm{M}_{\alpha}}$. A spike in learning occurs subsequent to the failure, but the controller learns quickly and learning returns to a normal level within 50 seconds. A small transient in angle of attack is also evident at the time of failure. 


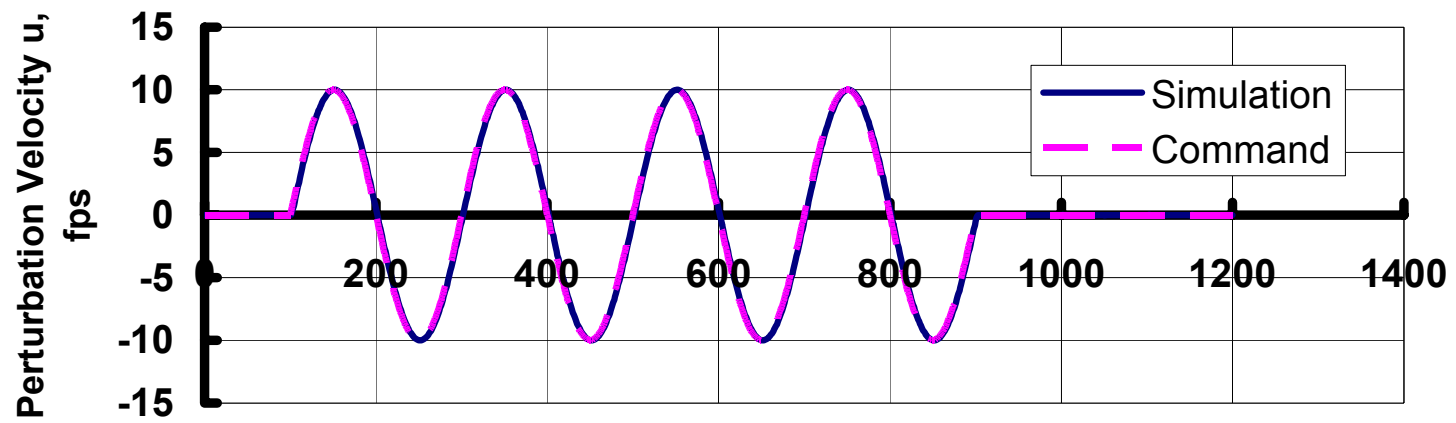

Time, Seconds

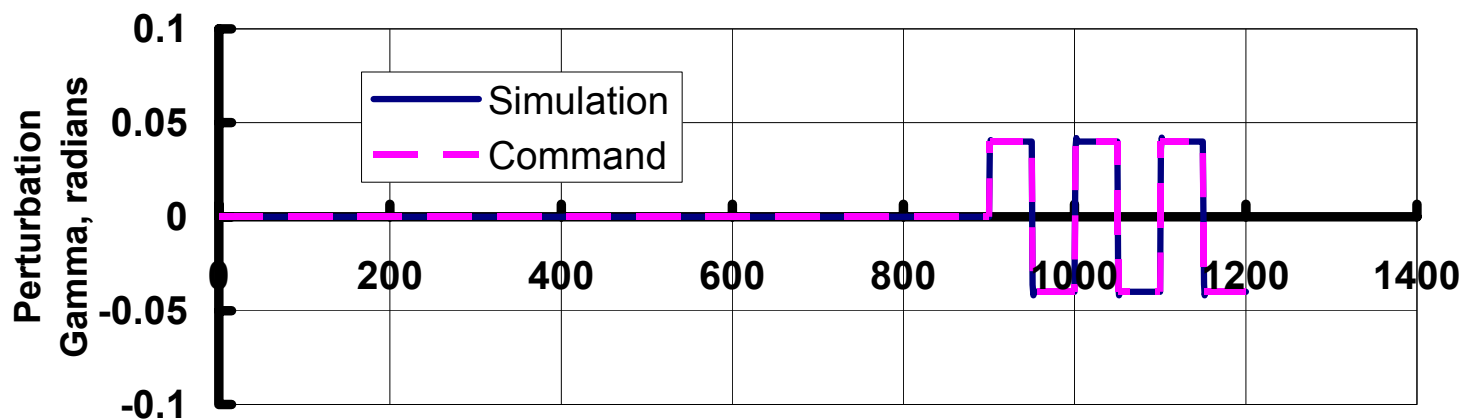

Time, Seconds

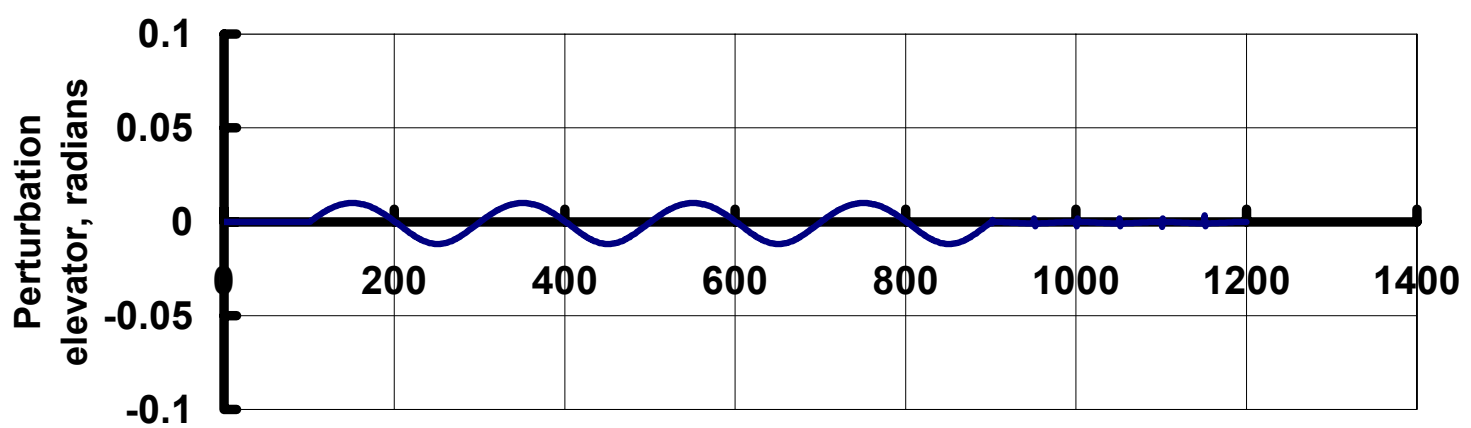

Time, Seconds

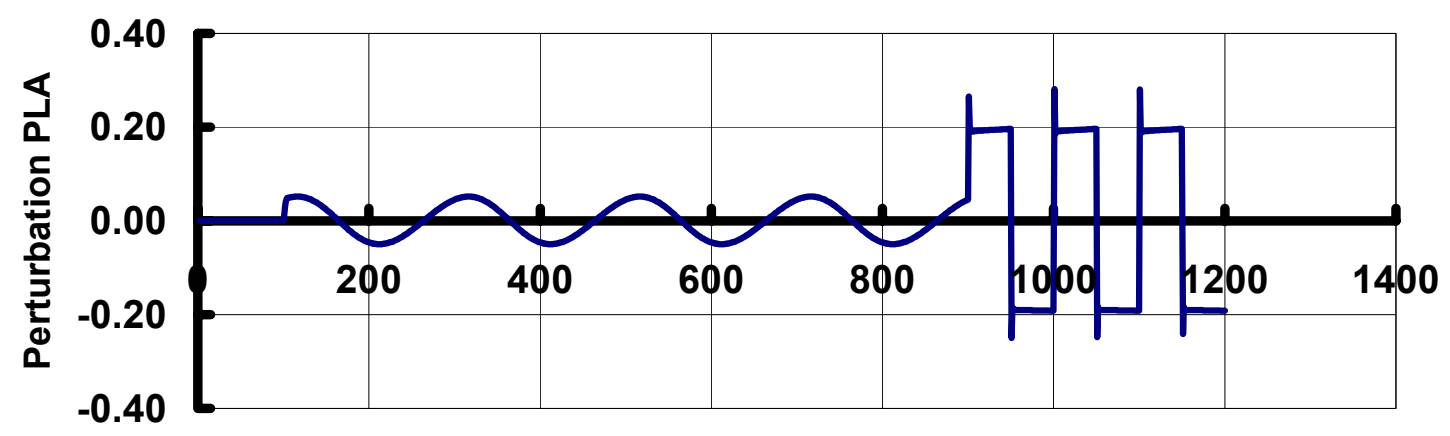

Time, Seconds

Figure 51 (a): Case 15 time history 

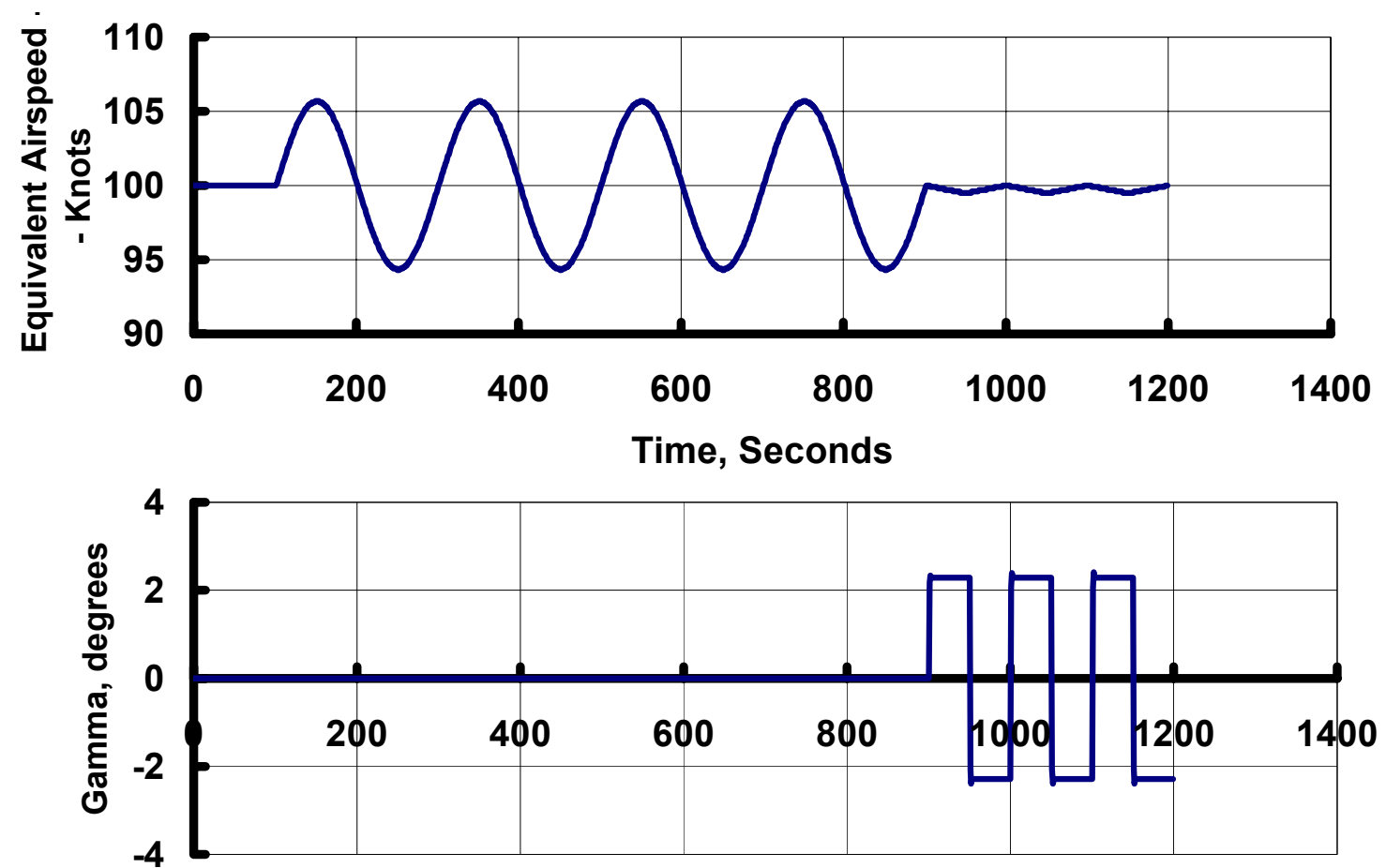

Time, Seconds

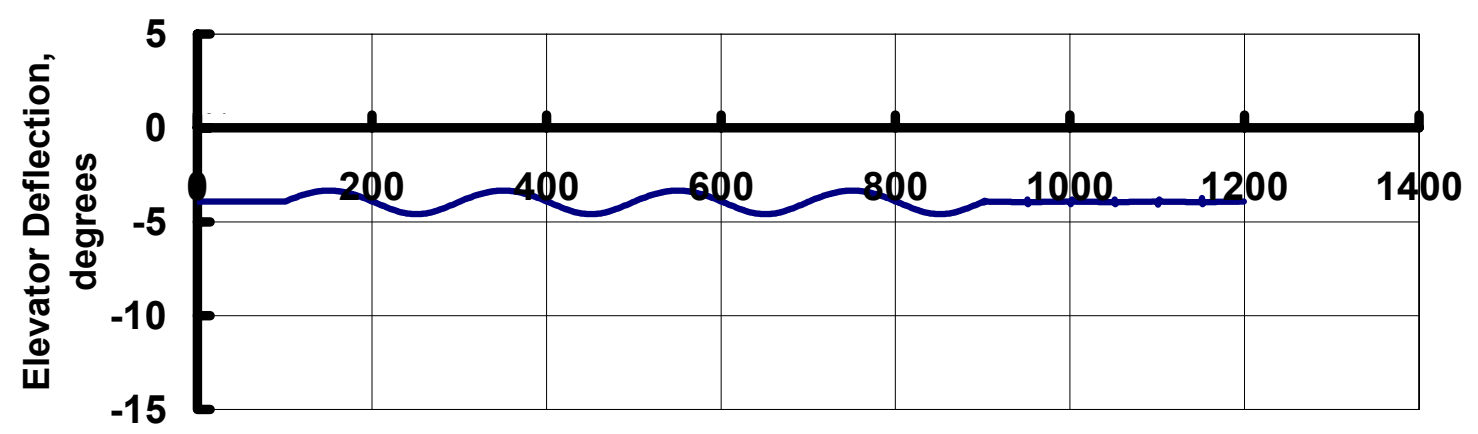

Time, Seconds

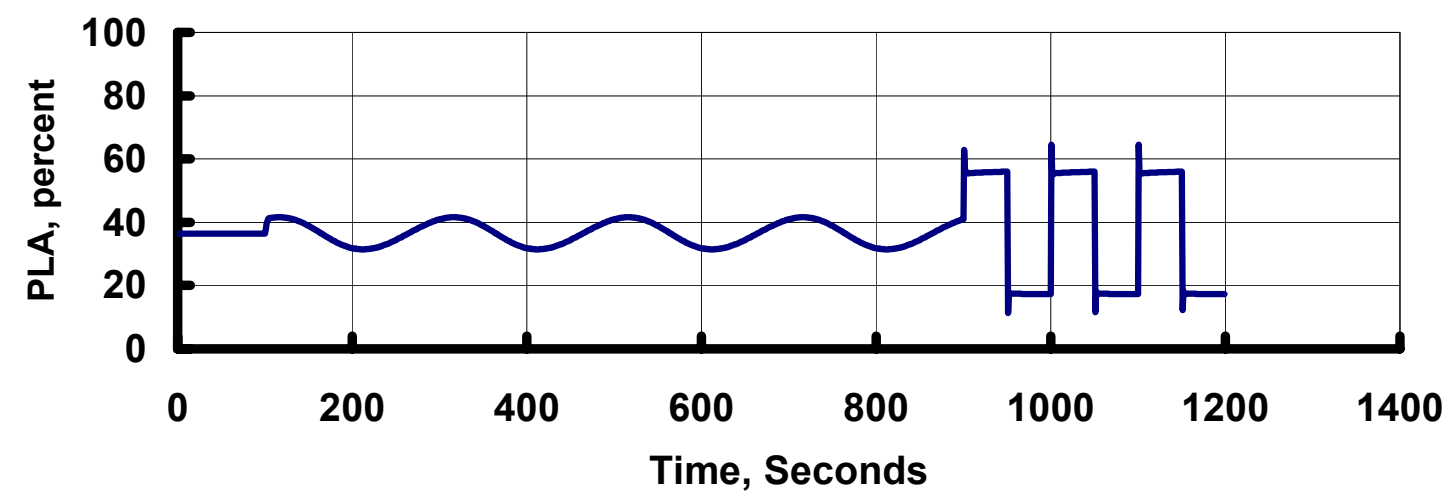

Figure 51 (b): Case 15 time history 

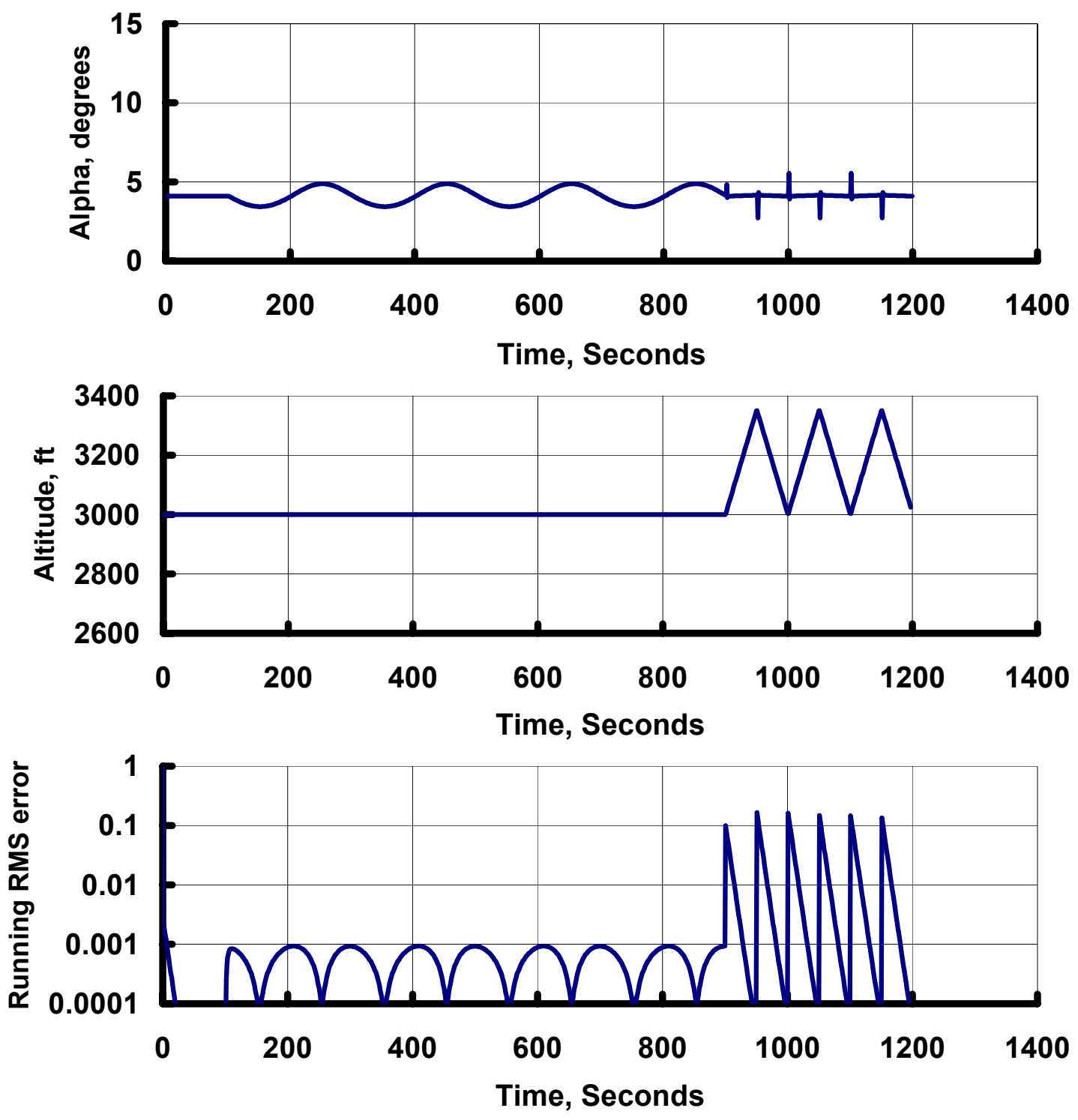

Figure 51 (c): Case 15 time history 


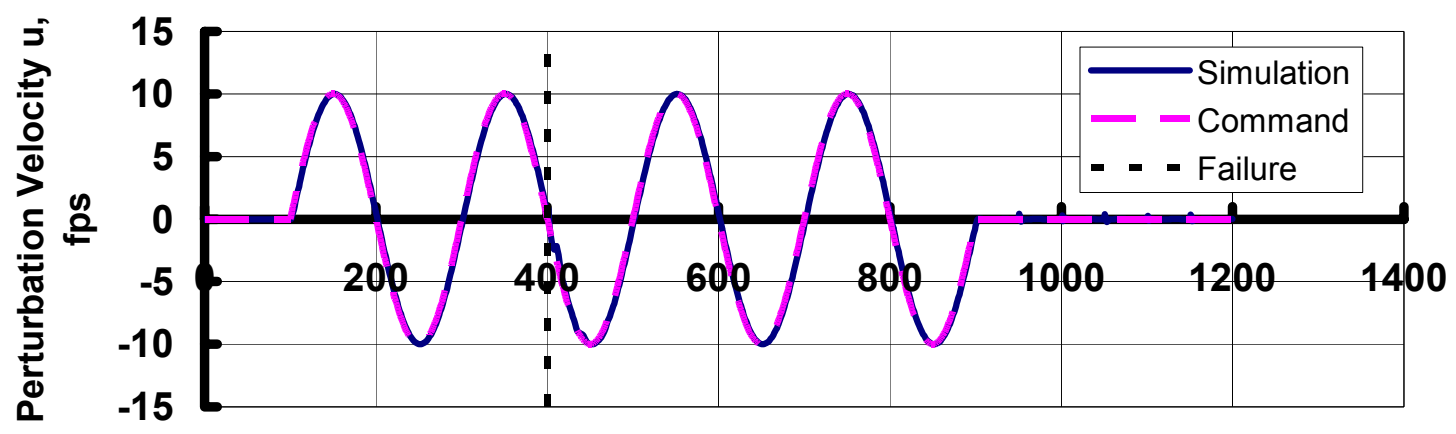

Time, Seconds

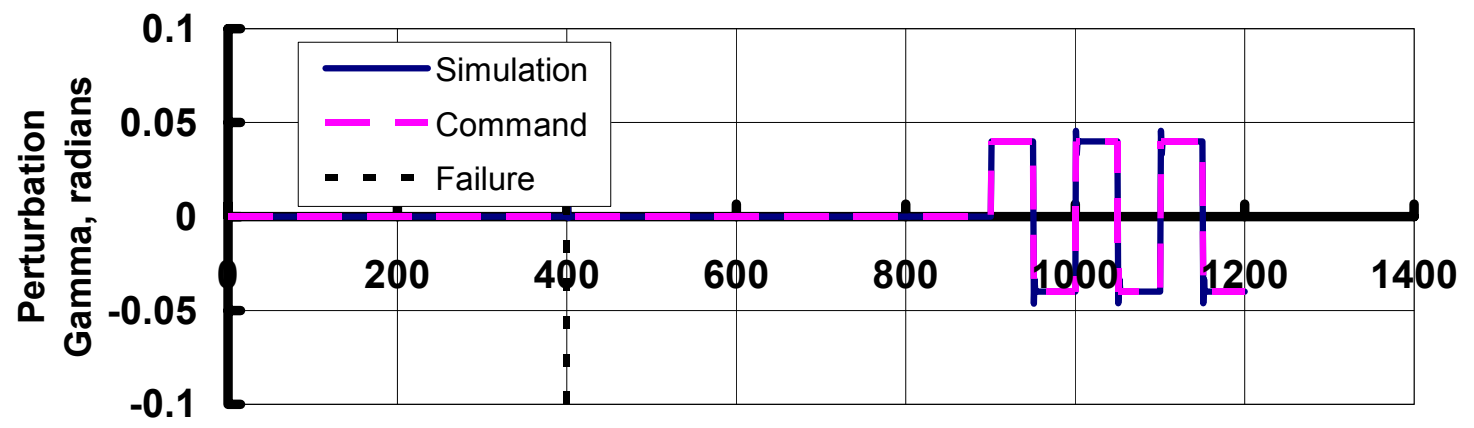

Time, Seconds

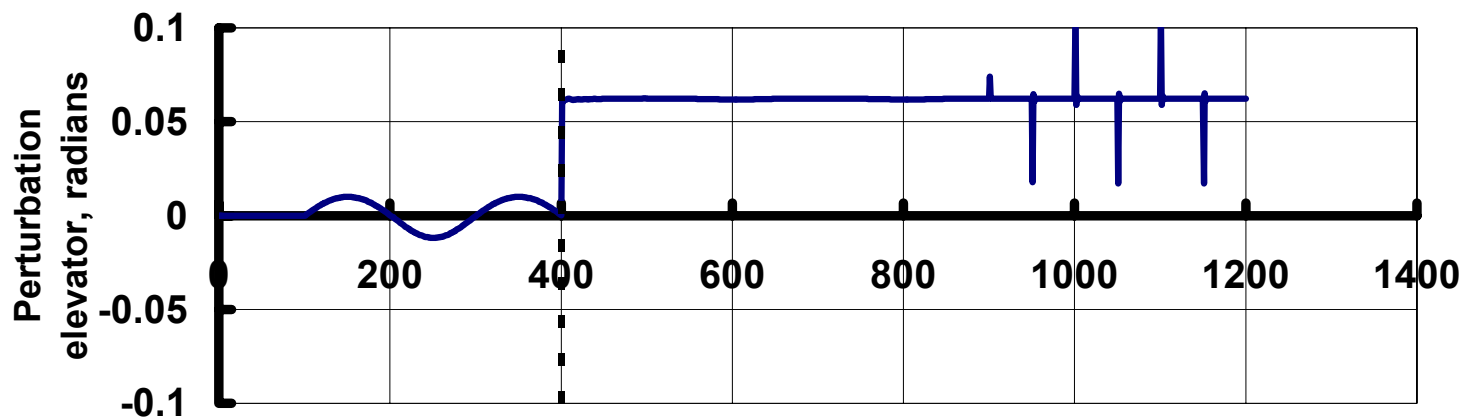

Time, Seconds

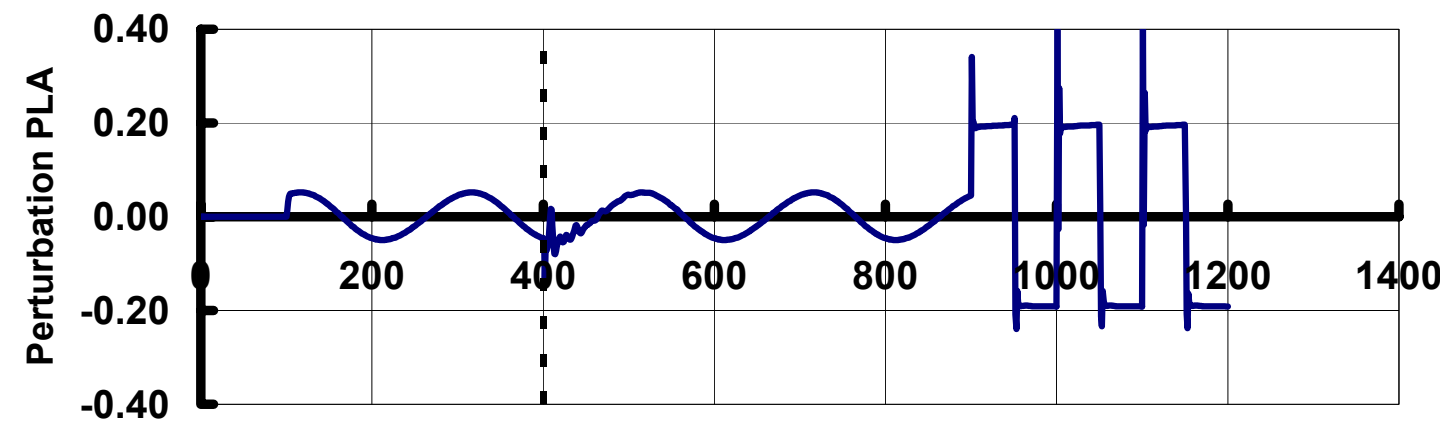

Time, Seconds

Figure 52 (a): Case 16 time history 

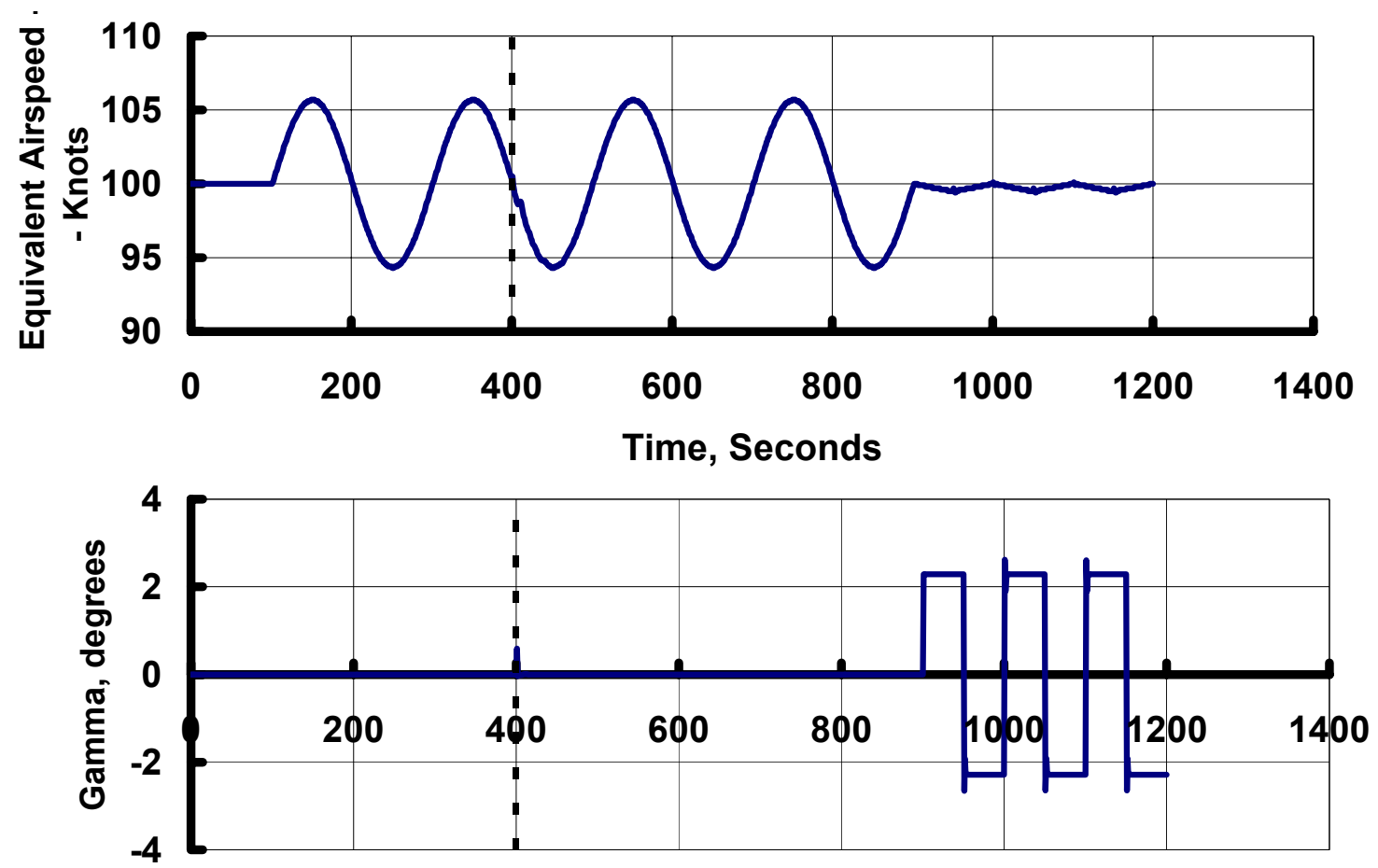

Time, Seconds

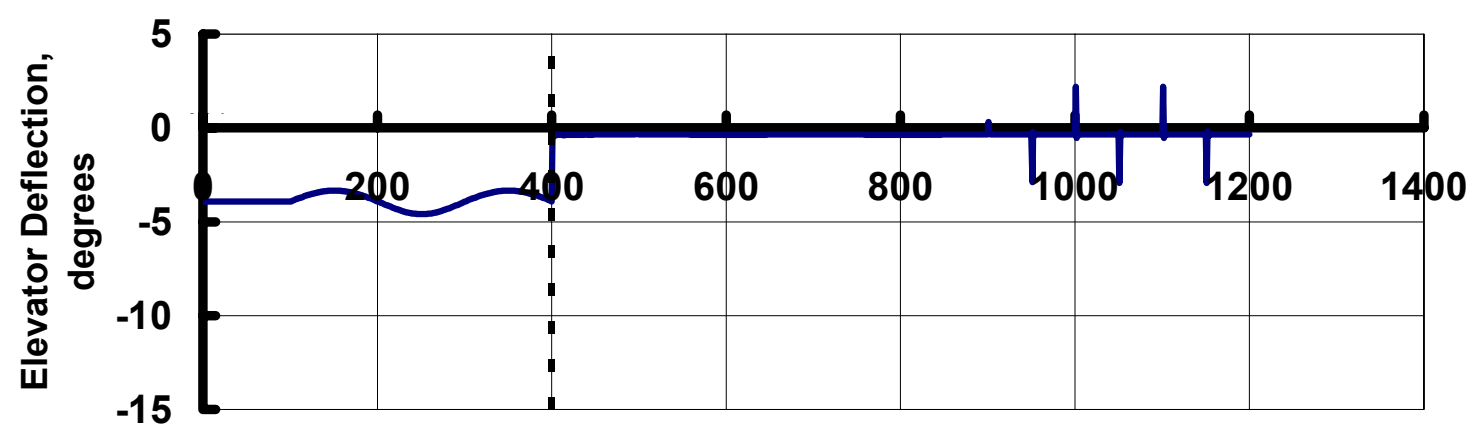

Time, Seconds

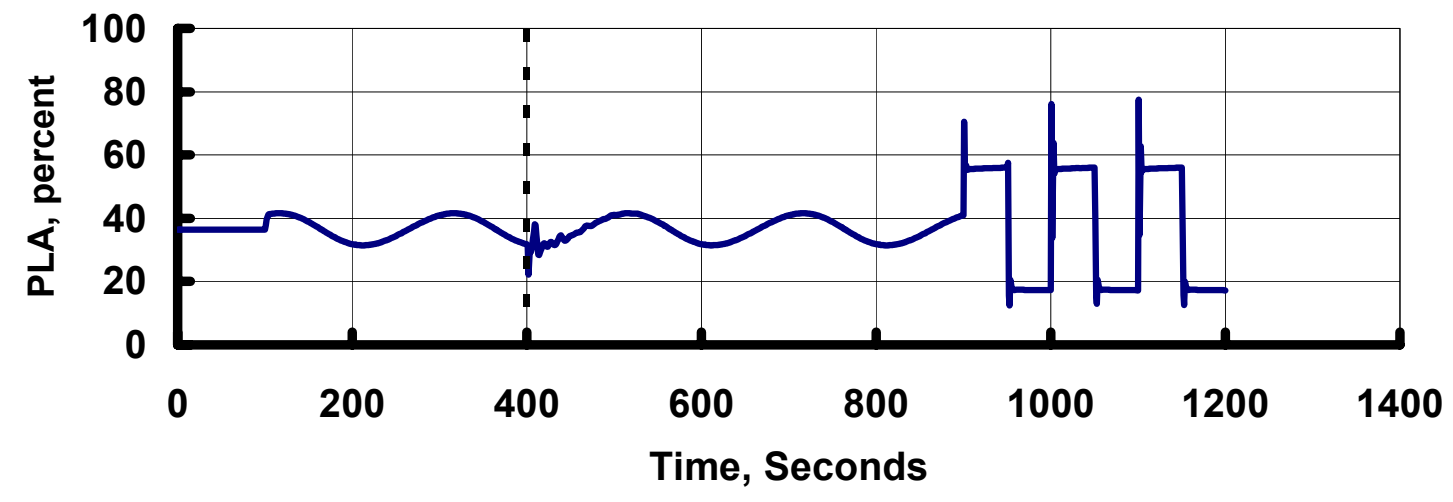

Figure 52 (b): Case 16 time history 

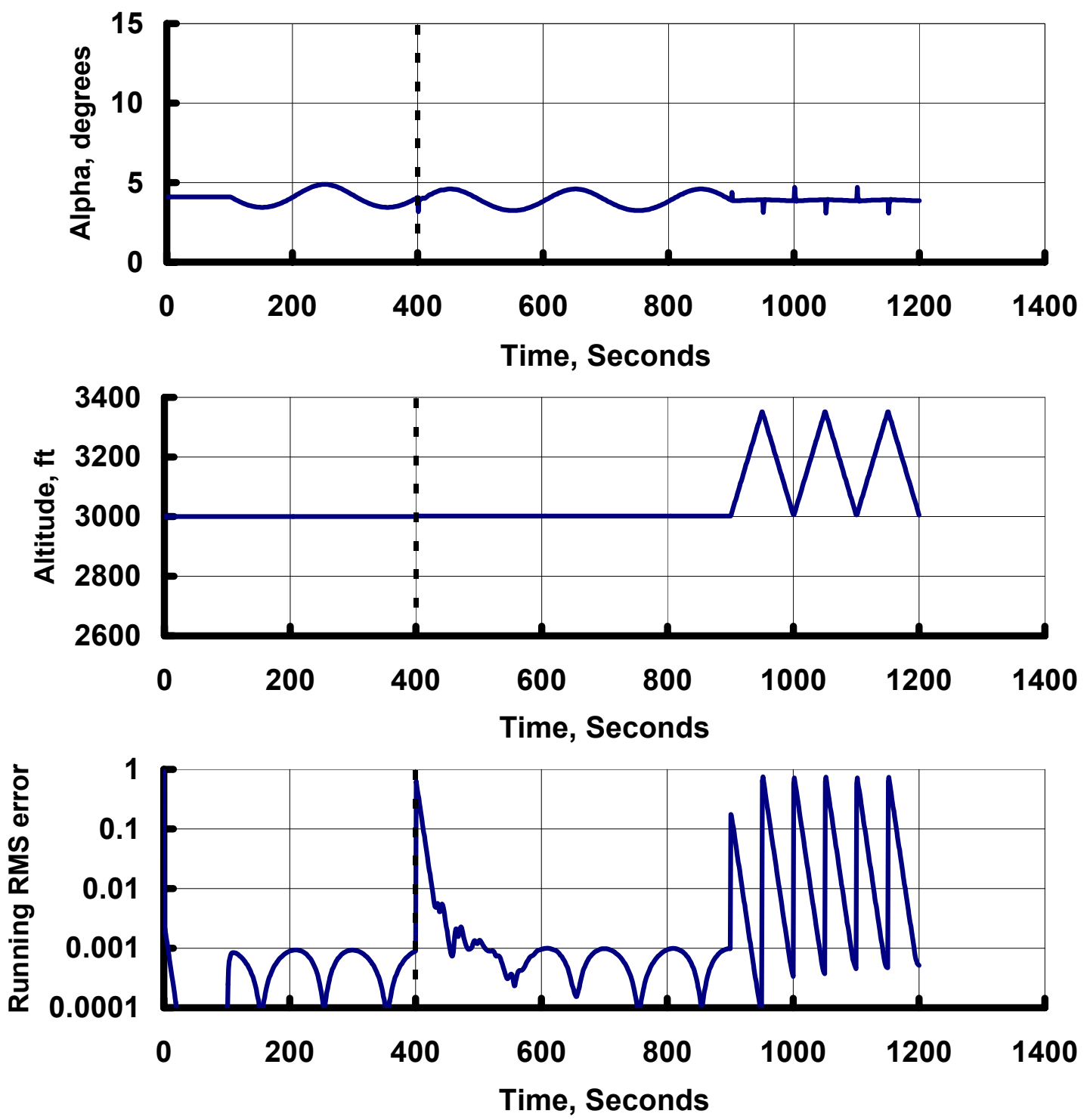

Figure 52 (c): Case 16 time history 


\subsection{Nonlinear Physical Plant Characteristics}

Cases 17 and 18 address nonlinear characteristics in the physical plant. All previous cases assumed linear aerodynamic characteristics. For these next two cases, the lift and pitching moment coefficients vary in an arbitrarily nonlinear fashion, as illustrated in Figures 53 and 54. However, the adaptive neural network is still pre-trained with linear aerodynamic characteristics.

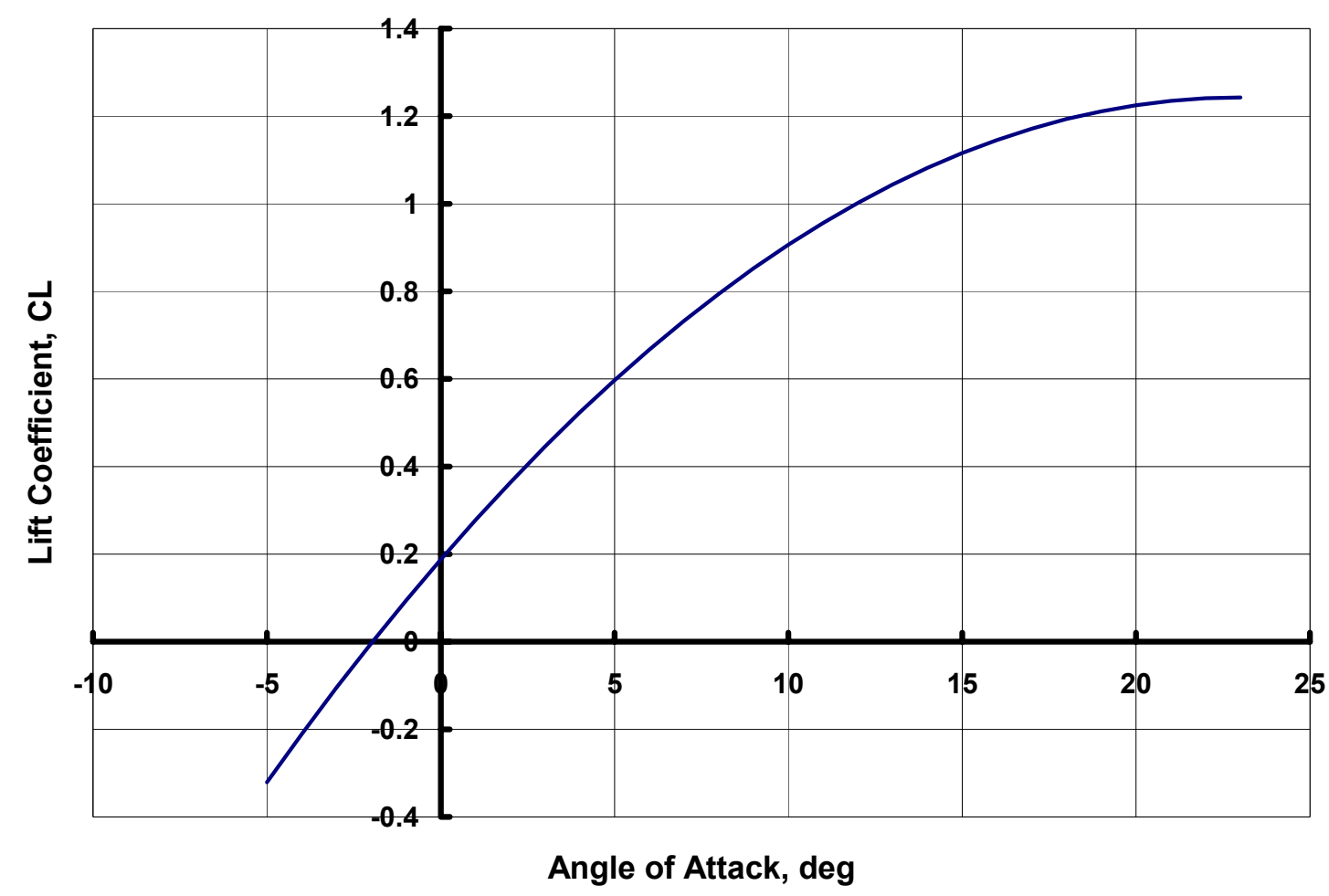

Figure 53: Simulated nonlinear lift coefficient characteristics 


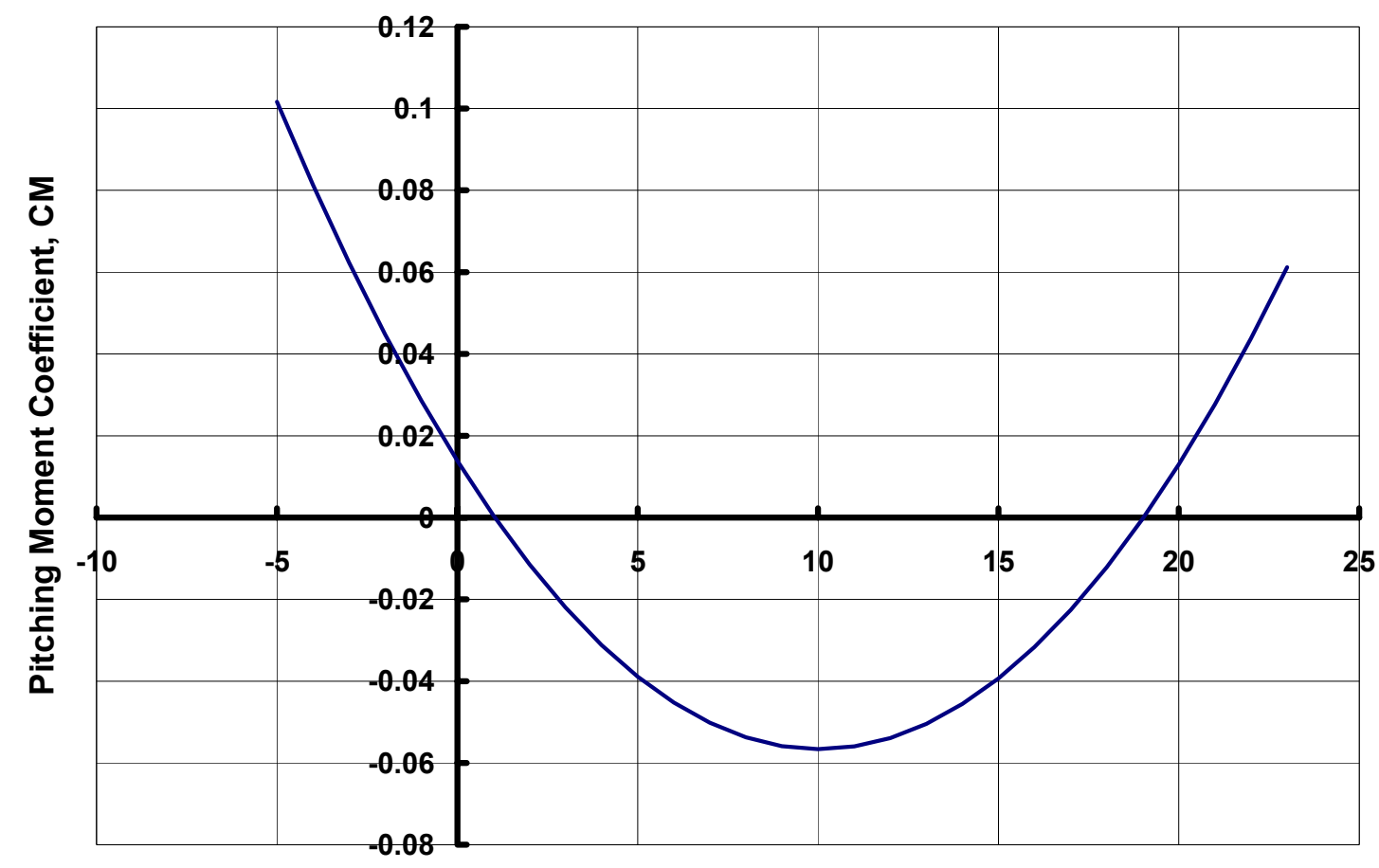

Angle of Attack, deg

Figure 54: Simulated nonlinear pitching moment coefficient characteristics

Case 17 (Figure 55) is commanded with the same variations in speed and flight-path as Case 15. However, these variations are about a new trim speed of 80 KEAS. This new trim speed is chosen such that the trim angle of attack of 9.47 degrees is at the threshold of the significant nonlinear behavior. As the angle of attack varies, the aircraft experiences significant variations in $\mathrm{C}_{\mathrm{L}_{\alpha}}$ and $\mathrm{C}_{\mathrm{M}_{\alpha}}$. No apparent difference in controller performance is evident from Case 15. This is a significant accomplishment considering that the adaptive neural network was pre-trained on the linear aircraft response, and it is adapting to the nonlinear aerodynamics in real-time.

Case 18 (Figure 56) is the same as Case 17, except that both $\mathrm{C}_{\mathrm{L}_{\delta \mathrm{c}}}$ and $\mathrm{C}_{\mathrm{M}_{\delta \mathrm{\delta e}}}$ are reduced by 50 percent at 400 seconds. A very small transient is evident at the time of the failure, but the 
controller maintains speed tracking accuracy well. This transient manifests itself as small variations in speed and PLA, but primarily in elevator deflection. The controller detects the need for a new trim elevator setting, in light of the reduced elevator authority, and smoothly adjusts. Later however, flight-path accuracy is somewhat degraded during the abrupt step changes in commanded flight-path. Some loss of precision is evident in the initial capture of the target flight-path angle. In the second period of the flight-path command square wave, the controller is unable to keep up, and control is lost. This is the only case tested where the controller was not capable of managing the airplane. As in Case 17, the network has been pre-trained with the linear aircraft response. It is speculated that if a rigorous pre-training of the network was undertaken using the nonlinear characteristics, this loss of control would be avoided. 


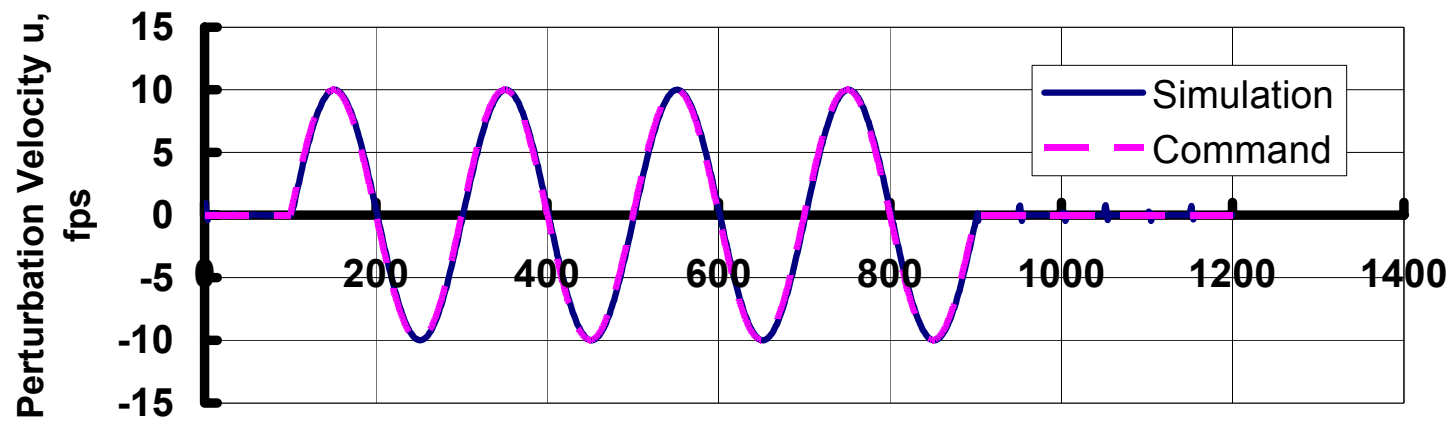

Time, Seconds

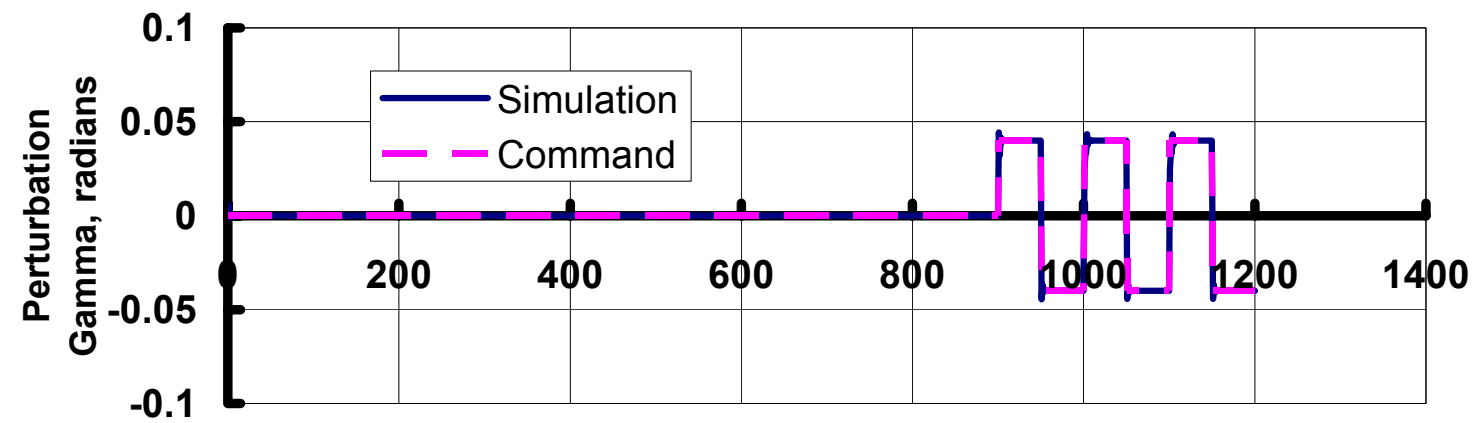

Time, Seconds

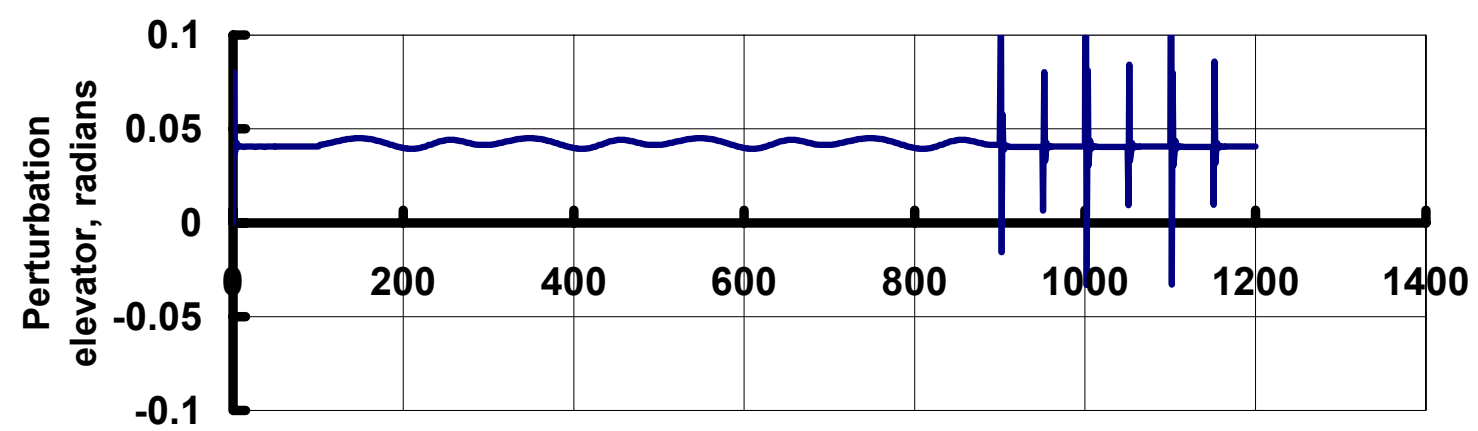

Time, Seconds

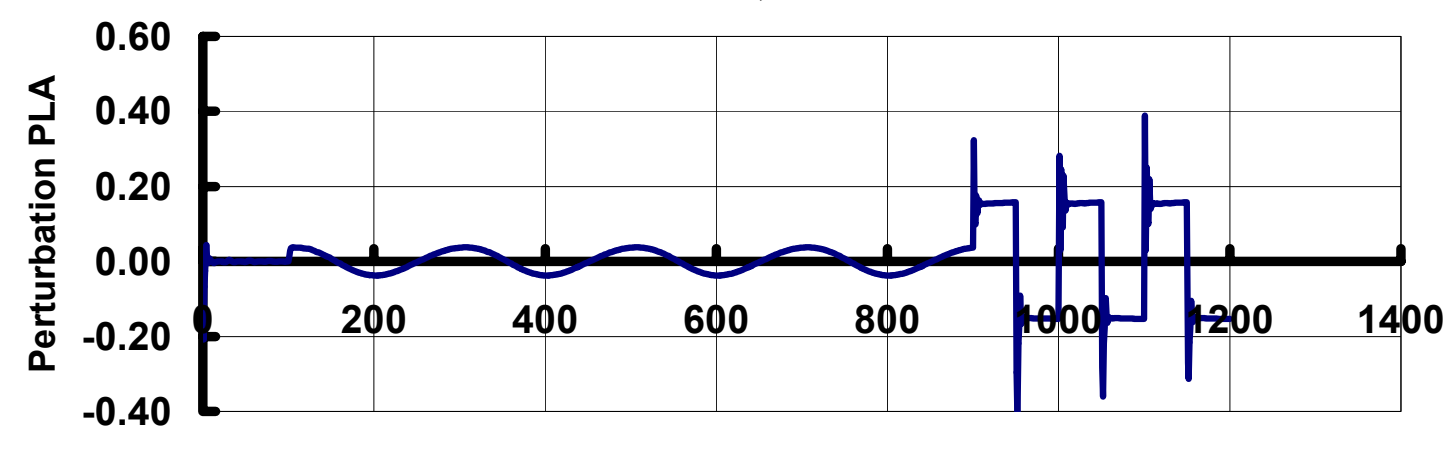

Time, Seconds

Figure 55 (a): Case 17 time history 

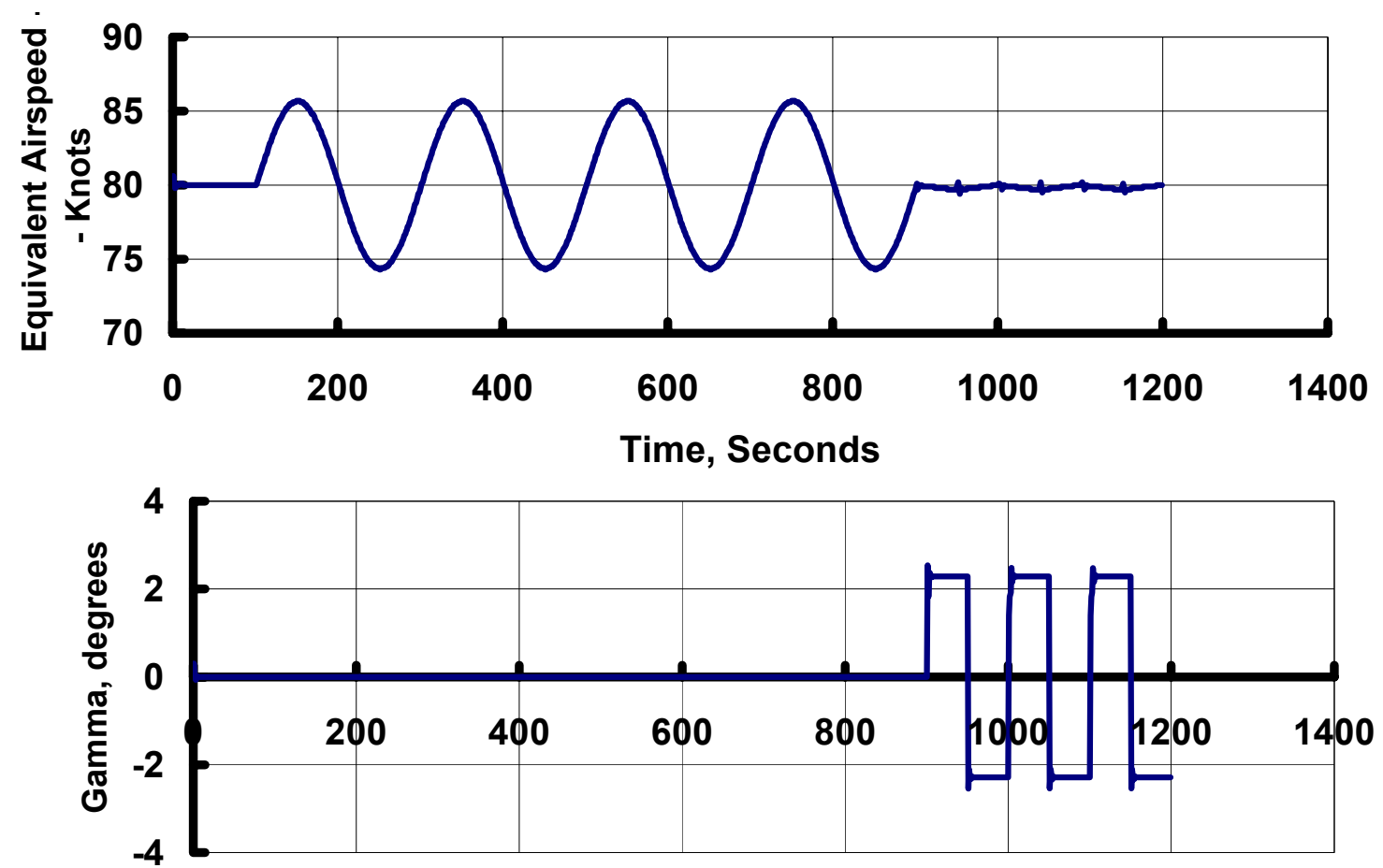

Time, Seconds

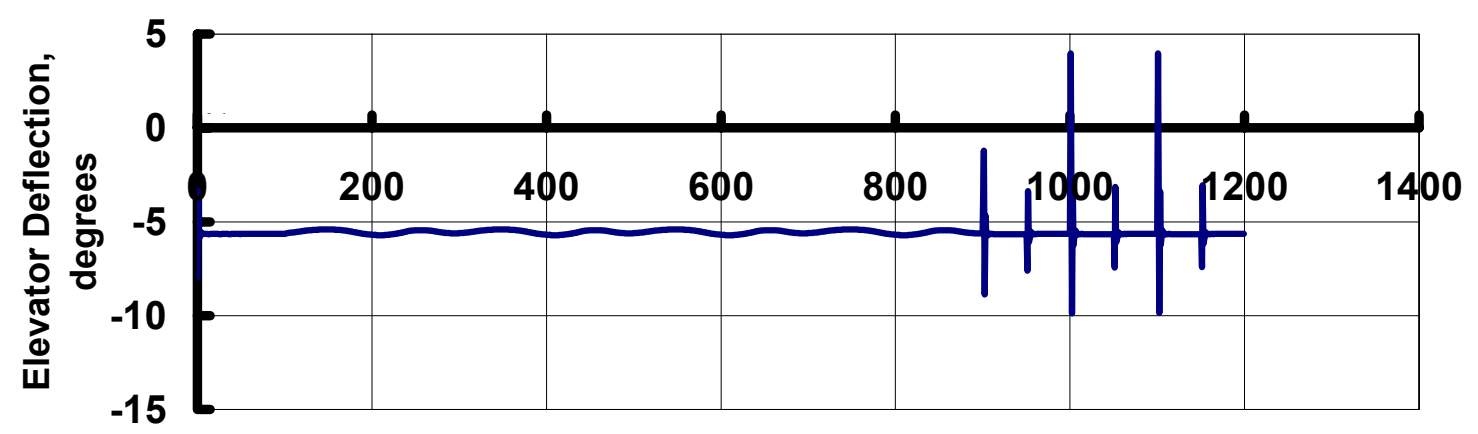

Time, Seconds

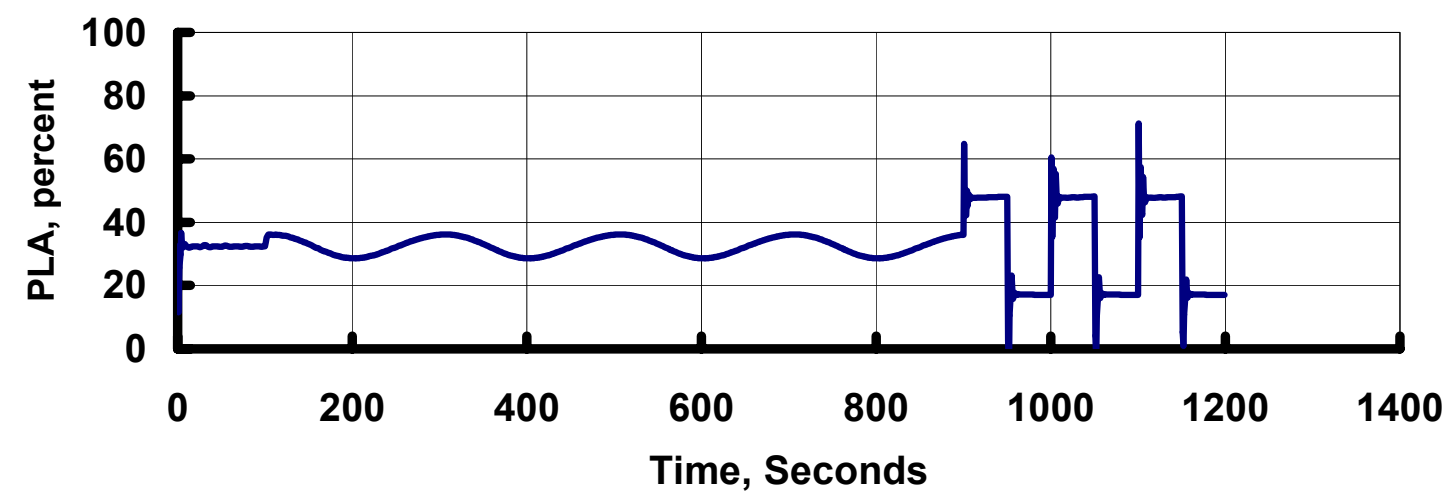

Figure 55 (b): Case 17 time history 

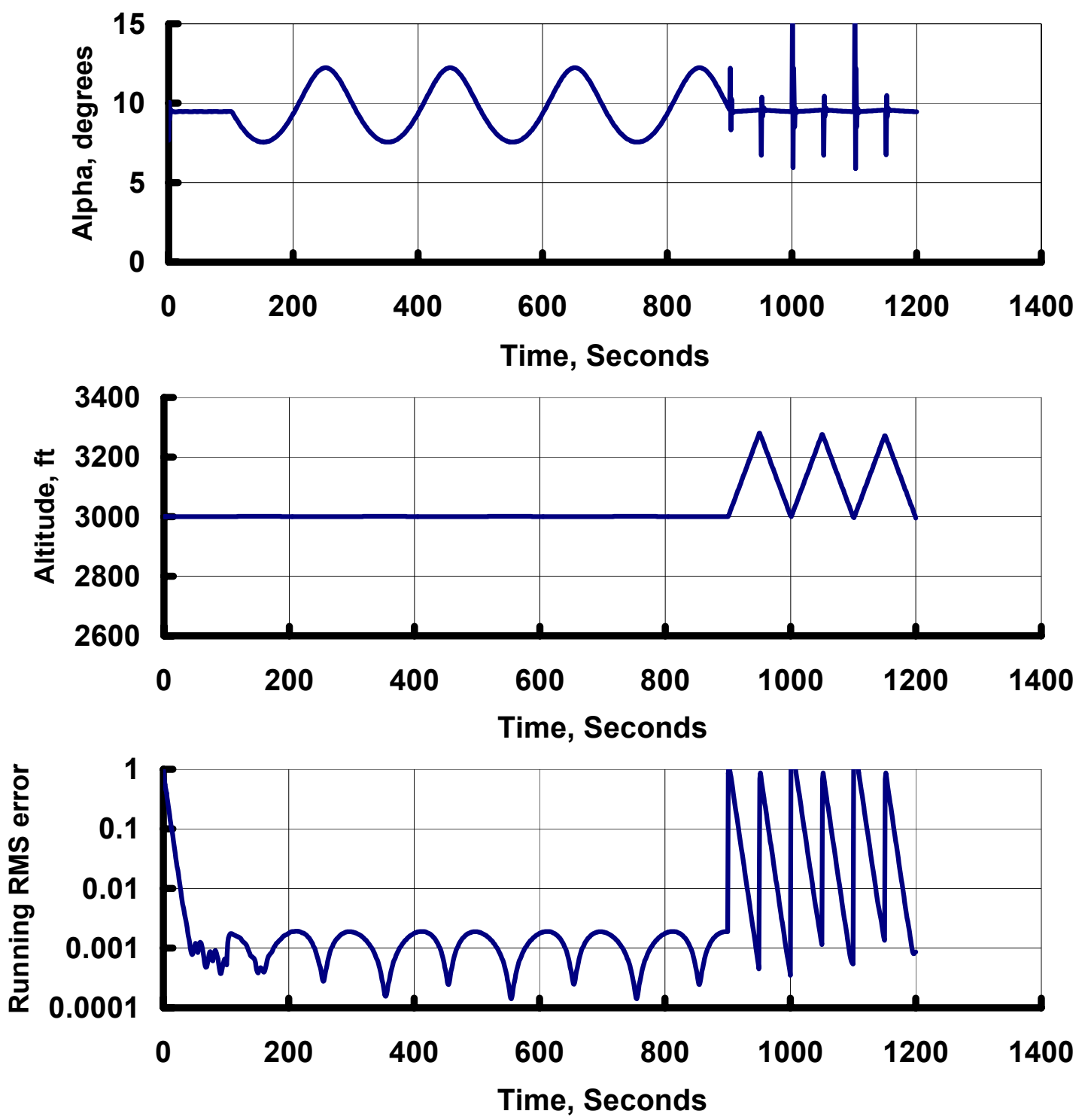

Figure 55 (c): Case 17 time history 


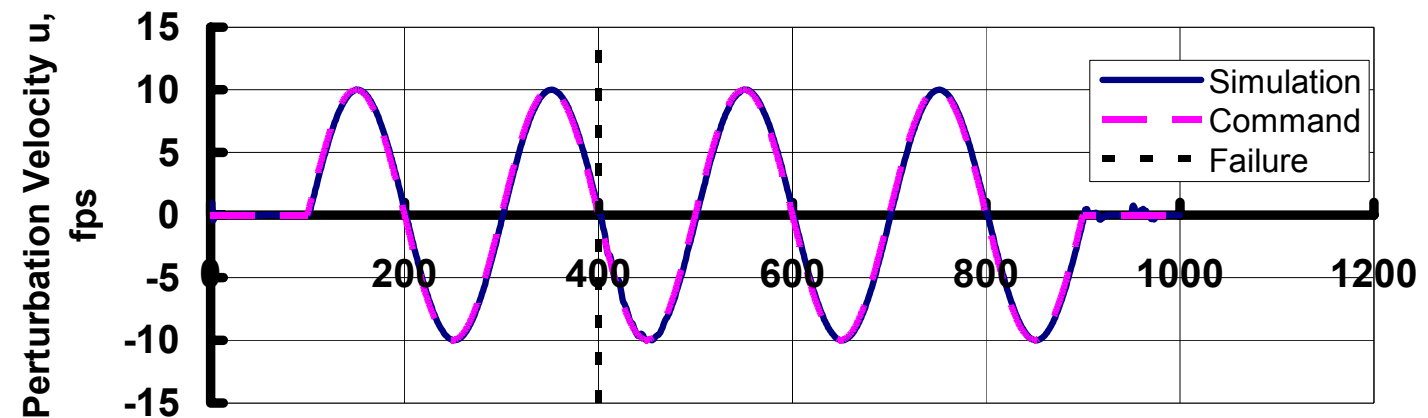

Time, Seconds

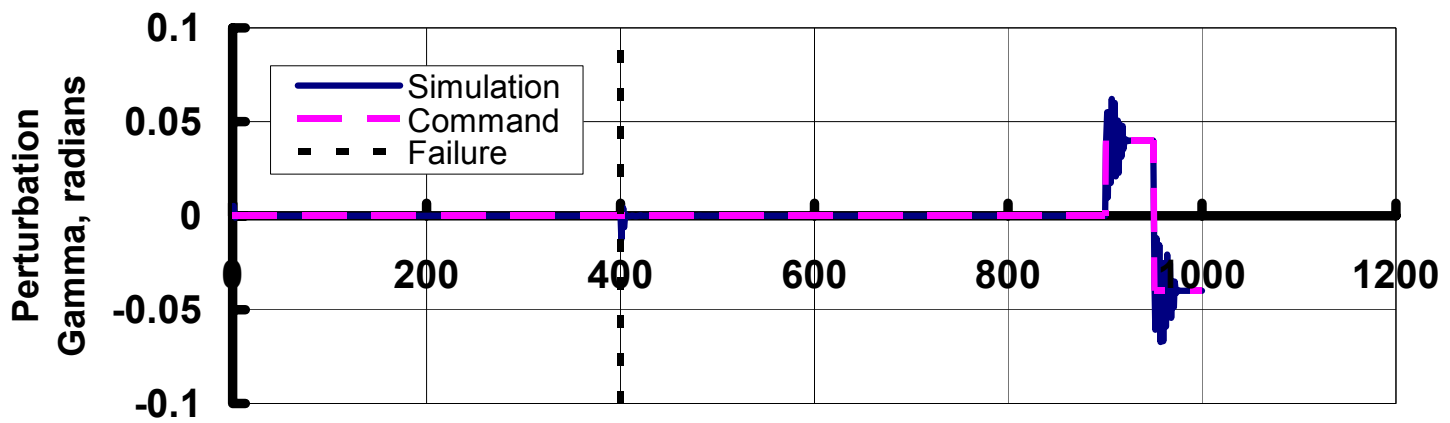

Time, Seconds

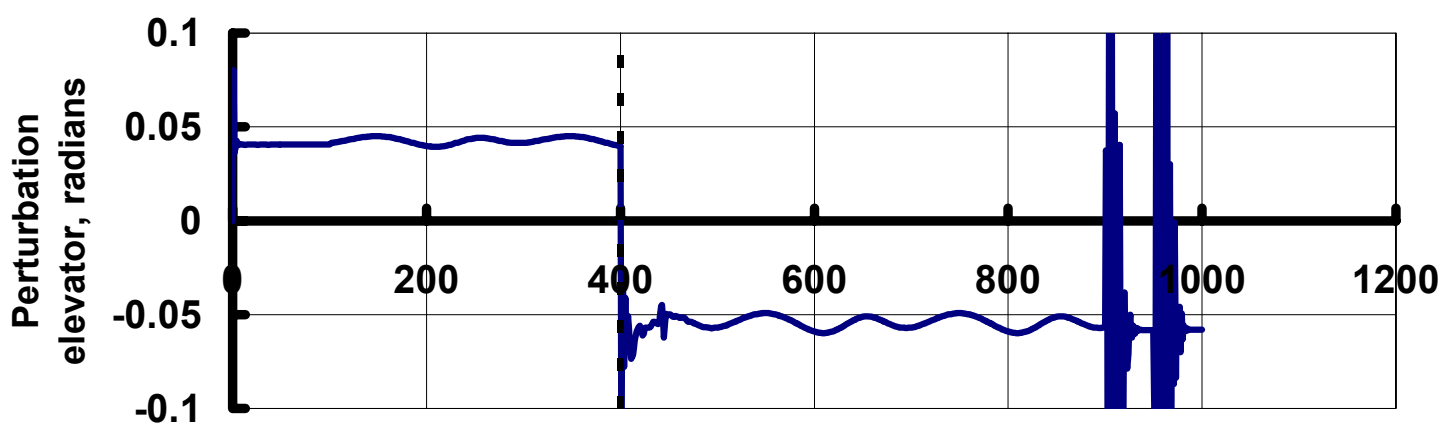

Time, Seconds

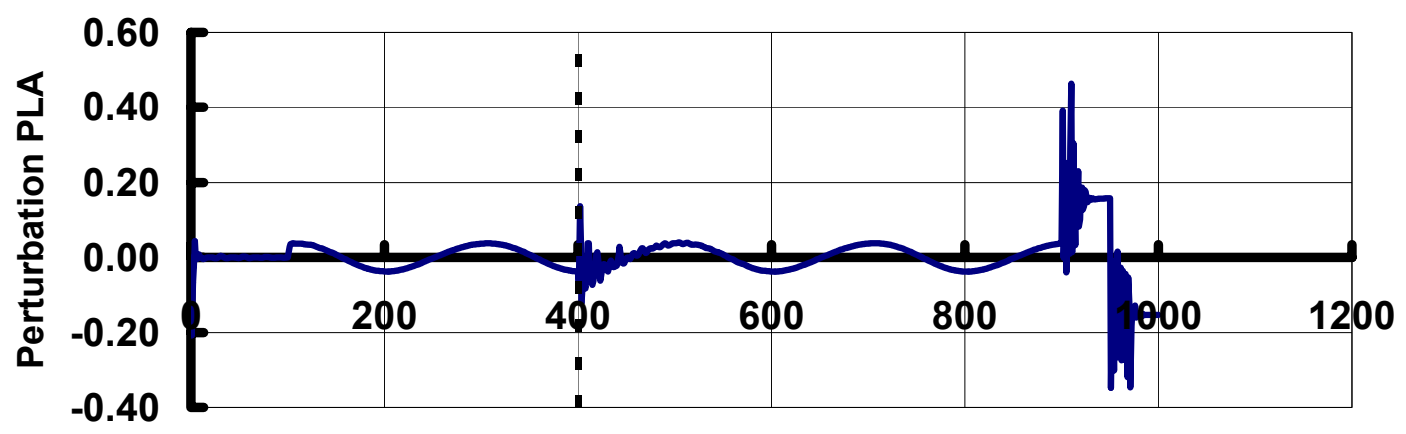

Time, Seconds

Figure 56 (a): Case 18 time history 

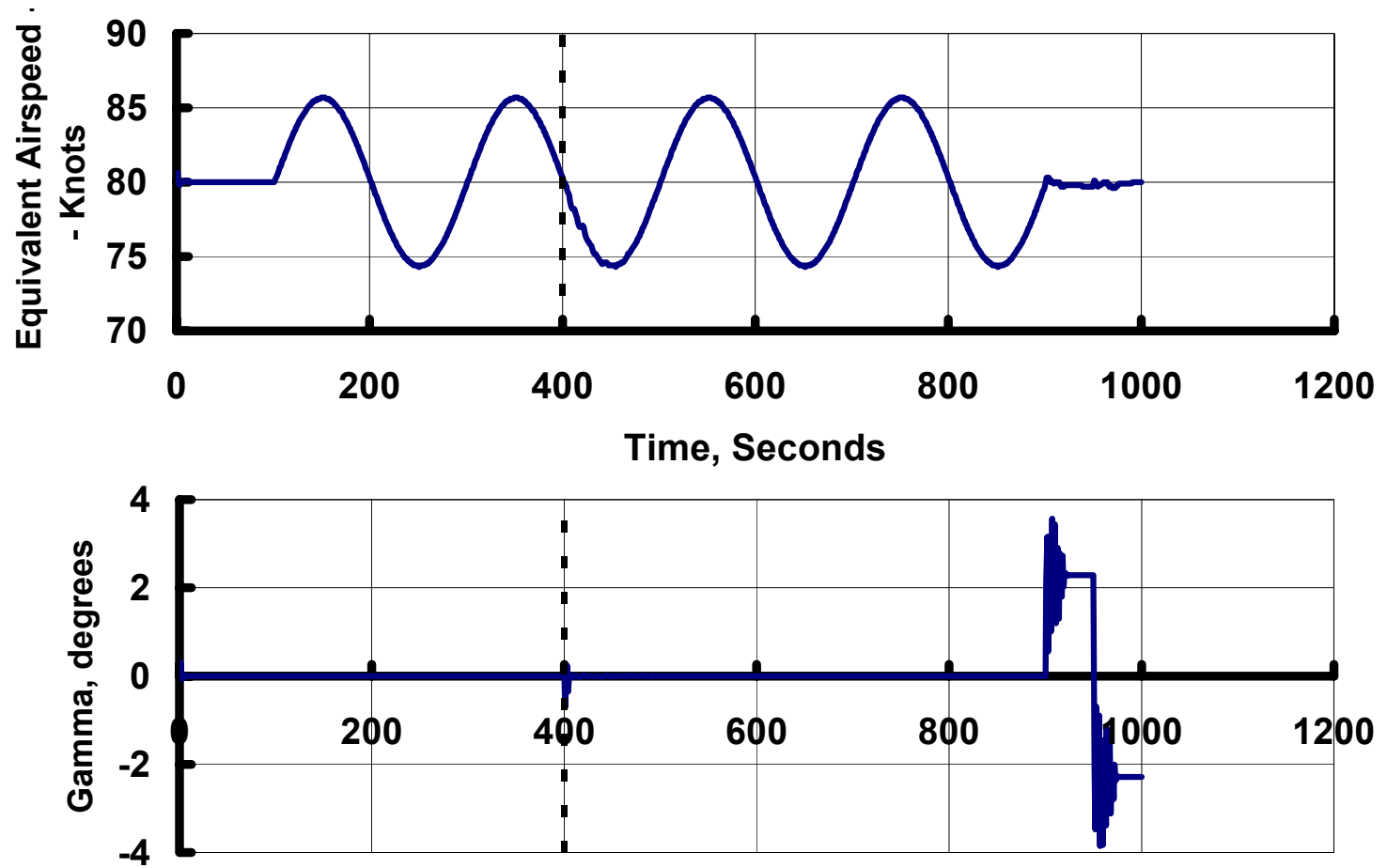

Time, Seconds

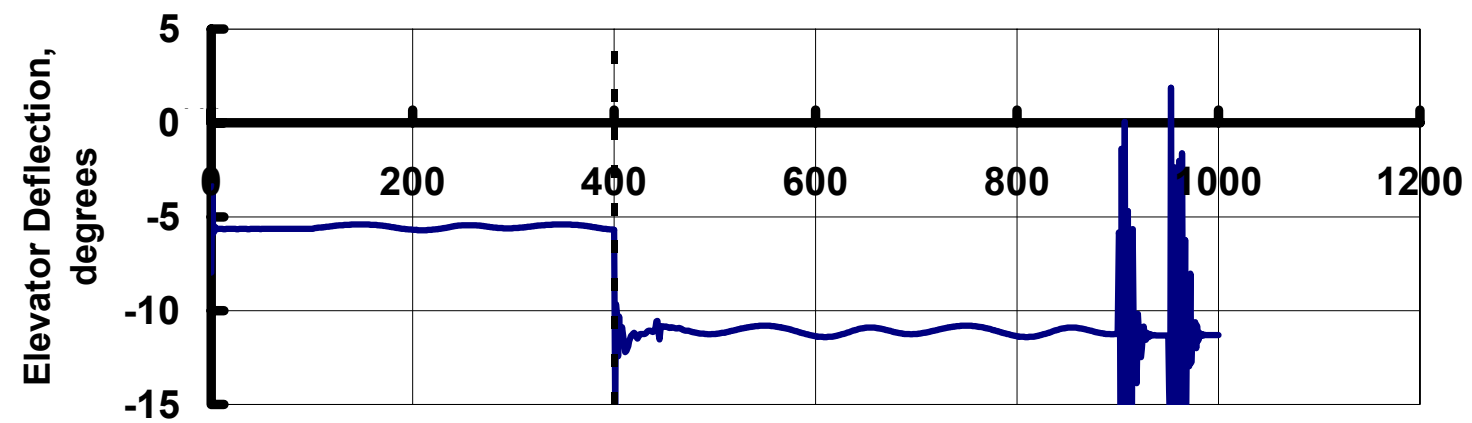

Time, Seconds

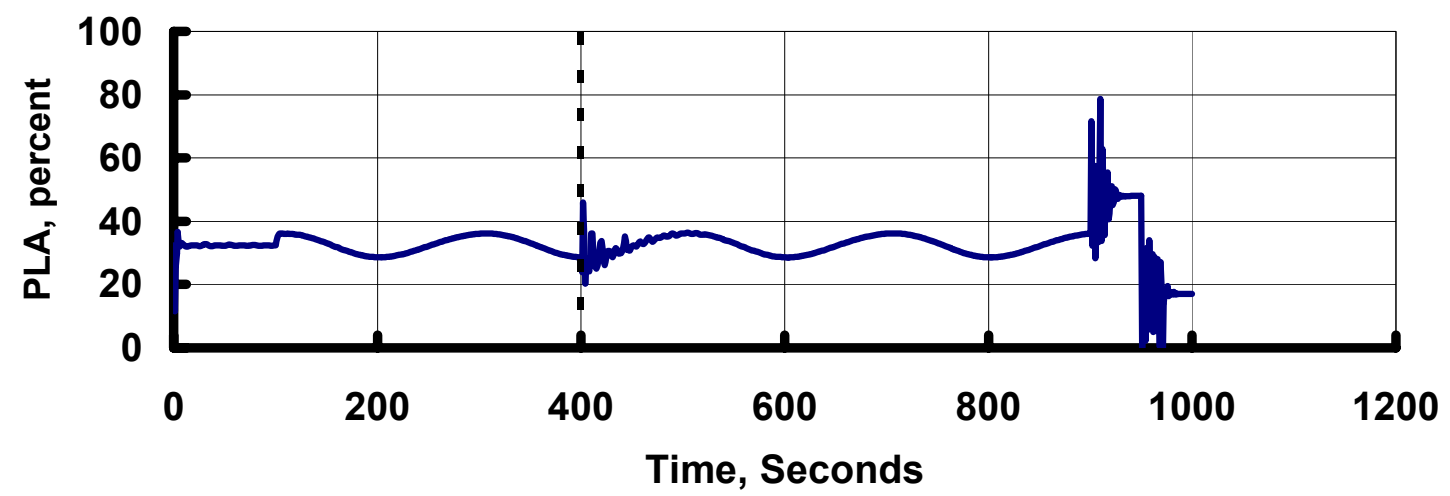

Figure 56 (b): Case 18 time history 

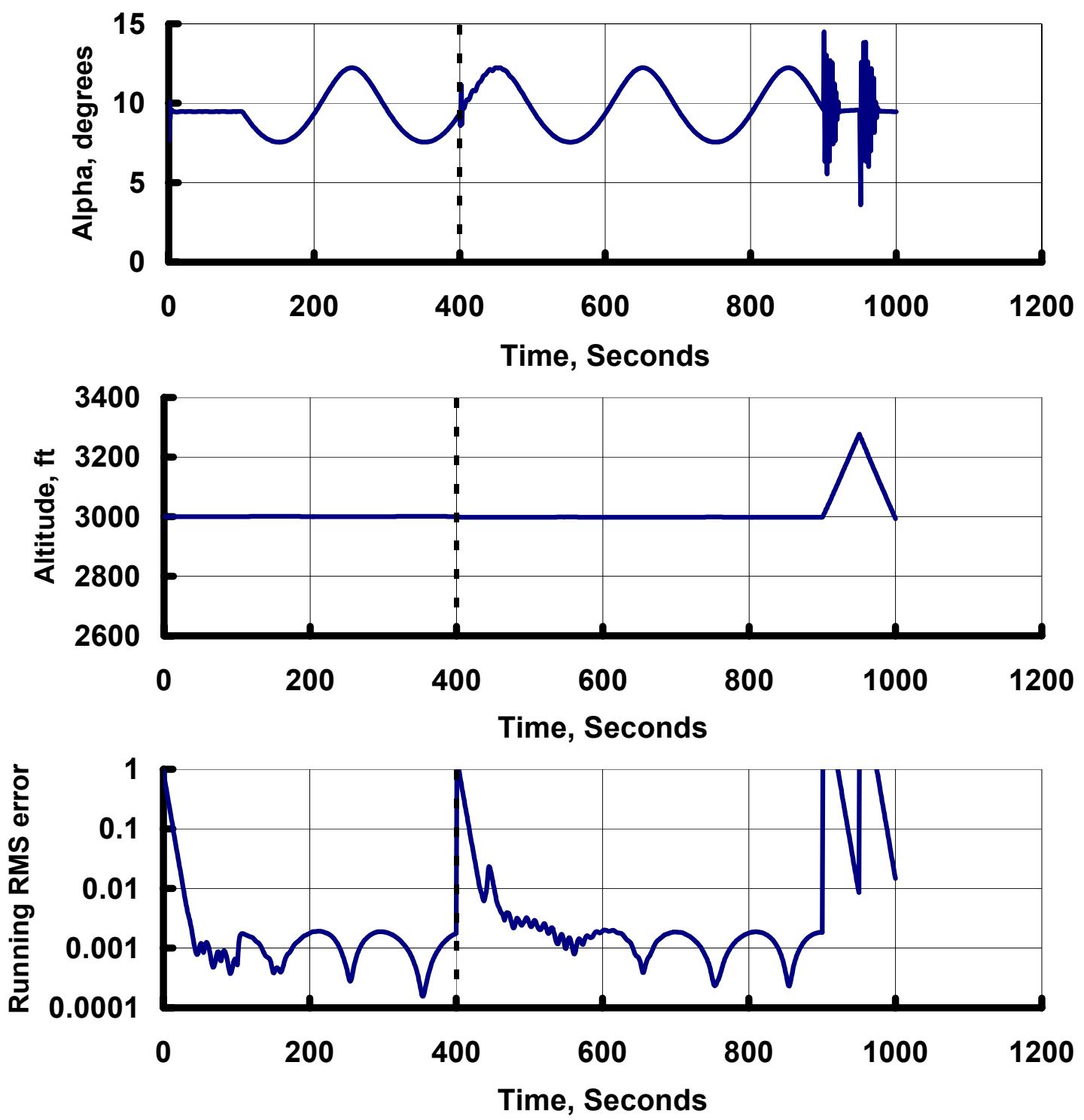

Figure 56 (c): Case 18 time history 


\subsection{Neural Network Failures}

A brief examination is undertaken of failures of the neural network itself. This discussion is outside the original scope of the research proposed for this dissertation but is a worthwhile addition nonetheless. Insights are obtained for design and potential certification of future control systems.

Failures in typical flight control systems consist of hardovers, oscillatory failures, or runaways resulting from integrator "wind-up." A beneficial feature of neural networks is that many employ activation functions that are self-limiting. In the current research, as an example, neurons use a hyperbolic tangent (tanh) activation function. The unscaled output of the neurons asymptotically approach plus-or-minus one as the sum of their inputs goes to plus-or-minus infinity. This is illustrated in Figure 57. Most importantly, the output neurons are likewise bounded. As a consequence of this, the output of the network is bounded. An unbounded windup failure is impossible. Hardovers will be limited in magnitude by the network output scaling. Oscillatory failures will likewise be bounded in amplitude by the output scaling.

Case 19 is the baseline scenario for the network failures examined. All controller functions operate normally. The command for this case is a long period ( $\mathrm{T}=200$ seconds) speed command of $10 \mathrm{feet} / \mathrm{sec}$ amplitude. Flight-path angle is commanded to be zero throughout. From examination of the perturbation velocity and perturbation gamma traces, it is seen that the output almost tracks the command perfectly. This should be expected since the frequency of the command is far less than the resonant frequency of the overall system. 


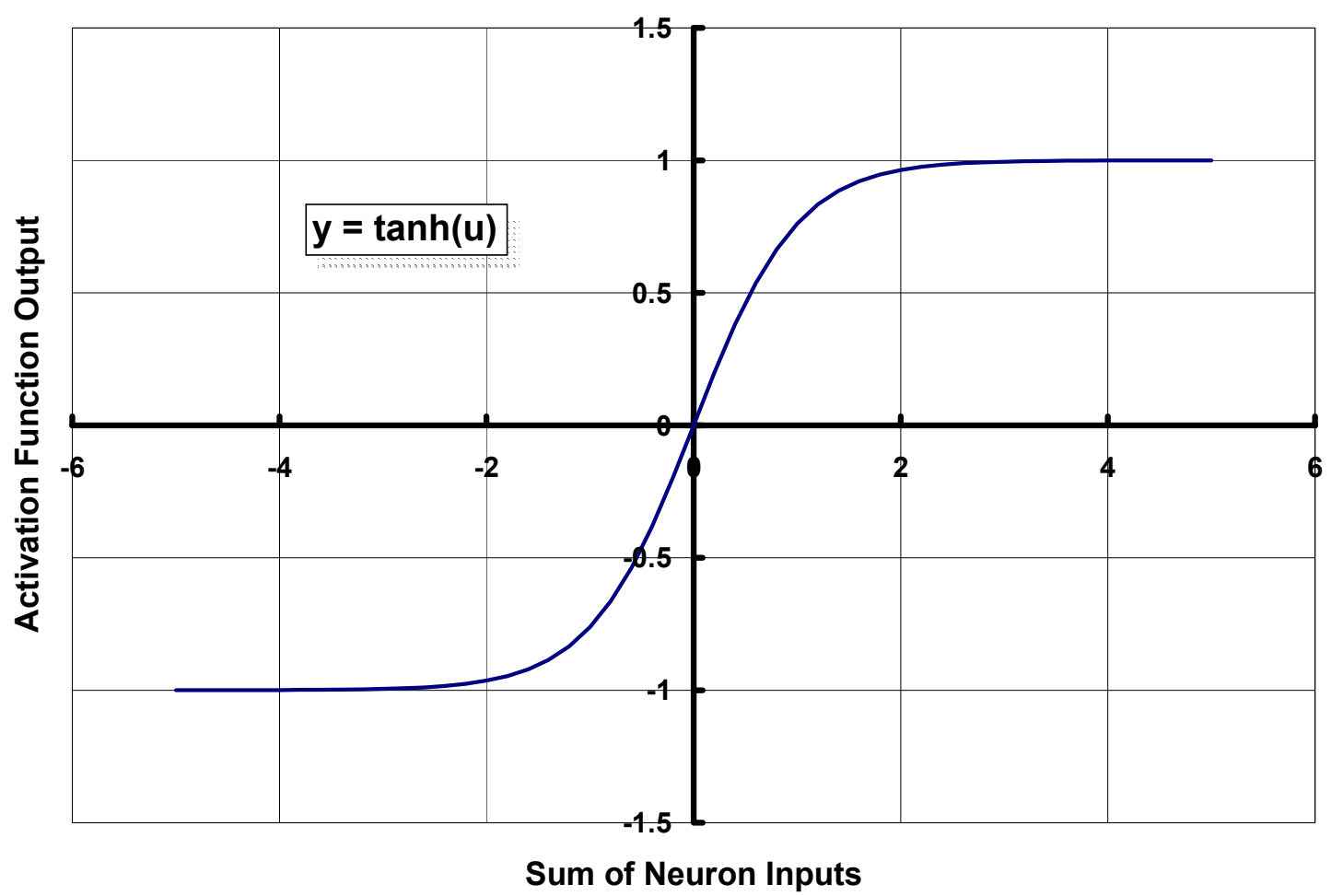

Figure 57: Neuron activation function self-limits output

Case 20 examines a neural network hardover on the $\Delta \dot{u}$ channel that occurs at 6 seconds. This hardover is assumed to go to the maximum output value permitted by the output scaling of the network. The $\Delta \dot{\alpha}$ signal remains functional. Figure 59 shows the resulting response. The simulation is run for 500 seconds to demonstrate there are no transients with long time constants. A steady-state error of approximately $26 \mathrm{feet} / \mathrm{sec}$ is visible in the perturbation velocity trace. While not trivial, this error could be trapped by software monitors that either enable a back-up algorithm for mitigation and/or annunciate the failure for pilot corrective action. Flight-path angle tracking error is less than one degree.

The RMS error time history for these failure cases should be interpreted with care. For Case 20 , the $\Delta \dot{\alpha}$ channel is learning and participating in control of the system normally. While a 
failure on the $\Delta \dot{\mathrm{u}}$ channel is simulated, the method of simulating the failure does not precluded that channel from learning normally. This is a consequence of how the failure is simulated. In practice, it is expected that the learning on a failed channel will be unpredictable and will depend on the nature of the failure.

Case 21 examines a $\Delta \dot{u}$ oscillatory failure at 6 seconds occurring at the overall system resonant frequency of .60 rad/sec. Once again, the amplitude of the error signal is assumed to be the maximum permitted by the output scaling. Figure 60 shows the resulting time histories. An oscillatory speed tracking error occurs at an amplitude of about 24 feet/sec. A small amplitude flight-path error occurs on the order of one-half degree.

Case 22 examines a neural network hardover on the $\Delta \dot{\alpha}$ channel that occurs at 6 seconds. This signal is assumed to go to the maximum output value permitted by output scaling of the network. The $\Delta \dot{\mathrm{u}}$ signal remains functional. Figure 61 shows the resulting response. For this case, speed is unaffected while flight-path angle experiences a steady-state error of only -..01 radians.

Case 23 is illustrated in Figure 62. This case looks at an oscillatory failure of the $\Delta \dot{\alpha}$ channel at 6 seconds. The failure has a frequency of $1.785 \mathrm{rand} / \mathrm{sec}$ to match the resonant frequency of the chosen flight-path angle characteristics. As seen in the $\Delta \dot{\alpha}$ hardover, no influence is detected in the speed tracking, and only a small periodic error less than one degree is noted in the flight-path angle tracking.

With these observations in place, questions arise to explain the differences in the effects of failures between $\Delta \dot{\mathrm{u}}$ and $\Delta \dot{\alpha}$. 


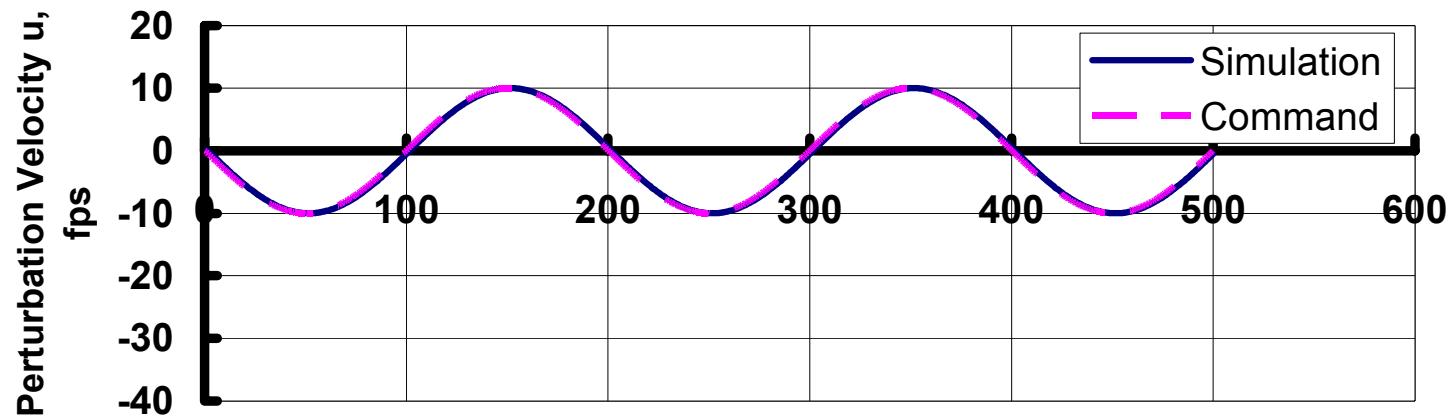

Time, Seconds

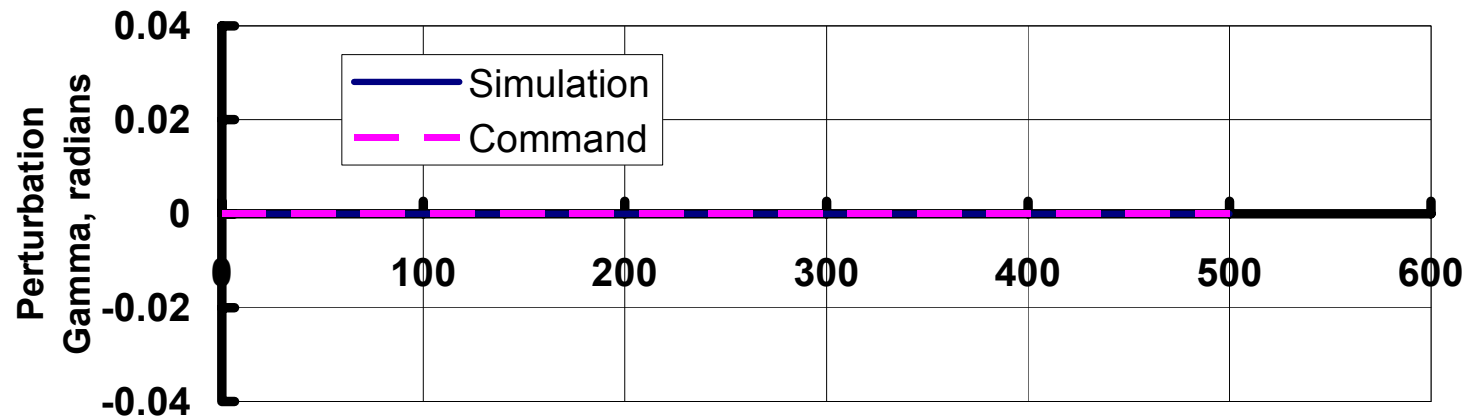

Time, Seconds

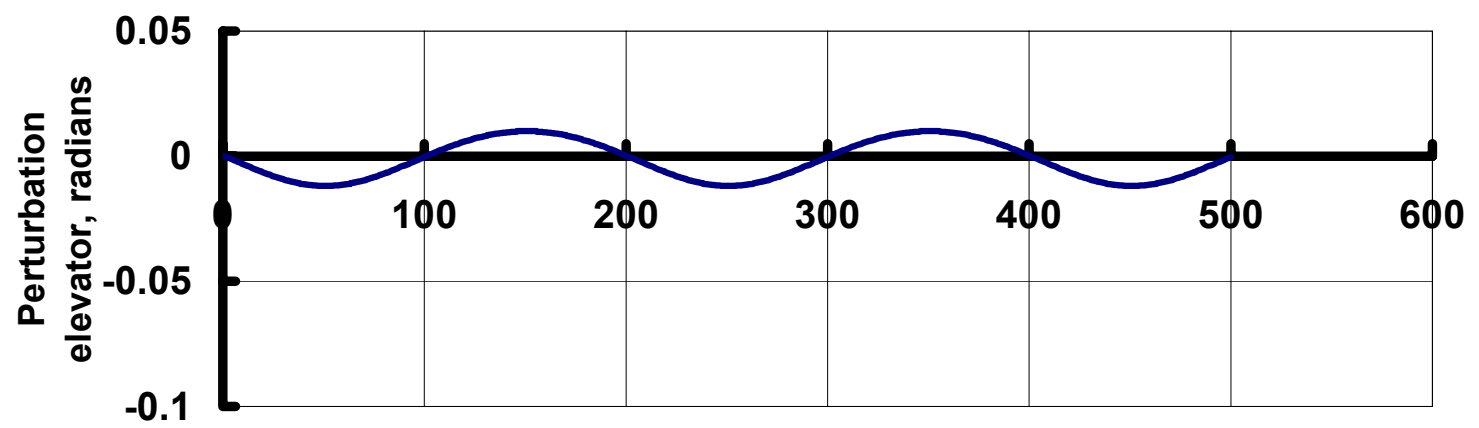

Time, Seconds

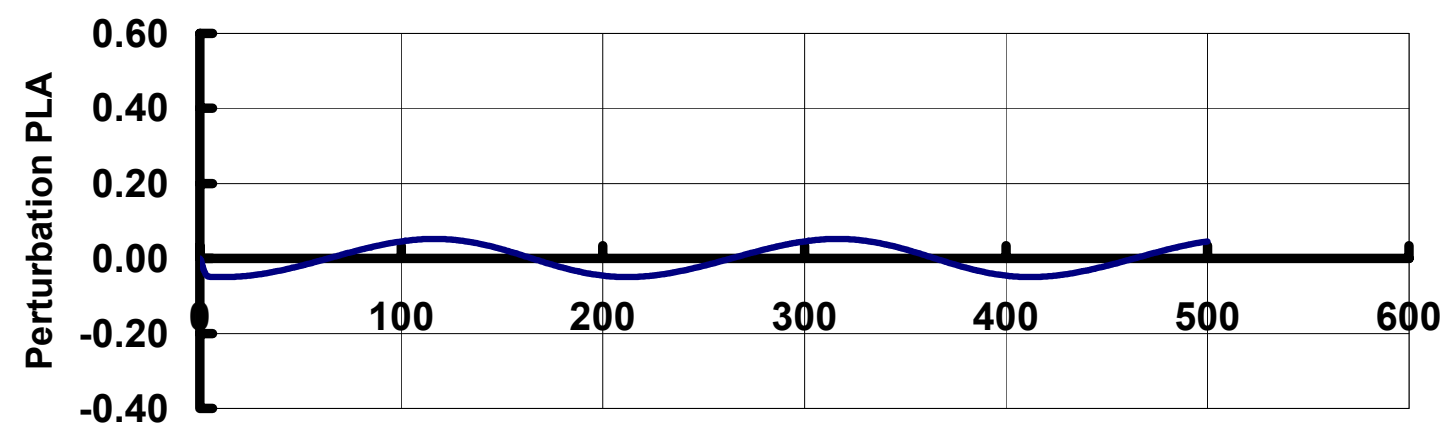

Time, Seconds

Figure 58 (a): Case 19 time history 

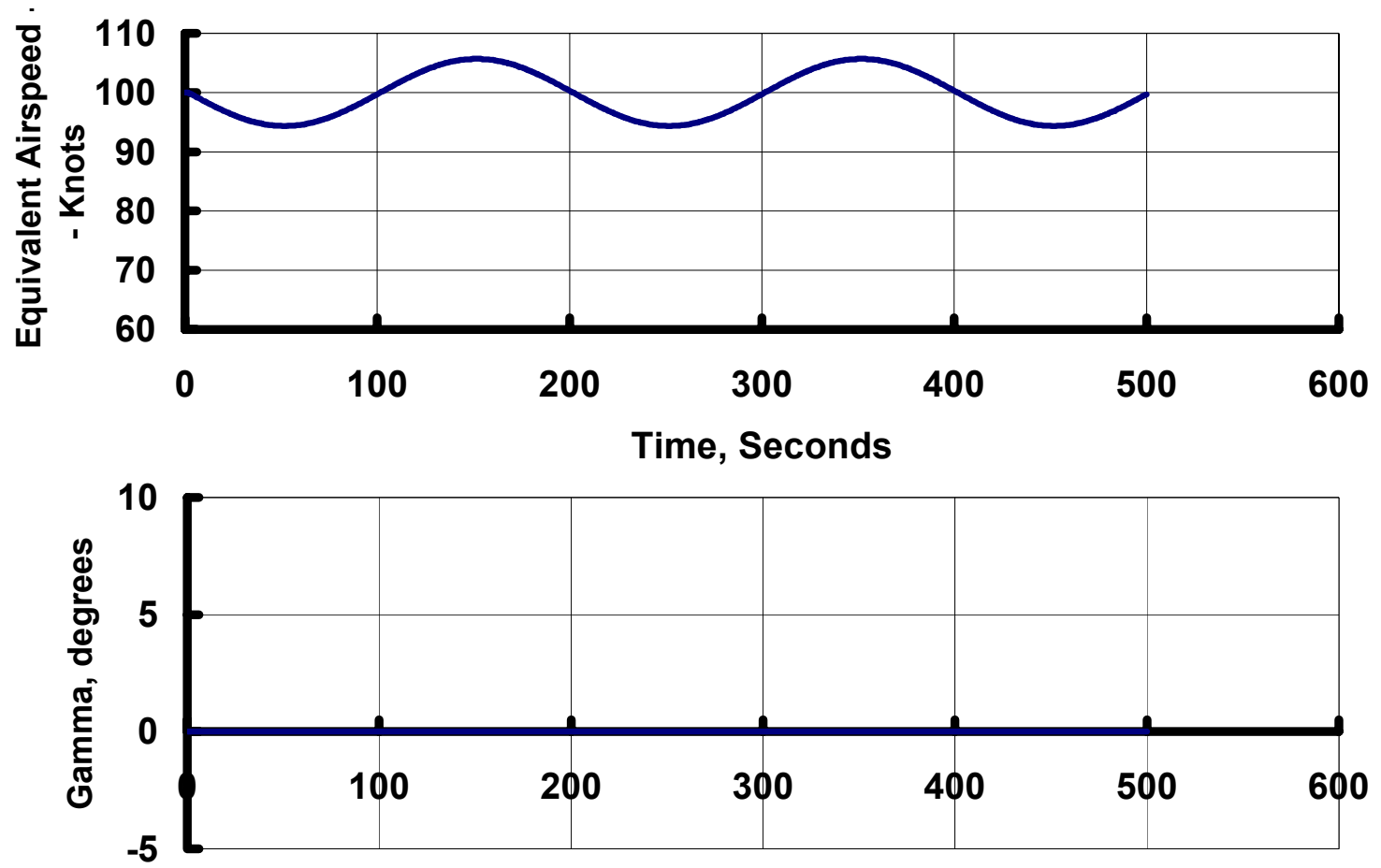

Time, Seconds

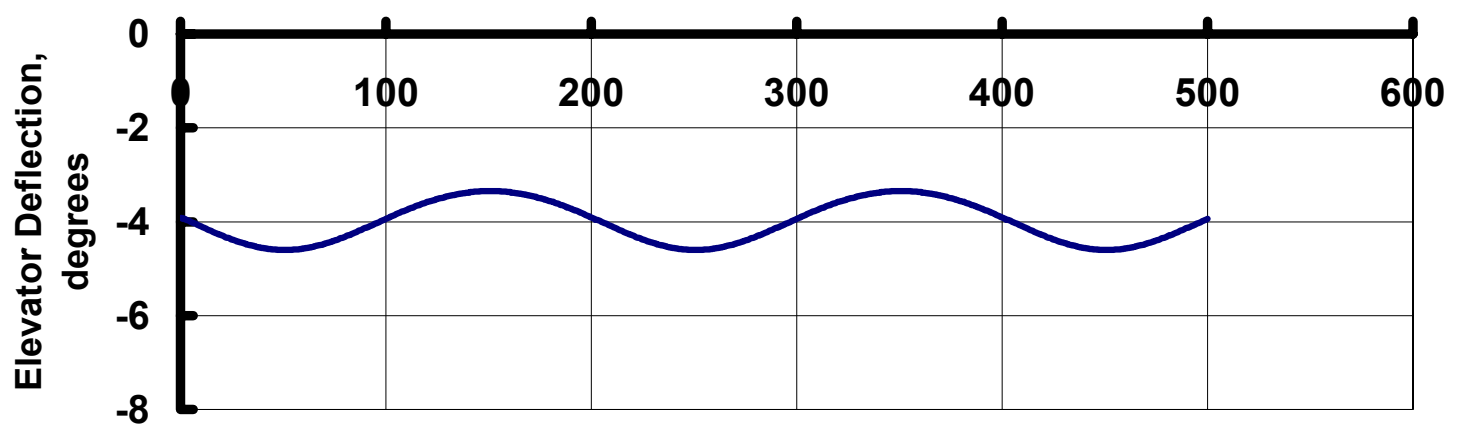

Time, Seconds

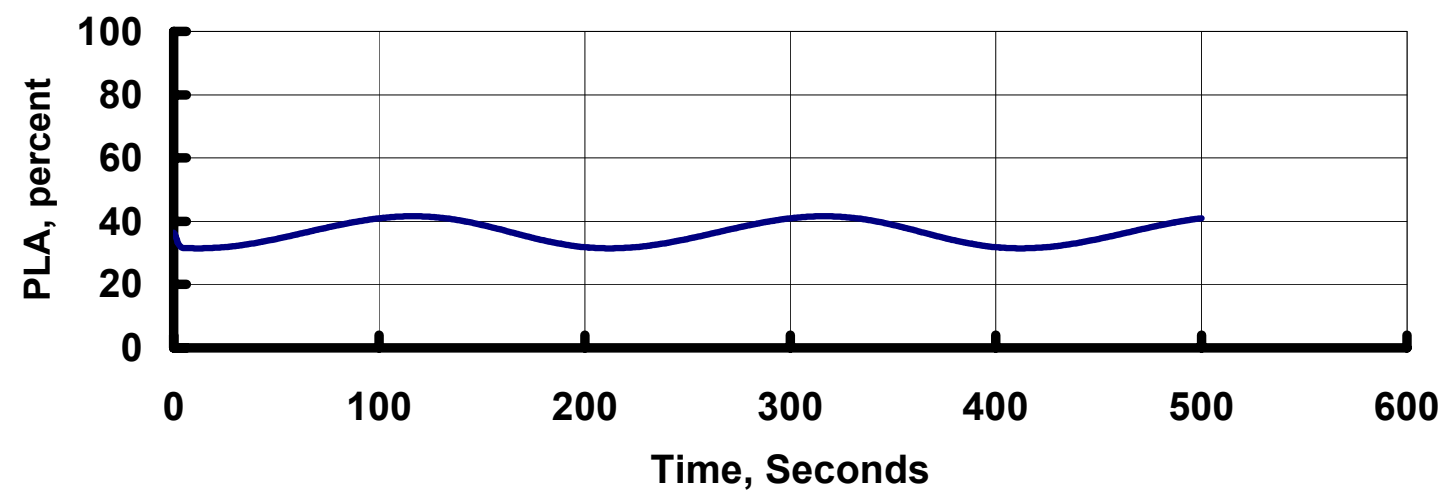

Figure 58 (b): Case 19 time history 

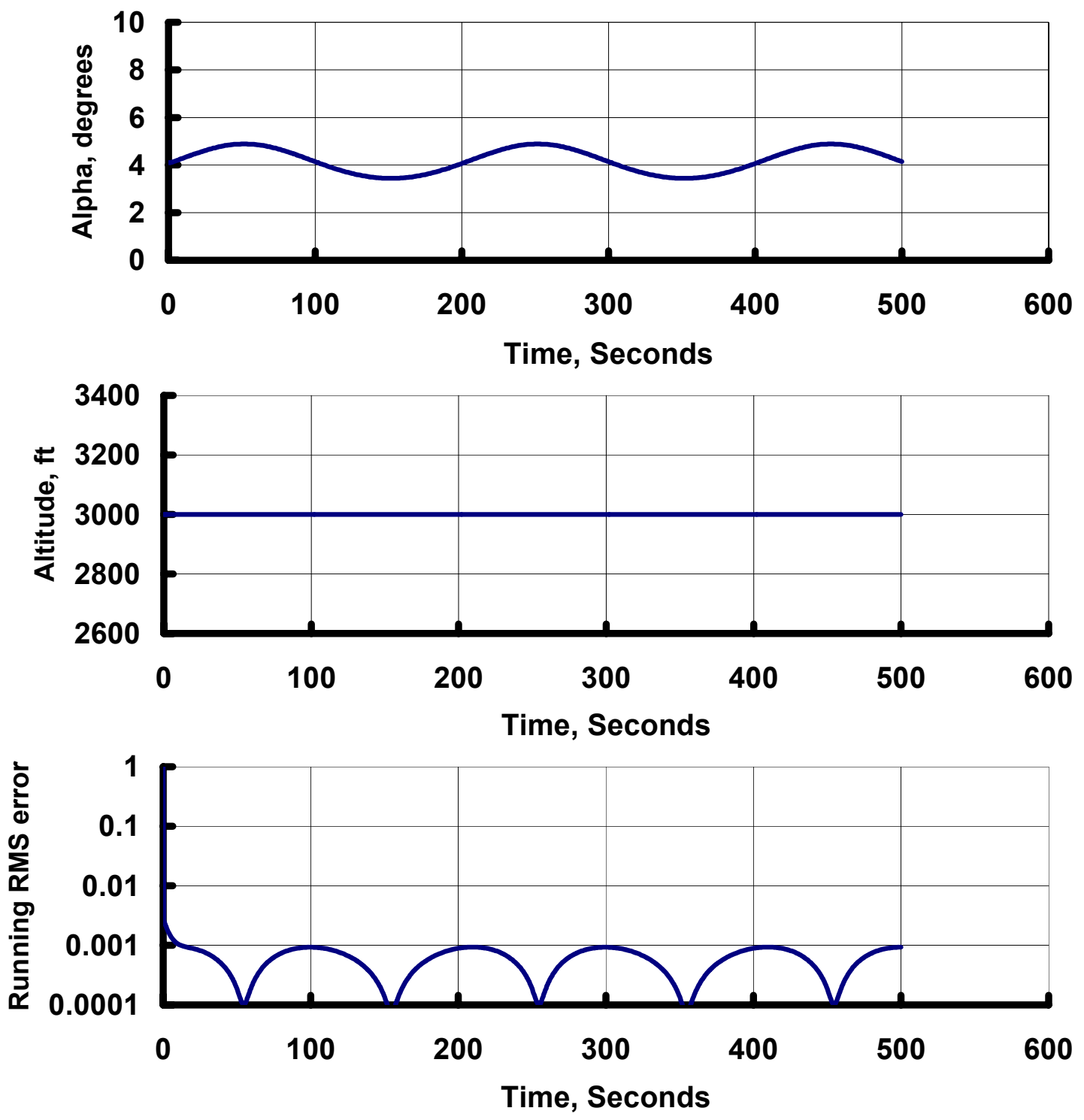

Figure 58 (c): Case 19 time history 


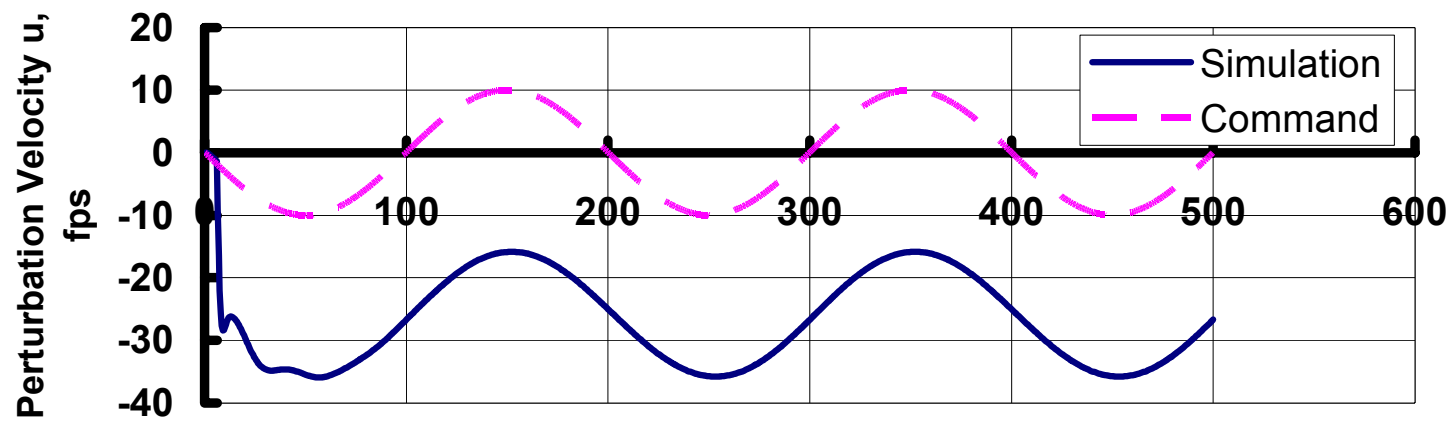

Time, Seconds

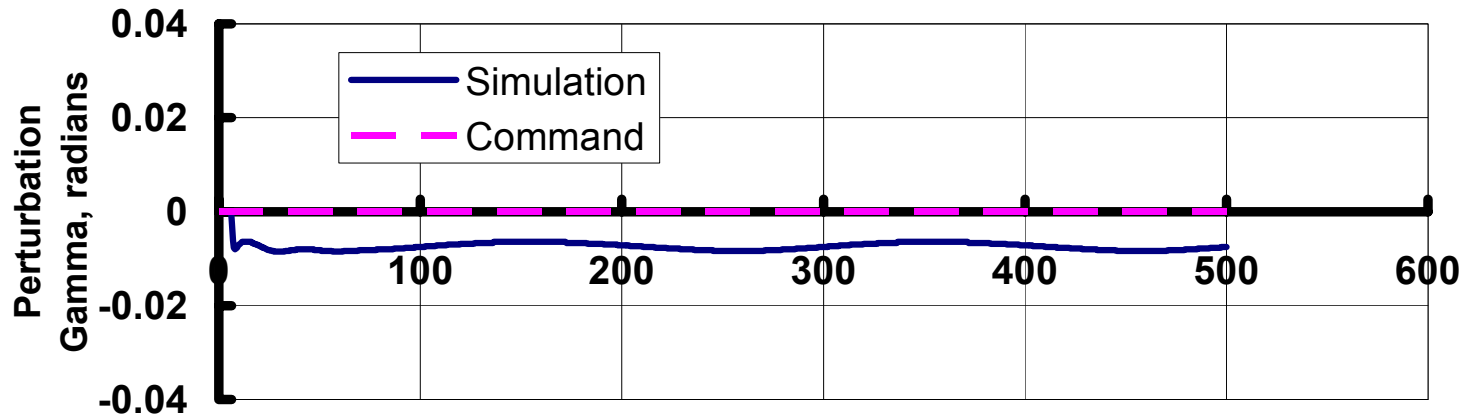

Time, Seconds

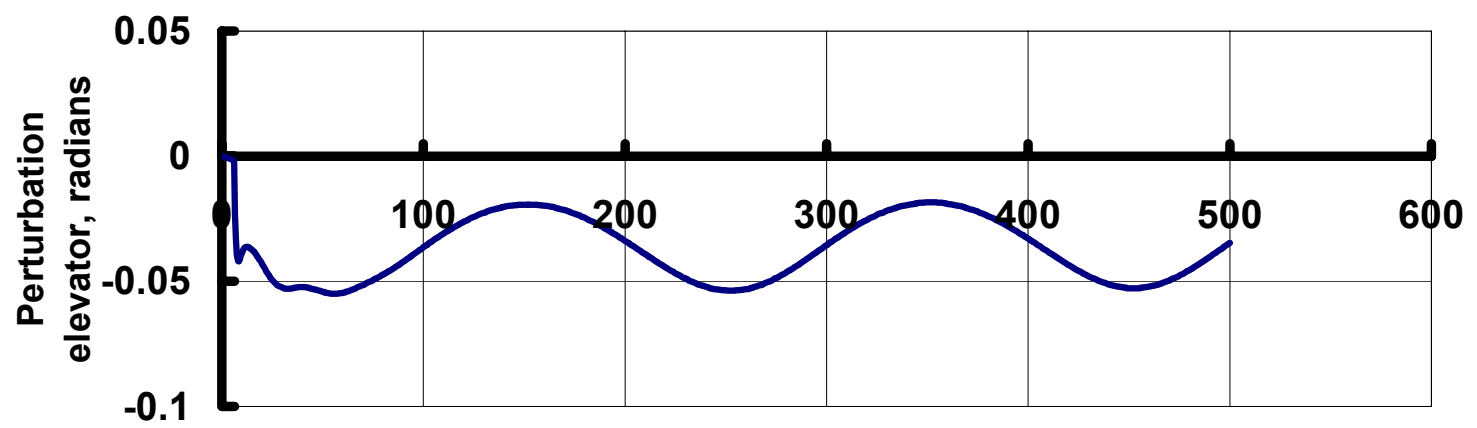

Time, Seconds

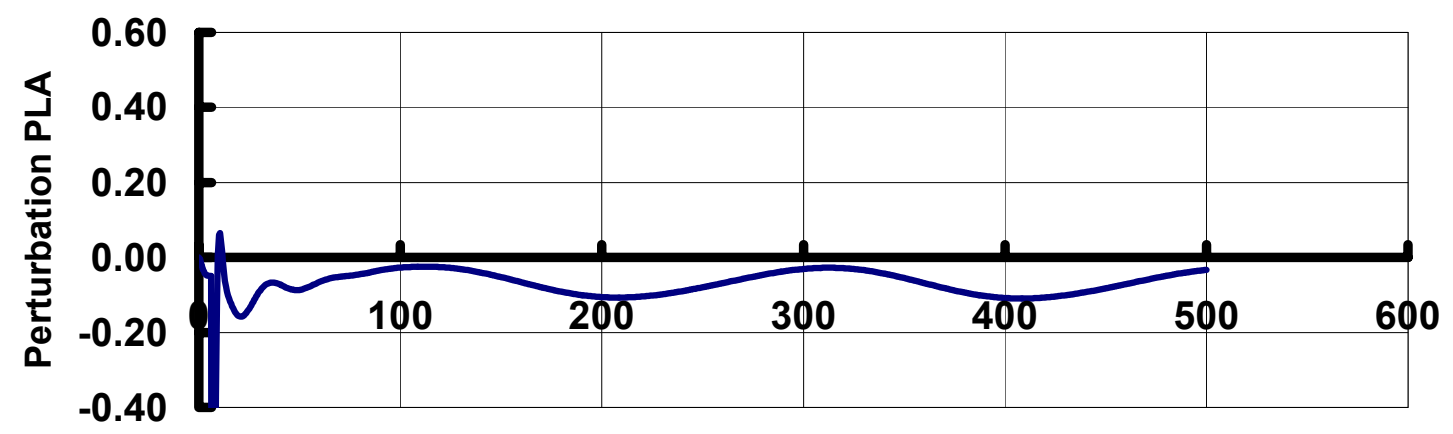

Time, Seconds

Figure 59 (a): Case 20 time history 

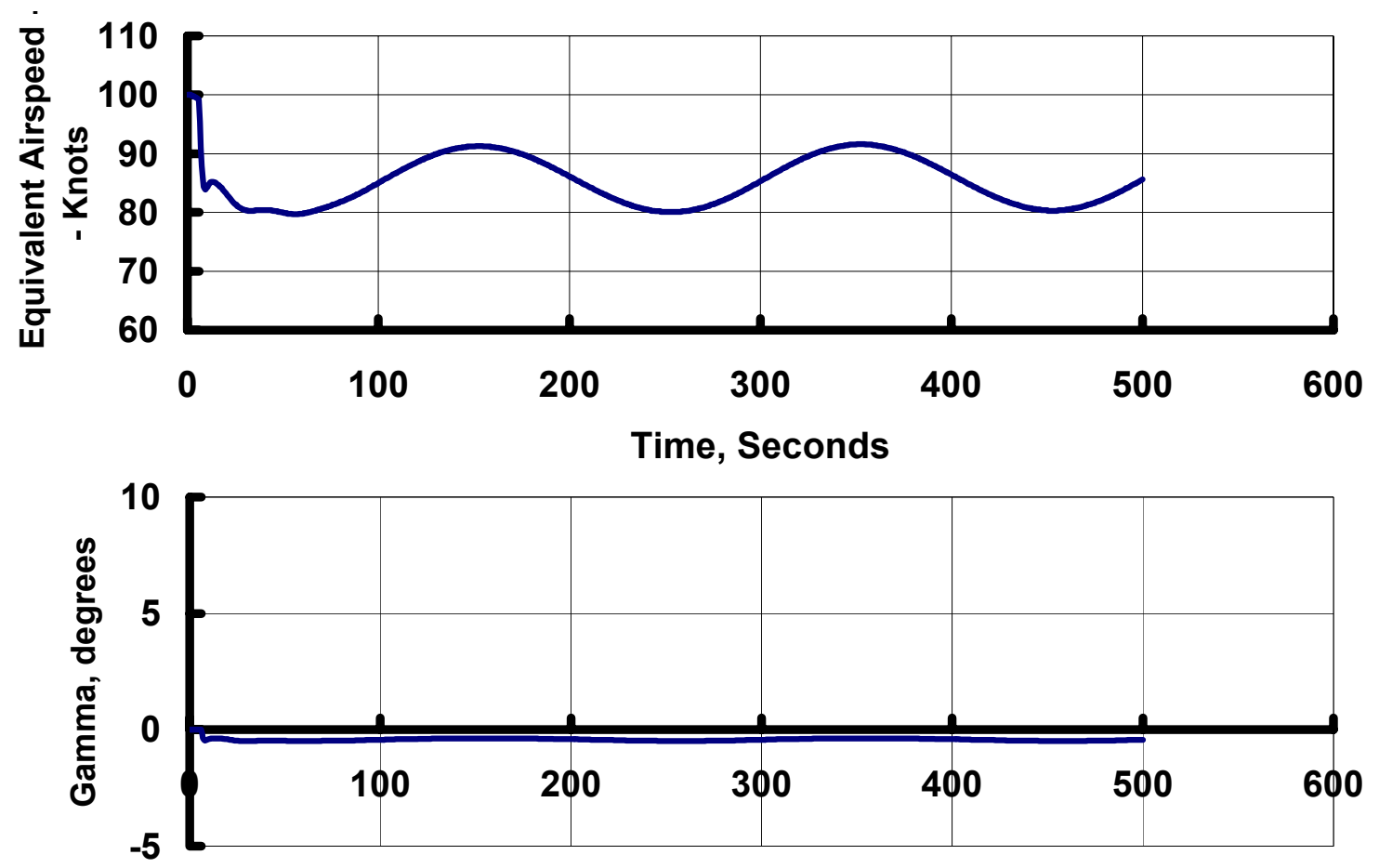

Time, Seconds

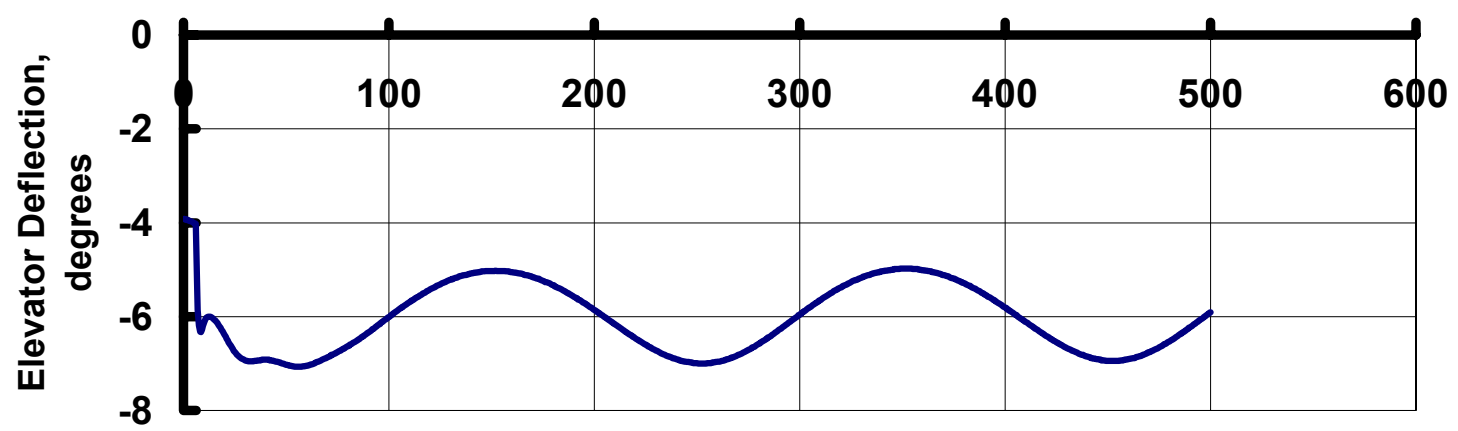

Time, Seconds

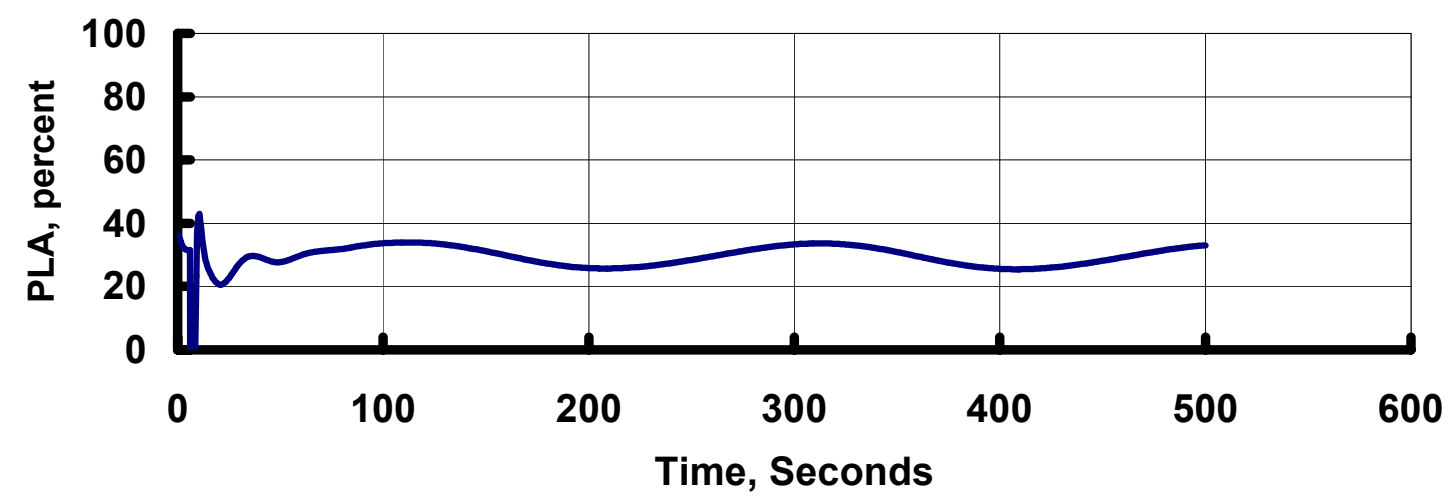

Figure 59 (b): Case 20 time history 

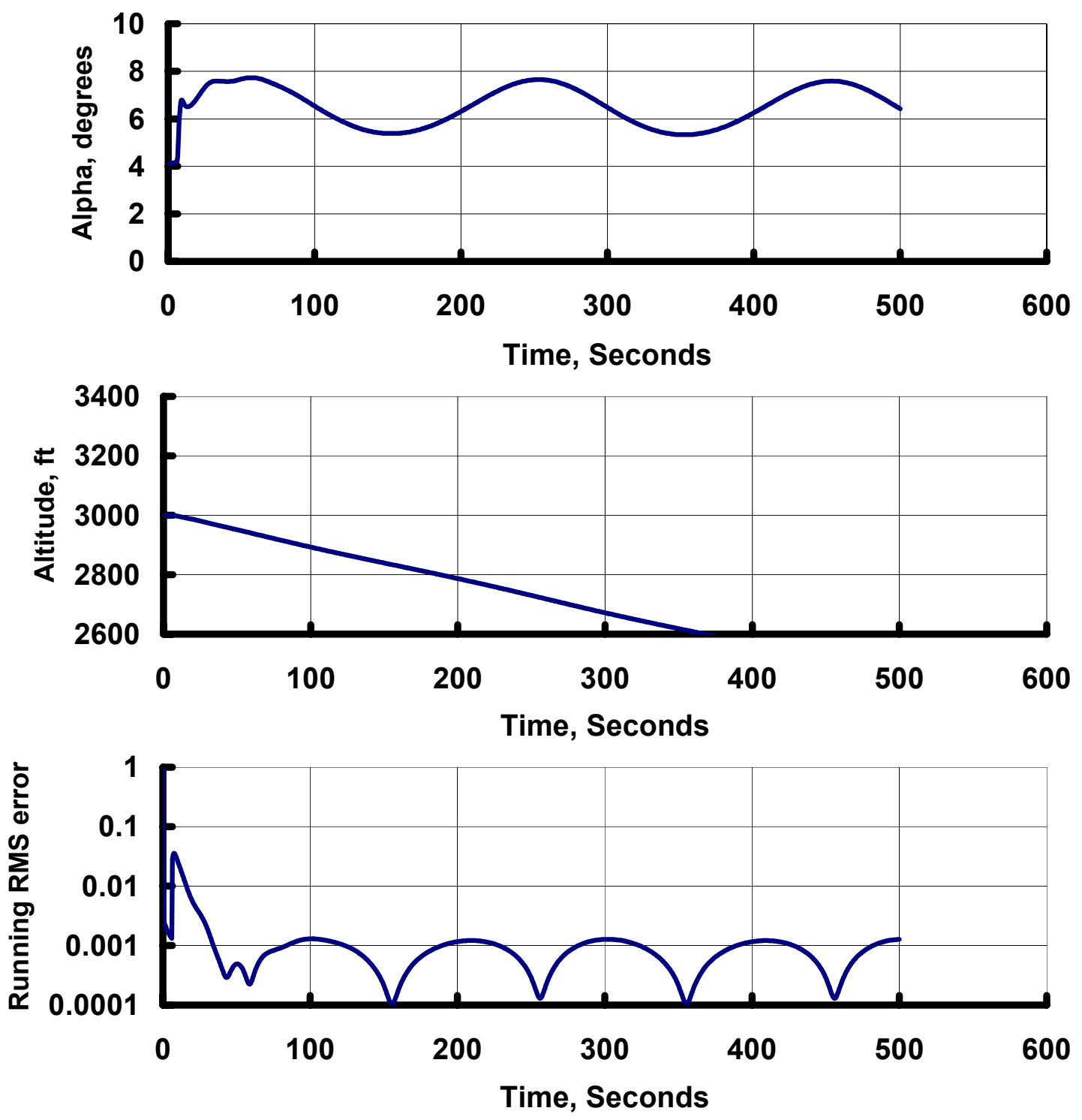

Figure 59 (c): Case 20 time history 
First and most easily explained is why $\Delta \dot{\mathbf{u}}$ failures influence speed and flight-path tracking, whereas $\Delta \dot{\alpha}$ failures only affect flight-path tracking. By referring back to the flightpath angle block diagram of Figure 20, it can be seen that the $\Delta \dot{\alpha}$ required to track the desired flight-path angle involves speed perturbations in the calculations. Errors in speed tracking will propagate into this function and create errors in flight-path tracking. The influence of this speed error is of the second-order when compared to flight-path errors and, consequently only a small error in flight-path angle results. In contrast, the speed control block diagram of Figure 18 has no angle of attack participation, and as a result, $\Delta \dot{\alpha}$ errors do not influence speed tracking. Of course, $\Delta \dot{\mathrm{u}}$ and $\Delta \dot{\alpha}$ failures each influence their respective tracking accuracy.

The question remains why the speed errors from a $\Delta \dot{\mathrm{u}}$ failure are significantly larger than the flight-path errors resulting from the $\Delta \dot{\alpha}$ failures, in relative terms. The answer is rather straight forward. The output scaling on $\Delta \dot{\mathrm{u}}$ is relatively large, approximately $16 \mathrm{feet} / \mathrm{sec}^{2}$ for the training data used in this investigation, or almost a half g. The influence of this scaling factor may be assessed by examining the dynamics of the speed controller. Figure 63 illustrates the speed controller with a neural network error, $\varepsilon(\mathrm{s})$, injected. Because of the equivalency of $\mathbf{G}^{-1}(\mathrm{~s}) \mathbf{P}_{\mathrm{A}}(\mathrm{s})$ to an integrator, this system can be viewed as a single-input single-output (SISO) system. The system transfer function can be readily derived. 


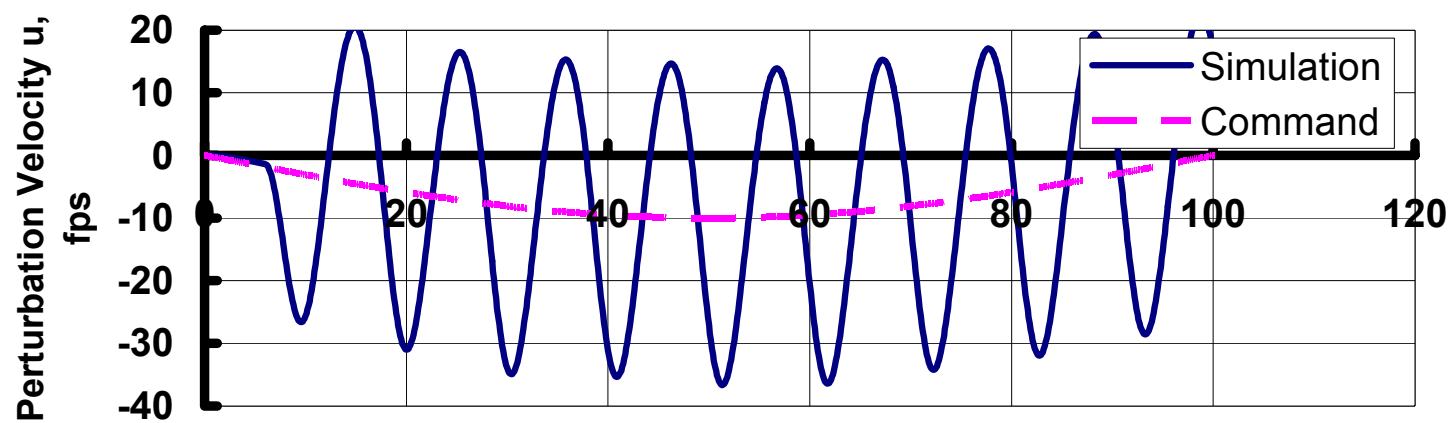

Time, Seconds

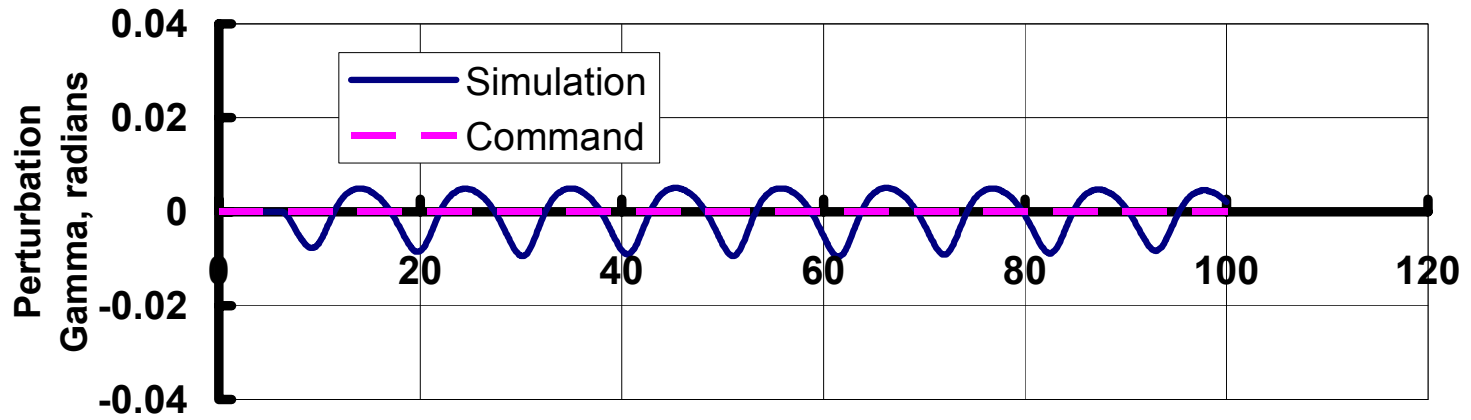

Time, Seconds

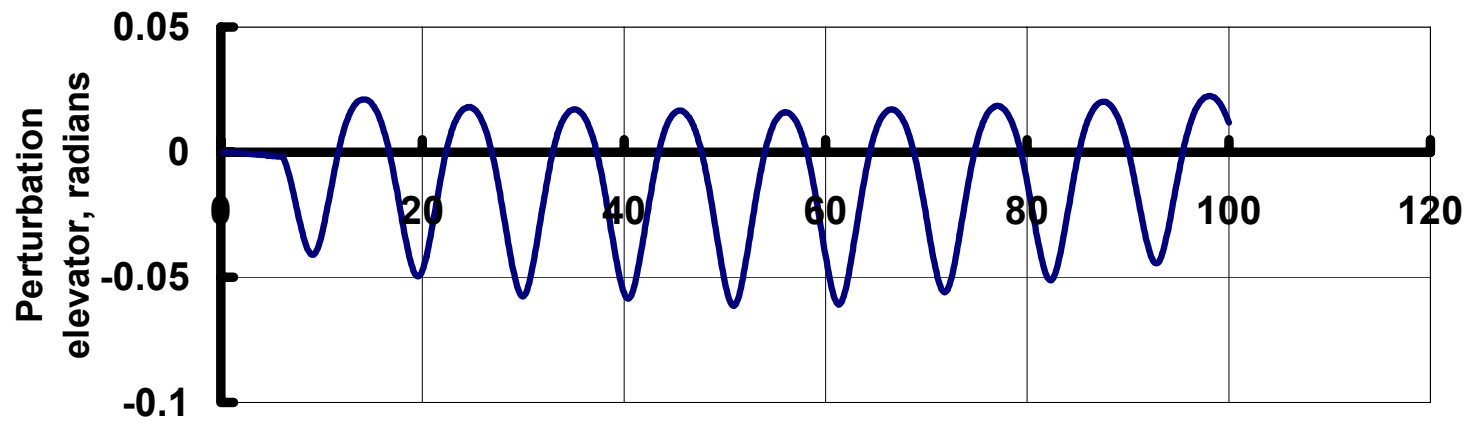

Time, Seconds

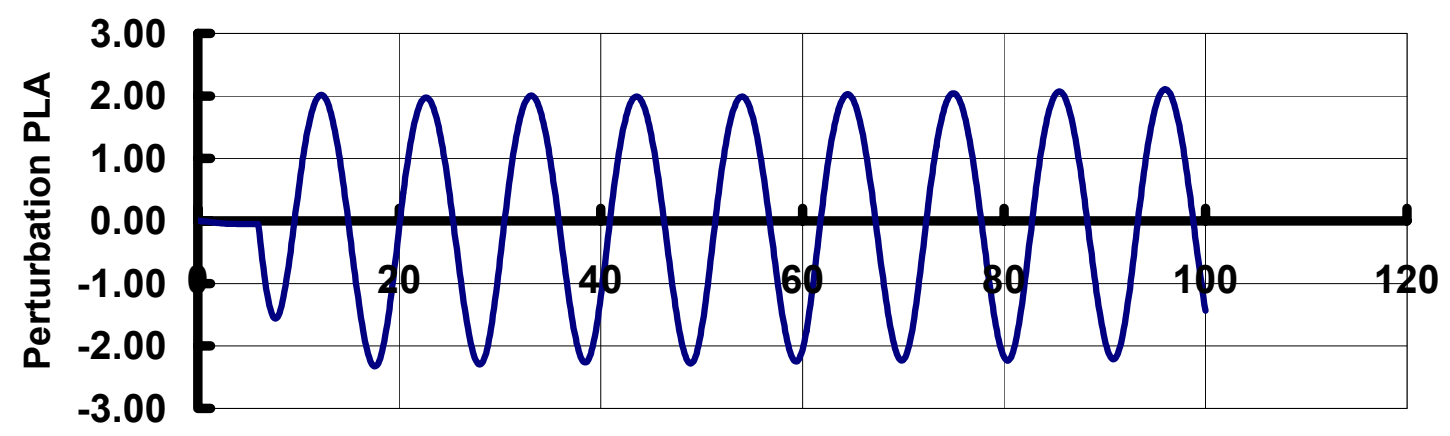

Time, Seconds

Figure 60 (a): Case 21 time history 

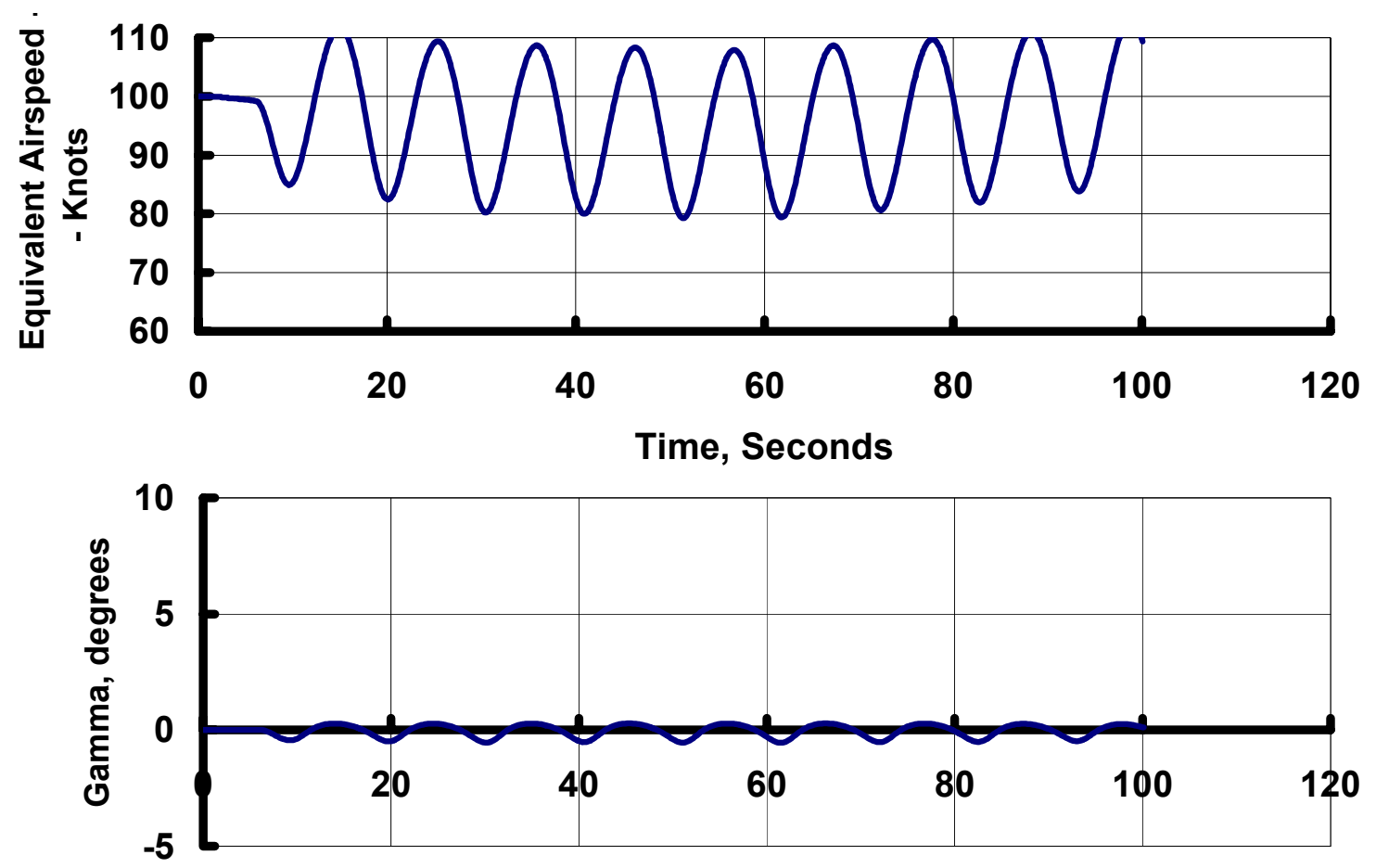

Time, Seconds

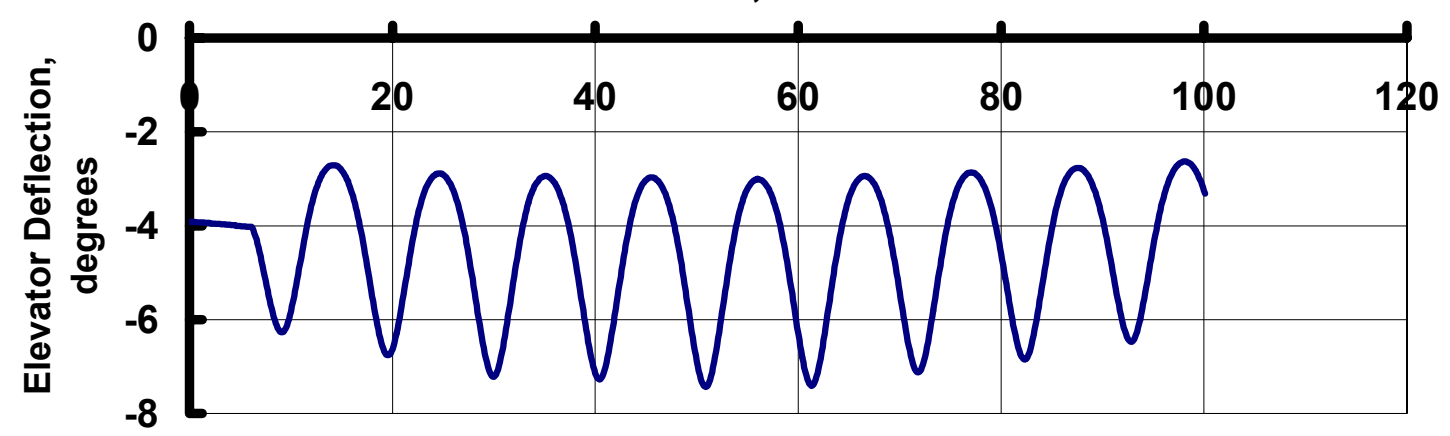

Time, Seconds

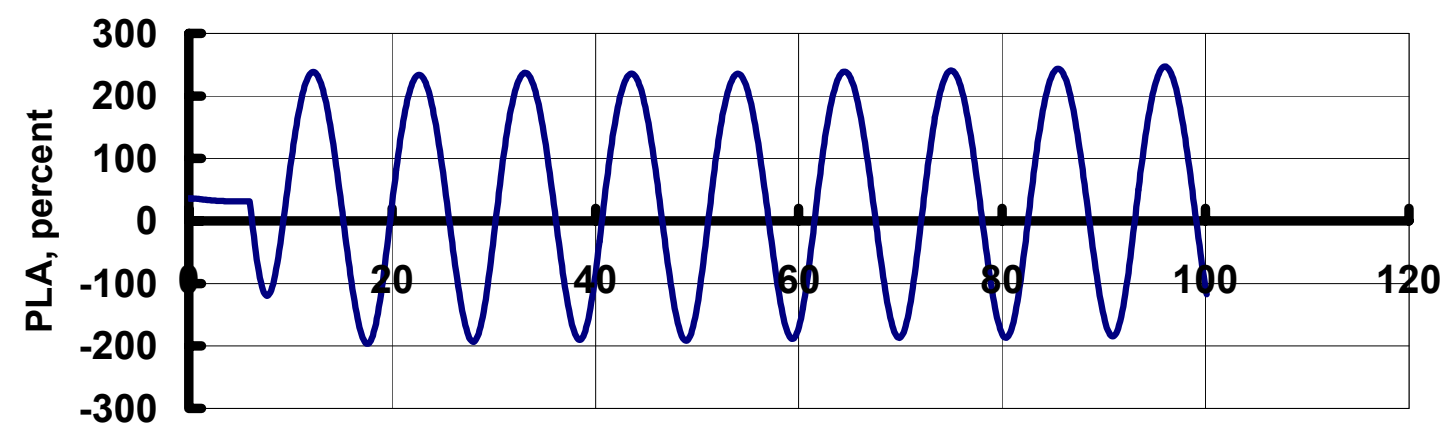

Time, Seconds

Figure 60 (b): Case 21 time history 

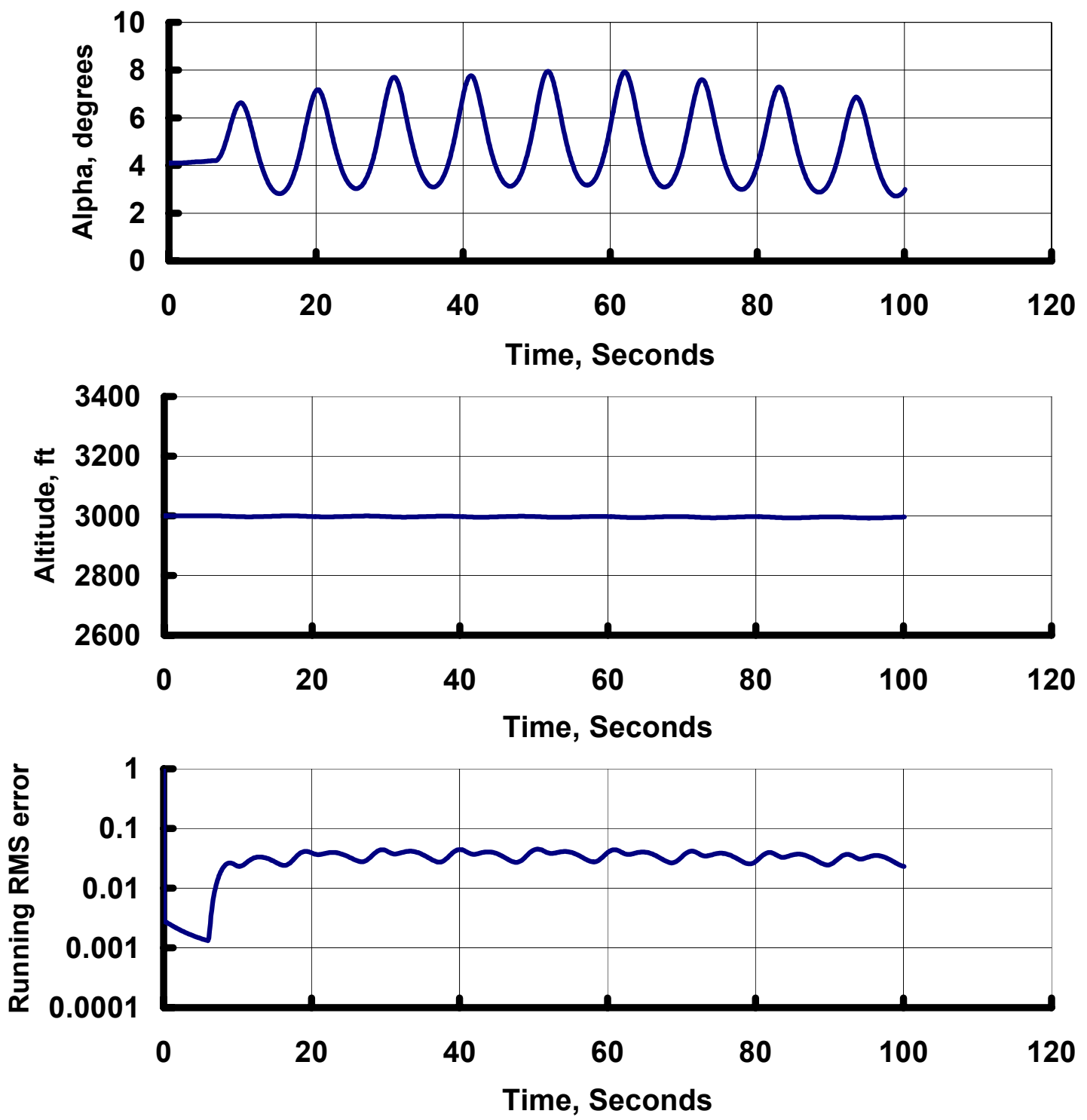

Figure 60 (c): Case 21 time history 


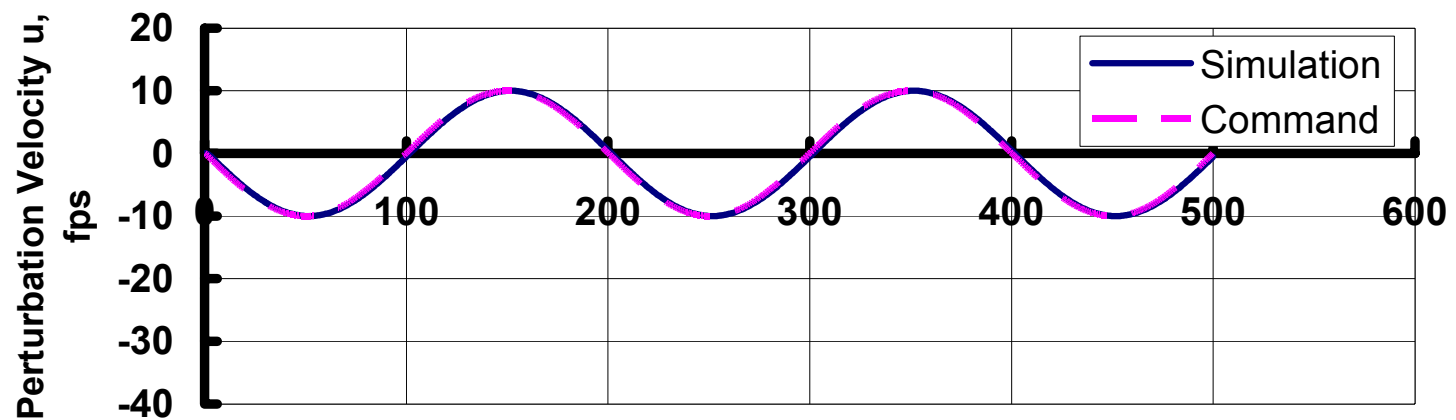

Time, Seconds

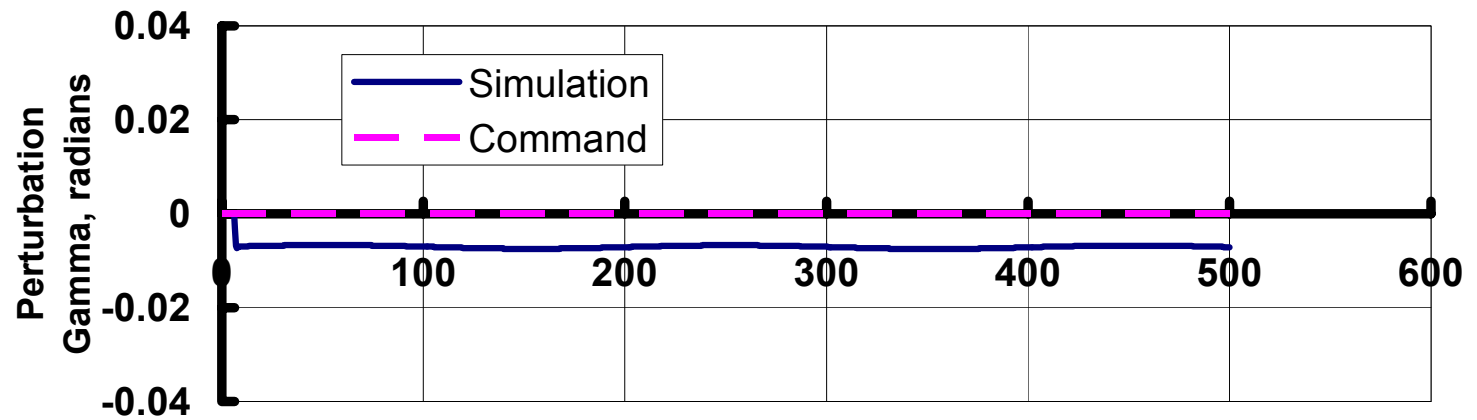

Time, Seconds

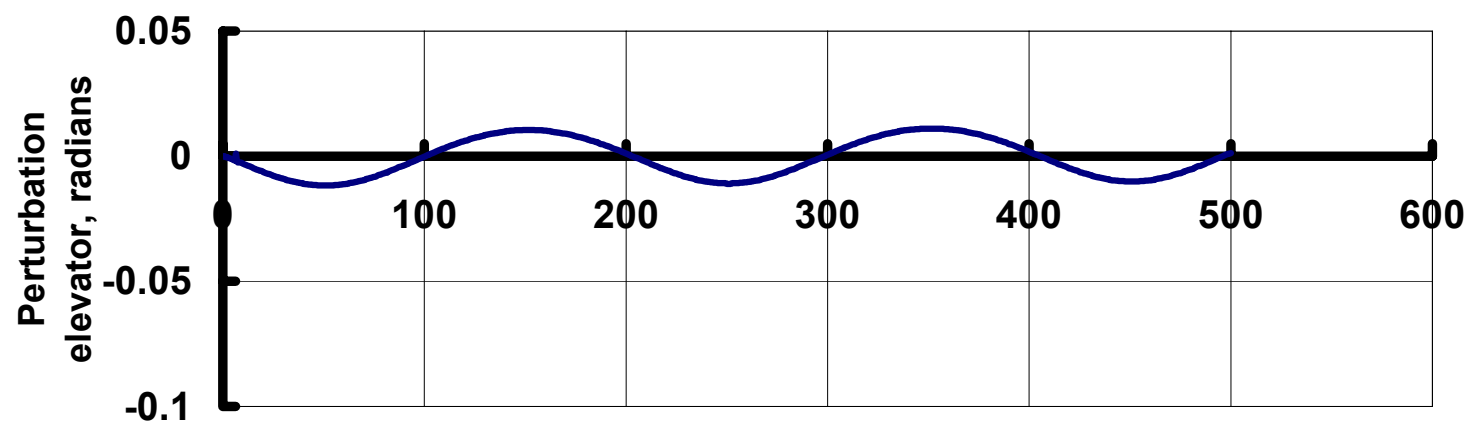

Time, Seconds

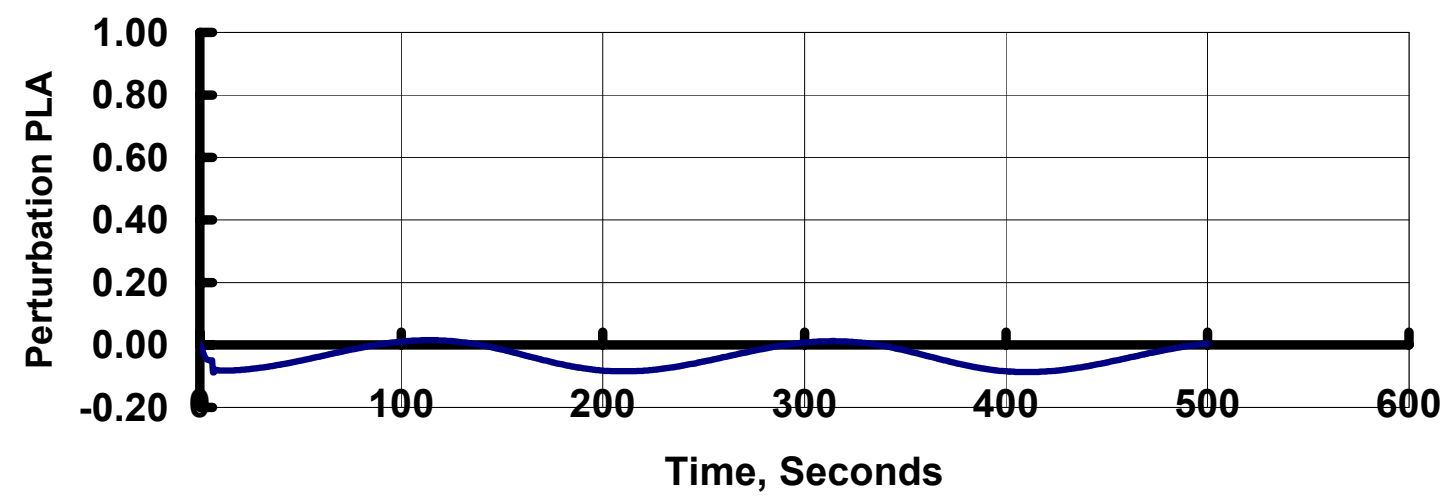

Figure 61 (a): Case 22 time history 

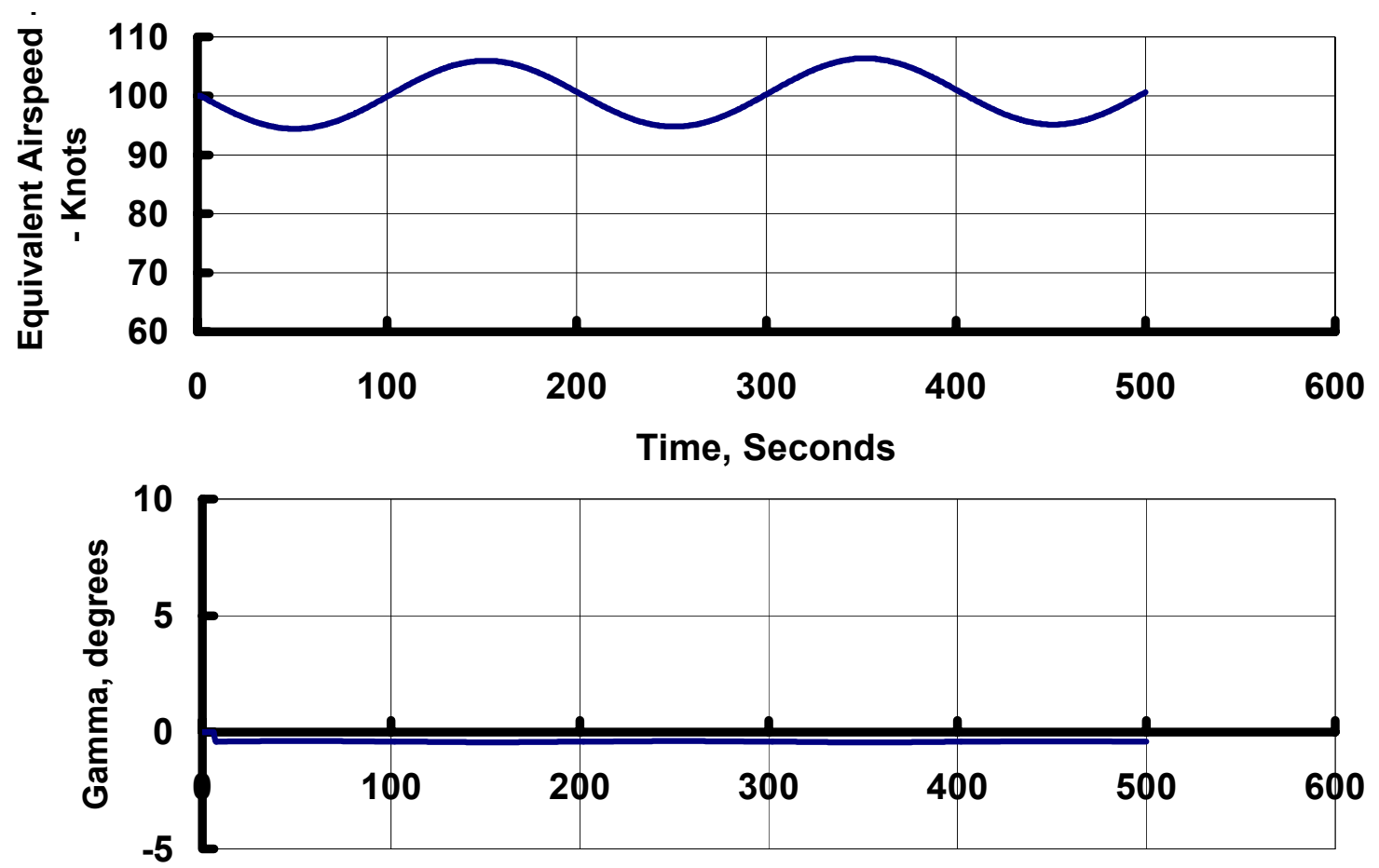

Time, Seconds

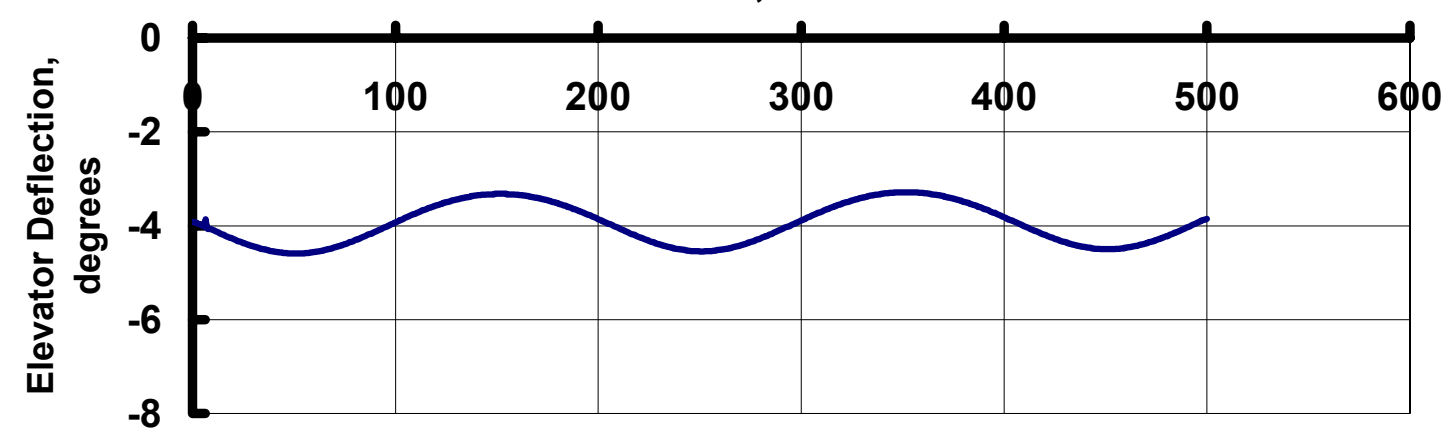

Time, Seconds

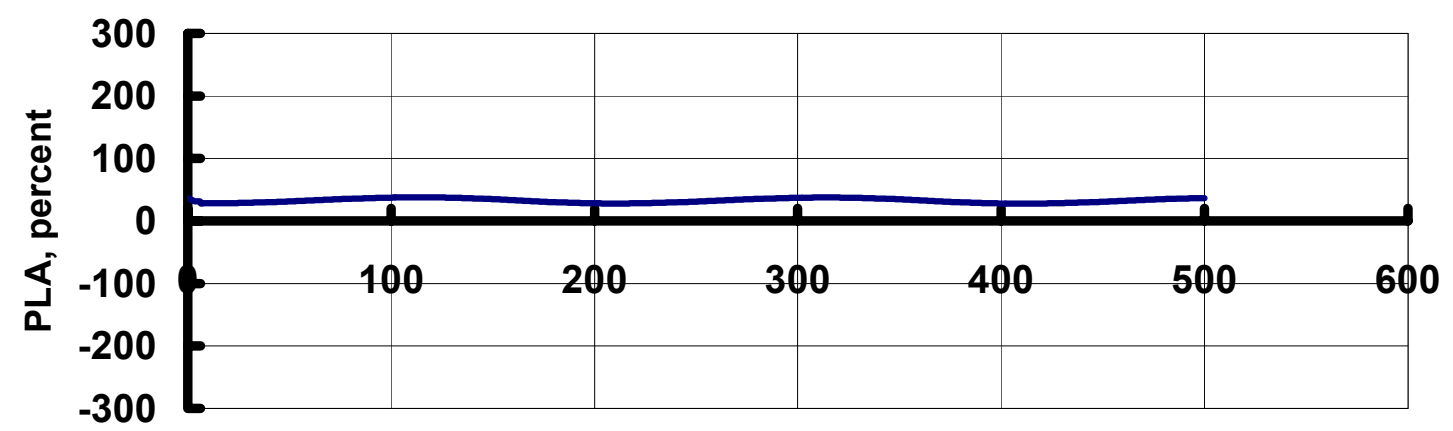

Time, Seconds

Figure 61 (b): Case 22 time history 

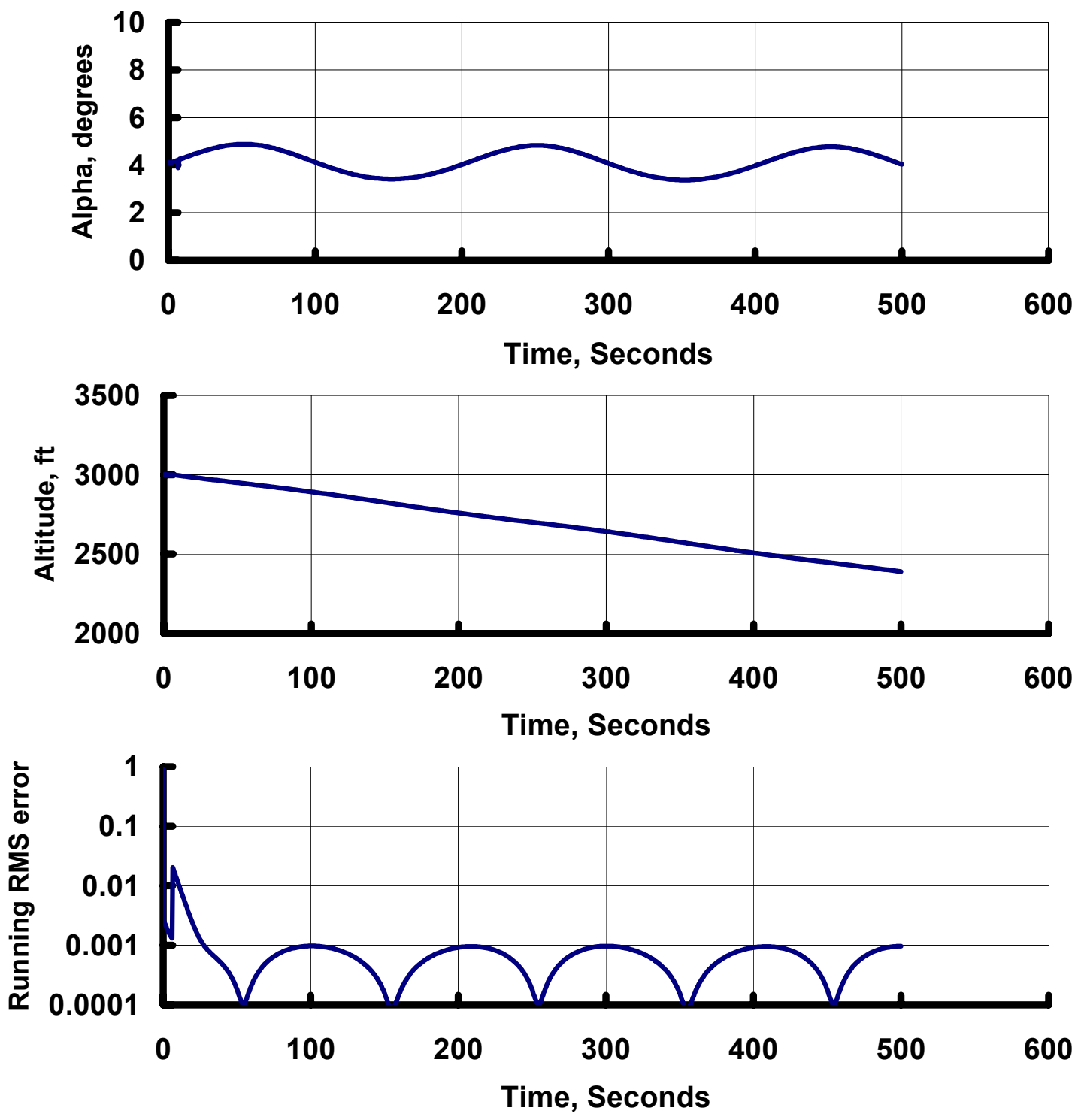

Figure 61 (c): Case 22 time history 


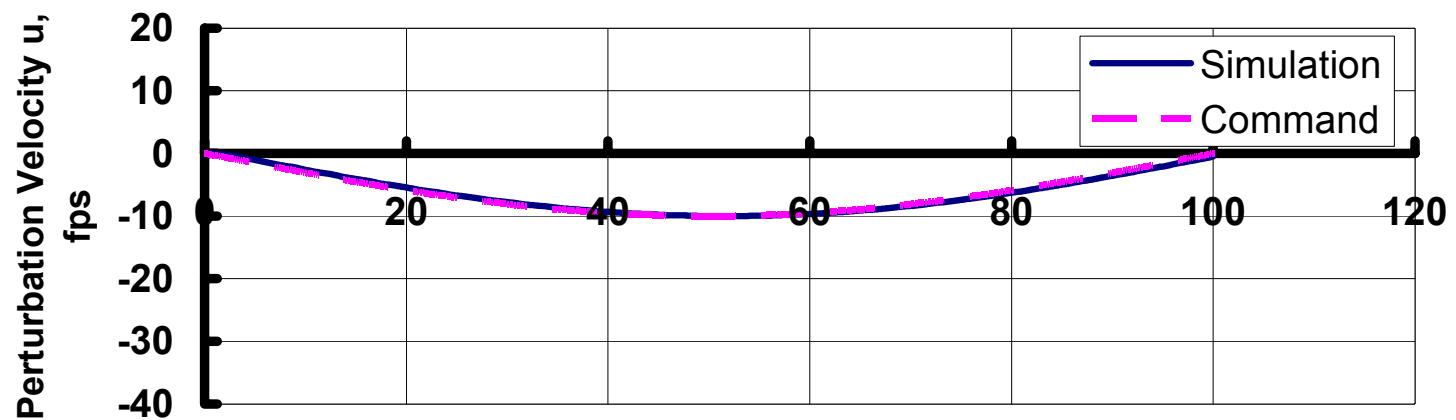

Time, Seconds

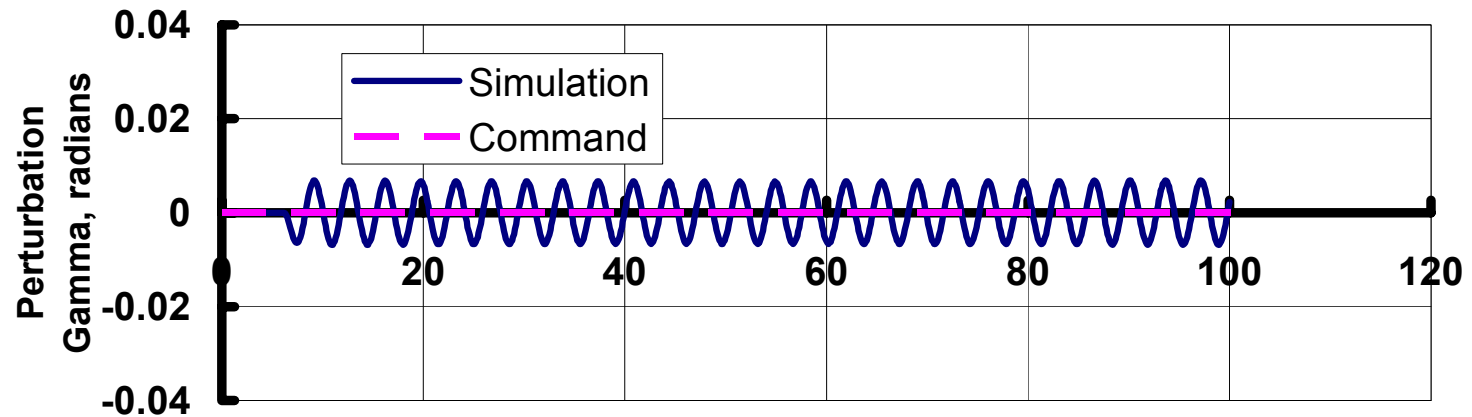

Time, Seconds

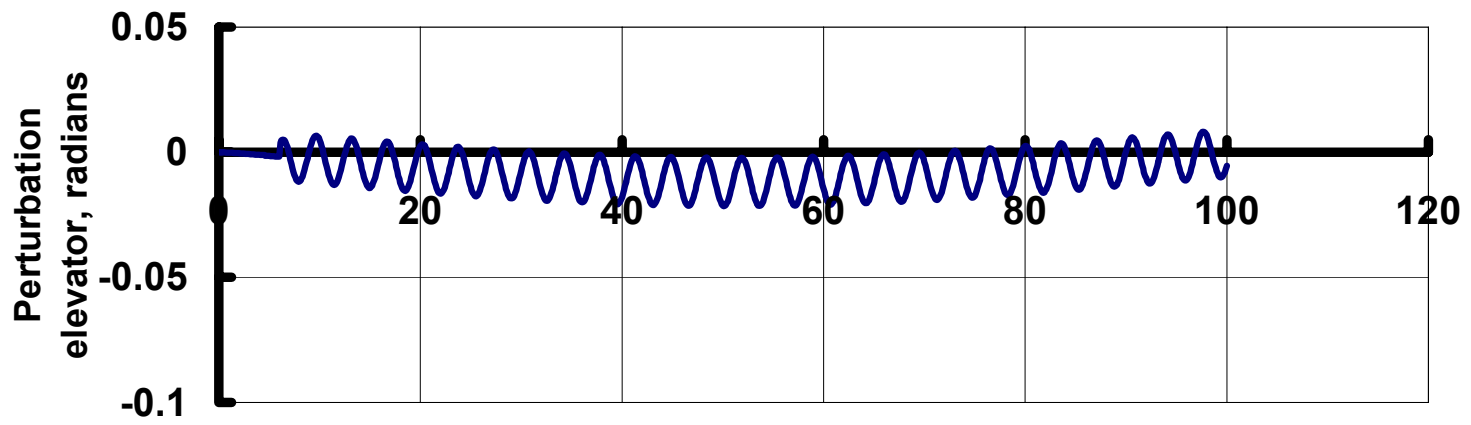

Time, Seconds

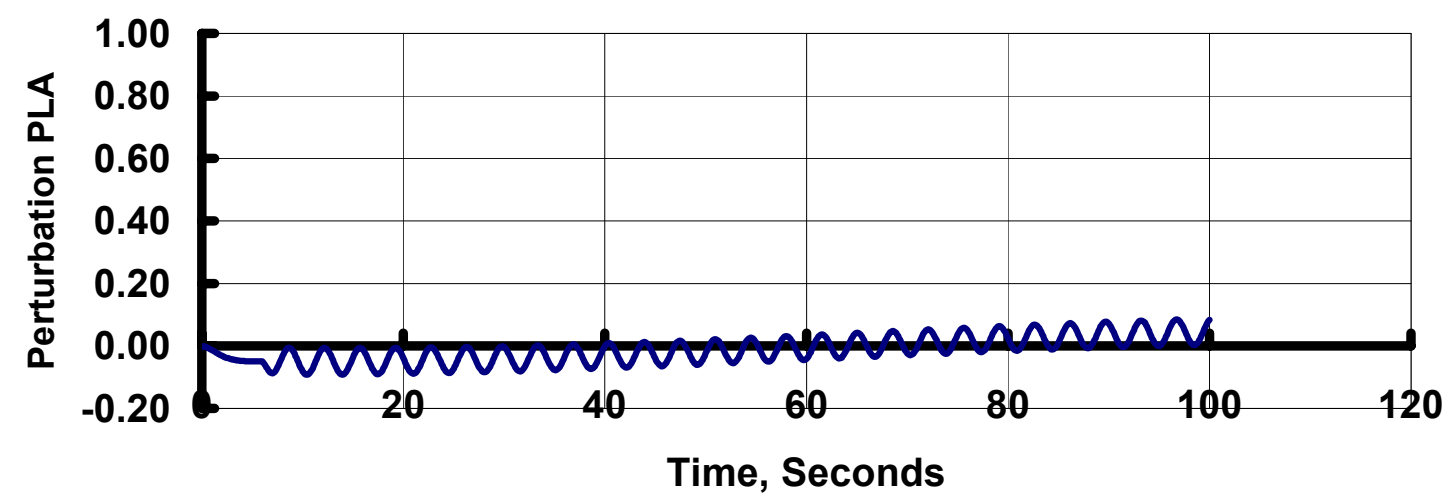

Figure 62 (a): Case 23 time history 

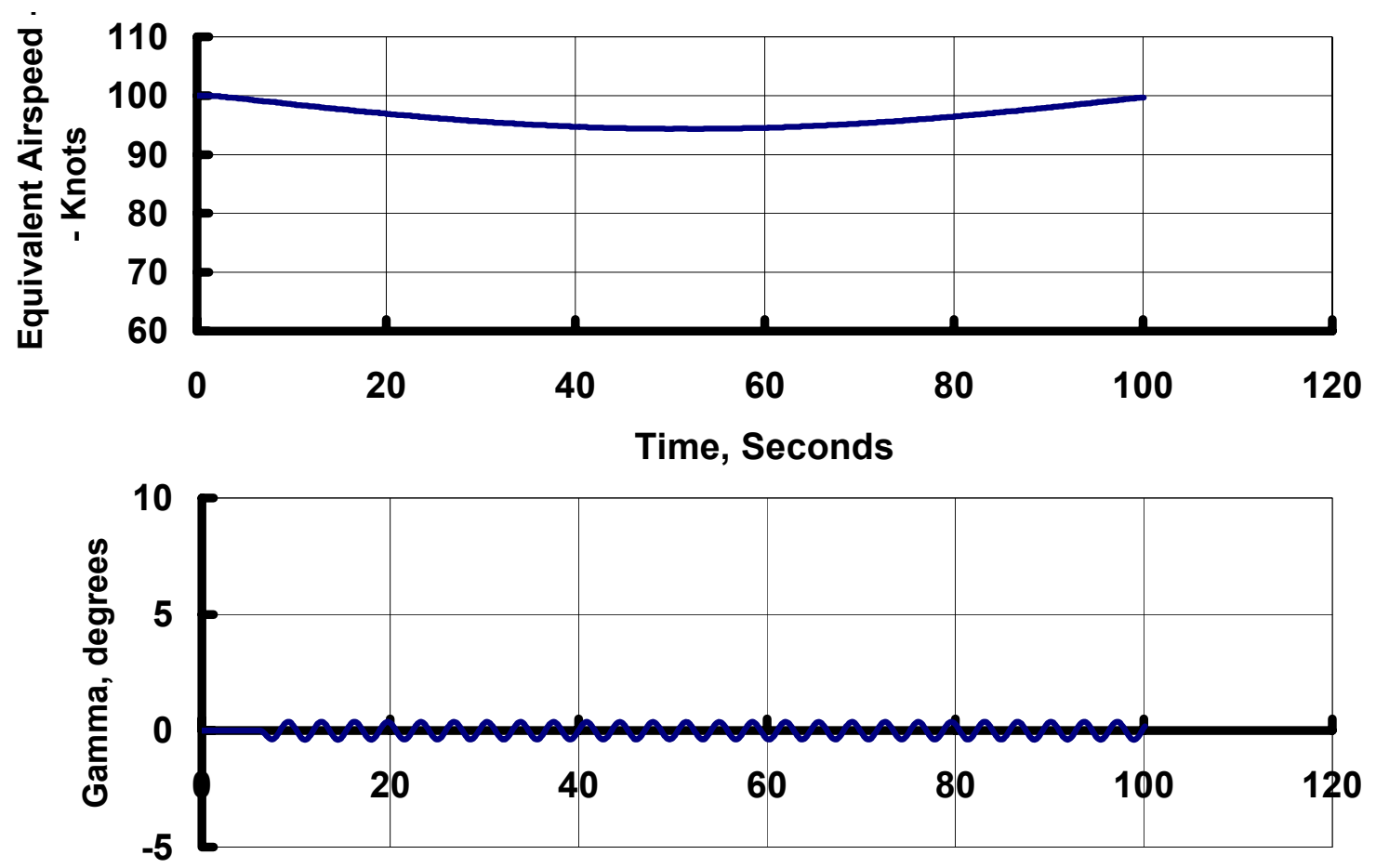

Time, Seconds

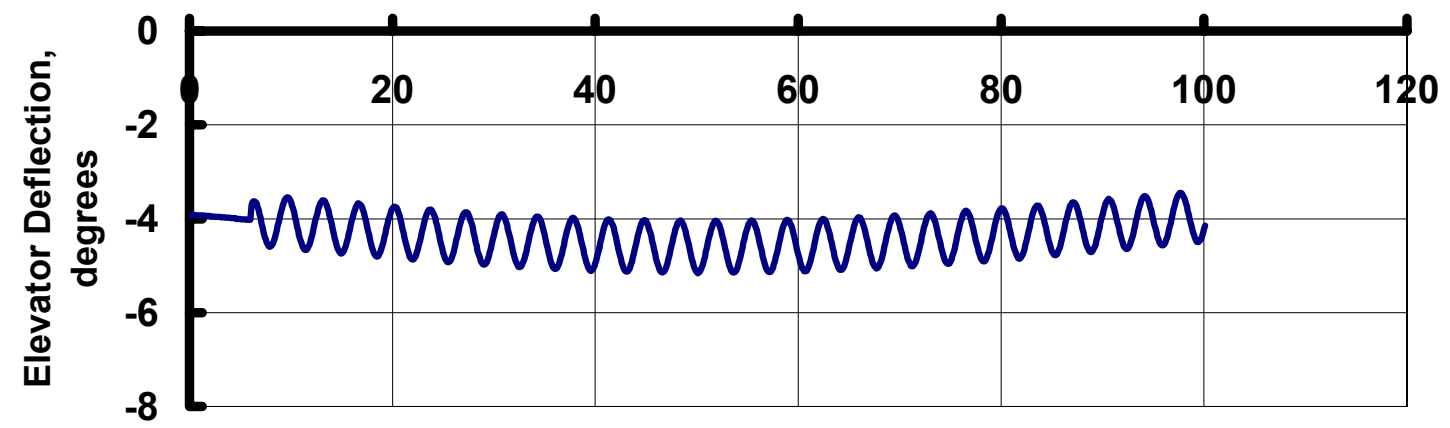

Time, Seconds

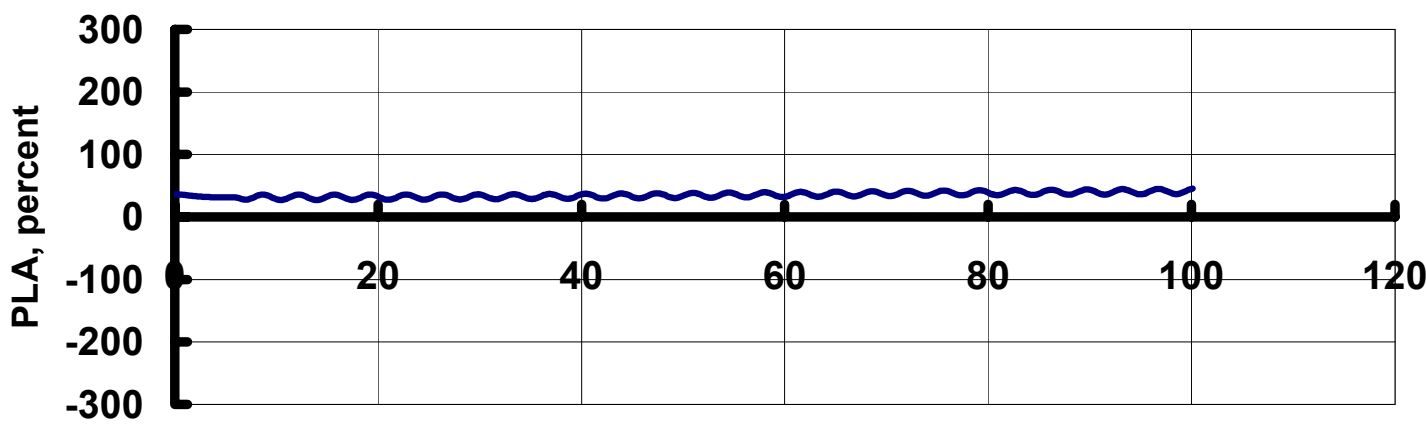

Time, Seconds

Figure 62 (b): Case 23 time history 

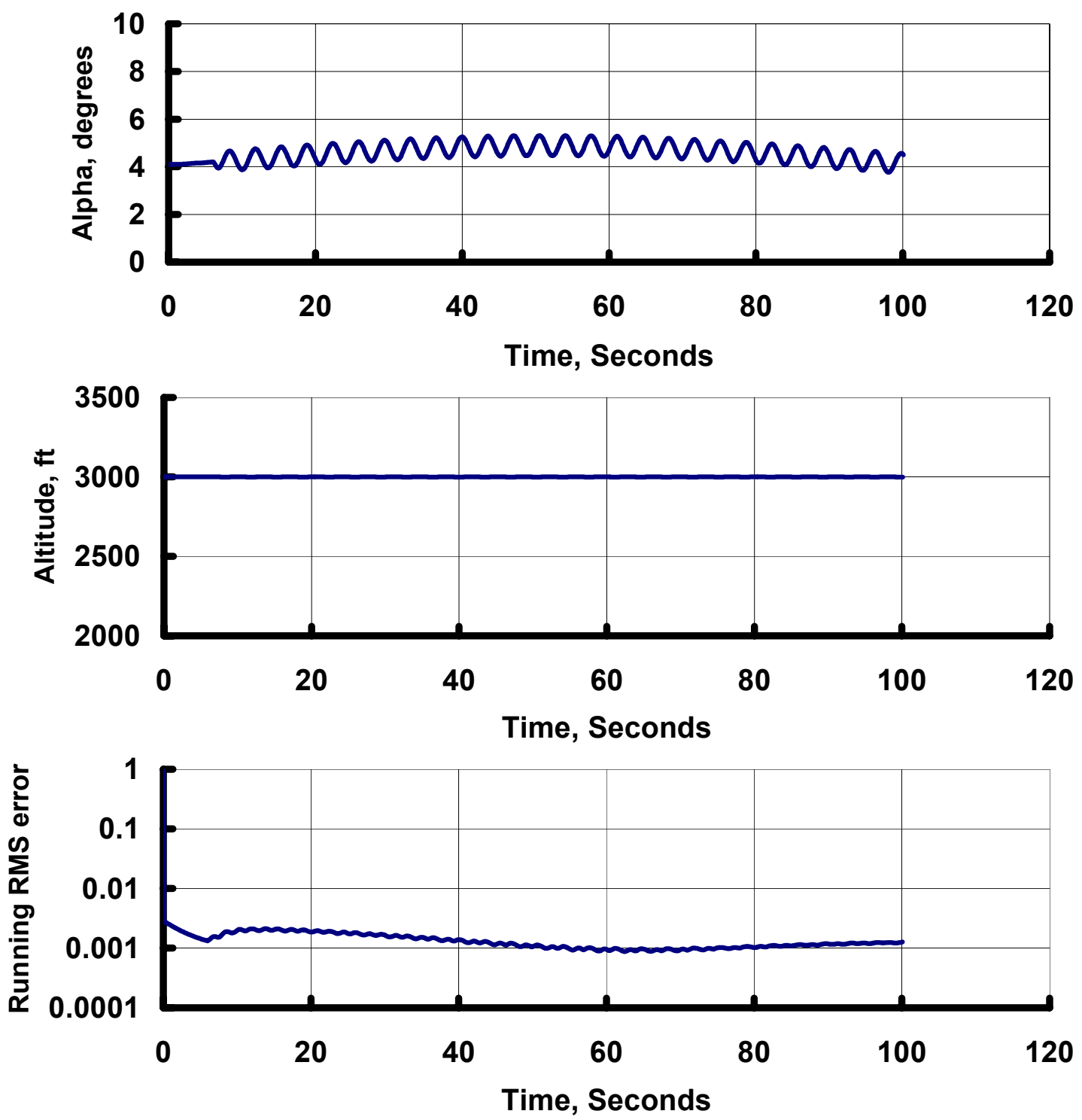

Figure 62 (c): Case 23 time history 


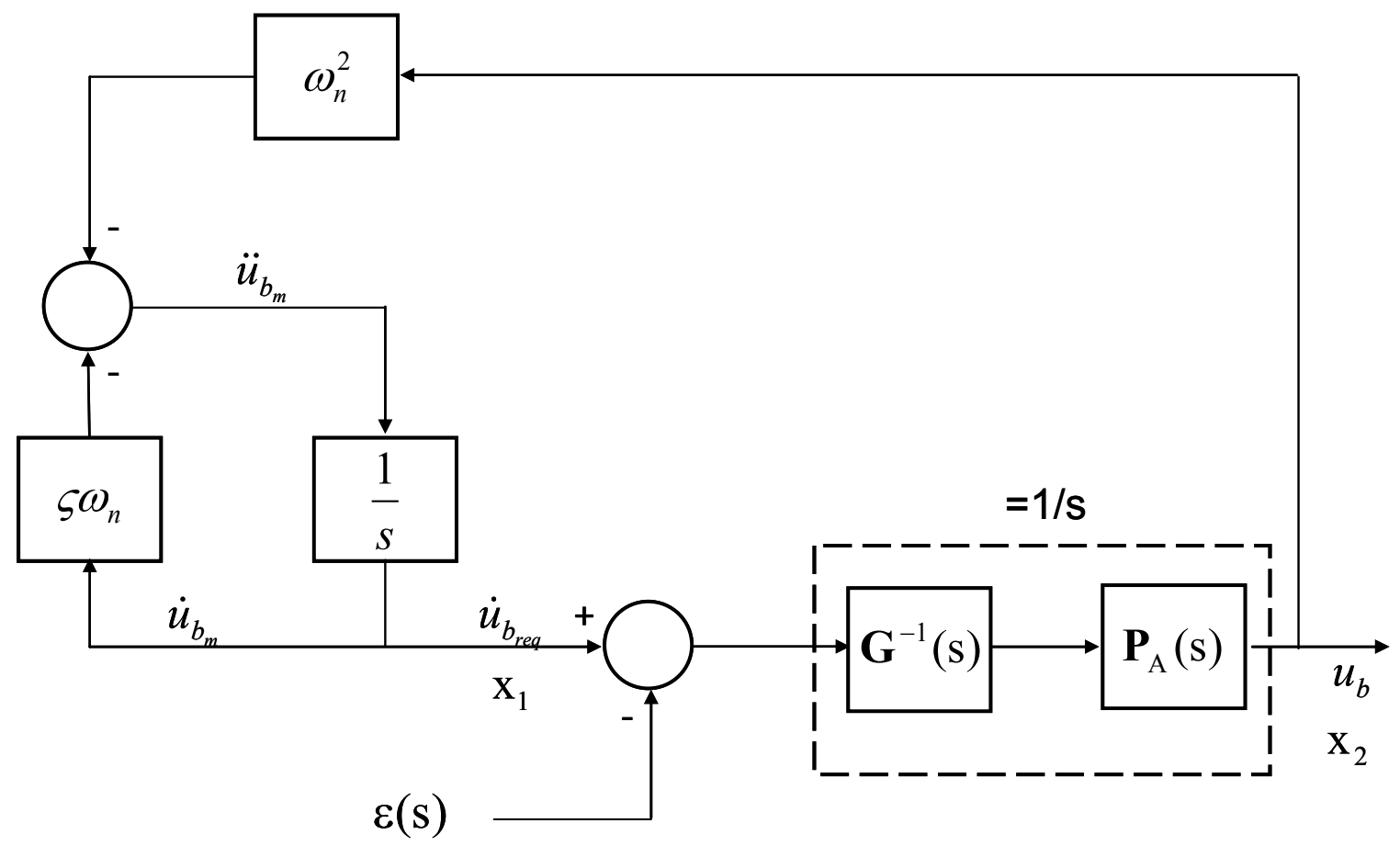

Figure 63: Speed controller block diagram with erroneous neural network signal

The state space representation of this system is

$$
\begin{gathered}
\left\{\begin{array}{l}
\dot{\mathrm{x}}_{1} \\
\dot{\mathrm{x}}_{2}
\end{array}\right\}=\left[\begin{array}{cc}
-2 \varsigma \omega_{\mathrm{n}} & -\omega_{\mathrm{n}}^{2} \\
1 & 0
\end{array}\right]\left\{\begin{array}{l}
\mathrm{x}_{1} \\
\mathrm{x}_{2}
\end{array}\right\}+\left[\begin{array}{c}
0 \\
-1
\end{array}\right] \varepsilon(\mathrm{t}) \\
\mathrm{u}_{\mathrm{b}}=\mathrm{x}_{2}
\end{gathered}
$$

The A matrix of this system is exactly the same as the A matrix for the $u$ channel of the linear prefilter in normal operation. The eigenvalues are determined by the characteristic equation

$$
\mathrm{s}^{2}+2 \varsigma \omega_{\mathrm{n}} \mathrm{s}+\omega_{\mathrm{n}}^{2}=0,
$$

which are similar to the nominal controller. 
The steady-state response of the system to a constant input $\varepsilon$ is

$$
\mathrm{u}_{\mathrm{b}}=-\frac{2 \varsigma}{\omega_{\mathrm{n}}} \varepsilon
$$

The steady-state response for a sinusoidal input $\varepsilon(\mathrm{t})=\sin \omega \mathrm{t}$ is

$$
\left|\mathrm{u}_{\mathrm{b}}\right|=|\varepsilon| \sqrt{\frac{\omega^{2}+\left(2 \varsigma \omega_{\mathrm{n}}\right)^{2}}{\left(\omega^{2}-\omega_{\mathrm{n}}^{2}\right)^{2}+\left(2 \varsigma \omega_{\mathrm{n}} \omega\right)^{2}}}
$$

For all sample cases, $\zeta=.80$ and $\omega_{\mathrm{n}}=1.0$. For the hardover case, $\varepsilon=16$ and the resulting steady-state error on $u_{\mathrm{b}}$ would be $25.6 \mathrm{feet} / \mathrm{sec}$. This theoretical error agrees quite well with the simulation results shown in Figure 59. For a sinusoidal input where $\varepsilon(t)=16 \sin (.6 \mathrm{t})$, the steady-state speed error amplitude would be $23.7 \mathrm{feet} / \mathrm{sec}$, which also agrees well with the simulation results shown in Figure 60. A Bode chart illustrating the speed sensitivity of the system response to $\Delta \dot{\mathrm{u}}$ failures is shown in Figure 64. It is common to associate critical oscillatory failures with the system resonant frequency; however, in this case the damping ratio is so high that for any frequency above zero, the system gain is decreasing.

A similar analysis can be undertaken for the flight-path angle control. However, this exercise is more complex because this channel of the control is not a single-input single-output system and is not conducive to a closed form expression for the errors. MATLAB ${ }^{\mathrm{TM}}$ is used to compute the frequency response. Figure 65 is a Bode chart showing the flight-path sensitivity to a $\Delta \dot{u}$ oscillatory failure.

The flight-path sensitivity to $\Delta \dot{\alpha}$ failures is calculated with MATLAB ${ }^{\mathrm{TM}}$ in the same manner. The flight-path angle sensitivity to oscillatory $\Delta \dot{\alpha}$ failures is shown in Figure 66 . As previously observed, $\Delta \dot{\alpha}$ failures have no effect on speed tracking. 


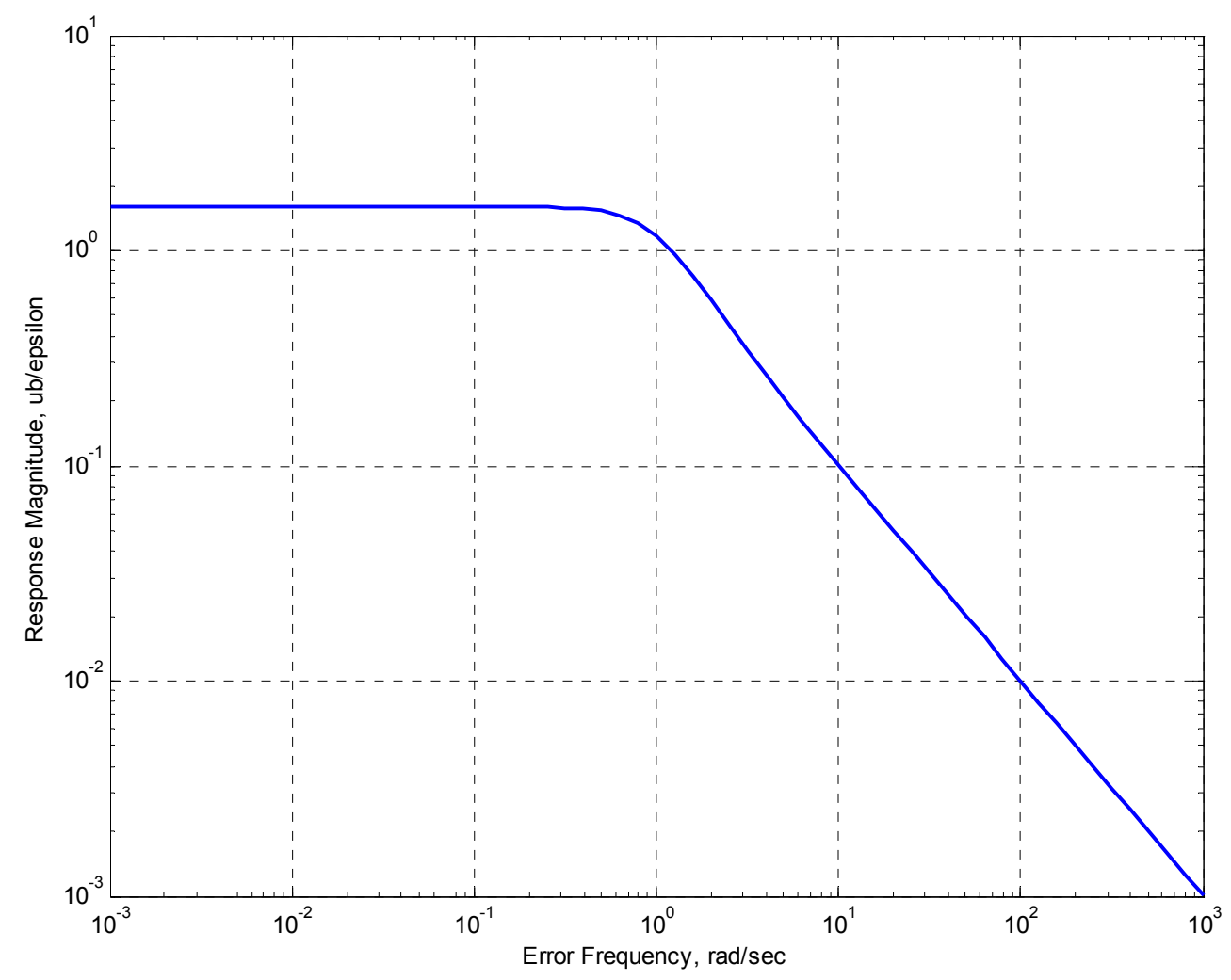

Figure 64: Speed sensitivity to oscillatory $\Delta \dot{\mathrm{u}}$ errors from the neural network

The key thought to take away from this discussion is that the overall system response to a network failure is proportional to the output scaling. The output scaling is derived from the range of $\Delta^{\prime}$ s accommodated during training. A network of this architecture designed to accommodate aggressive maneuvering will be susceptible to large bounded speed errors in the event of an undetected uncorrected network malfunction. A corollary to this is that the designer can make the response to undetected network benign if the control system is designed for benign maneuvers. It must be reiterated that the training maneuvers used throughout all of these sample cases were specifically chosen to be aggressive for the purposes of exploring the boundaries of this design paradigm. A network trained specifically for an easy-to-fly General Aviation aircraft 
would likely be designed for docile flight, with lower values experienced for $\Delta \dot{u}$ and $\Delta \dot{\alpha}$. This sort of flight would permit smaller scaling factors for $\Delta \dot{u}$ and $\Delta \dot{\alpha}$, and consequently the controller would experience less susceptibility to speed tracking errors in the event of a neural network failure.

Thus, two new design parameters emerge for the design of the control system: scale factors for $\Delta \dot{\mathrm{u}}$ and scale factors for $\Delta \dot{\alpha}$. These parameters determine the aggressiveness accommodated by the neural network, with the tradeoff that large scaling factors for $\Delta \dot{\mathrm{u}}$ will result in speed excursions proportional to $\Delta \dot{u}$ in the event of a neural network failure of the $\Delta \dot{\mathrm{u}}$ channel. As already discussed, the parameters $\zeta_{u}, \omega_{n_{u}}, \zeta_{\gamma}$, and $\omega_{n_{\gamma}}$ determine the responsiveness of the system. 


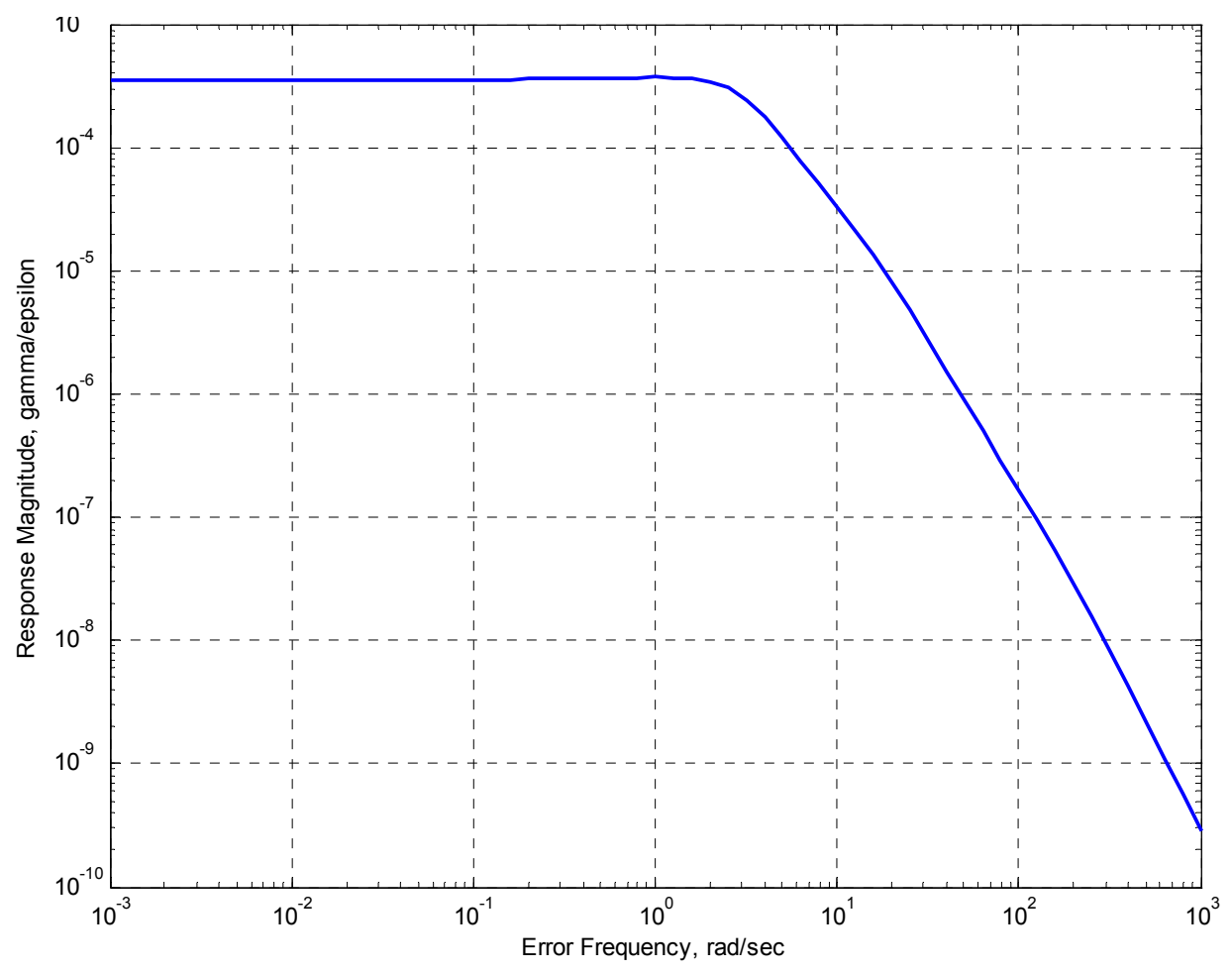

Figure 65: Flight-path sensitivity to oscillatory $\Delta \dot{\mathrm{u}}$ errors from the neural network

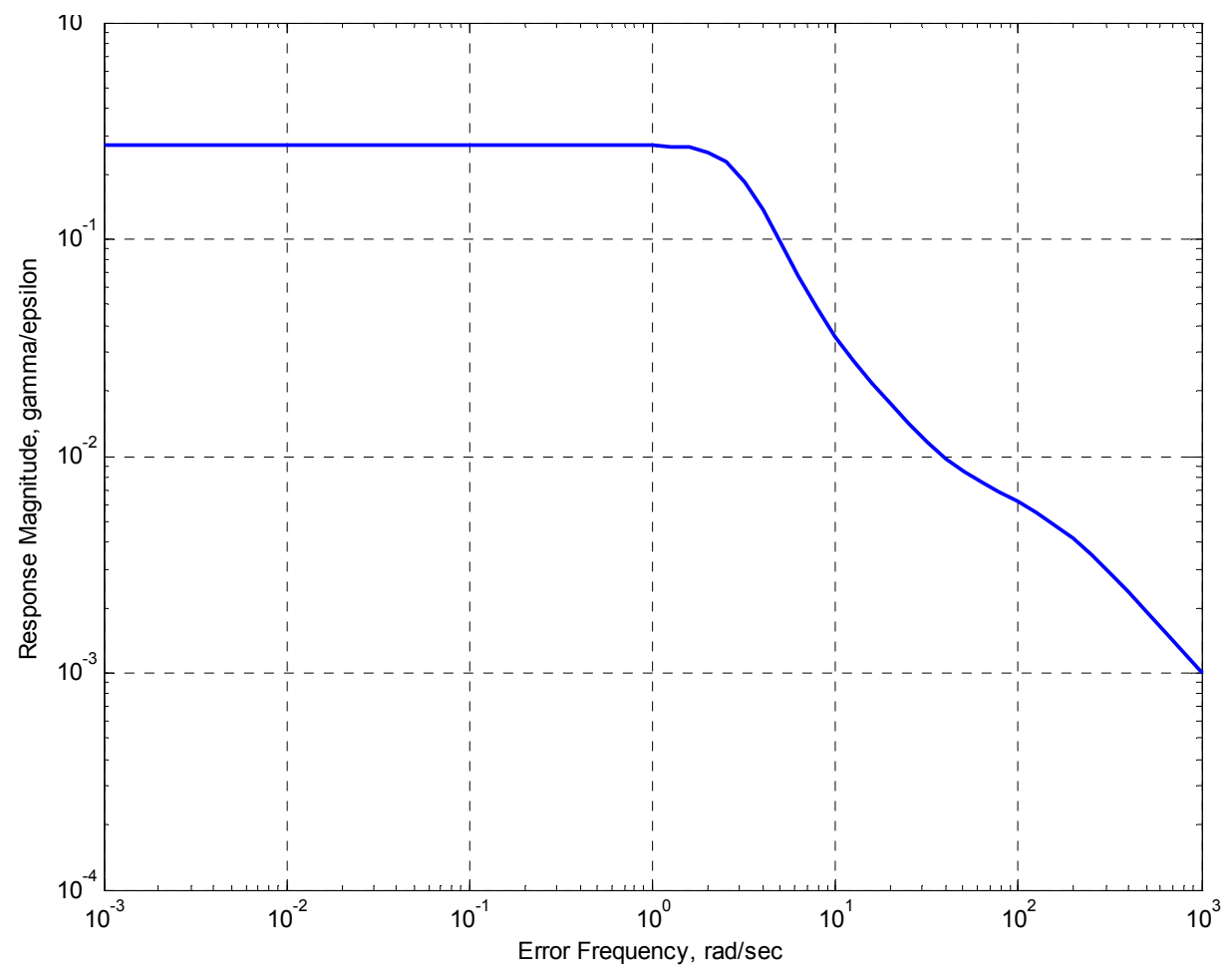

Figure 66: Flight-path sensitivity to oscillatory $\Delta \dot{\alpha}$ errors from the neural network 


\section{CHAPTER 5}

\section{CONCLUSIONS}

\subsection{Summary of Results}

An adaptive controller is developed that meets the research objectives identified in subchapter 1.2. By building upon the work done by Calise, Rysdyk, Steck, Rokhsaz, and others, the present work extends the state of the art by implementing a closed-form expression for the inverse transfer function and using a recursive neural network to account for dynamics in the modeling and inversion errors.

Simulation results are shown to substantiate the current controller does the following:

1. Separates the normally coupled modes of the aircraft. The classic phugoid and short period modes typical of General Aviation aircraft are eliminated.

2. Enables independent control of speed and flight-path angle. Abrupt commands in one parameter create only minor transients in the other.

3. Produces an overall system response that matches the desired characteristics of an arbitrary linear system.

4. Quickly adapts to unexpected changes in the physical plant. Changes in plant stability and controllability are accommodated equally well.

5. Executes quickly, enabling the overall simulation to run 1000 times faster than real time. All sample cases show that the controller performs its intended function. Only one case indicates any degradation from the desired performance. This exception is a challenging case involving nonlinear aerodynamics where linear aerodynamics is expected, reduced control authority, and abrupt square-wave inputs. 
A brief examination of the consequences of failures in the adaptive neural network is undertaken. All cases examined are bounded. No cases are seen where total loss of control occurs. Speed excursions are seen to be the primary outcome from neural network failures. These speed excursions may be a simple offset from commanded speed or an oscillatory variation, depending on the nature of the failure. Flight-path angle is shown to be more robust to network failures.

The overall objectives for a General Aviation controller are satisfied as well. Coupled with a cockpit controls configuration such as in Hawker Beechcraft Corporation's CJ-144, the current controller will permit the pilot to directly control the high-level performance variables, speed and flight-path angle, in an intuitive fashion. Training time and time spent on maintaining proficiency should be reduced. Safety is enhanced by enabling predictable flying characteristics regardless of the aircraft failure state. These attributes should contribute to encouraging more people to enter General Aviation by reducing some of the significant barriers to personal flight.

\subsection{Recommendations for Future Study}

The objectives for this research are met. However, several areas of interest surfaced in the course of this research. While outside the scope of this effort, investigation of the following topics might be of interest to others:

1. Implement the current controller on CJ-144. Funding for this effort will be required. It would be of interest to compare the current controller in back-to-back flights with the baseline CJ-144 controller and the present WSU Adaptive Bias Controller derived by Steck and Rokhsaz [12]. 
2. Develop an improved linear prefilter using robust control theory. It would be interesting to develop an $\mathrm{H}_{2}$ or $\mathrm{H}_{\infty}$ controller to mitigate some resonance evident arising from the gamma filter within the linear prefilter.

3. Extend the current method to include lateral-directional modes of the aircraft.

4. Investigate the resilience to other nonlinear effects known to be present in real airplane implementations. Of particular interest would be the effects of time delays in aircraft actuators (both first-order lags and pure time delays) and mechanical backlash.

5. Investigate the use of observers to synthesize certain aircraft state variables, with the goal of reducing the sensor suite required on the airplane.

6. Consider a committee network to provide redundancy in the neural network.

While not an exhaustive list, these are the topics that are most evident from the current research. 
REFERENCES 


\section{REFERENCES}

[1] General Aviation Statistical Databook 2005, General Aviation Manufacturers Association, 2006.

[2] Holmes, B.J., Durham, M.H., and Tarry, S., "The Small Aircraft Transportation System Concept and Technologies," AIAA 2003-2510, 2003.

[3] Beringer, D.B., "Applying Performance-Controlled Systems, Fuzzy Logic, and Fly-byWire Controls to General Aviation," DOT/FAA/AM-02/7, 2002.

[4] Duerksen, N., "Fuzzy Logic Adaptive Decoupled Flight Controls for General Aviation Airplane", Doctoral Dissertation, Department of Aerospace Engineering, Wichita State University, Spring 1996.

[5] Calise, A.J,. and Rysdyk, R.T., "Nonlinear Adaptive Flight Control Using Neural Networks," IEEE Control Systems, December 1998, pp. 14-25.

[6] MacFarland, M.B., and Calise, A.J., "Adaptive Nonlinear Control of Agile Antiair Missiles Using Neural Networks," IEEE Transactions On Control Systems Technology, Vol. 8, No. 5, September 2000, pp. 749-756.

[7] Hovakimyan, N., Nardi, F. Calise, A.J., and Kim N., "Adaptive Output Feedback Control of Uncertain Nonlinear Systems Using Single-Hidden-Layer Neural Networks," IEEE Transactions On Neural Networks, Vol. 13, No. 6, November 2002, pp 1420-1431.

[8] Rysdyk, R., and Calise, A.J., "Robust Nonlinear Adaptive Flight Controls for Consistent Handling Qualities," IEEE Transactions On Control Systems Technology, Vol. 13, No. 6, November 2005, pp. 896-910.

[9] Rysdyk, R., and Agarwal, R.K., "Nonlinear Adaptive Flight-path and Speed Control Using Energy Principles," AIAA 2002-4440, August 2002.

[10] Lambregts, A., "Vertical flight-path and Speed Autopilot Design using Total Energy Principles," AIAA Conference Proceedings, AIAA Paper 83-2239CP, August 1983.

[11] Steck, J., and Rokhsaz, K., "Simulation and Flight Test Assessment of Safety Benefits and Certification Aspects of Advanced Flight Control Systems," Briefing paper for FAA Project Restart Meeting, November 2001.

[12] Pesonen, U.J. et al., "Adaptive Neural Network Inverse Controller for General Aviation Safety," Journal Of Guidance, Control, And Dynamics, Vol. 27, No. 3, May-June 2004, pp. 434-443. 
[13] Steck, J. et al., "Simulation and Flight Test Assessment of Safety Benefits and Certification Aspects of Advanced Flight Control Systems," U.S. Department of Transportation Report DOT/FAA/AR-00-C-WSU-00-14, December 2002.

[14] Steck, J.E., Rokhsaz, K., Pesonen, U.J., Mochrie, S., and Maxfield, M. "Pilot Evaluation of an Adaptive Controller on a General Aviation SATS Testbed Aircraft," AIAA 20045239, August 2004.

[15] Hess, D.E., Faller, W.E., Fu, T.C., and Ammeen, E.S., "Improved Simulation of Ship Maneuvers Using Recursive Neural Networks," AIAA 2006-1481, 2006.

[16] Anon., Beechcraft Bonanza F33A/F33C Acrobatic Pilot's Operating Handbook, Beech Aircraft Company, 1994.

[17] Zhou, K., Essentials of Robust Control, Prentice Hall, New Jersey, 1998.

[18] Roskam, J., Airplane Flight Dynamics and Automatic Flight Controls, DAR Corporation, Lawrence, Kansas, 2001.

[19] Fausett, L., Fundamentals of Neural Networks, Prentice-Hall, Upper Saddle River, New Jersey, 1994.

[20] Deitel, H.M., C: How to Program, Pearson Education Inc., New Jersey, 2004.

[21] Burl, J.B., Linear Optimal Control, Addison-Wesley, Menlo Park, California, 1999.

[22] Brogan, W., Modern Control Theory, Prentice-Hall, New Jersey, 1991.

[23] Kreyszig, E., Advanced Engineering Mathematics, John Wiley and Sons, New York, 1999.

[24] Deng, H., and Li, H., "A Novel Neural Approximate Inverse Control for Unknown Nonlinear Discrete Dynamical Systems," IEEE Transactions on Systems, Man and Cybernetics -- Part B: Cybernetics, Vol. 35, No. 1, February 2005, pp. 115-123.

[25] Davis, C.J., "Solving and Inverse Control Problem using Predictive Methods with Application to an Aircraft Accident," AIAA-99-4220, 1998.

[26] Deb, D., Tao, G., Burkholder, J.O., and Smith, D., "An Adaptive Inverse Control Scheme for Synthetic Jet Actuator Arrays," AIAA 2005-7170, 2005.

[27] Lou, K.Y., and Bryson, A.E., "Inverse and Optimal Control for Precision Aerobatic Maneuvers," Journal of Guidance, Control, and Dynamics, Vol. 19, No. 2, March-April 1996, pp. 483-488. 
[28] Carberra, J.B.D., and Nerendra, K.S., "Issues in the Application of Neural Networks for Tracking Based on Inverse Control," IEEE Transactions On Automatic Control, Vol. 44, No. 11, pp. 2007-2027.

[29] de Rivals-Mazeres, G., Yim, W., Mora-Camino, F., and Singh, S.N., "Dual Mode Inverse Control and Stabilization of Flexible Space Robots," AIAA-97-3559, 1997.

[30] Bruce, K.R., "Integrated Autopilot/Autothrottle Based on a Total Energy Control Concept: Design and Evaluation of Additional Autopilot Modes," NASA Contractor Report 4131, 1988.

[31] Kurdjukov, A.P., Natchinkina, G.N., and Shevtchenko, A.M., "Energy Approach to Flight Control," AIAA-98-4211, 1998. 


\section{APPENDICES}




\section{APPENDIX A}

\section{STATE SPACE REPRESENTATION FOR THE LINEARIZED AIRCRAFT MODEL}

The state space equations for the linear representation of the airplane, $\mathbf{P}_{\mathrm{A}}(\mathrm{s})$, are derived in this appendix. The state space representation is an essential ingredient in solving for the inverse transfer function, $\mathbf{G}^{-1}(\mathrm{~s})$.

The starting point for this derivation is the perturbed longitudinal equations of motion, found in equations (5.30a), (5.30b), and (5.30c) of Roskam [18]:

$$
\begin{gathered}
\dot{\mathrm{u}}=-\mathrm{g} \theta \cos \theta_{1}+\mathrm{X}_{\mathrm{u}} \mathrm{u}+\mathrm{X}_{\mathrm{T}_{\mathrm{u}}} \mathrm{u}+\mathrm{X}_{\alpha} \alpha+\mathrm{X}_{\delta_{\mathrm{e}}} \delta_{\mathrm{e}} \\
\mathrm{U}_{1} \dot{\alpha}-\mathrm{U}_{1} \dot{\theta}=-\mathrm{g} \theta \sin \theta_{1}+\mathrm{Z}_{\mathrm{u}} \mathrm{u}+\mathrm{Z}_{\alpha} \alpha+\mathrm{Z}_{\dot{\alpha}} \dot{\alpha}+\mathrm{Z}_{\mathrm{q}} \dot{\theta}+\mathrm{Z}_{\delta_{\mathrm{e}}} \delta_{\mathrm{e}} \\
\ddot{\theta}=\mathrm{M}_{\mathrm{u}} \mathrm{u}+\mathrm{M}_{\mathrm{T}_{\mathrm{u}}} \mathrm{u}+\mathrm{M}_{\alpha} \alpha+\mathrm{M}_{\dot{\alpha}} \dot{\alpha}+\mathrm{M}_{\mathrm{q}} \dot{\theta}+\mathrm{M}_{\delta_{\mathrm{e}}} \delta_{\mathrm{e}}
\end{gathered}
$$

The definition of the dimensional stability derivatives can be found in Roskam, Table 5.1.

Equations (A1), (A2), and (A3) can be rearranged to move all terms involving accelerations to the left-hand side. Control derivatives related to thrust are also incorporated.

$$
\begin{gathered}
\dot{\mathrm{u}}=\left(\mathrm{X}_{\mathrm{u}}+\mathrm{X}_{\mathrm{T}_{\mathrm{u}}}\right) \mathrm{u}+\mathrm{X}_{\alpha} \alpha-\mathrm{g} \theta \cos \theta_{1}+\mathrm{X}_{\delta_{\mathrm{e}}} \delta_{\mathrm{e}}+\mathrm{X}_{\delta_{\mathrm{t}}} \delta_{\mathrm{t}} \\
\left(\mathrm{U}_{1}-\mathrm{Z}_{\dot{\alpha}}\right) \dot{\alpha}=\mathrm{Z}_{\mathrm{u}} \mathrm{u}+\mathrm{Z}_{\alpha} \alpha+\left(\mathrm{Z}_{\mathrm{q}}+\mathrm{U}_{1}\right) \dot{\theta}-\mathrm{g} \theta \sin \theta_{1}+\mathrm{Z}_{\delta_{\mathrm{e}}} \delta_{\mathrm{e}}+\mathrm{Z}_{\delta_{\mathrm{t}}} \delta_{\mathrm{t}} \\
\ddot{\theta}=\mathrm{M}_{\mathrm{u}} \mathrm{u}+\mathrm{M}_{\mathrm{T}_{\mathrm{u}}} \mathrm{u}+\mathrm{M}_{\alpha} \alpha+\mathrm{M}_{\dot{\alpha}} \dot{\alpha}+\mathrm{M}_{\mathrm{q}} \dot{\theta}+\mathrm{M}_{\delta_{\mathrm{e}}} \delta_{\mathrm{e}}+\mathrm{M}_{\delta_{\mathrm{t}}} \delta_{\mathrm{t}}
\end{gathered}
$$

Equations (A4), (A5), and (A6) can now be rewritten in matrix format. Note that row four in equation (A7) simply expresses the relationship that $q=\dot{\theta}$. 


$$
\begin{aligned}
& {\left[\begin{array}{cccc}
1 & 0 & 0 & 0 \\
0 & \left(\mathrm{U}_{1}-\mathrm{Z}_{\dot{\alpha}}\right) & 0 & 0 \\
0 & -\mathrm{M}_{\dot{\alpha}} & 1 & 0 \\
0 & 0 & 0 & 1
\end{array}\right] \frac{\mathrm{d}}{\mathrm{dt}}\left\{\begin{array}{l}
\mathrm{u} \\
\alpha \\
\mathrm{q} \\
\theta
\end{array}\right\}=\left[\begin{array}{cccc}
\left(\mathrm{X}_{\mathrm{u}}+\mathrm{X}_{\mathrm{T}_{\mathrm{u}}}\right) & \mathrm{X}_{\alpha} & 0 & -\mathrm{g} \cos \theta_{1} \\
\mathrm{Z}_{\mathrm{u}} & \mathrm{Z}_{\alpha} & \left(\mathrm{Z}_{\mathrm{q}}+\mathrm{U}_{1}\right) & -\mathrm{g} \sin \theta_{1} \\
\left(\mathrm{M}_{\mathrm{u}}+\mathrm{M}_{\mathrm{T}_{\mathrm{u}}}\right) & \left(\mathrm{M}_{\alpha}+\mathrm{M}_{\mathrm{T}_{\alpha}}\right) & \mathrm{M}_{\mathrm{q}} & 0 \\
0 & 0 & 1 & 0
\end{array}\right]\left\{\begin{array}{l}
\mathrm{u} \\
\alpha \\
\mathrm{q} \\
\theta
\end{array}\right\}} \\
& +\left[\begin{array}{cc}
X_{\delta_{e}} & X_{\delta_{t}} \\
Z_{\delta_{e}} & Z_{\delta_{e}} \\
M_{\delta_{e}} & M_{\delta_{t}} \\
0 & 0
\end{array}\right]\left\{\begin{array}{l}
\delta_{e} \\
\delta_{t}
\end{array}\right\} \\
& \text { Let } \mathbf{M}_{1}=\left[\begin{array}{cccc}
1 & 0 & 0 & 0 \\
0 & \left(\mathrm{U}_{1}-\mathrm{Z}_{\dot{\alpha}}\right) & 0 & 0 \\
0 & -\mathrm{M}_{\dot{\alpha}} & 1 & 0 \\
0 & 0 & 0 & 1
\end{array}\right] \\
& \mathbf{A}_{1}=\left[\begin{array}{cccc}
\left(\mathrm{X}_{\mathrm{u}}+\mathrm{X}_{\mathrm{T}_{\mathrm{u}}}\right) & \mathrm{X}_{\alpha} & 0 & -\mathrm{g} \cos \theta_{1} \\
\mathrm{Z}_{\mathrm{u}} & \mathrm{Z}_{\alpha} & \left(\mathrm{Z}_{\mathrm{q}}+\mathrm{U}_{1}\right) & -\mathrm{g} \sin \theta_{1} \\
\left(\mathrm{M}_{\mathrm{u}}+\mathrm{M}_{\mathrm{T}_{\mathrm{u}}}\right) & \left(\mathrm{M}_{\alpha}+\mathrm{M}_{\mathrm{T}_{\alpha}}\right) & \mathrm{M}_{\mathrm{q}} & 0 \\
0 & 0 & 1 & 0
\end{array}\right] \\
& \mathbf{B}_{1}=\left[\begin{array}{cc}
\mathrm{X}_{\delta_{\mathrm{e}}} & \mathrm{X}_{\delta_{\mathrm{t}}} \\
\mathrm{Z}_{\delta_{\mathrm{e}}} & \mathrm{Z}_{\delta_{\mathrm{e}}} \\
\mathrm{M}_{\delta_{\mathrm{e}}} & \mathrm{M}_{\delta_{\mathrm{t}}} \\
0 & 0
\end{array}\right] \\
& \mathbf{x}=\left\{\begin{array}{l}
u \\
\alpha \\
q \\
\theta
\end{array}\right\}, \\
& \text { and } \mathbf{u}=\left\{\begin{array}{l}
\delta_{\mathrm{e}} \\
\delta_{\mathrm{t}}
\end{array}\right\} \text {. }
\end{aligned}
$$

Using this notation,

$$
\mathbf{M}_{1} \dot{\mathbf{x}}=\mathbf{A}_{1} \mathbf{x}+\mathbf{B}_{1} \mathbf{u}
$$


Both sides of equation (A12) can be pre-multiplied by $\mathbf{M}_{1}^{-1}$.

$$
\mathbf{M}_{1}^{-1} \mathbf{M}_{1} \dot{\mathbf{x}}=\mathbf{M}_{1}^{-1} \mathbf{A}_{1} \mathbf{x}+\mathbf{M}_{1}^{-1} \mathbf{B}_{1} \mathbf{u}
$$

Using the following definitions

$$
\begin{aligned}
\mathbf{A}_{\mathrm{A}} & \equiv \mathbf{M}_{1}^{-1} \mathbf{A}_{1} \\
\mathbf{B}_{\mathrm{A}} & \equiv \mathbf{M}_{1}^{-1} \mathbf{B}_{1}
\end{aligned}
$$

equation (A13) can be rewritten as

$$
\dot{\mathbf{x}}=\mathbf{A}_{\mathrm{A}} \mathbf{x}+\mathbf{B}_{\mathrm{A}} \mathbf{u}
$$

Equation (A16) is the desired form for the first part of the state space representation. To complete the state space representation, an expression for $\mathbf{y}$ is required in the form of

$$
\mathbf{y}=\mathbf{C}_{\mathrm{A}} \mathbf{x}+\mathbf{D}_{\mathrm{A}} \mathbf{u}
$$

For the case where it is desired for $\mathbf{y}$ to be the state variables $\mathrm{u}, \alpha, \mathrm{q}$, and $\theta$ :

$$
\mathbf{C}_{\mathrm{A}}=\left[\begin{array}{llll}
1 & 0 & 0 & 0 \\
0 & 1 & 0 & 0 \\
0 & 0 & 1 & 0 \\
0 & 0 & 0 & 1
\end{array}\right]=\mathbf{I}
$$

and

$$
\mathbf{D}_{\mathrm{A}}=\mathbf{0}
$$

For the case where it is desired for $\mathbf{y}$ to be only $\mathrm{u}$ and $\gamma$ :

$$
\mathbf{C}_{\mathrm{A}}=\left[\begin{array}{cccc}
1 & 0 & 0 & 0 \\
0 & -1 & 0 & 1
\end{array}\right]
$$

and

$$
\mathbf{D}_{\mathrm{A}}=\mathbf{0}
$$


For the case where it is desired for $\mathbf{y}$ to be $u$ and $\alpha$ :

$$
\mathbf{C}_{\mathrm{A}}=\left[\begin{array}{llll}
1 & 0 & 0 & 0 \\
0 & 1 & 0 & 0
\end{array}\right]
$$

and

$$
\mathbf{D}_{\mathrm{A}}=\mathbf{0}
$$

An augmented matrix can also be constructed that includes the state variables and $\gamma$ :

$$
\mathbf{C}_{\mathrm{A}}=\left[\begin{array}{cccc}
1 & 0 & 0 & 0 \\
0 & 1 & 0 & 0 \\
0 & 0 & 1 & 0 \\
0 & 0 & 0 & -1 \\
0 & -1 & 0 & -\frac{1}{1}
\end{array}\right]
$$

and

$$
\mathbf{D}_{\mathrm{A}}=\mathbf{0}
$$

In any of these cases, a complete state space representation of the linearized aircraft model is available. This state space model can be abbreviated with the notation

$$
\mathbf{P}_{\mathrm{A}}(\mathrm{s})=\left[\begin{array}{c|c}
\mathbf{A}_{\mathrm{A}} & \mathbf{B}_{\mathrm{A}} \\
\hline \mathbf{C}_{\mathrm{A}} & \mathbf{D}_{\mathrm{A}}
\end{array}\right]
$$




\section{APPENDIX B}

\section{EQUATIONS OF MOTION USED IN MODELING AIRCRAFT FLIGHT DYNAMICS}

Equations (B1) through (B37) summarize the equations utilized in the simulation to solve the flight dynamics of the aircraft. Note that full coupling between axes is included. These equations are adapted from those found in Roskam [18]. Subscript s denotes a stability axis property. Subscript b denotes a body axis property.

$$
\begin{gathered}
\alpha=\tan ^{-1}\left(\frac{\mathrm{W}_{\mathrm{b}}}{\mathrm{U}_{\mathrm{b}}}\right) \\
\beta=\tan ^{-1}\left(\frac{\mathrm{V}_{\mathrm{b}}}{\mathrm{U}_{\mathrm{b}}}\right) \\
\mathrm{V}_{\mathrm{p}}=\sqrt{\mathrm{U}_{\mathrm{b}}^{2}+\mathrm{V}_{\mathrm{b}}^{2}+\mathrm{W}_{\mathrm{b}}^{2}} \\
\mathrm{P}_{\mathrm{s}}=\mathrm{P}_{\mathrm{b}} \cos \alpha+\mathrm{P}_{\mathrm{b}} \sin \alpha \\
\mathrm{R}_{\mathrm{s}}=-\mathrm{R}_{\mathrm{b}} \sin \alpha+\mathrm{R}_{\mathrm{b}} \cos \alpha \\
\dot{\alpha}=\frac{\left(\dot{\mathrm{W}}_{\mathrm{b}} \mathrm{U}_{\mathrm{b}}-\mathrm{W}_{\mathrm{b}} \dot{\mathrm{U}}_{\mathrm{b}}\right)}{\mathrm{U}_{\mathrm{b}}^{2}} \\
\hat{\dot{\alpha}}=\frac{\dot{\alpha} \overline{\mathrm{c}}}{2 \mathrm{~V}_{\mathrm{p}}} \\
\hat{\mathrm{p}}=\frac{\mathrm{P}_{\mathrm{s}} \mathrm{b}}{2 \mathrm{~V}_{\mathrm{p}}} \\
2 \mathrm{~V}_{\mathrm{p}} \\
\mathrm{Q}_{\mathrm{c}}
\end{gathered}
$$




$$
\begin{aligned}
& \hat{r}=\frac{R_{s} b}{2 V_{p}} \\
& \mathrm{C}_{\mathrm{L}}=\mathrm{C}_{\mathrm{L}_{0}}+\mathrm{C}_{\mathrm{L}_{\alpha}} \alpha+\mathrm{C}_{\mathrm{L}_{\delta_{\mathrm{e}}}} \delta_{\mathrm{e}}+\mathrm{C}_{\mathrm{L}_{\delta_{\mathrm{tab}}}} \delta_{\text {tab }}+\mathrm{C}_{\mathrm{L}_{\dot{\alpha}}} \hat{\dot{\alpha}}+\mathrm{C}_{\mathrm{L}_{\mathrm{q}}} \hat{\mathrm{q}} \\
& \mathrm{C}_{\mathrm{M}}=\mathrm{C}_{\mathrm{M}_{0}}+\mathrm{C}_{\mathrm{M}_{\alpha}} \alpha+\mathrm{C}_{\mathrm{M}_{\delta_{\mathrm{e}}}} \delta_{\mathrm{e}}+\mathrm{C}_{\mathrm{M}_{\delta_{\text {tab }}}} \delta_{\text {tab }}+\mathrm{C}_{\mathrm{M}_{\dot{\alpha}}} \hat{\dot{\alpha}}+\mathrm{C}_{\mathrm{M}_{\mathrm{q}}} \hat{\mathrm{q}} \\
& \mathrm{C}_{\mathrm{D}}=\mathrm{C}_{\mathrm{D}_{0}}+\mathrm{C}_{\mathrm{D}_{\mathrm{K}}} \mathrm{C}_{\mathrm{L}}^{2} \\
& T=f\left(P L A, V_{p}, \rho\right) \\
& \mathrm{C}_{\mathrm{y}}=\mathrm{C}_{\mathrm{y}_{0}}+\mathrm{C}_{\mathrm{y}_{\beta}} \beta+\mathrm{C}_{\mathrm{y}_{\delta_{\mathrm{r}}}} \delta_{\mathrm{r}}+\mathrm{C}_{\mathrm{y}_{\delta_{\mathrm{a}}}} \delta_{\mathrm{a}}+\mathrm{C}_{\mathrm{y}_{\mathrm{p}}} \hat{\mathrm{p}}+\mathrm{C}_{\mathrm{y}_{\mathrm{r}}} \hat{\mathrm{r}}
\end{aligned}
$$

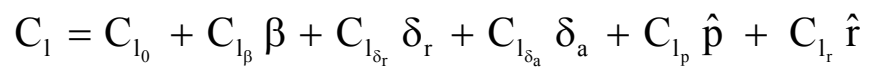

$$
\begin{aligned}
& \mathrm{C}_{\mathrm{n}}=\mathrm{C}_{\mathrm{n}_{0}}+\mathrm{C}_{\mathrm{n}_{\beta}} \beta+\mathrm{C}_{\mathrm{n}_{\delta_{\mathrm{r}}}} \delta_{\mathrm{r}}+\mathrm{C}_{\mathrm{n}_{\delta_{\mathrm{a}}}} \delta_{\mathrm{a}}+\mathrm{C}_{\mathrm{n}_{\mathrm{p}}} \hat{\mathrm{p}}+\mathrm{C}_{\mathrm{n}_{\mathrm{r}}} \hat{\mathrm{r}} \\
& \mathrm{F}_{\mathrm{x}_{\mathrm{b}}}=\overline{\mathrm{q}} \mathrm{S}\left(\mathrm{C}_{\mathrm{L}} \sin \alpha-\mathrm{C}_{\mathrm{D}} \cos \alpha\right)-\mathrm{W} \sin \Theta+\mathrm{T} \cos \alpha \\
& \mathrm{F}_{\mathrm{y}_{\mathrm{b}}}=\overline{\mathrm{q}} \mathrm{SC}_{\mathrm{y}}+\mathrm{W} \sin \Phi \cos \Theta \\
& \mathrm{F}_{\mathrm{z}_{\mathrm{b}}}=\overline{\mathrm{q}} \mathrm{S}\left(-\mathrm{C}_{\mathrm{L}} \cos \alpha-\mathrm{C}_{\mathrm{D}} \sin \alpha\right)+\mathrm{W} \cos \Theta \cos \Phi+\mathrm{T} \sin \alpha \\
& \mathrm{L}_{\mathrm{s}}=\overline{\mathrm{q}} \mathrm{SbC}_{1} \\
& \mathrm{M}_{\mathrm{s}}=\overline{\mathrm{q}} \mathrm{S} \overline{\mathrm{c}} \mathrm{C}_{\mathrm{M}} \\
& \mathrm{N}_{\mathrm{s}}=\overline{\mathrm{q}} \mathrm{SbC}_{\mathrm{n}} \\
& \mathrm{L}_{\mathrm{b}}=\mathrm{L}_{\mathrm{s}} \cos \alpha-\mathrm{N}_{\mathrm{s}} \sin \alpha \\
& \mathrm{M}_{\mathrm{b}}=\mathrm{M}_{\mathrm{s}} \\
& \mathrm{N}_{\mathrm{b}}=\mathrm{L}_{\mathrm{s}} \sin \alpha+\mathrm{N}_{\mathrm{s}} \cos \alpha \\
& \dot{\mathrm{U}}_{\mathrm{b}}=\mathrm{F}_{\mathrm{x}_{\mathrm{b}}} / \mathrm{m}+\mathrm{V}_{\mathrm{b}} \mathrm{R}_{\mathrm{b}}-\mathrm{W}_{\mathrm{b}} \mathrm{Q}_{\mathrm{b}} \\
& \dot{\mathrm{V}}_{\mathrm{b}}=\mathrm{F}_{\mathrm{y}_{\mathrm{b}}} / \mathrm{m}-\mathrm{U}_{\mathrm{b}} \mathrm{R}_{\mathrm{b}}+\mathrm{W}_{\mathrm{b}} \mathrm{P}_{\mathrm{b}}
\end{aligned}
$$




$$
\begin{aligned}
& \dot{\mathrm{W}}_{\mathrm{b}}=\mathrm{F}_{\mathrm{z}} / \mathrm{m}+\mathrm{U}_{\mathrm{b}} \mathrm{Q}_{\mathrm{b}}-\mathrm{V}_{\mathrm{b}} \mathrm{P}_{\mathrm{b}} \\
& \dot{\mathrm{P}}_{\mathrm{b}}=\frac{\left(\begin{array}{c}
\mathrm{I}_{\mathrm{zz}} \mathrm{L}_{\mathrm{b}}+\mathrm{I}_{\mathrm{xz}} \mathrm{N}_{\mathrm{b}}+\left(\mathrm{I}_{\mathrm{xz}} \mathrm{I}_{\mathrm{zz}}-\mathrm{I}_{\mathrm{yy}} \mathrm{I}_{\mathrm{xz}}+\mathrm{I}_{\mathrm{xz}}^{2}\right) \mathrm{P}_{\mathrm{b}} \mathrm{Q}_{\mathrm{b}}+ \\
\left(\mathrm{I}_{\mathrm{yy}} \mathrm{I}_{\mathrm{zz}}-\mathrm{I}_{\mathrm{zz}}^{2}-\mathrm{I}_{\mathrm{xz}}^{2}\right) \mathrm{R}_{\mathrm{b}} \mathrm{Q}_{\mathrm{b}}
\end{array}\right)}{\left(\mathrm{I}_{\mathrm{xx}_{\mathrm{b}}} \mathrm{I}_{\mathrm{zz} \mathrm{b}}-\mathrm{I}_{\mathrm{xz} \mathrm{b}}^{2}\right)} \\
& \dot{Q}_{b}=\frac{M_{s}-\left(I_{x_{b}}-I_{z_{b}}\right) P_{b} R_{b}-I_{x_{b}}\left(P_{b}^{2}-R_{b}^{2}\right)}{I_{y_{b}}} \\
& \dot{R}_{b}=\frac{\left(\begin{array}{c}
I_{\mathrm{xz}_{\mathrm{b}}} \mathrm{L}_{\mathrm{b}}+\mathrm{I}_{\mathrm{xx}} \mathrm{N}_{\mathrm{b}}+\left(\mathrm{I}_{\mathrm{xz}}^{2}-\mathrm{I}_{\mathrm{yy}} \mathrm{I}_{\mathrm{xx}}+\mathrm{I}_{\mathrm{xx}}^{2}\right) \mathrm{P}_{\mathrm{b}} \mathrm{Q}_{\mathrm{b}}+ \\
\left(\mathrm{I}_{\mathrm{yy}} \mathrm{I}_{\mathrm{xz}}-\mathrm{I}_{\mathrm{zz}} \mathrm{I}_{\mathrm{xz}}-\mathrm{I}_{\mathrm{xz}} \mathrm{I}_{\mathrm{xx}}\right) \mathrm{R}_{\mathrm{b}} \mathrm{Q}_{\mathrm{b}}
\end{array}\right)}{\left(\mathrm{I}_{\mathrm{xx}_{\mathrm{b}}} \mathrm{I}_{\mathrm{zz}_{\mathrm{b}}}-\mathrm{I}_{\mathrm{xz}_{\mathrm{b}}}^{2}\right)} \\
& \dot{\Phi}=\mathrm{P}_{\mathrm{b}}+\mathrm{Q}_{\mathrm{b}} \sin \Phi \tan \Theta+\mathrm{R}_{\mathrm{b}} \cos \Phi \tan \Theta \\
& \dot{\Theta}=Q_{b} \cos \Phi-R_{b} \sin \Phi \\
& \dot{\Psi}=\left(\mathrm{Q}_{\mathrm{b}} \sin \Phi+\mathrm{R}_{\mathrm{b}} \cos \Phi\right) / \cos \Theta \\
& \dot{\mathrm{h}}=\mathrm{U}_{\mathrm{b}} \sin \Theta-\mathrm{V}_{\mathrm{b}} \cos \Theta \sin \Phi-\mathrm{W}_{\mathrm{b}} \cos \Theta \cos \Phi
\end{aligned}
$$

An Adams predictor-corrector method is used for solving these equations. For each computation cycle, the values of the state variables are predicted using four prior timeframes. These predicted values are then used to start an iterative calculation of the equations of motion for this timeframe. Iteration is required because, in general, all state variables are functions of one another. The iteration continues until the value of each state variable converges. Practice shows that four iterations are sufficient. After four iterations, a final correction step takes place.

The predictor-corrector method is not self-starting. However, it can be started by assuming that the system is in equilibrium, and all accelerations are zero. Initial conditions for rates and displacements are determined from the solution for the initial trim state. These initial 
conditions are used to preload the predictor-corrector, so that the algorithm has values for four "fictitious" time states prior to the starting time, all with the same equilibrium values. 


\section{Appendix C}

\section{Program Listing}

A complete listing of the simulation computer code follows. This program is written in ANSI C for portability. The individual functions are listed alphabetically.

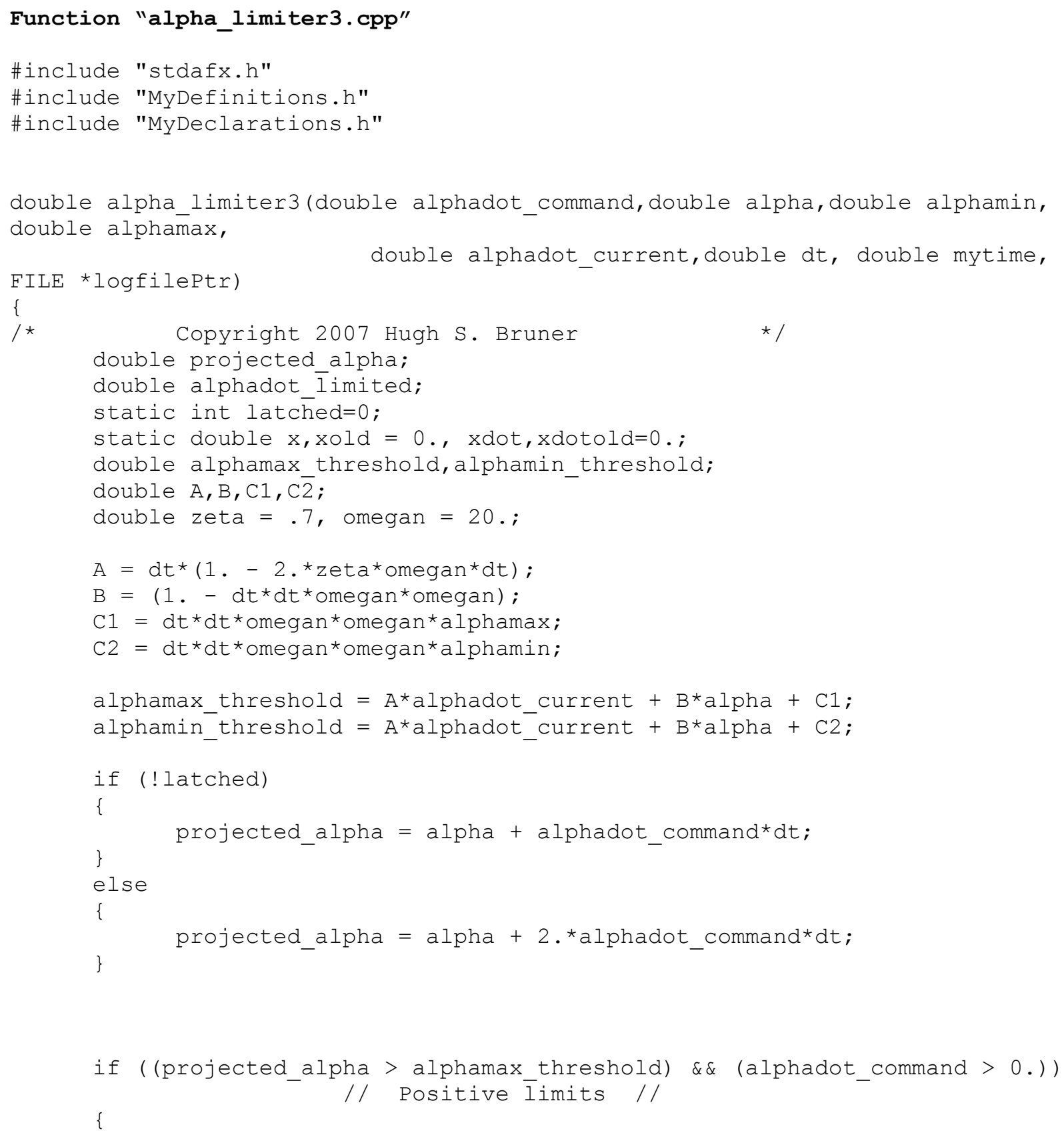




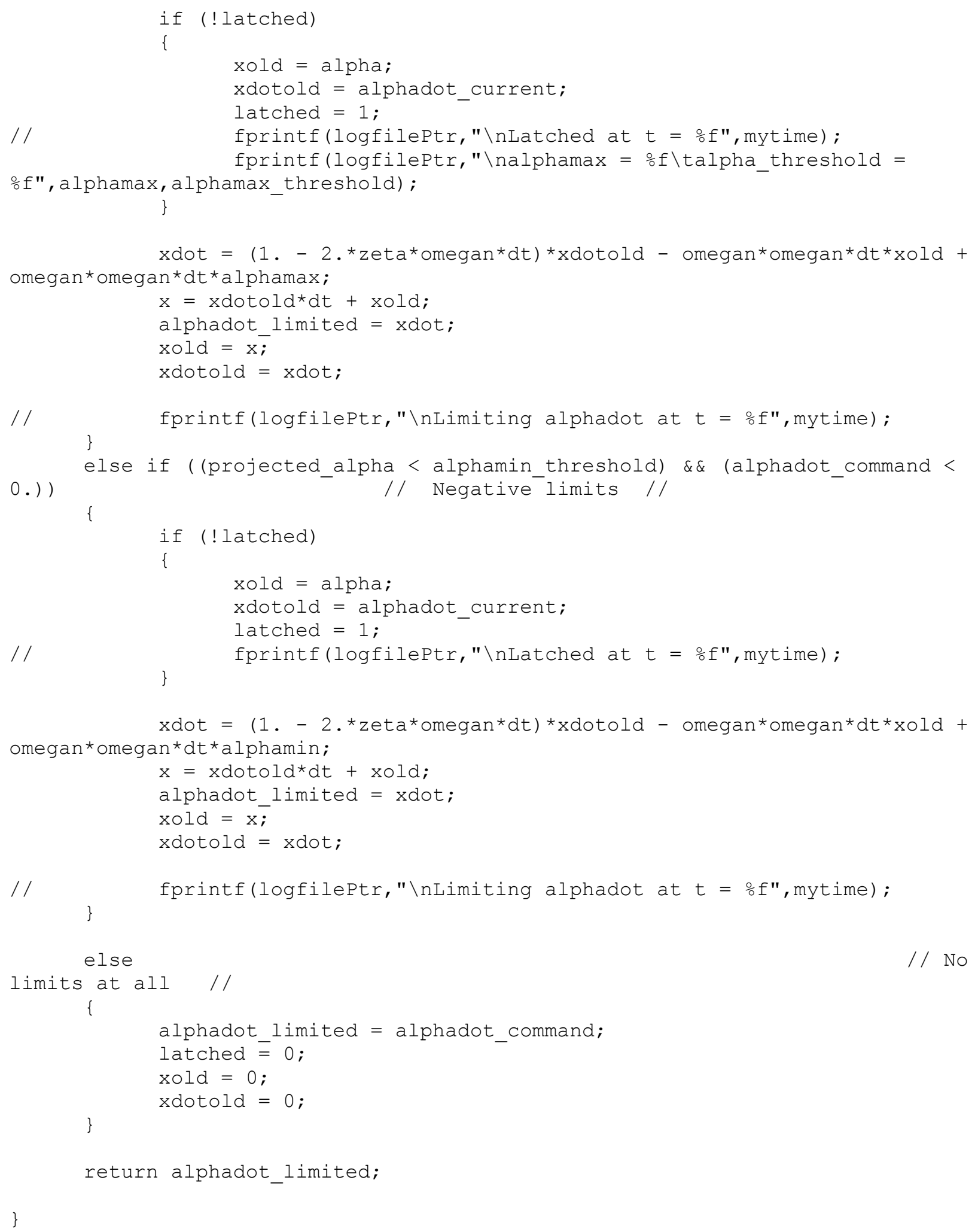




\section{Function "autopilot.cpp"}

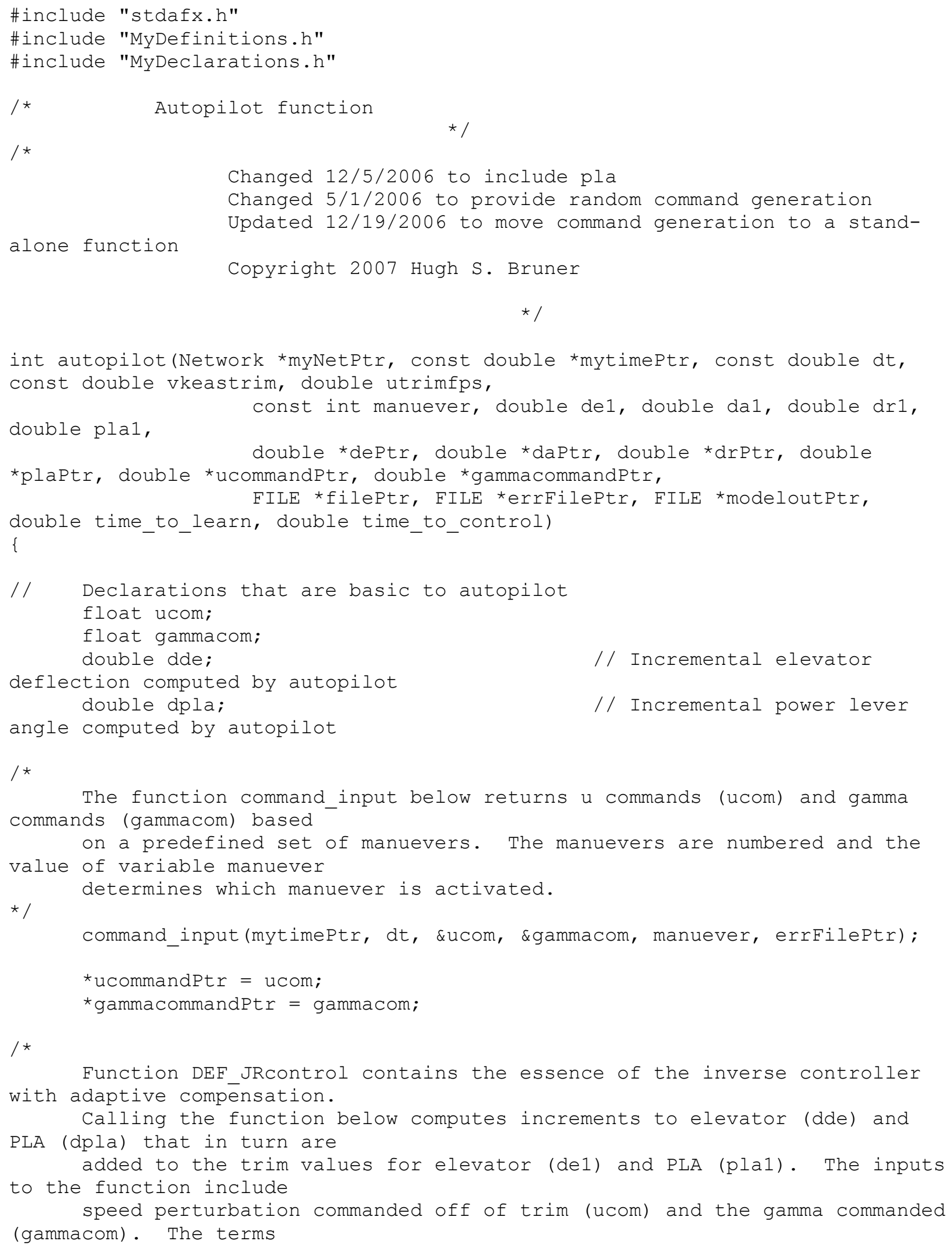




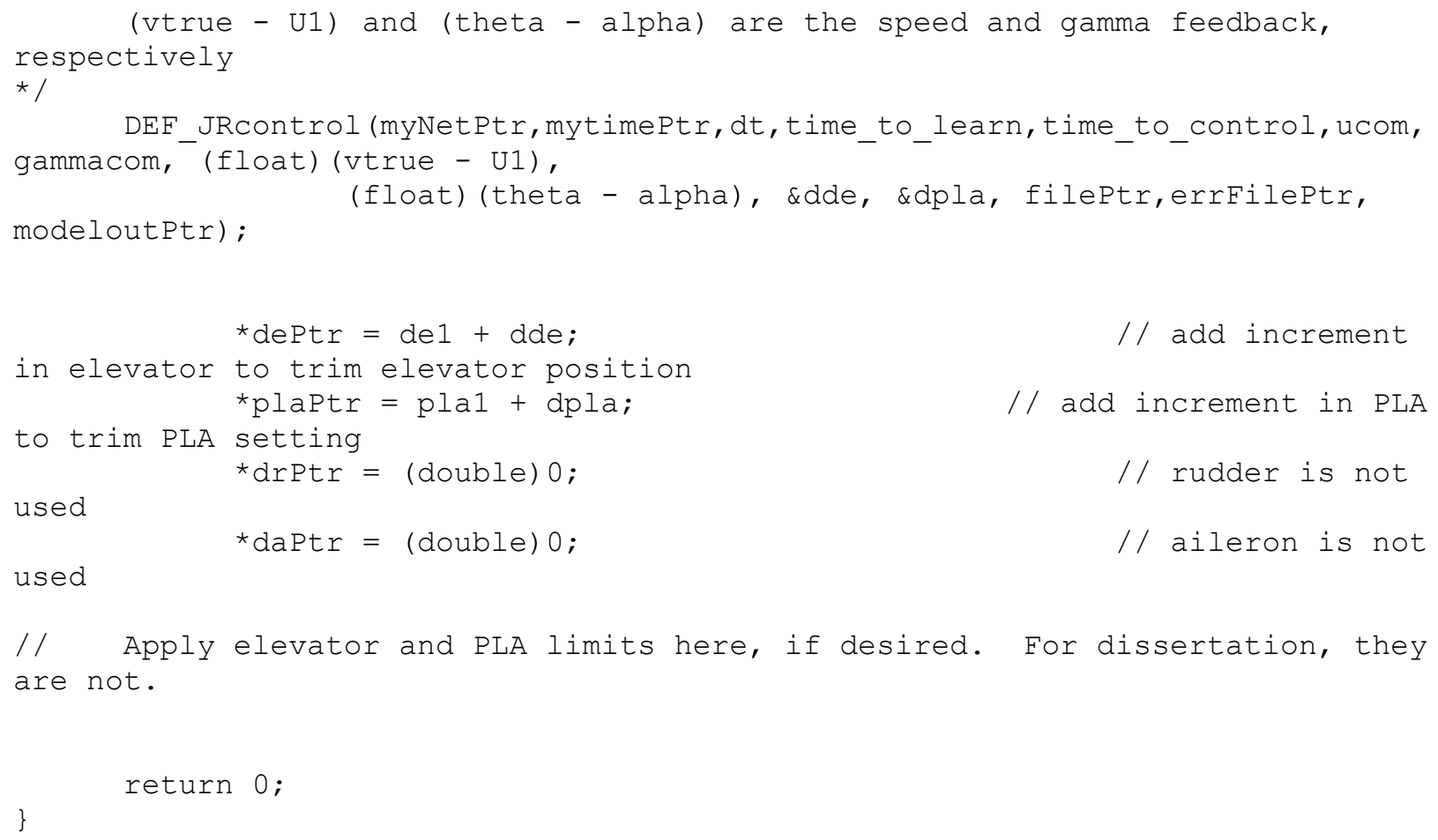




\section{Function "backprop.cpp"}

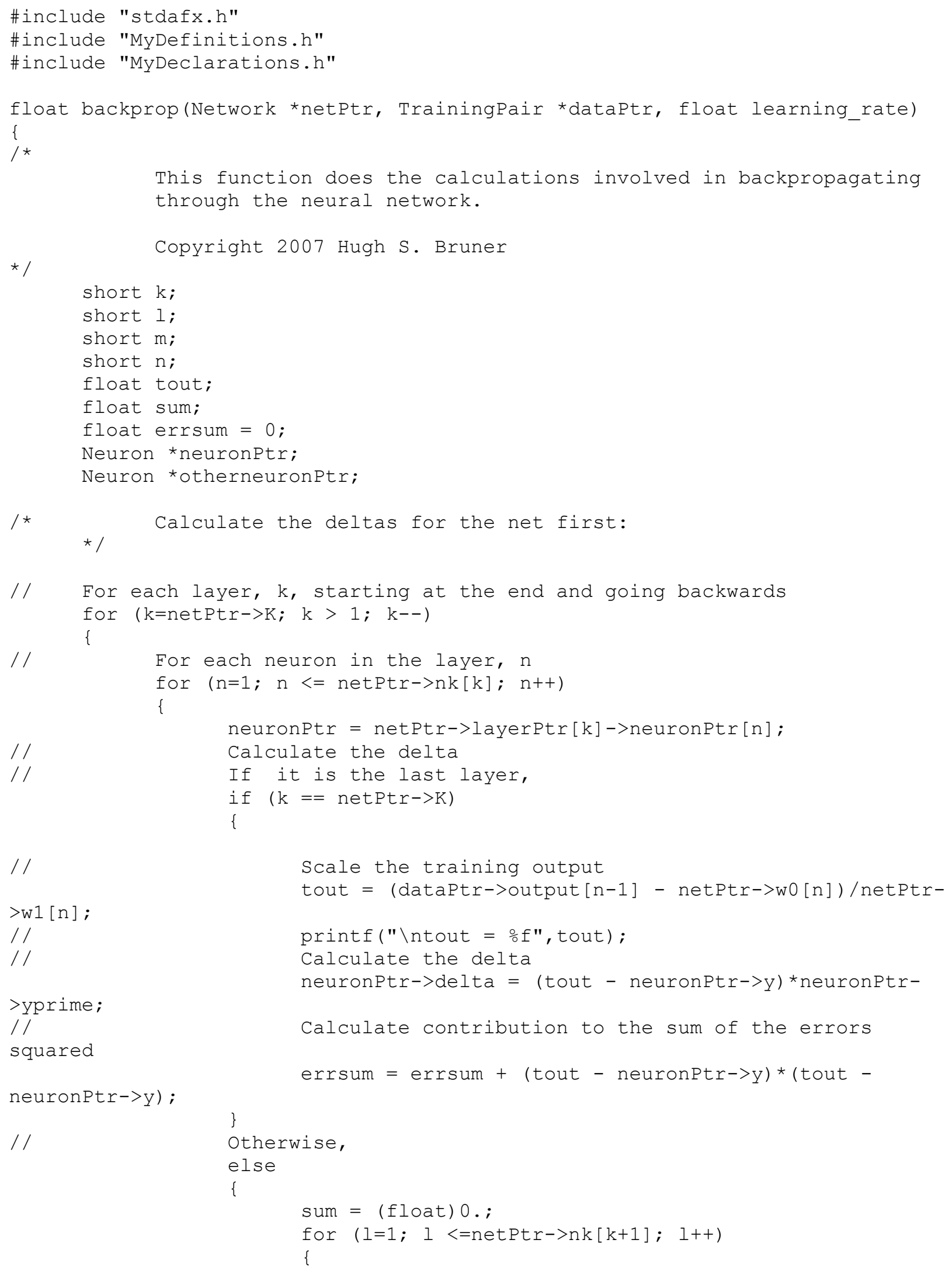




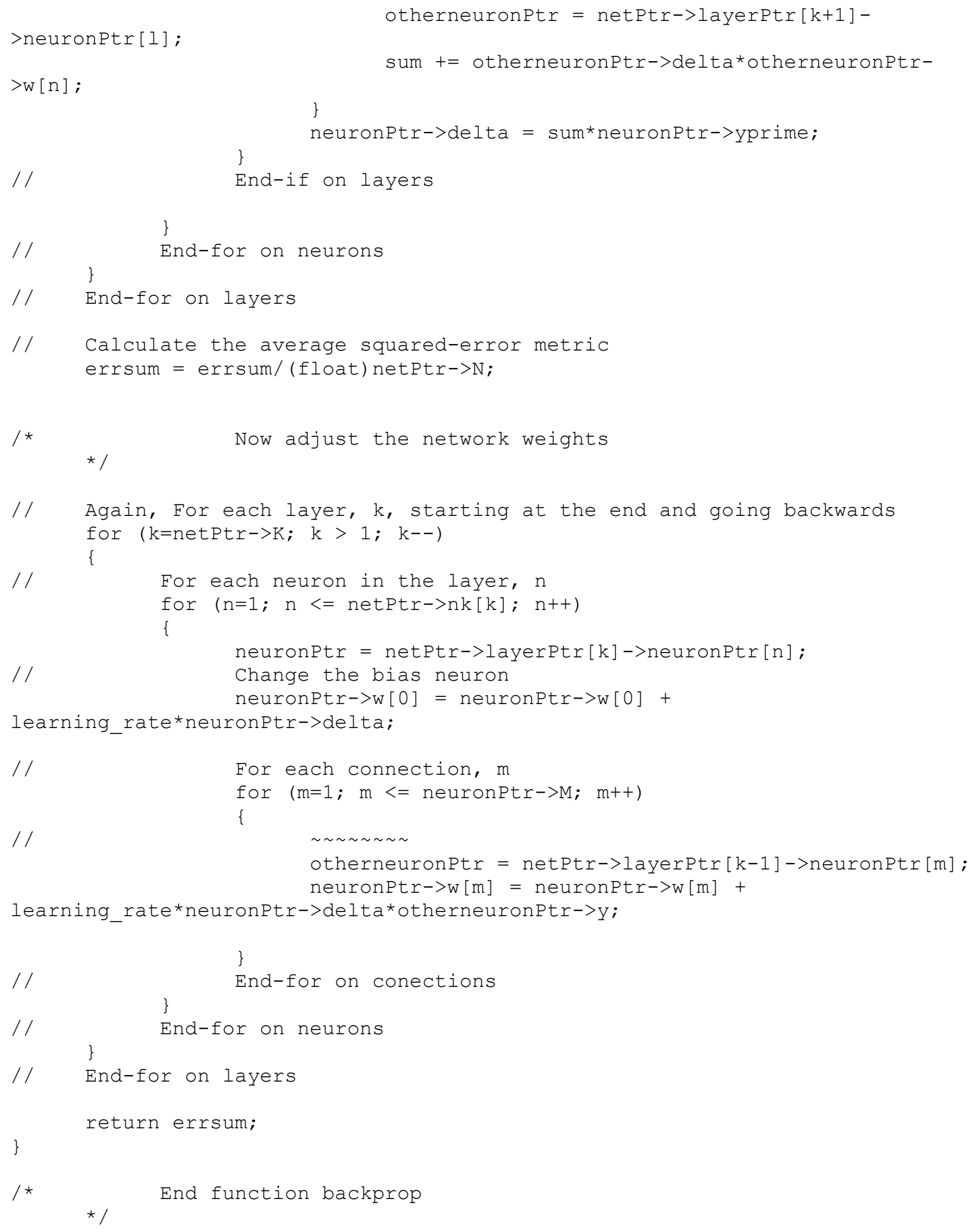




\section{Function "case_data_write.cpp"}

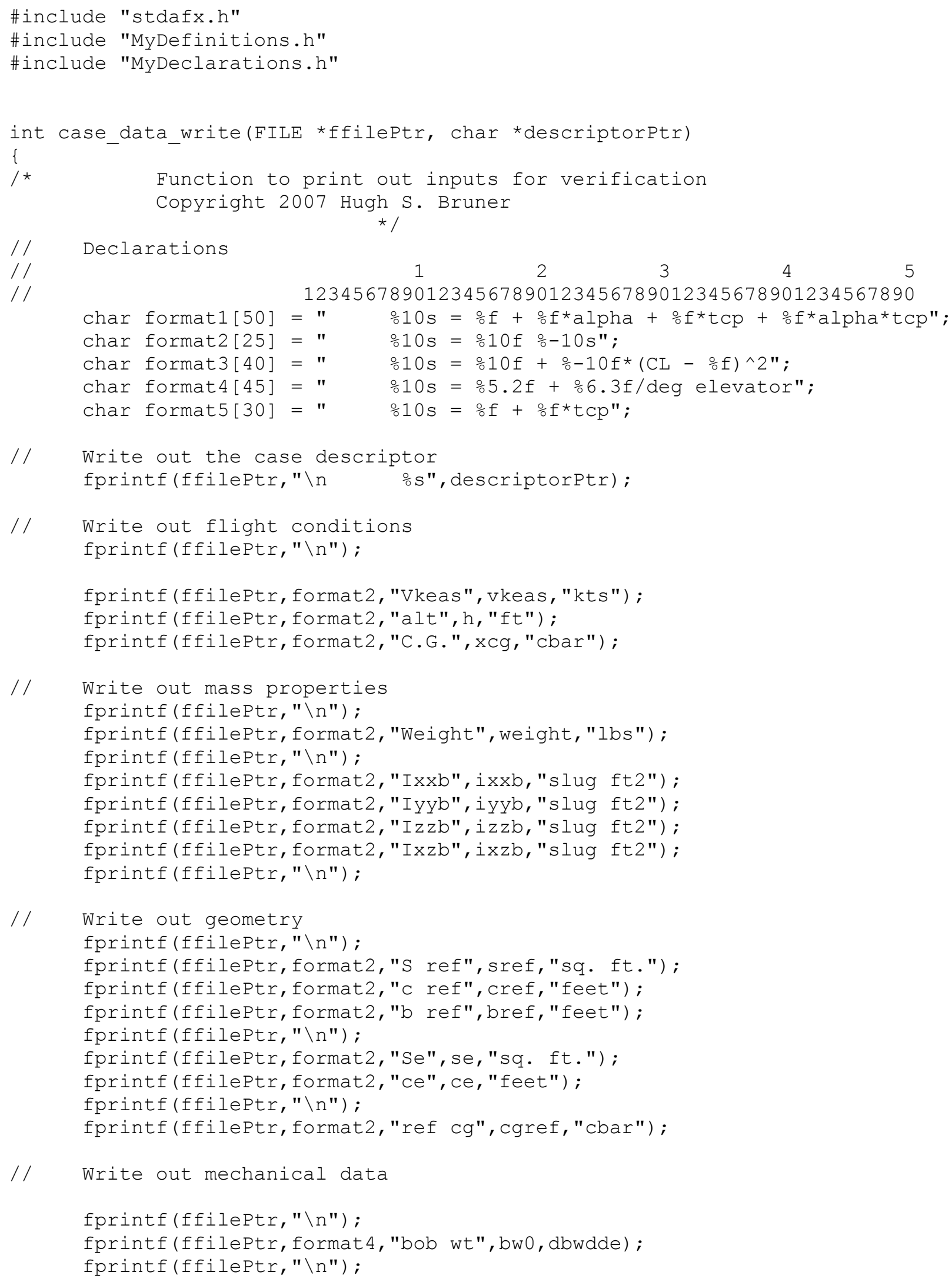


fprintf(ffileptr, format4,"dn spring", ds0, ddsdde) ;

// Write out longitudinal stability data

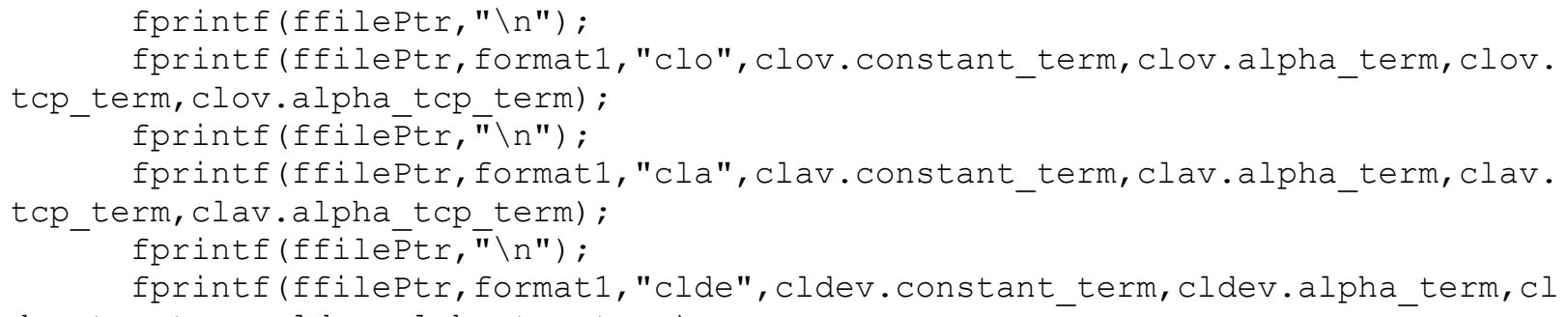

dev.tcp term, cldev.alpha top term);

fprintf (ffilePtr, "\n");

fprintf (ffilePtr, format1, "cldetab", cldetabv.constant_term, cldetabv.alph

a_term, cldetabv.tcp_term, cldetabv.alpha_tcp_term);

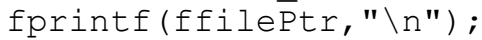

fprintf (ffileptr, format1,"cladot", cladotv.constant_term, cladotv.alpha_t

erm, cladotv.tcp_term, cladotv.alpha_tcp_term);

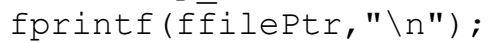

fprintf (ffilePtr, format1, "clq",clqv.constant_term, clqv.alpha_term, clqv.

tcp_term, clqv.alpha_tcp_term);

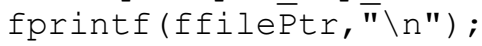

fprintf (ffileptr," \n");

fprintf (ffileptr, format1,"cmo",cmov.constant_term, cmov.alpha_term, cmov.

tcp_term, cmov.alpha_tcp_term);

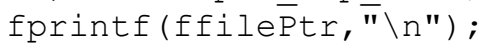

fprintf (ffileptr, format1,"cma", cmav.constant_term, cmav.alpha term, cmav. tcp_term, cmav.alpha_tcp_term);

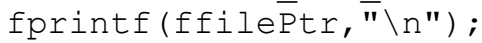

fprintf (ffilePtr, format1, "cmde", cmdev.constant_term, cmdev.alpha_term, cm

dev.tcp_term, cmdev.alpha_tcp_term);

fōpintf (ffileptr, "\n");

fprintf (ffilePtr, format1, "cmdetab", cmdetabv.constant_term, cmdetabv.alph a_term, cmdetabv.tcp_term, cmdetabv.alpha_tcp_term);

fprintf (ffileptr,"\n");

fprintf (ffileptr,format1,"cmadot", cmadotv.constant_term, cmadotv.alpha_t

erm, cmadotv.tcp_term, cmadotv.alpha_tcp_term);

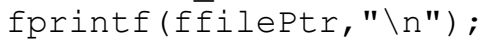

fprintf(ffileptr, format1,"cmq", cmqv.constant_term, cmqv.alpha_term, cmqv.

tcp term, cmqv.alpha tcp term);

fprintf (ffile $\bar{P} t r, \bar{n} " \backslash n$ );

fprintf (ffileptr," $\backslash \mathrm{n} ")$;

fprintf (ffileptr, format3,"cd",cdo, cdk, cli) ;

fprintf (ffileptr," \n");

fprintf(ffileptr,format5,"eps0", epsv.constant_term, epsv.tcp_term) ;

fprintf (ffileptr," $\backslash n "$ );

fprintf (ffileptr, format5, "epsa", epsv.alpha_term, epsv.alpha_tcp_term);

fprintf (ffileptr," \n");

fprintf (ffileptr, format2,"cheo", cheo," /rad") ;

fprintf (ffileptr, format2,"chea", chea," /rad") ;

fprintf (ffileptr," $\backslash \mathrm{n} ")$;

fprintf (ffilePtr, format2,"chede", chede,"/rad") ; 


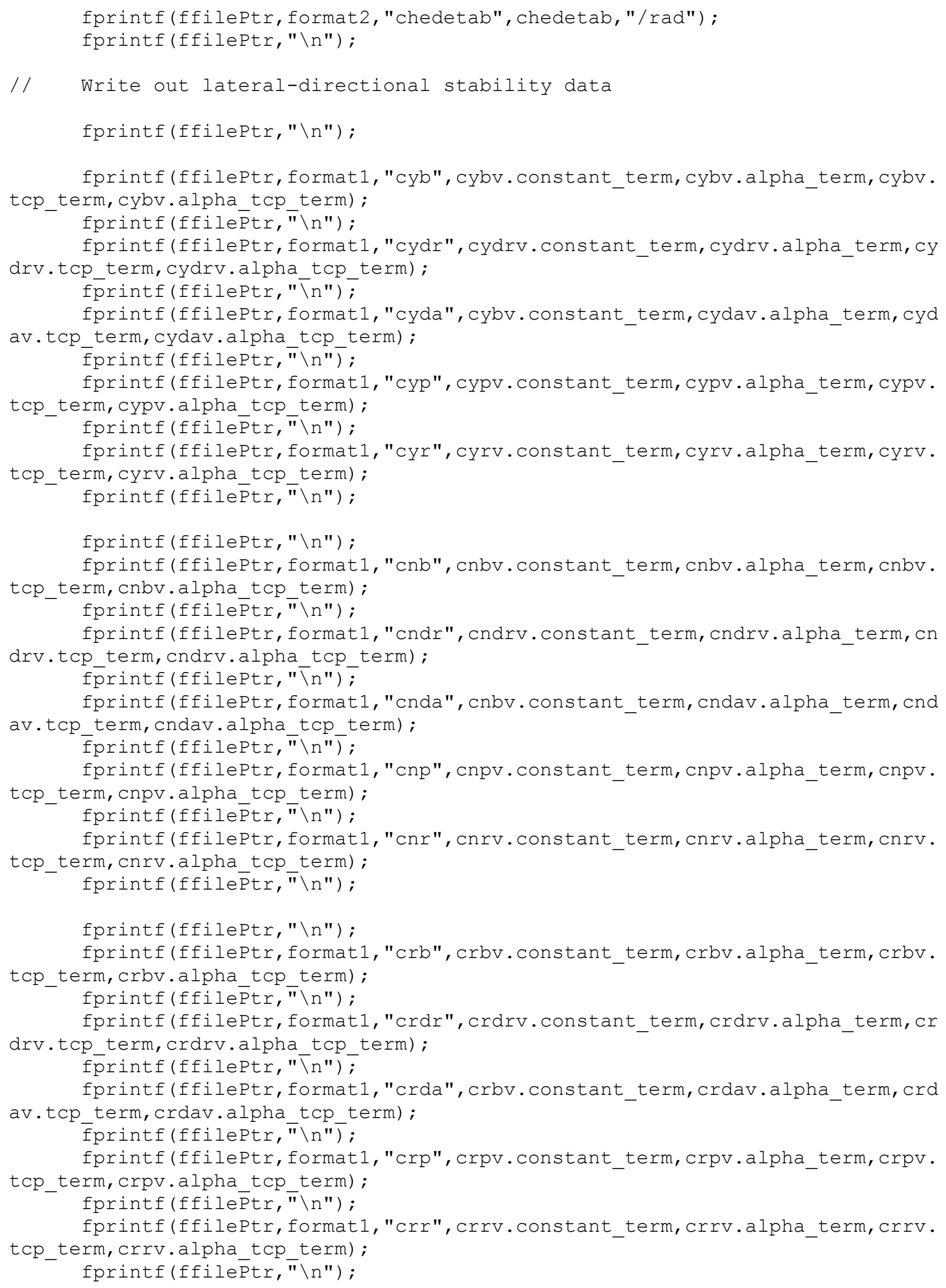




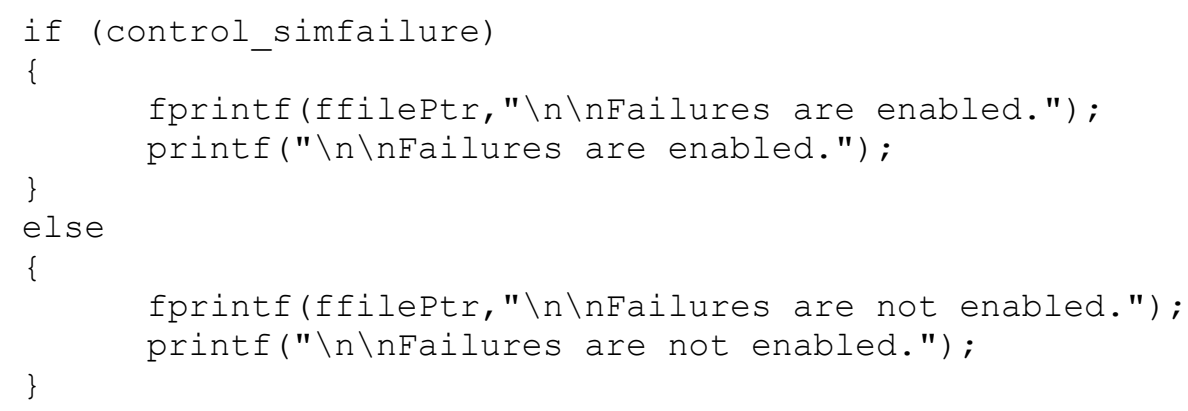




\section{Function "CJ144_data.cpp"}

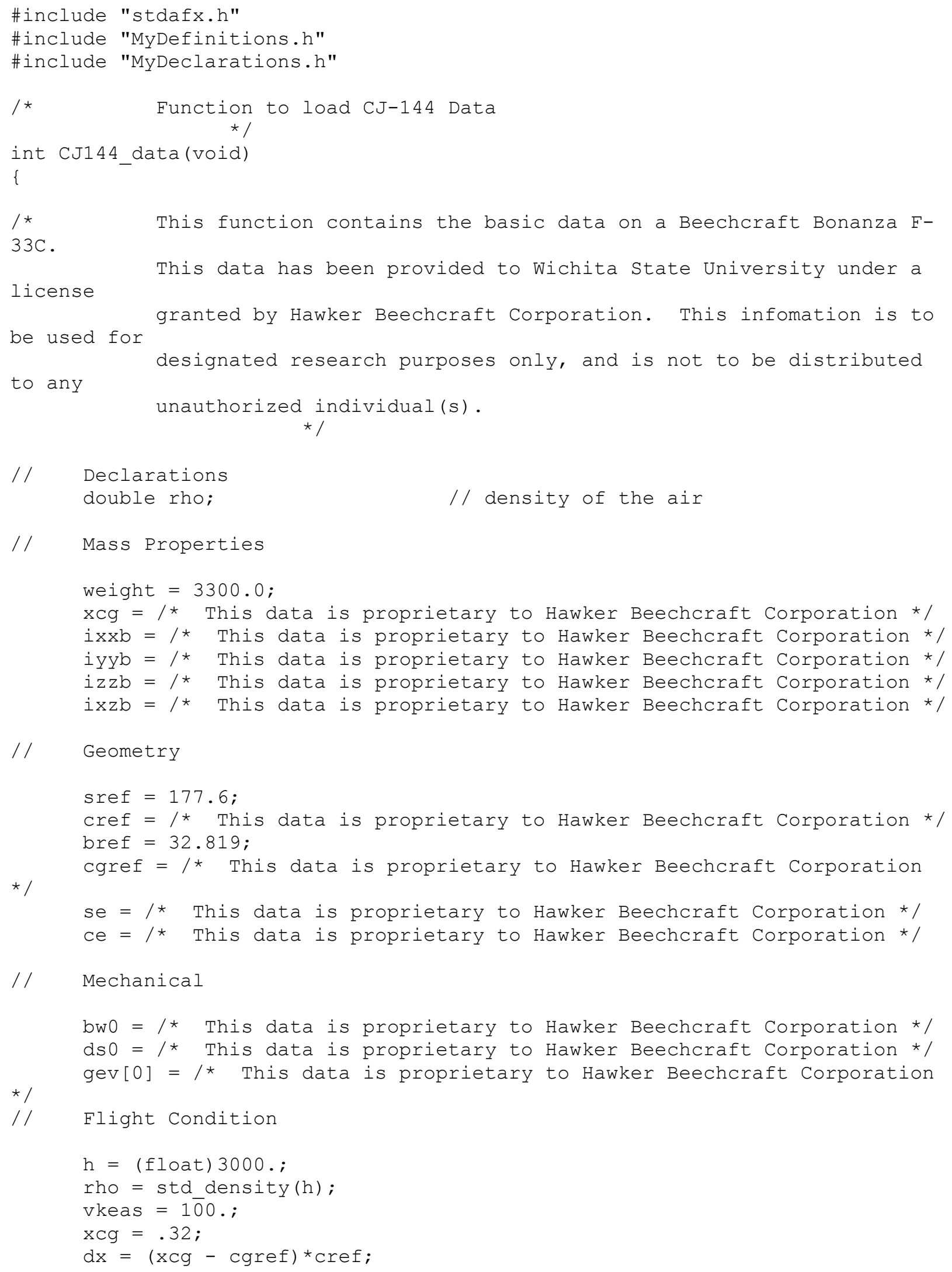




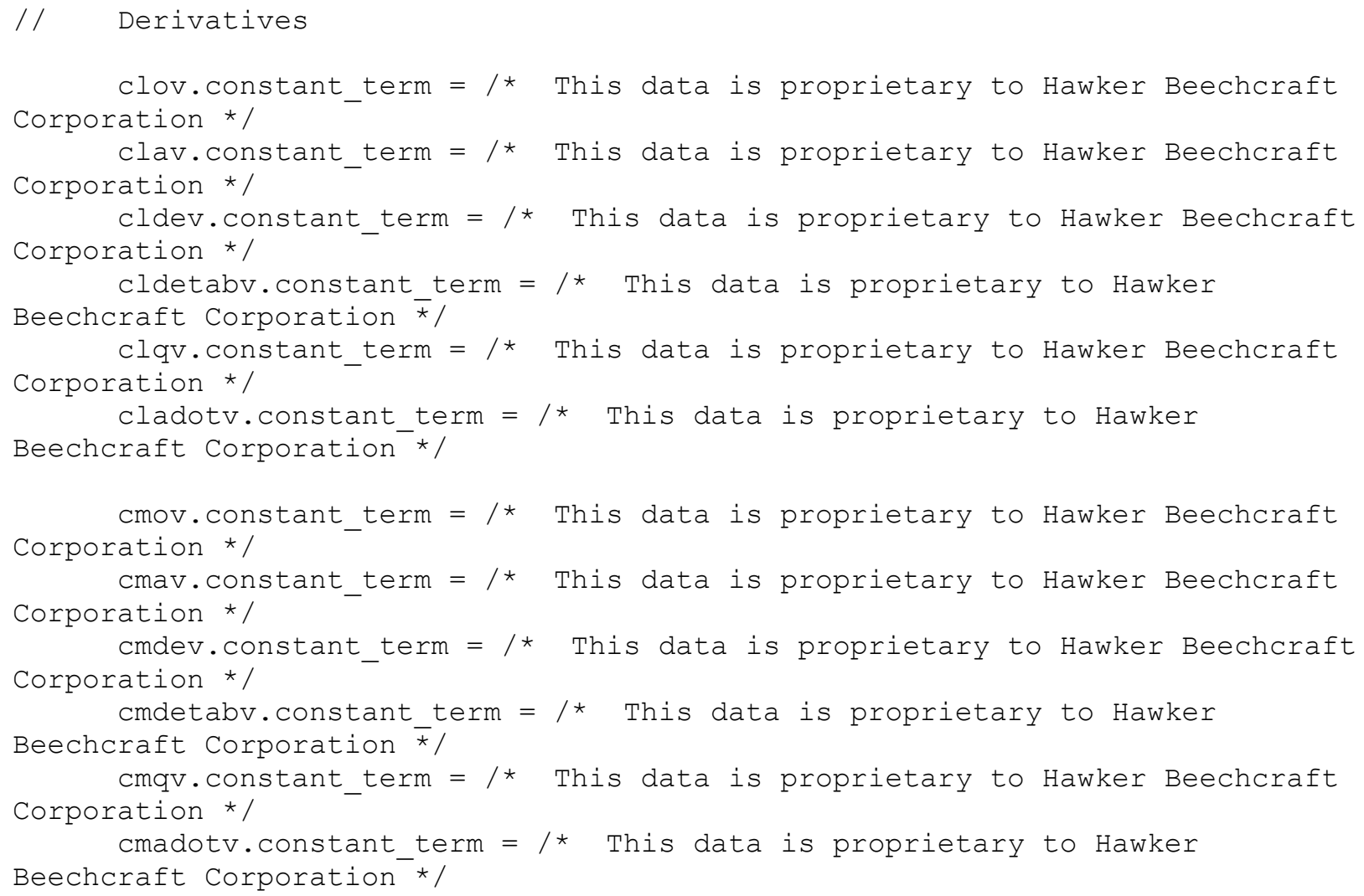




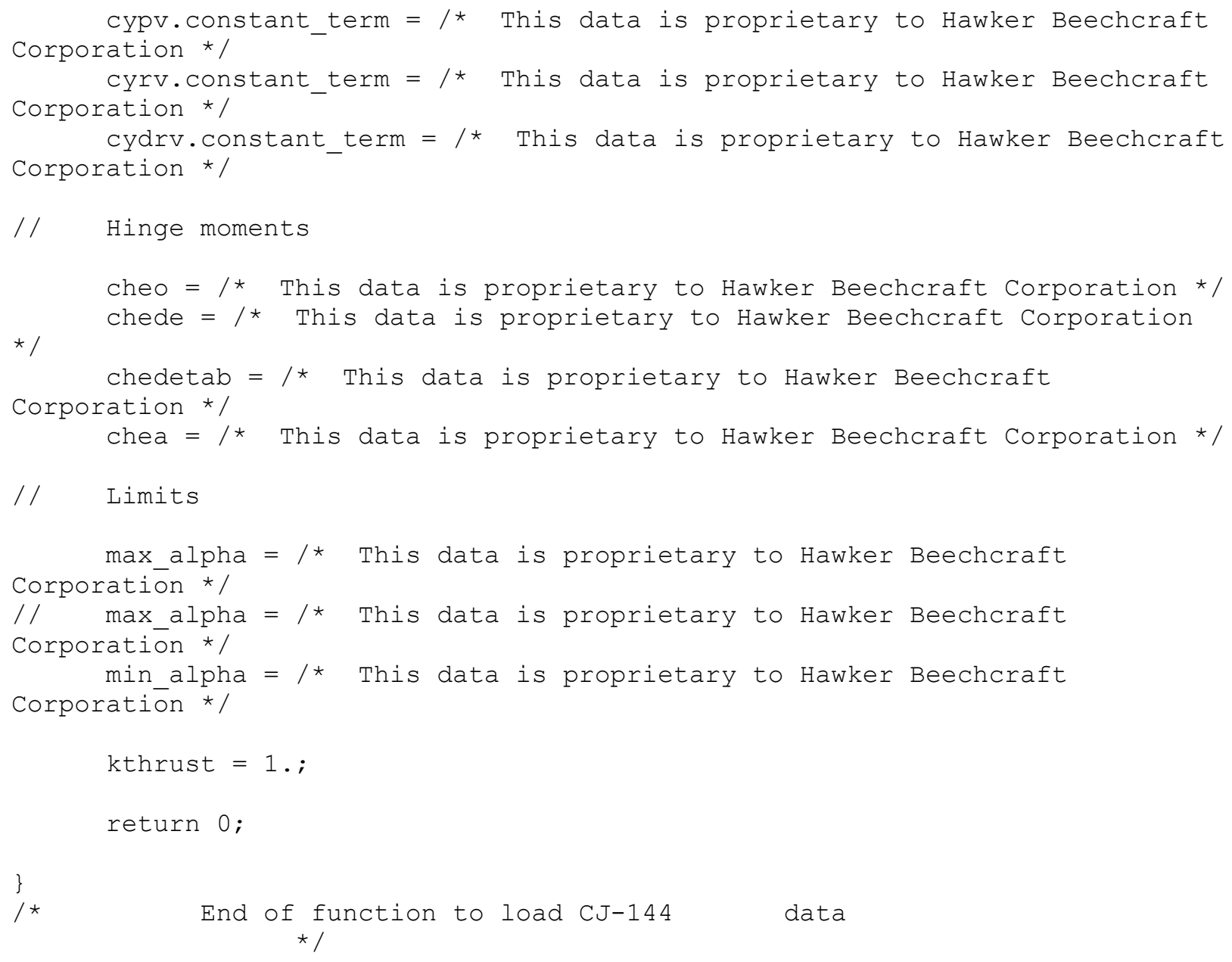




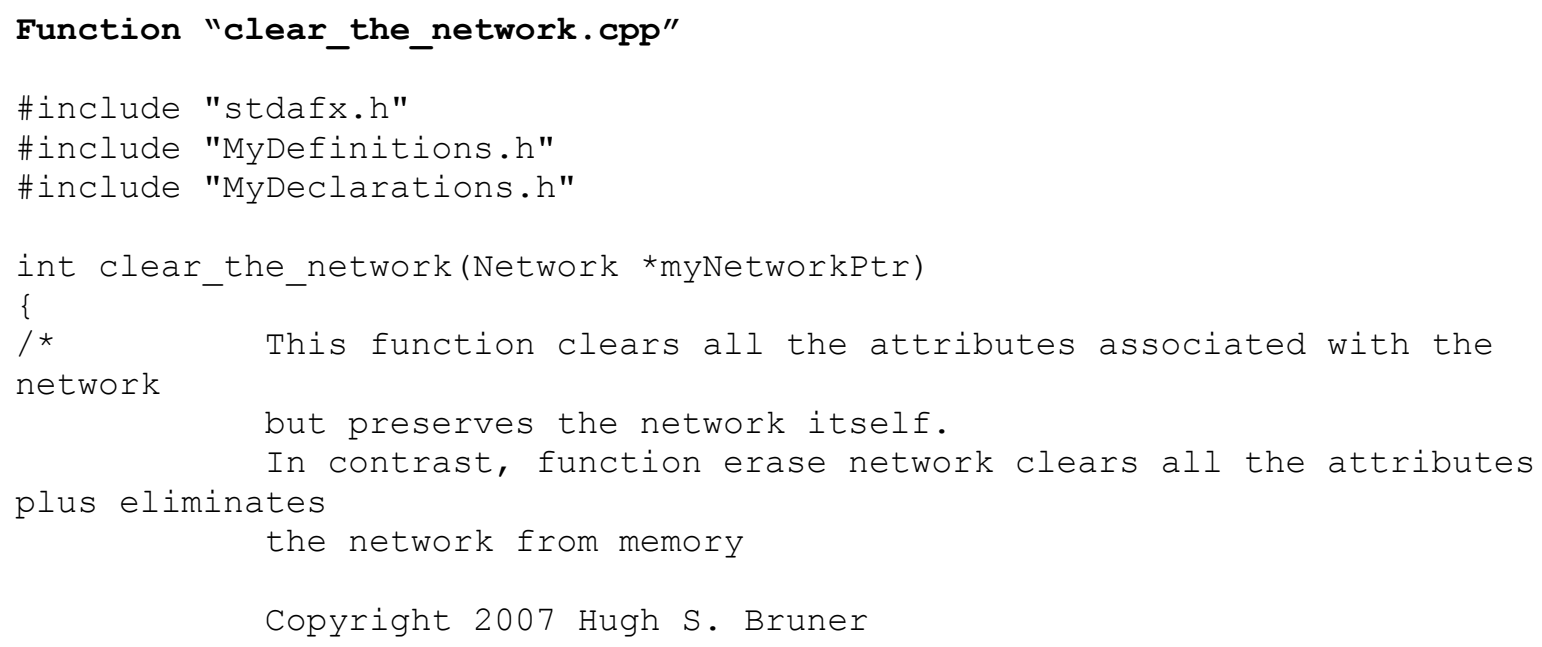




\section{Function "command_input.cpp"}

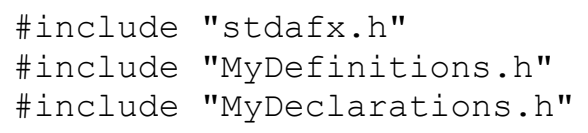

through of a simulation 


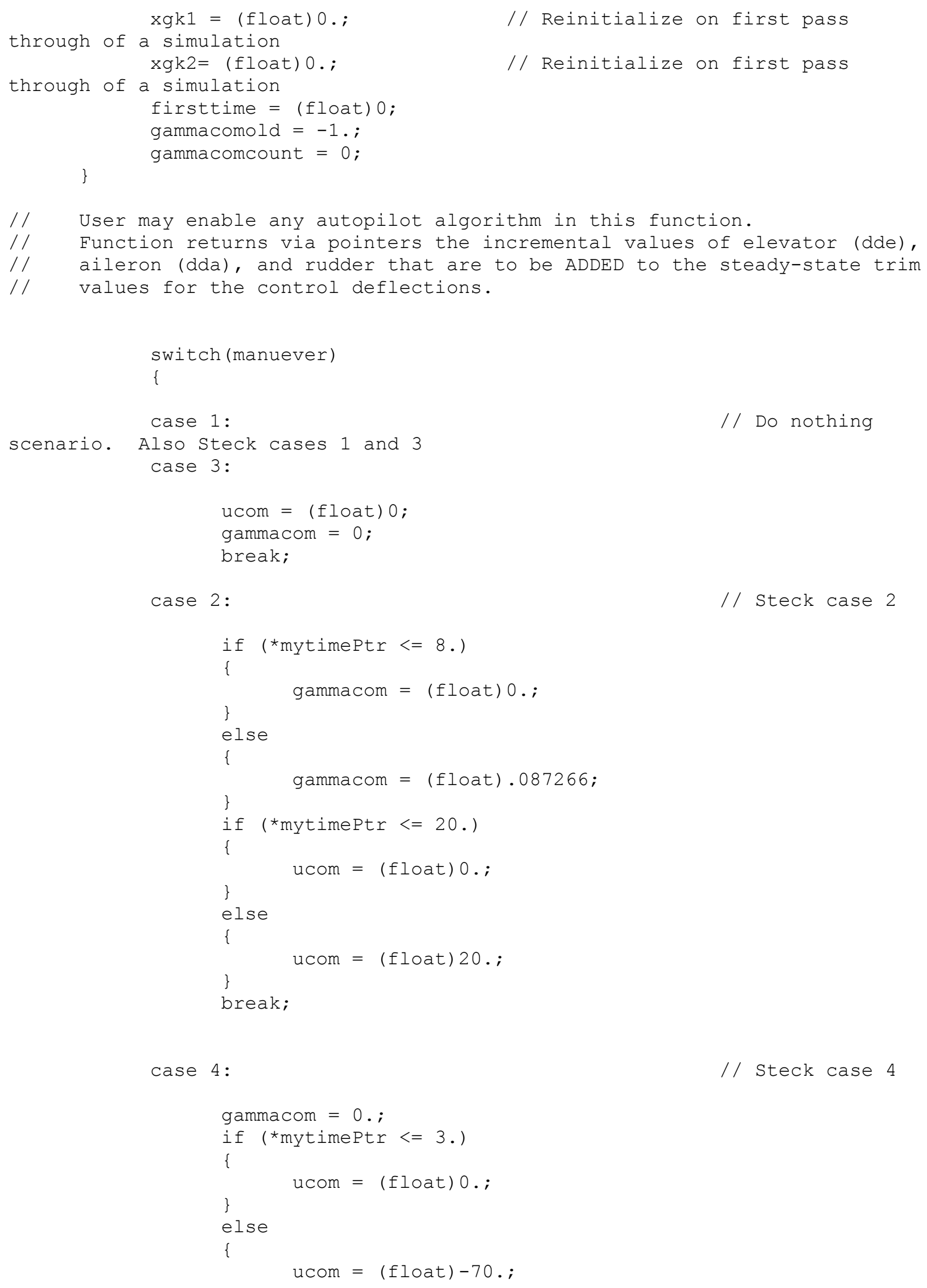


\}

break;

case 5:

// Hard pull up

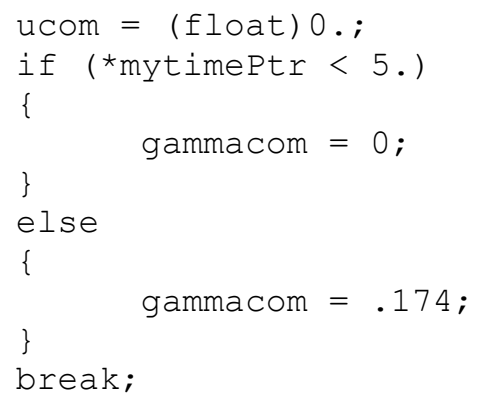

/ /

/ /

xuk2) /denomu;

xgk2) /denomg;

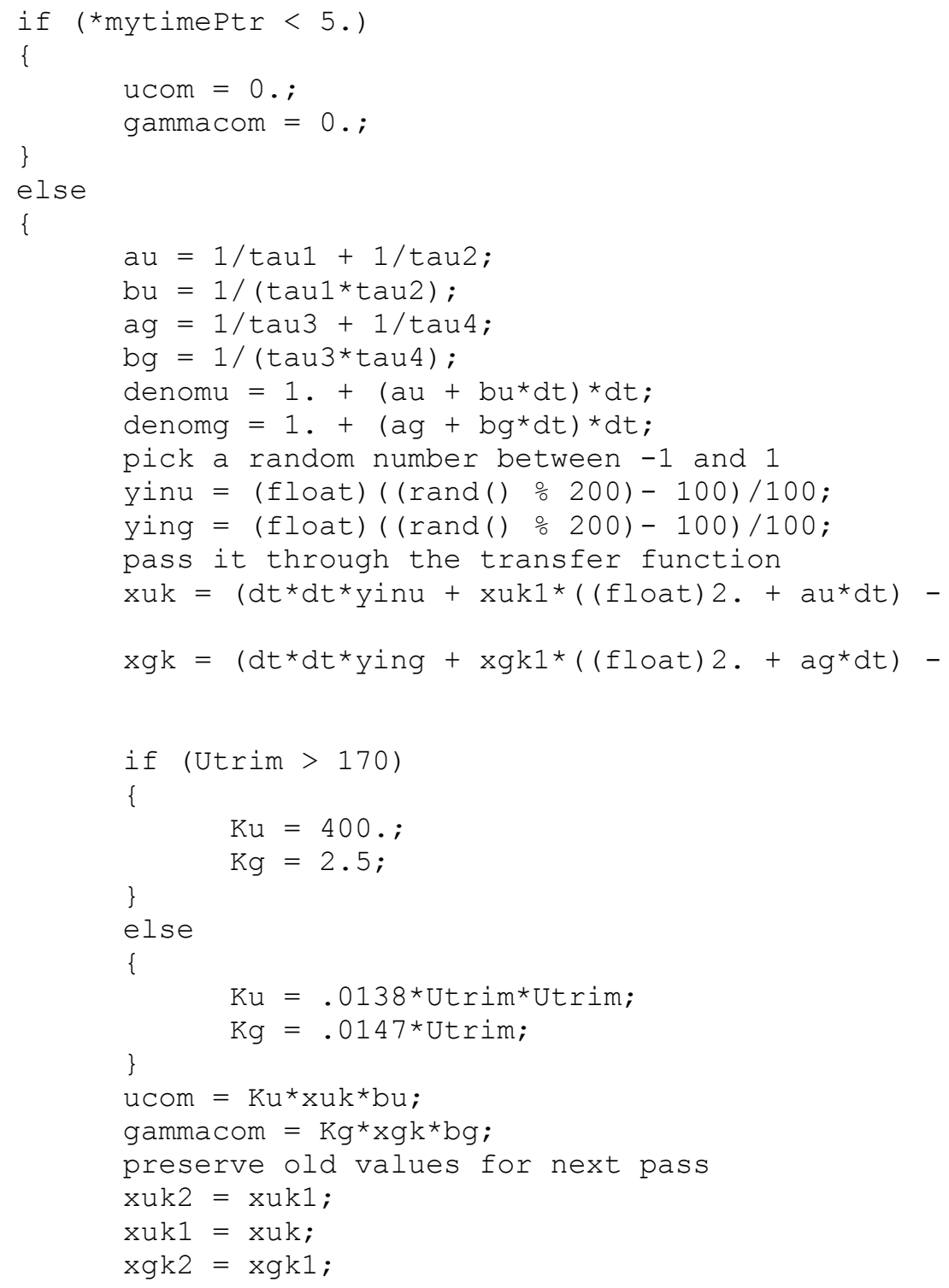




$$
\mathrm{xgk} 1=\mathrm{xgk}
$$

\}

break;

case 7:

// Increasing

step functions gamma then speed

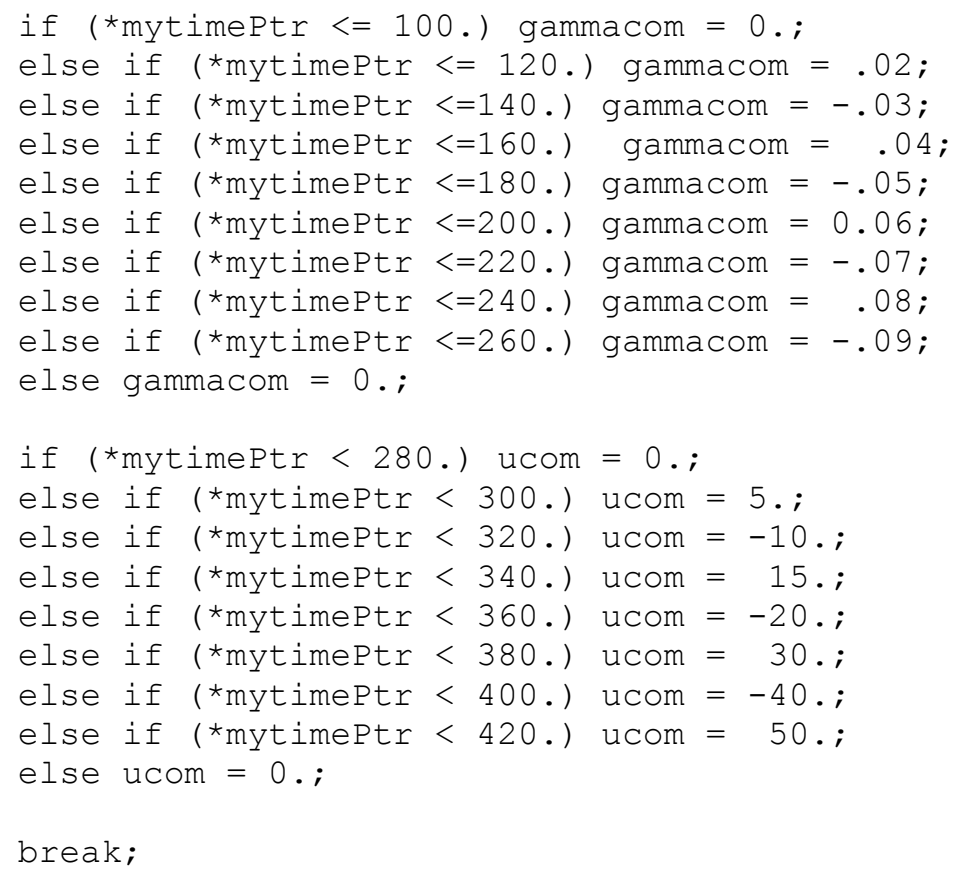


square wave on speed and gamma

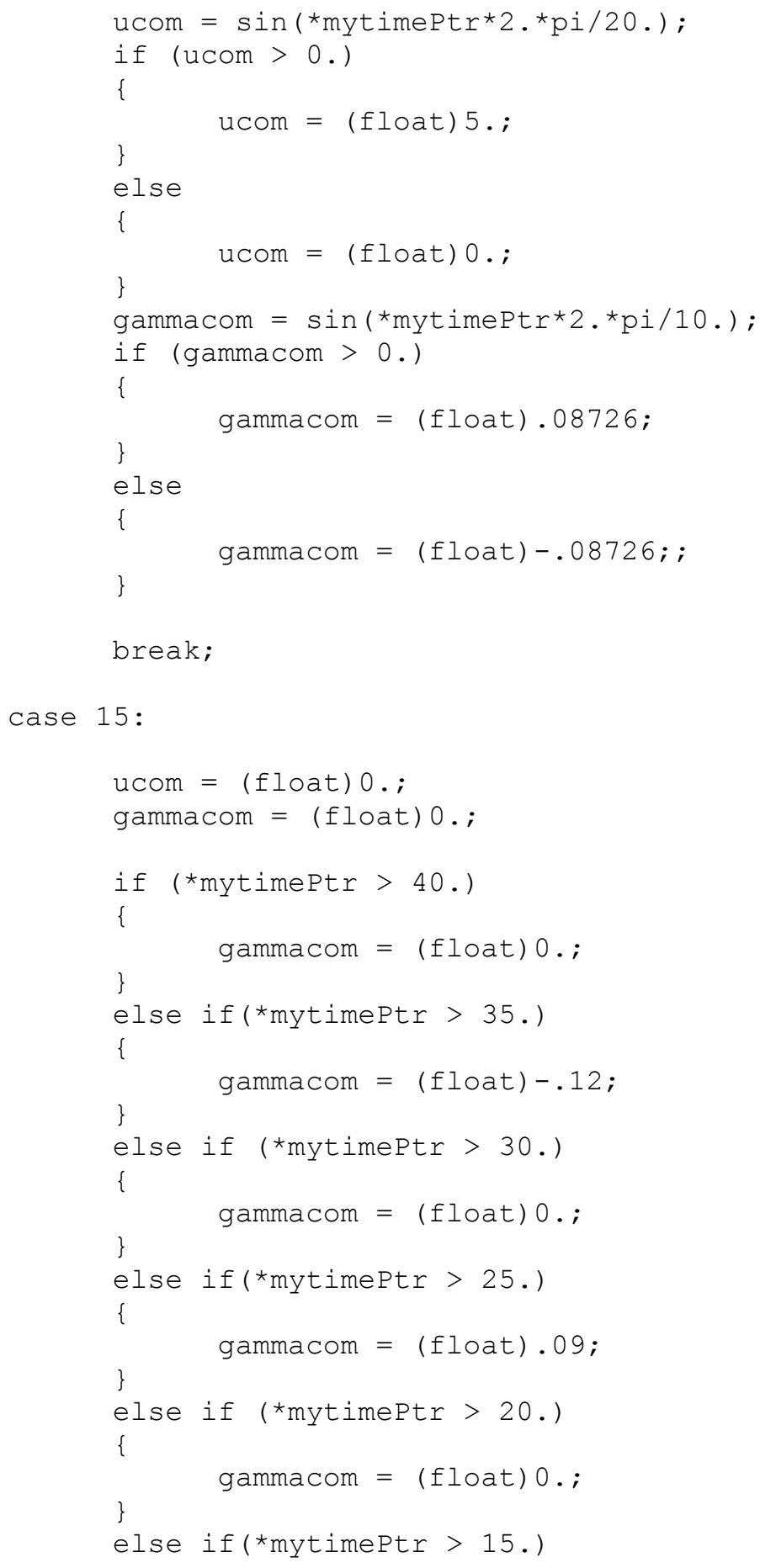




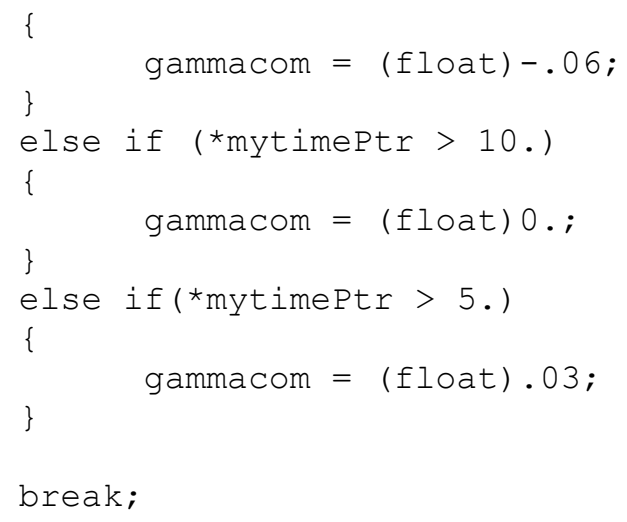




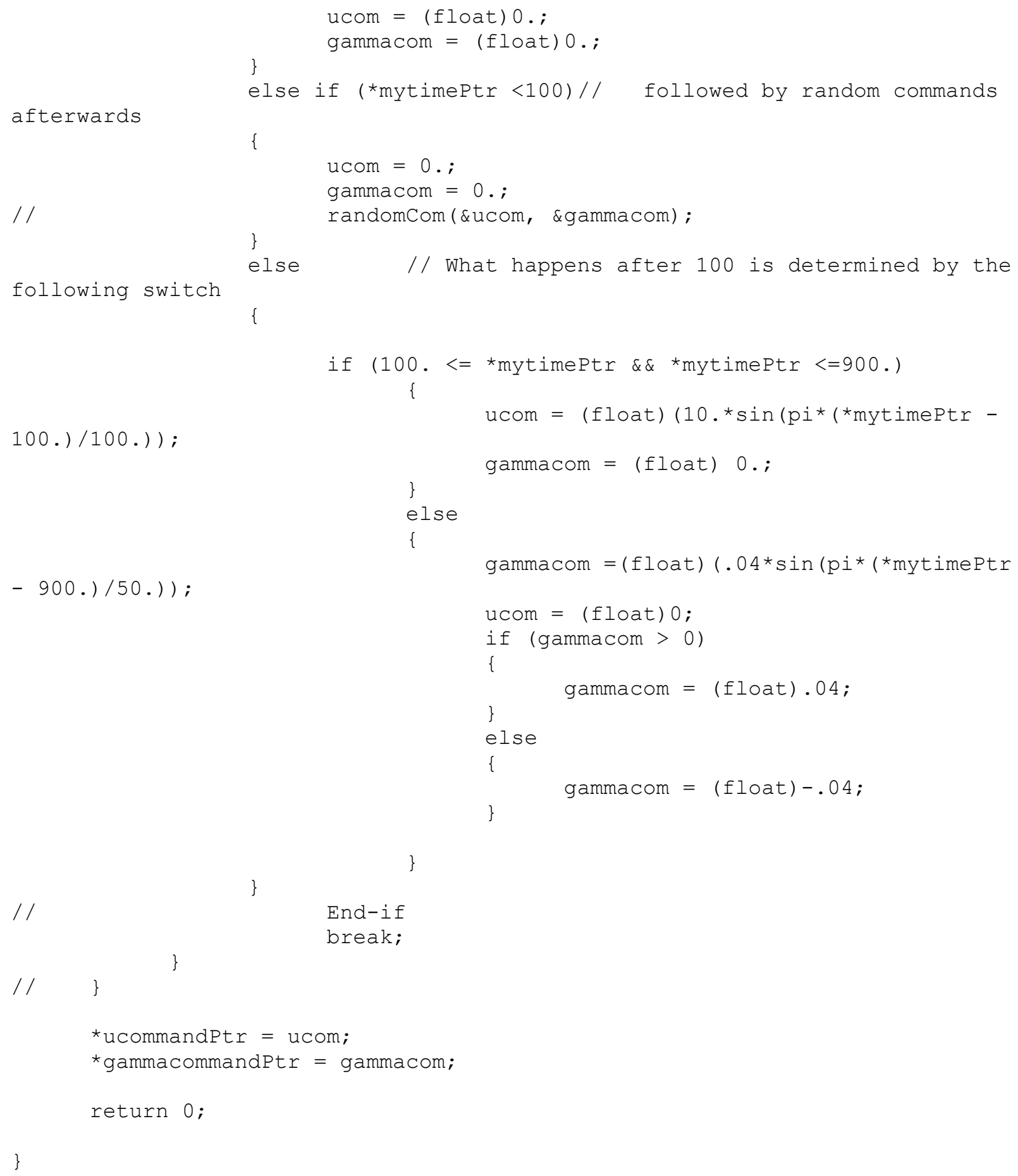




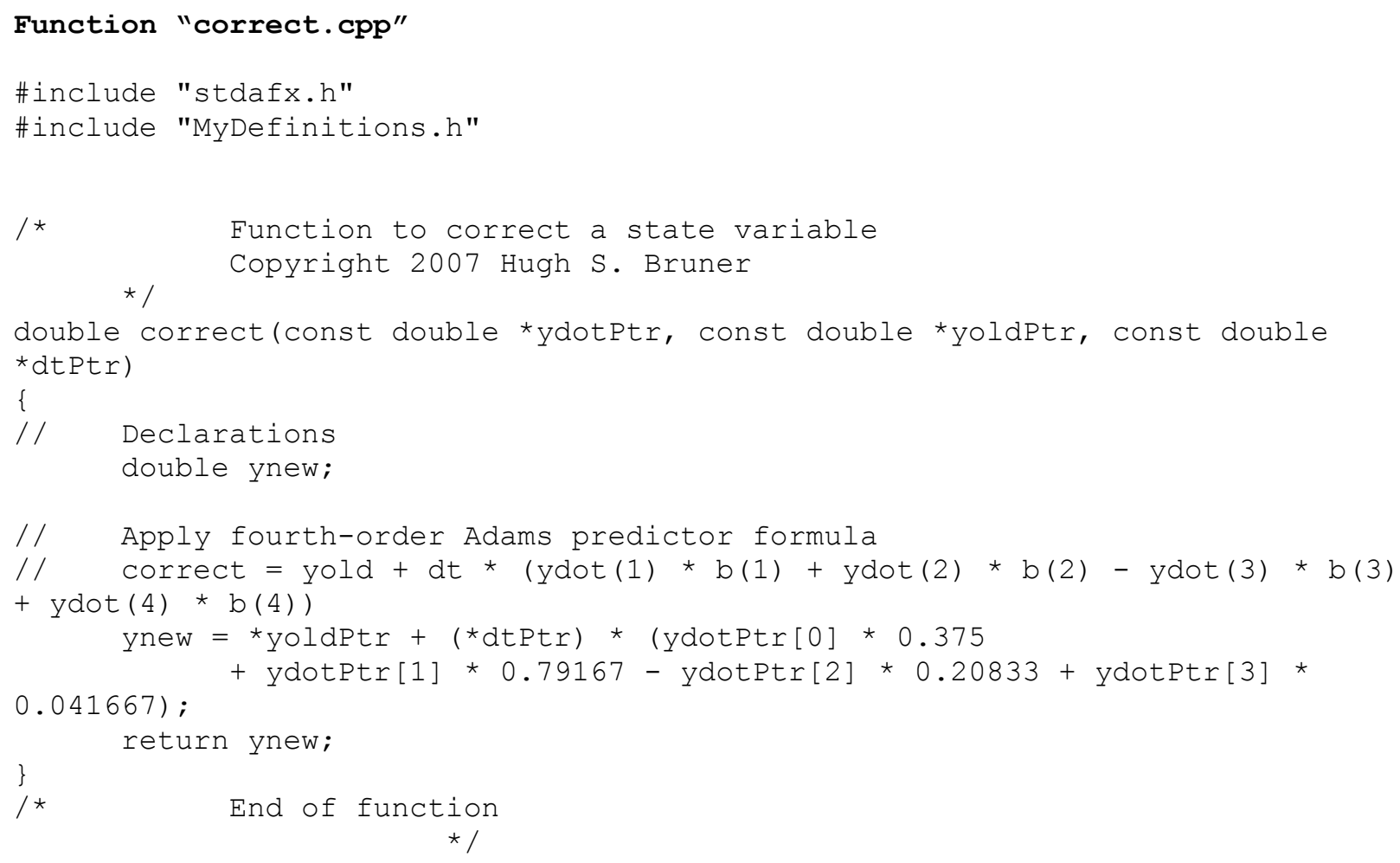




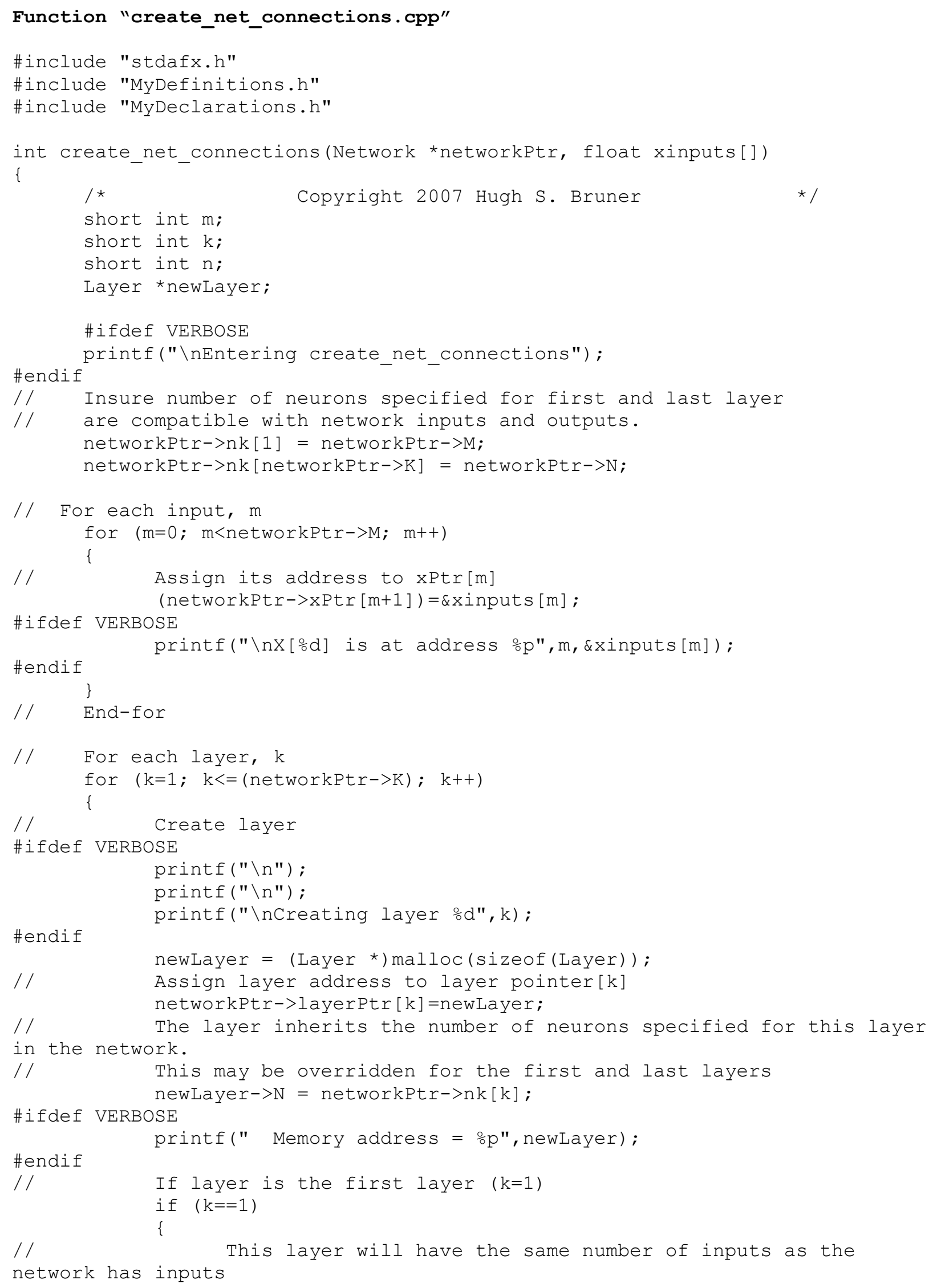




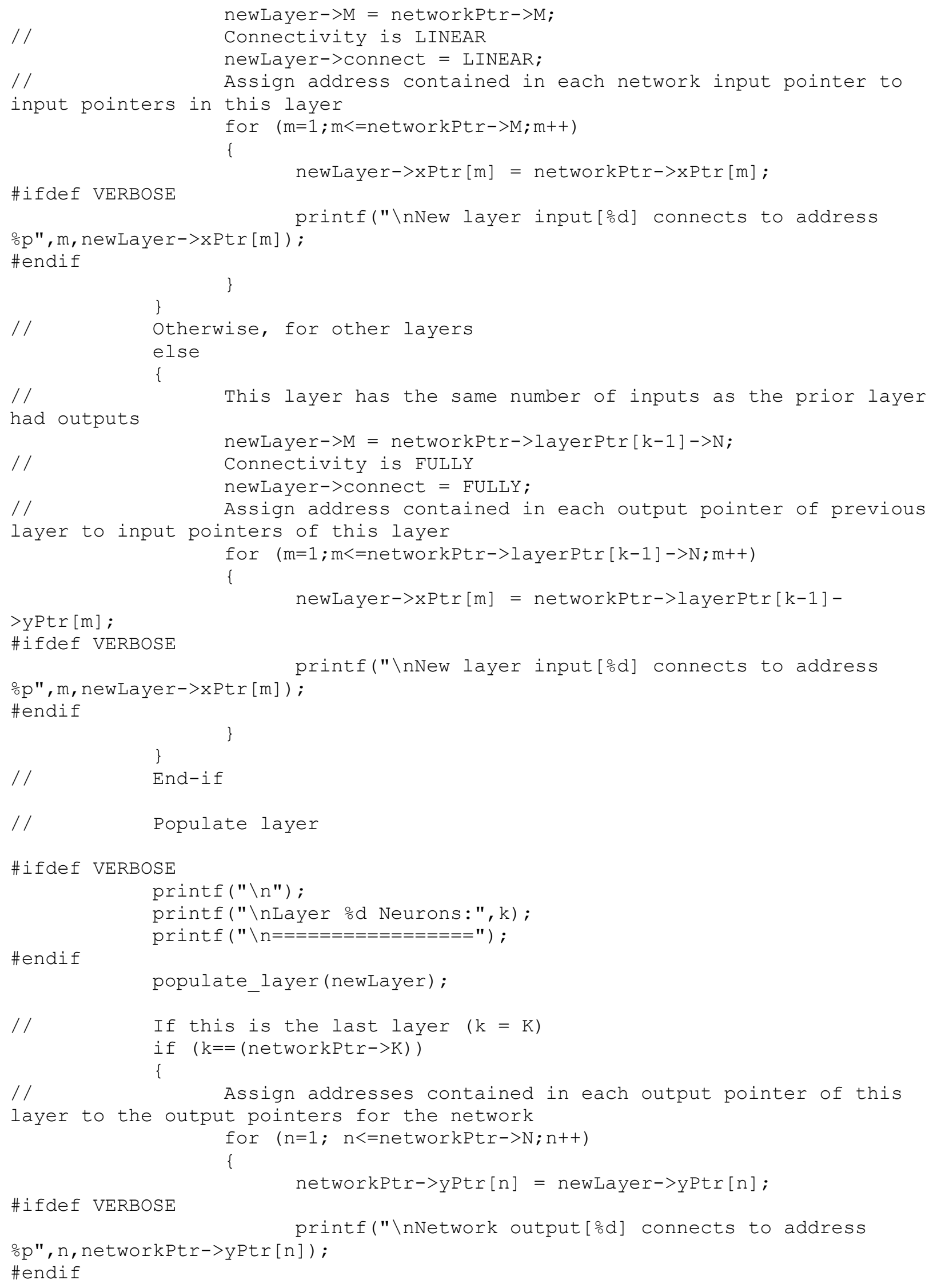




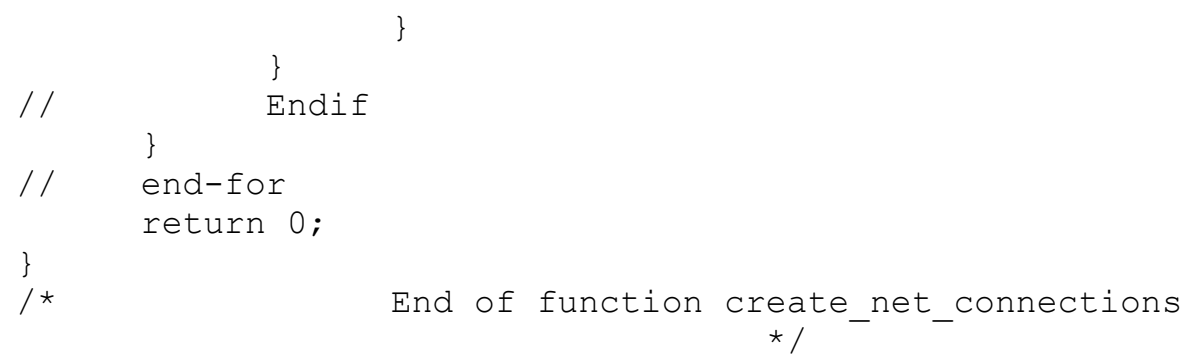




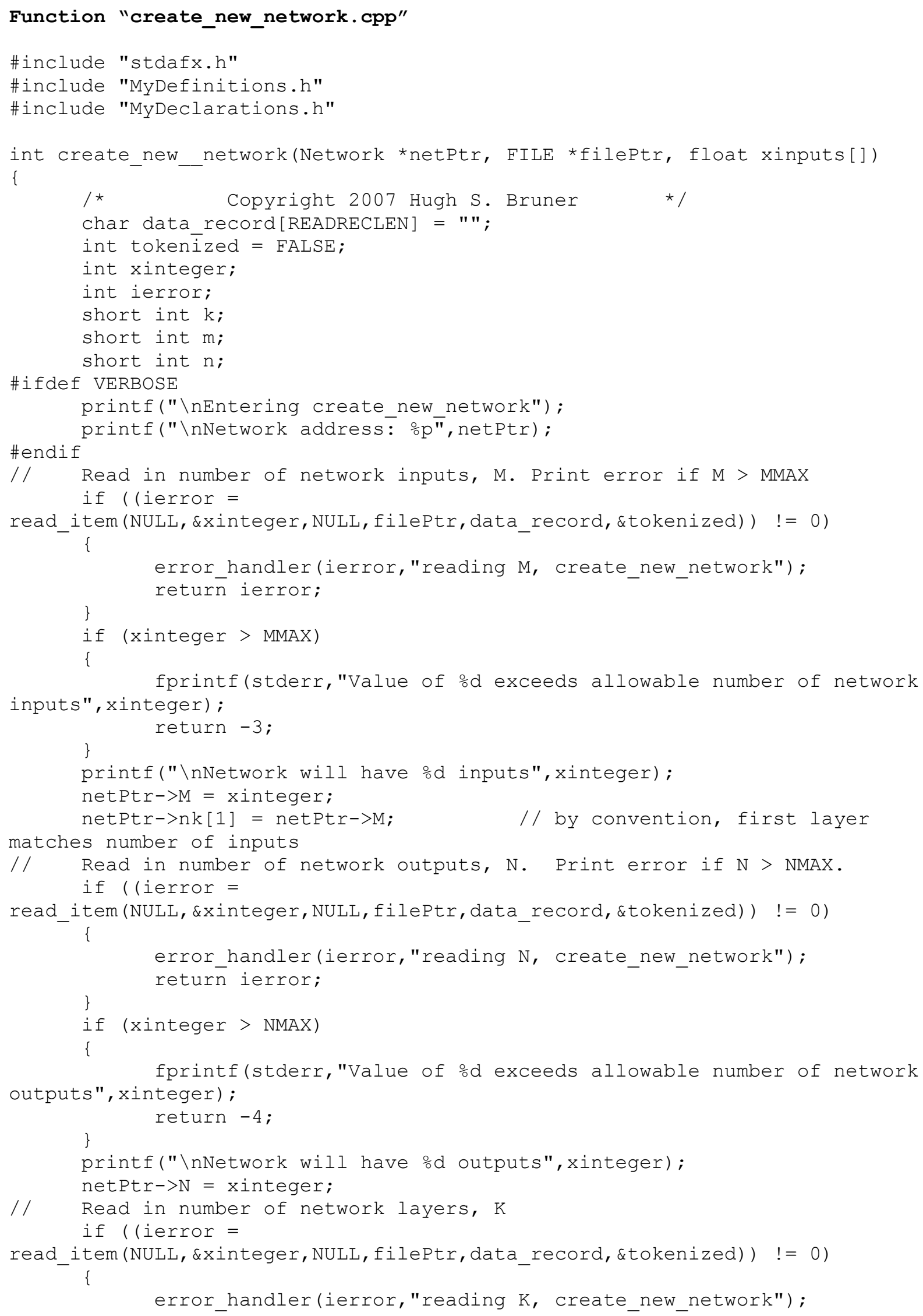




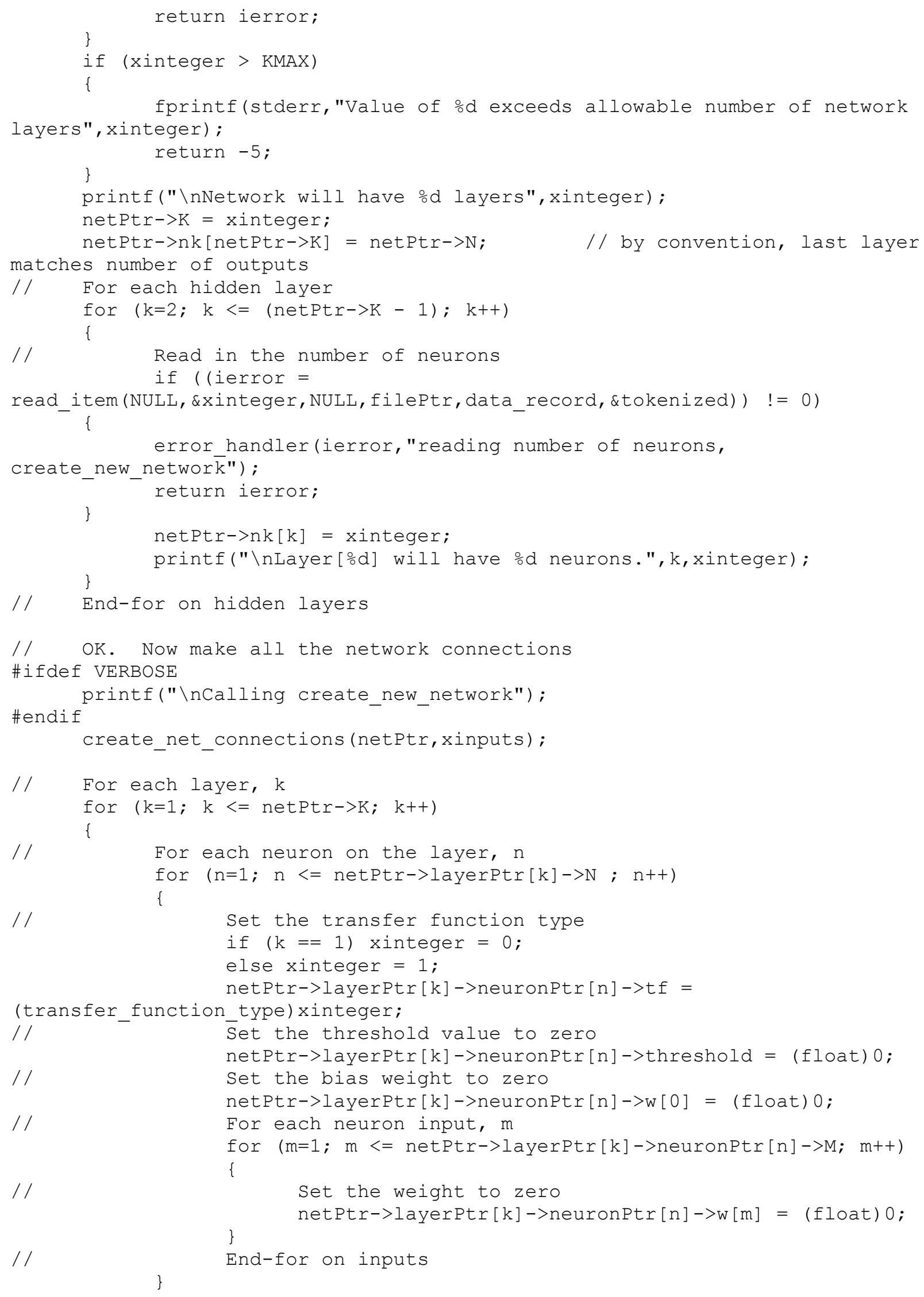




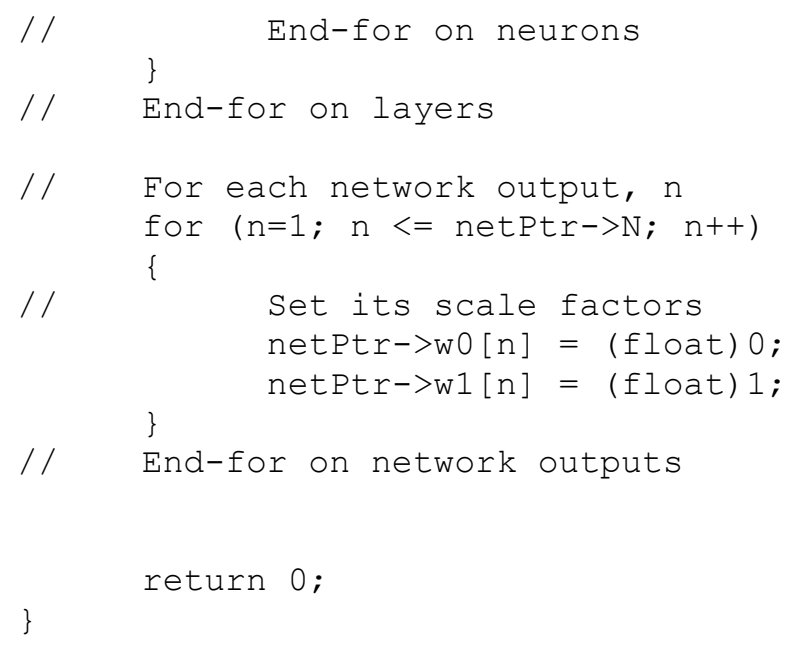




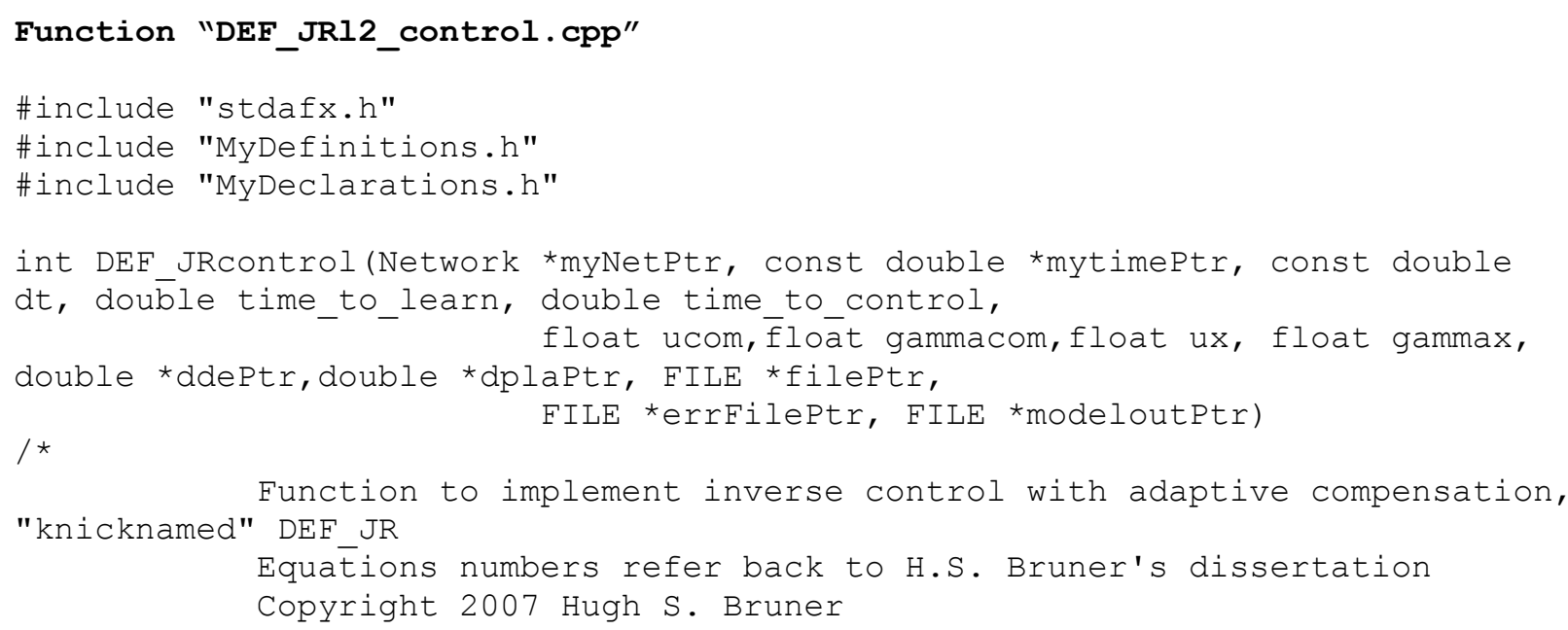




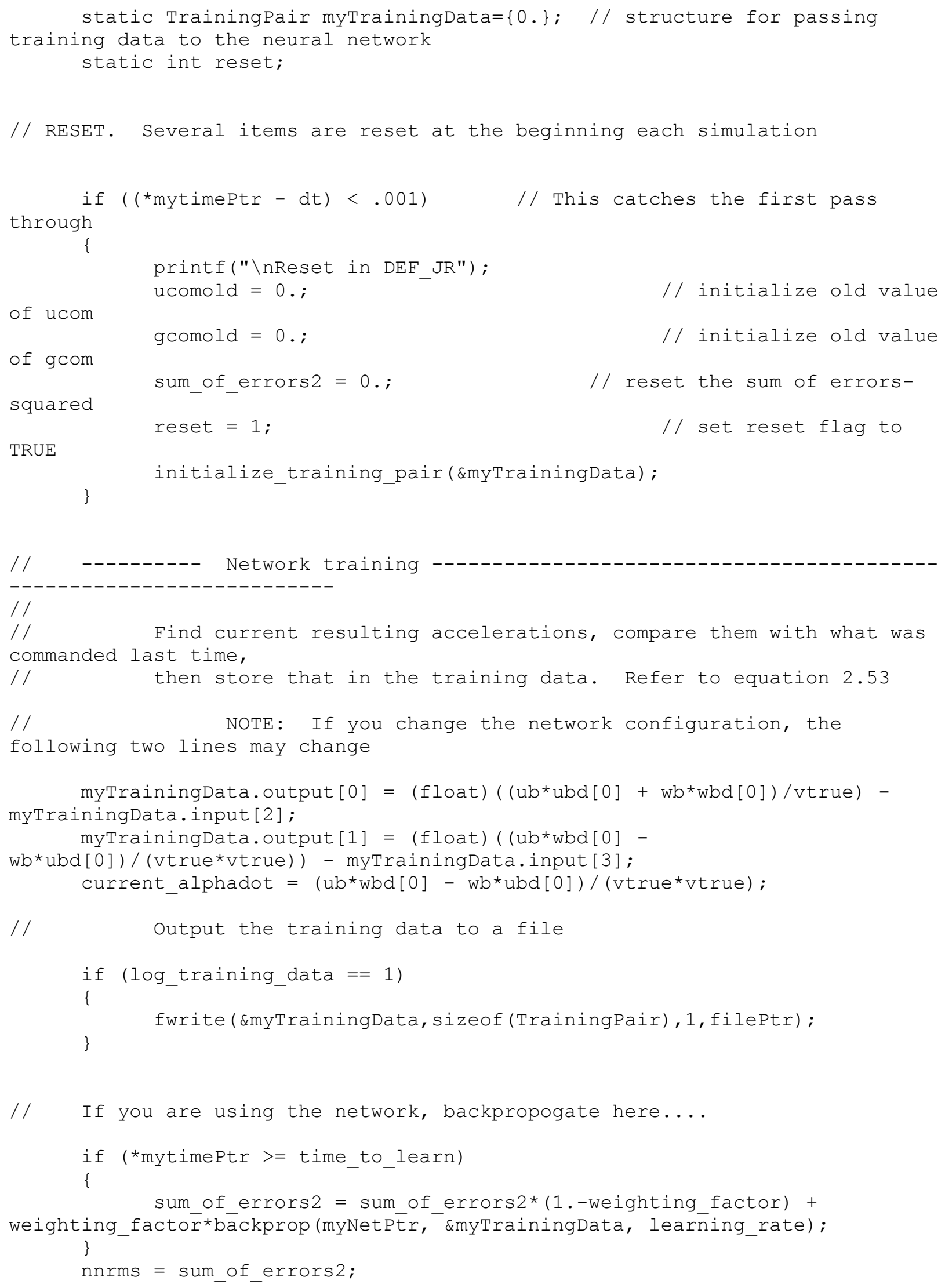




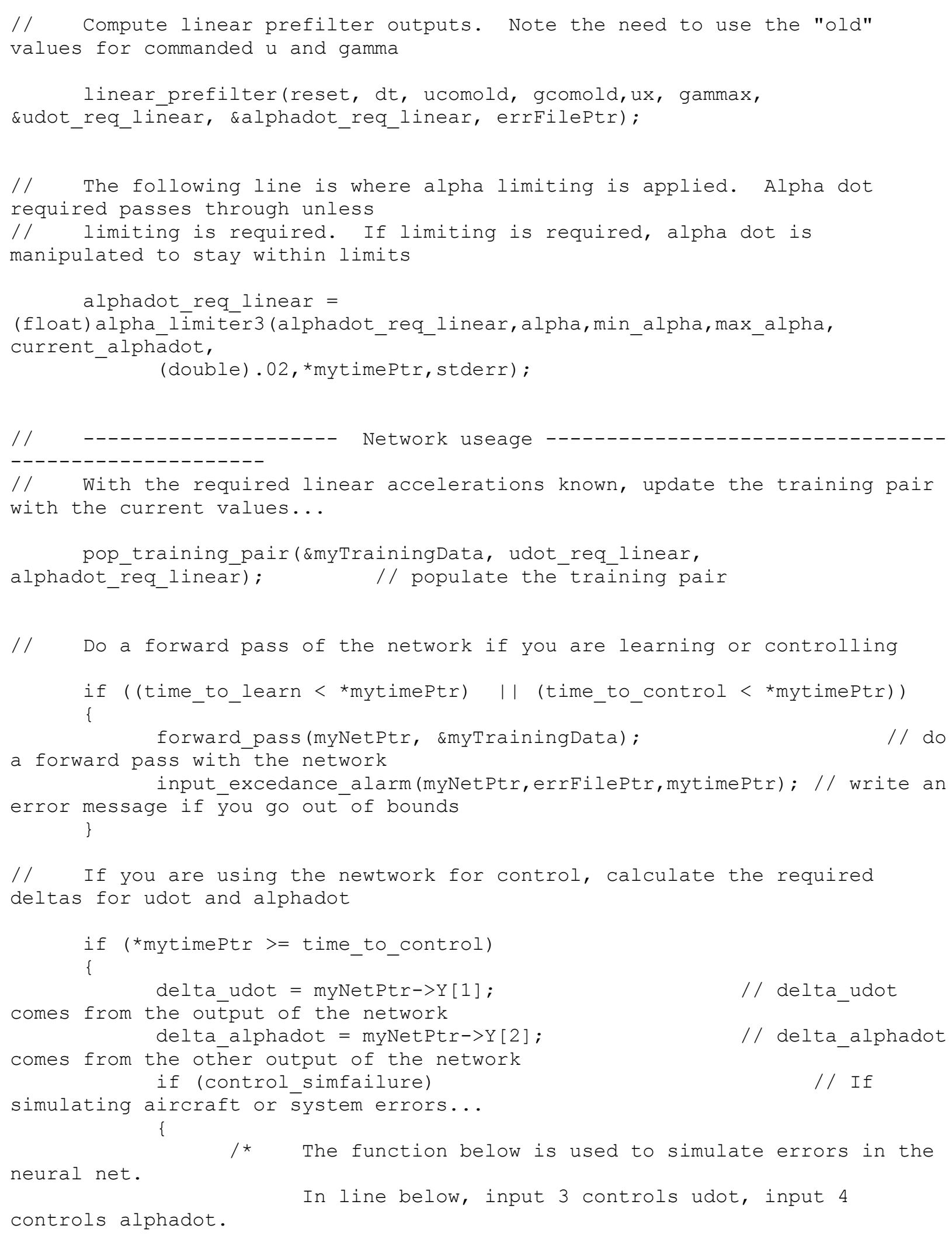




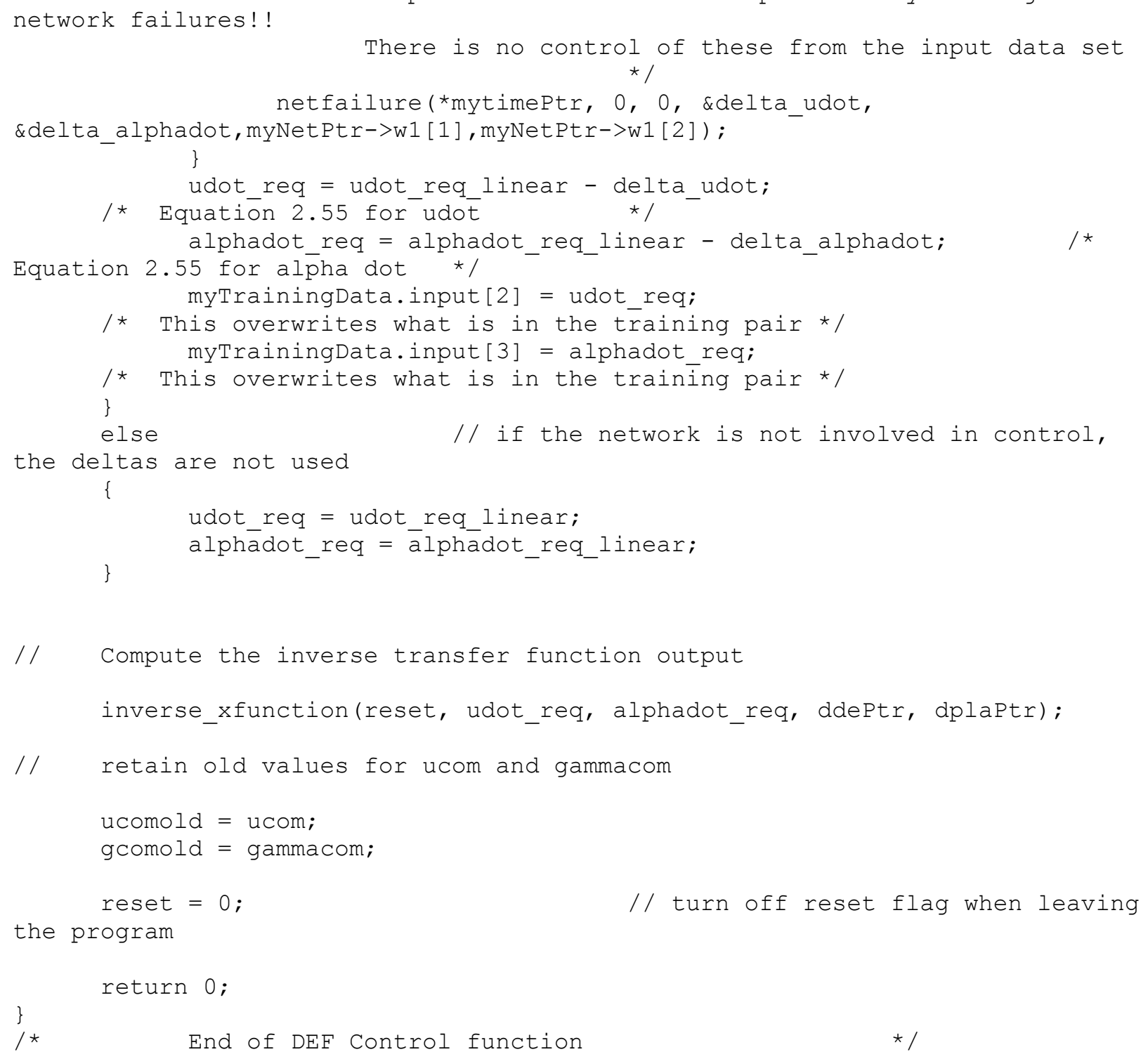




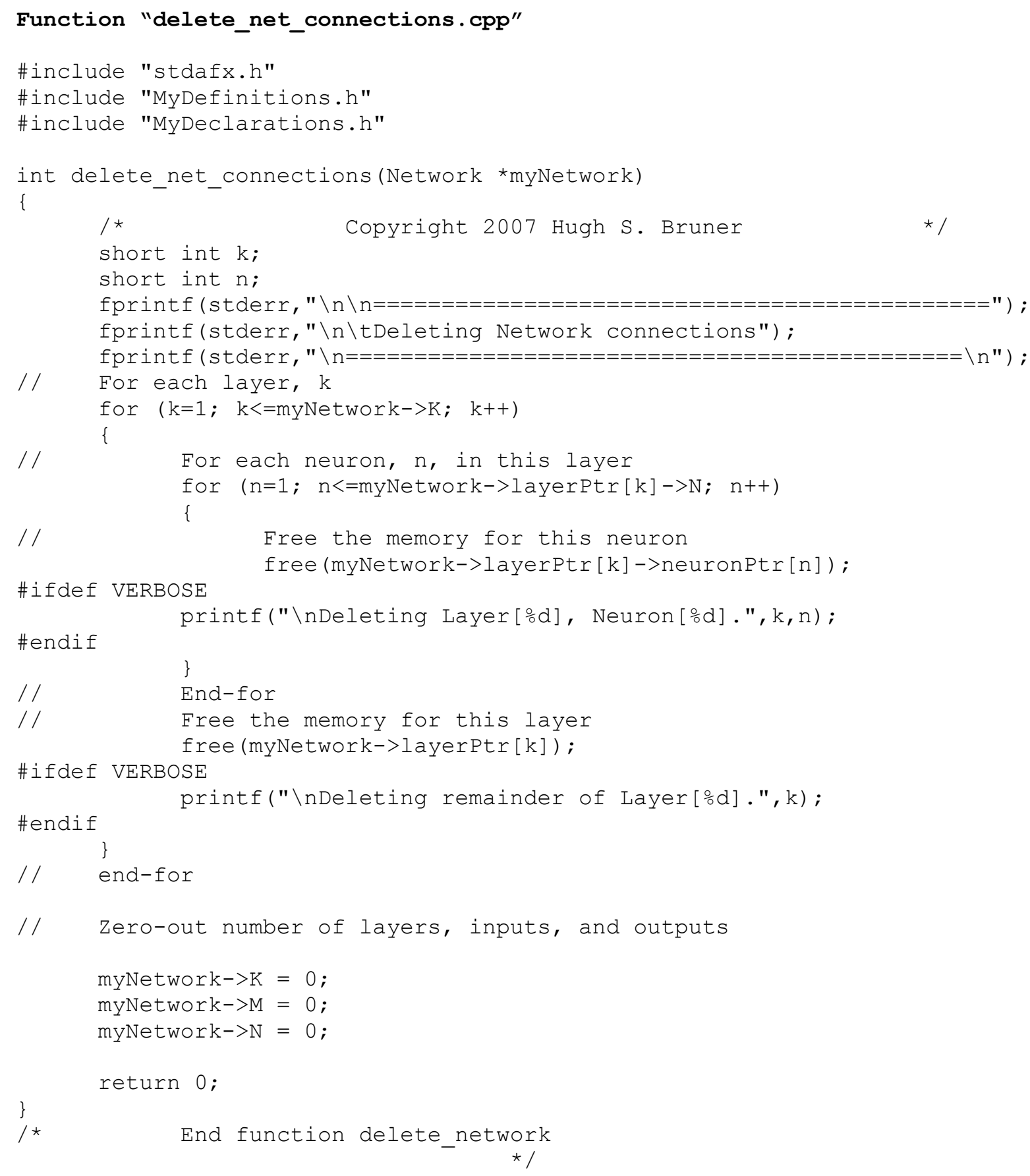




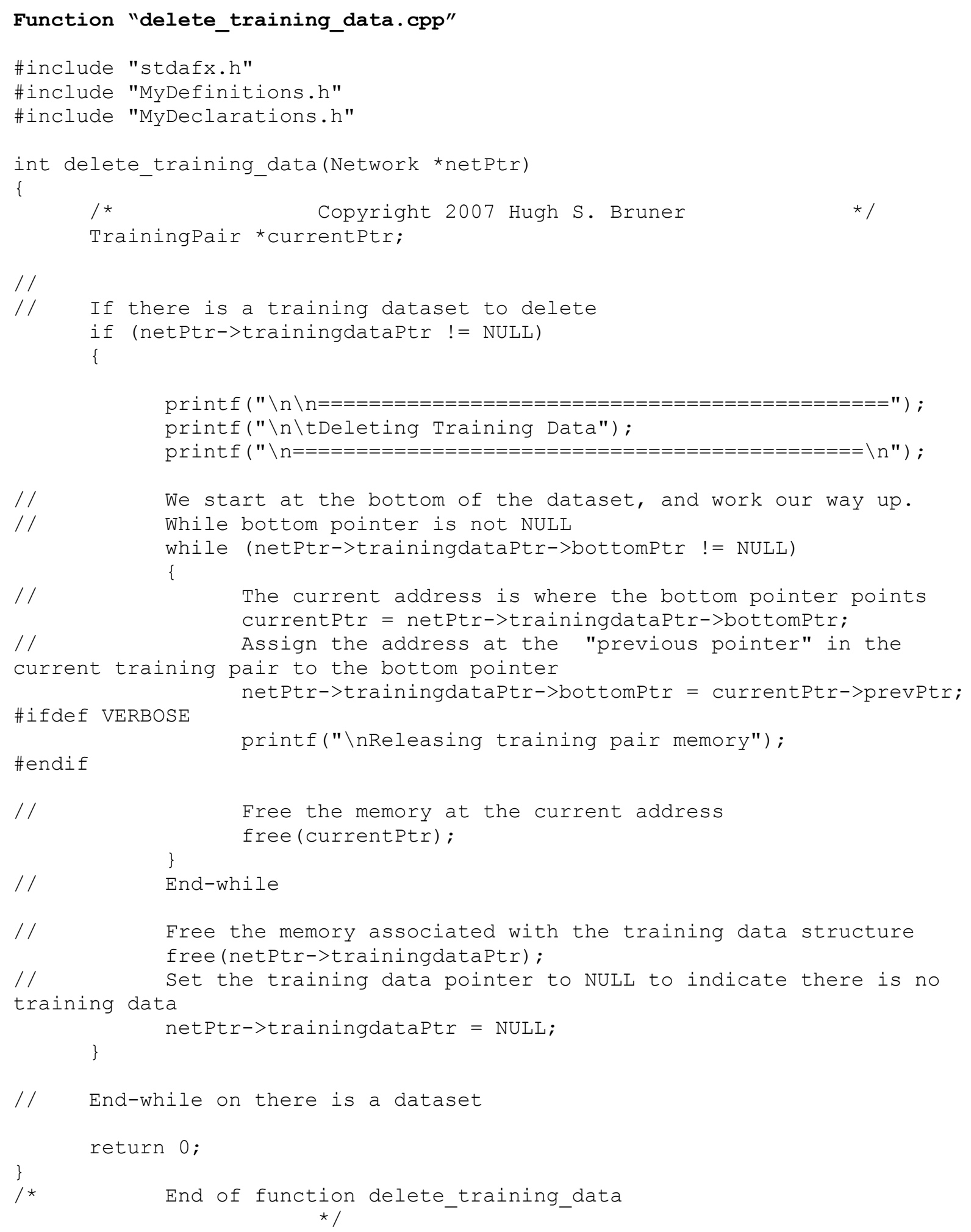




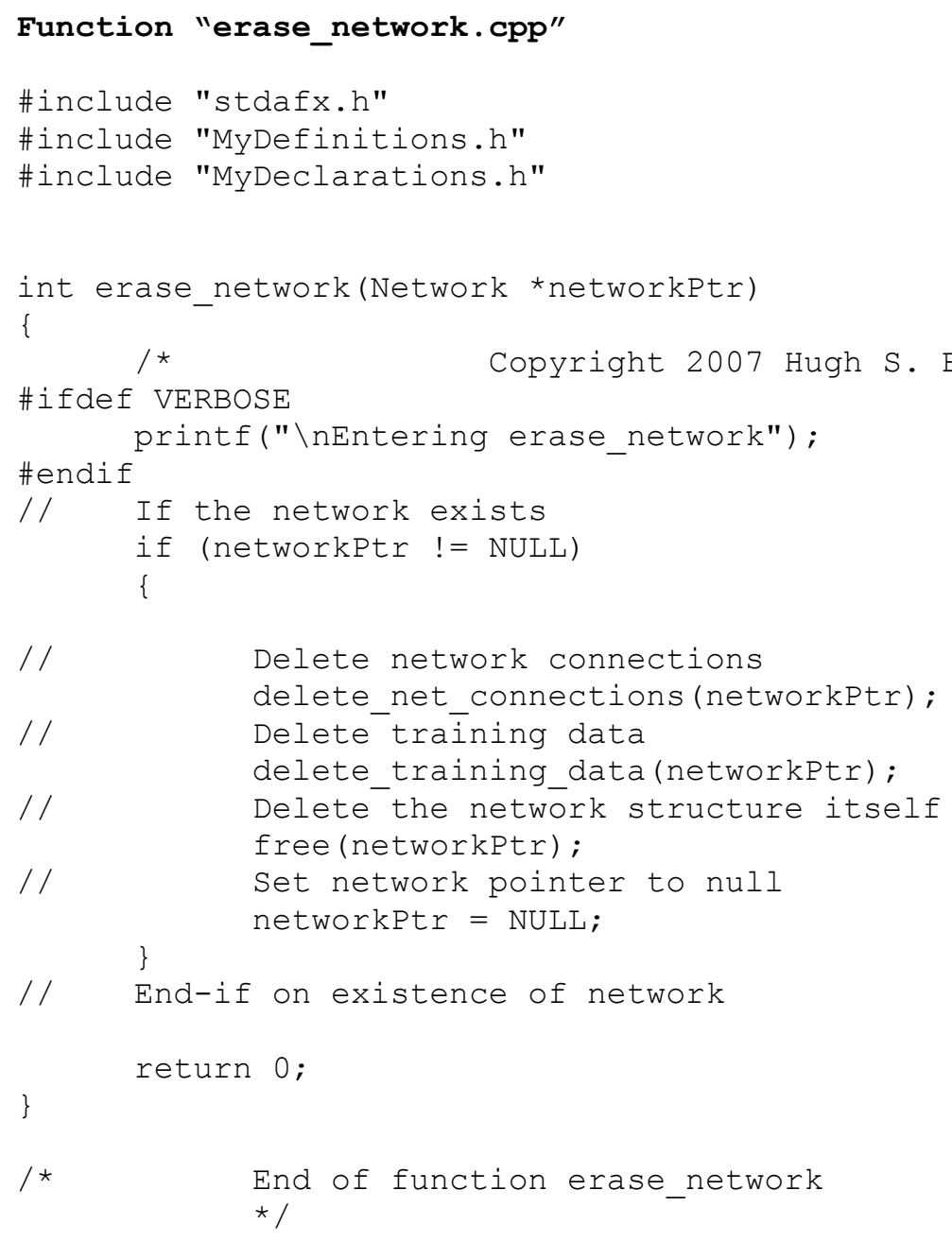




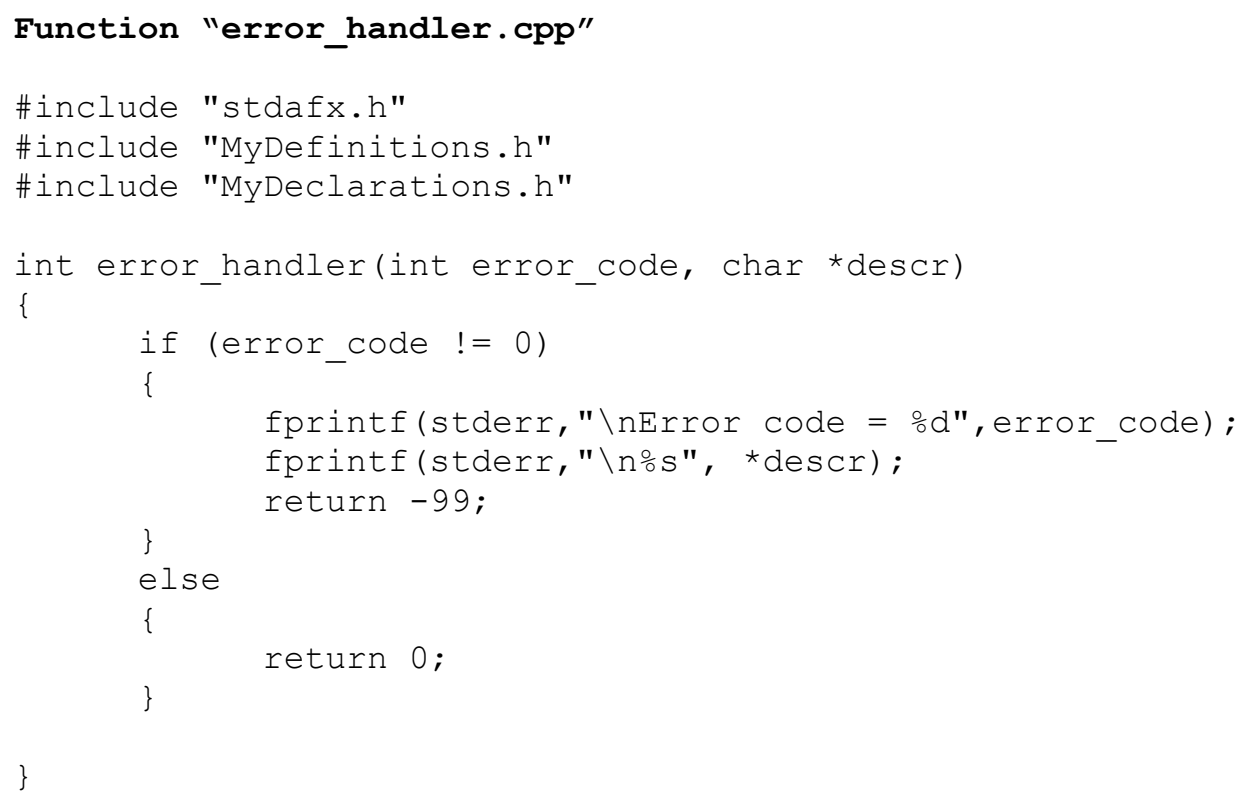




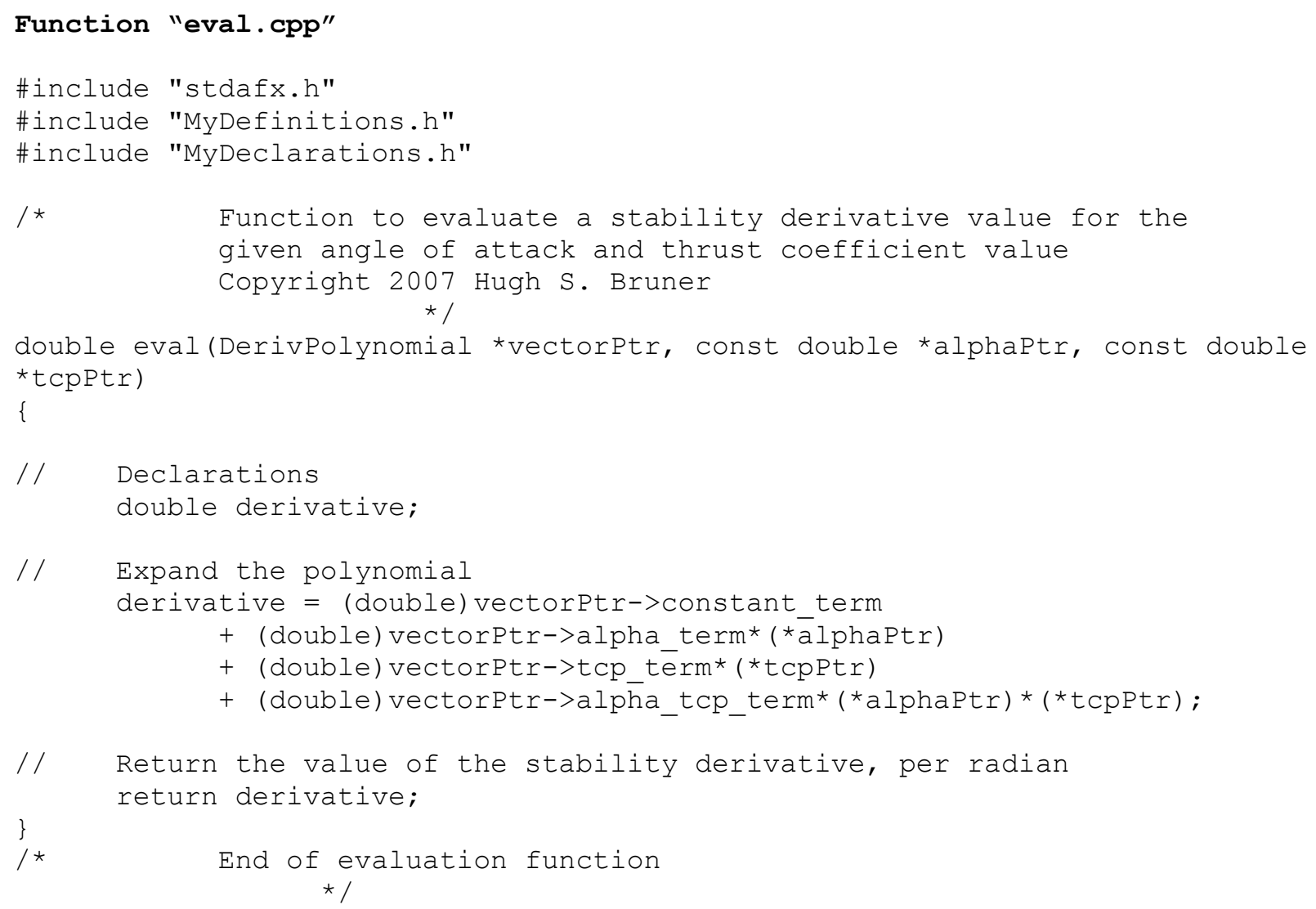




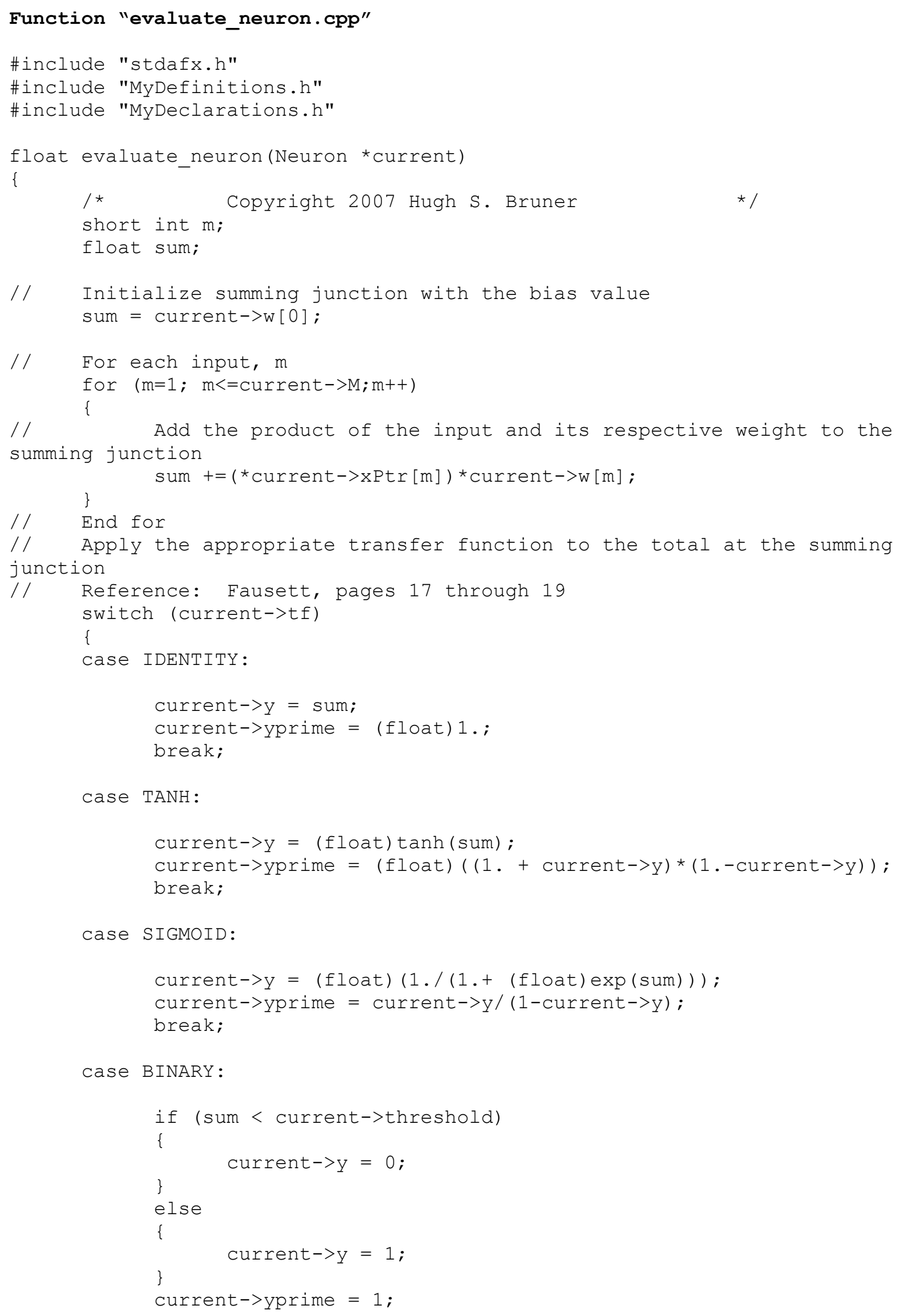




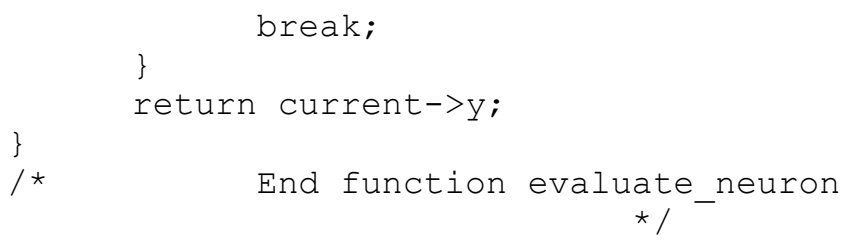




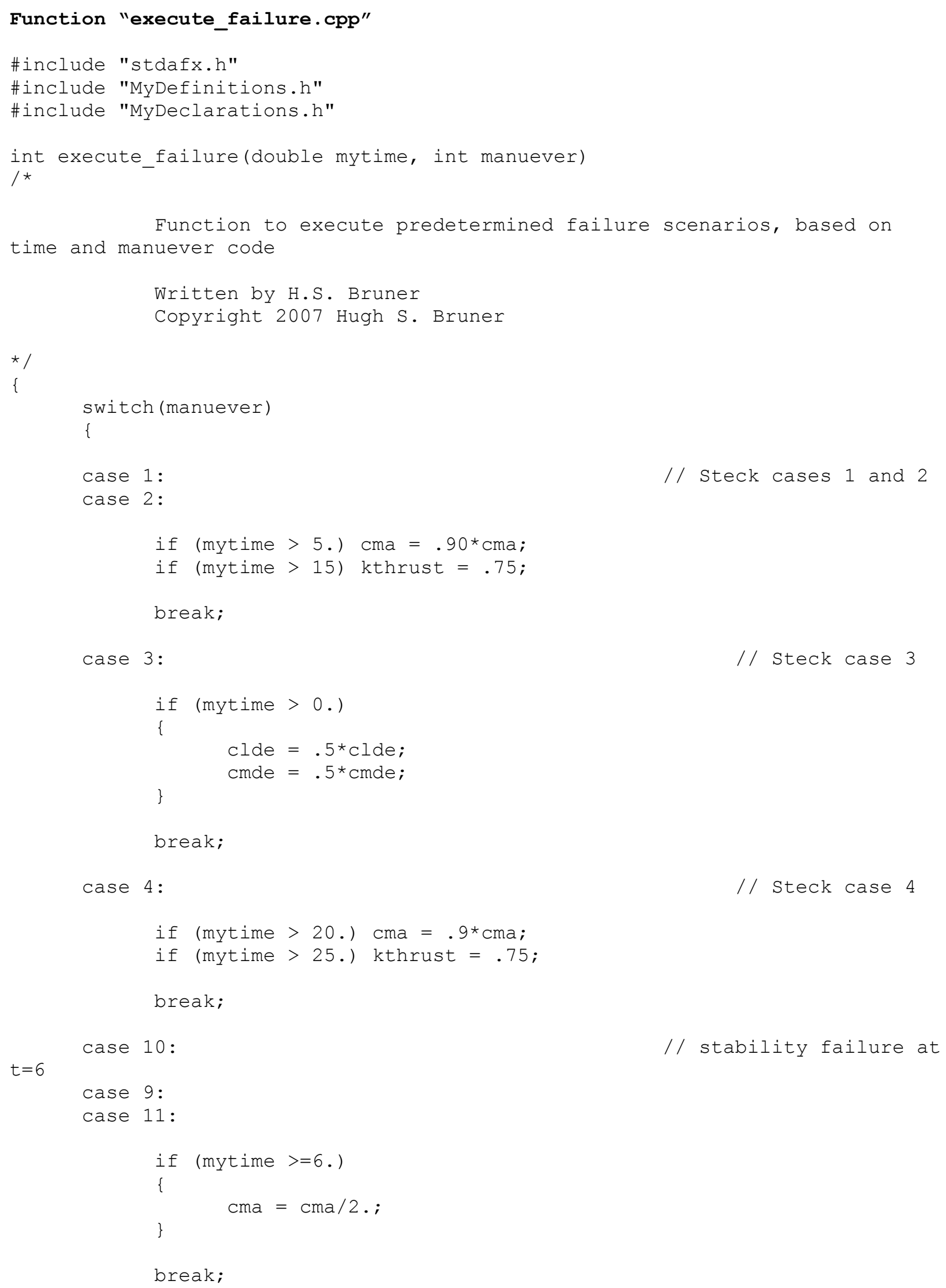




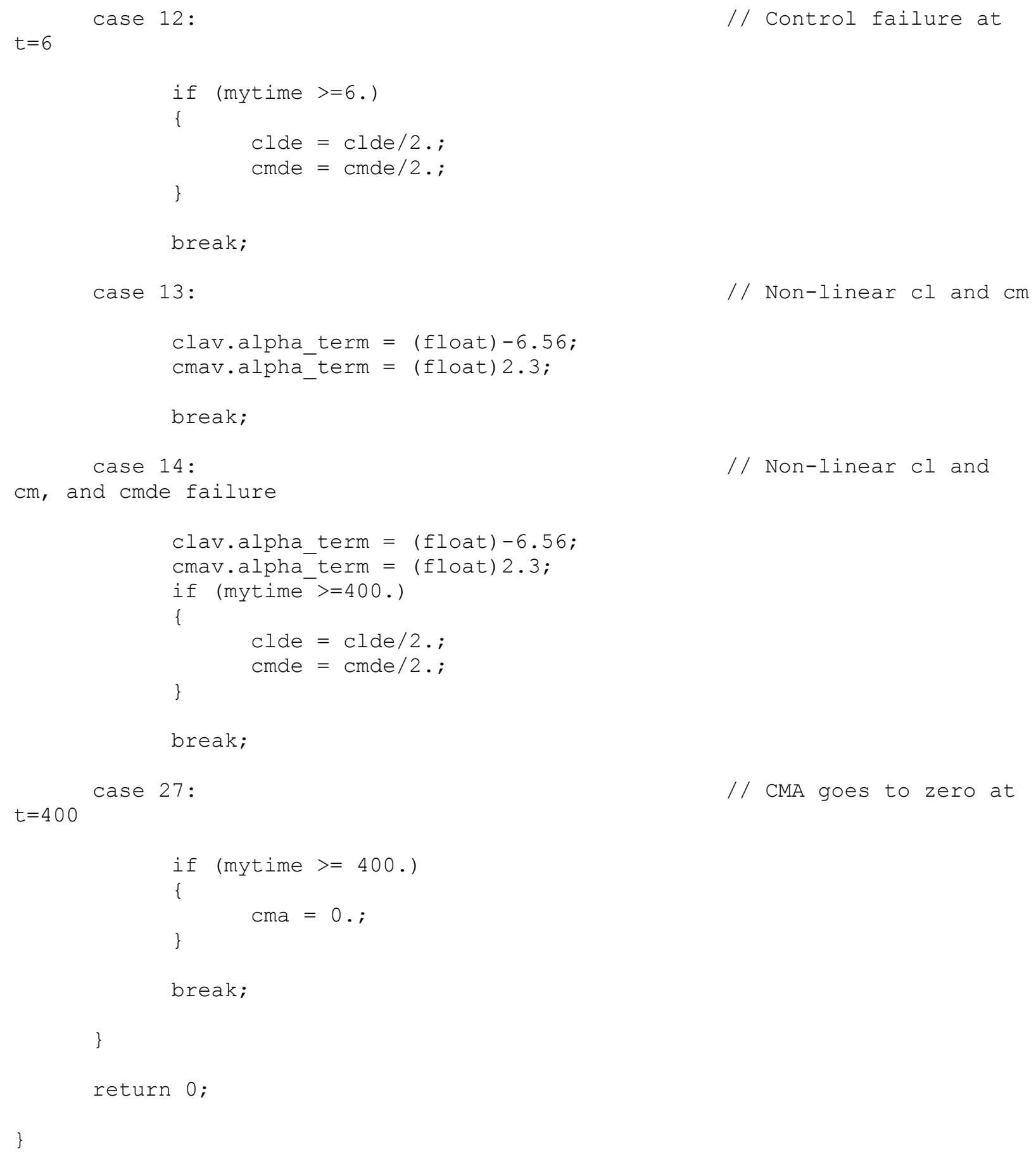




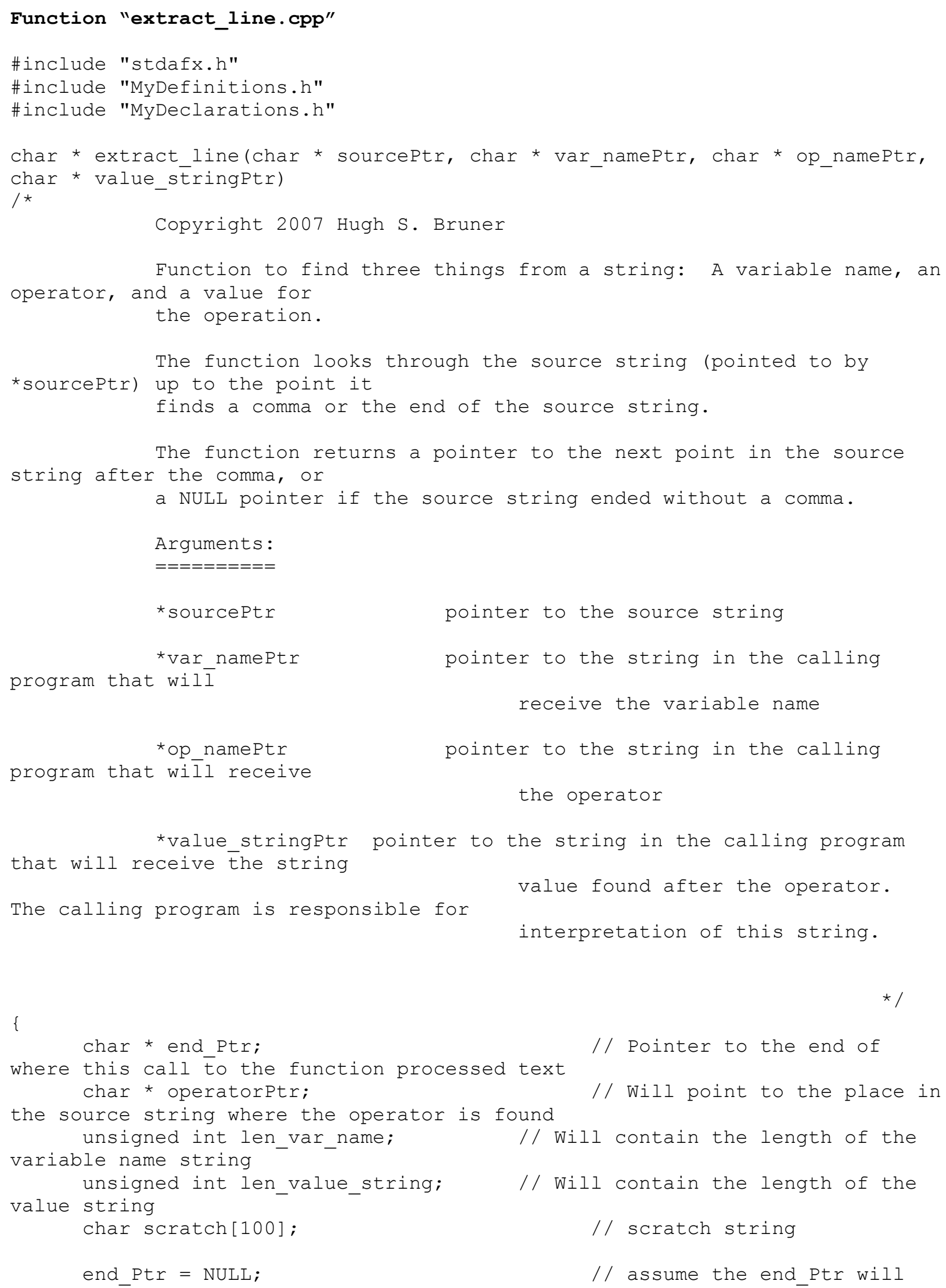




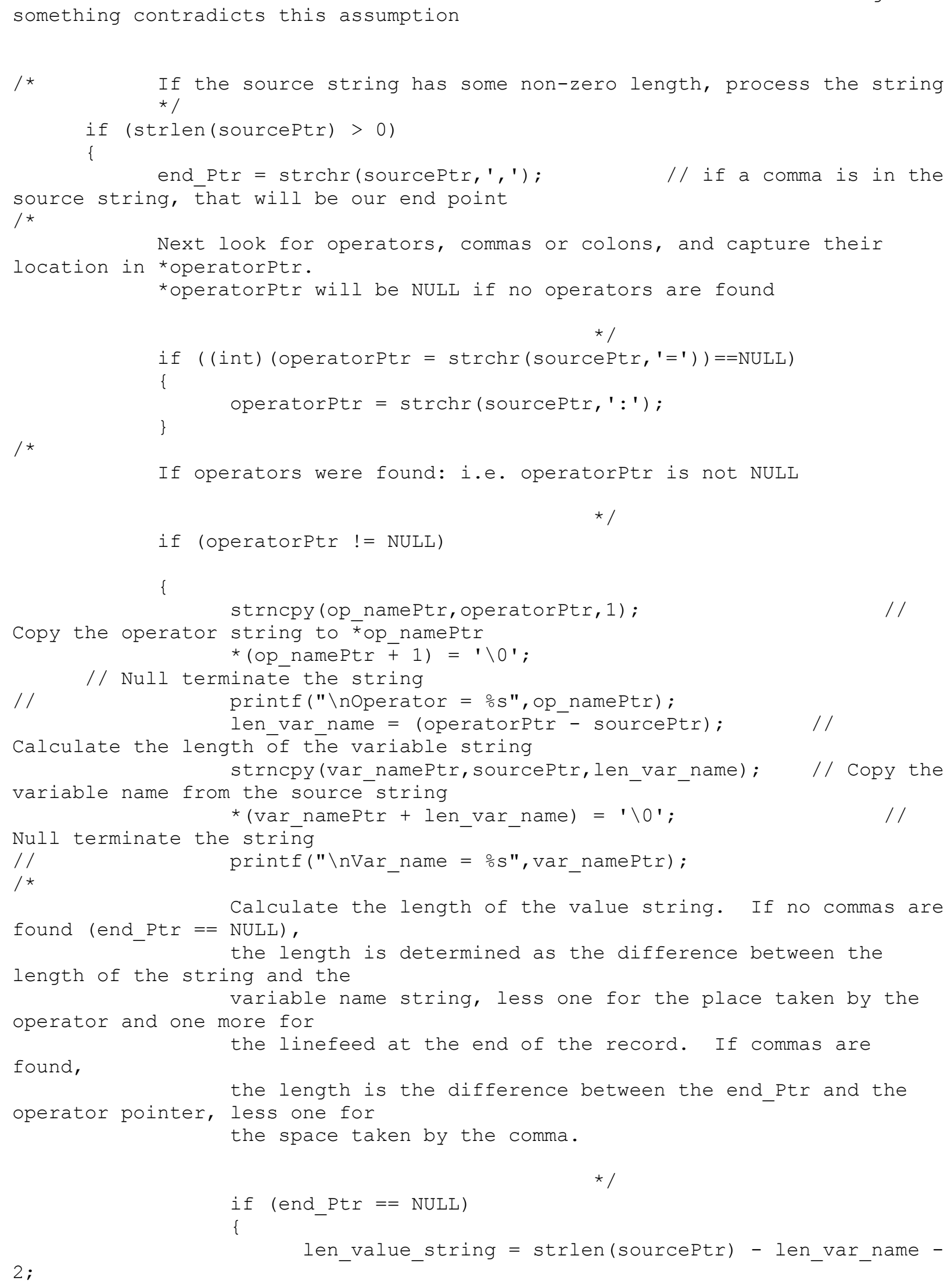




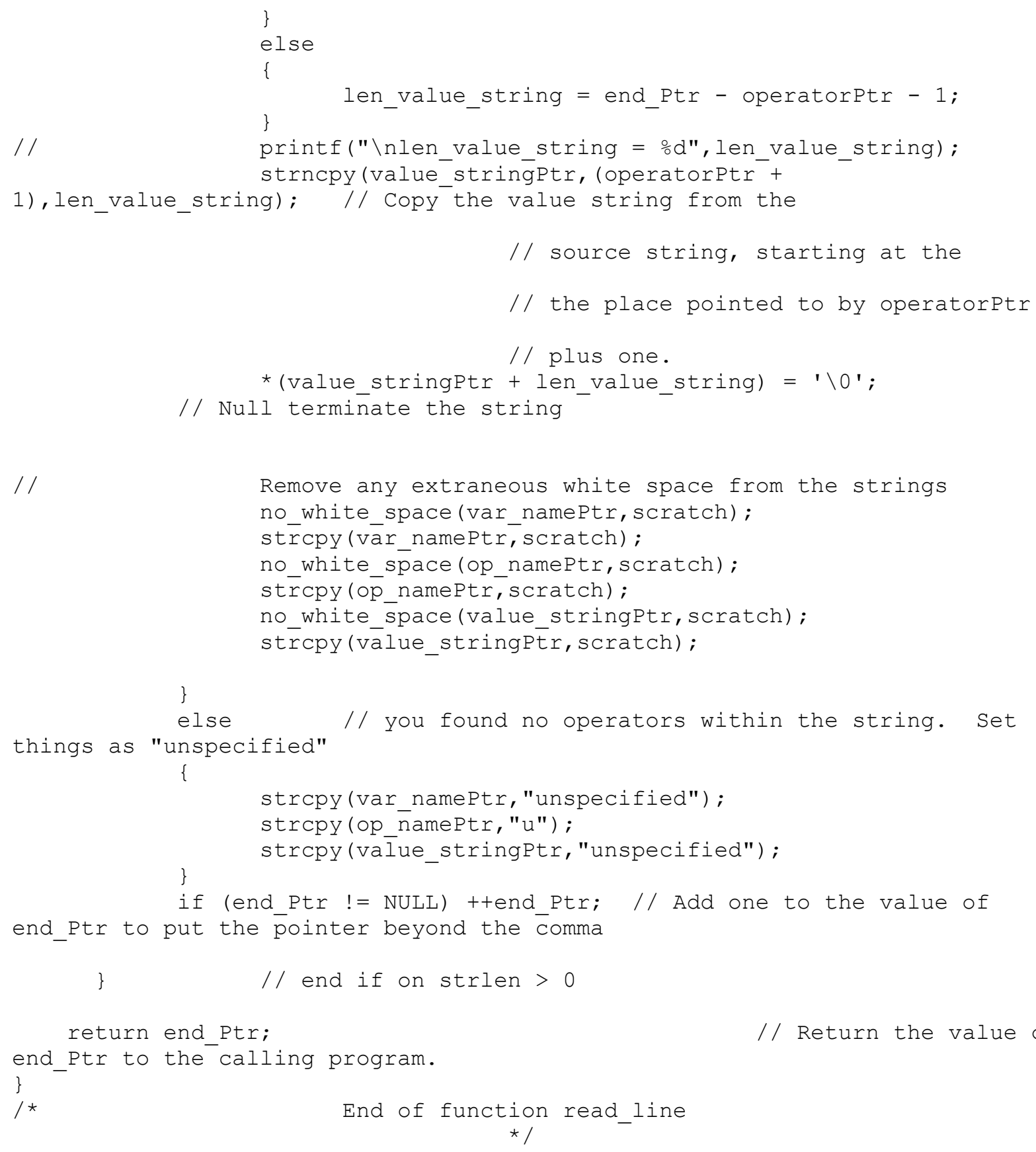




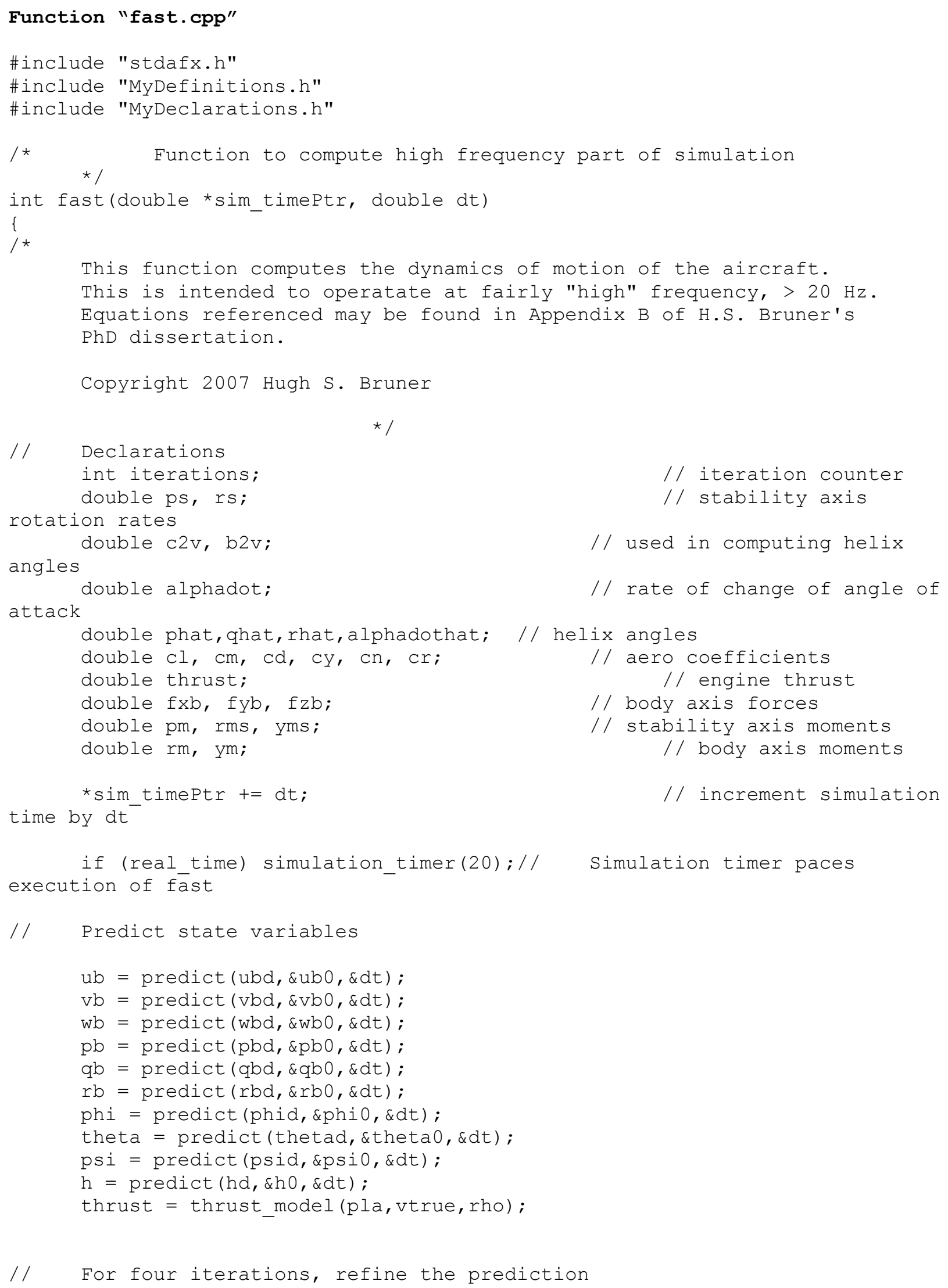




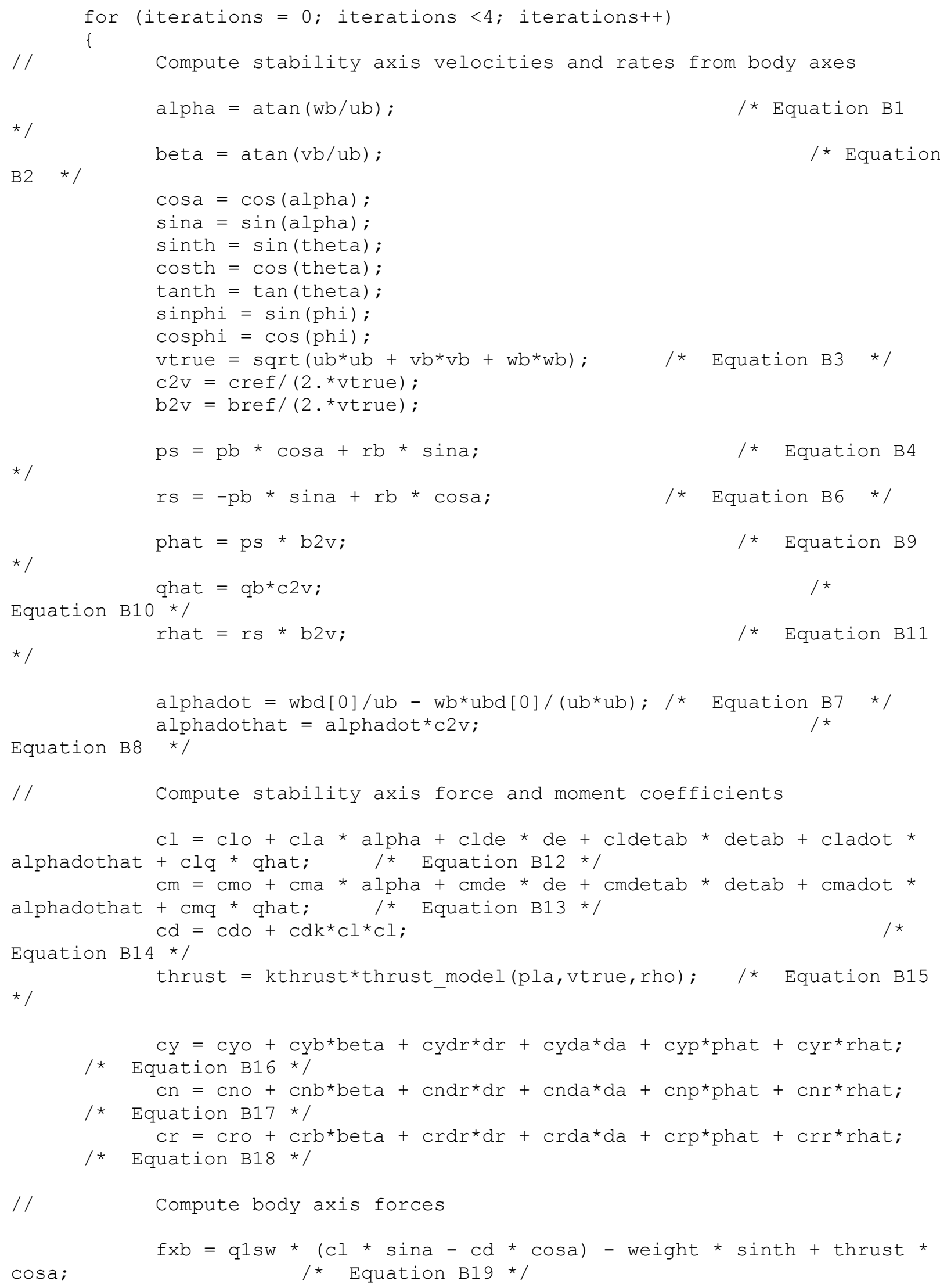




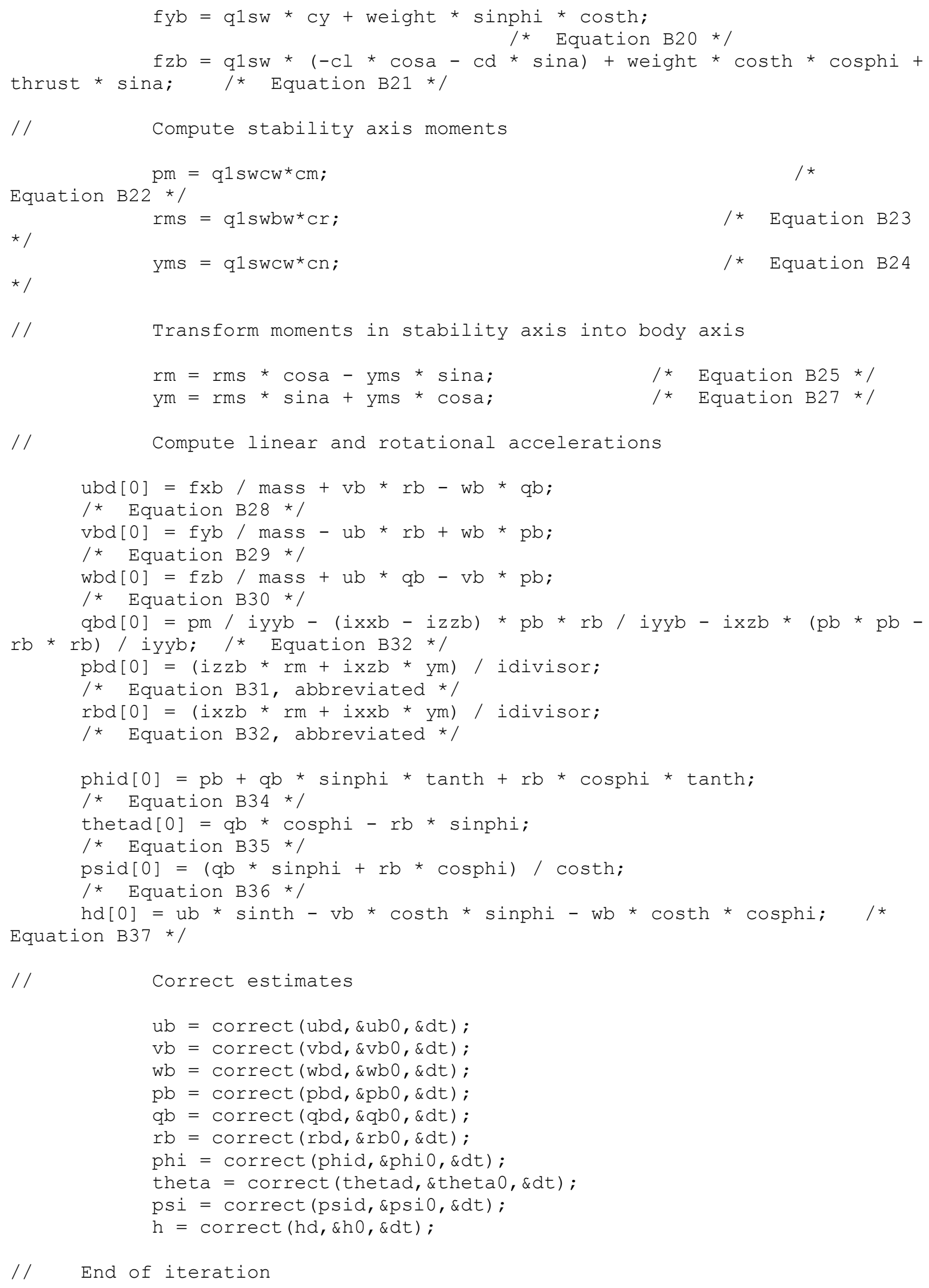




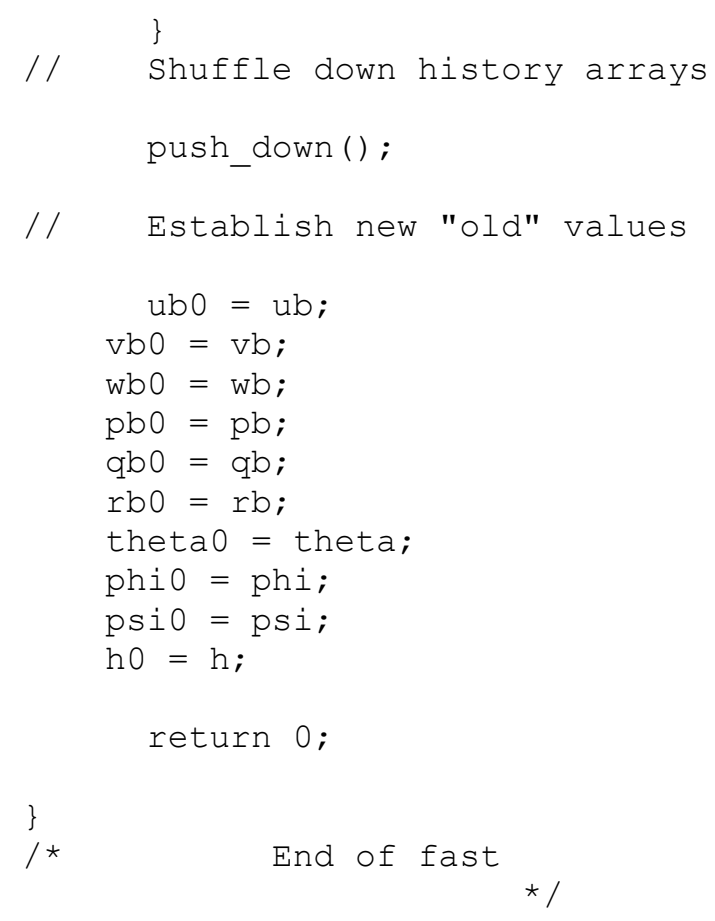




\section{Function "fetrim.cpp"}

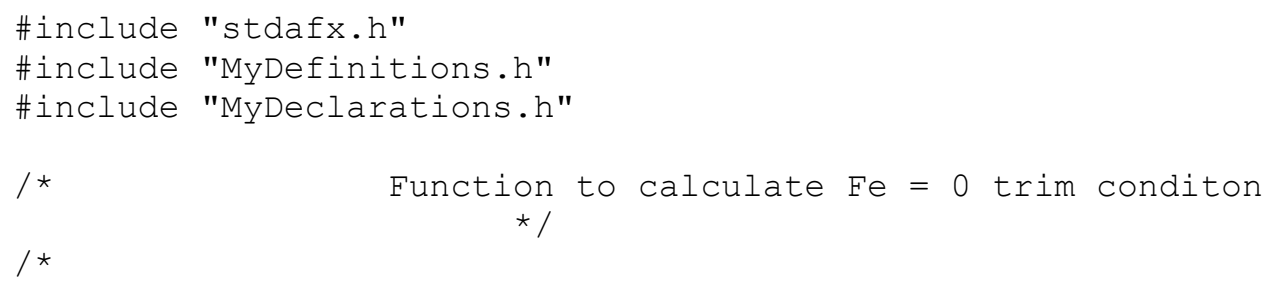

\#endif 
// Iterate until the change in each iteration is within tolerance iterations $=0$;

while (change > eps)

\{

iterations ++ ;

// Compute things that are functions of de and alpha

$\mathrm{fbw}=\mathrm{bw0}+\mathrm{dbwdde} \mathrm{de}$;

$\mathrm{fds}=\mathrm{ds} 0+\mathrm{ddsdde}^{\star} \mathrm{de} 1$

ge $=\operatorname{gev}[0]+\operatorname{de} 1 *(\operatorname{gev}[1]+\operatorname{del}(\operatorname{gev}[2]+\operatorname{de} 1 * \operatorname{gev}[3]))$;

clo = eval (\&clov, \&alpha1, \&tcp); // update parameters

with new alpha and tcp

cla $=\operatorname{eval}(\& c l a v$, \&alphal, \&tcp);

clde = eval (\&cldev, \&alphal, \&tcp);

cldetab = eval $(\& c l$ detabv, \&alphal, \&tcp $)$;

cmo $=\operatorname{eval}(\& \mathrm{cmov}$, \&alpha1, \&tcp);

$\mathrm{cma}=\operatorname{eval}(\& \mathrm{cmav}$, \&alphal, \&tcp);

cmde $=\operatorname{eval}(\& \mathrm{cmdev}, \& a l p h a 1, \& t c p) ;$

cmdetab = eval (\&cmdetabv, \&alpha1, \&tcp);

eps $0=$ epsv.constant term + epsv.tcp term*tcp;

epsa = epsv.alpha te $\overline{r m}+$ epsv.alpha tcp term*tcp;

// $\quad \operatorname{tcp}=\operatorname{cdl} / \cos (\mathrm{alph} a 1)$;

$/ / \quad$ thrust $=t_{c p}^{*} q 1 \mathrm{sw}$;

// Load array that represent the matrix equation for trim

$\mathrm{a}[0][0]=\mathrm{cla}$;

$\mathrm{a}[0][1]=$ clde;

$\mathrm{a}[0][2]=$ cldetab;

$\mathrm{a}[1][0]=\mathrm{cma}$;

$\mathrm{a}[1][1]=$ cmde;

$\mathrm{a}[1][2]=$ cmdetab;

$\mathrm{a}[2][0]=\operatorname{chea}^{*}(1 .-\mathrm{epsa})$;

$\mathrm{a}[2][1]=$ chede;

$\mathrm{a}[2][2]=$ chedetab;

$\mathrm{y}[0]=\mathrm{Cl} 1-\mathrm{ClO} ;$

$\mathrm{y}[1]=-\mathrm{cmo}-\mathrm{dx}{ }^{*} \mathrm{Cl} 1$;

$y[2]=-c h e o+c h e a * e p s 0-(f b w+f d s) /\left(q 1 * s e^{\star} c e^{\star} g e\right)$;

solve3by3 $(a, x, y)$;

absolute

change = fabs $(x[1]-d e 1) ; \quad$ // change is defined by the

change in elevator during this iteration

// value of

alpha1 $=\mathrm{x}[0]$;

de1 $1=x[1]$;

\}

detabl $1=x[2]$;

de $=$ de 1 ;

$\operatorname{detab}=\operatorname{detab} 1$

alpha = alphal

$\mathrm{pla}=$ (double)100*thrust/thrust_model((double)100., vtrue, rho); 


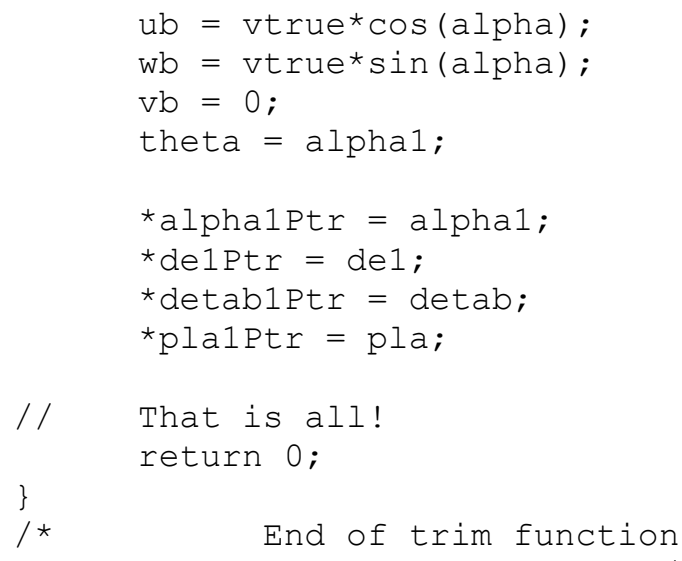




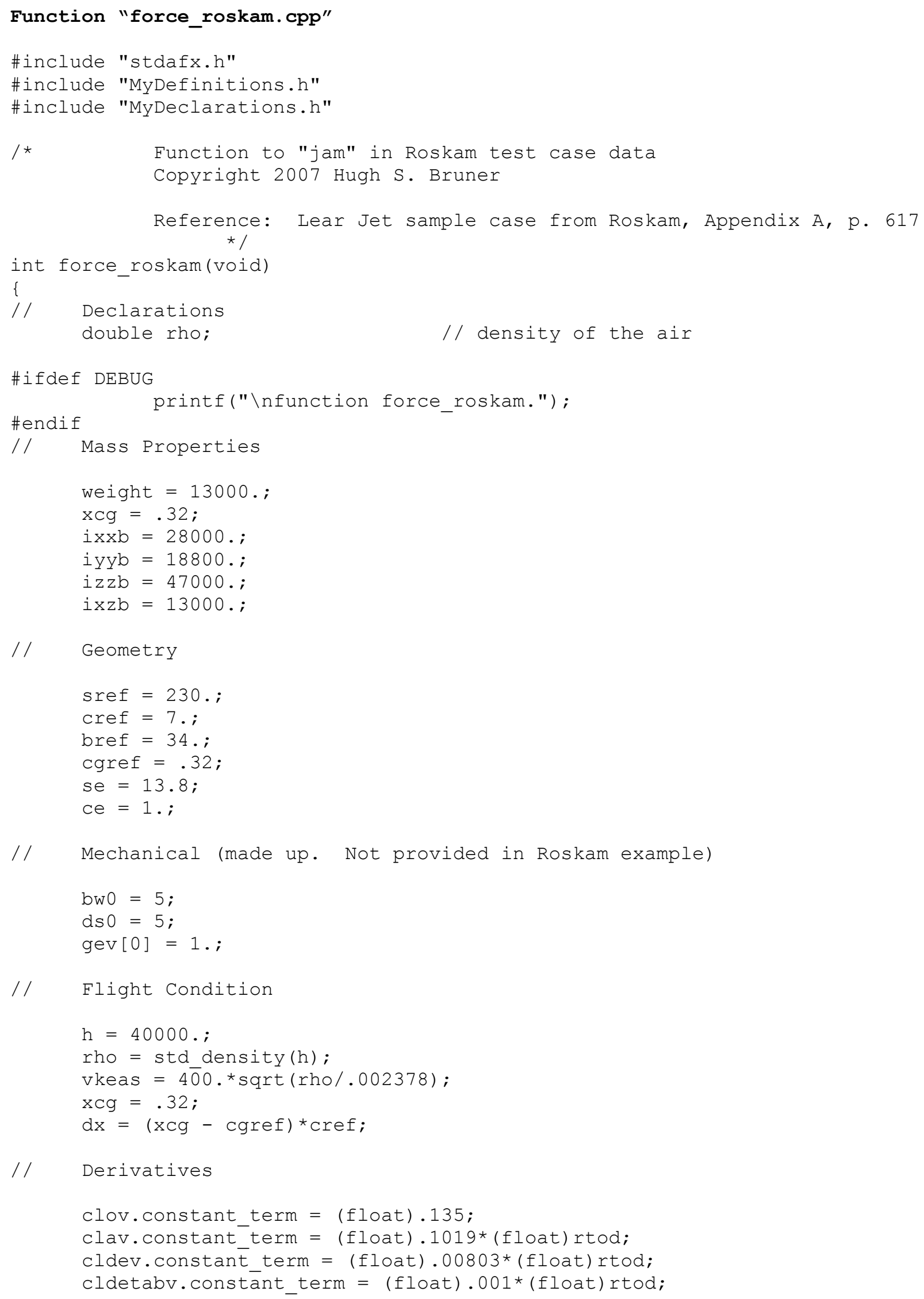




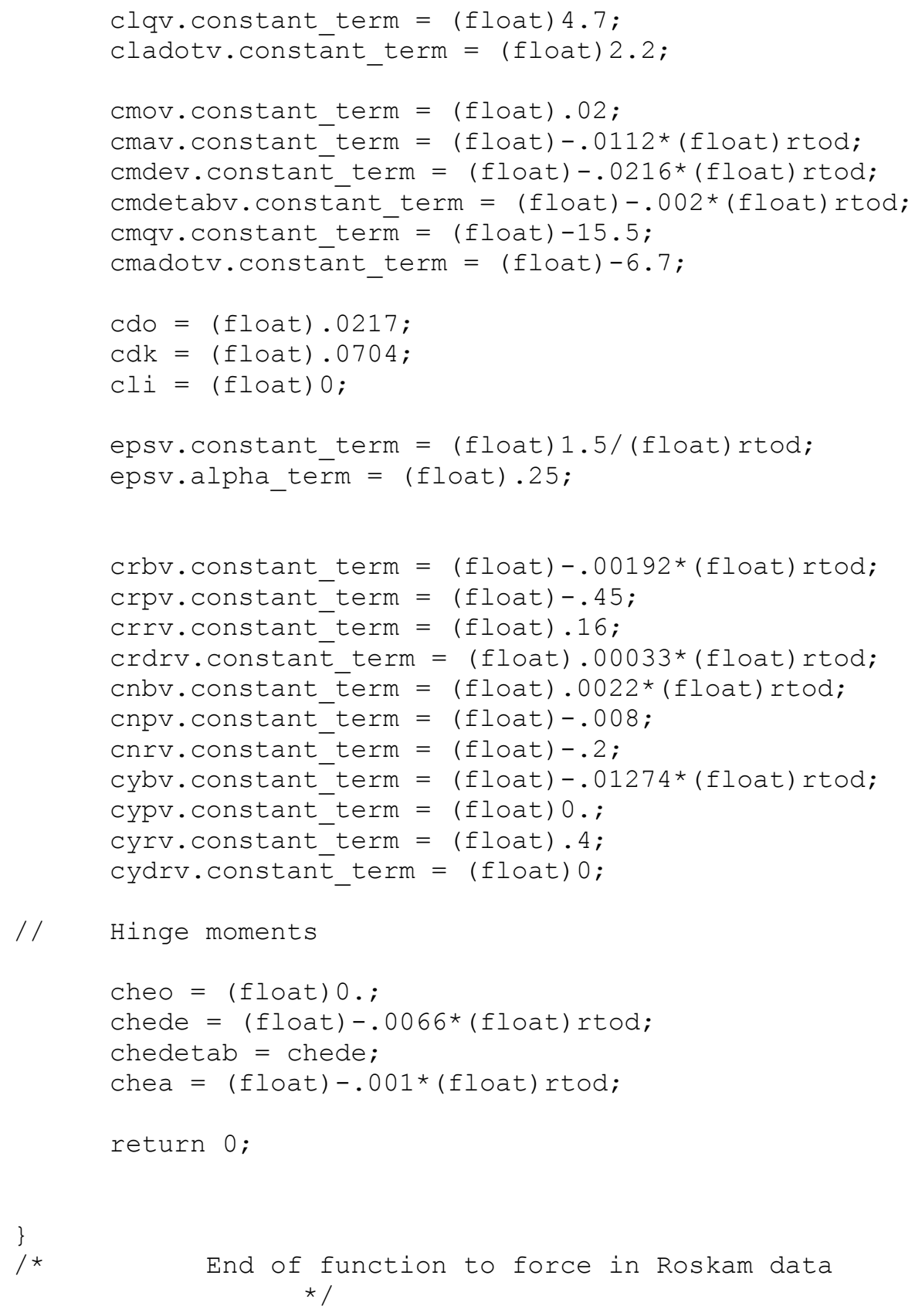




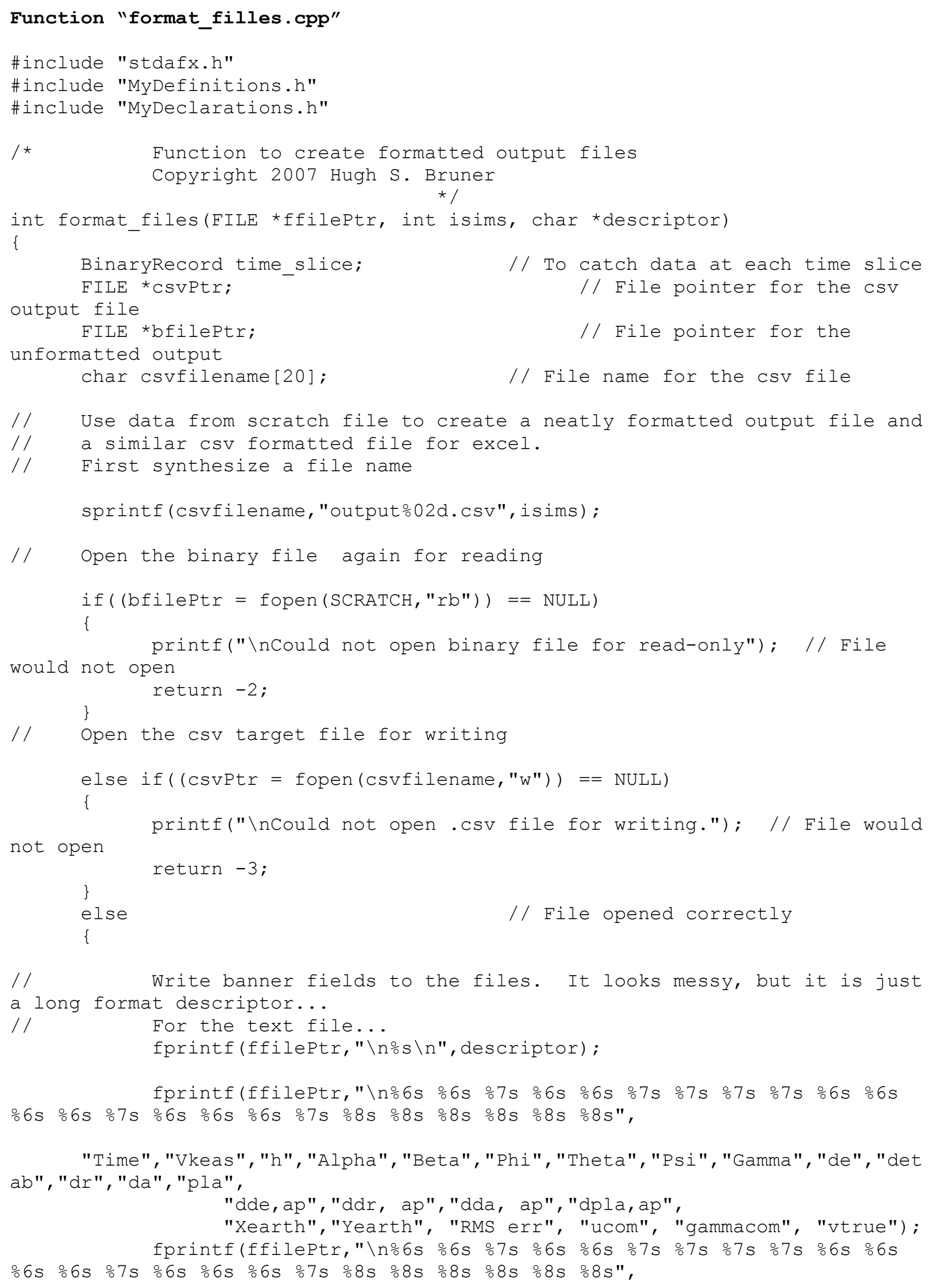




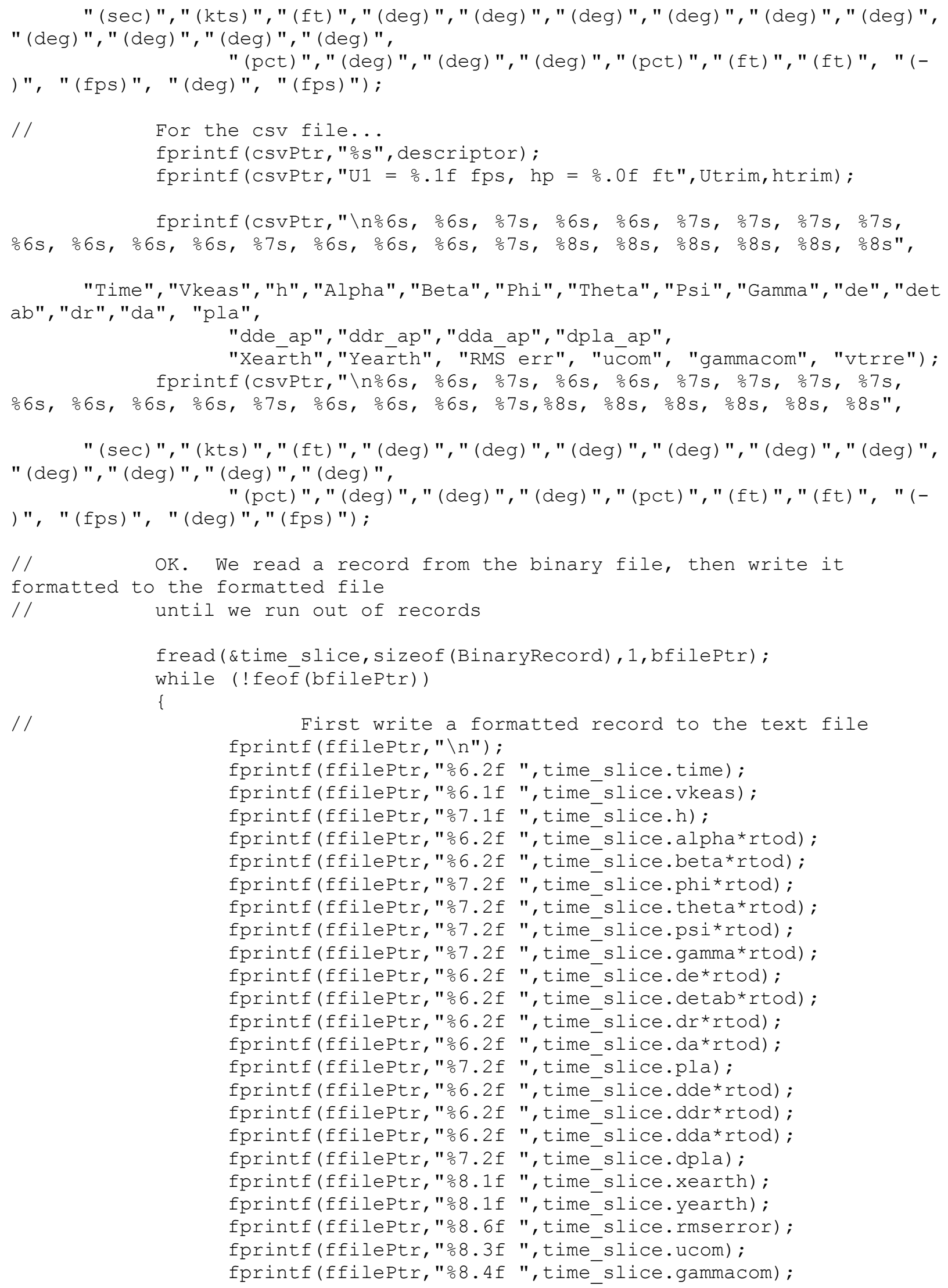


fprintf(ffileptr,"으의 ",time_slice.vtrue);

//

Then write a formatted record to the csv file fprintf (csvPtr," $\backslash \mathrm{n} "$ ) ;

fprintf(csvPtr,"으.2f, ",time_slice.time);

fprintf(csvPtr,"⒍1f, ", time_slice.vkeas);

fprintf(csvPtr,"ㄱ.1f, ", time-slice.h);

fprintf (csvPtr,"⒍2f, ", time slice.alpha*rtod);

fprintf(csvPtr,"⒍2f, ", time_slice.beta*rtod);

fprintf(csvPtr,"ㄱ‥2f, ", time_slice.phi*rtod);

fprintf (csvPtr,"으.2f, ", time-slice.theta*rtod);

fprintf(csvPtr,"그.2f, ", time_slice.psi*rtod);

fprintf(csvPtr,"으.2f, ", time_slice.gamma*rtod);

fprintf(csvPtr,"⒍2f, ", time_slice.de*rtod);

fprintf(csvPtr,"으.2f, ", time_slice.detab*rtod);

fprintf (csvPtr,"\%6.2f, ", time_slice.dr*rtod);

fprintf(csvPtr,"⒍2f, ", time_slice.da*rtod);

fprintf(csvPtr,"그.2f, ", time_slice.pla);

fprintf(csvPtr,"\%6.2f, ",time_slice.dde*rtod);

fprintf(csvPtr,"\%6.2f, ",time_slice.ddr*rtod);

fprintf(csvPtr,"⒍2f, ", time_slice.dda*rtod);

fprintf(csvPtr,"\%7.2f, ", time_slice.dpla);

fprintf (csvPtr,"ㅇ․․ f, ", time slice.xearth);

fprintf (csvPtr,"ㅇ․ㄷ, ", time_slice.yearth);

fprintf(csvPtr,"ㅇ․6f, ", time_slice.rmserror);

fprintf(csvPtr,"ㅇ․ㅇ, ", time_slice.ucom);

fprintf(csvPtr,"ㅇ‥4f, ", time_slice.gammacom);

fprintf(csvPtr,"\%8.3f, ", time_slice.vtrue);

//

Try to read another binary record

fread(\&time_slice,sizeof (BinaryRecord), 1,bfilePtr);

// $\quad$ End of while loop on reading records

// Close output files

fclose (bfilePtr);

fclose (csvPtr);

return 0 ;

\}

clause on file opens

\}

/*

End of function to output formatted files

* 


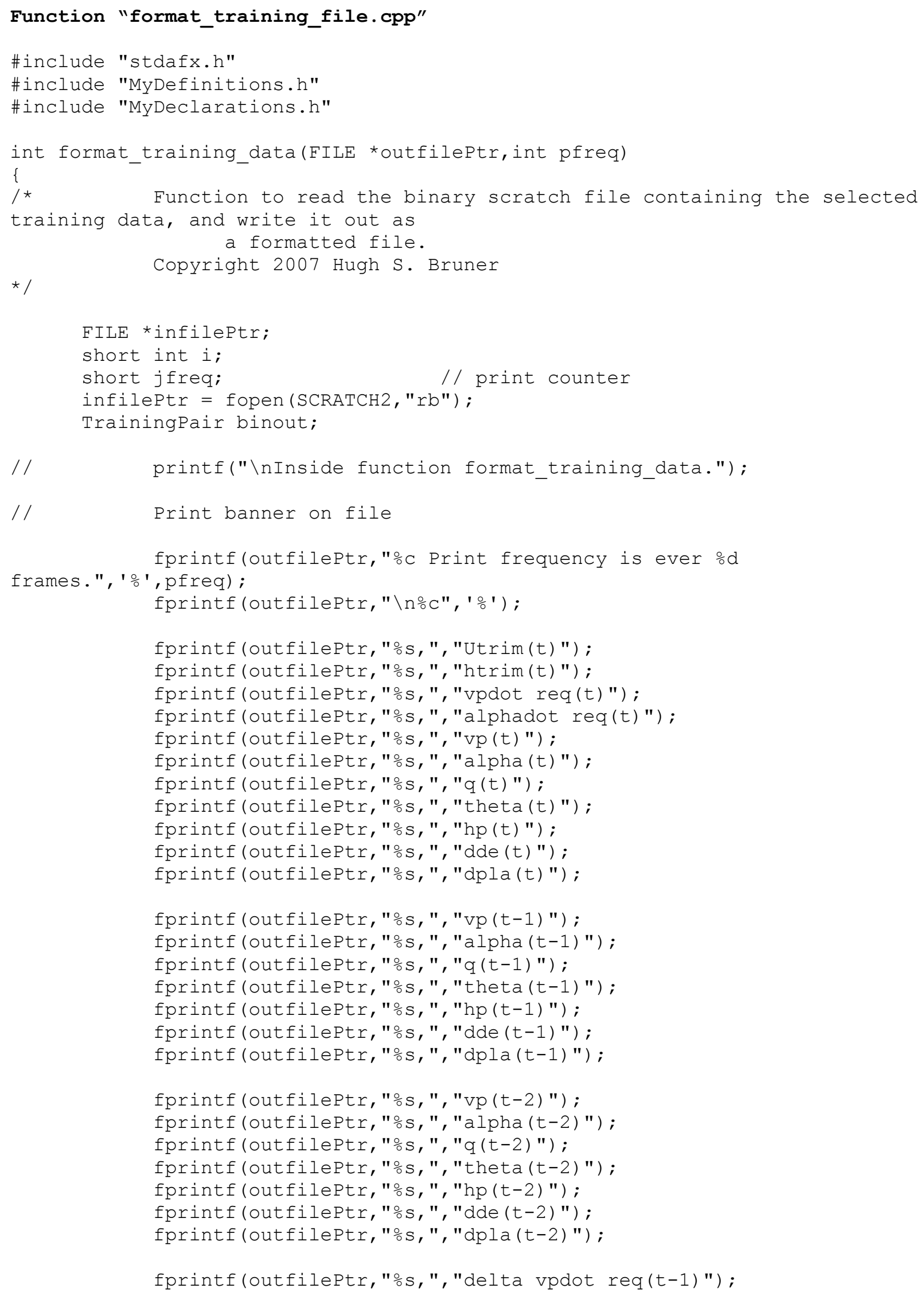




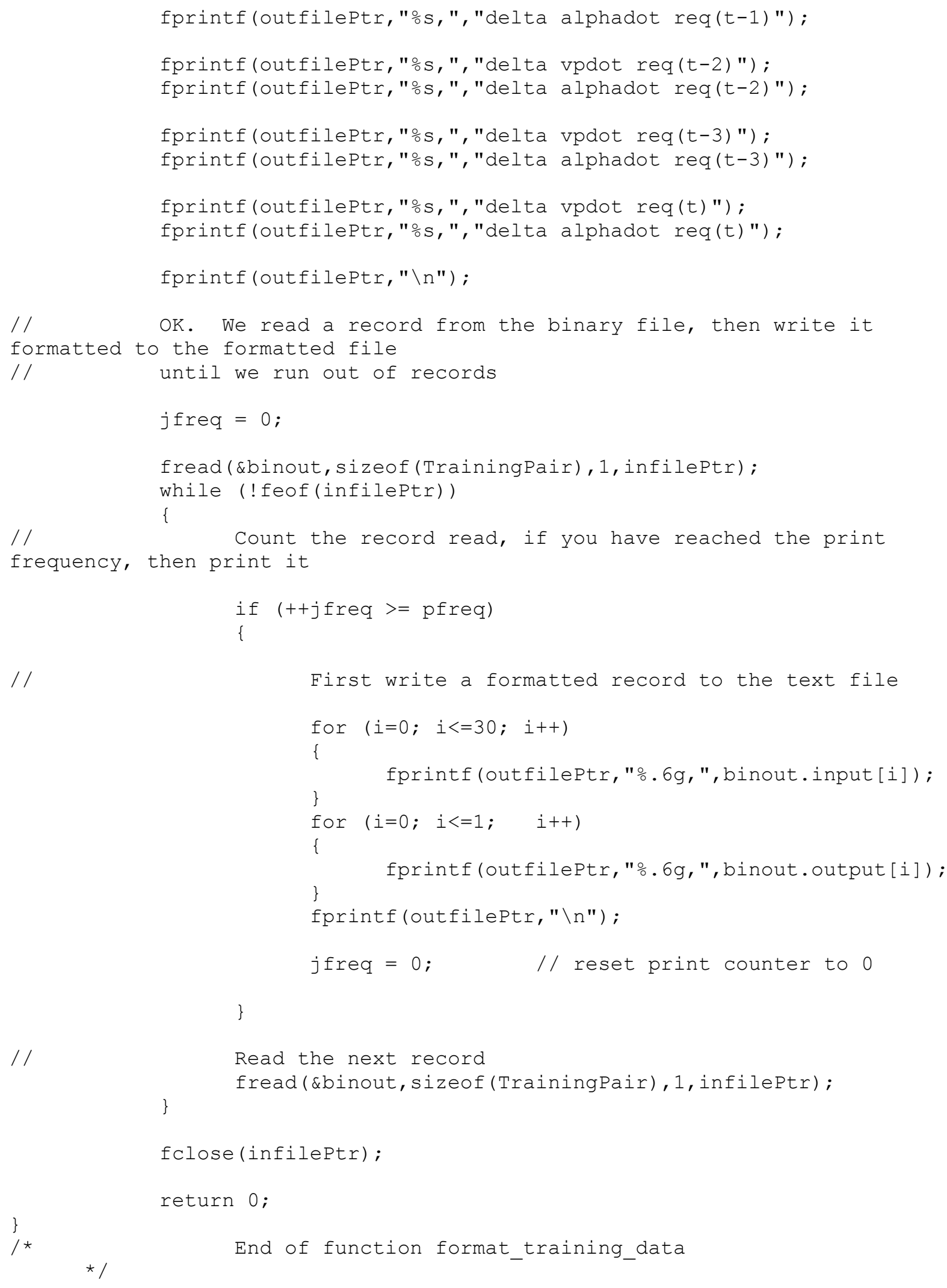




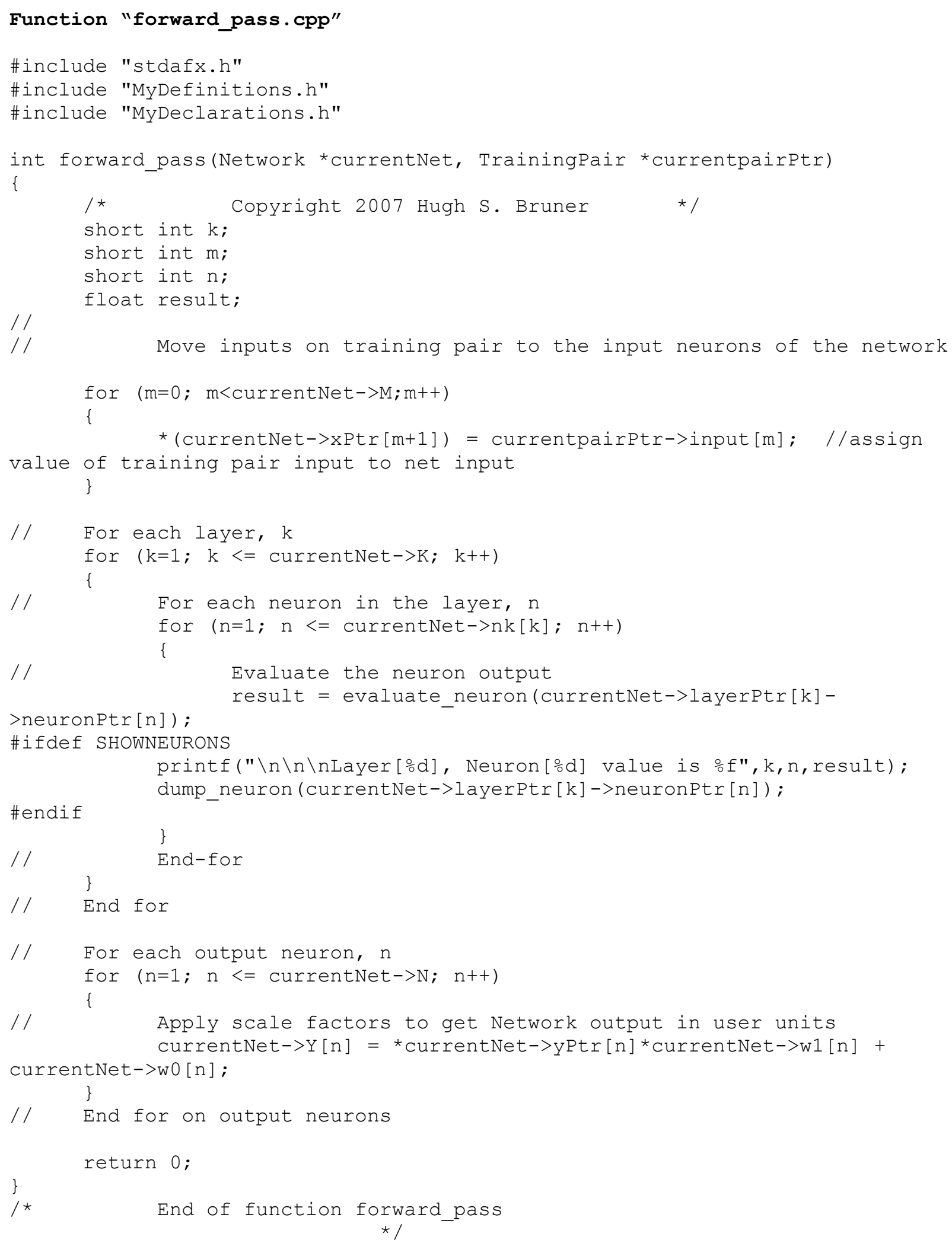




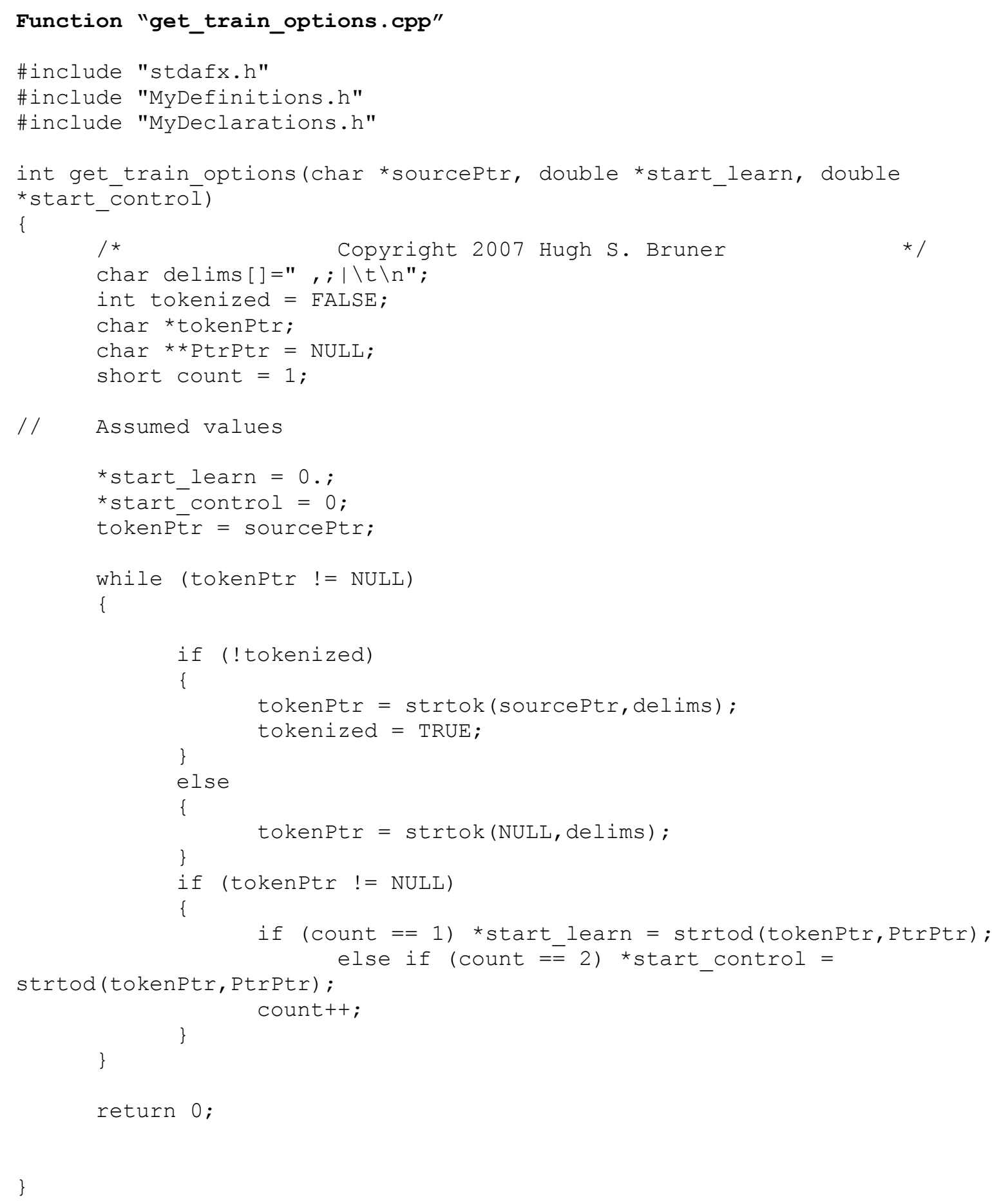




\section{Function "globals.cpp"}

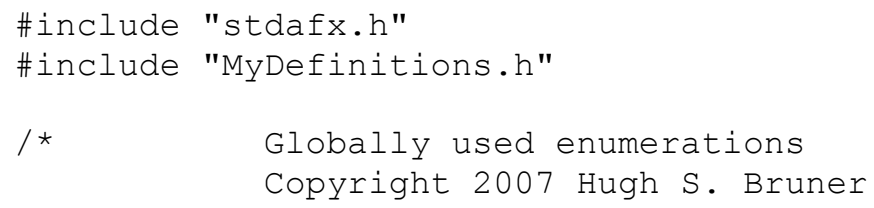

// Global declarations

FILE *testoutPtr;

short int real time $=0$;

whether running realtime or not

short int log_training_data $=1$;

not to log training data

int control printfreq $=1$;

frequency of printing output during sim

int control_ptrainfreq $=100$;

frequency of printing training data during sim

int control simfailure=0;

whether or not to execute failures during sim double nnrms;

error.

// Global variables

\section{// Simulation:}

DerivPolynomial $\mathrm{clov}=\{0\},. \operatorname{clav}=\{0\},. \operatorname{cldev}=\{0$.$\} ;$

DerivPolynomial cldetabv $=\{0\},. \operatorname{cladotv}=\{0\},. \operatorname{clqv}=\{0$.$\} ;$

DerivPolynomial $\mathrm{cmov}=\{0\},. \operatorname{cmav}=\{0\},. \operatorname{cmdev}=\{0$.$\} ;$

Derivpolynomial cmdetabv $=\{0\},. \operatorname{cmadotv}=\{0\},. \operatorname{cmqv}=\{0$.$\} ;$

DerivPolynomial cyov $=\{0\},. \operatorname{cybv}=\{0\},. \operatorname{cydrv}=\{0$.$\} ;$

DerivPolynomial $\operatorname{cydav}=\{0\},. \operatorname{cypv}=\{0\},. \operatorname{cyrv}=\{0$.$\} ;$

DerivPolynomial $\operatorname{cnov}=\{0\},. \operatorname{cnbv}=\{0\},. \operatorname{cndrv}=\{0$.$\} ;$

DerivPolynomial $\operatorname{cndav}=\{0\},. \operatorname{cnpv}=\{0\},. \operatorname{cnrv}=\{0$.$\} ;$

DerivPolynomial $\operatorname{crov}=\{0\},. \operatorname{crbv}=\{0\},. \operatorname{crdrv}=\{0$.$\} ;$

DerivPolynomial $\operatorname{crdav}=\{0\},. \operatorname{crpv}=\{0\},. \operatorname{crrv}=\{0$.$\} ;$

DerivPolynomial epsv=\{0.\};

double cheo, chea, chede, chedetab;

moment characteristics

double max_alpha, min_alpha;

double weight, $x c g, d x$, mass, ixxb, iyyb, izzb, ixzb, idivisor;

properties

double sref, cref, bref, cgref;

Reference geometry

double se, ce;

elevator area and elevator chord

double fds, flow, ds0, ddsdde, bw0, dbwdde;

downspring values
// elevator hinge

// Mass

/ /

/ /

// bobweight and 


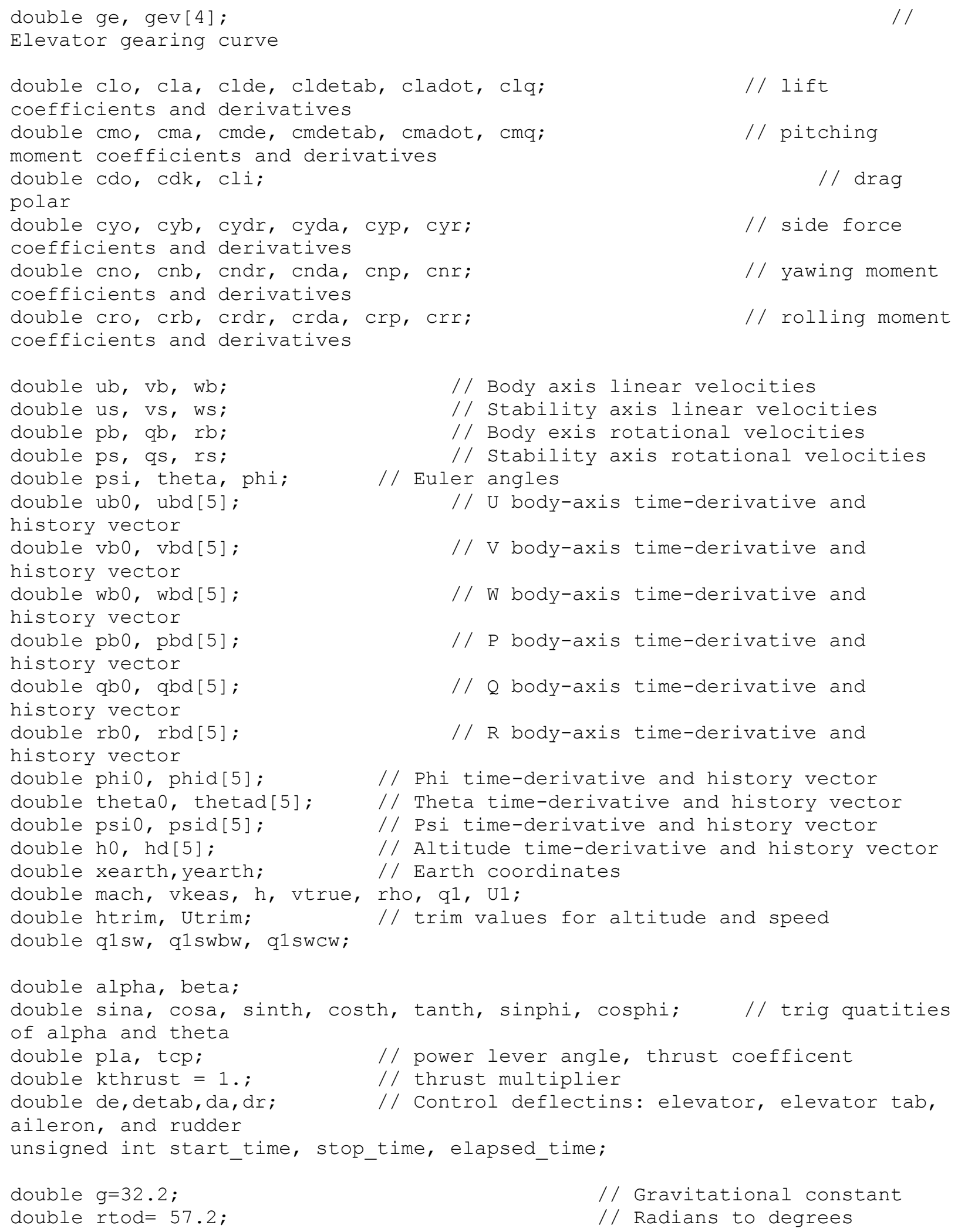




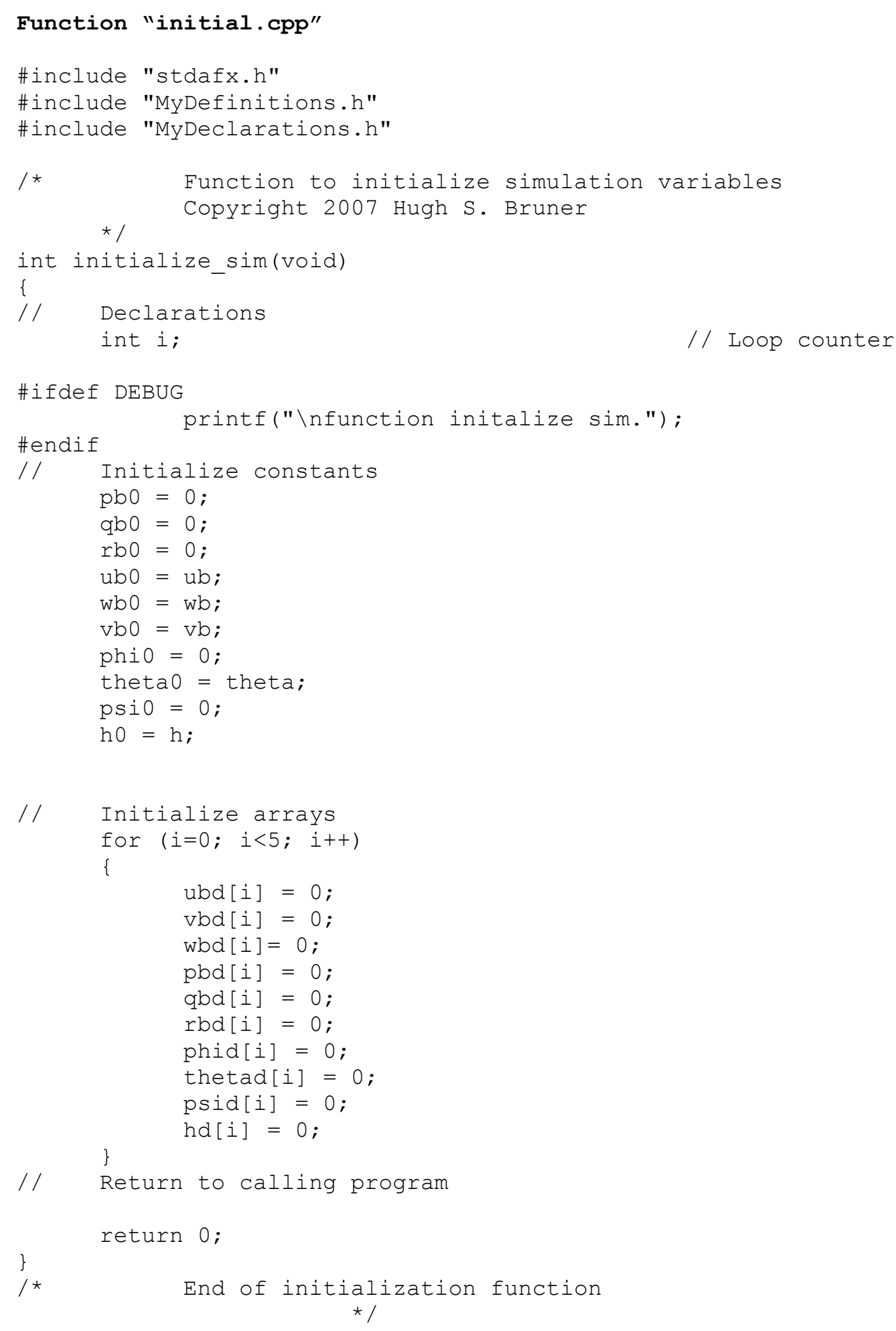




\section{Function "initialize_by_code.cpp"}

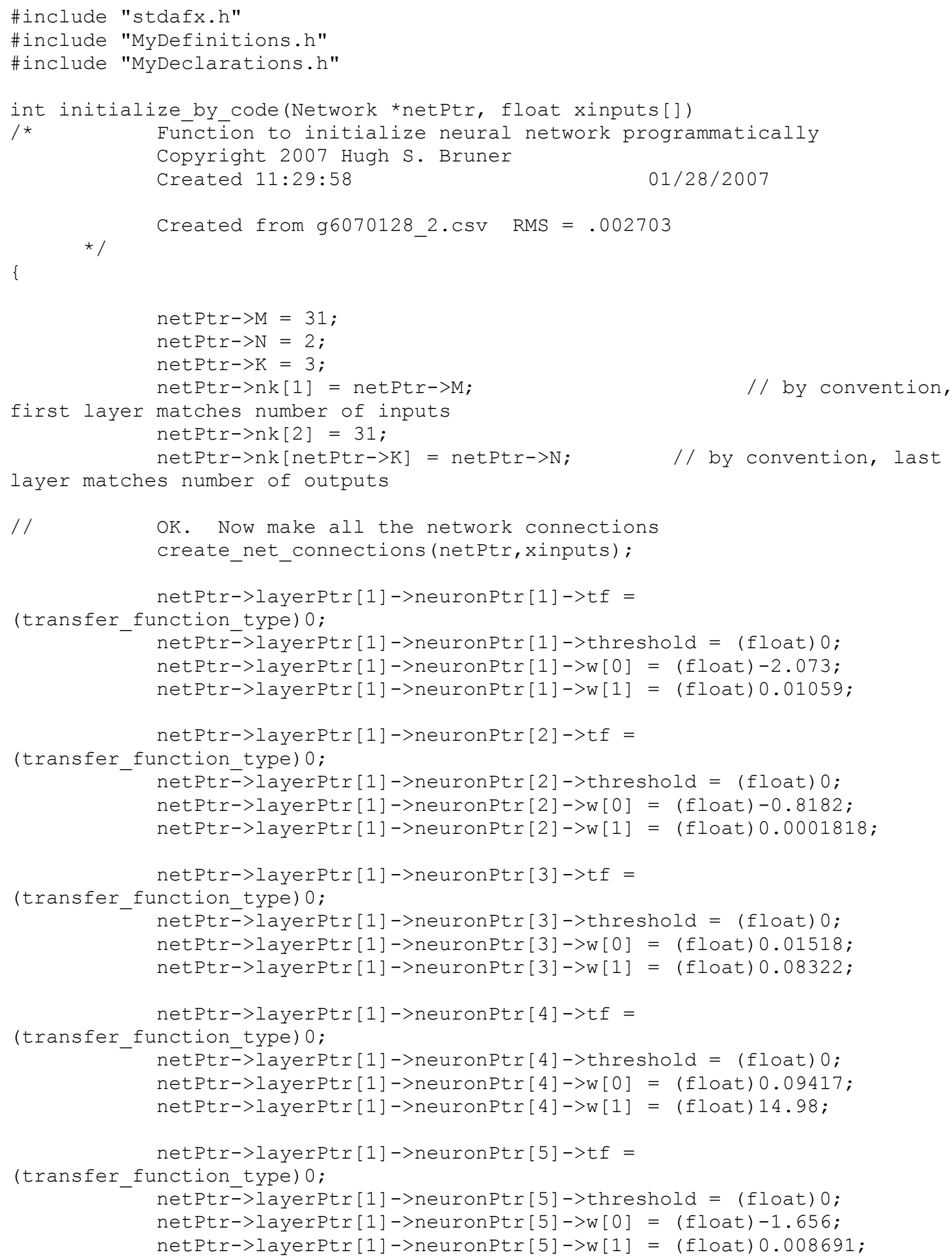




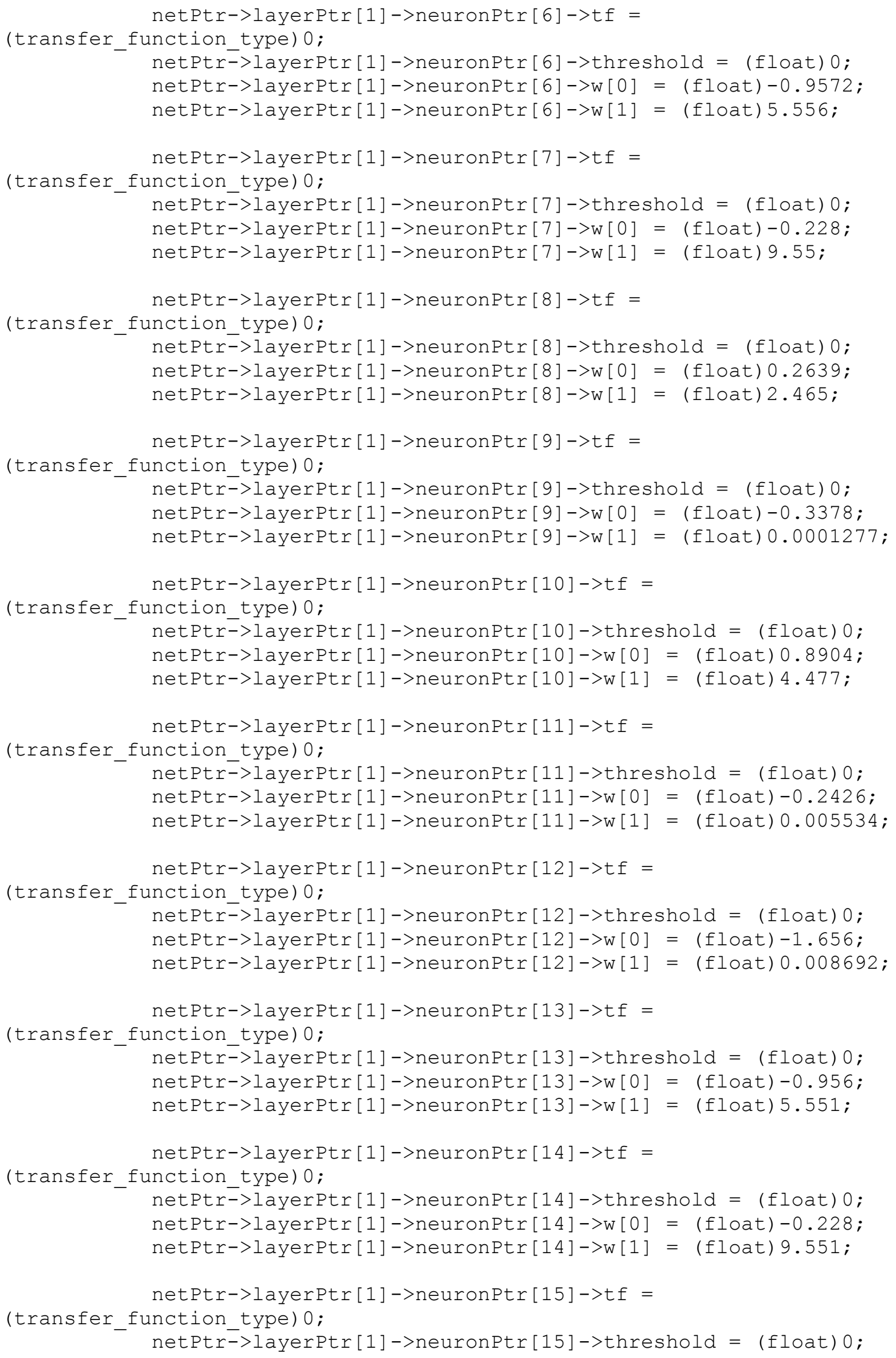




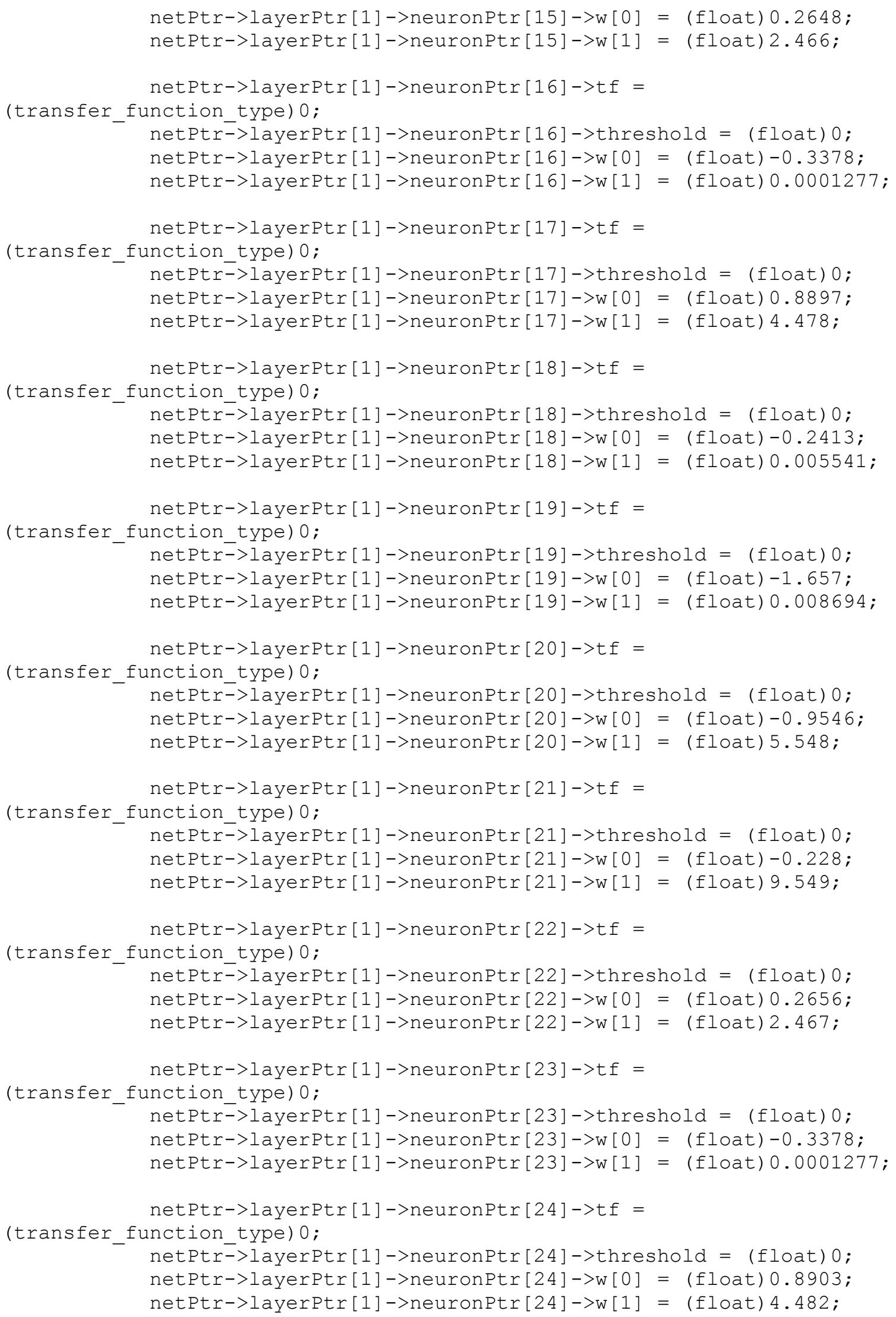




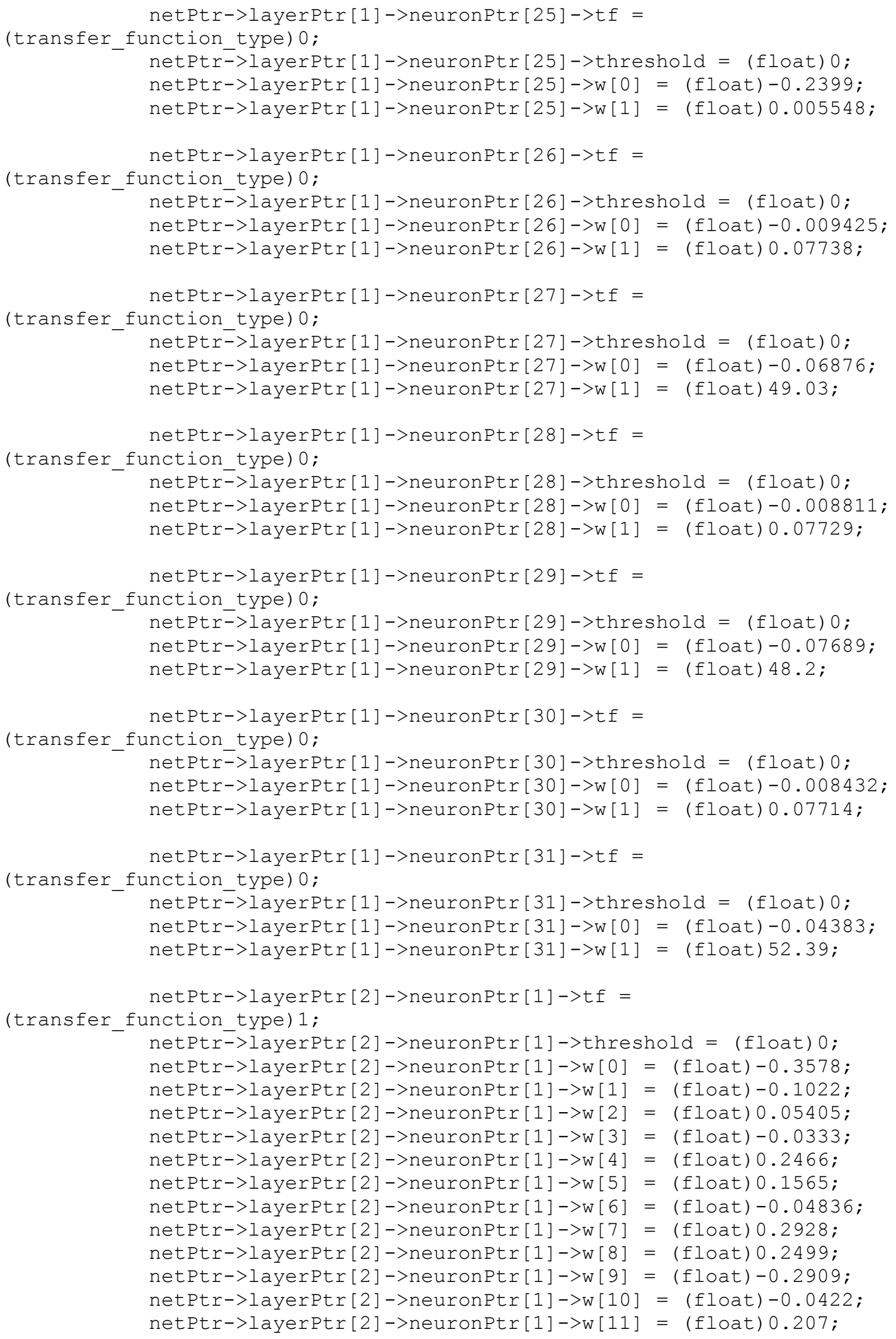




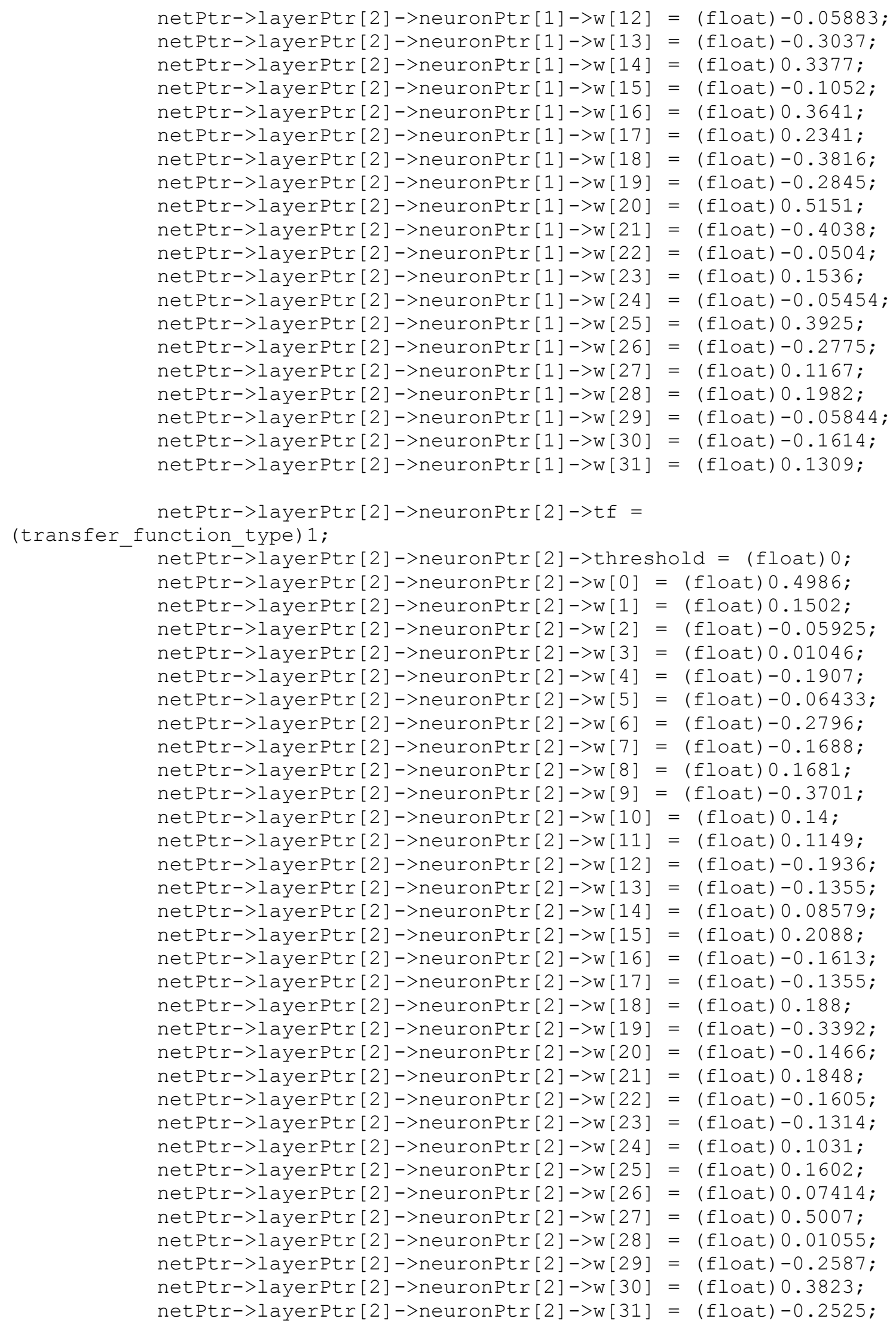




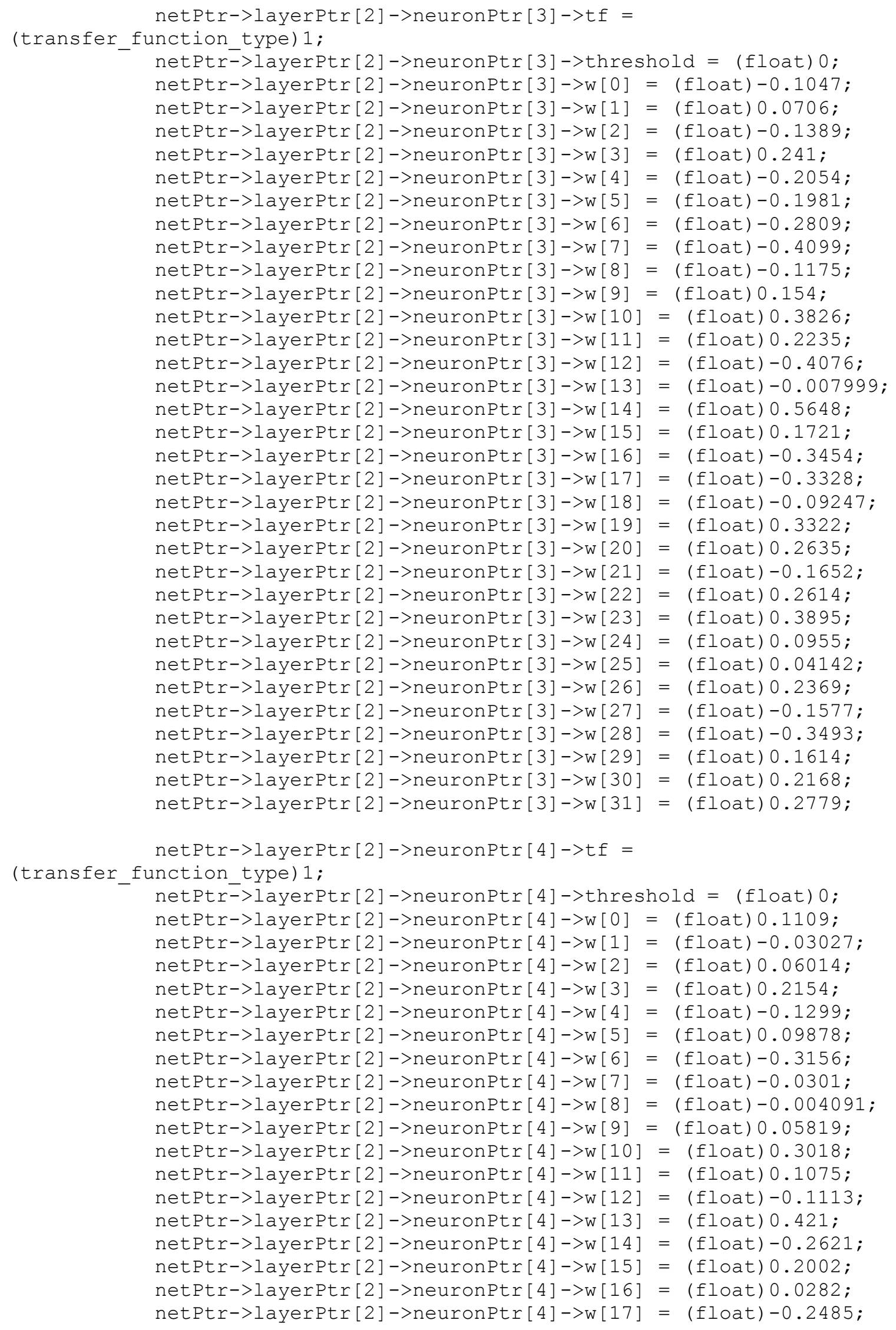




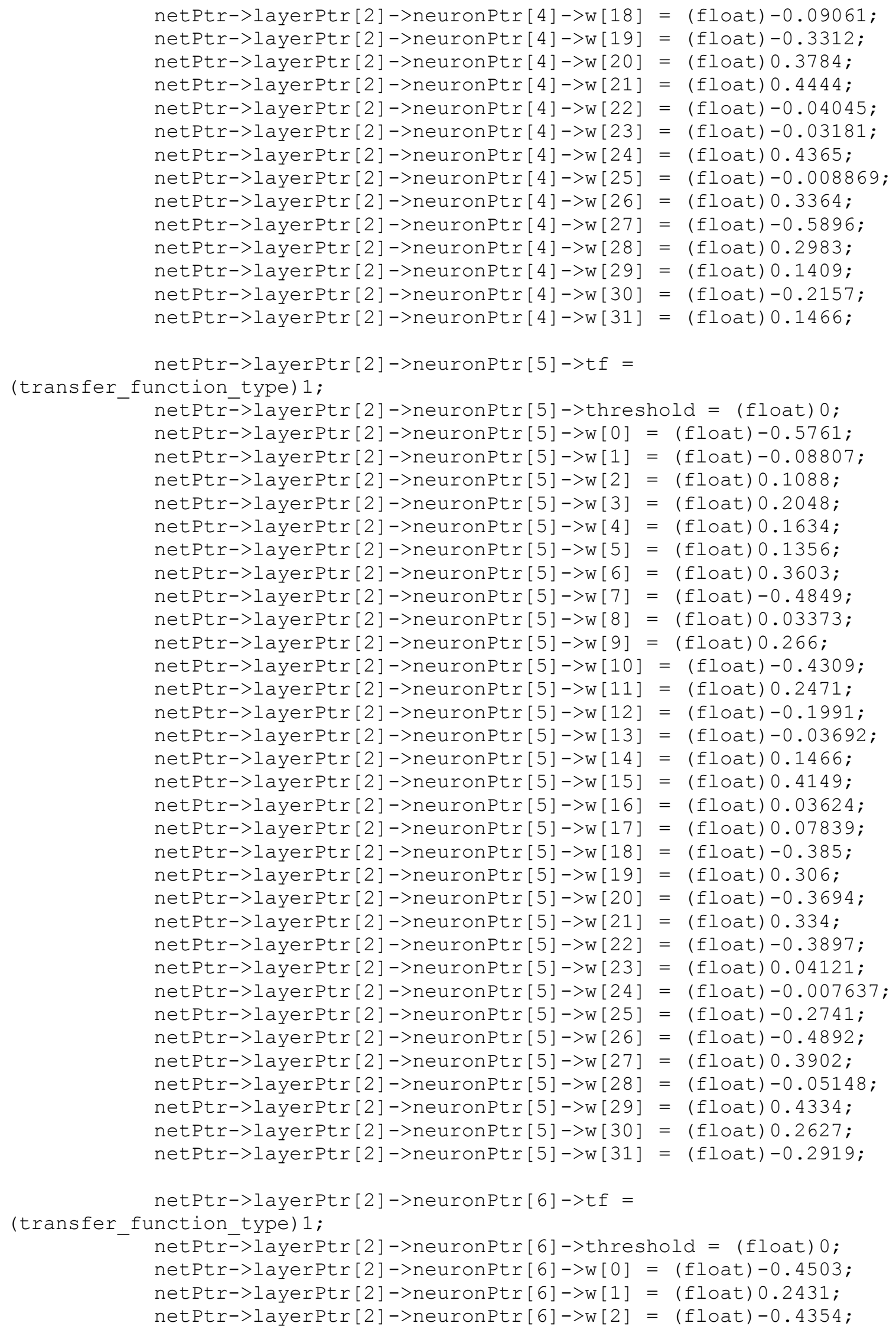




$$
\begin{aligned}
& \text { netPtr->layerPtr[2]->neuronPtr[6]->w[3] = (float) } 0.2328 \text {; } \\
& \text { netPtr->layerPtr[2] ->neuronPtr[6]->w[4] = (float) } 0.1662 \text {; } \\
& \text { netPtr->layerPtr[2]->neuronPtr[6]->W[5] = (float) }-0.1451 \text {; } \\
& \text { netPtr->layerPtr[2]->neuronPtr[6]->w[6] = (float) } 0.4627 \text {; } \\
& \text { netPtr->layerPtr[2]->neuronPtr[6]->w[7] = (float) }-0.3663 \text {; } \\
& \text { netPtr->layerPtr[2]->neuronPtr[6]->w[8] = (float) }-0.4208 \text {; } \\
& \text { netPtr->layerPtr[2]->neuronPtr[6]->w[9] = (float)0.194; } \\
& \text { netPtr->layerPtr [2]->neuronPtr[6]->W[10] = (float) }-0.03267 \text {; } \\
& \text { netPtr->layerPtr [2]->neuronPtr [6]->w[11] = (float) }-0.2498 \text {; } \\
& \text { netPtr->layerPtr[2]->neuronPtr[6]->w[12] = (float)0.3106; } \\
& \text { netPtr->layerPtr[2]->neuronPtr[6]->w[13] = (float) }-0.4114 \text {; } \\
& \text { netPtr->layerPtr[2] ->neuronPtr[6]->W[14] = (float) } 0.4027 \text {; } \\
& \text { netPtr->layerPtr [2] ->neuronPtr[6]->w[15] = (float) }-0.3256 \text {; } \\
& \text { netPtr->layerPtr[2]->neuronPtr[6]->w[16] = (float) } 0.184 ; \\
& \text { netPtr->layerPtr[2]->neuronPtr[6]->w[17] = (float) } 0.4999 ; \\
& \text { netPtr->layerPtr[2] }->\text { neuronPtr [6] }->\mathrm{w}[18]=\text { (float) }-0.09063 \text {; } \\
& \text { netPtr->layerPtr [2] ->neuronPtr[6]->W[19] = (float) }-0.1401 \text {; } \\
& \text { netPtr->layerPtr[2]->neuronPtr[6]->W[20] = (float) }-0.1057 \text {; } \\
& \text { netPtr->layerPtr[2]->neuronPtr[6]->w[21] = (float)0.08788; } \\
& \text { netPtr->layerPtr[2]->neuronPtr[6]->w[22] = (float) }-0.1655 \text {; } \\
& \text { netPtr->layerPtr[2]->neuronPtr[6]->w[23] = (float) } 0.4385 \text {; } \\
& \text { netPtr->layerPtr[2]->neuronPtr[6]->w[24] = (float) }-0.3917 \text {; } \\
& \text { netPtr->layerPtr[2]->neuronPtr[6]->w[25] = (float)0.2579; } \\
& \text { netPtr }->\text { layerPtr [2]->neuronPtr [6]->W[26] = (float) }-0.03475 \text {; } \\
& \text { netPtr }->\text { layerPtr [2]->neuronPtr[6]->W[27] = (float) } 0.358 \text {; } \\
& \text { netPtr->layerPtr[2]->neuronPtr[6]->w[28] = (float) } 0.1021 \text {; } \\
& \text { netPtr->layerPtr[2]->neuronPtr[6]->w[29] = (float) }-0.2207 \text {; } \\
& \text { netPtr->layerPtr[2]->neuronPtr[6]->W[30] = (float) } 0.1079 \text {; } \\
& \text { netPtr->layerPtr [2]->neuronPtr[6]->w[31] = (float) }-0.0738 \text {; }
\end{aligned}
$$

(transfer_function_type) 1 ;

netPtr->layerPtr[2]->neuronPtr[7]->tf =

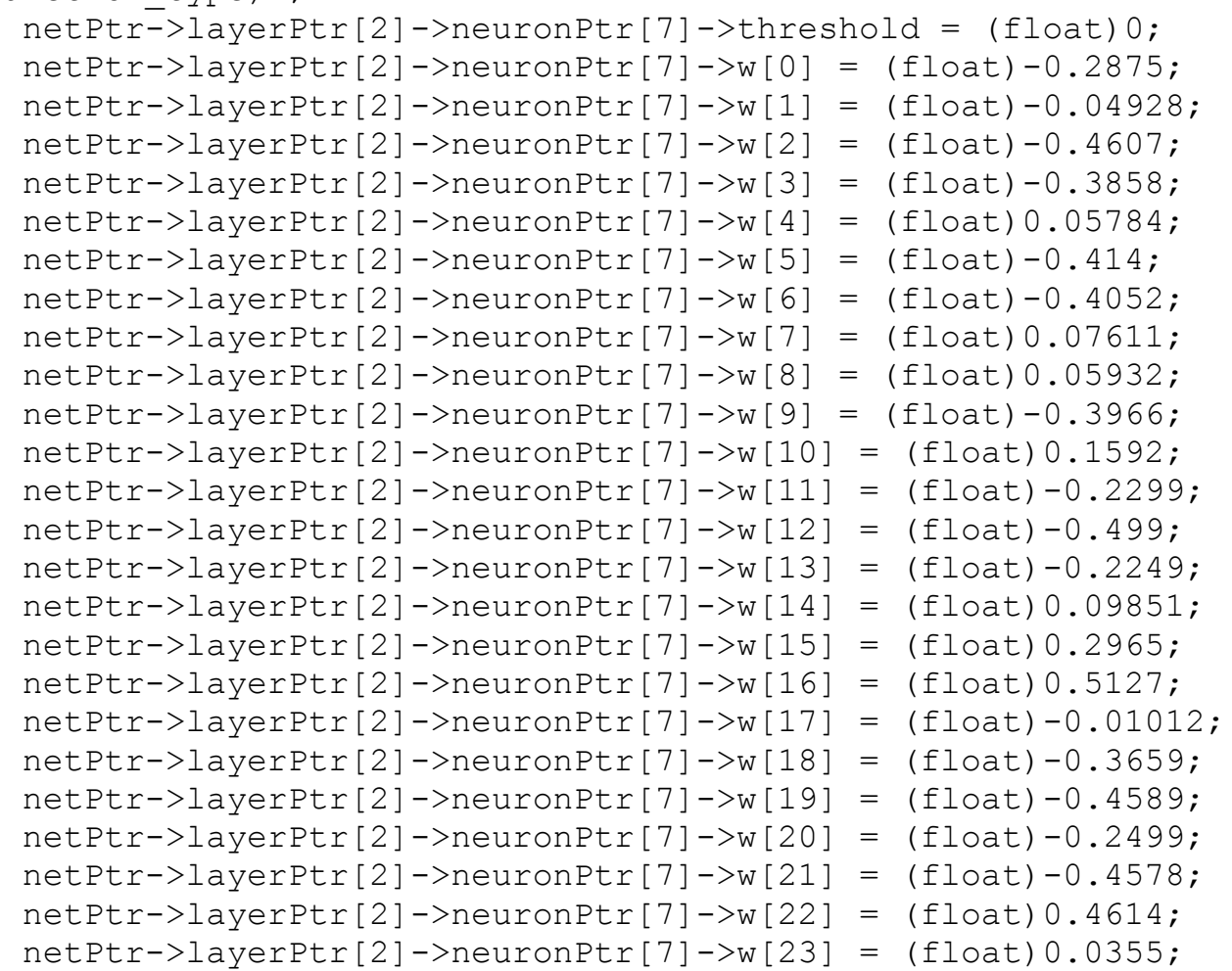




\begin{tabular}{|c|c|c|}
\hline & 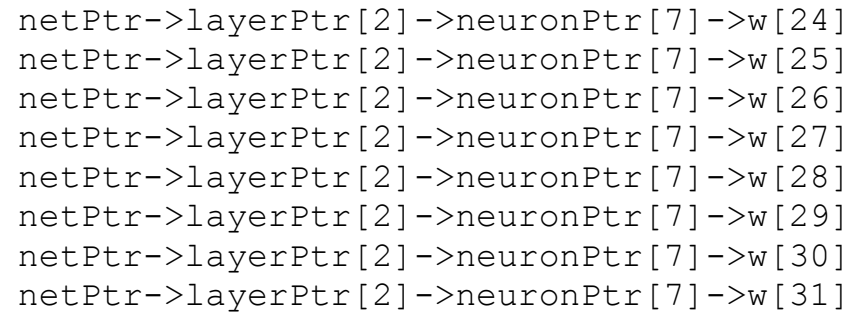 & $\begin{array}{l}=(\text { float }) 0.4522 ; \\
=(\text { float })-0.05924 ; \\
=(\text { float })-0.06682 ; \\
=(\text { float }) 0.1799 ; \\
=(\text { float }) 0.08489 ; \\
=(\text { float }) 0.4091 ; \\
=(\text { float }) 0.04114 ; \\
=(\text { float })-0.008181 ;\end{array}$ \\
\hline & 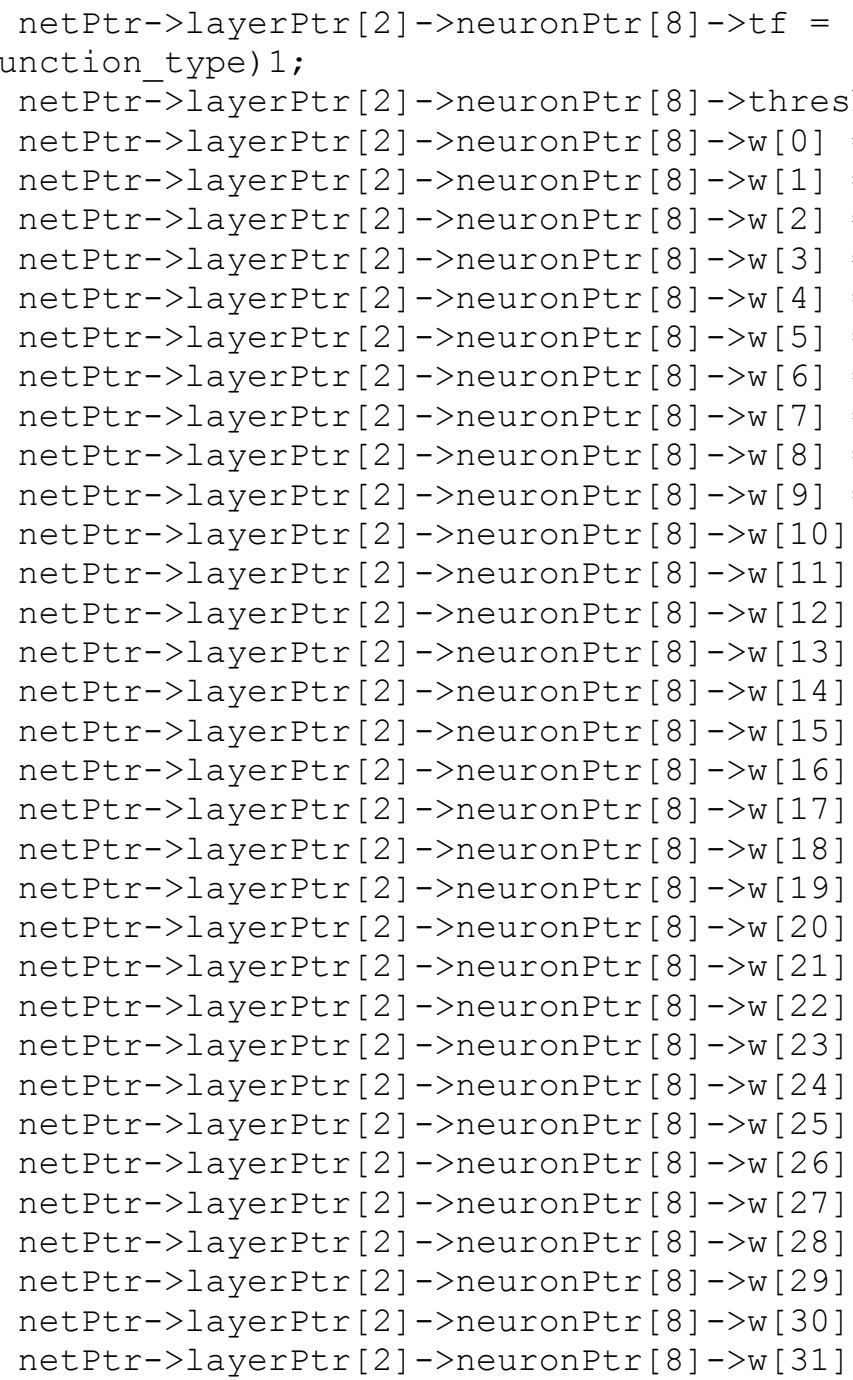 & 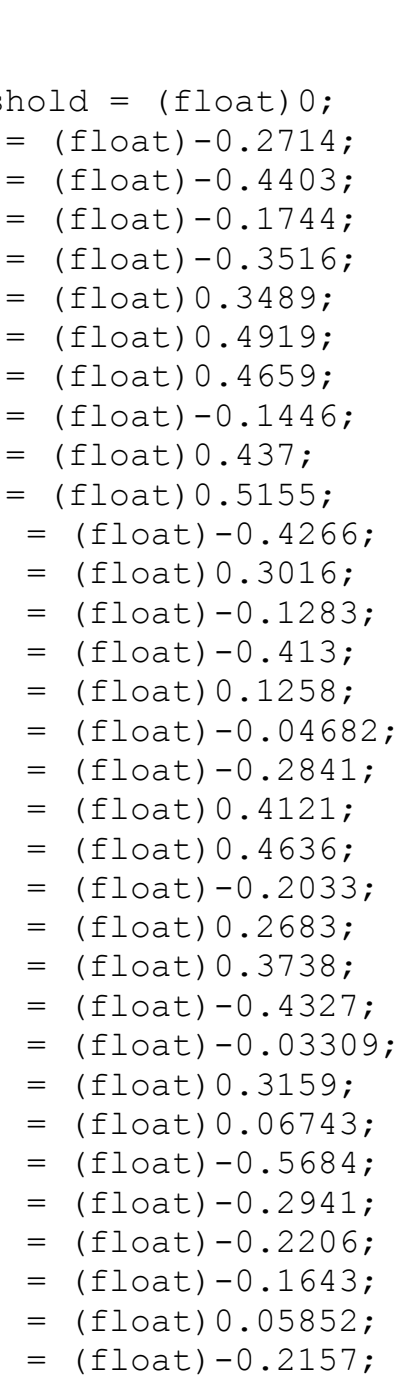 \\
\hline 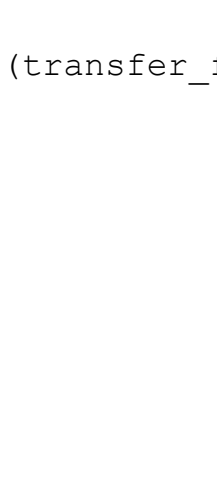 & 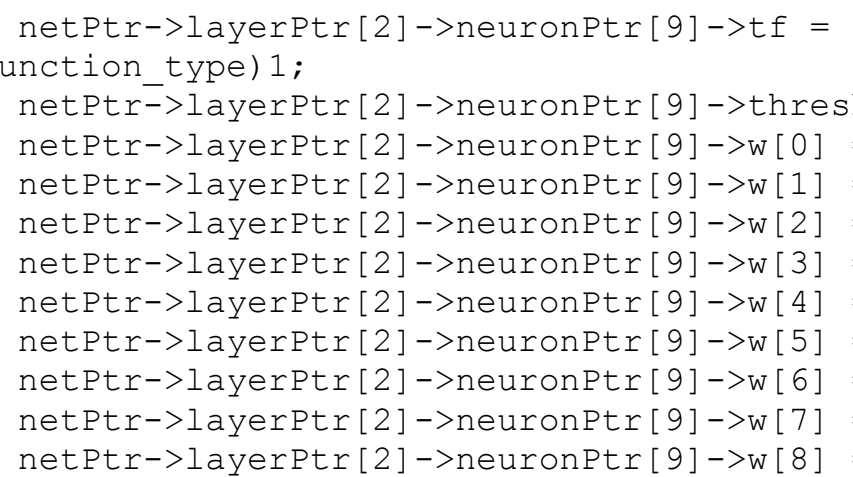 & 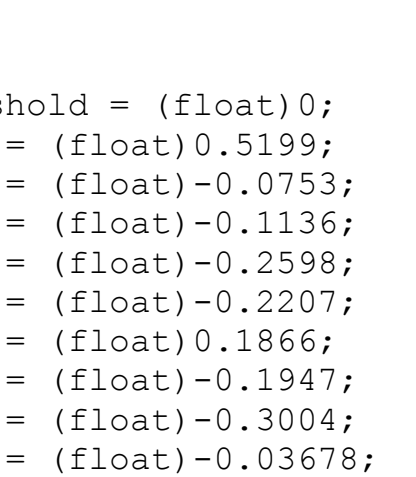 \\
\hline
\end{tabular}




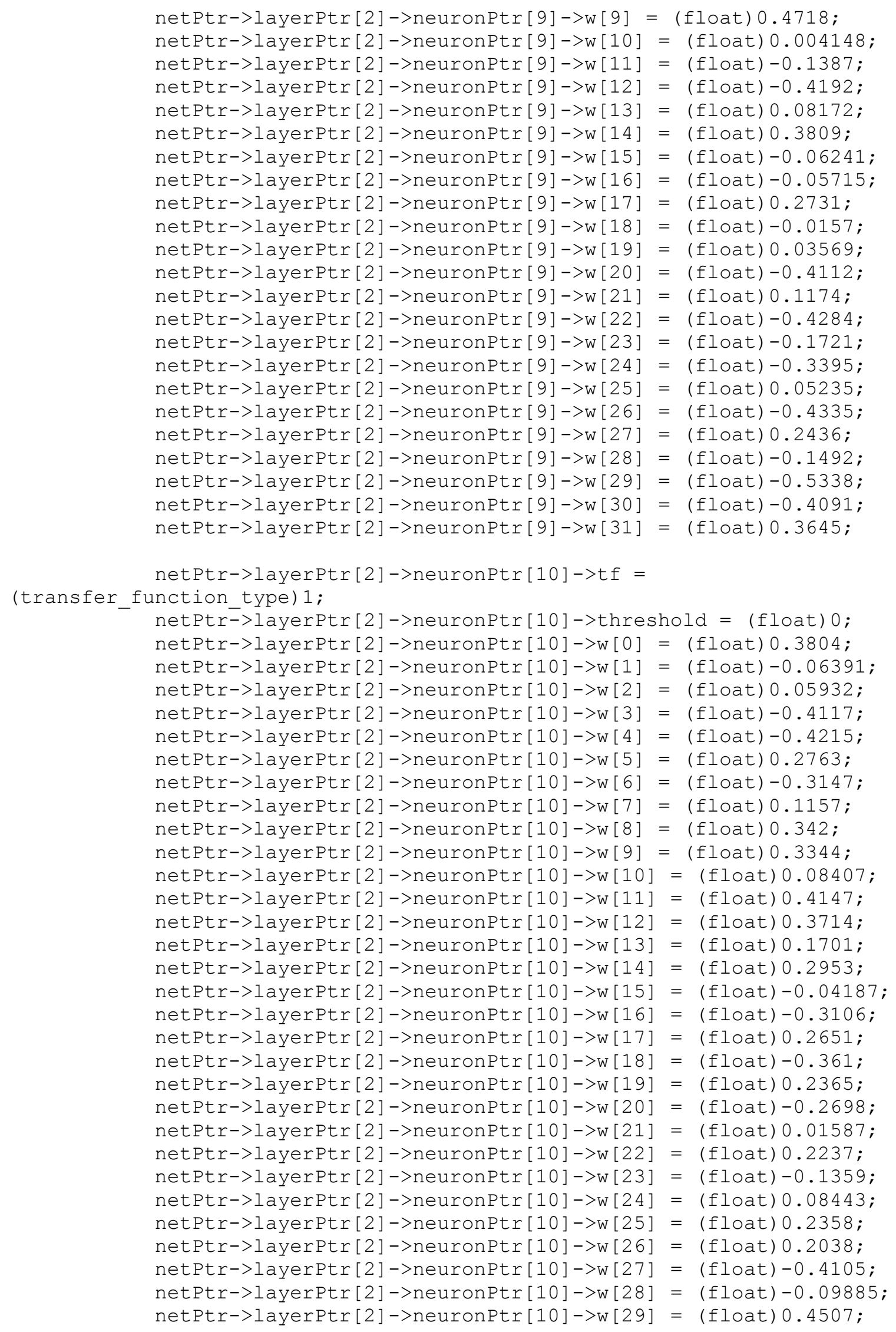




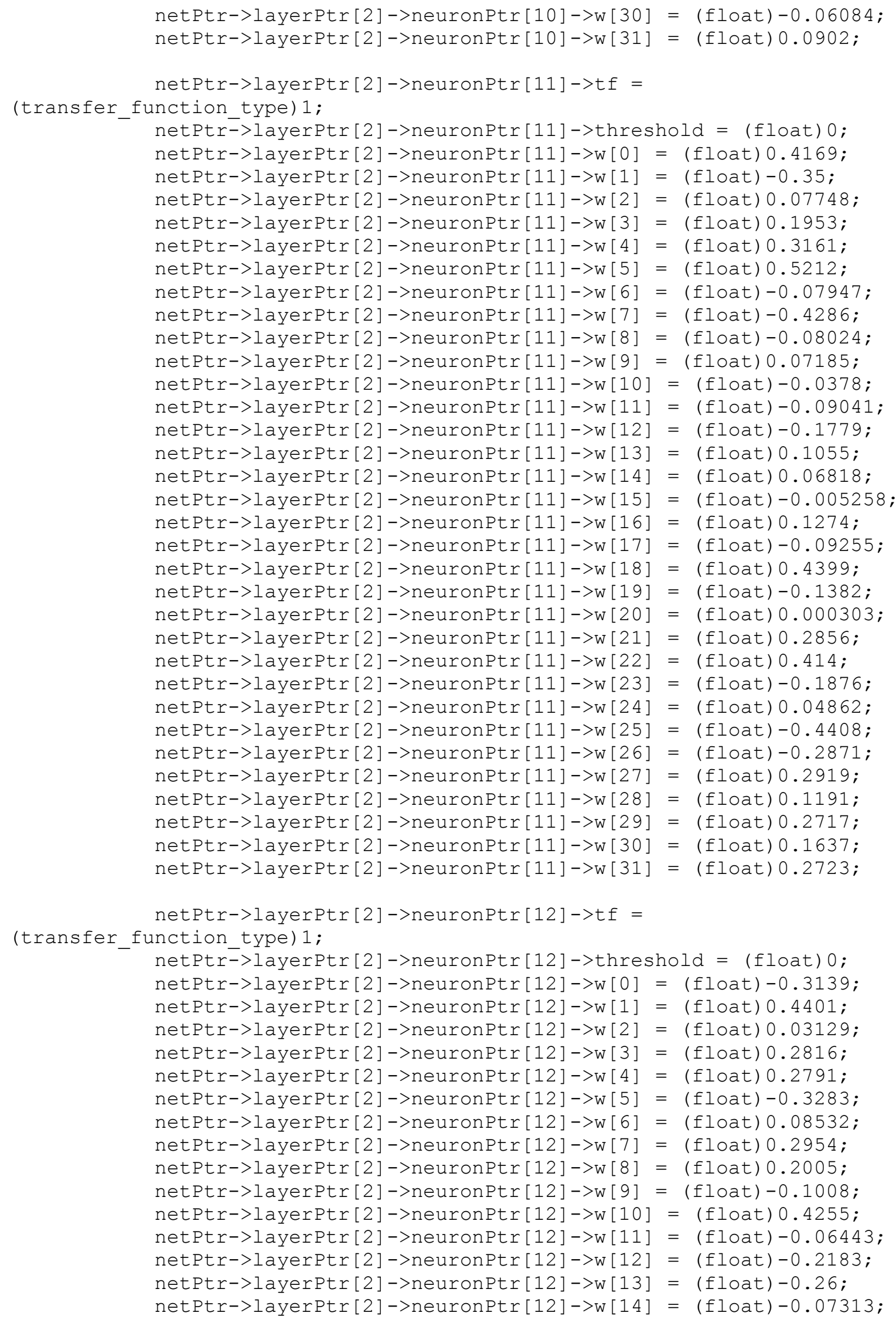




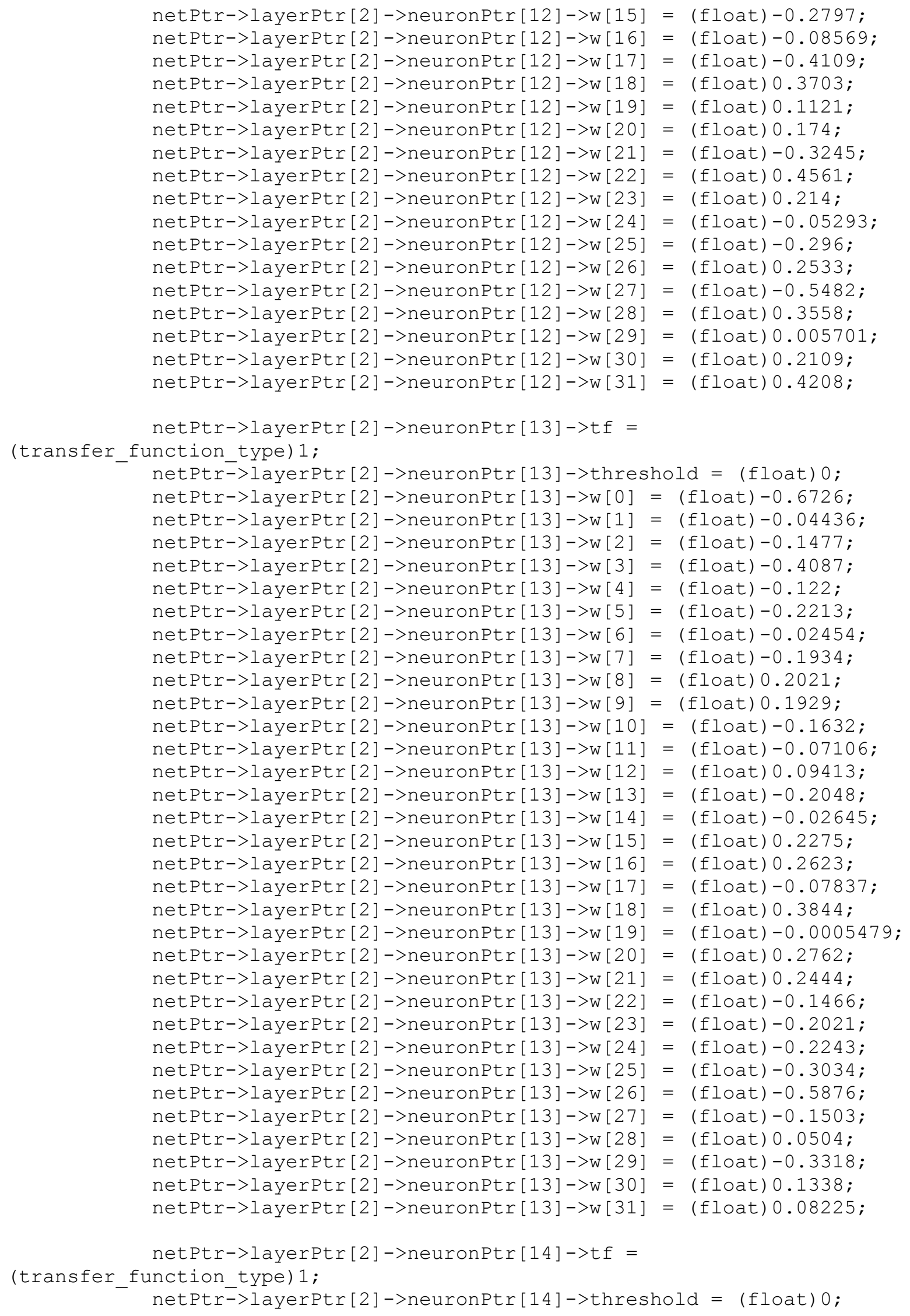




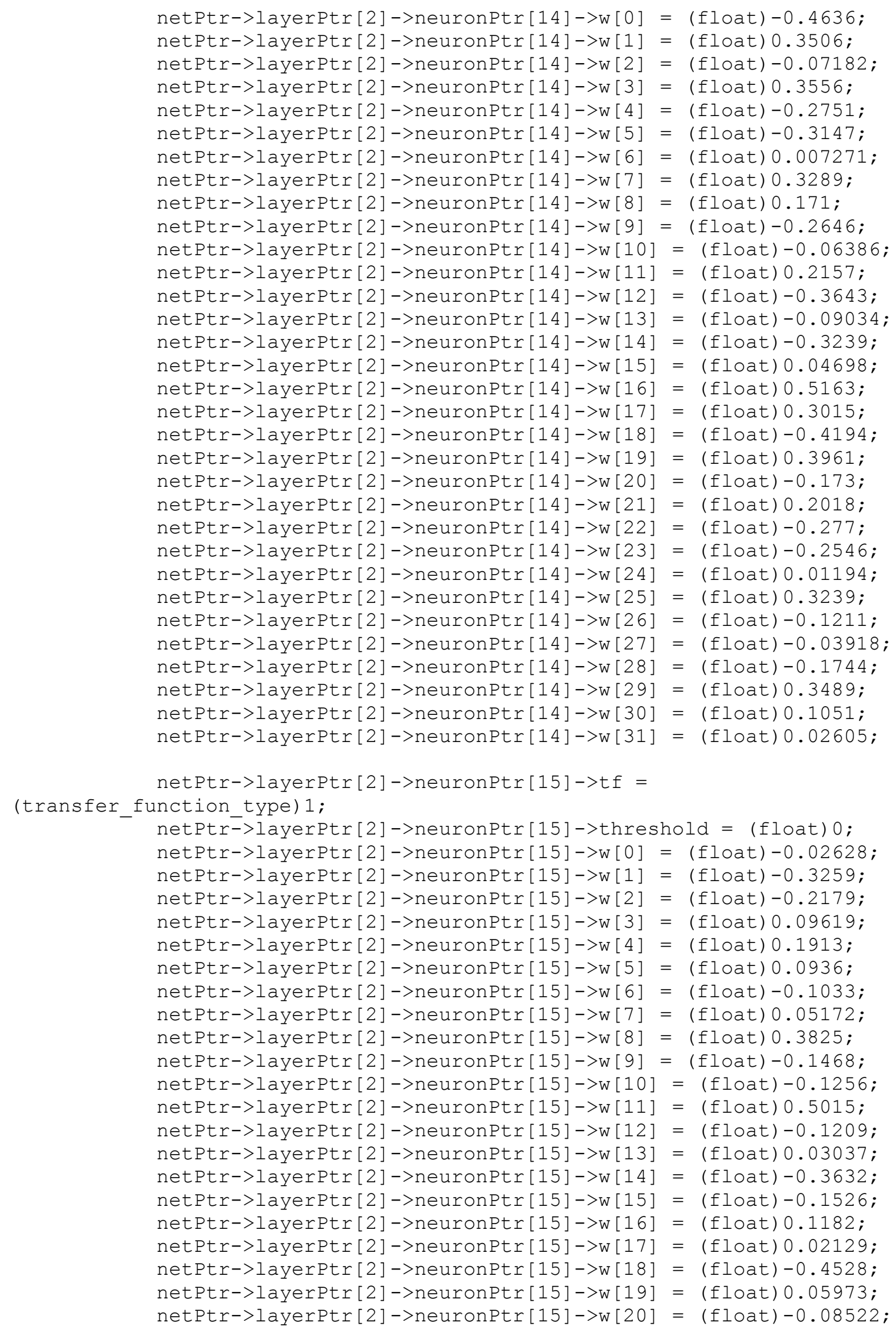




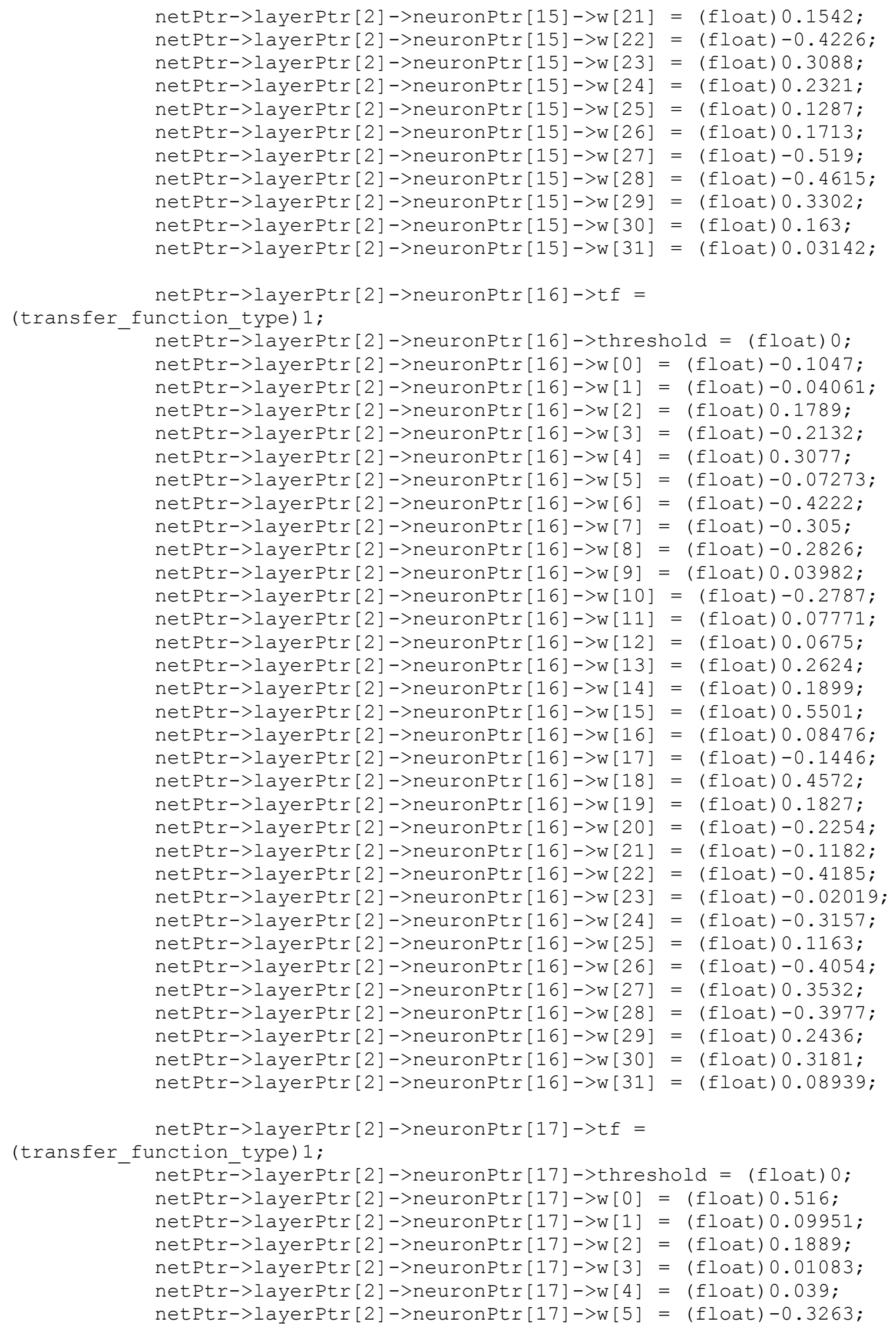




\begin{tabular}{|c|c|c|}
\hline & 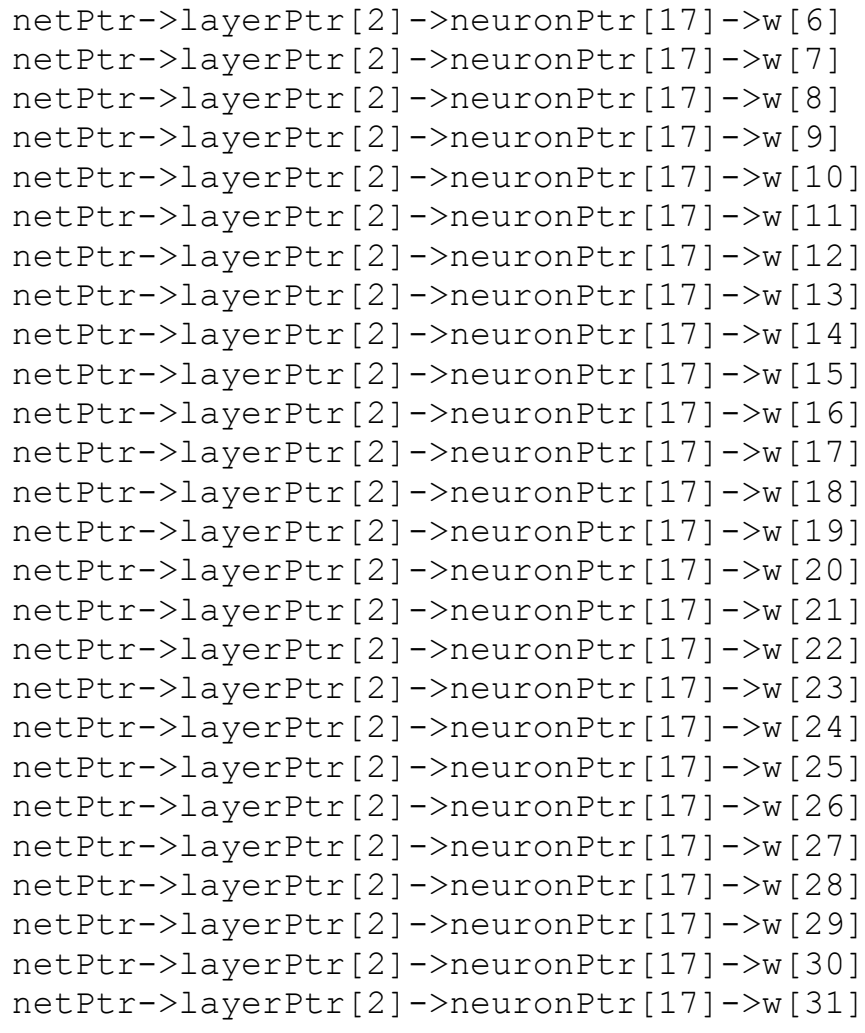 & 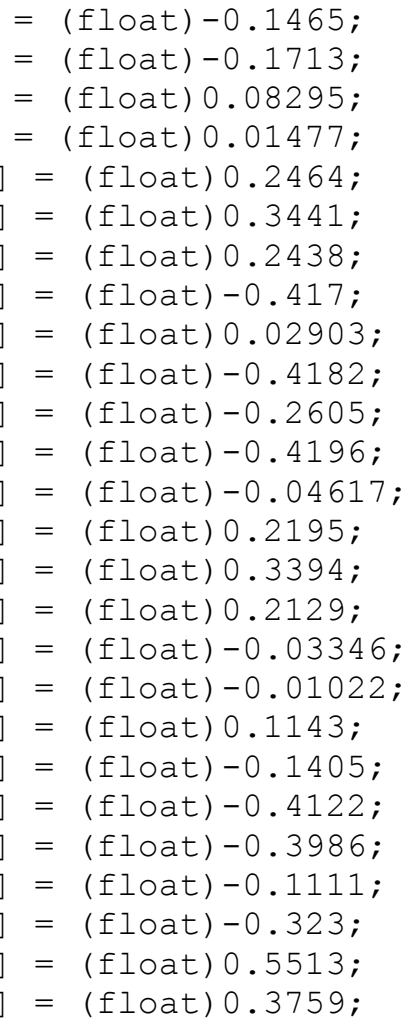 \\
\hline & 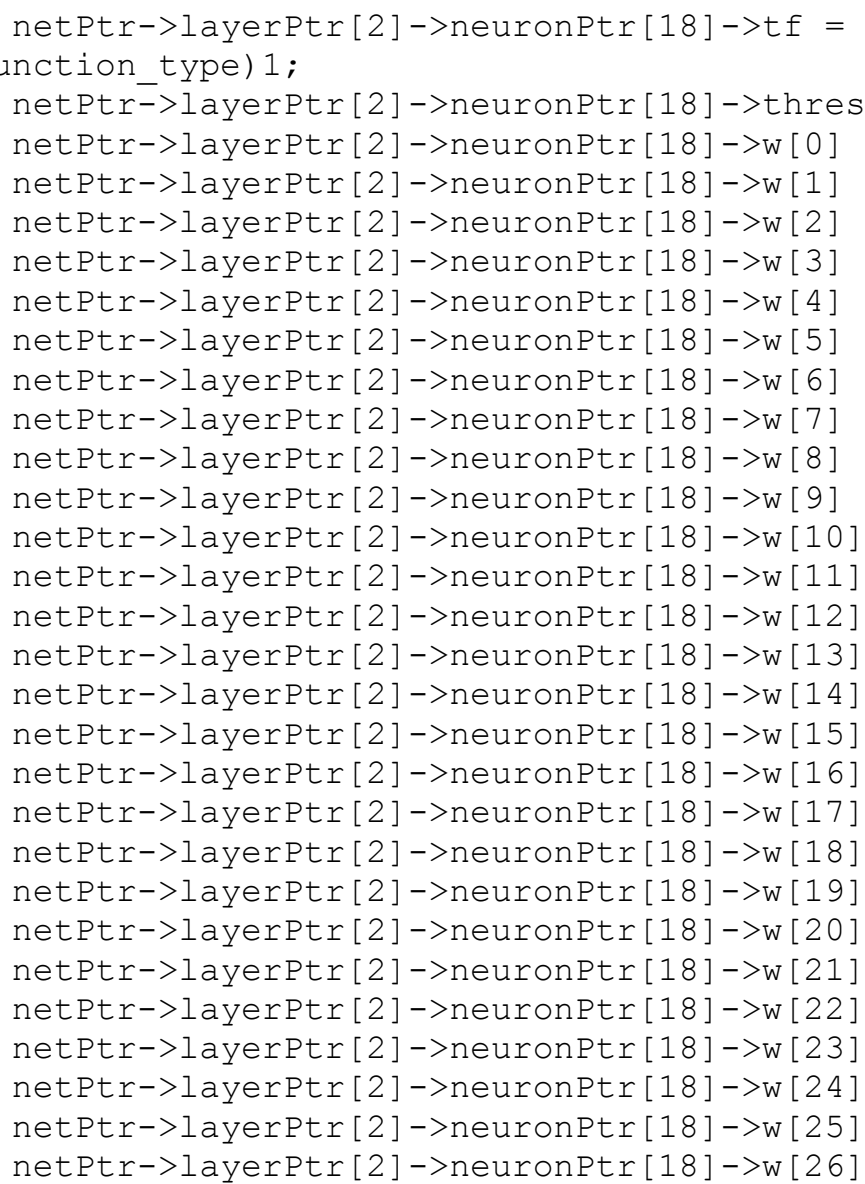 & 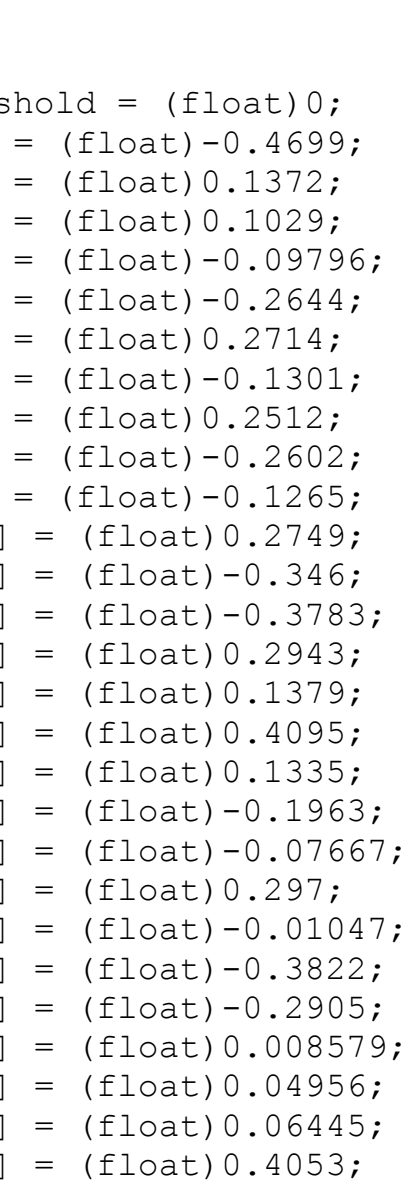 \\
\hline
\end{tabular}




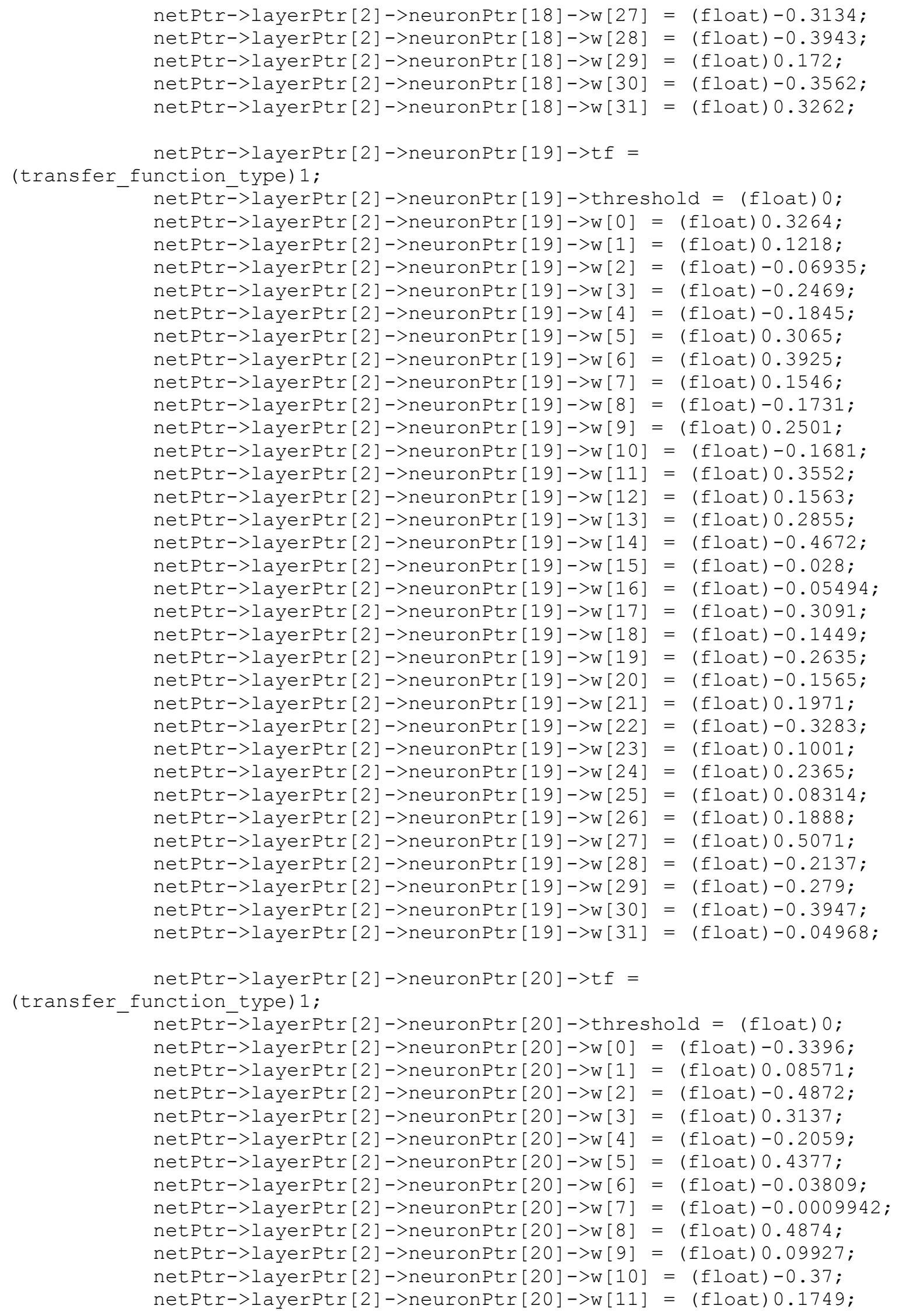




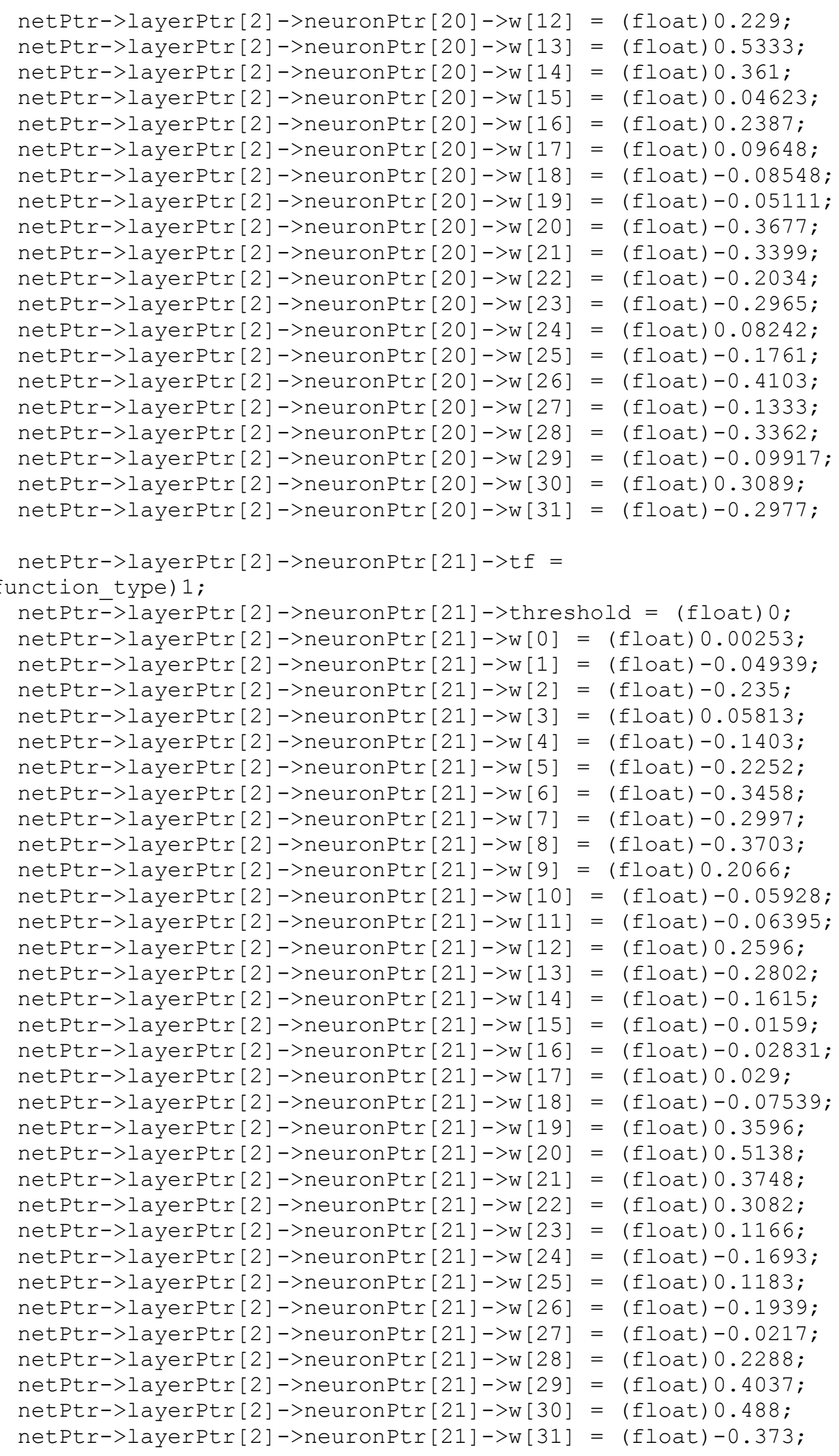


netPtr->layerPtr[2]->neuronPtr[22]->tf $=$ (transfer_function_type) 1 ;

netPtr->layerPtr[2]->neuronPtr[22]->threshold = (float) 0 ;

netPtr->layerPtr[2]->neuronPtr[22]->W[0] = (float) -0.1933 ;

netPtr->layerPtr[2]->neuronPtr[22]->w[1] = (float)0.4039;

netPtr->layerPtr[2]->neuronPtr[22]->w[2] = (float)0.04174;

netPtr->layerPtr [2]->neuronPtr[22]-> $[3]=$ (float) -0.2977 ;

netPtr->layerPtr[2]->neuronPtr[22]->W[4] = (float)0.2226;

netPtr->layerPtr [2]->neuronPtr[22]-> $[5]=$ (float) -0.3972 ;

netPtr->layerPtr[2]->neuronPtr[22]->w[6] = (float) -0.0442 ;

netPtr->layerPtr[2]->neuronPtr[22]->w[7] = (float) $0.3561 ;$

netPtr->layerPtr[2]->neuronPtr[22] $->\mathrm{w}[8]=$ (float) $0.3264 ;$

netPtr->layerPtr[2]->neuronPtr[22] $>\mathrm{w}[9]=$ (float) 0.2983 ;

netPtr->layerPtr[2]->neuronPtr[22]->w[10] = (float) 0.355 ;

netPtr->layerPtr[2]->neuronPtr[22]->w[11] = (float) -0.2022 ;

netPtr->layerPtr[2]->neuronPtr[22]->w[12] = (float) -0.1024 ;

netPtr->layerPtr [2]->neuronPtr[22]->W[13] = (float) $0.2011 ;$

netPtr->layerPtr[2]->neuronPtr[22]->w[14] = (float) 0.0407 ;

netPtr->layerPtr[2]->neuronPtr[22]->w[15] = (float)-0.2201;

netPtr->layerPtr[2]->neuronPtr[22]->W[16] = (float) -0.4167 ;

netPtr->layerPtr[2]->neuronPtr[22]->w[17] = (float) $0.432 ;$

netPtr->layerPtr[2]->neuronPtr[22]->w[18] = (float) -0.3286 ;

netPtr->layerPtr[2]->neuronPtr[22]->w[19] = (float) -0.0525 ;

netPtr->layerPtr[2]->neuronPtr[22]->W[20] = (float) -0.4294 ;

netPtr->layerPtr[2]->neuronPtr[22]->W[21] = (float) -0.1638 ;

netPtr->layerPtr[2]->neuronPtr[22]->w[22] = (float) -0.3525 ;

netPtr $->$ layerPtr[2]->neuronPtr[22]->w[23] = (float) 0.4783 ;

netPtr->layerPtr[2]->neuronPtr[22]->W[24] = (float) -0.3454 ;

netPtr $->$ layerPtr [2] $->$ neuronPtr [22] $->\mathrm{w}[25]=$ (float) -0.3255 ;

netPtr->layerPtr [2]->neuronPtr[22]->w[26] = (float) $0.482 ;$

netPtr->layerPtr[2]->neuronPtr[22]->w[27] = (float)0.4483;

netPtr->layerPtr[2] ->neuronPtr[22] $\rightarrow \mathrm{w}[28]=$ (float) -0.134 ;

netPtr->layerPtr[2] ->neuronPtr[22]->W[29] = (float) -0.2467 ;

netPtr->layerPtr[2]->neuronPtr[22]->w[30] = (float) -0.3447 ;

netPtr->layerPtr[2]->neuronPtr[22]->w[31] = (float) 0.03224 ;

netPtr->layerPtr[2]->neuronPtr[23]->tf $=$

(transfer_function_type) 1 ;

netPtr->layerPtr[2]->neuronPtr[23]->threshold $=($ float) 0 ;

netPtr->layerPtr [2]->neuronPtr[23]-> $[0]=$ (float) -0.209 ;

netPtr->layerPtr [2]->neuronPtr[23]-> $[1]=$ (float) 0.5087 ;

netPtr->layerPtr[2]->neuronPtr[23]->w[2] = (float)0.04873;

netPtr->layerPtr[2]->neuronPtr[23]->W[3] = (float) 0.3322 ;

netPtr->layerPtr[2]->neuronPtr[23]->W[4] = (float) -0.08582 ;

netPtr->layerPtr[2]->neuronPtr[23]->W[5] = (float) -0.2017 ;

netPtr $->$ layerPtr [2]->neuronPtr[23]->W[6] = (float) -0.4945 ;

netPtr->layerPtr[2]->neuronPtr[23]->w[7] = (float)0.00568;

netPtr->layerPtr[2]->neuronPtr[23]->w[8] = (float) -0.3671 ;

netPtr->layerPtr[2]->neuronPtr[23]->w[9] = (float) -0.1641 ;

netPtr->layerPtr[2]->neuronPtr[23]->W[10] = (float) 0.04256 ;

netPtr $->$ layerPtr [2]->neuronPtr[23]->W[11] = (float) 0.3561 ;

netPtr->layerPtr[2]->neuronPtr[23]->W[12] = (float) -0.3865 ;

netPtr->layerPtr[2]->neuronPtr[23]->w[13] = (float) 0.34 ;

netPtr->layerPtr[2]->neuronPtr[23]->w[14] = (float) -0.05907 ;

netPtr $->$ layerPtr [2]->neuronPtr $[23]->\mathrm{W}[15]=$ (float) -0.272 ;

netPtr->layerPtr [2]->neuronPtr[23]->w[16] = (float)0.3398;

netPtr->layerPtr[2]->neuronPtr[23]->w[17] = (float)0.239; 


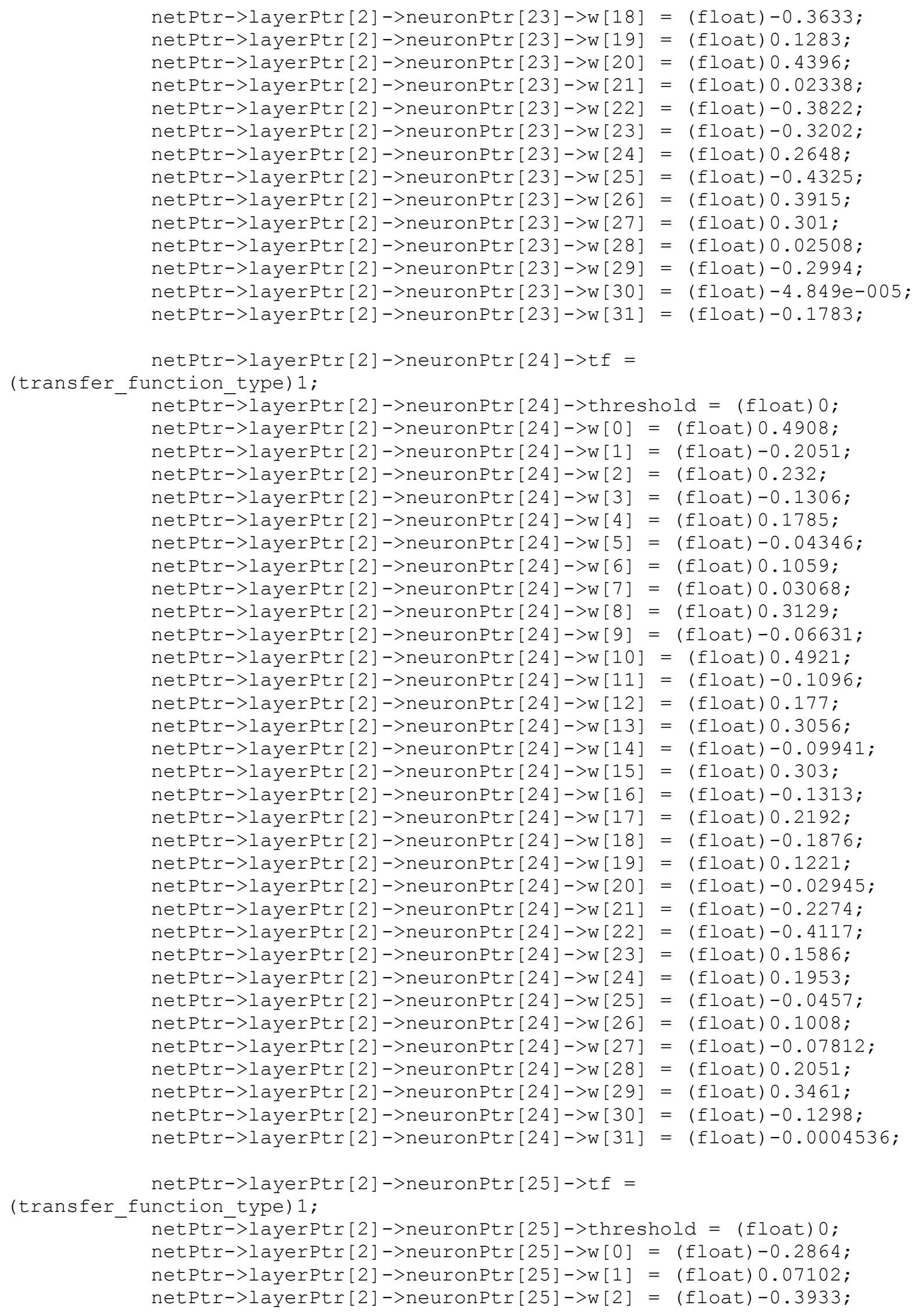




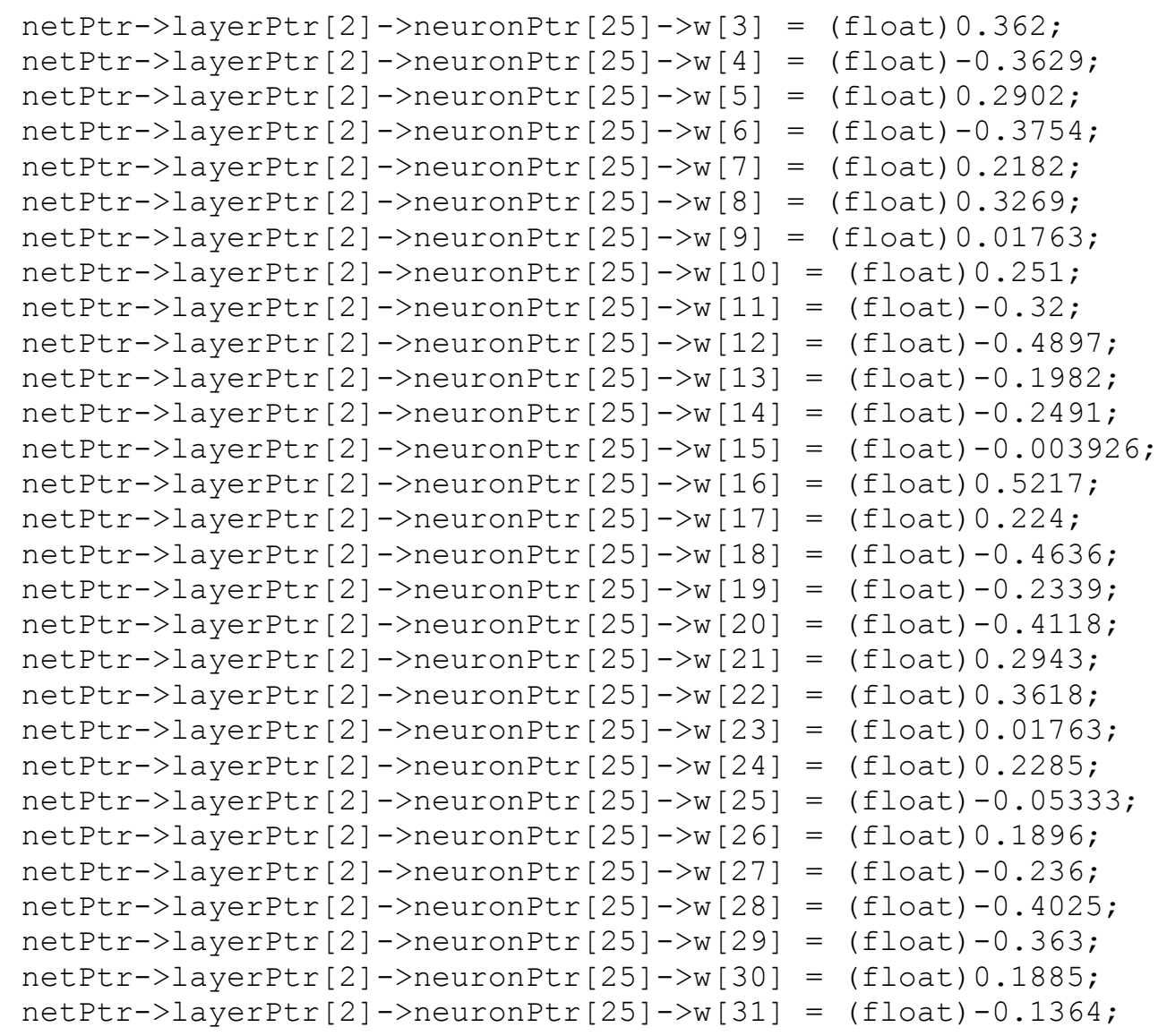

(transfer_function_type) 1 ;

netPtr->layerPtr[2]->neuronPtr[26]->tf =

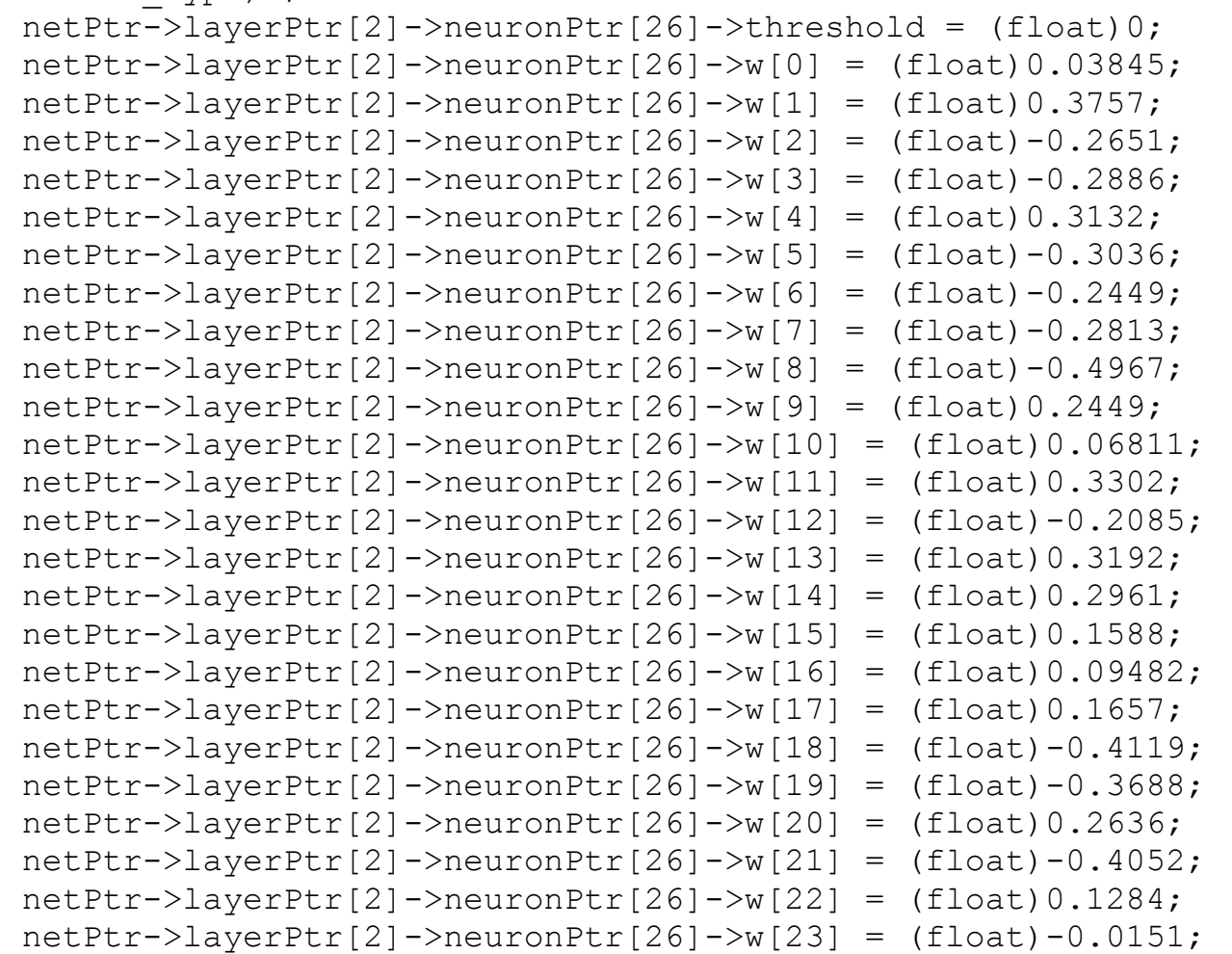




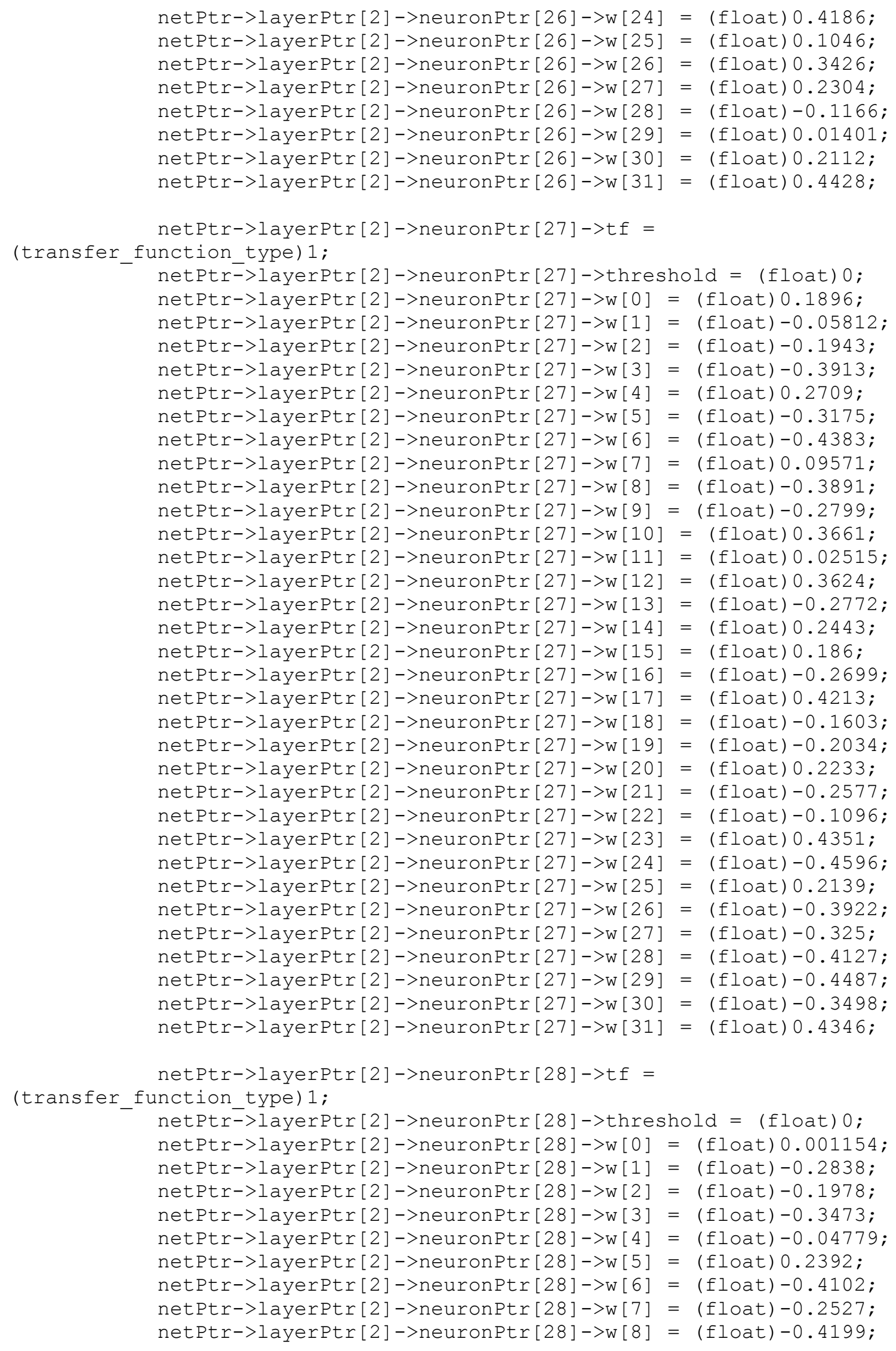




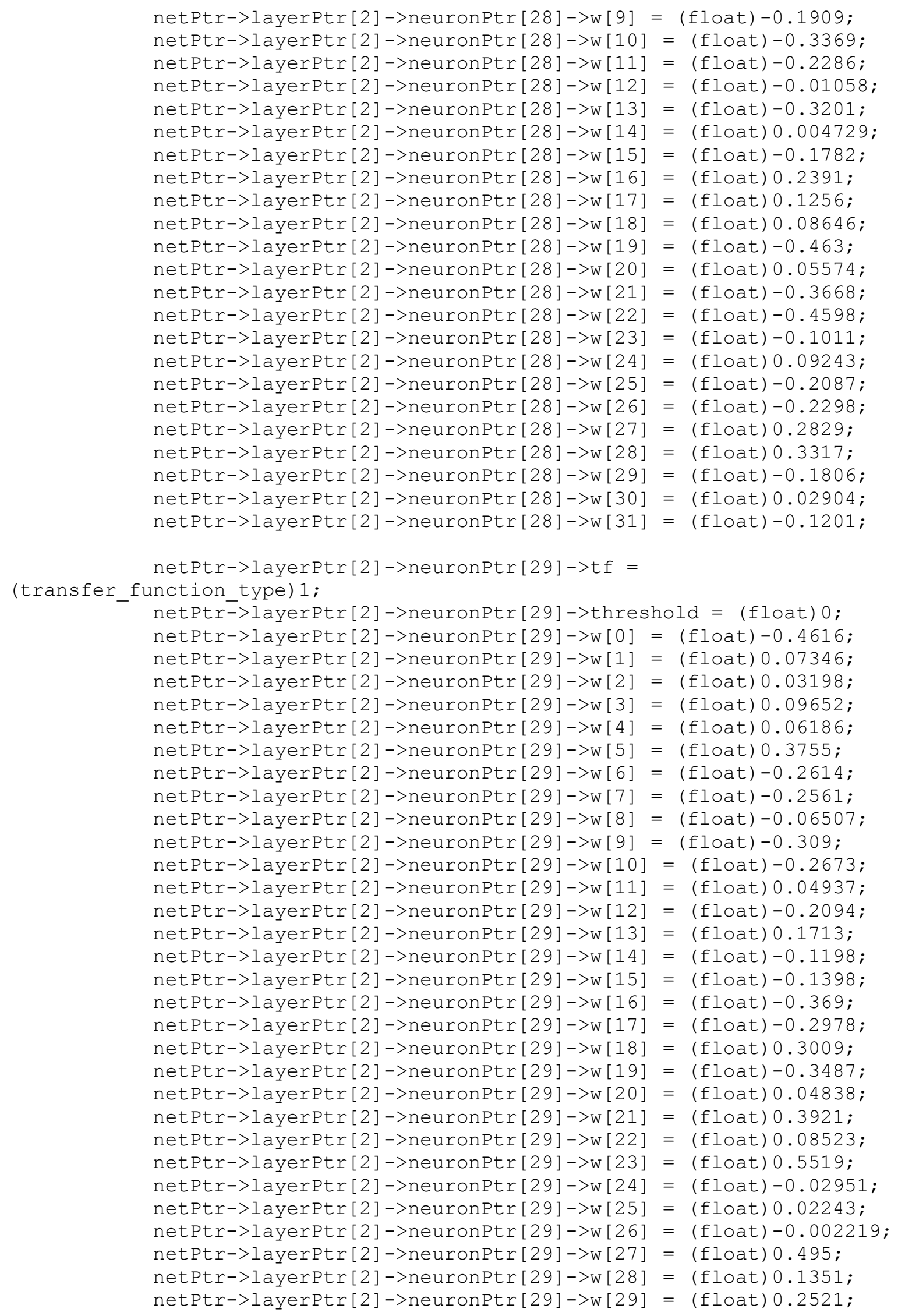




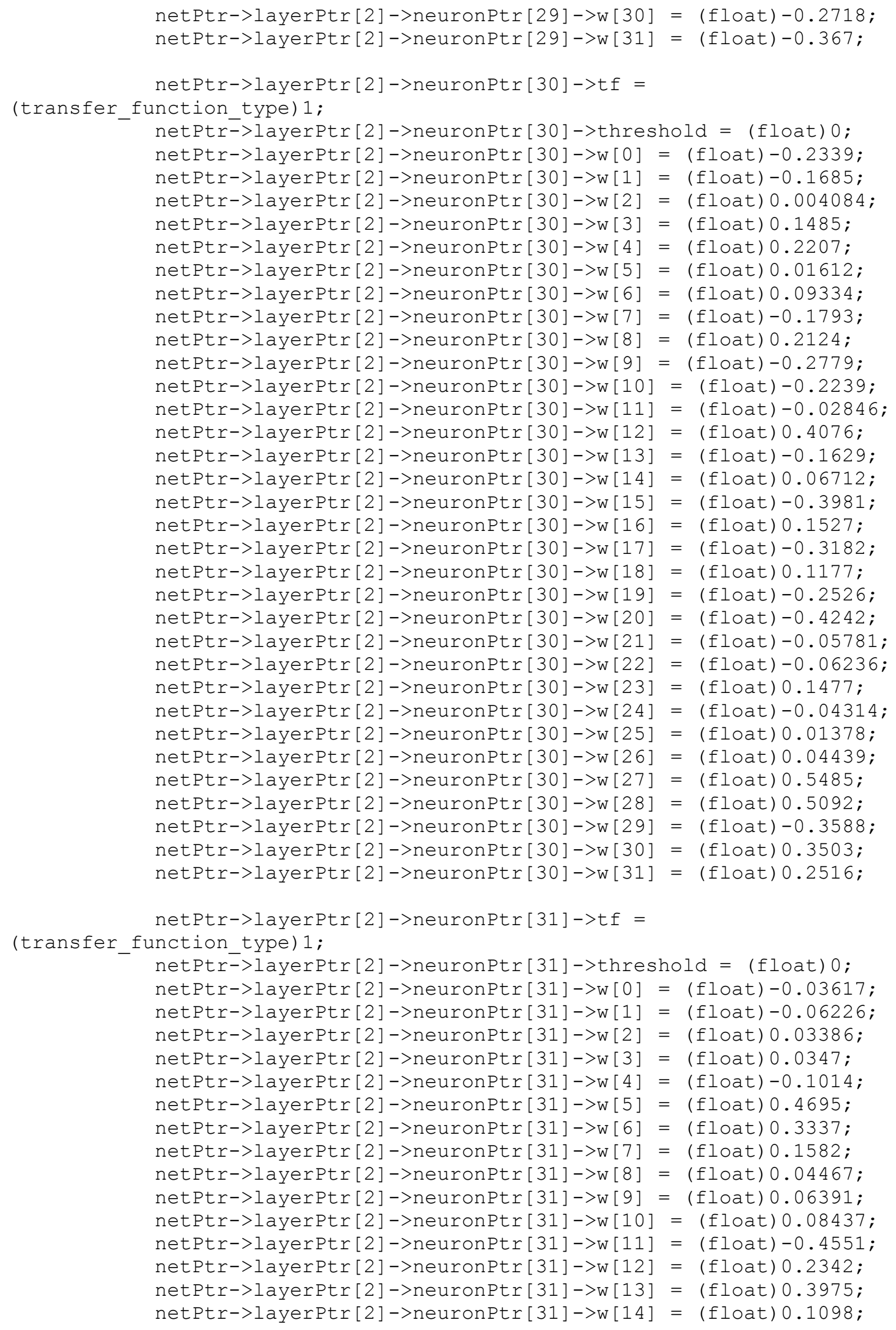




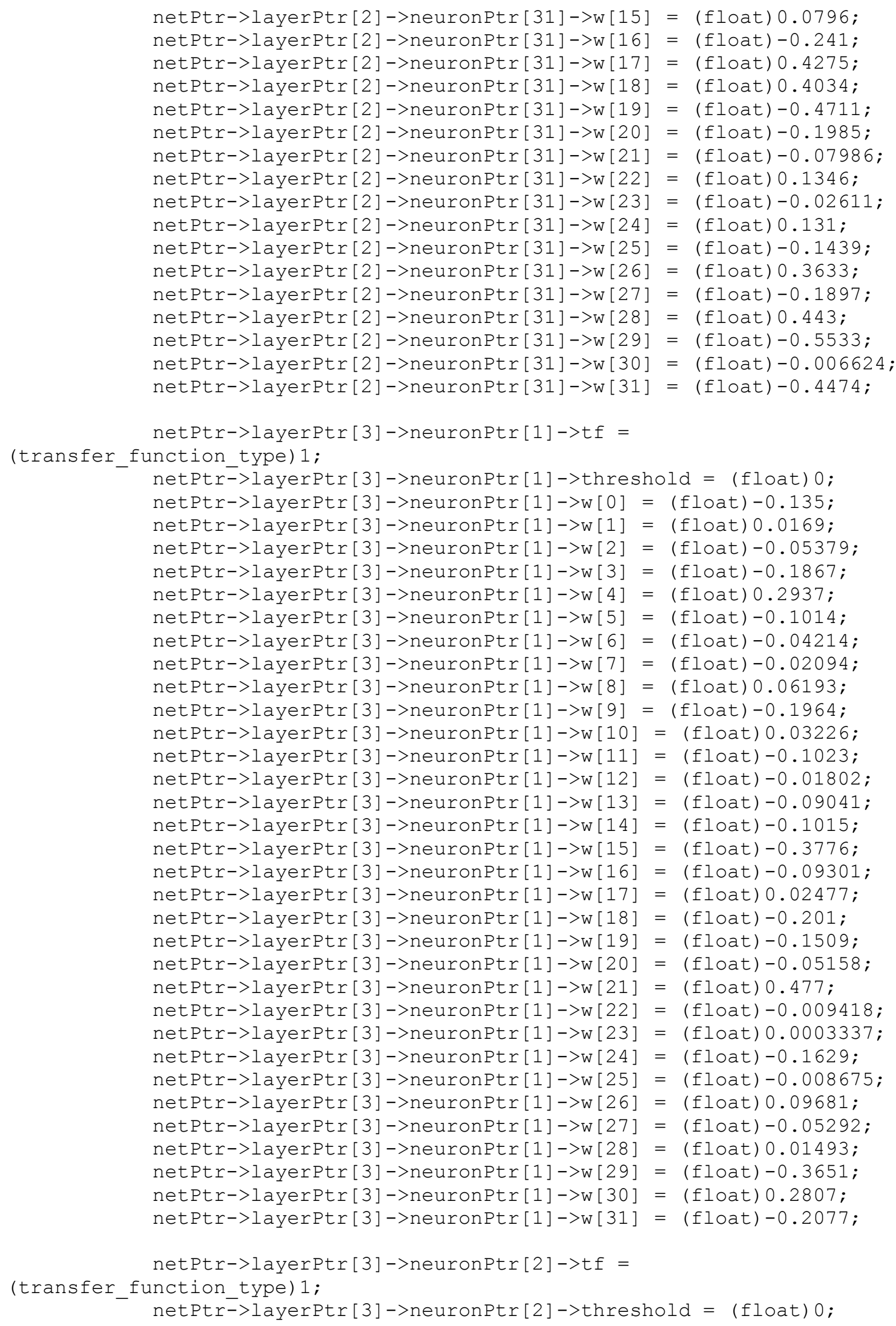




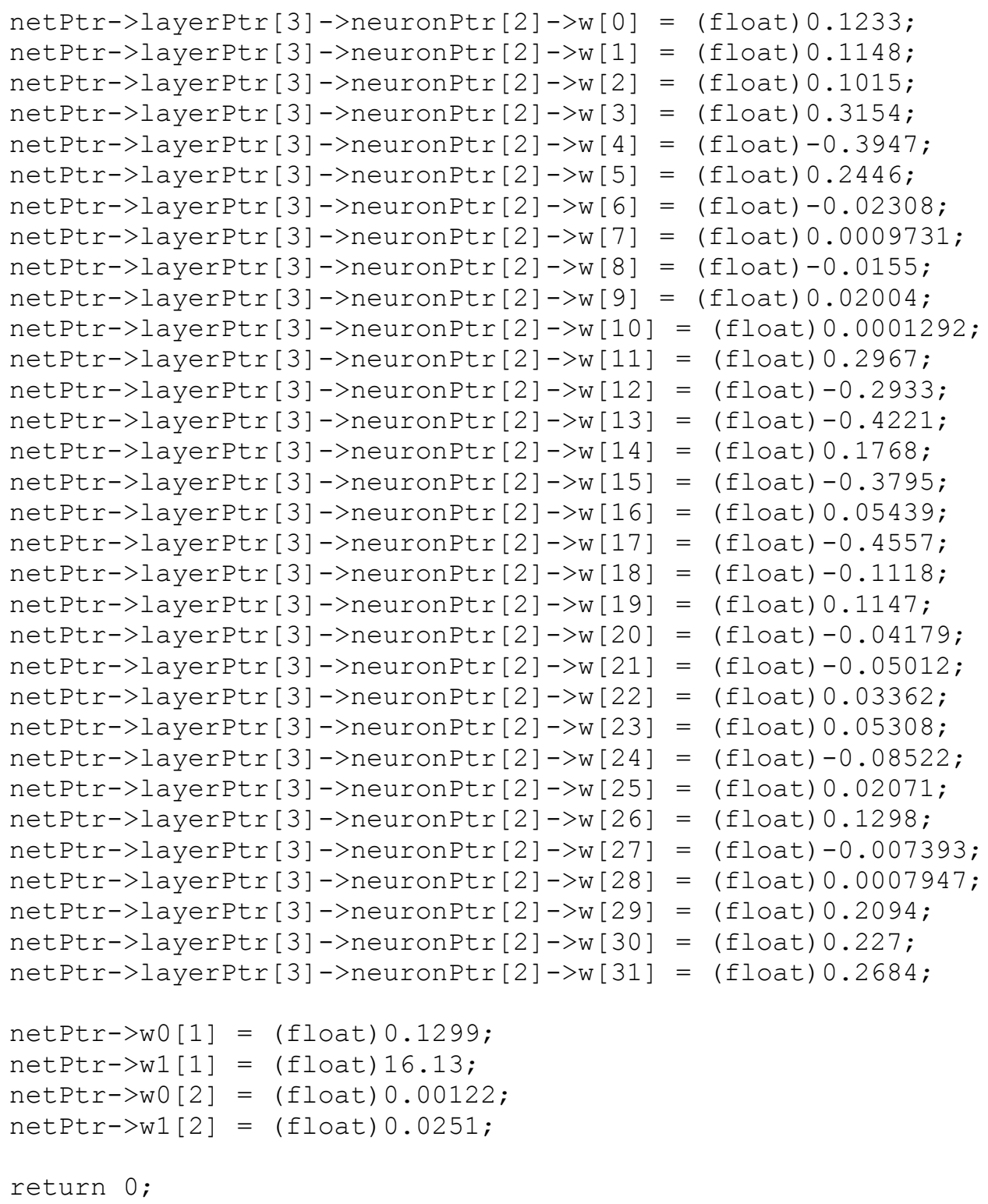




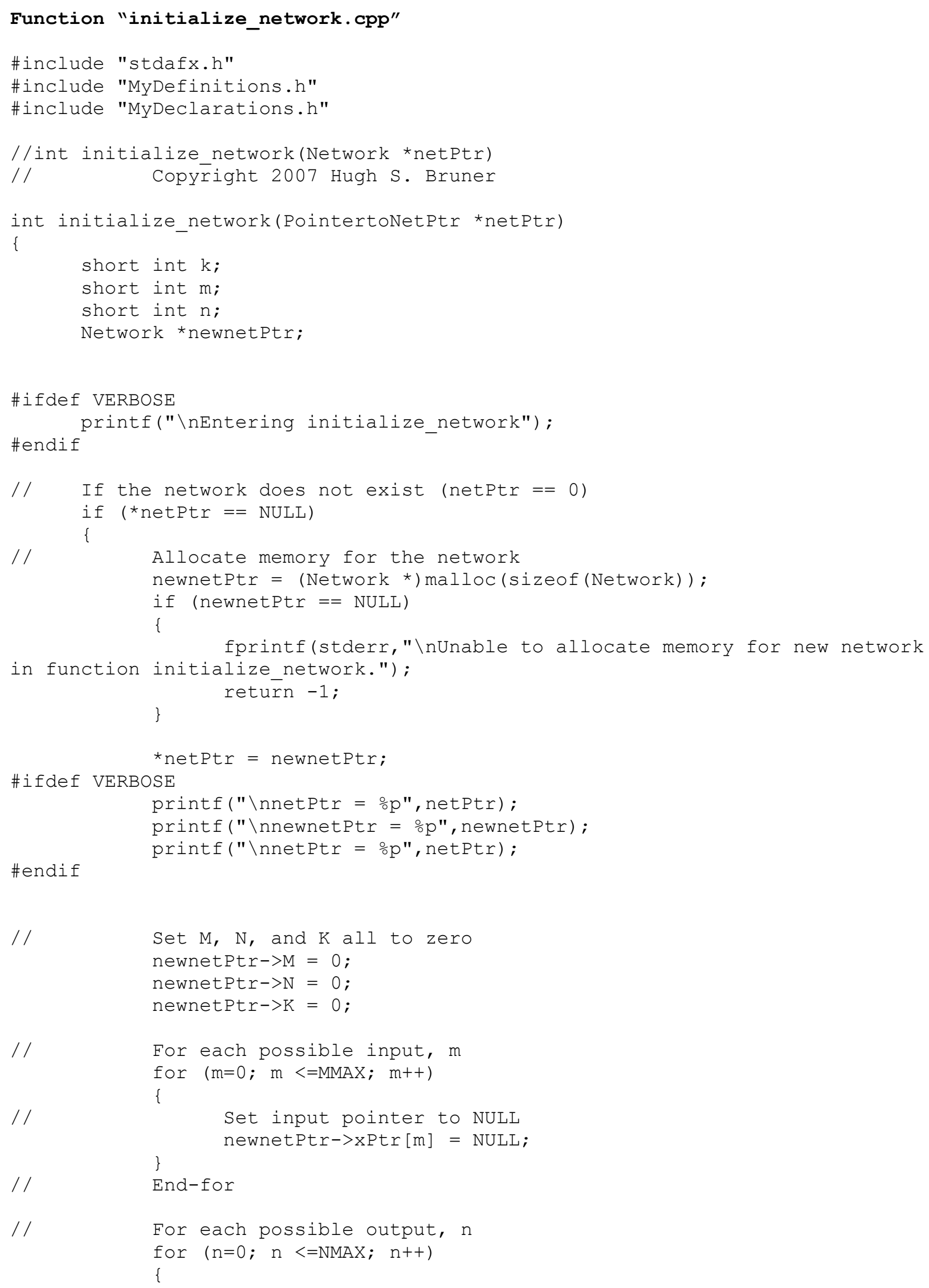




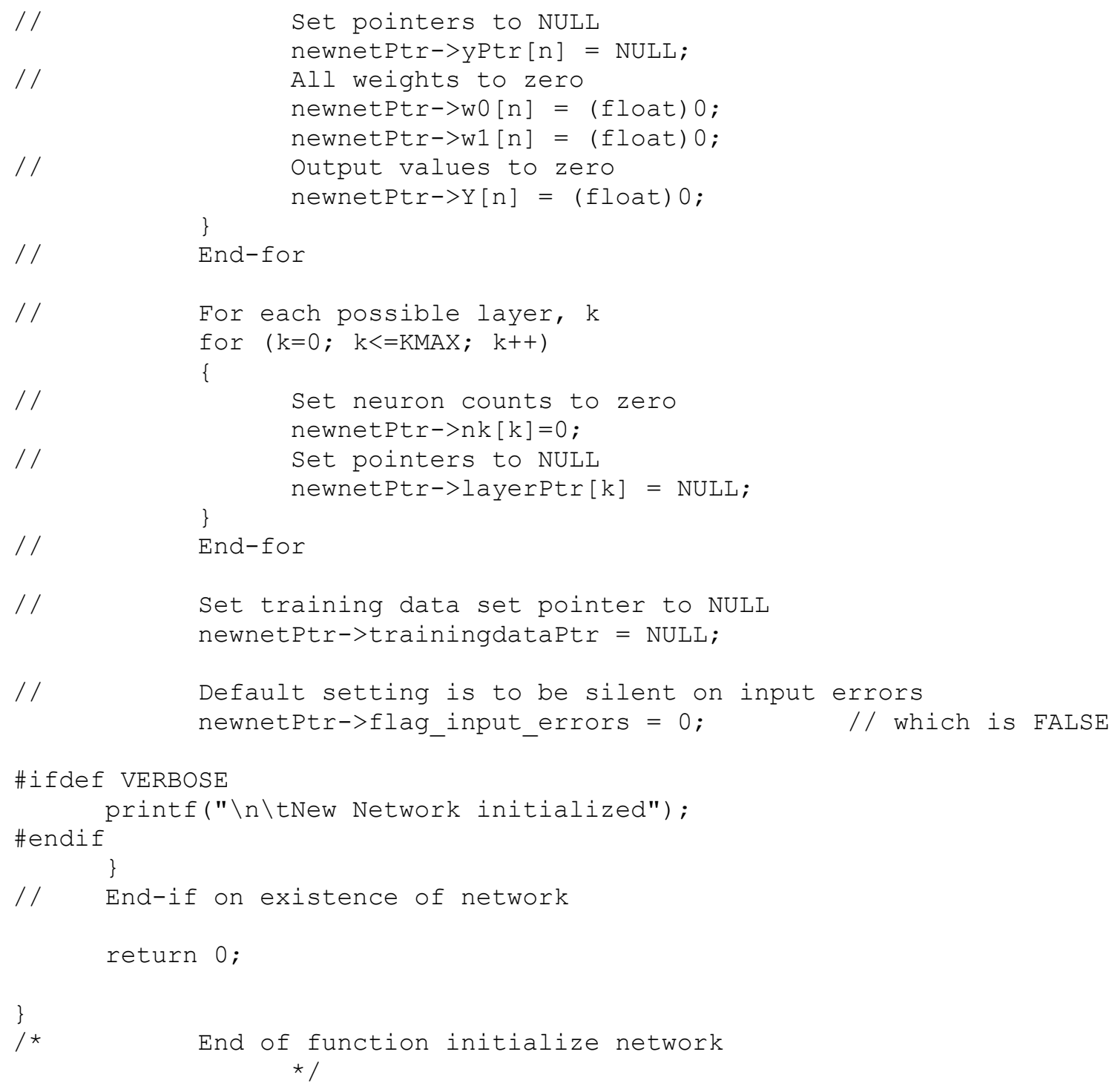




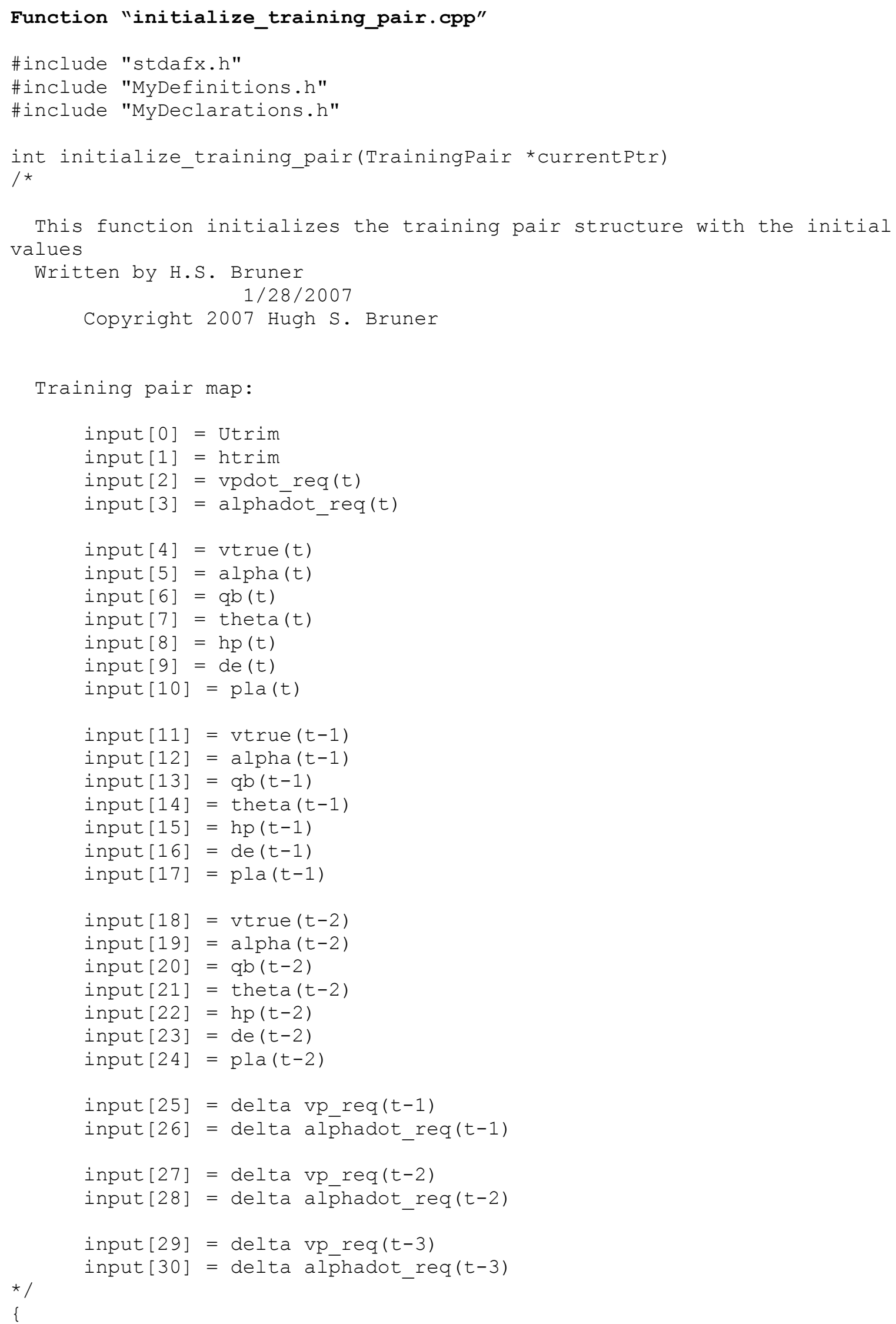




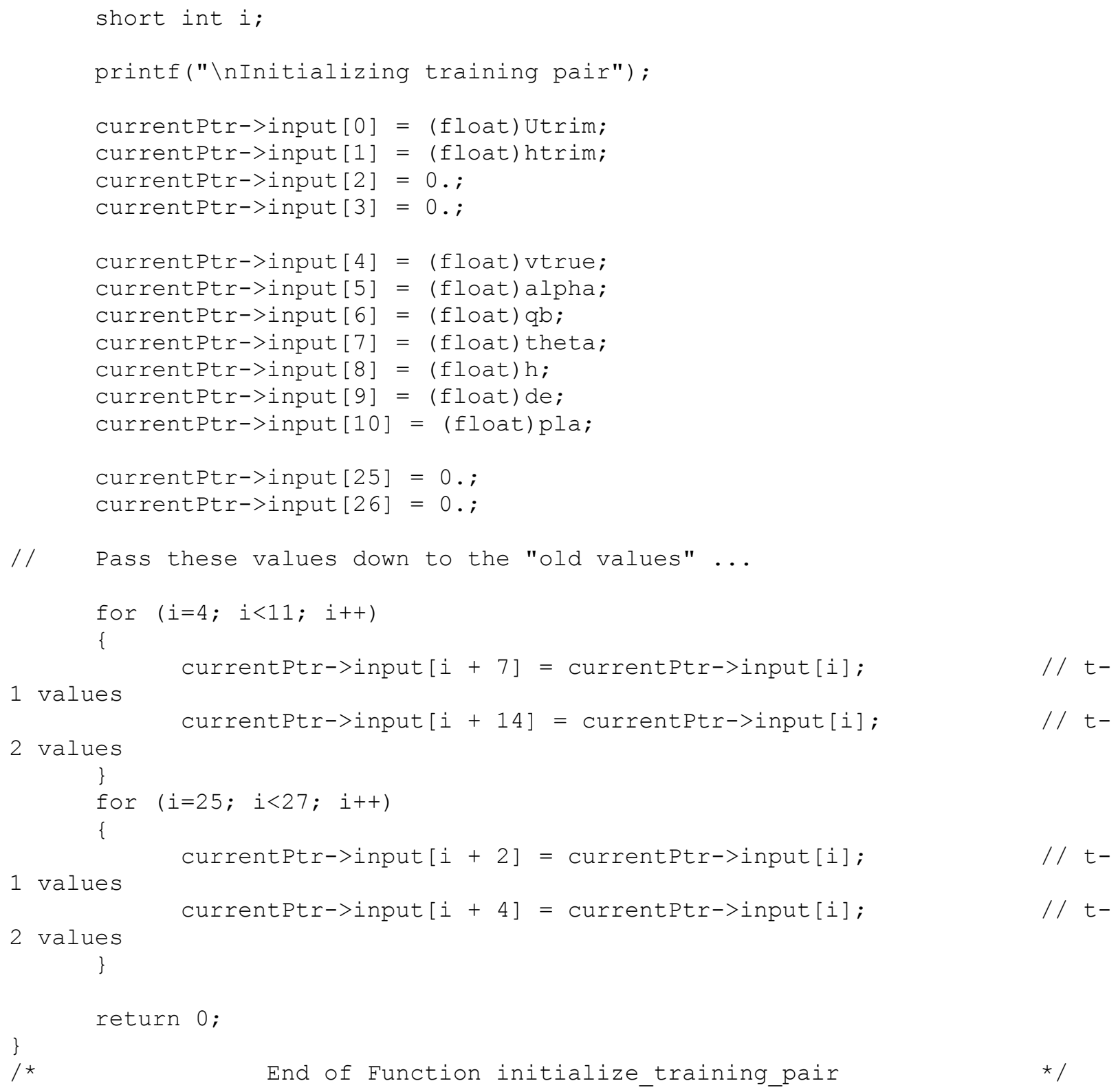




\section{input_excedance_alarm.cpp"}

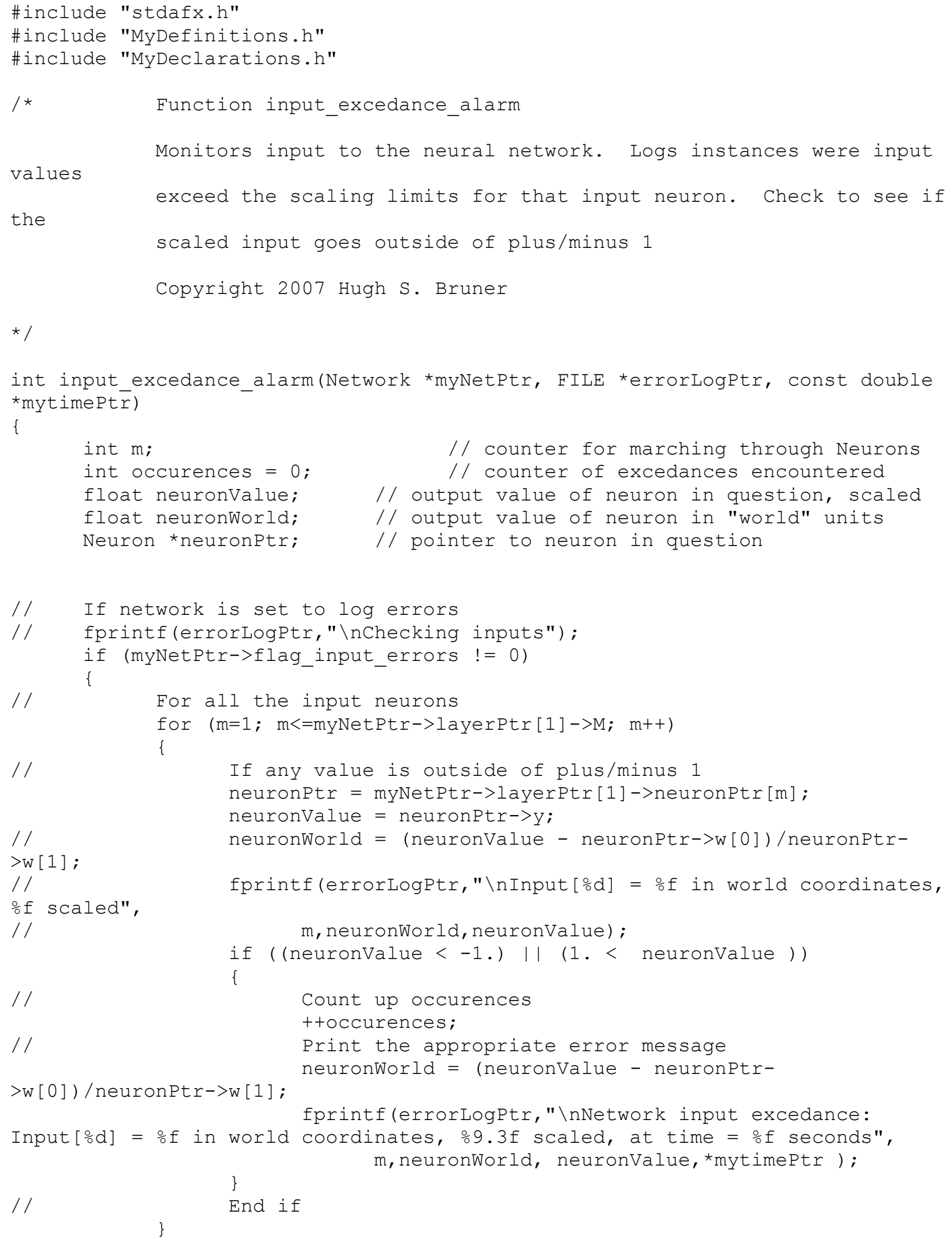




$\begin{array}{ll}/ / & \text { End for } \\ / / & \begin{array}{l}\text { Endif } \\ \text { return occurences; }\end{array} \\ 3 & \text { End of function }\end{array}$




\section{Function "inverse_xfunction.cpp"}

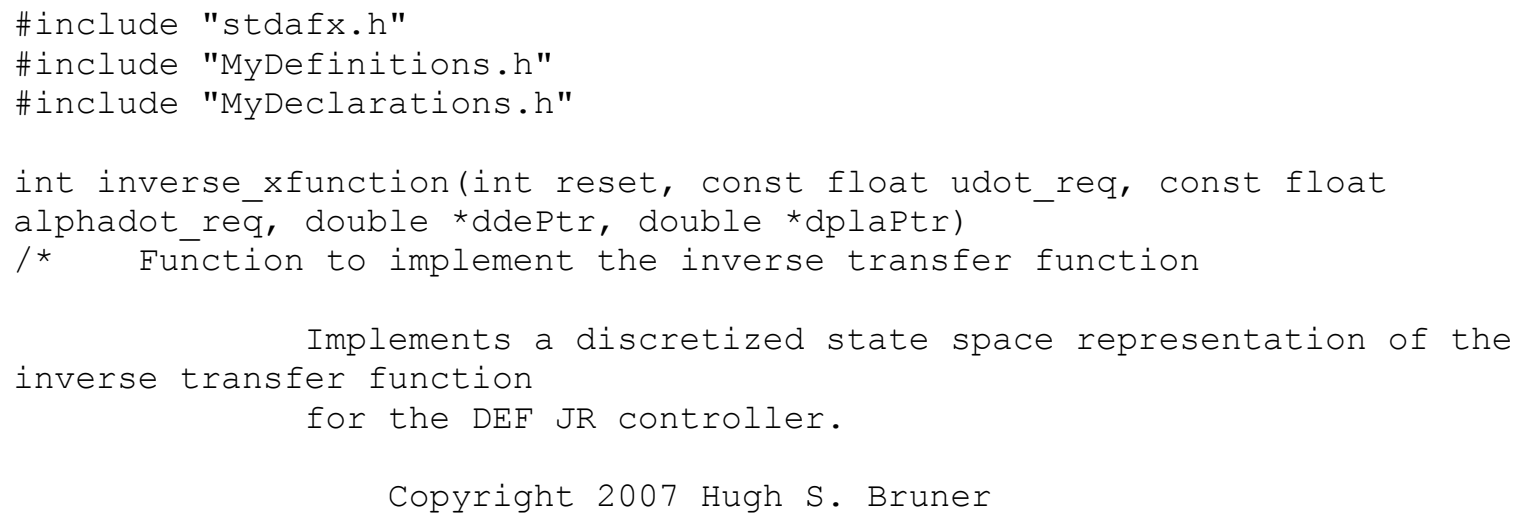




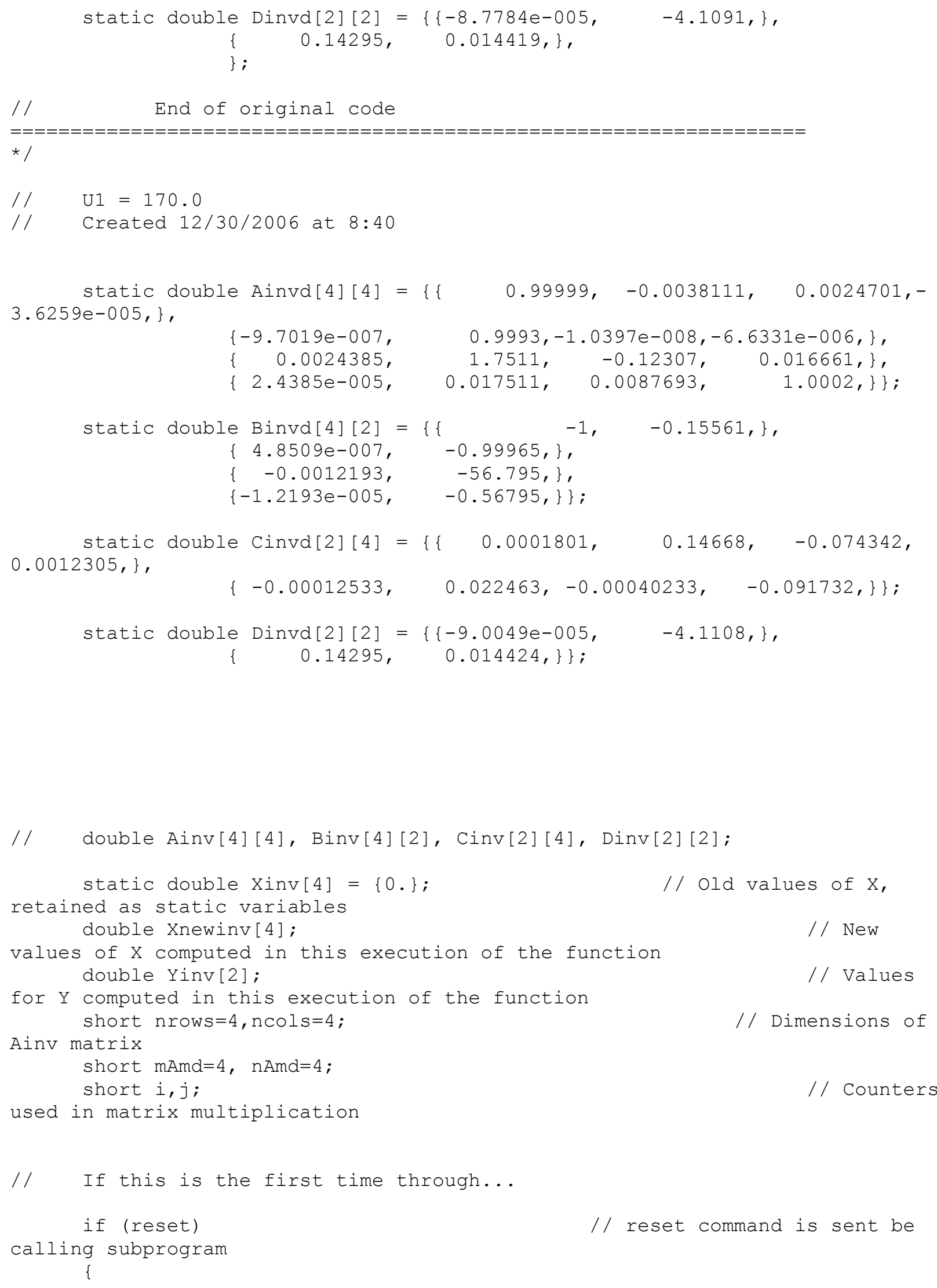




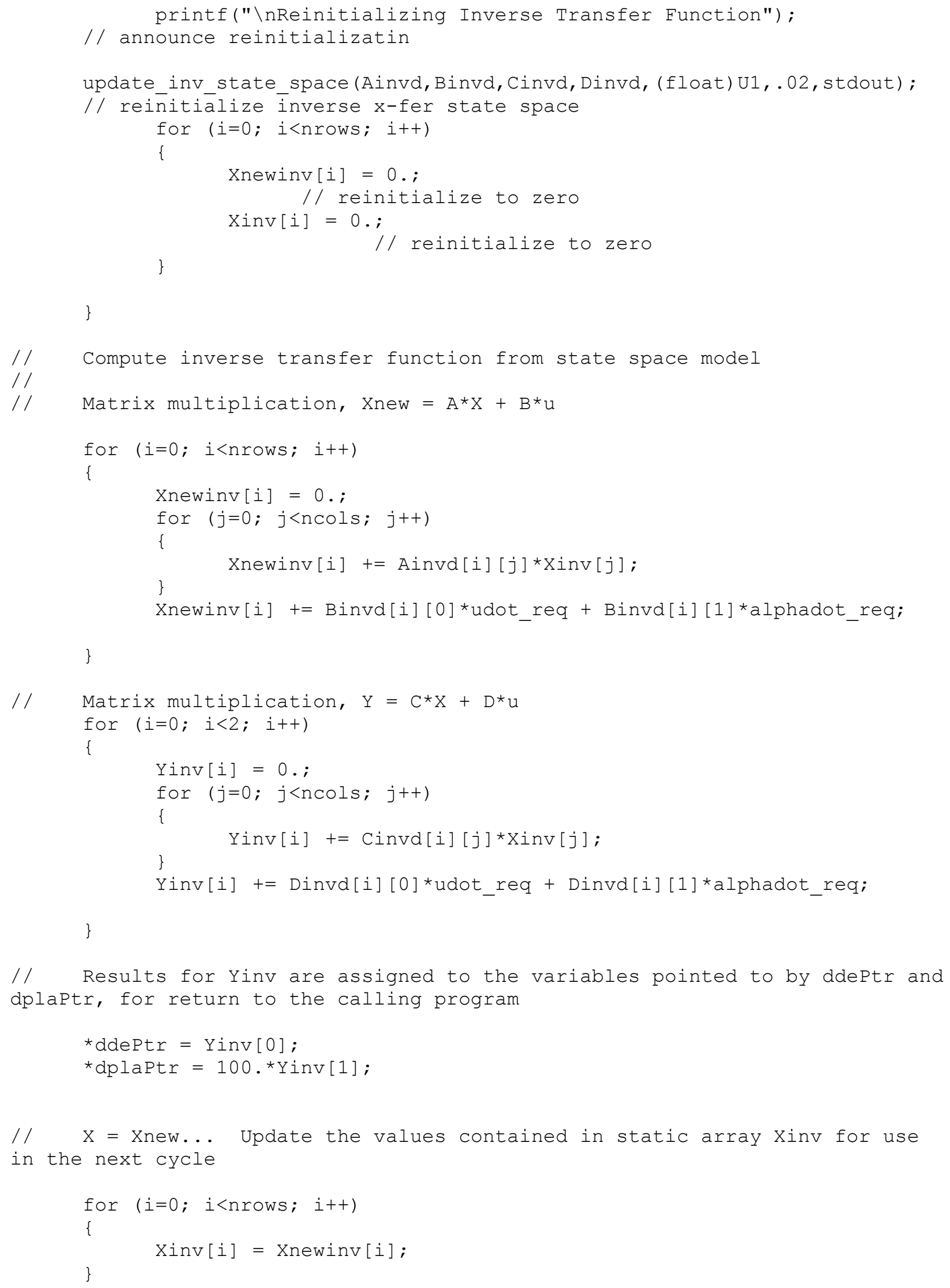




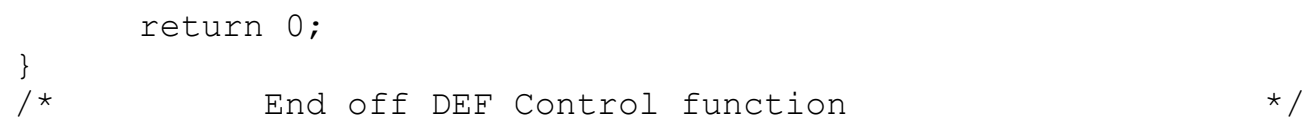




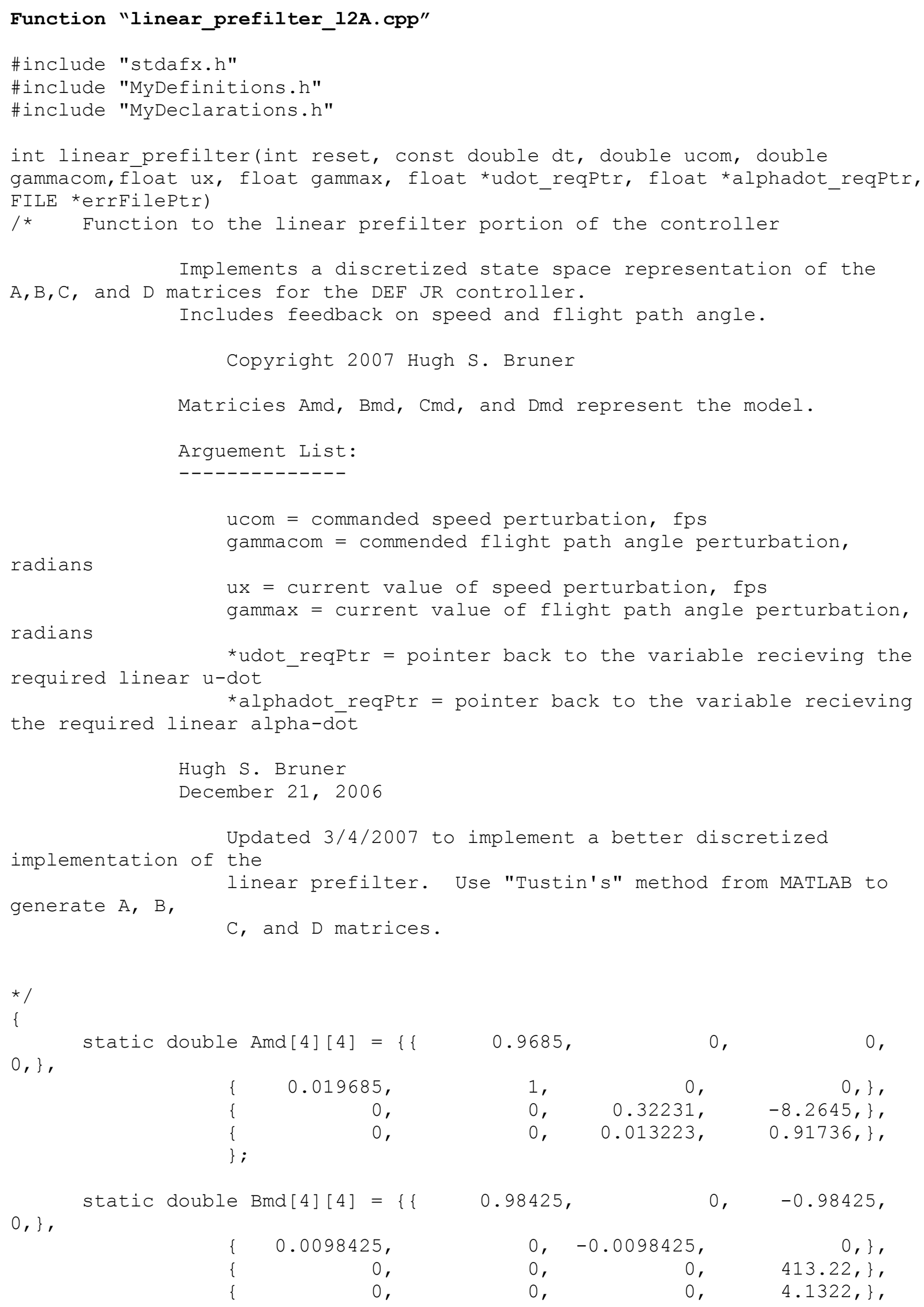


\} ;

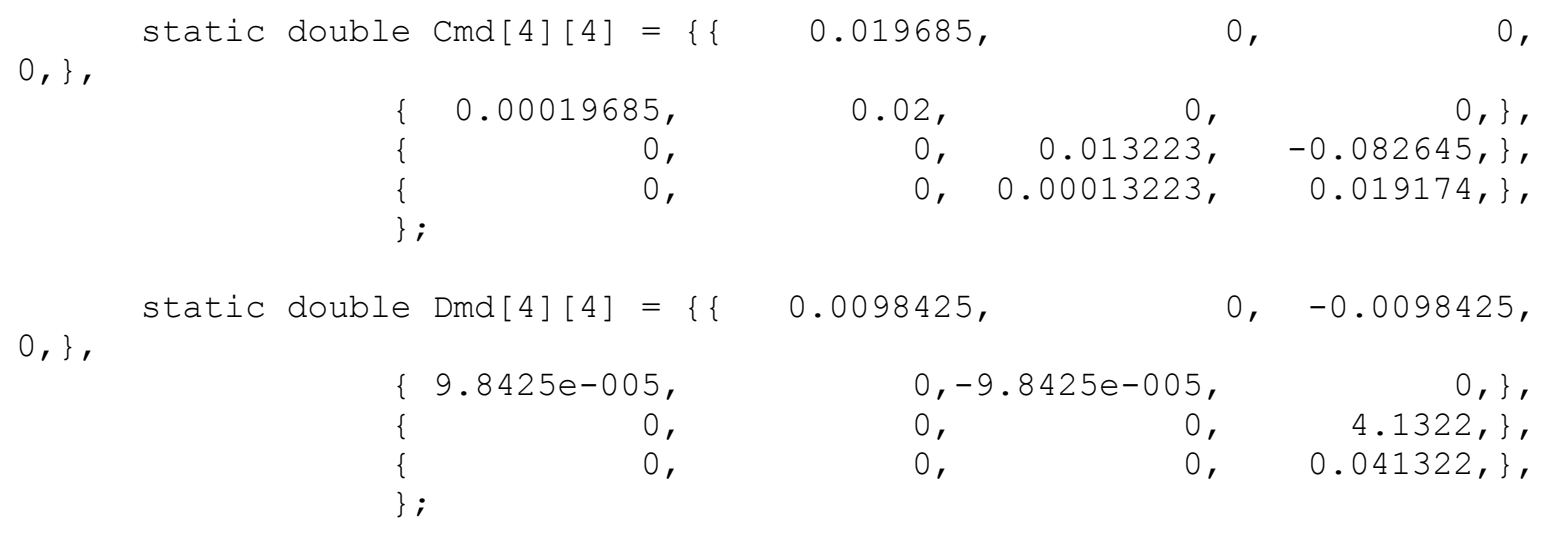

static double Xm[4]=\{0.\};

// old

values of $X$, retained as static variables

static double ucomold $=0 .$, gcomold $=0 . ; \quad / /$ old values of

command variables, retained as static variables double Xnewm[4];

values of $X$ computed in this execution of the function double Ym[2];

// New

Values for $Y$ computed in this execution of the function

short $\mathrm{mAmd}=4$, $\mathrm{nAmd}=4$;

short $i, j$;

Counters used in matrix multiplication

// static int matrix_done=0;

value if the matricies have been computed

// double alphadot, gamma, gammadot; // Flight path parameters

double lambda1, lambda2;

double zetag $=.707$, omegang $=2.50$;

double Ymid[4];

the calling program

// if a reset command was sent from

\{

printf("\nNew Linear Prefilter Model calculated");

// announce a reset taking place

for $(i=0 ; i<4 ; i++)$

\{

$$
\mathrm{Xm}[\mathrm{i}]=0 . ;
$$

// reinitialize Xm to zero

\}

ucomold $=0 . ;$

// reinitialize ucomold

gcomold $=0 . ;$

\}

// reinitialize gcomold

// Compute model response

// 


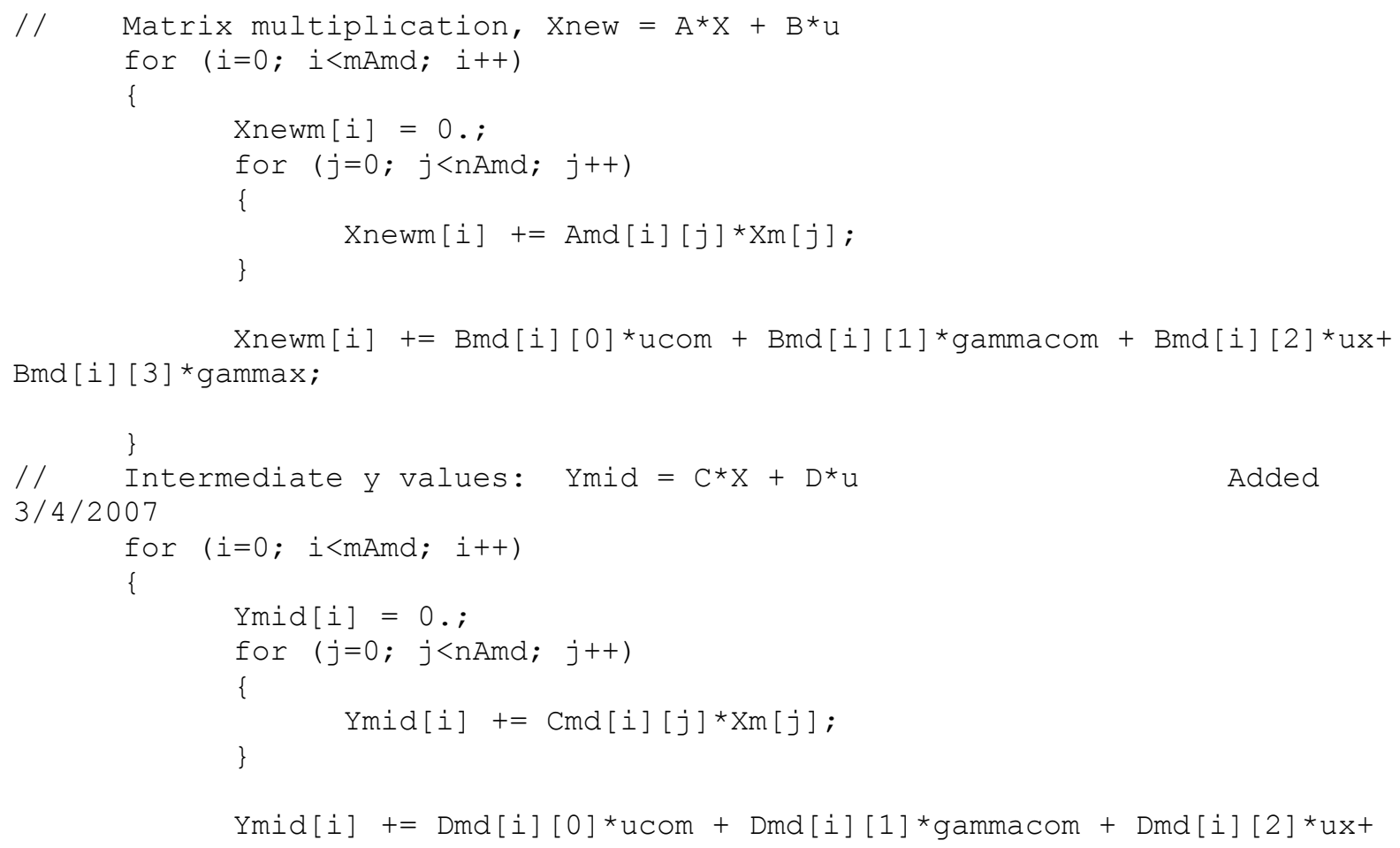




\section{Function "load_existing_network.cpp"}

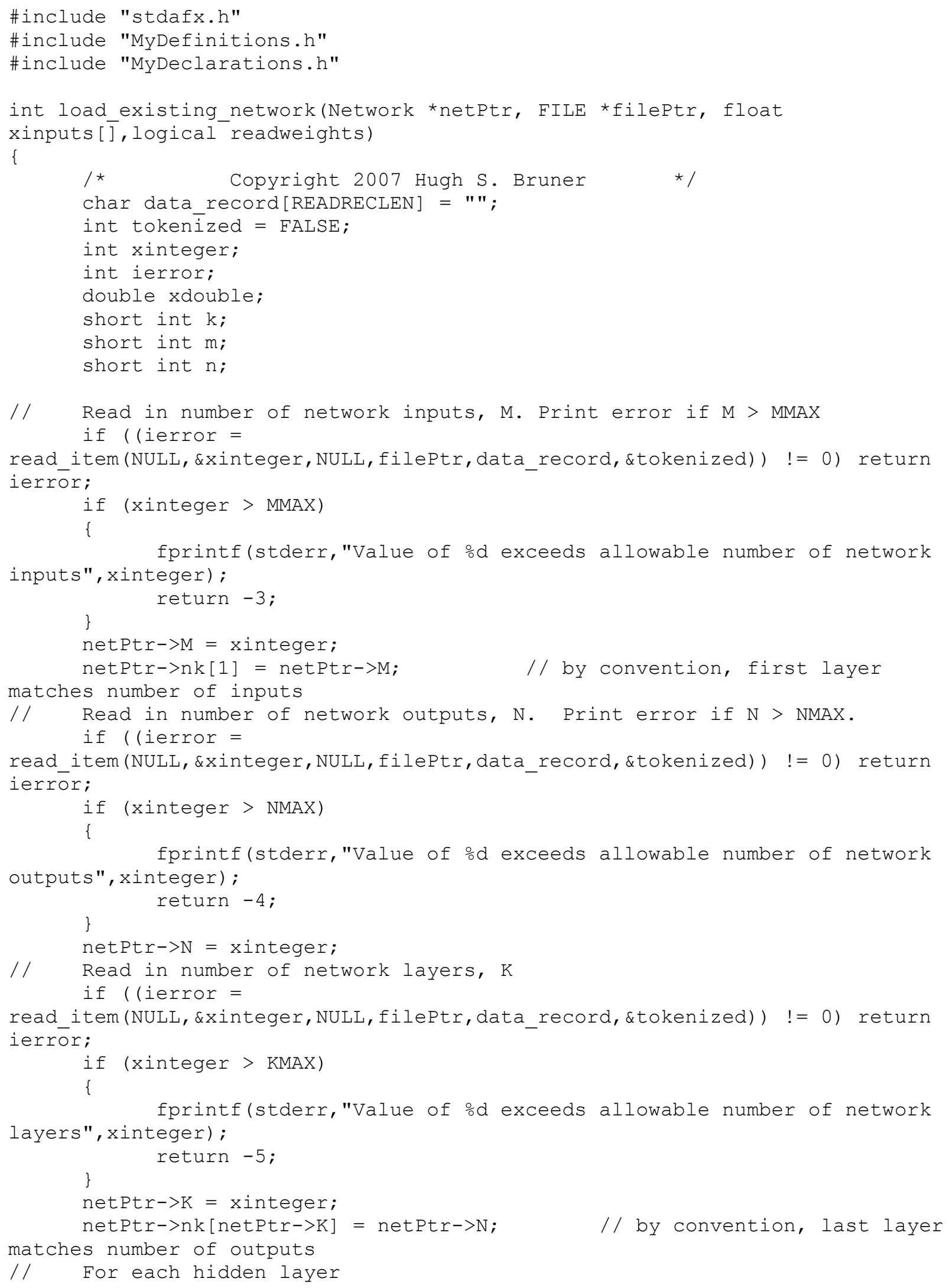




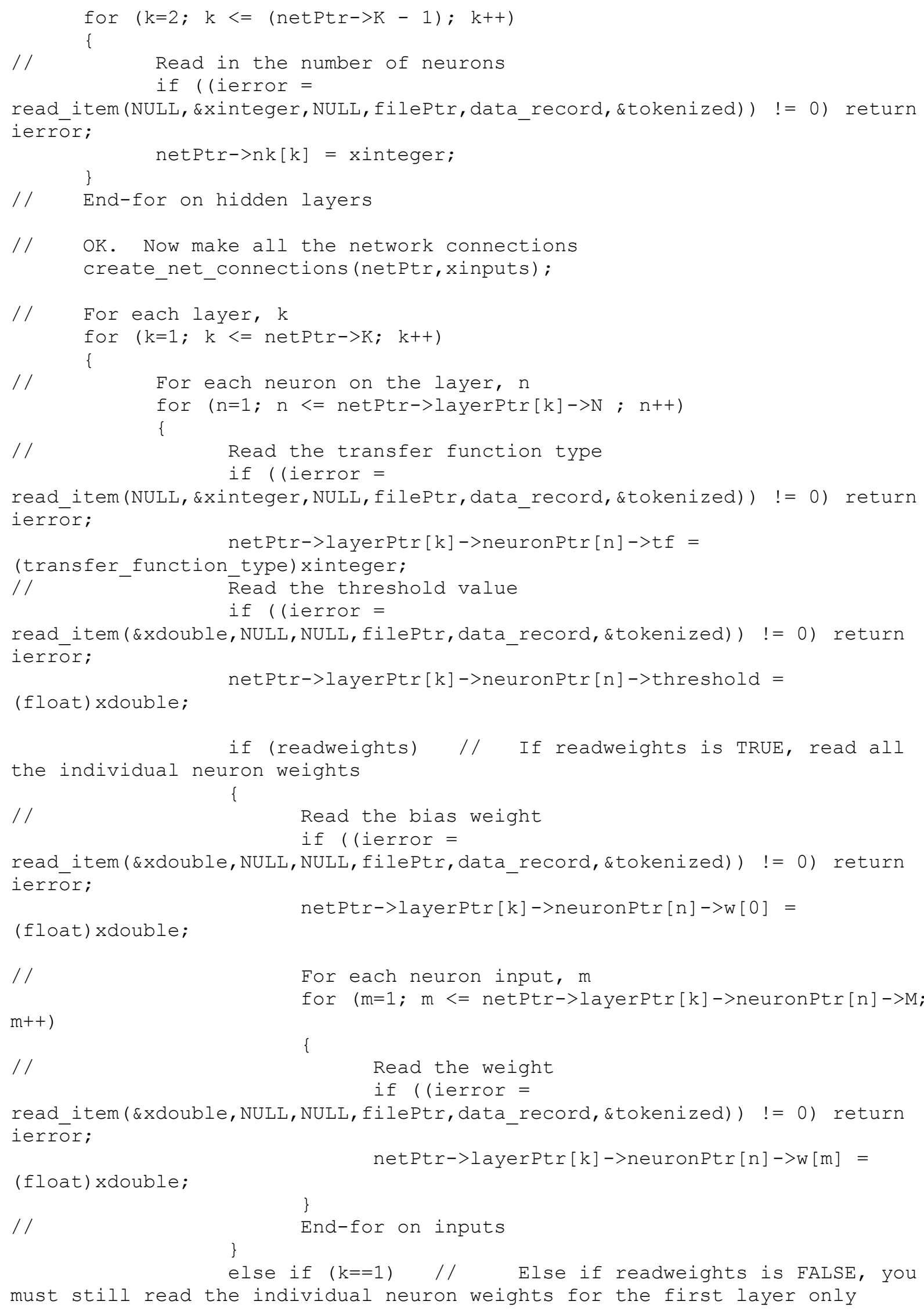




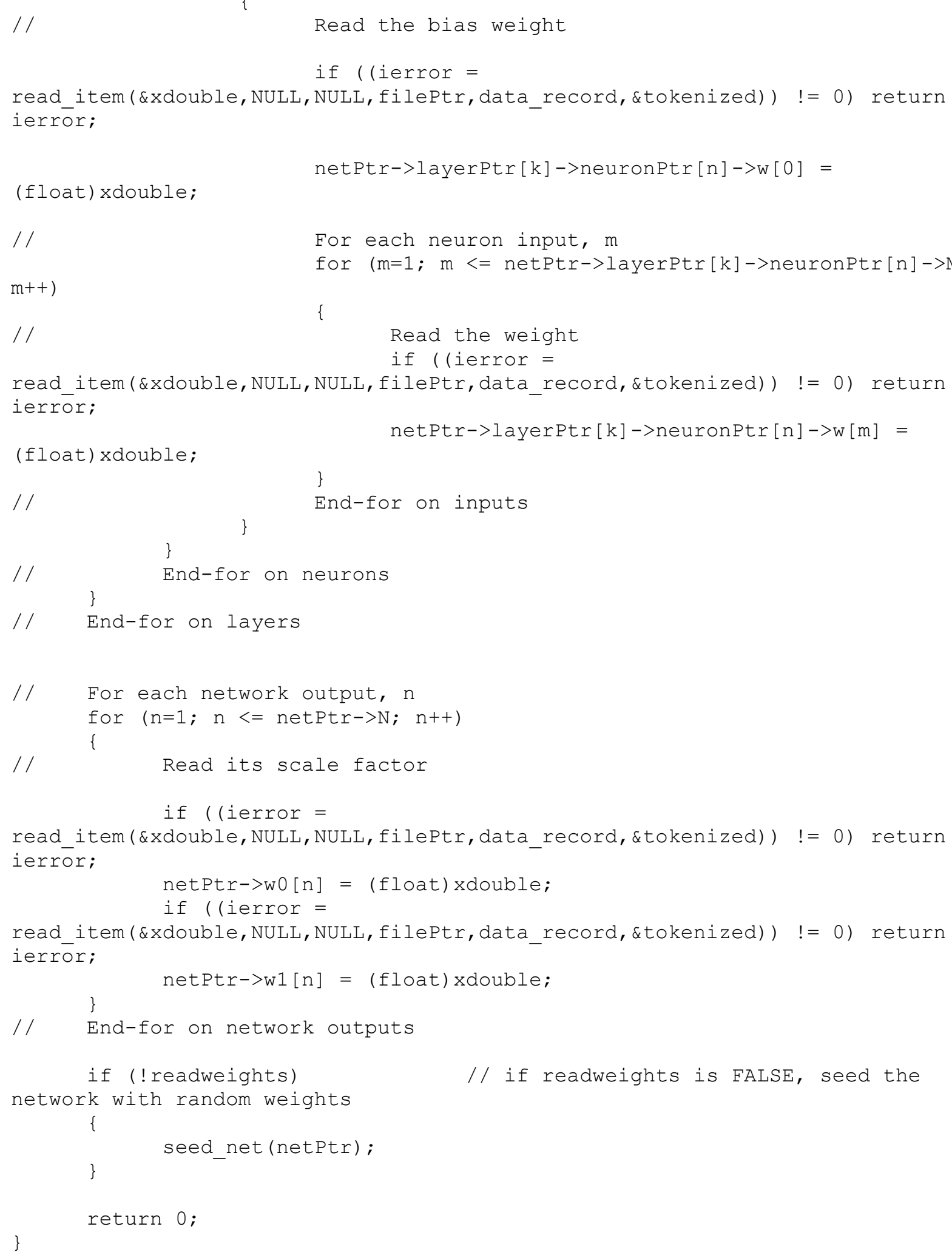




\section{Function "MyDeclarations.h"}

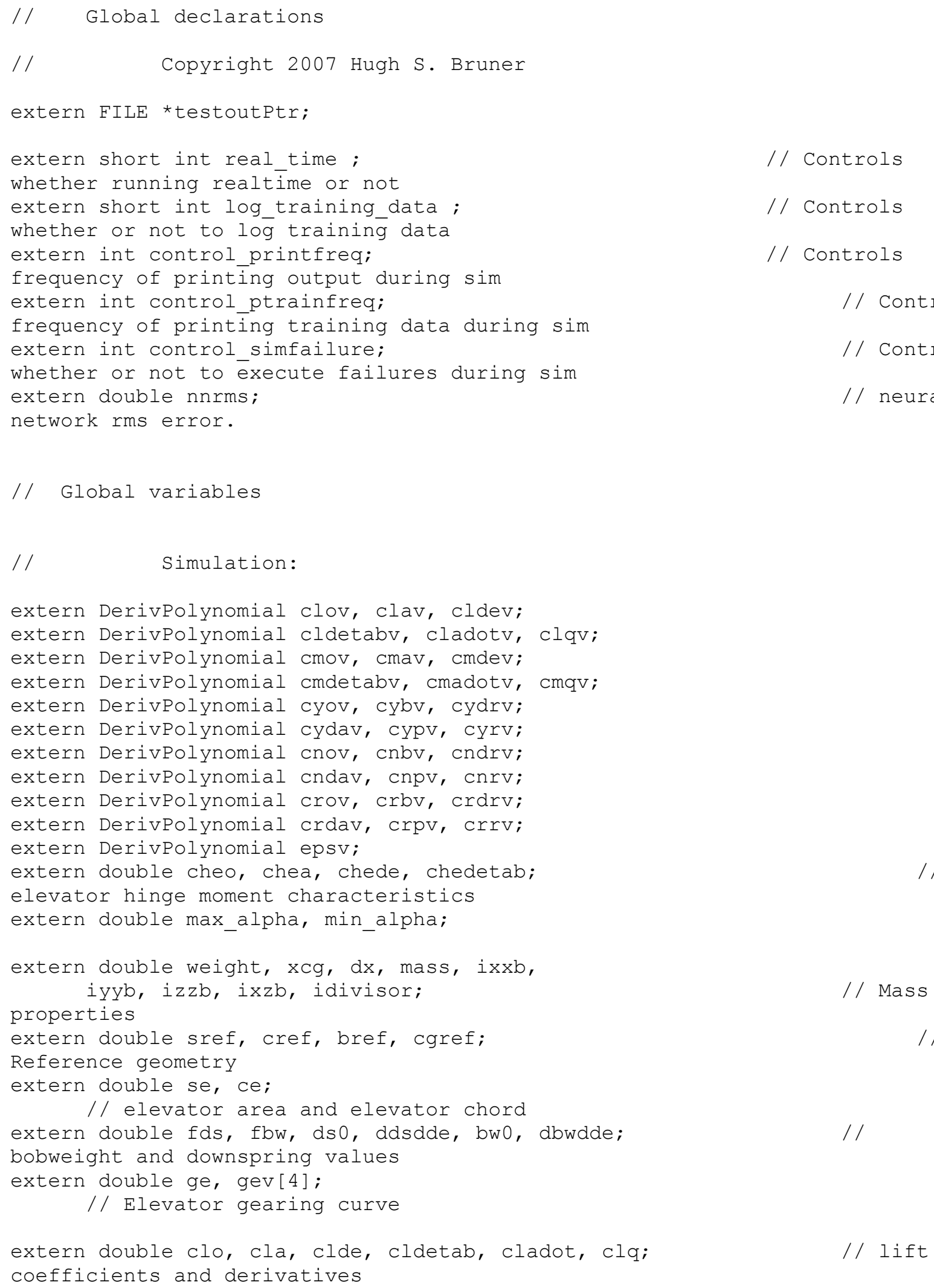




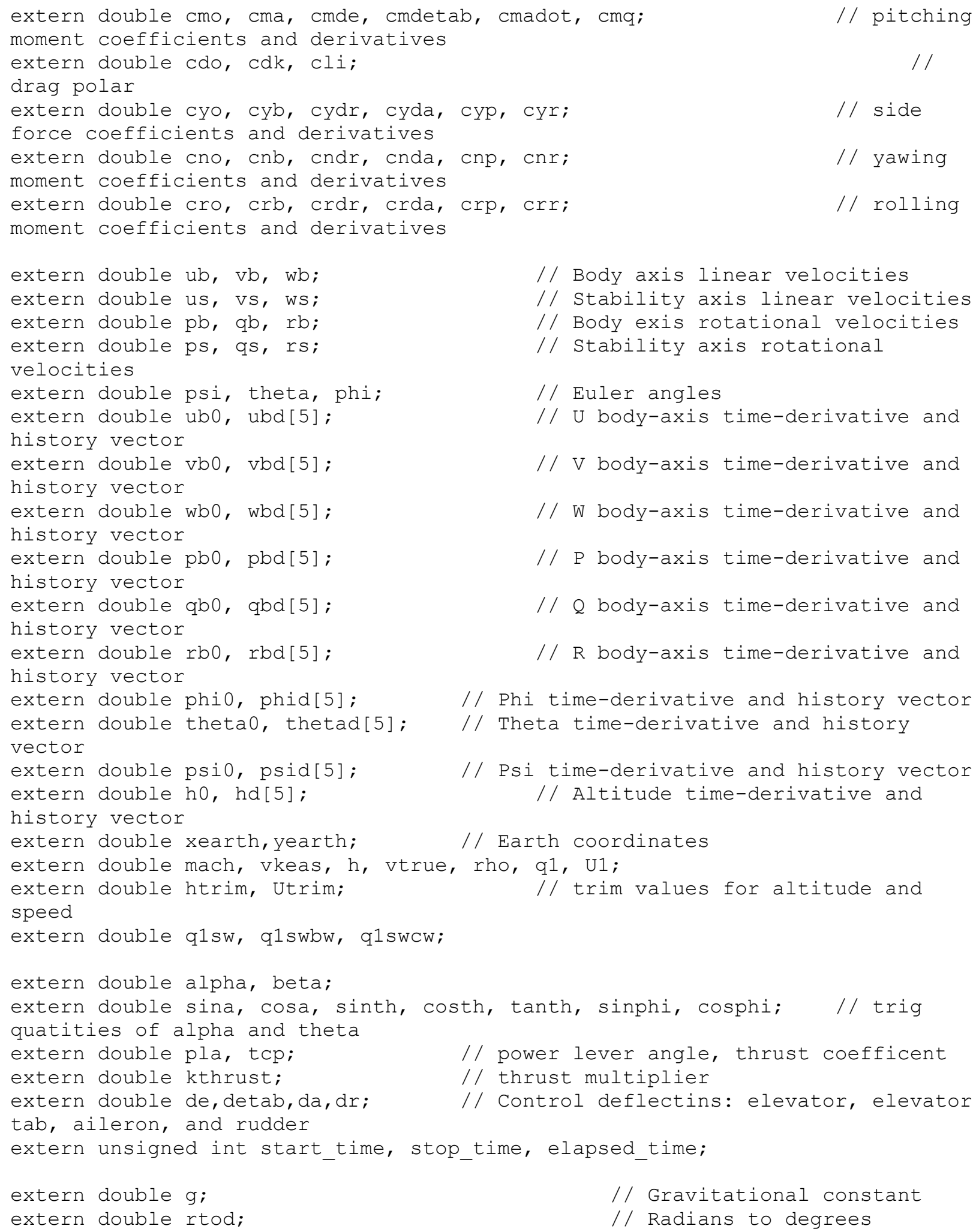




\section{Function "MyDefinitions.h"}

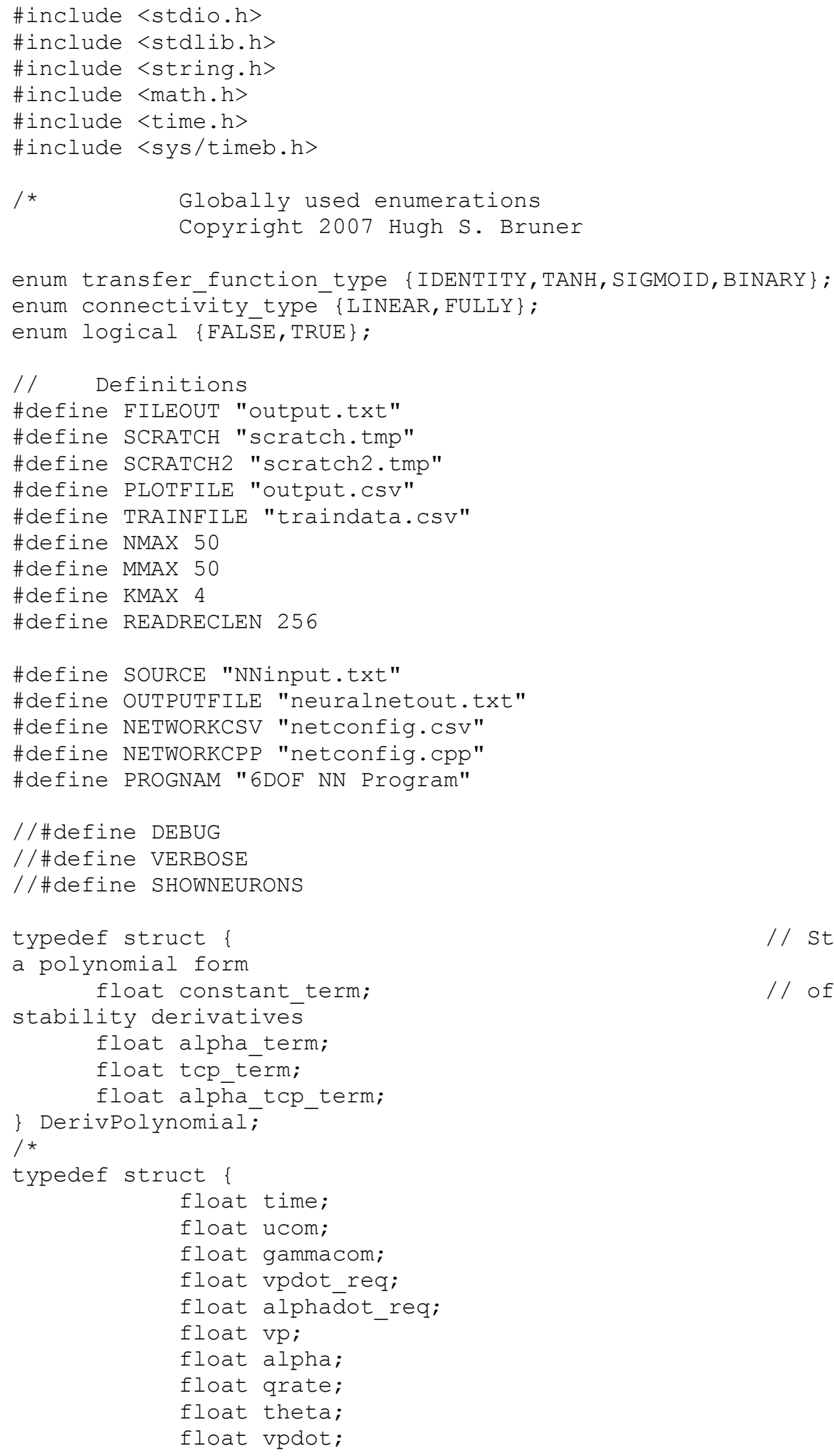




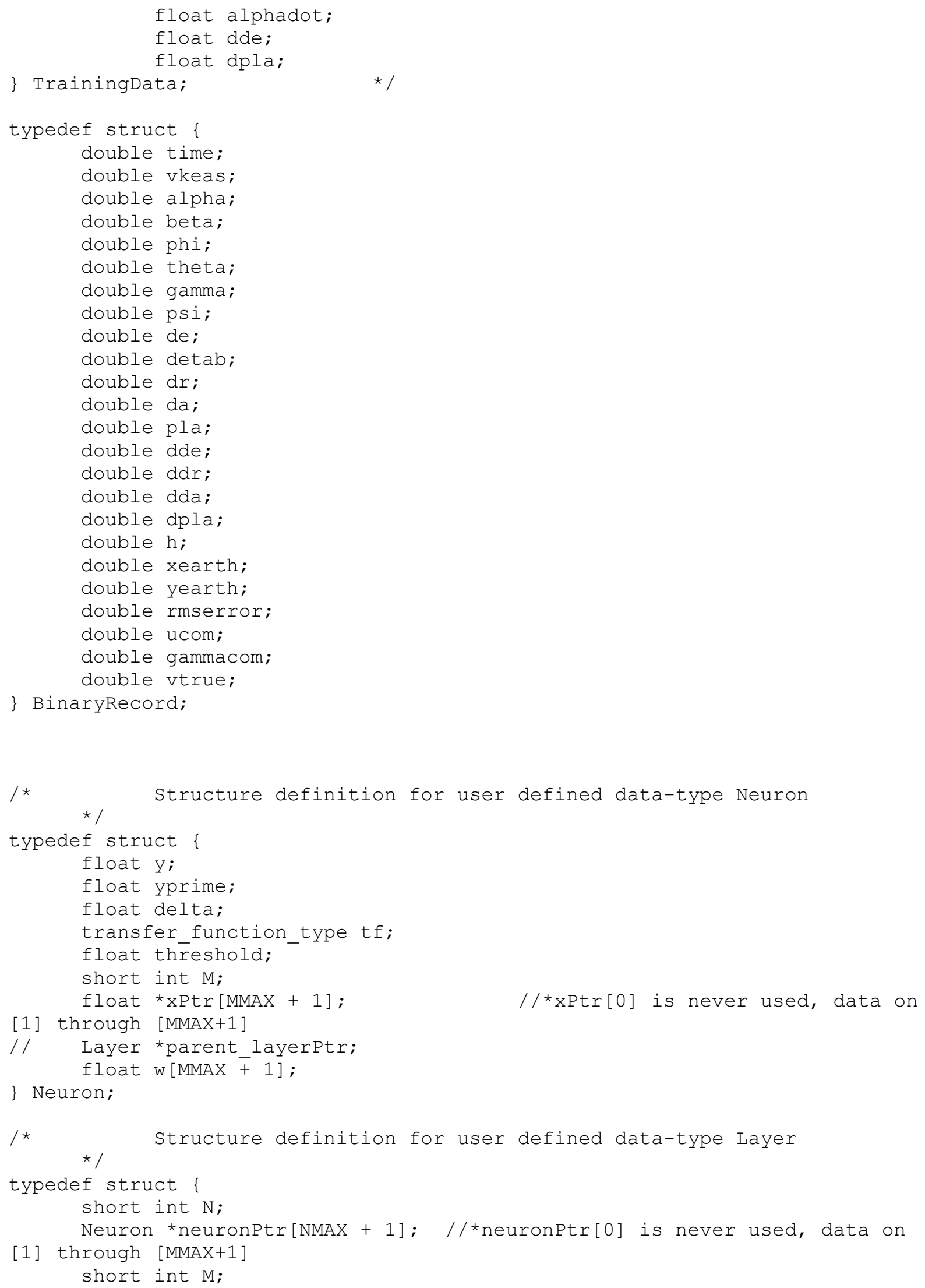




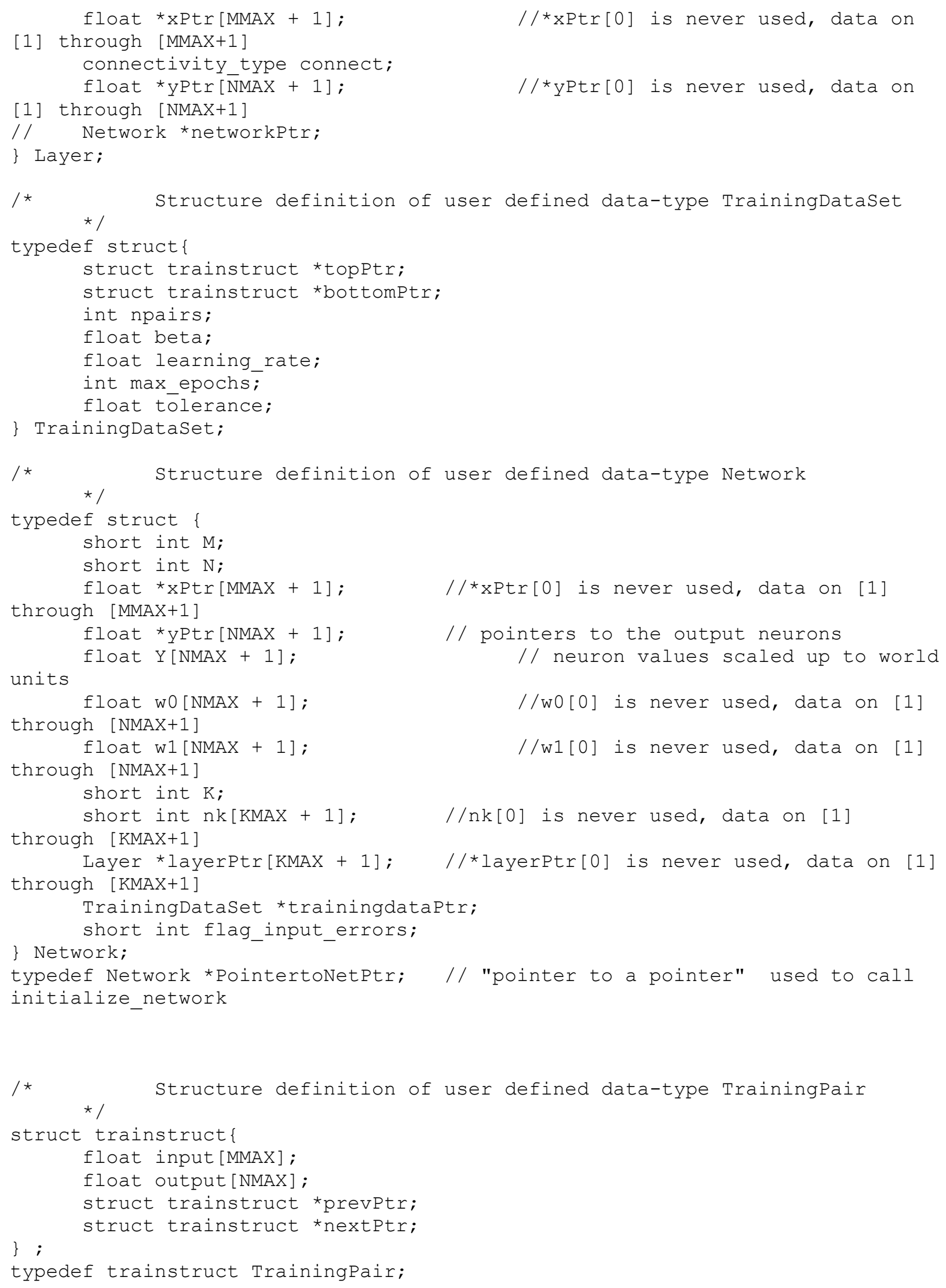


double eval (DerivPolynomial *, const double *, const double *); double predict (const double *, const double *, const double *); double correct (const double *, const double *, const double *); double std_temp (double hp);

double std_pressure (double hp);

double std density (double hp);

int initialize sim(void);

int fast (double * , double dt);

int push down (void);

int CJ14 $\overline{4}$ data(void);

int force roskam(void);

int case $\bar{d}$ ata write (FILE *ffilePtr, char *descriptorPtr);

int fetrim(double *alphalPtr, double *delPtr, double *detablPtr, double *pla1Ptr);

int solve3by3 (double a[3][3], double x[3], double y[3]);

int autopilot (Network *myNetPtr, const double *mytimePtr, const double dt, const double vkeastrim, double utrimfps, const int manuever, double de1, double dal, double drl,

double pla1, double *dePtr, double *daPtr, double *drPtr, double *plaptr, double *ucommandPtr, double *gammacommandPtr, Flle *filePtr, FILE *errfilePtr, FILE *modeloutPtr, double

time to learn, double time to control);

int format files(FILE *ffileptr, int isims, char *descriptor); int simulation_timer(int wait_millisec);

double thrust model(double PLA, double vtrue_fps, double air_density);

int DEF_JRcontrol (Network *myNetPtr, const double *mytimePtr, const double dt, doubjle time_to_learn, double time_to_control, float ucom, $\overline{\text { float }}$ gammacom, float ux, float gammax, double *ddePtr, double *dplaPtr, FILE *filePtr, FILE *errfilePtr, FILE *modeloutPtr);

int write_out_training(TrainingPair myTrainingData, FILE *filePtr);

int pop_training_pair(TrainingPair *currentPtr, float vpdot_req, float alphadot_req);

int format_training_data(FILE *outfilePtr,int pfreq);

int forward $\bar{d}$ pass (NeĒwork * currentNet, TrainingPair * currentpairPtr);

float backprop(Network *netPtr, TrainingPair *dataPtr, float learning_rate);

int simNN(FILE *inputPtr, Network *myNetPtr, double const timemax, double

time to learn, double time to control);

int $\bar{N}$ Nexec (char *sourcefilēetri);

char * extract_line (char * sourcePtr, char * var_namePtr, char * op_namePtr, char * value_stringPtr);

int read_actions(FILE *inputPtr, FILE *outputPtr, PointertoNetPtr *netPtrPtr, char direct_object[], float xinputs[]);

int print_the_network_csv(Network *netPtr, FILE *logfilePtr);

int print_the_network_Ccode (Network *netPtr, FILE *logfilePtr);

int print_the_network (Network *netPtr, FILE *outputPtr);

int no_white_space (const char *sourcePtr, char *targetPtr);

int clear_thé_network (Network *myNetworkPtr);

int erase network (Network *networkPtr);

int initiāize network(PointertoNetPtr *netPtr);

int error_handler(int error_code, char *descr);

int creatē_new_network (Netwōork *netPtr, FILE *filePtr, float xinputs[]);

int load existing network(Network *netPtr, FILE *filePtr, float

xinputs $[\overline{]}$, logical readweights);

int delete_net_connections (Network *myNetwork); 


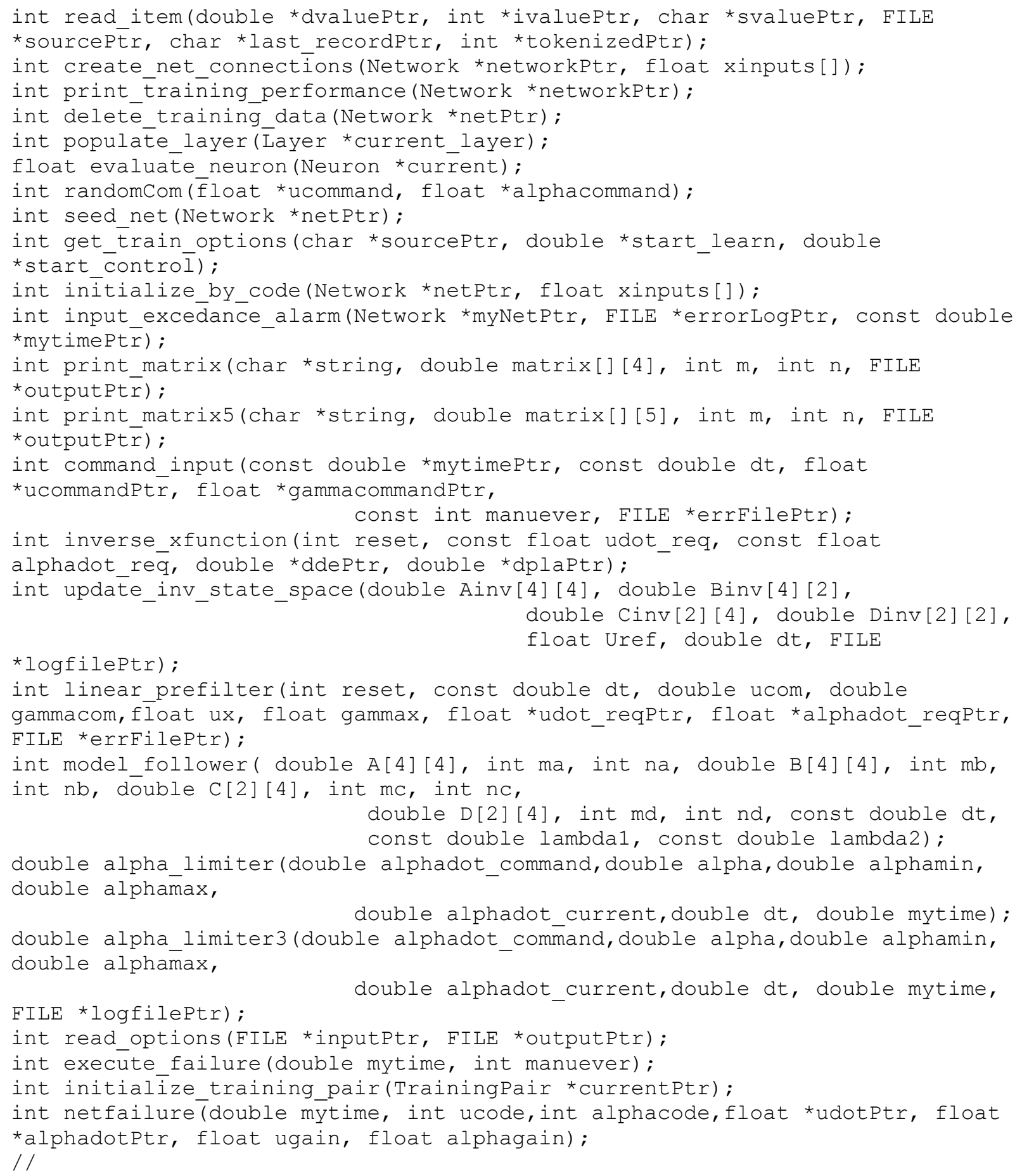




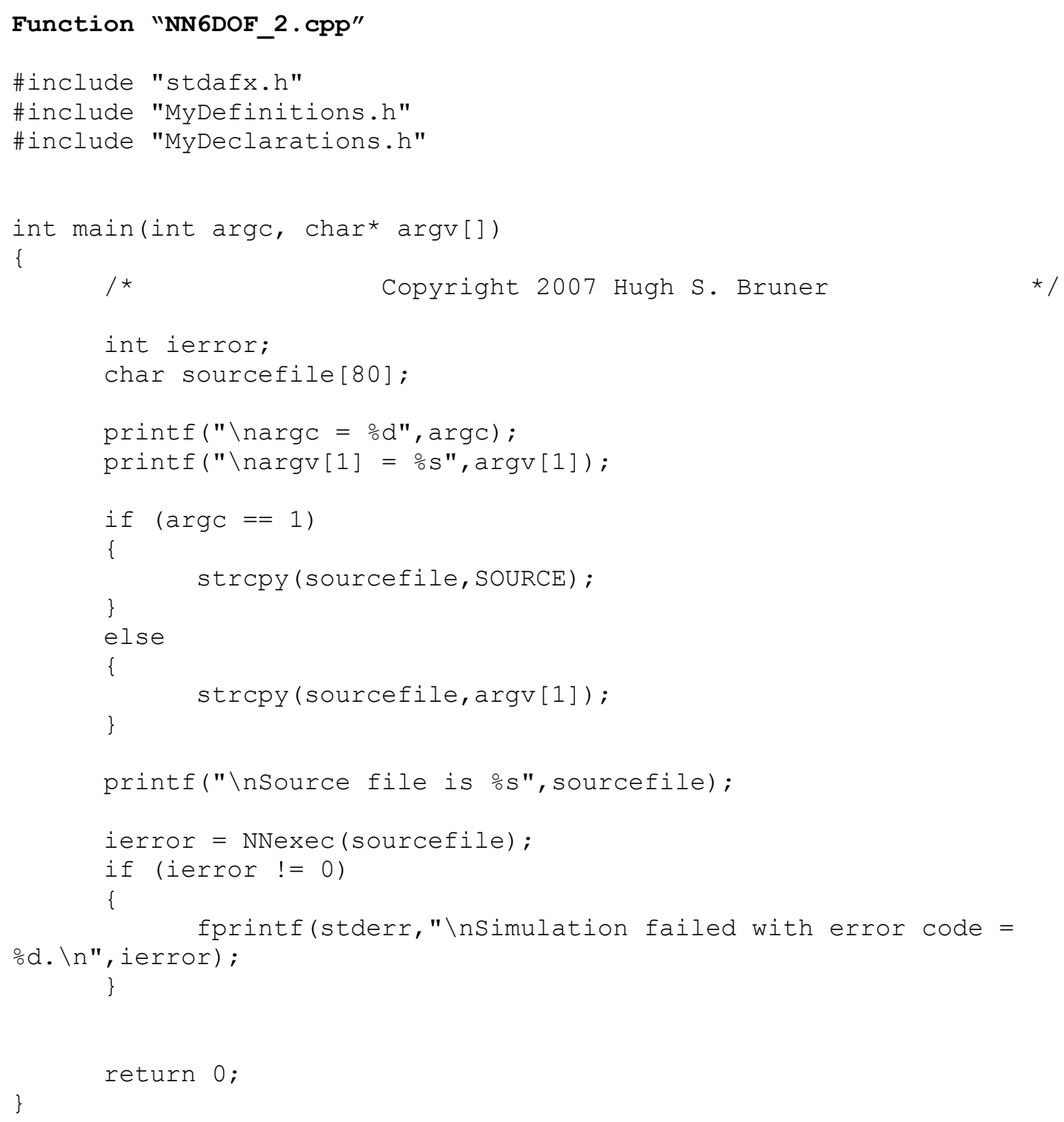




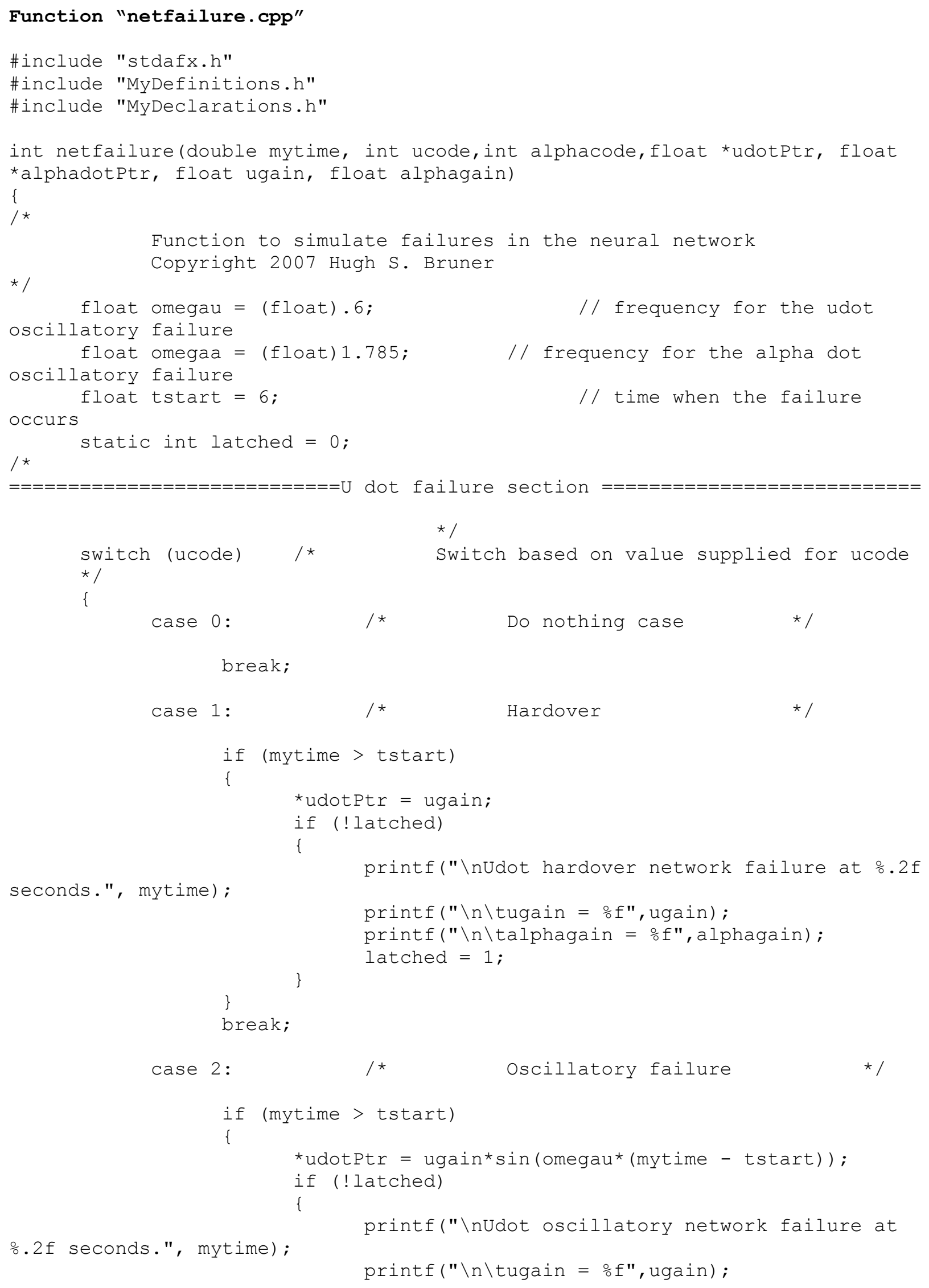




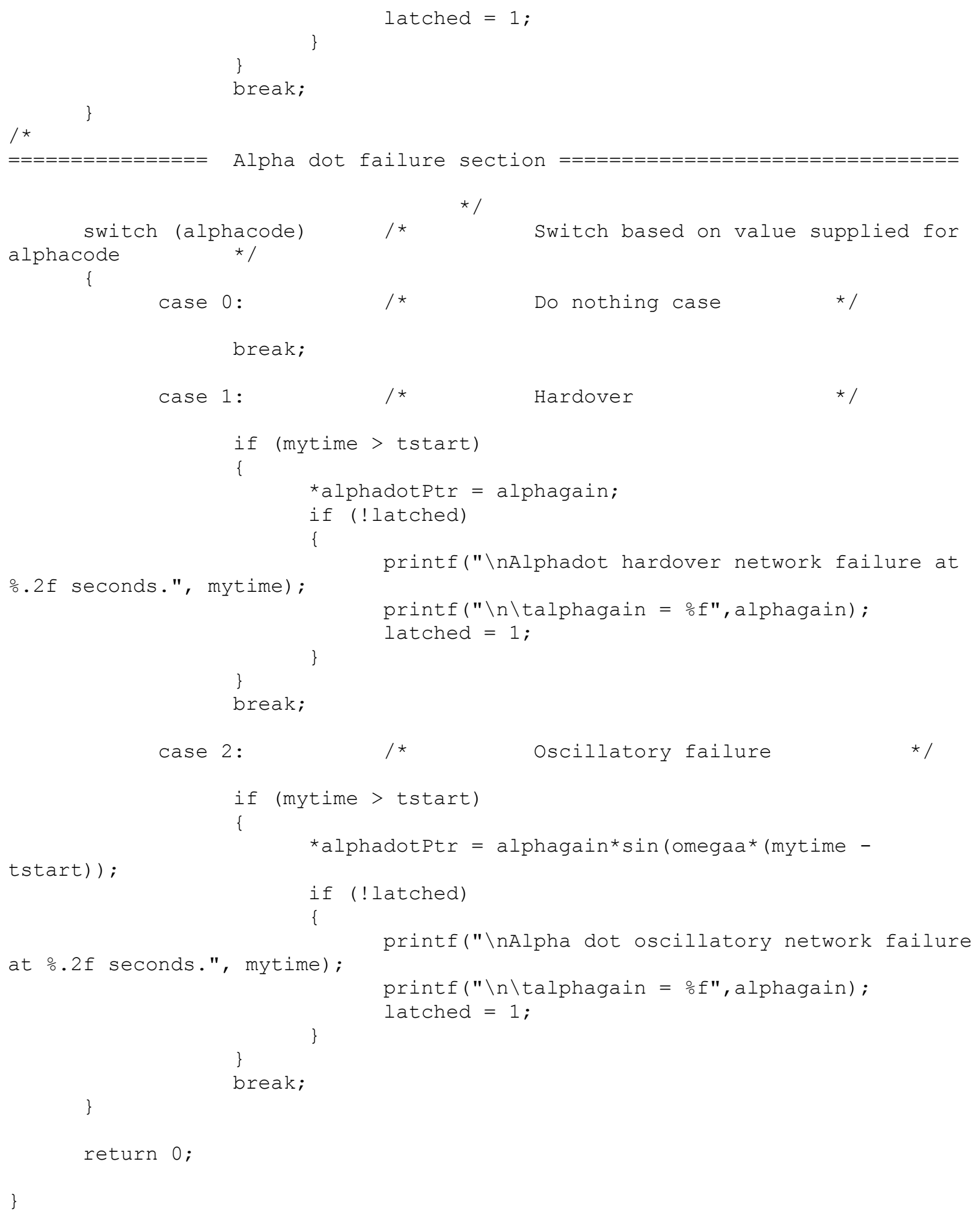




\section{Function "NNexec.cpp"}

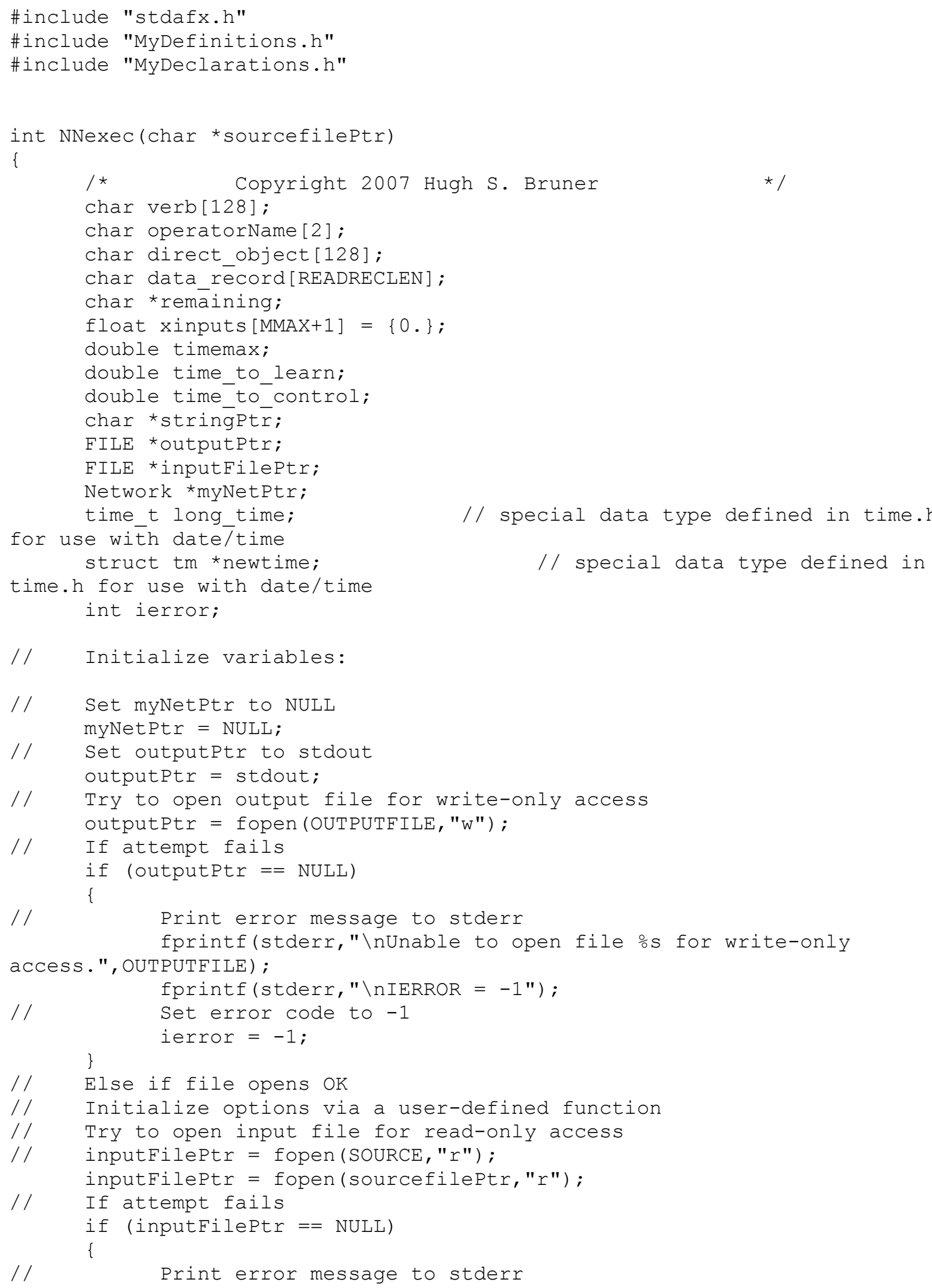




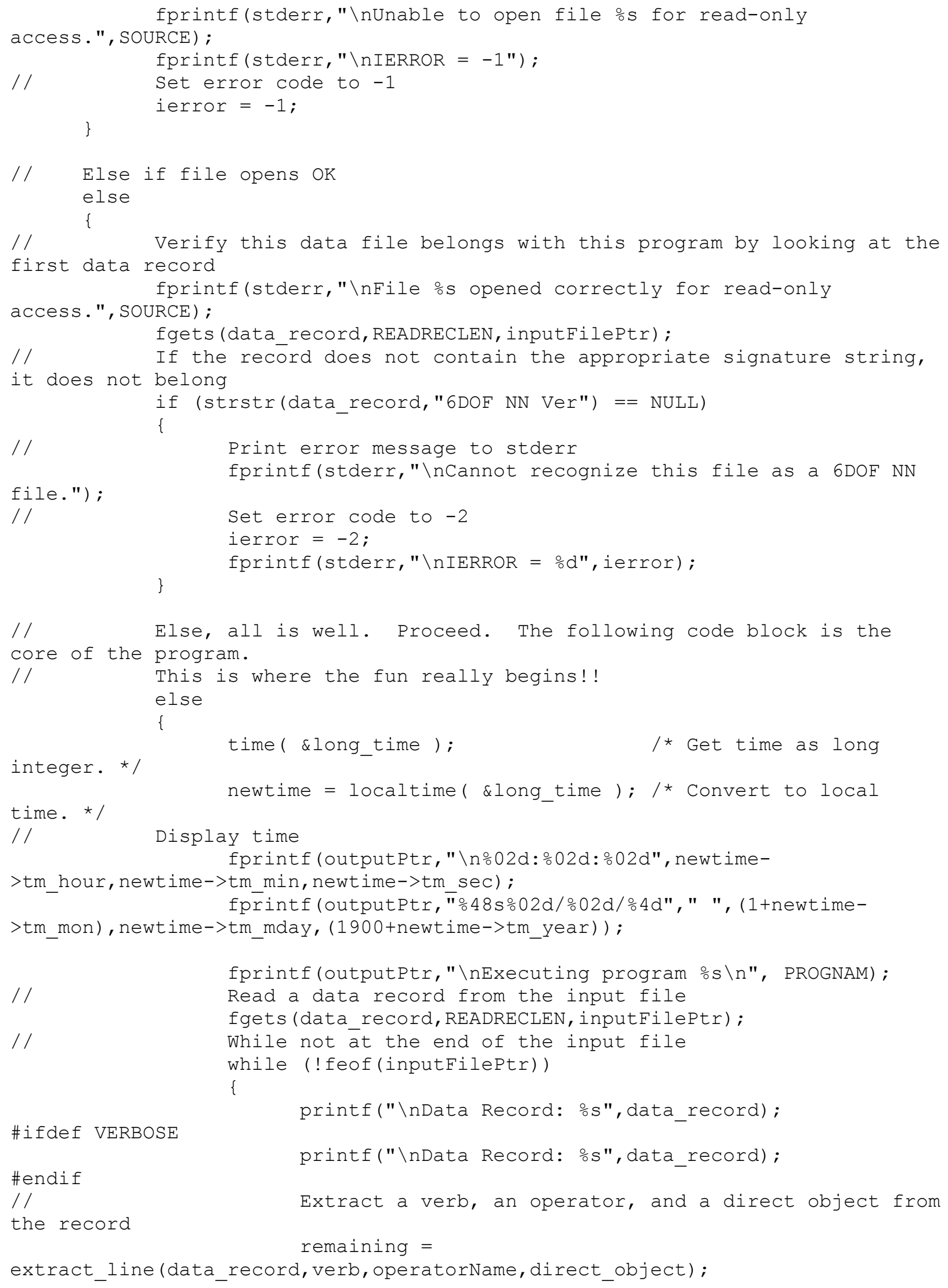




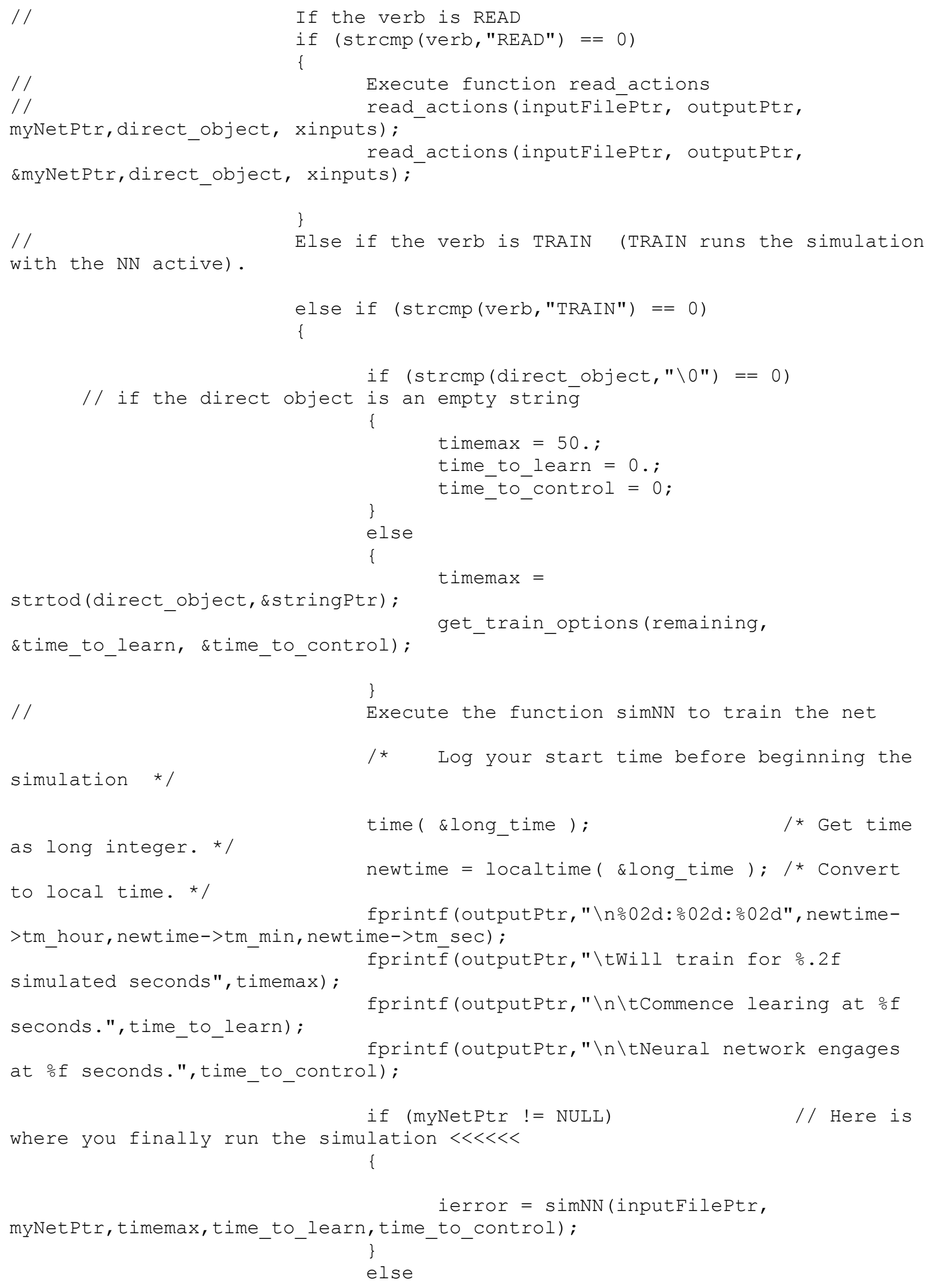




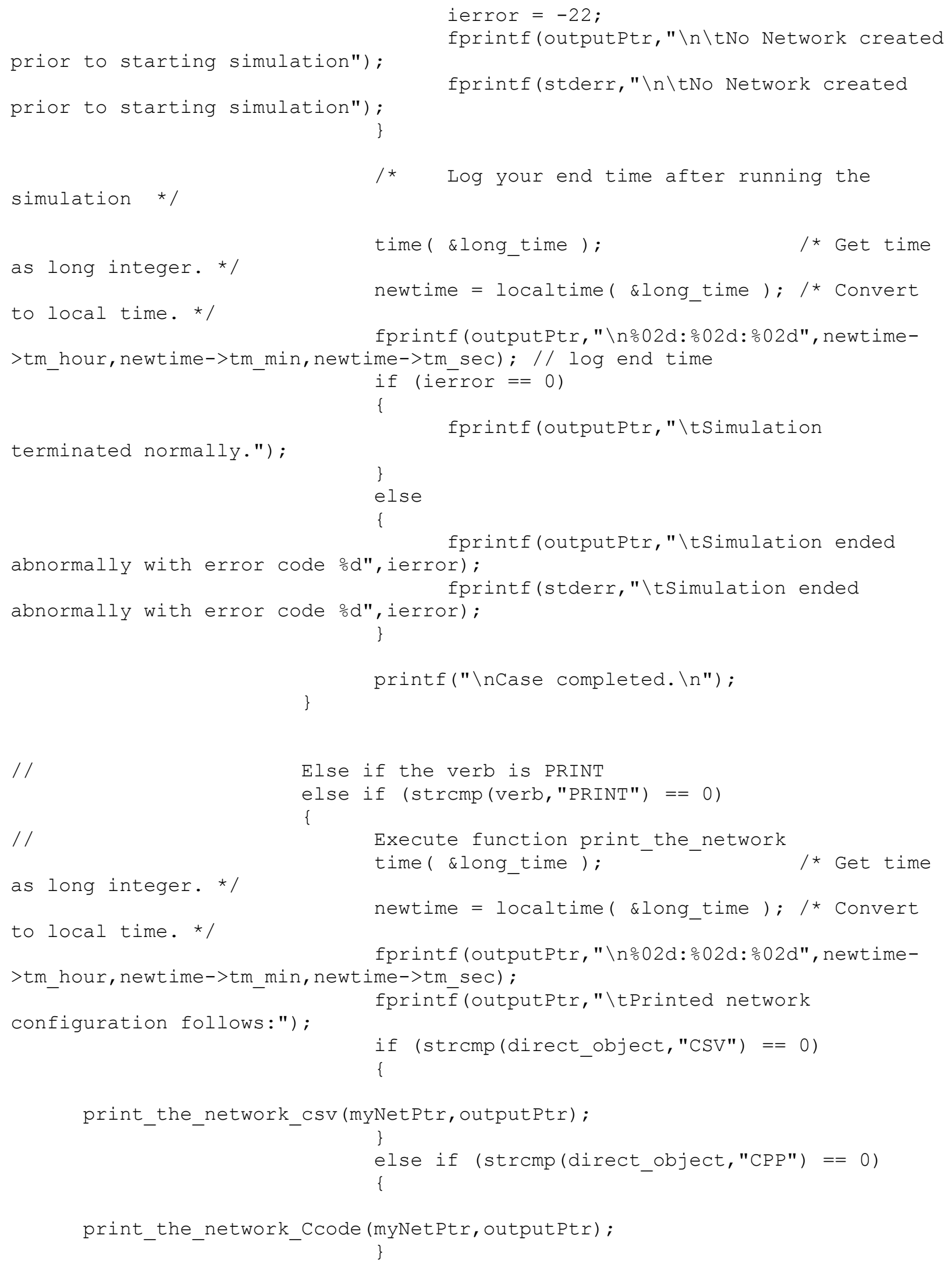




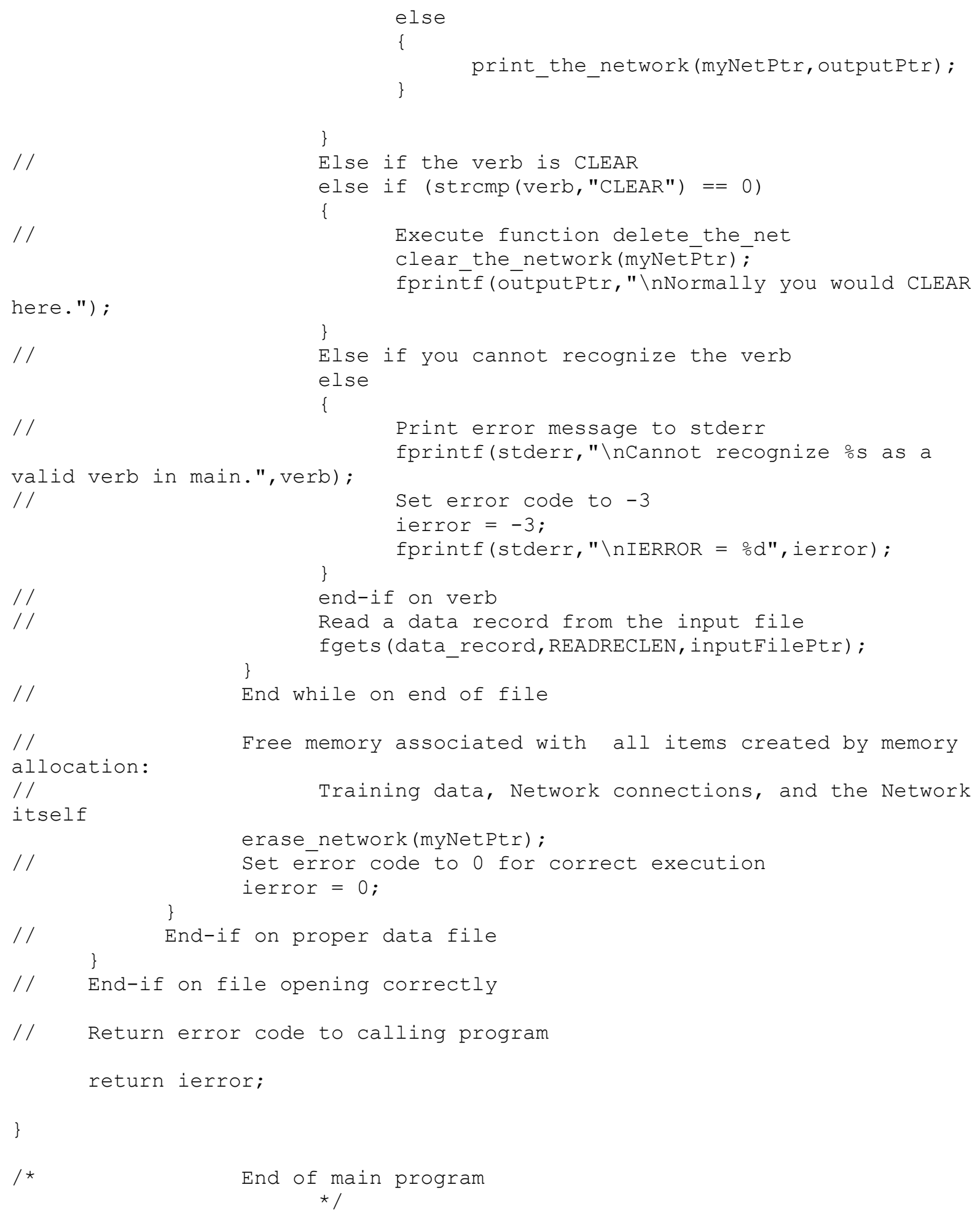




\section{Function "no_white_space.cpp"}

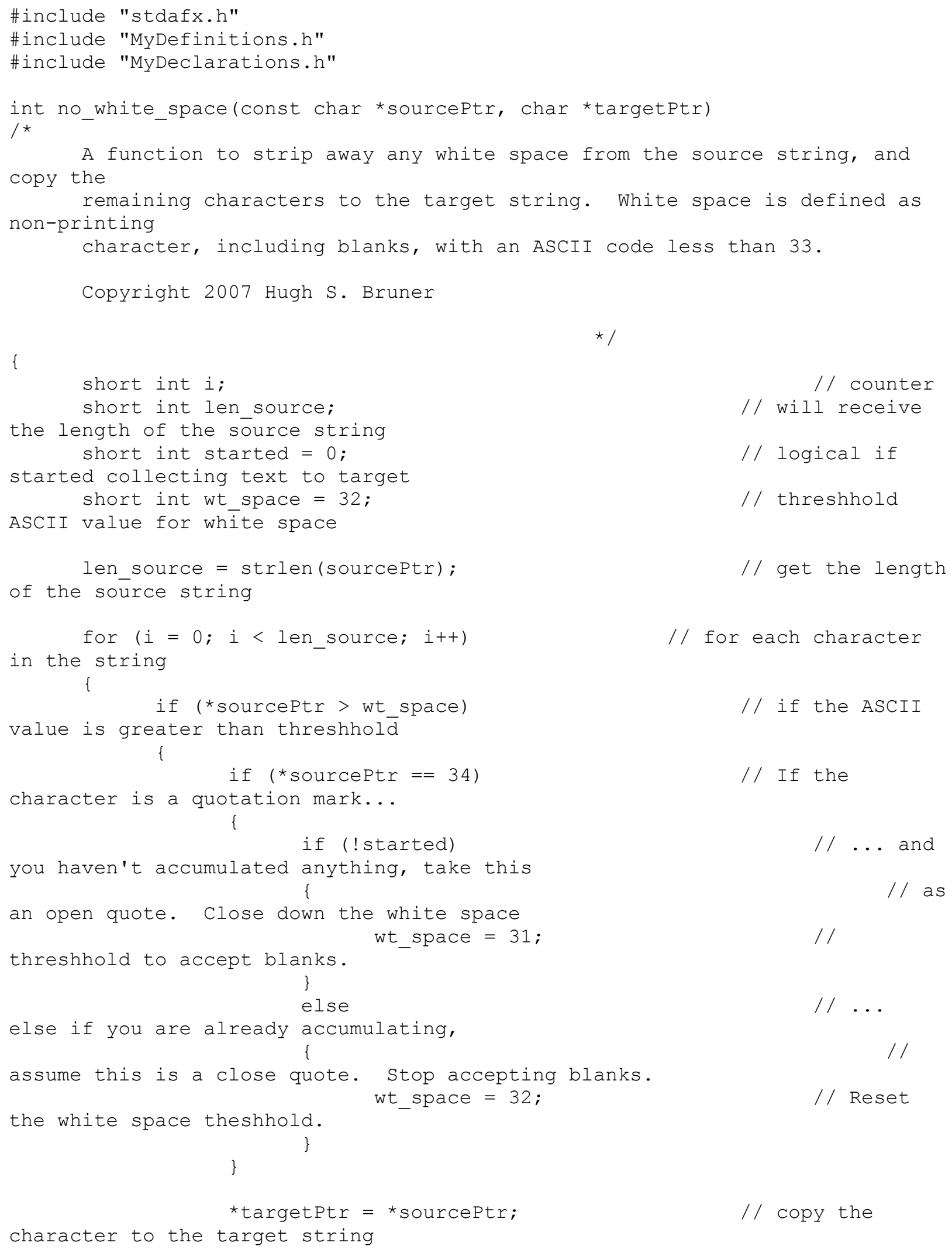




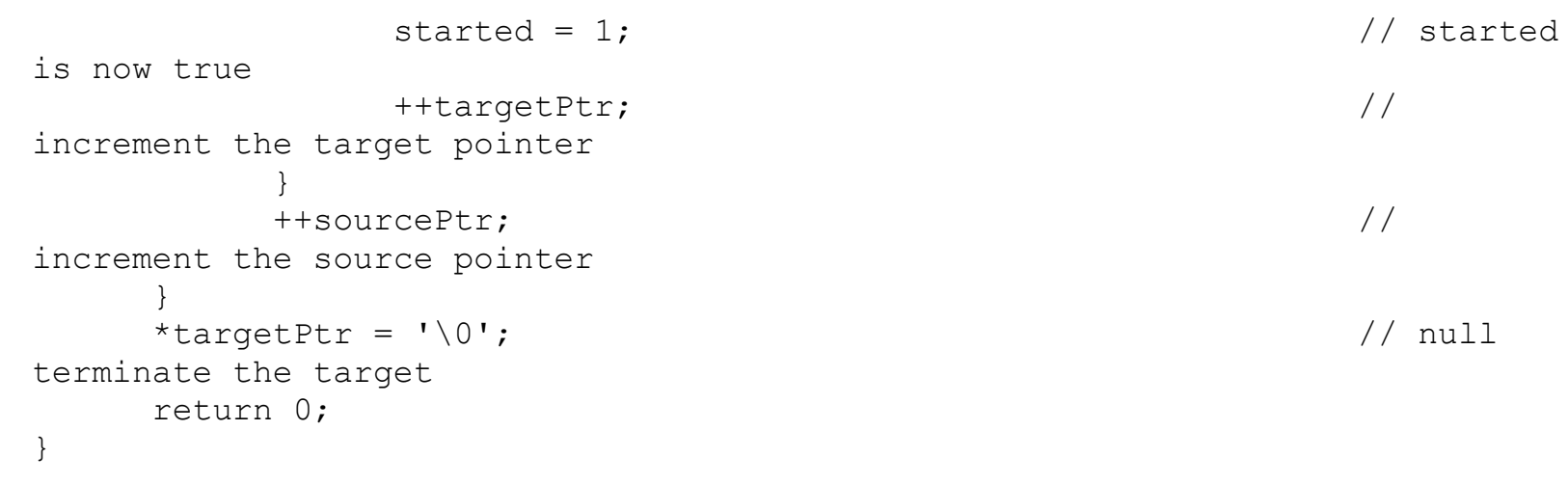




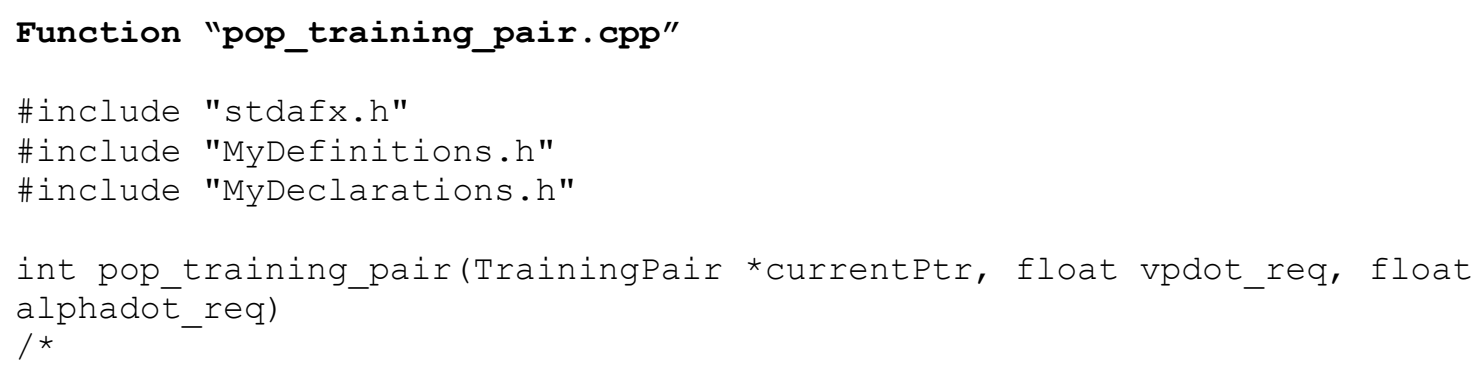




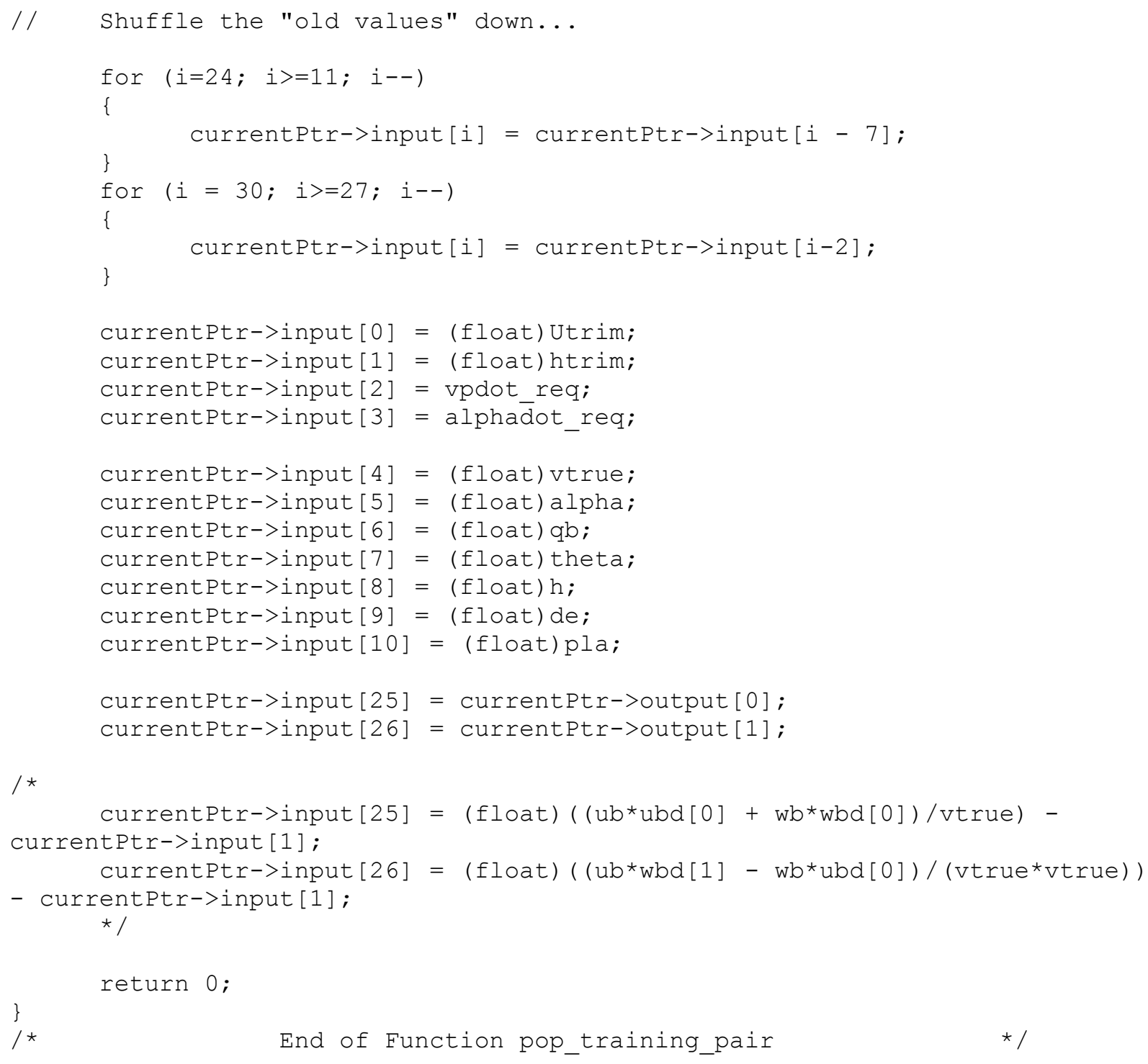




\section{Function "populate_layer.cpp"}

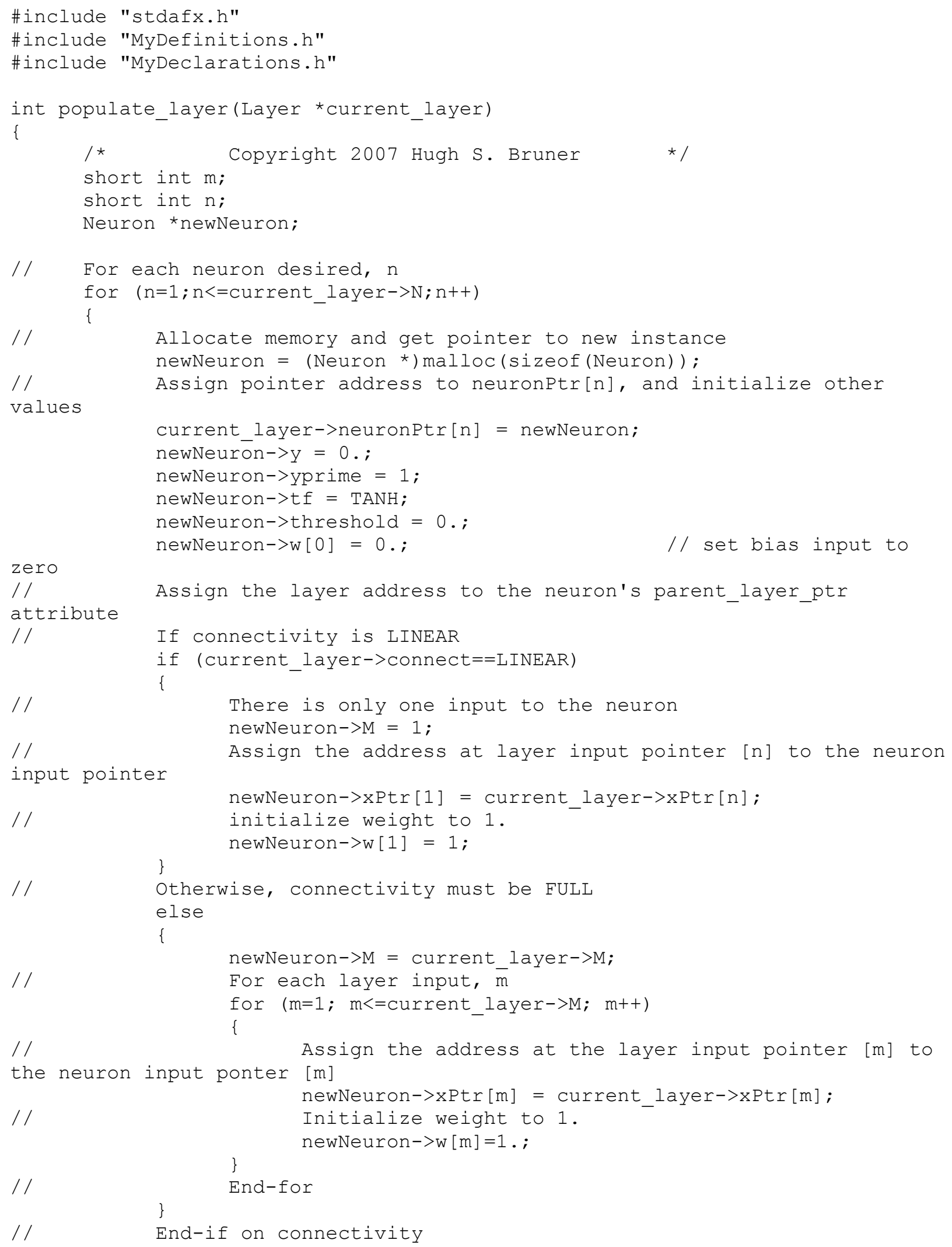


// Assign the memory address of the neuron output to layer output pointer $[\mathrm{n}]$

\#ifdef VERBOSE

current_layer->yPtr $[\mathrm{n}]=\&$ (newNeuron->y);

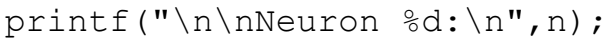

\#endif

dump_neuron(newNeuron) ;

/ $\quad$ end-for on neurons

return 0 ;

\}

/* End function populate_layer 


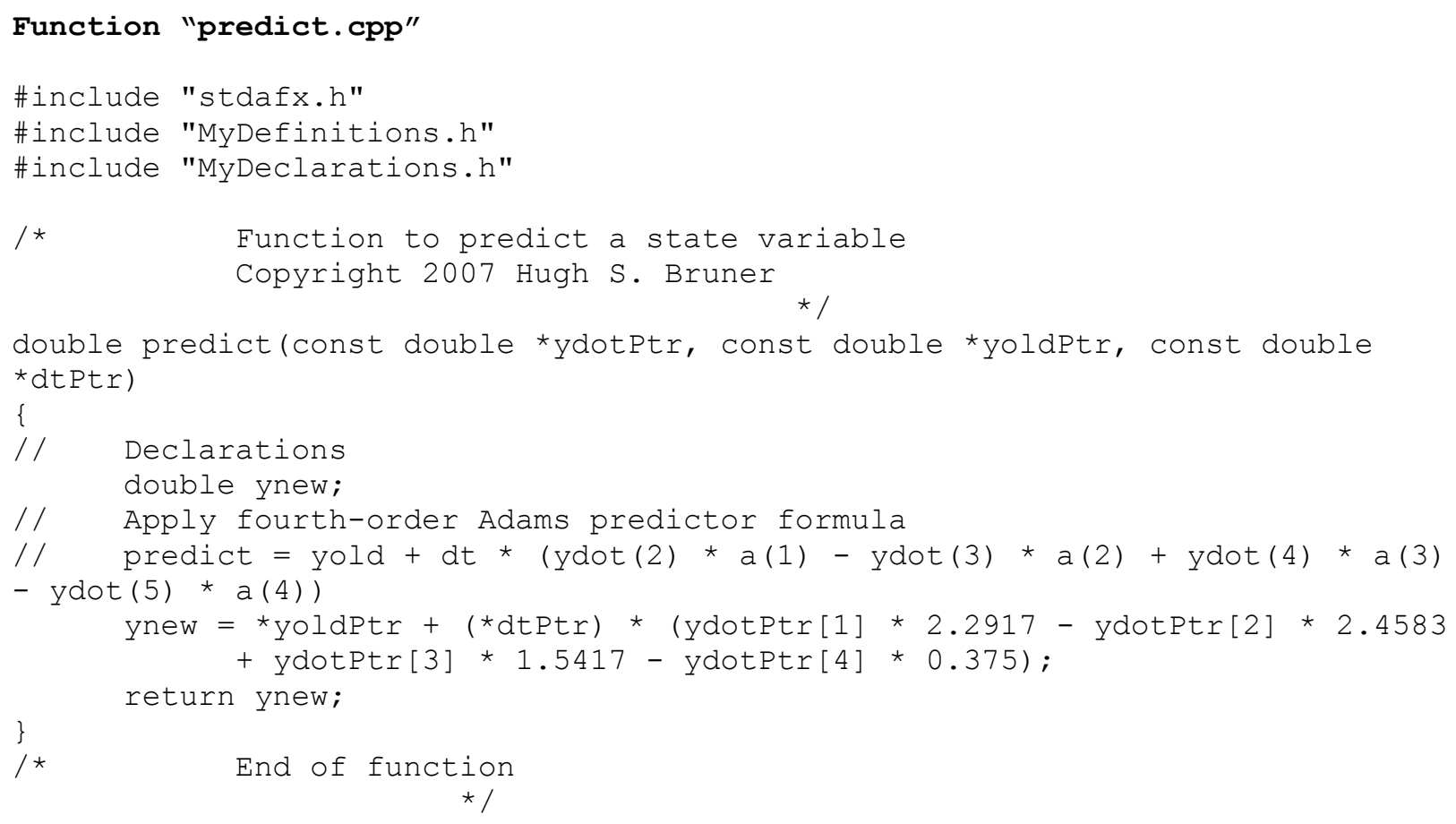




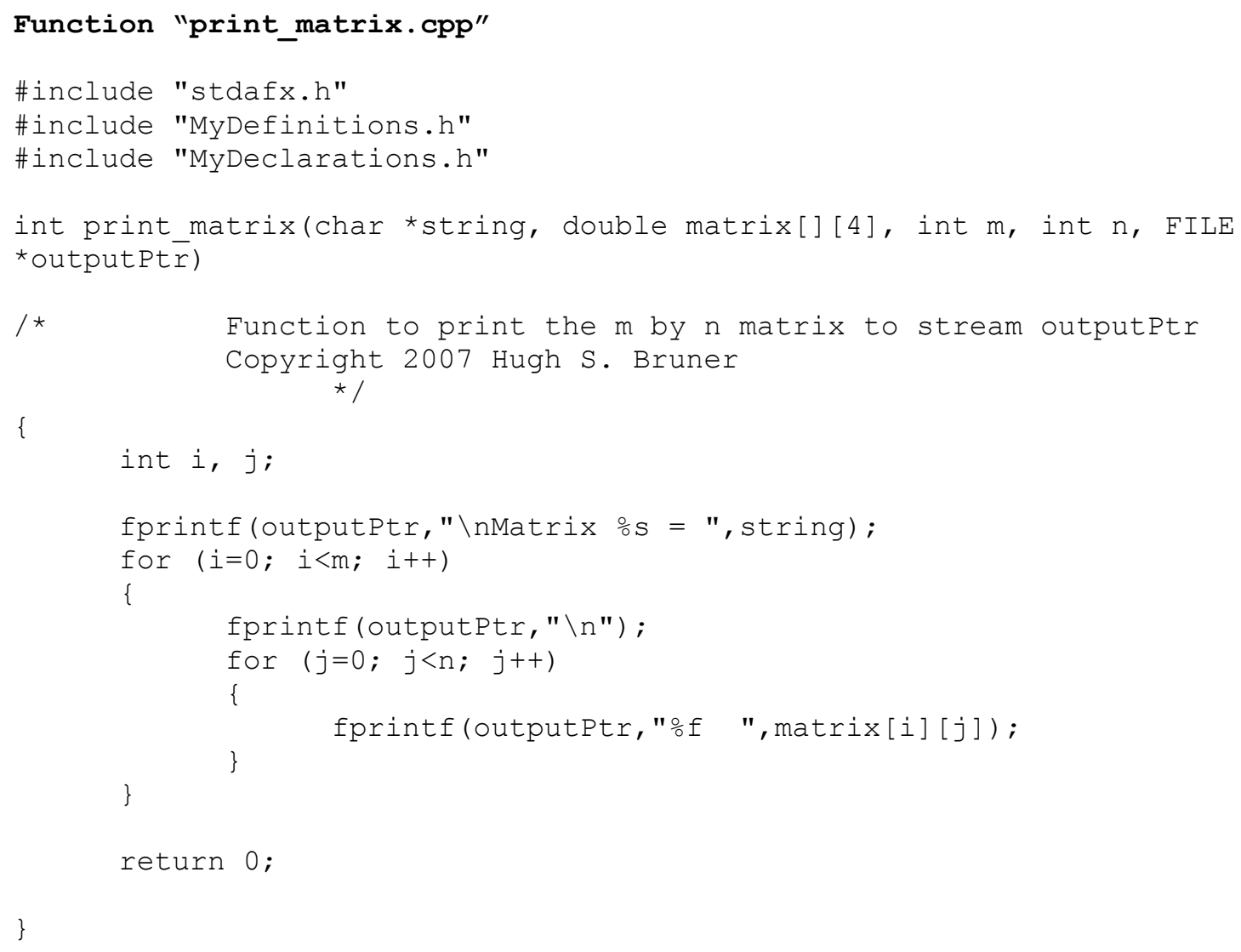




\section{Function "print_the_network.cpp"}

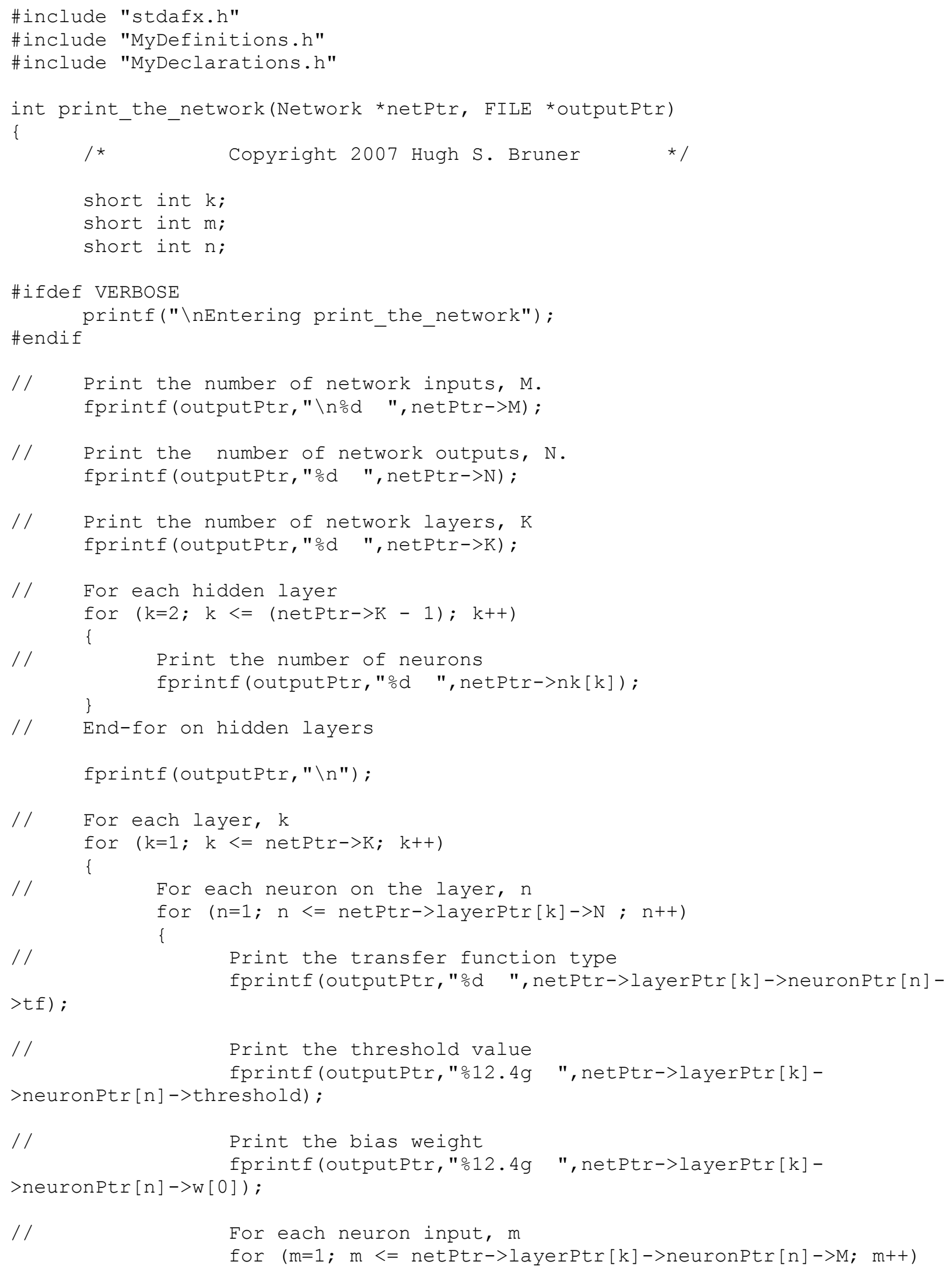




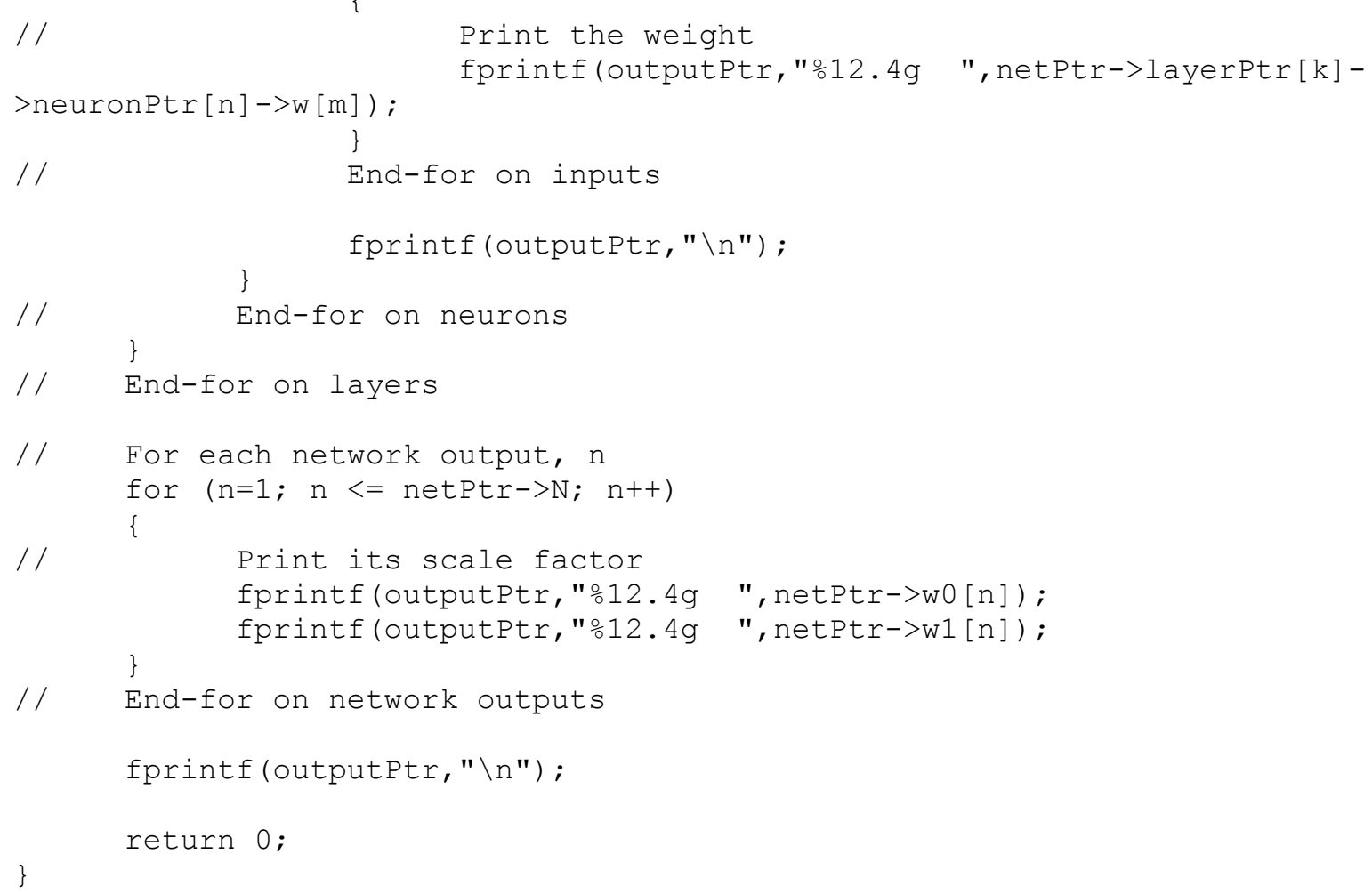




\section{Function "print_the_network_Ccode.cpp"}

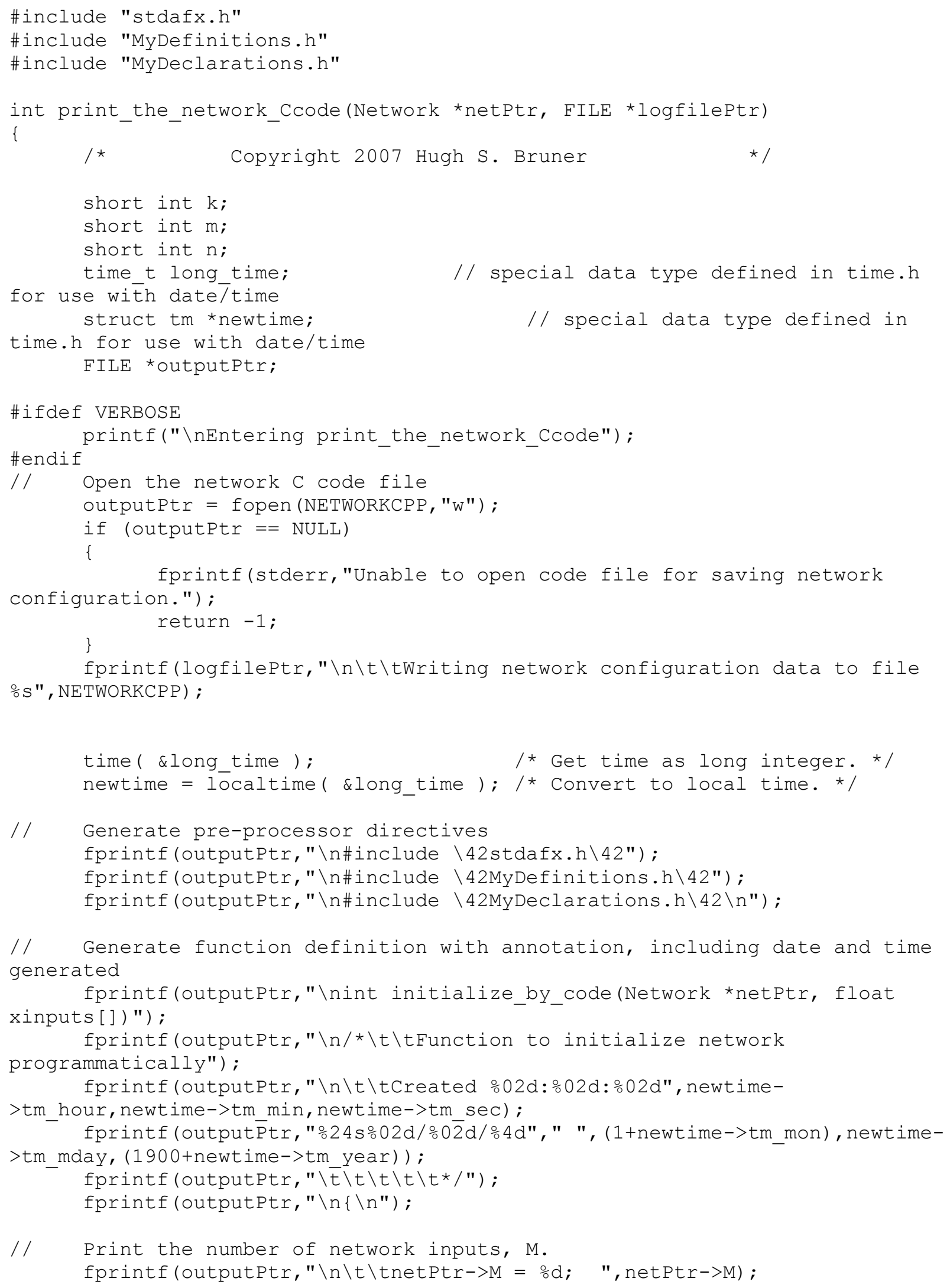


// Print the number of network outputs, N.

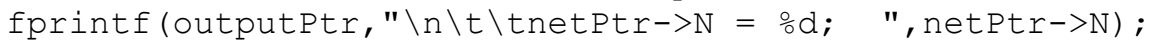

// Print the number of network layers, $\mathrm{K}$

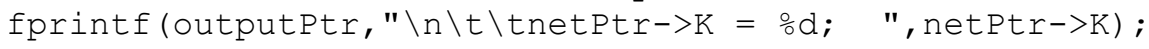

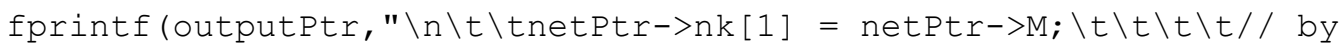

convention, first layer matches number of inputs");

// For each hidden layer

for $(\mathrm{k}=2 ; \mathrm{k}<=$ (netPtr $->\mathrm{K}-1) ; \mathrm{k}++$ )

// $\quad$ Print the number of neurons

$>\mathrm{nk}[\mathrm{k}])$

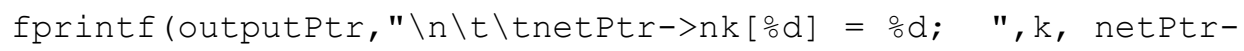

// End-for on hidden layers

// Insert necessary code verbatim

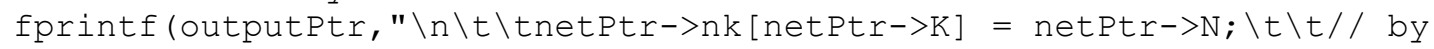

convention, last layer matches number of outputs");

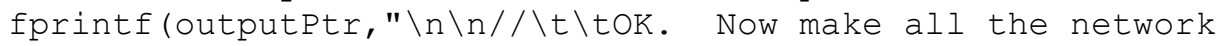
connections");

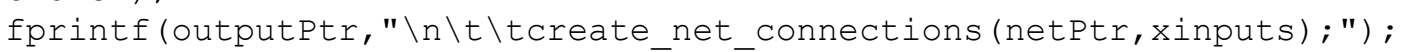

fprintf (outputPtr," \n");

// For each layer, k

for $(\mathrm{k}=1 ; \mathrm{k}<=$ netPtr $->\mathrm{k} ; \mathrm{k}++)$

I/ For each neuron on the layer, $\mathrm{n}$

for $(\mathrm{n}=1 ; \mathrm{n}<=$ netPtr->layerPtr $[\mathrm{k}]->\mathrm{N} ; \mathrm{n}++)$

/ $\{$ Print the transfer function type

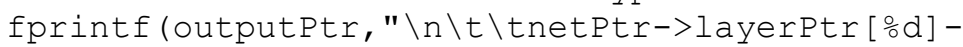

>neuronPtr [\%d]->tf = (transfer_function_type) $\% d ; \quad$ ", k, n, netPtr->layerPtr[k]$>$ neuronPtr $[\mathrm{n}]->\operatorname{tf})$;

// Print the threshold value

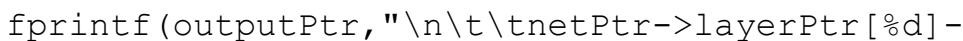

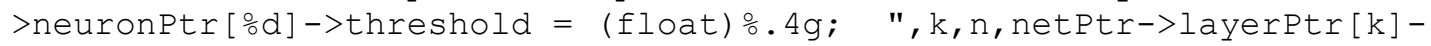

>neuronPtr $[\mathrm{n}]->$ threshold);

// $\quad$ Print the bias weight

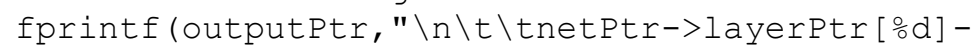

$>$ neuronPtr $[\circ \mathrm{d}]->\mathrm{w}[0]=$ (float) $\%$. $4 \mathrm{~g} ; \quad \mathrm{k}, \mathrm{k}, \mathrm{n}$, netPtr->layerPtr $[\mathrm{k}]->$ neuronPtr $[\mathrm{n}]-$ $>\mathrm{W}[0])$;

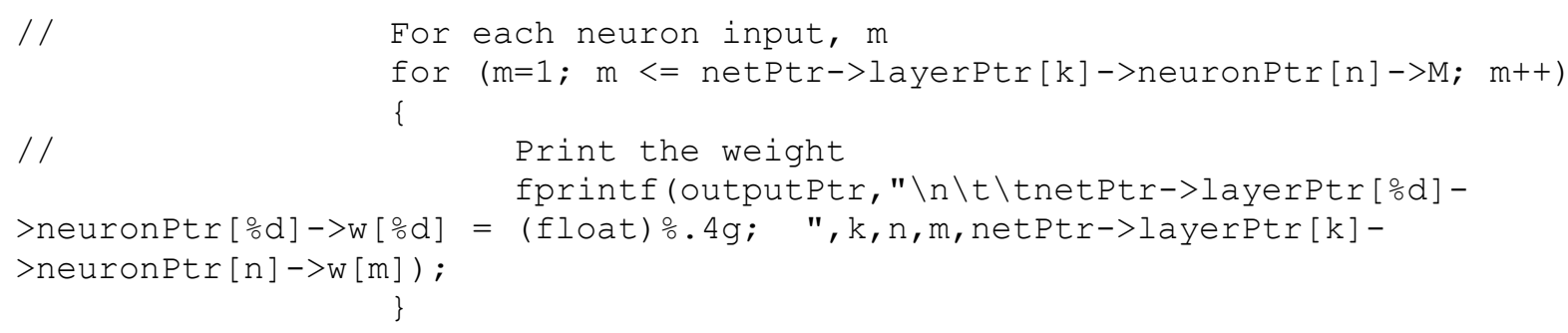




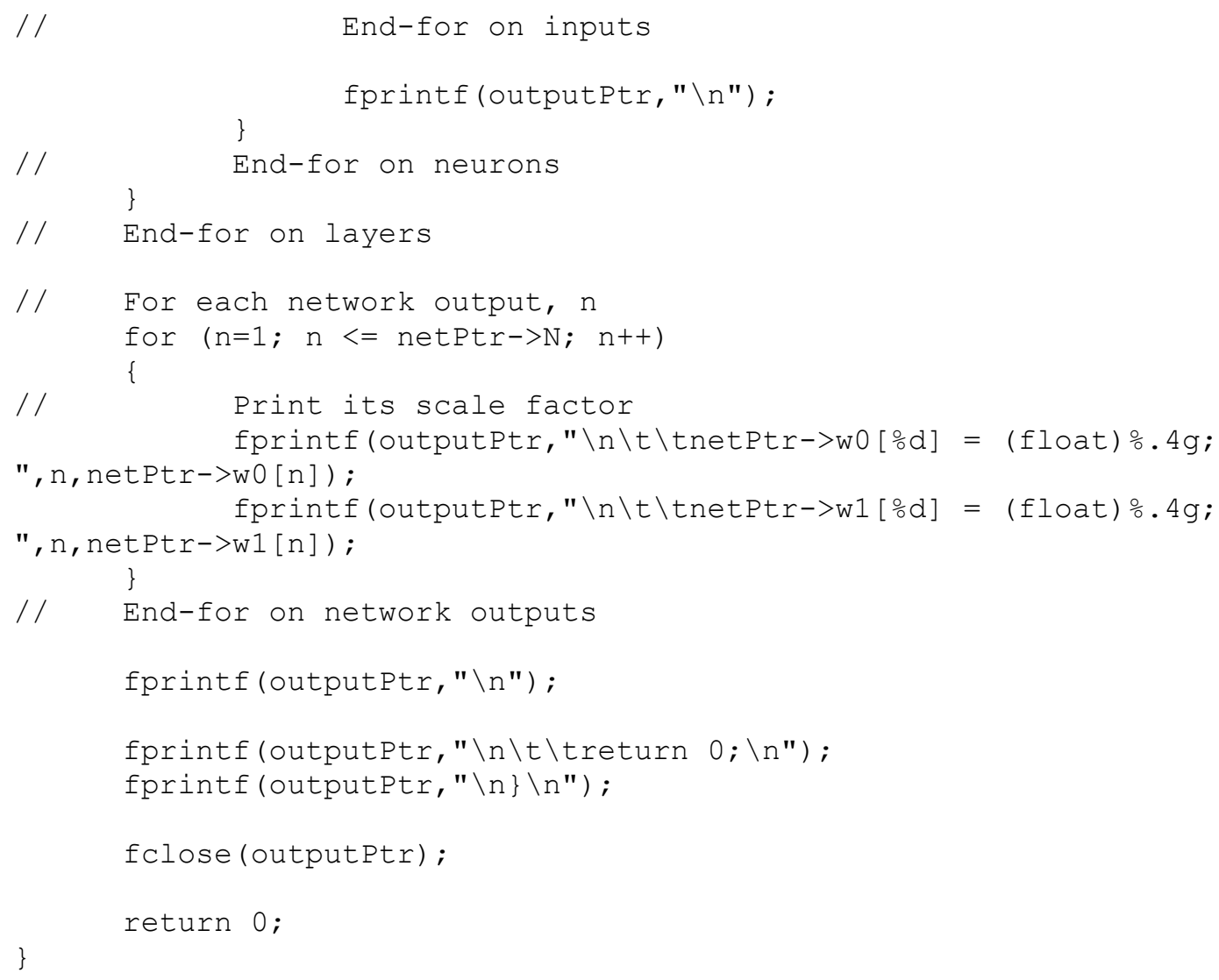




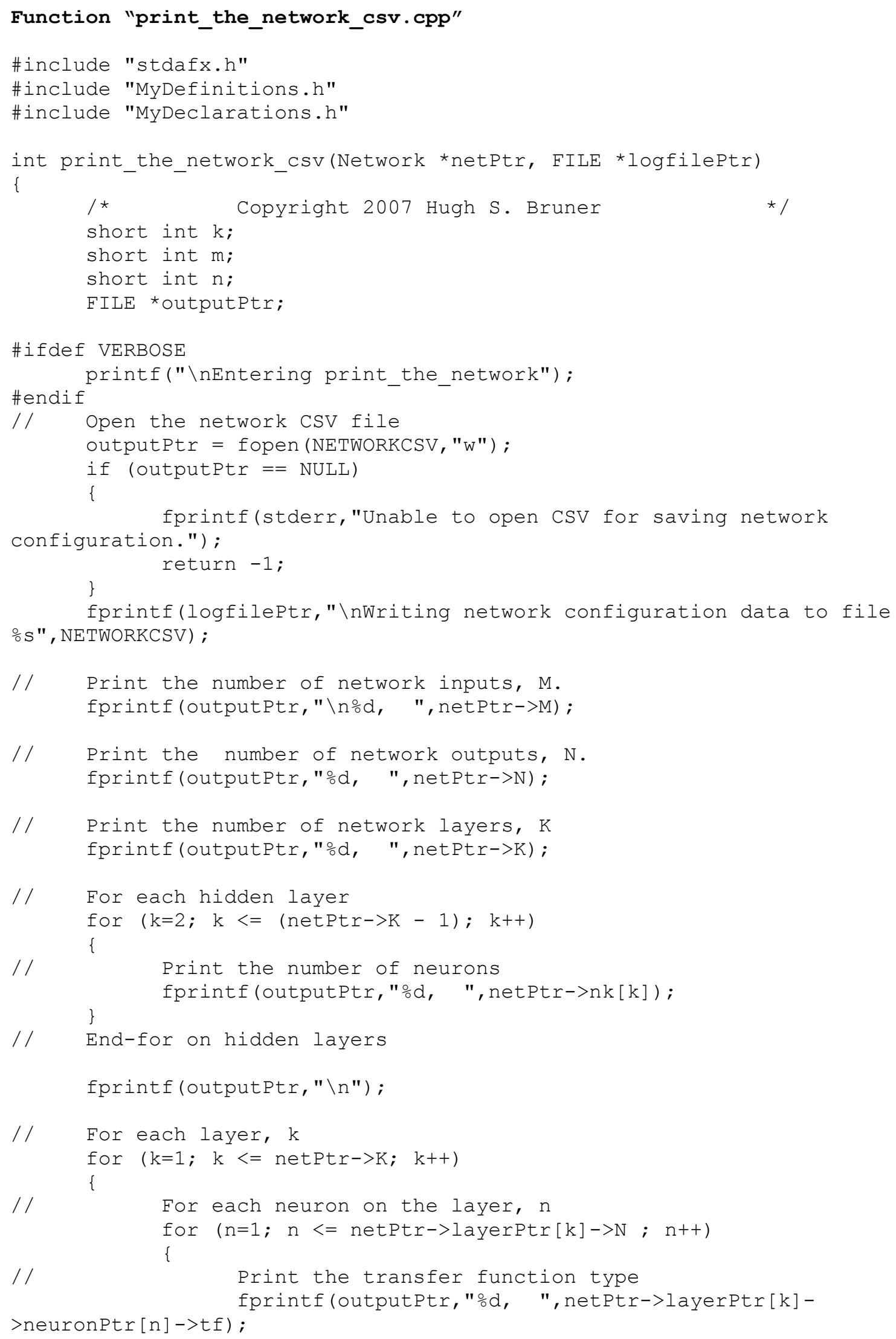




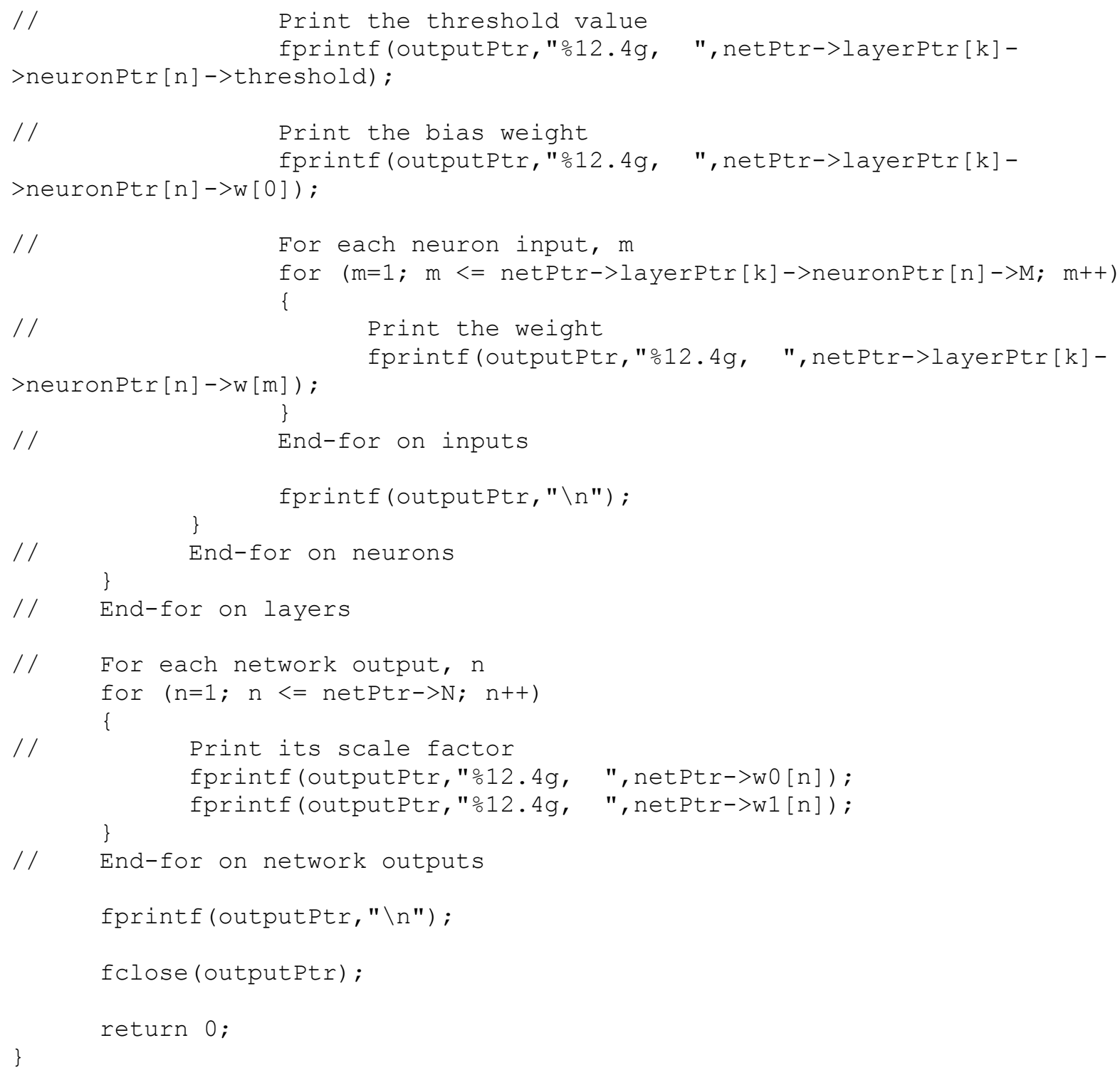




\section{Function "push_down.cpp"}

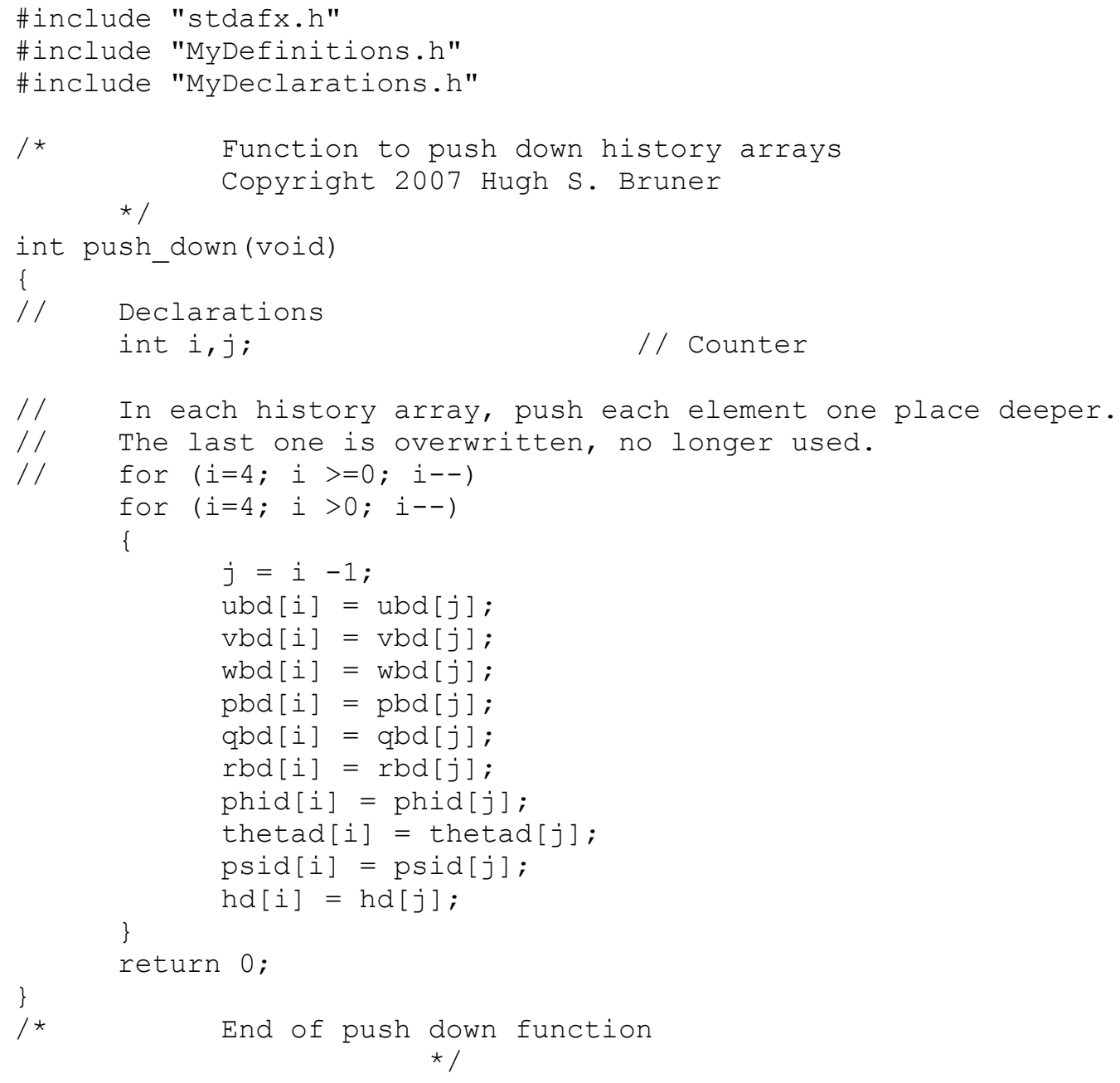




\section{Function "RandomCom. cpp"}

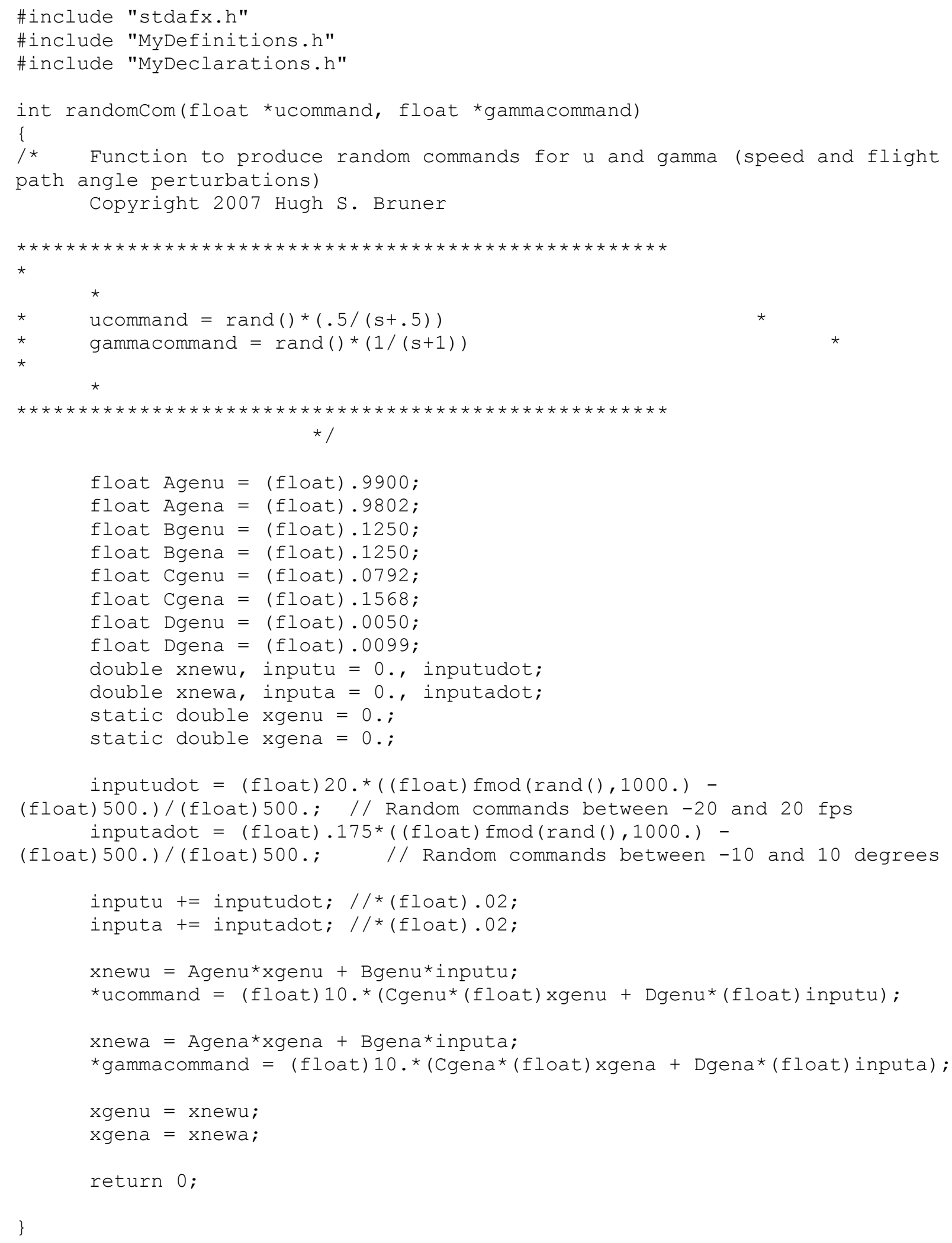




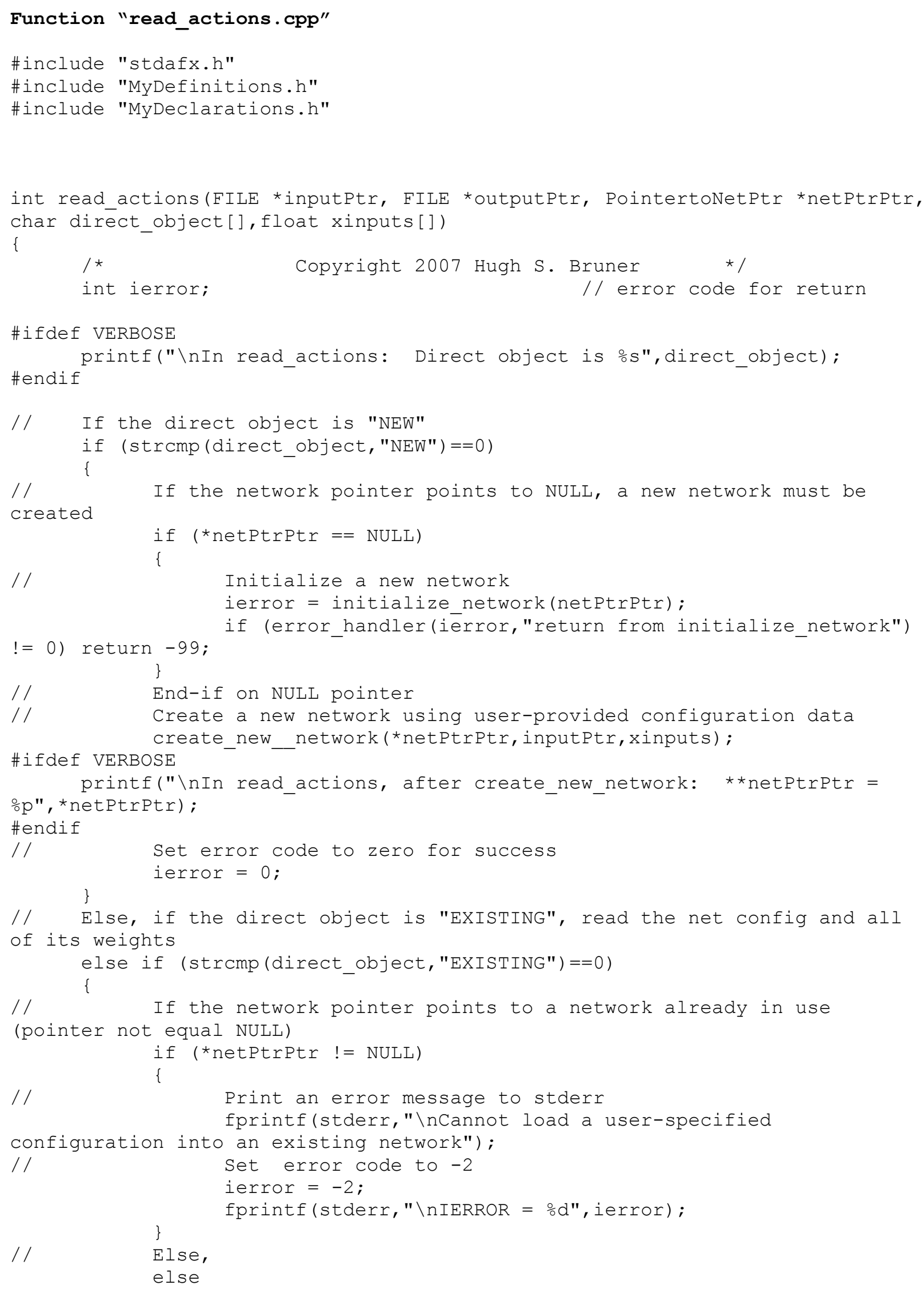




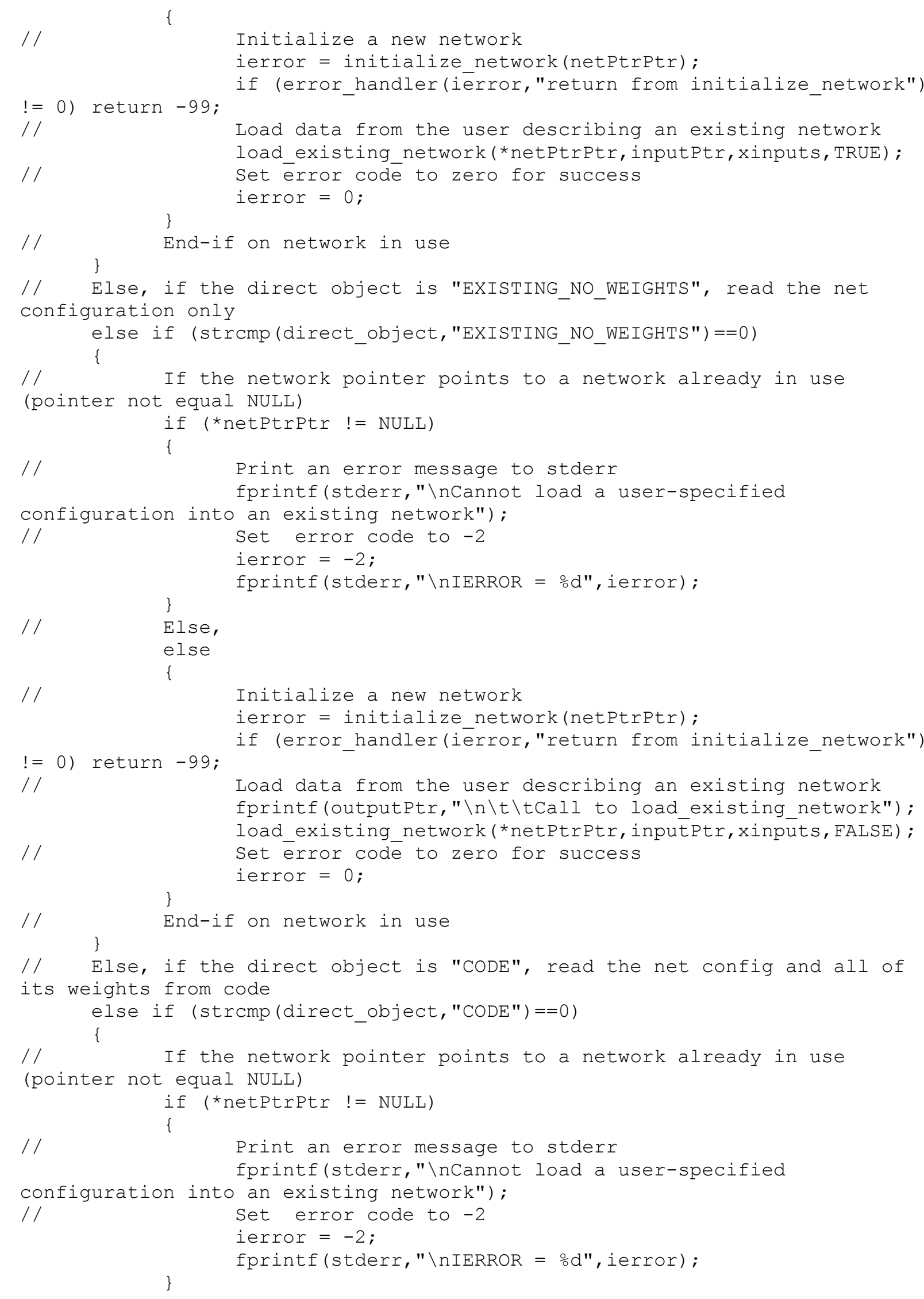




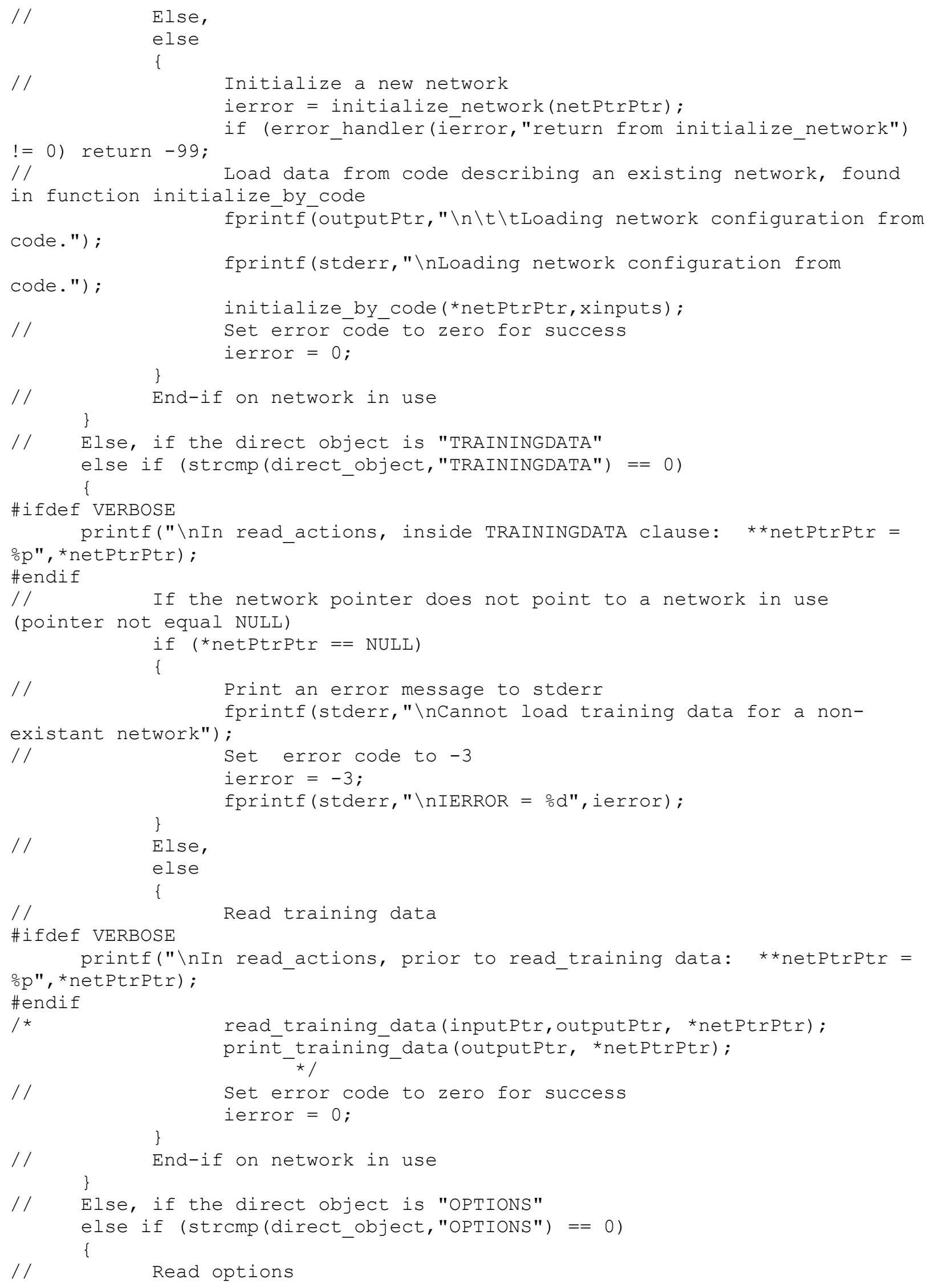




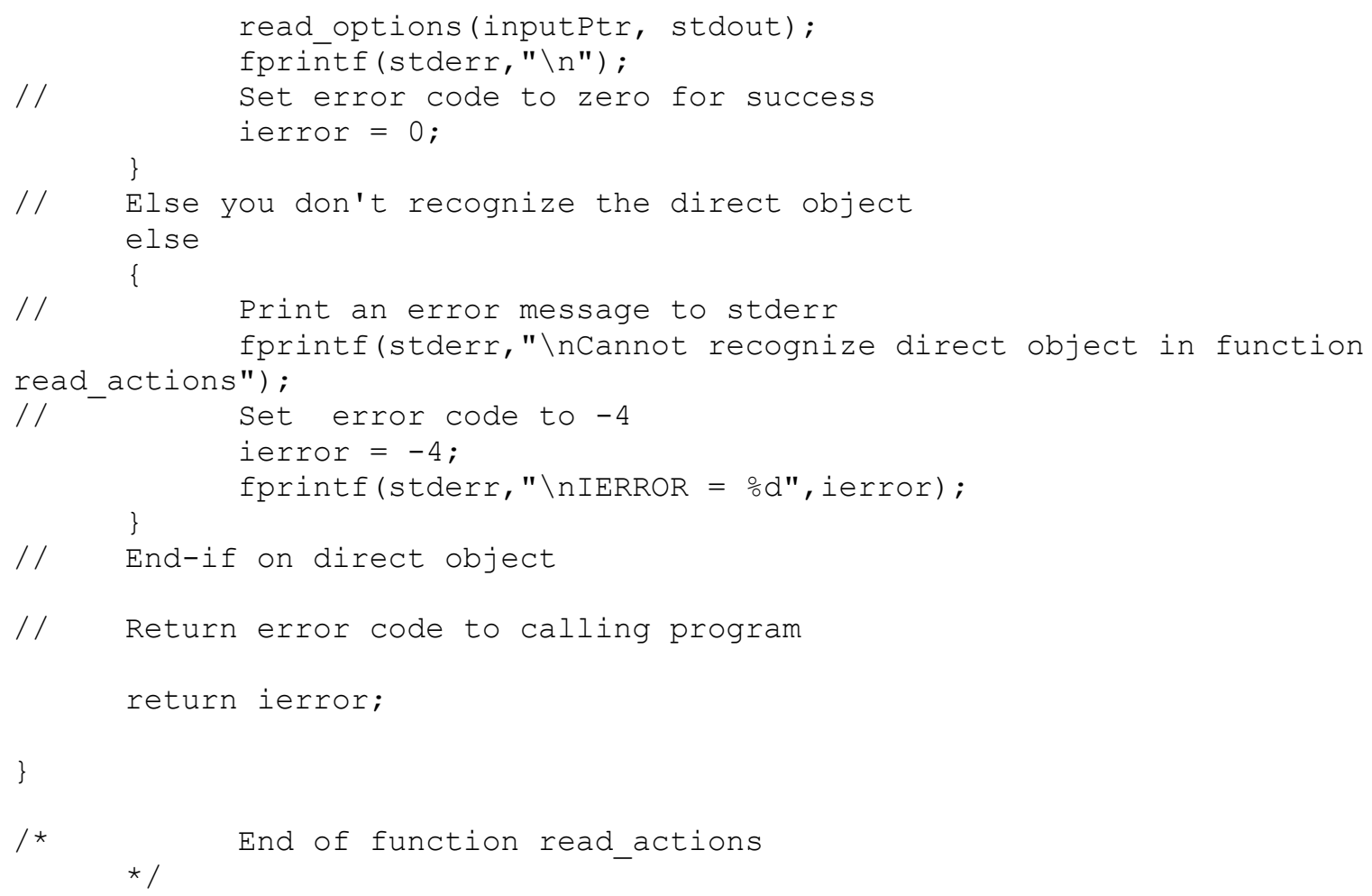




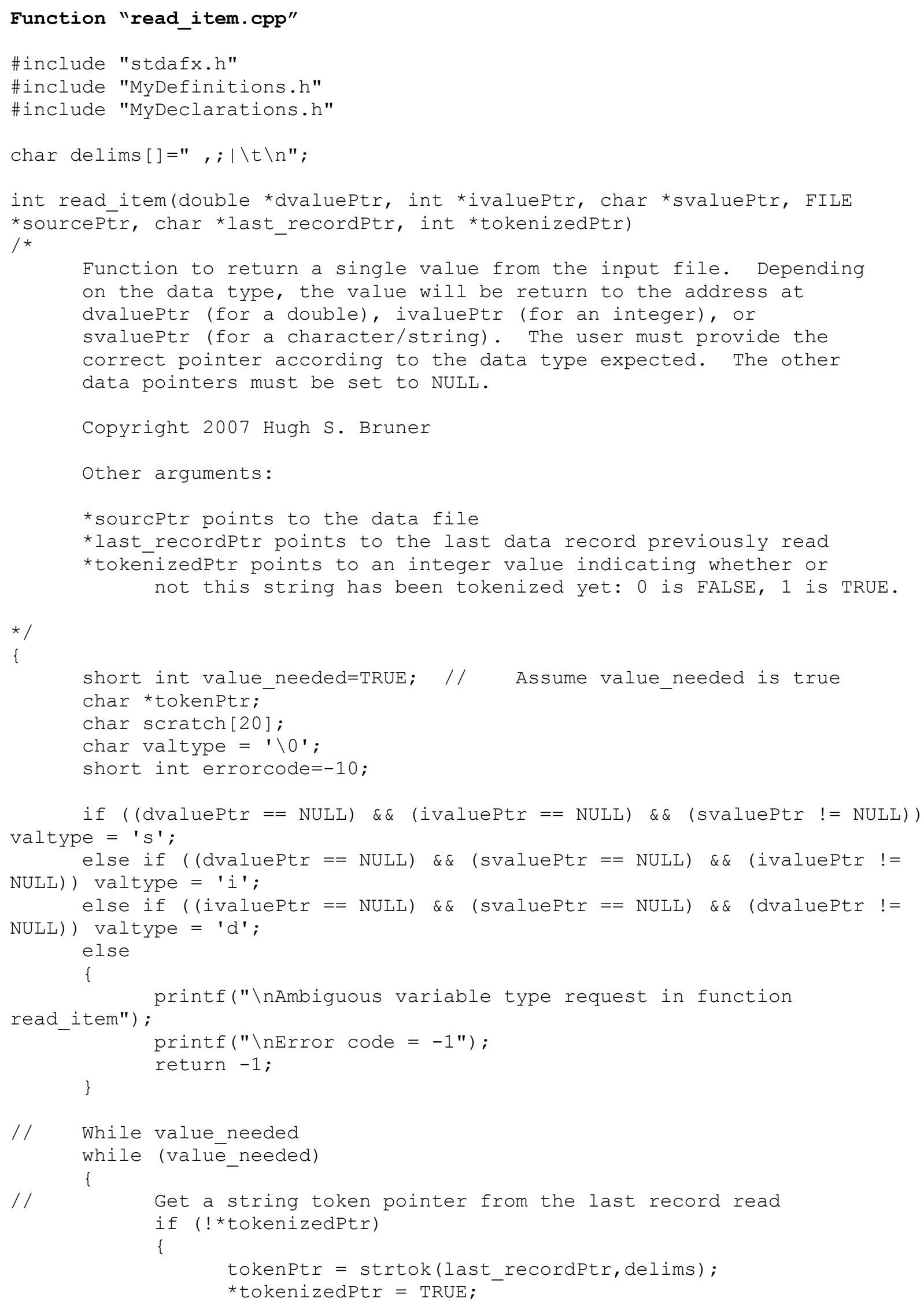




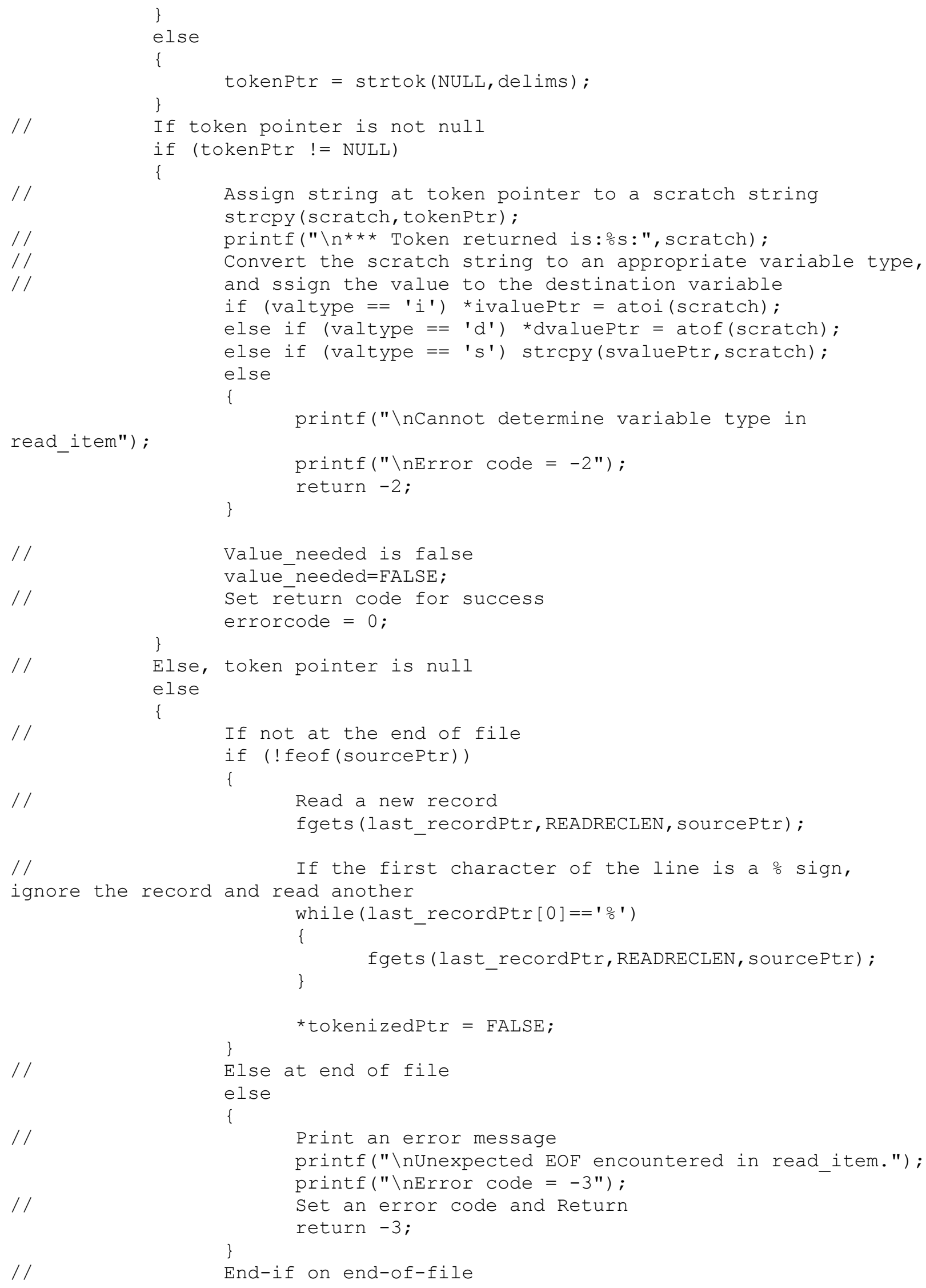




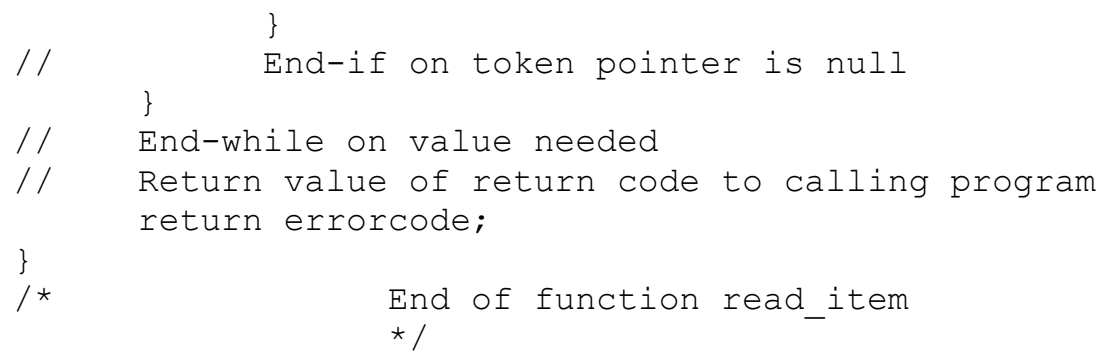




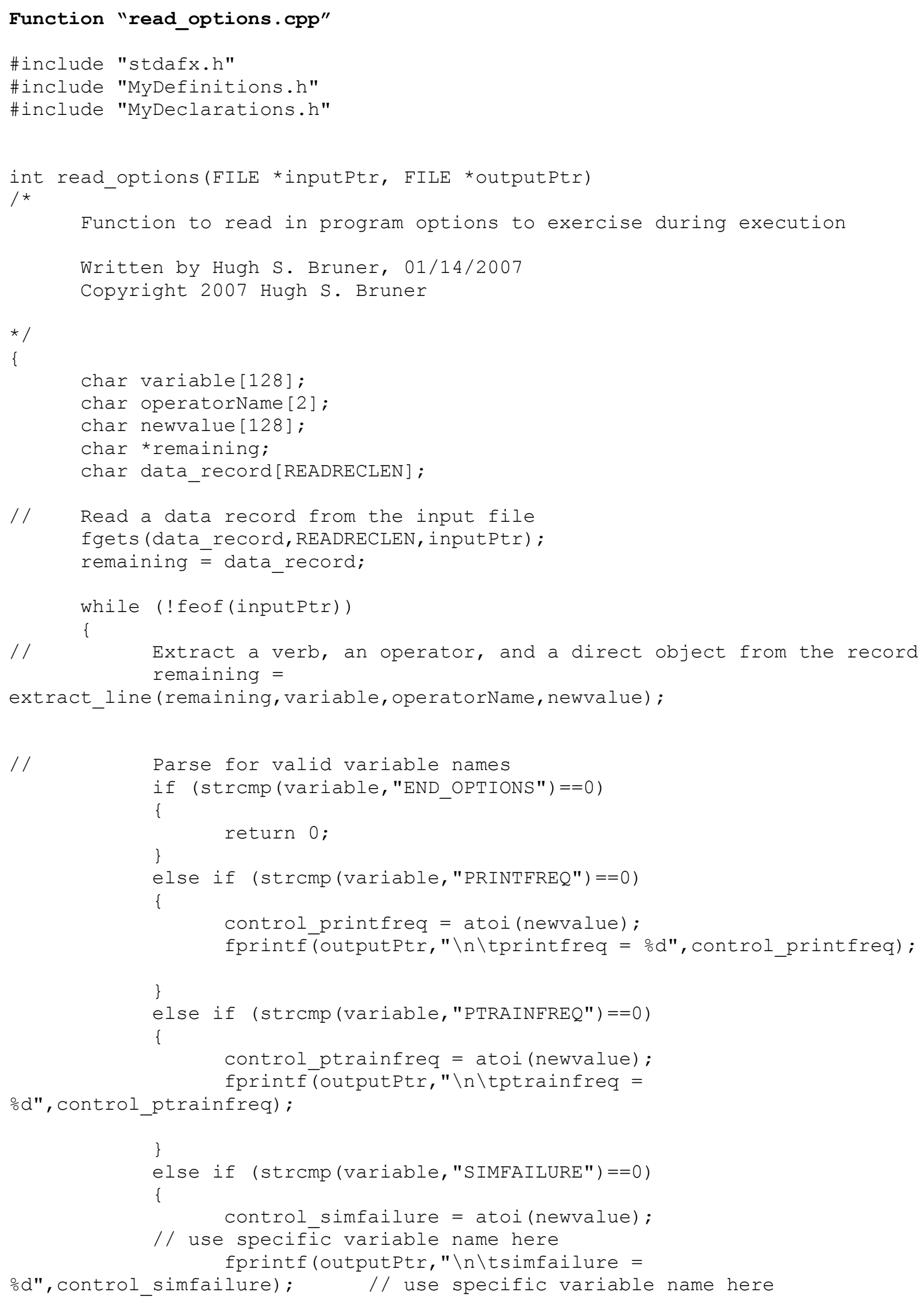




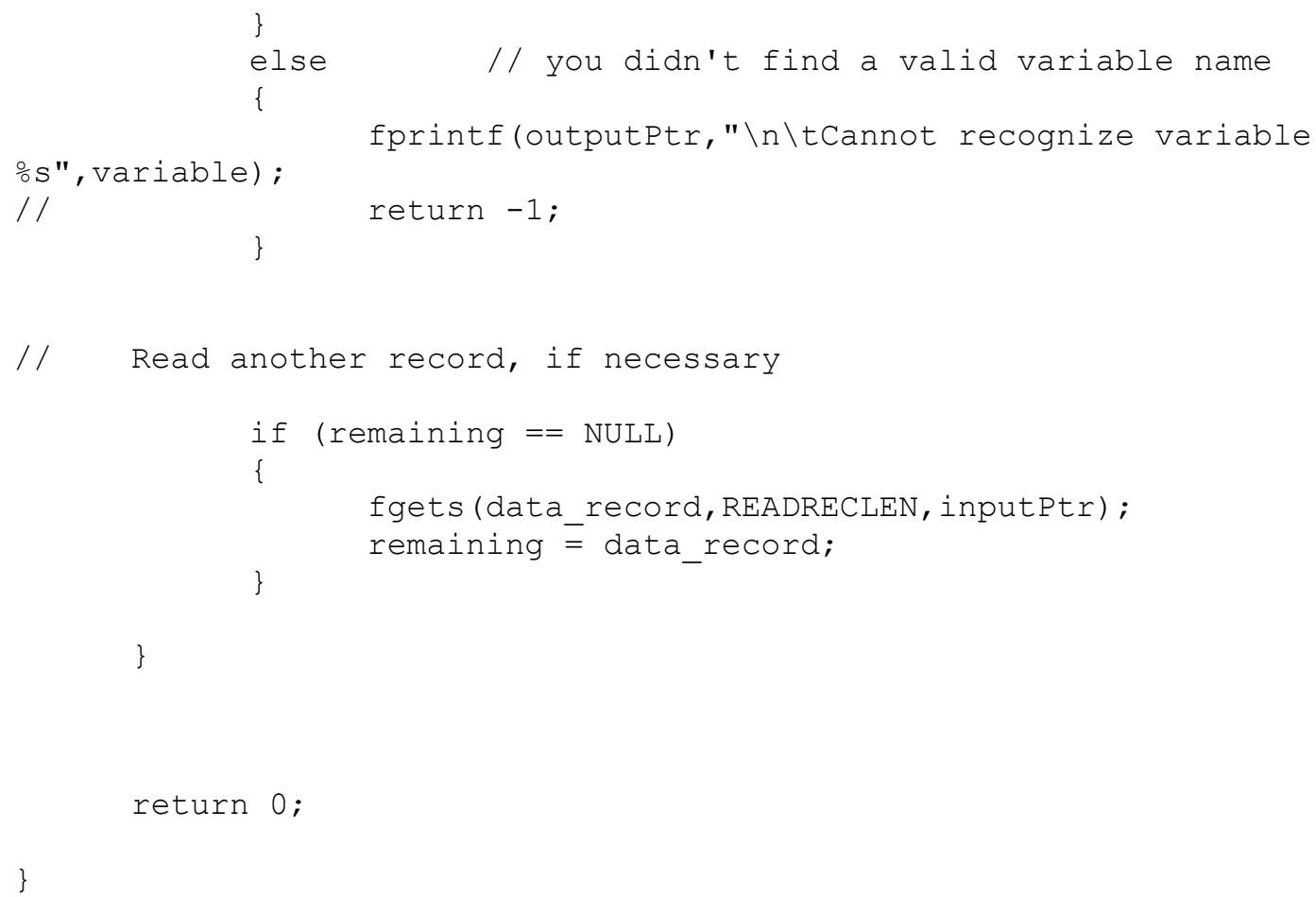




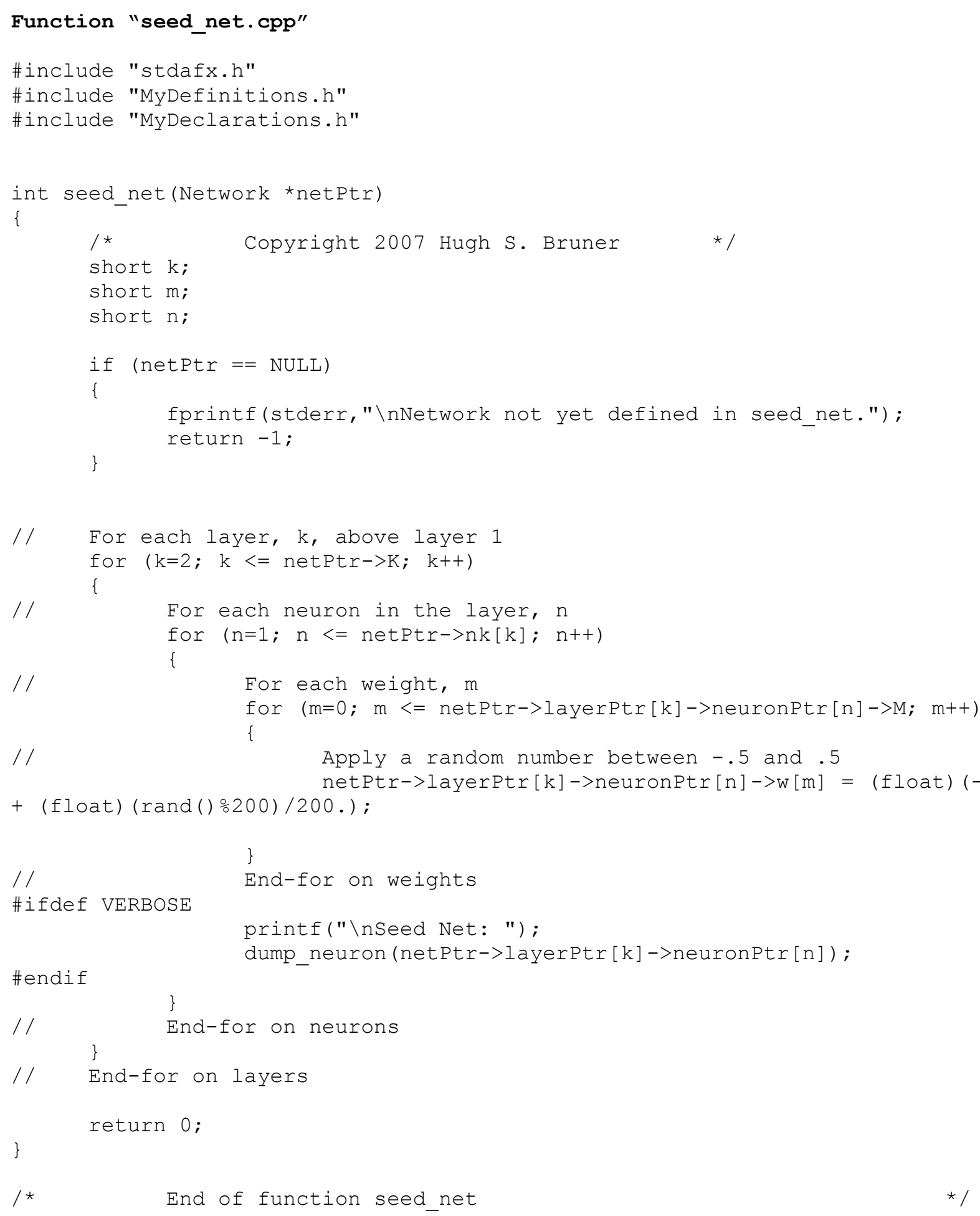




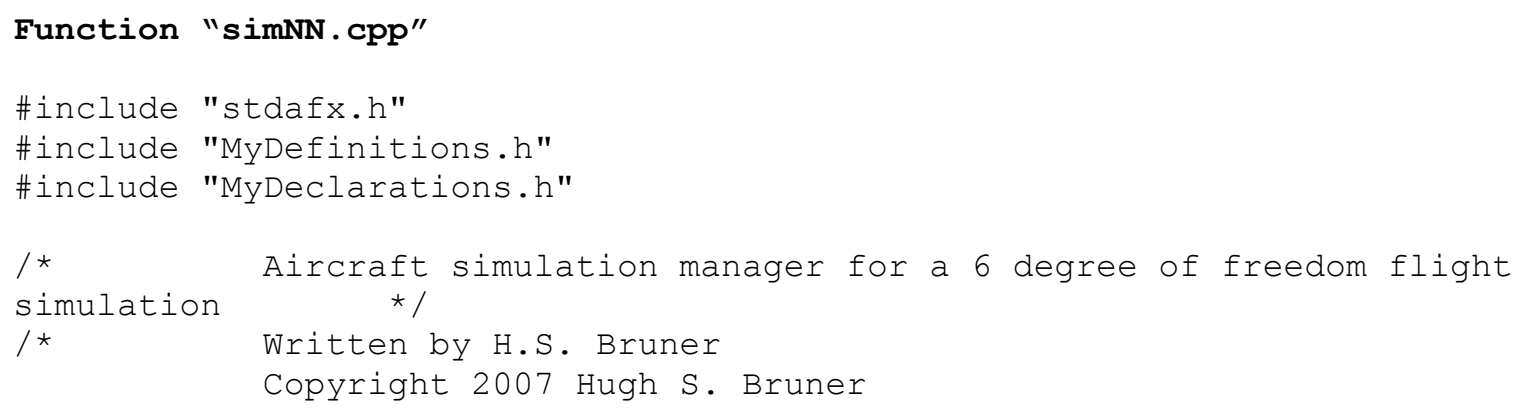


char case_descriptor[120];

description of the case to be read
// string that contains a

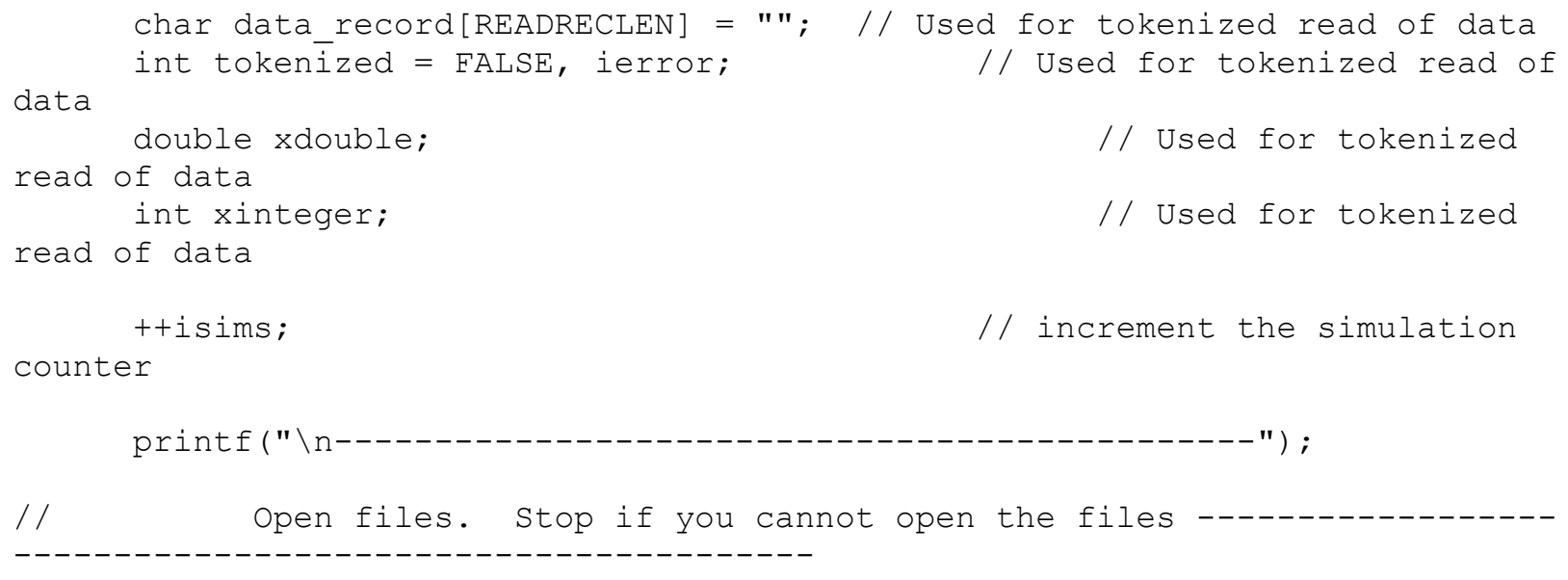

/ / End of opening files 


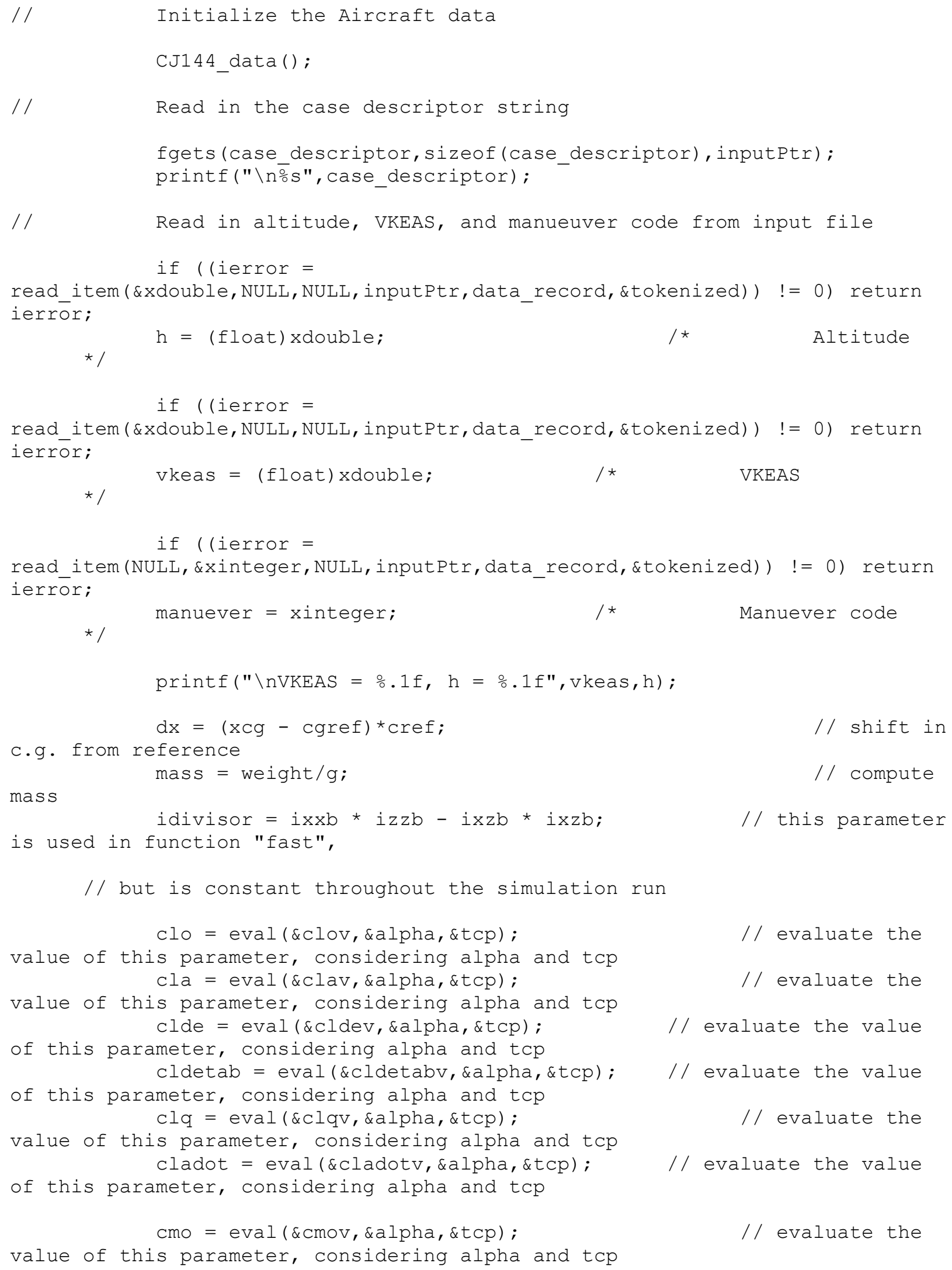


$\mathrm{cma}=\operatorname{eval}(\& \mathrm{cmav}$, \&alpha, \&tcp);

value of this parameter, considering alpha and tcp cmde = eval (\&cmdev, \&alpha, \&tcp);

of this parameter, considering alpha and tcp cmdetab = eval (\&cmdetabv, \&alpha, \&tcp) ;

of this parameter, considering alpha and tcp $\mathrm{cmq}=\operatorname{eval}(\& \mathrm{cmqv}$, \&alpha, \&tcp) ;

value of this parameter, considering alpha and tcp cmadot $=$ eval $(\&$ cmadotv, \&alpha, \&tcp) ;

of this parameter, considering alpha and tcp

cyb = eval (\&cybv, \&alpha, \&tcp);

value of this parameter, considering alpha and tcp cyp = eval (\&cypv, \&alpha, \&tcp) ;

value of this parameter, considering alpha and tcp cyr = eval (\&cyrv, \&alpha, \&tcp) ;

value of this parameter, considering alpha and tcp cydr = eval (\&cydrv, \&alpha, \&tcp);

of this parameter, considering alpha and tcp cyda $=\operatorname{eval}(\& c y d a v$, \&alpha, \&tcp);

of this parameter, considering alpha and tcp

cnb = eval (\&cnbv, \&alpha, \&tcp) ;

value of this parameter, considering alpha and tcp $c n p=\operatorname{eval}(\& c n p v$, \&alpha, \&tcp) ;

value of this parameter, considering alpha and tcp cnr = eval (\&cnrv, \&alpha, \&tcp);

value of this parameter, considering alpha and tcp cndr = eval (\&cndrv, \&alpha, \&tcp) ;

of this parameter, considering alpha and tcp cnda $=\operatorname{eval}(\& c n d a v$, \&alpha, \&tcp);

of this parameter, considering alpha and tcp

$\mathrm{crb}=\operatorname{eval}($ \&crbv, \&alpha, \&tcp) ;

value of this parameter, considering alpha and tcp $\operatorname{crp}=\operatorname{eval}(\& c r p v$, \&alpha, \&tcp) ;

value of this parameter, considering alpha and tcp crr = eval (\&crrv, \&alpha, \&tcp) ;

value of this parameter, considering alpha and tcp crdr $=\operatorname{eval}(\& c r d r v, \& a l p h a, \& t c p)$;

of this parameter, considering alpha and tcp crda $=\operatorname{eval}(\& c r d a v$, \&alpha, \&tcp) ;

of this parameter, considering alpha and tcp

rho $=$ std density $(\mathrm{h})$;

density of air for this altitude

vtrue $=1.689{ }^{*}$ vkeas $/$ sqrt $($ rho/.0023769);

airspeed, givn the KEAS for trim

$\mathrm{U} 1$ = vtrue;

equals true airspeed

$q 1=.5{ }^{*}$ rho*vtrue*vtrue;

dynamic pressure

$\mathrm{q} 1 \mathrm{sw}=\mathrm{q} 1{ }^{*}$ sref

frequently repeated calculations. Do now to save time later.

q1swbw = q1sw*bref;

frequently repeated calculations. Do now to save time later.
// evaluate the

// evaluate the value

// evaluate the value

// evaluate the

// evaluate the value

// evaluate the

// evaluate the

// evaluate the

// evaluate the value

// evaluate the value

// evaluate the

// evaluate the

// evaluate the

// evaluate the value

// evaluate the value

// evaluate the

// evaluate the

// evaluate the

// evaluate the value

// evaluate the value

// compute the

// compute true

/ / U1

/ / compute

II 


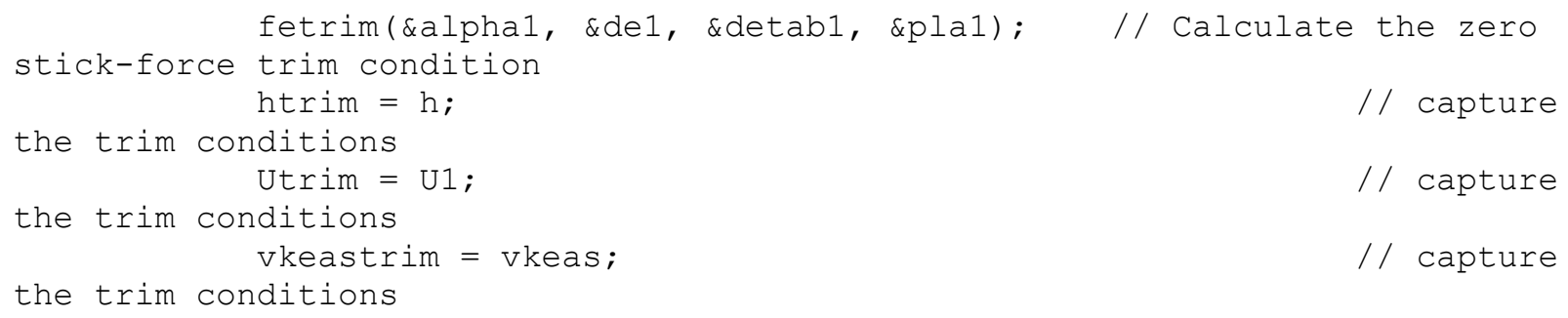




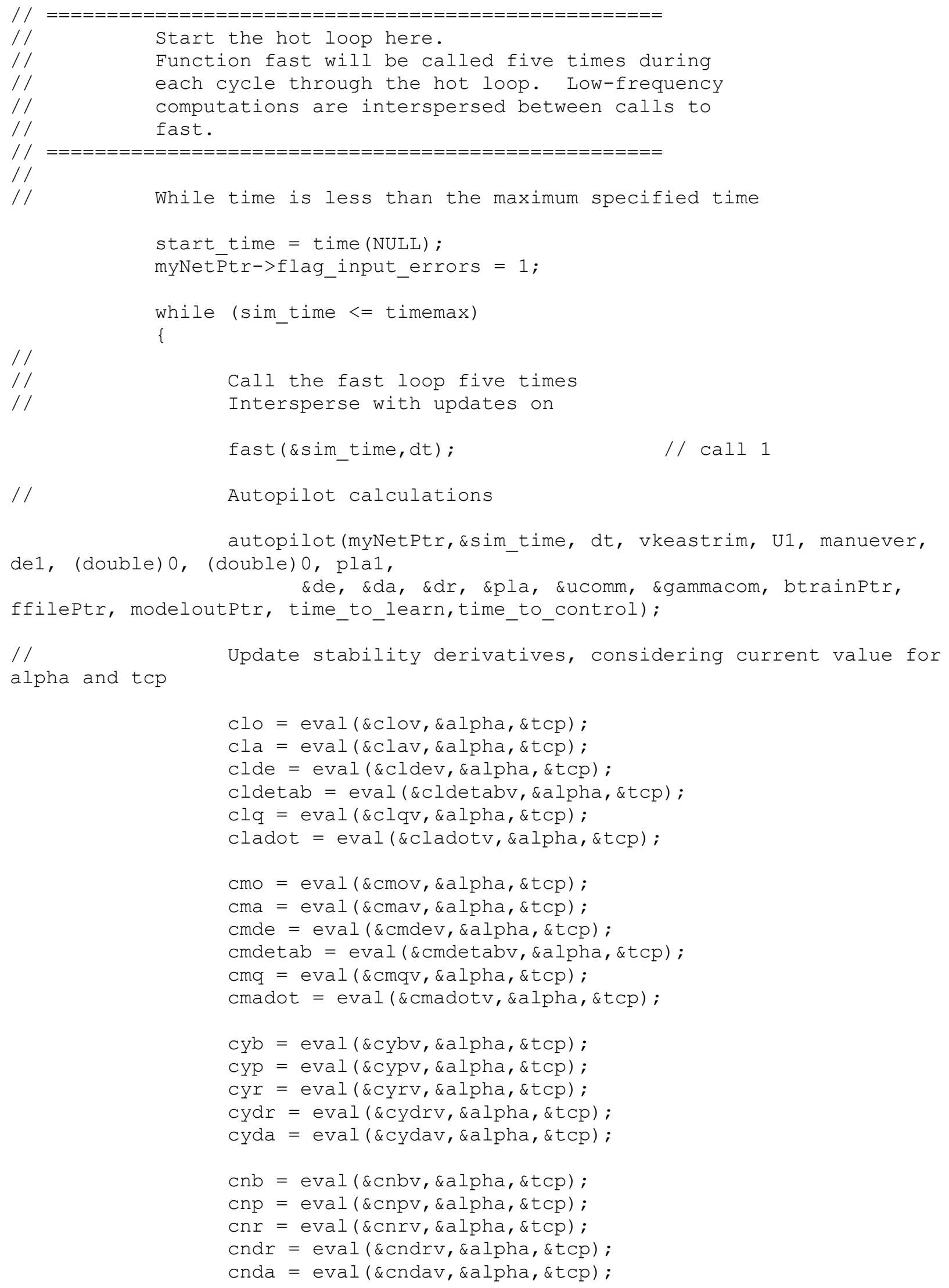




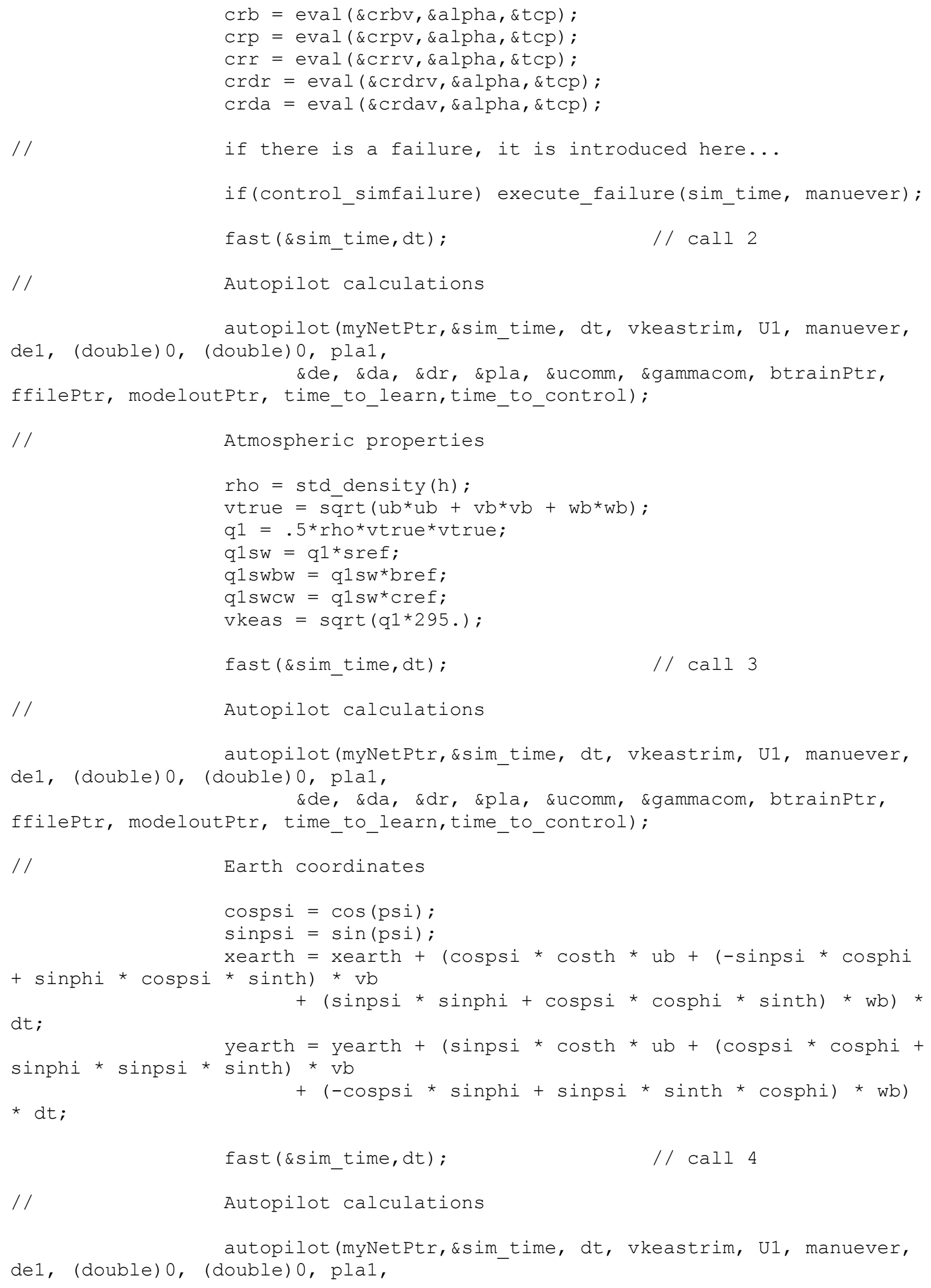


\&de, \&da, \&dr, \&pla, \&ucomm, \&gammacom, btrainPtr,

ffileptr, modeloutptr, time_to_learn,time_to_control);

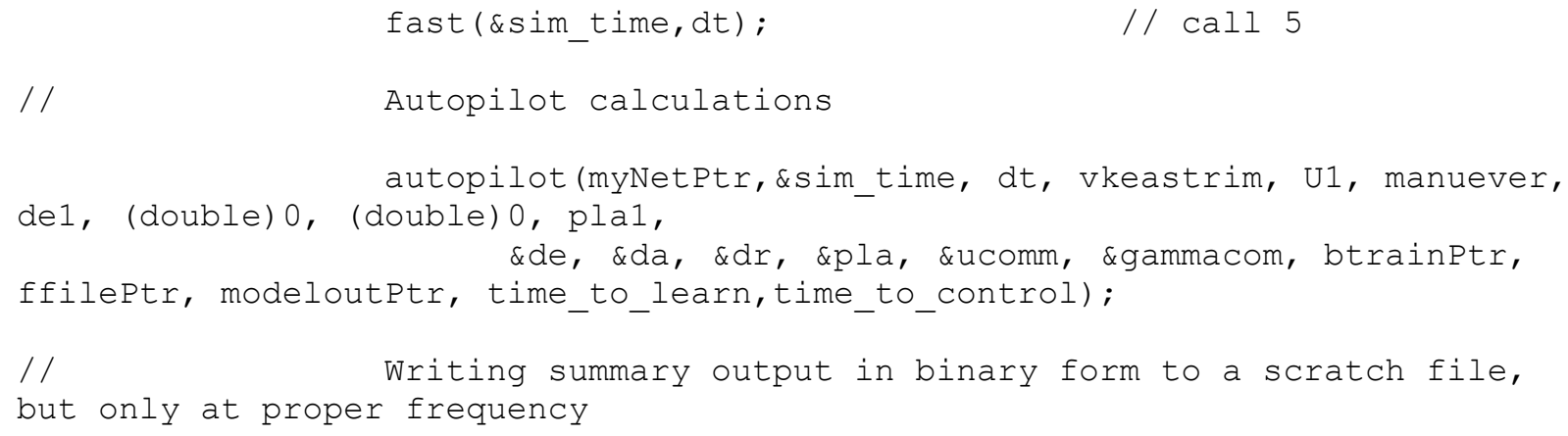




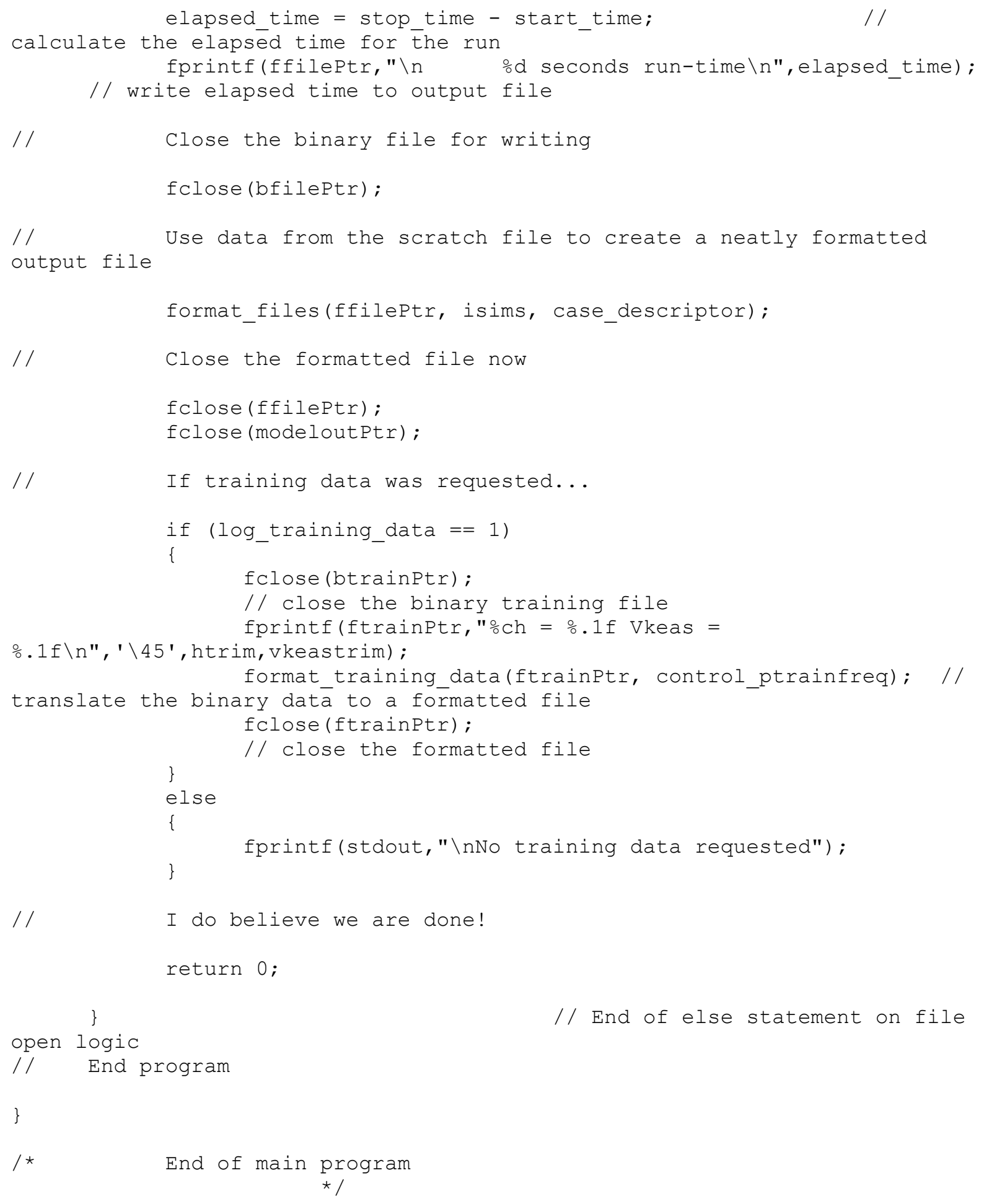




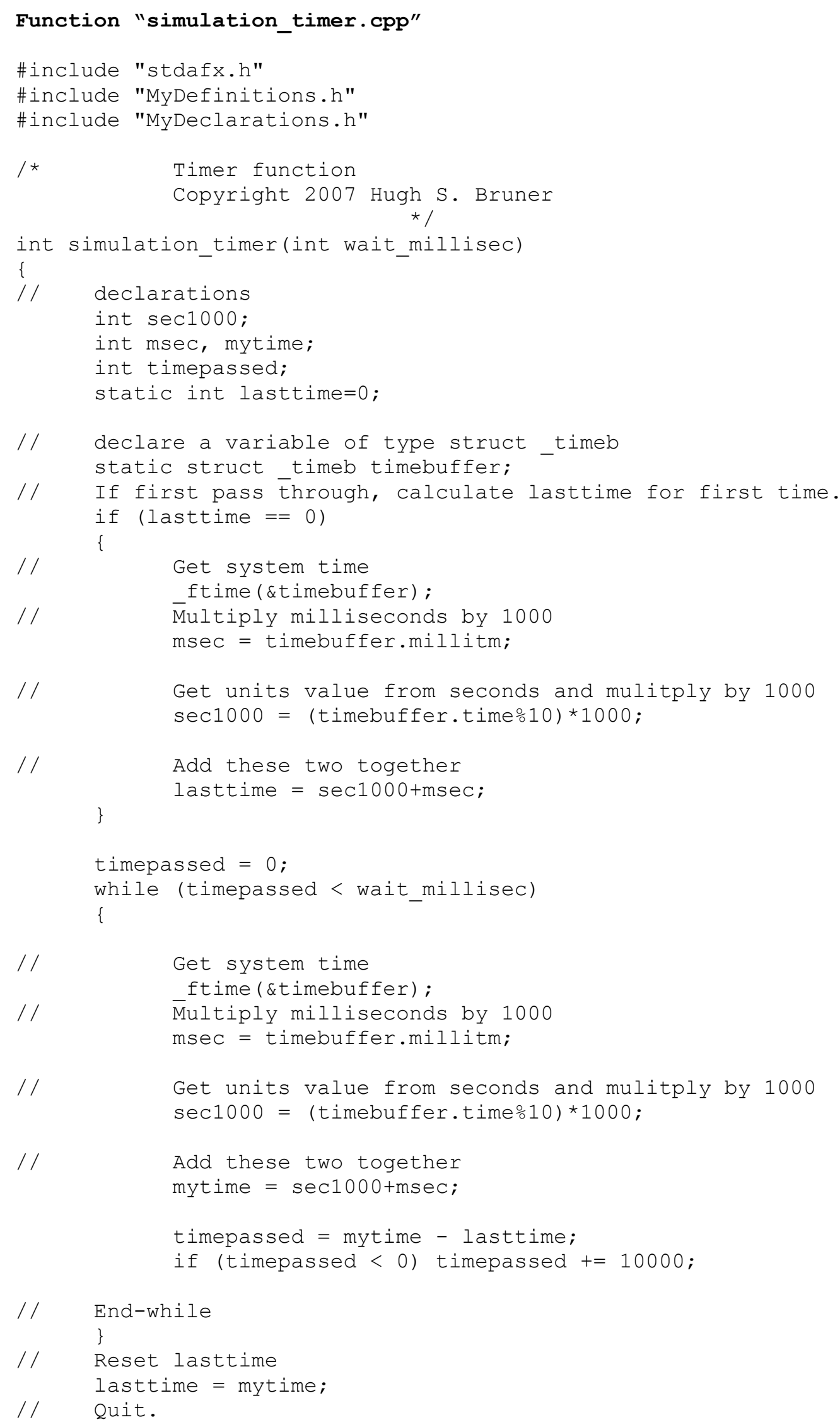


return 0 ; 


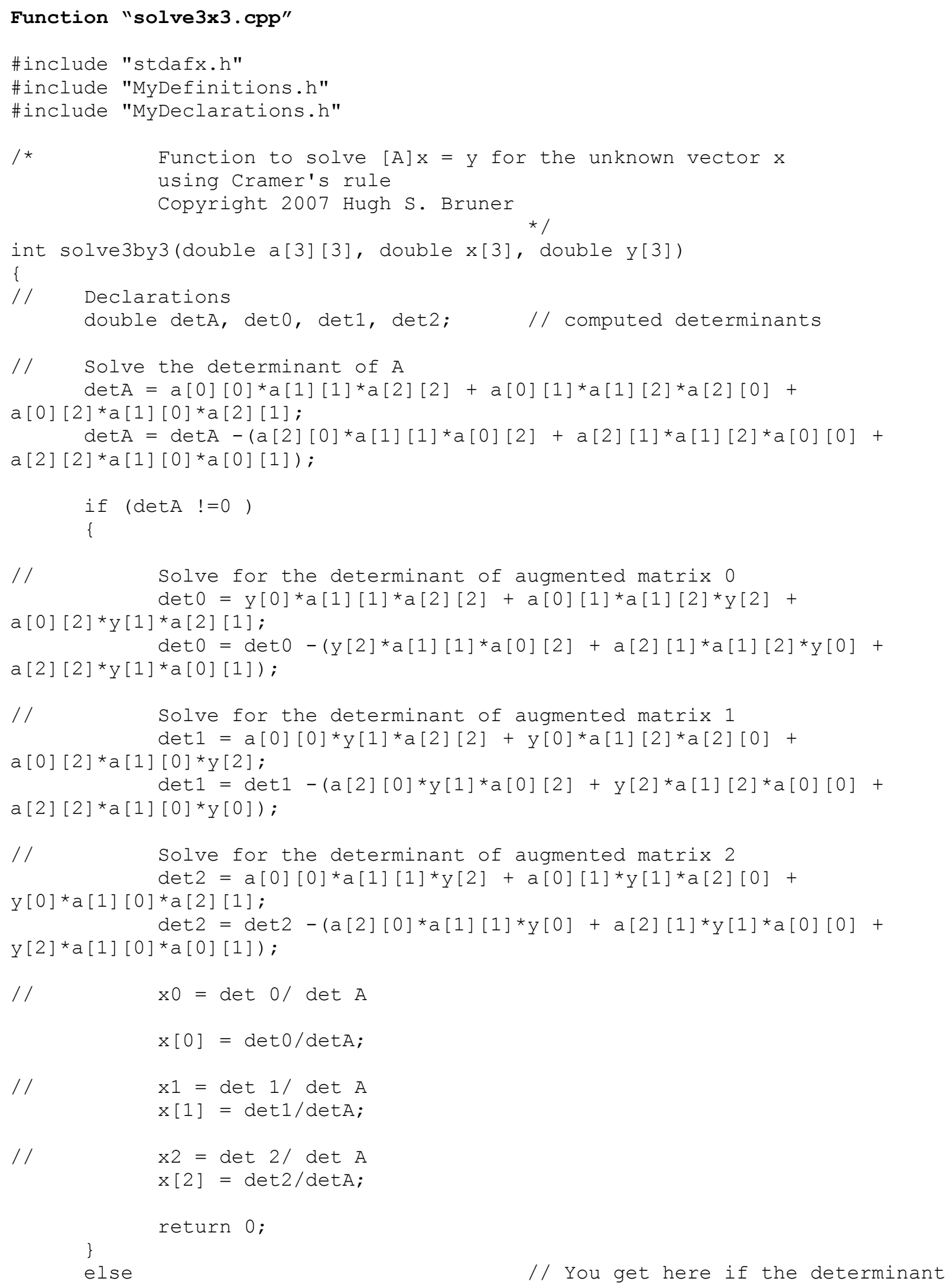


printf("\nDeterminant of $\mathrm{A}$ is zero in function solve3by3."); return -1;

// That's all!

\}

/*

End of function solve3by3

*/ 


\section{Function "std_atmos.cpp"}

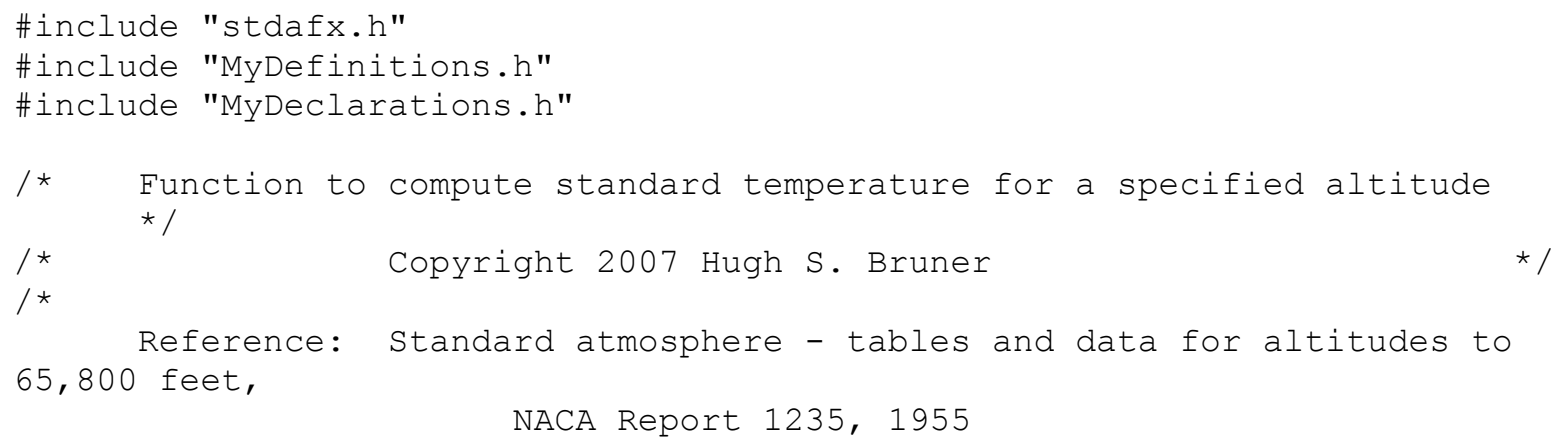


NACA Report 1235, 1955

double std_density(double h)

* /

\{

double $\mathrm{k} 1=6.87535 \mathrm{e}-6$;

double k2=4.80634e-5;

double density;

// If $h$ is below the tropopause $(36,089$ feet) then

if $(\mathrm{h}<36089$.$) density =.0023769 *$ pow $((1-\mathrm{k} 1 * \mathrm{~h}), 4.2561)$;

// else use the high altitude equation

else density $=.00070611 * \exp (-\mathrm{k} 2 *(\mathrm{~h}-36089))$;

// return the calculated value

\}

return density; 


\section{Function "thrust_model.cpp"}

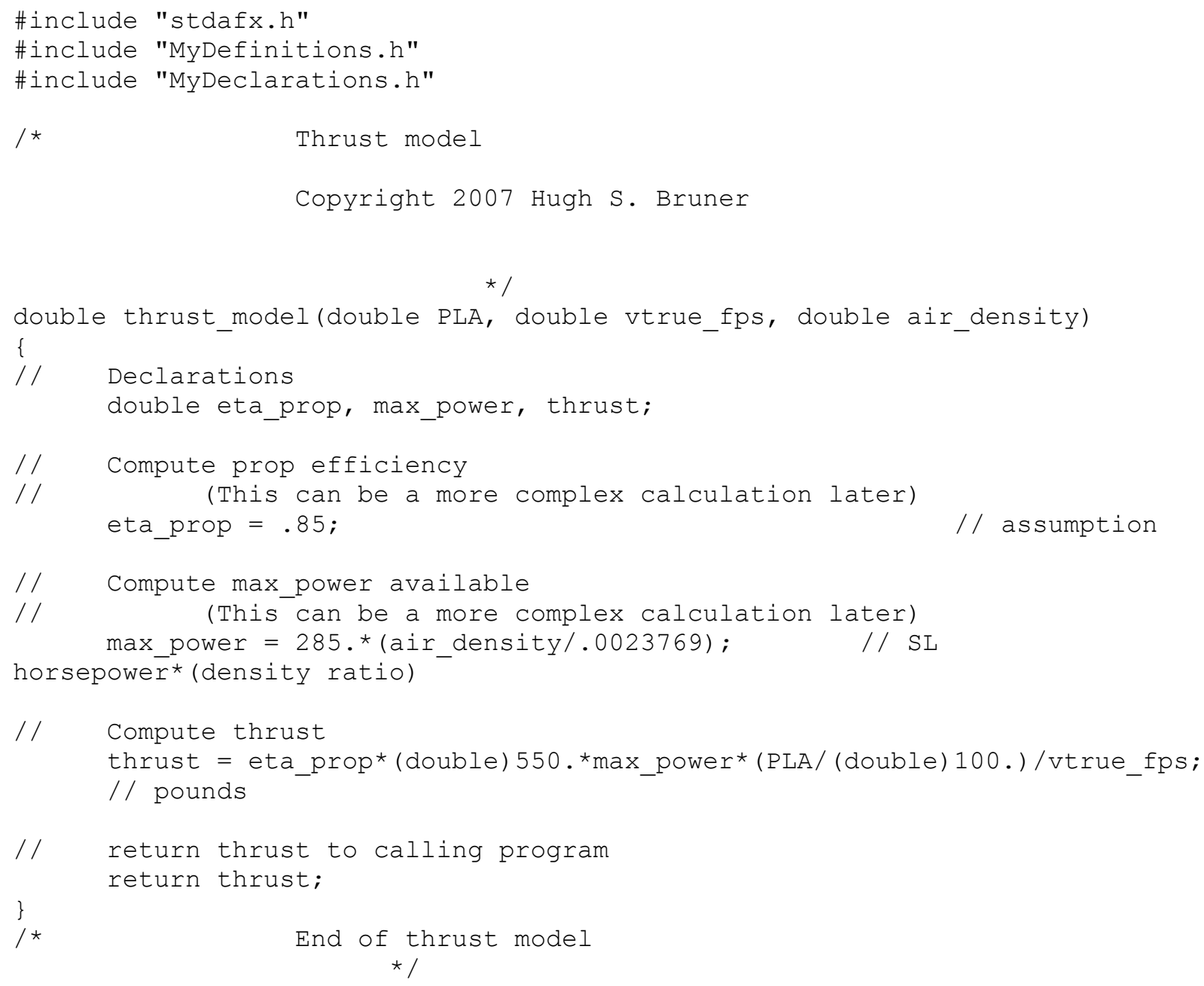




\section{Function "update_inv_state_space.cpp"}

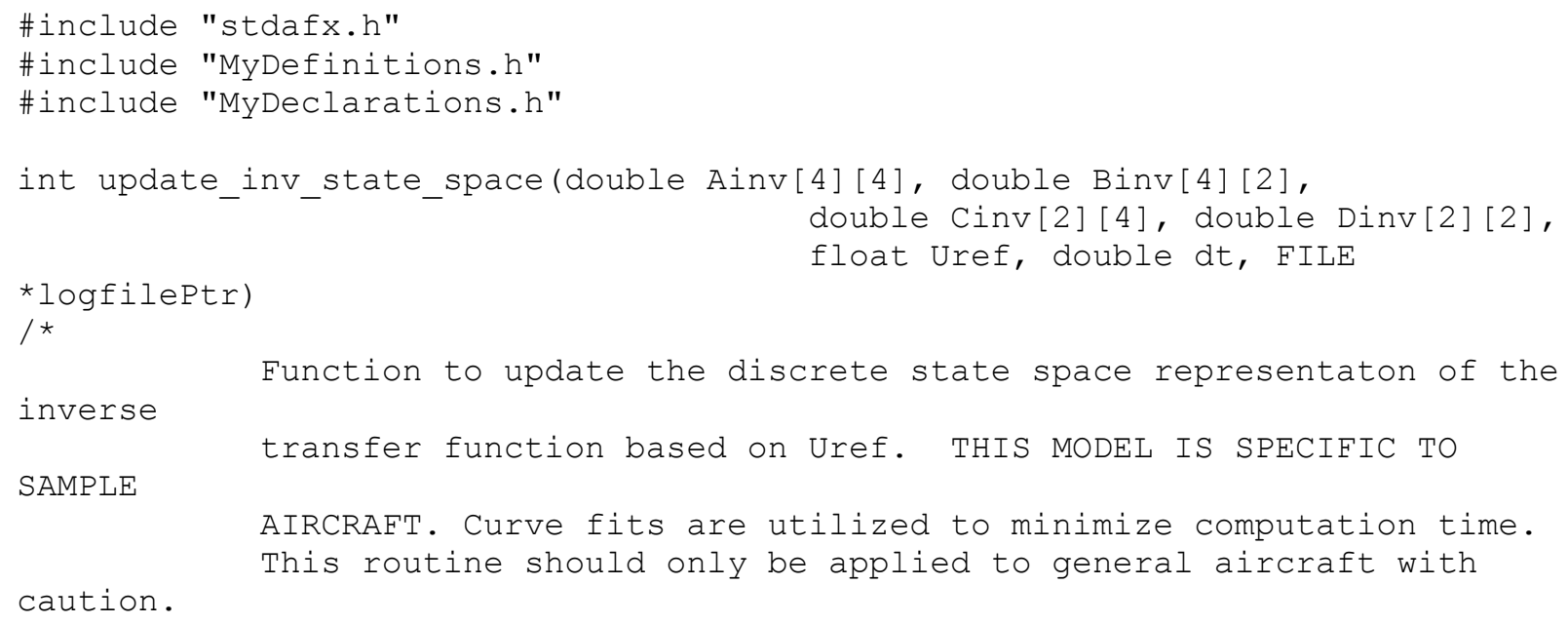




\begin{tabular}{|c|c|c|c|}
\hline $\begin{array}{l}1.94342 \mathrm{E}-06 \\
-1655.130196 \\
-9.11762 \mathrm{E}-07 \\
95.92485517\end{array}$ & $\begin{array}{l}-0.001927679, \\
0.000708776,\end{array}$ & $\begin{array}{l}-0.185771206 \\
-0.200159962,\end{array}$ & $\begin{array}{l}66.55999093, \\
20.28805436,\end{array}$ \\
\hline $\begin{array}{l}-3.52681 \mathrm{E}-10, \\
-0.480270499, \\
3.40111 \mathrm{E}-08, \\
-16.06159753, \\
1.94245 \mathrm{E}-08, \\
-16.55611886, \\
-1.75584 \mathrm{E}-08, \\
-5.035655039,\end{array}$ & $\begin{array}{l}3.00937 \mathrm{E}-07, \\
0.000154824, \\
-1.92703 \mathrm{E}-05, \\
1.29111 \mathrm{E}-05,\end{array}$ & $\begin{array}{l}-0.000100729, \\
-0.012747373, \\
0.008140766, \\
0.996546499,\end{array}$ & $\begin{array}{l}0.017129439, \\
0.597033475, \\
0.665747387, \\
0.358191636,\end{array}$ \\
\hline $\begin{array}{l}2.68231 \mathrm{E}-10, \\
0.538191281, \\
2.90171 \mathrm{E}-08, \\
1460.289464,\end{array}$ & $\begin{array}{l}-1.94295 \mathrm{E}-07, \\
1.26541 \mathrm{E}-05,\end{array}$ & $\begin{array}{l}-0.999944325 \\
-0.033613217\end{array}$ & $\begin{array}{l}-0.007873919 \\
-29.83748628\end{array}$ \\
\hline $\begin{array}{l}4.69749 \mathrm{E}-10, \\
0.791849949, \\
8.09309 \mathrm{E}-09, \\
33.15757478,\end{array}$ & $\begin{array}{l}-3.77296 \mathrm{E}-07 \\
-7.04237 \mathrm{E}-06\end{array}$ & $\begin{array}{l}0.000113382, \\
-0.997401277\end{array}$ & $\begin{array}{l}-0.015253098 \\
-0.413720457\end{array}$ \\
\hline $\begin{array}{l}1.76351 \mathrm{E}-08, \\
24.01471298, \\
9.64408 \mathrm{E}-05, \\
-80491.81989,\end{array}$ & $\begin{array}{l}-1.50476 E-05, \\
-0.104777537,\end{array}$ & $\begin{array}{l}0.005036652, \\
-58.09263113\end{array}$ & $\begin{array}{l}-0.856499023 \\
3248.455214\end{array}$ \\
\hline $\begin{array}{l}1.76351 \mathrm{E}-10, \\
0.24014713, \\
9.64408 \mathrm{E}-07, \\
-804.9181989,\end{array}$ & $\begin{array}{l}-1.50476 E-07, \\
-0.001047775,\end{array}$ & $\begin{array}{l}5.03665 E-05, \\
-0.580926311,\end{array}$ & $\begin{array}{l}-0.00856499 \\
32.48455214\end{array}$ \\
\hline $\begin{array}{l}\text { 2.39358E-08, } \\
60.37331964, \\
5.45087 \mathrm{E}-07, \\
643.8932847, \\
2.22017 \mathrm{E}-07, \\
-164.3586712, \\
8.7951 \mathrm{E}-07,-0.0007 \\
1669.650217,\end{array}$ & $\begin{array}{l}-1.98711 E-05, \\
-0.000505754, \\
-0.00021794, \\
715776, \quad 0\end{array}$ & $\begin{array}{l}0.006314563, \\
0.217432246, \\
0.090931658, \\
657264,\end{array}$ & $\begin{array}{l}-0.941228671, \\
-3.876508453, \\
-21.92177077, \\
8673301,\end{array}$ \\
\hline $\begin{array}{l}-1.36658 \mathrm{E}-09, \\
-1.680342435, \\
-5.11367 \mathrm{E}-08, \\
-1.98814302, \\
1.64029 \mathrm{E}-09, \\
0.723967273, \\
2.55338 \mathrm{E}-08, \\
101.1694261,\end{array}$ & $\begin{array}{l}-3.64726 \mathrm{E}-07, \\
0.000143908, \\
-1.40394 \mathrm{E}-06, \\
-0.000563057,\end{array}$ & $\begin{array}{l}8.28019 E-05, \\
-0.000824977, \\
-0.000225732, \\
0.00752846,-1\end{array}$ & $\begin{array}{l}-0.008259727, \\
0.062930077, \\
-0.001767297, \\
2424088,\end{array}$ \\
\hline $\begin{array}{l}-1.24021 E-08 \\
-30.44418306\end{array}$ & $1.02096 \mathrm{E}-05$ & -0.00322186 & 0.477345631 \\
\hline
\end{tabular}


9565.023116 ,
$1.0147 \mathrm{E}-05,-0.010092857$,
4.267611697,
-1126.22127,

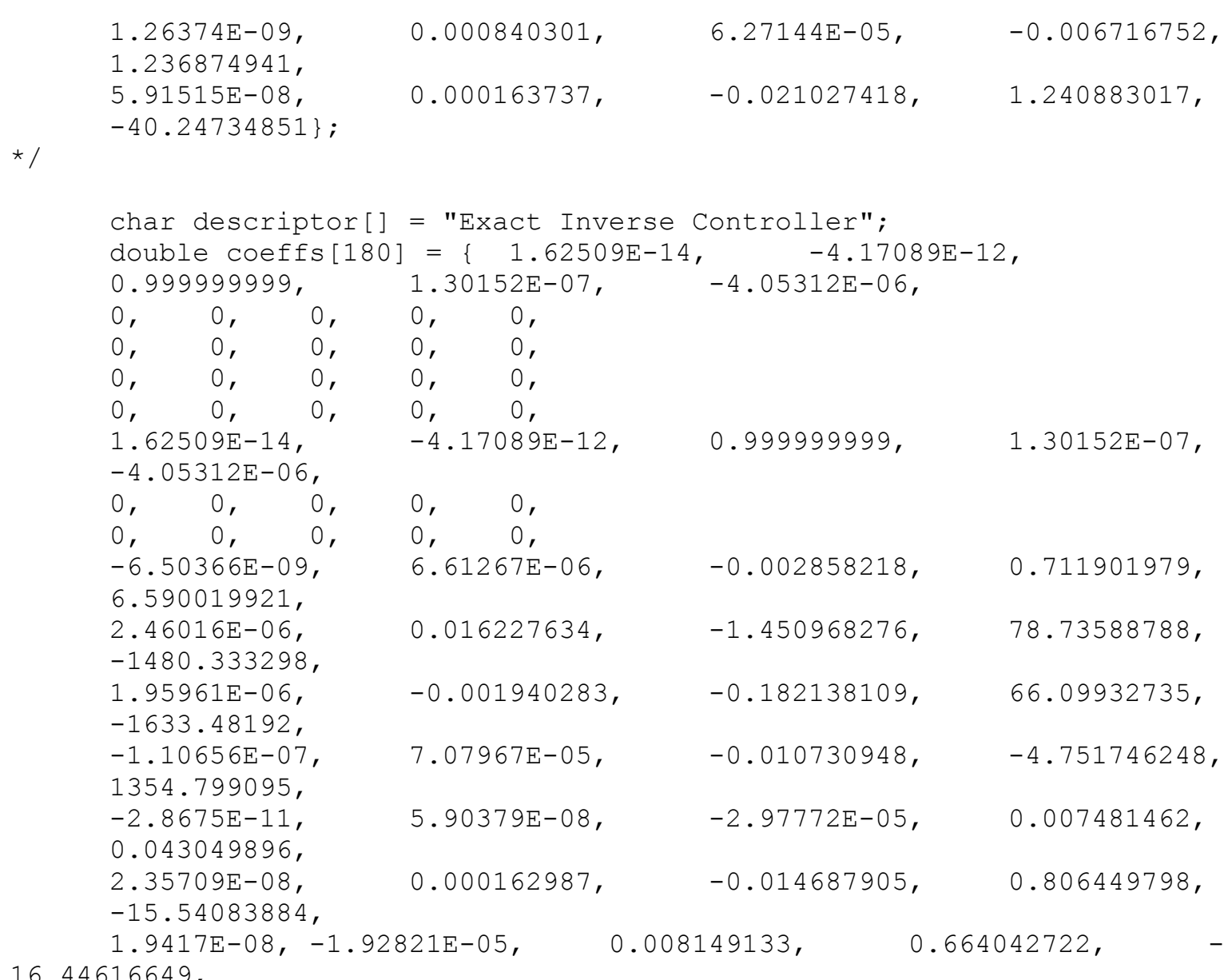
16.44616649 ,

$-1.87851 \mathrm{E}-09$,

1.29513E-06,

0.999733972,

-0.029467766,

12.8196868,

$-1.62509 \mathrm{E}-14$,

$4.17089 \mathrm{E}-12$,

-0.999999999,

$-1.30152 \mathrm{E}-07$,

4.05312E-06,

$0, \quad 0, \quad 0, \quad 0, \quad 0$,

$0, \quad 0, \quad 0, \quad 0, \quad 0$,

$-1.62509 \mathrm{E}-14$,

4.17089E-12, $\quad-0.999999999, \quad-1.30152 \mathrm{E}-07$,

4.05312E-06,

3.52613E-09,

-3.110346617 ,

$9.63602 \mathrm{E}-05$,

-81274.9572 ,

$-8.94547 \mathrm{E}-11$,

-0.187076916 ,

$9.63899 \mathrm{E}-07$,

-812.6784882 ,

$1.63227 \mathrm{E}-08$,

49.05236514,

-3.48242E-06, $\quad 0.001471937, \quad-0.360590366$,

$-0.10472806, \quad-58.1248125, \quad 3254.154347$,

$5.3555 \mathrm{E}-07$,

6.67899E-08, $-1.42535 \mathrm{E}-05, \quad-7.90274 \mathrm{E}-05$,

322.6146011 ,

2.19889E-07,

$-167.8942146$

$-0.001047449, \quad-0.581214892, \quad 32.53885814$,

$-1.39208 \mathrm{E}-05, \quad 0.004577177, \quad-0.714667496$,

$-0.000483242, \quad 0.203505211, \quad-0.775477582$,

$-0.000216167, \quad 0.090389311, \quad-21.8495734$, 


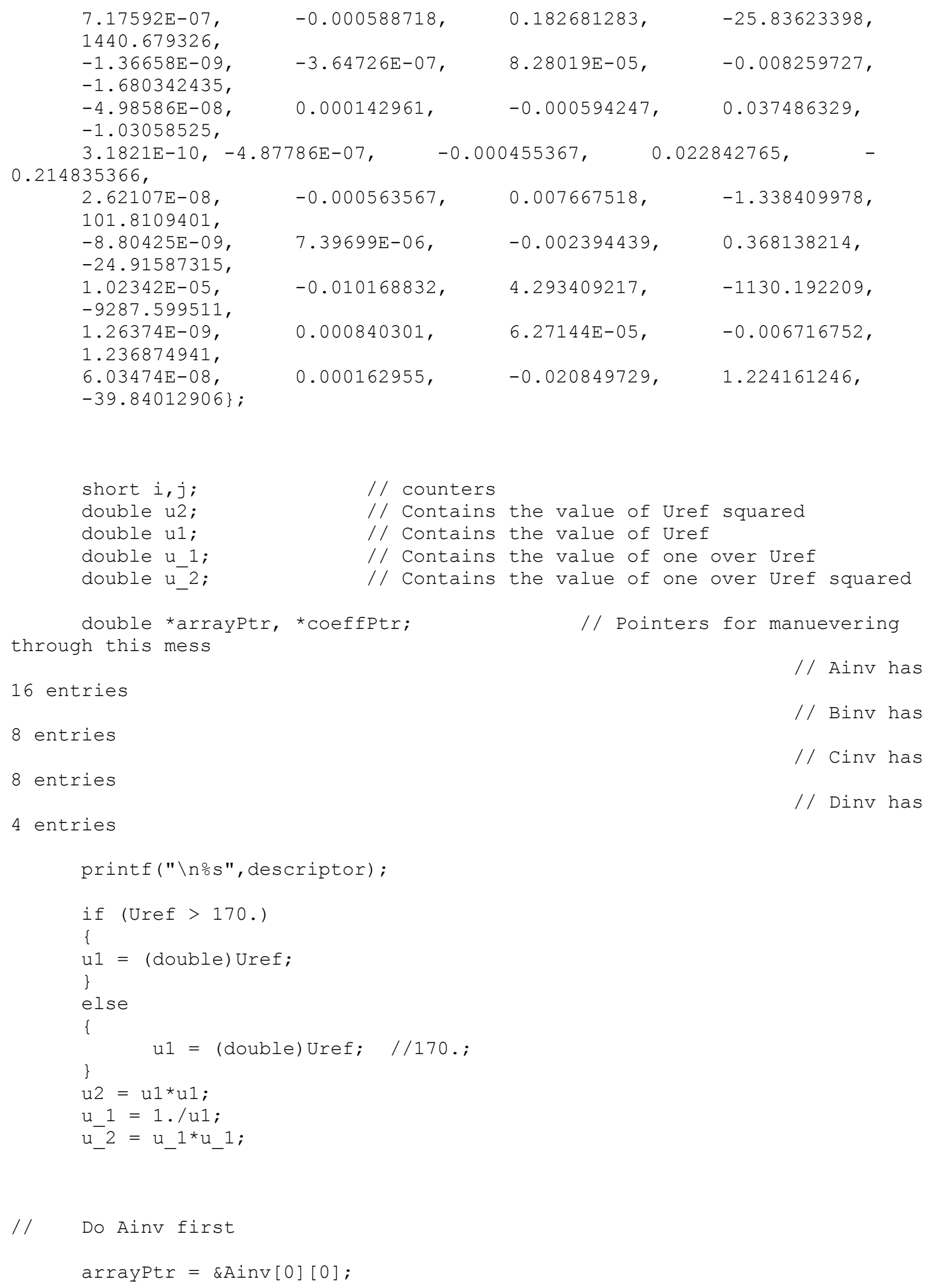




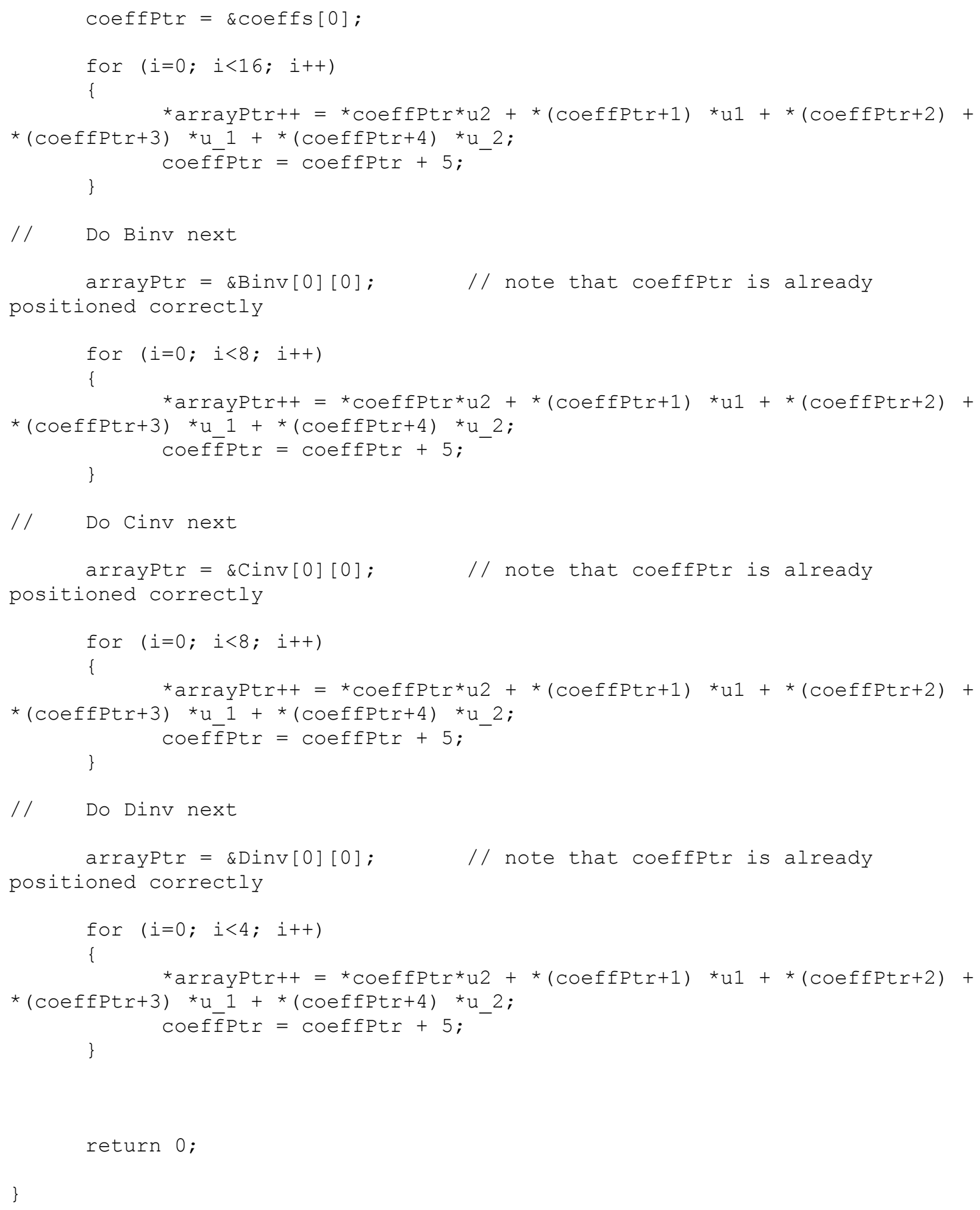




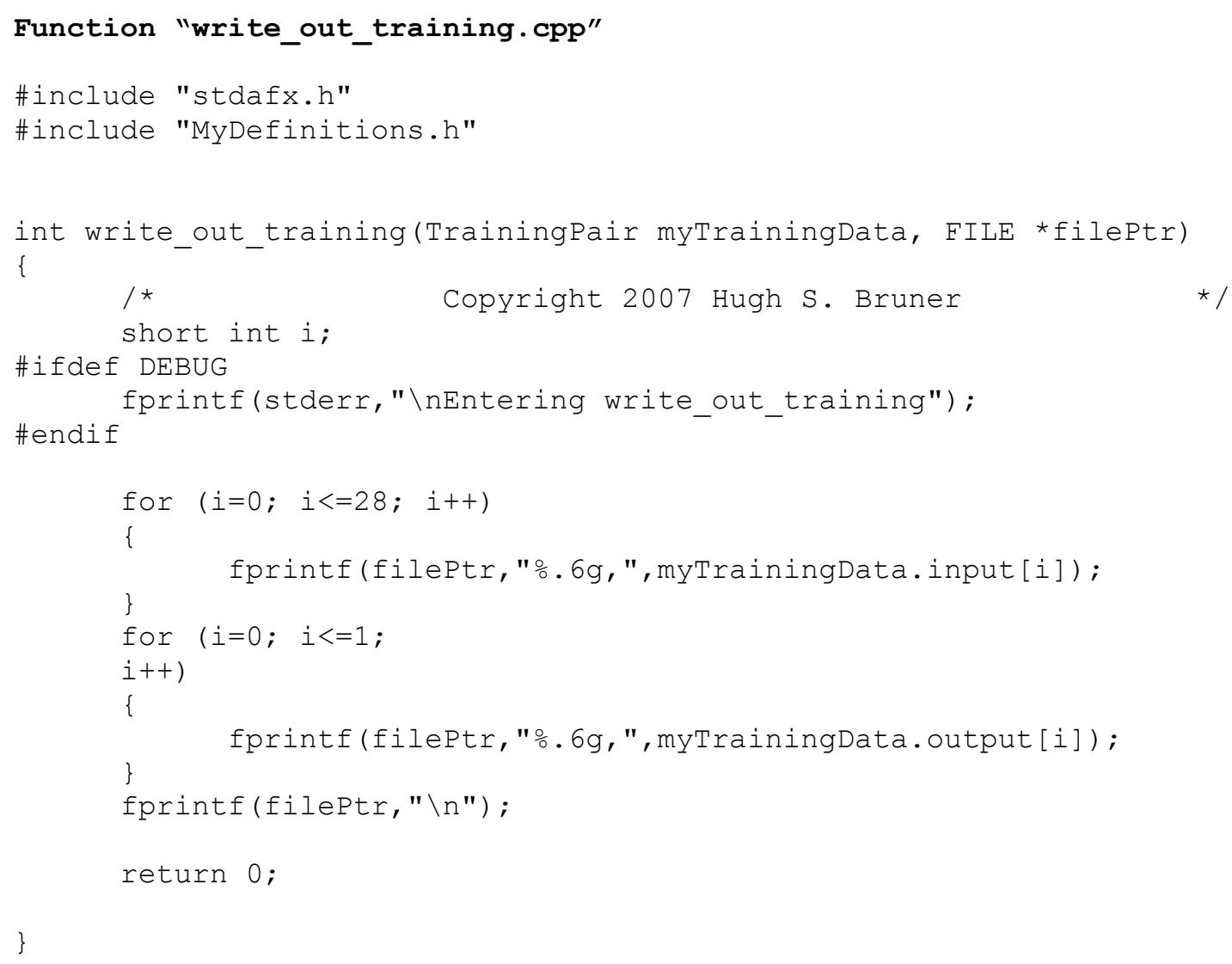

